in Proceedings of Symposia in Pure Mathematics 70 (2002) editors M. Fried and Y. Ihara, 1999 von Neumann Symposium on Arithmetic Fundamental Groups and Noncommutative Algebra at MSRI, 79-221.

Proceedings of Symposia in Pure Mathematics

\title{
Hurwitz monodromy, spin separation and higher levels of a Modular Tower
}

\author{
Paul Bailey and Michael D. Fried
}

\begin{abstract}
Each finite $p$-perfect group $G$ ( $p$ a prime) has a universal central $p$-extension coming from the $p$ part of its Schur multiplier. Serre gave a StiefelWhitney class approach to analyzing spin covers of alternating groups ( $p=$ 2) aimed at geometric covering space problems that included their regular realization for the Inverse Galois Problem.

A special case of a general result is that any finite simple group with a nontrivial $p$ part to its Schur multiplier has an infinite string of perfect centerless group covers exhibiting nontrivial Schur multipliers for the prime $p$. Sequences of moduli spaces of curves attached to $G$ and $p$, called Modular Towers, capture the geometry of these many appearances of Schur multipliers in degeneration phenomena of Harbater-Mumford cover representatives. These are modular curve tower generalizations. So, they inspire conjectures akin to Serre's open image theorem, including that at suitably high levels we expect no rational points.

Guided by two papers of Serre's, these cases reveal common appearance of spin structures producing $\theta$-nulls on these moduli spaces. The results immediately apply to all the expected Inverse Galois topics. This includes systematic exposure of moduli spaces having points where the field of moduli is a field of definition and other points where it is not.
\end{abstract}

\section{Contents}

List of Tables 81

1. Overview. related fundamental groups and preliminaries 81

1.1. Quick summary of results 82

1.2. Classical paths on $U_{2} \quad 85$

1.3. Results of this paper 86

1.4. General results and divergences with modular curves 89

2. Presenting $H_{4}$ as an extension $\quad 95$

2.1. Notation and the groups $B_{r}, H_{r}$ and $M_{r} \quad 95$

2.2. Geometrically interpreting $M_{4}$ and $\bar{M}_{4} \quad 96$

2000 Mathematics Subject Classification. Primary 11F32, 11G18, 11R58; Secondary 20B05, 20C25, 20D25, 20E18, 20F34.

Thm. 2.9] from a 1987 preprint of M. Fried, includes observations of J. Thompson. D. Semmen corrected a faulty first version of Prop. 5.4 and made the computations of Lem. 9.17 Support for Fried came from NSF \#DMS-9970676 and a senior research Alexander von Humboldt award. 
2.3. Proof of Prop. $\sqrt{2.3} \quad 98$

2.4. Statement of the main presentation theorem 100

2.5. Start of Theorem 2.9 proof 101

2.6. Properties of $\mathcal{Q}$; combinatorics of $\sqrt{2.117} 103$

2.7. The shift $\gamma_{1}=q_{1} q_{2} q_{1}$ and the middle twist $\gamma_{\infty}=q_{2} \quad 106$

2.8. General context with modular curve illustrations 107

2.9. $p$-lifting property and using sh-incidence $\quad 110$

2.10. The sh-incidence matrix 111

3. Nielsen classes and the Inverse Galois Problem 112

3.1. Equivalences on covers of $\mathbb{P}^{1} \quad 113$

3.2. Motivation from the Inverse Galois Problem 114

$\begin{array}{lll}3.3 . & \text { Frattini group covers } & 116\end{array}$

3.4. One prime at a time 118

3.5. Refined s-equivalence from $N_{S}(G . C) \quad 120$

$\begin{array}{ll}\text { 3.6. } p \text {-perfect groups and fine moduli } & 122\end{array}$

3.7. $\mathrm{SL}_{2}(\mathbb{C})$ action on Hurwitz spaces and covers of $\Lambda_{r}$ and $J_{r} \quad 124$

3.8. Monodromy groups of $\psi: \mathcal{H} \rightarrow U_{r}$ and $\psi^{\text {rd }}: \mathcal{H}^{\text {rd }} \rightarrow J_{r} \quad 126$

$\begin{array}{ll}\text { 4. Moduli and reduced Modular Towers } & 129\end{array}$

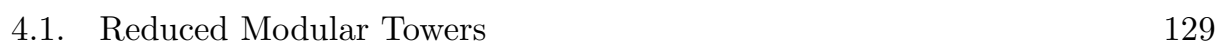

4.2. $j$-line covers when $r=4 \quad 130$

4.3. Reduced moduli spaces: b-fine and fine 131

4.4. Points on a Modular Tower 133

5. Group theorv of an $A_{5}$ Modular Tower 136

$\begin{array}{ll}\text { 5.1. Frattini curve covers } & 137\end{array}$

5.2. The normalizer of a $p$-Svlow and Loewv displav 137

5.3. Producing ${ }_{2} \tilde{A}_{5} \quad 140$

5.4. Cusps and a theorem of Serre 143

5.5. Further Modular Towers for $A_{5}, p=2$ and $r=4 \quad 144$

$\begin{array}{ll}\text { 5.6. Modular Towers from } p \text {-split data } & 147\end{array}$

5.7. Criteria for $G_{k}$ faithful on $M_{k}$ and appearance of $\mathbf{1}_{c \text {. }}$

6. Real points on Hurwitz spaces 149

6.1. Diophantine properties of a Modular Tower 149

6.2. Bounding levels with $K$ points $\quad 150$

6.3. Remainder of Thm. 6.1proof 151

6.4. Real points when $r=4 \quad 153$

6.5. Level $0 \mathbb{R}$ points on the $\left(A_{5}, \mathbf{C}_{24}\right)$ Modular Tower 156

7. Cusps on a Modular Tower 158

7.1. Fundamental domains and cusp data 158

7.2. Cusp notation 160

7.3. H-M and near H-M reps. in $\mathrm{Ni}\left(G_{1}, \mathbf{C}_{24}\right)$

8. Cusp widths and the genus of components 165

8.1. Orbit genus and fixed points of $\gamma_{0}$ and $\gamma_{1} \quad 166$

$\begin{array}{lll}\text { 8.2. Subtle conjugations } & 169\end{array}$

8.3. Length two and four cusp widths 171

8.4. Cusp widths of length 6 and $10 \quad 173$

8.5. The sh-incidence matrix at level 1 176

8.6. Real points on level 1 of the $\left(A_{5} . \mathbf{C}_{24}\right)$ Modular Tower 178

9. Completing level 1 of an $A_{5}$ Modular Towen 181 
9.1. Diophantine implications 181

9.2. Working with $\left|\mathbf{S}^{+} \mathbf{9 5}\right| 182$

9.3. Schur multipliers detect two $H_{4}$ orbits on $\mathrm{Ni}_{1}=\mathrm{Ni}\left(G_{1} \cdot \mathbf{C}_{24}\right.$

9.4. Spin separation and two $\bar{M}_{4}$ orbits on $\mathrm{Ni}_{1}^{\text {rd }} \quad 188$

$\begin{array}{ll}\text { 9.5. Understanding spin separation } & 193\end{array}$

9.6. Inductive steps in going to higher Modular Tower levels 196

9.7. Generalizing the notion of H-M reps. 197

Appendix A. Coset description of a fundamental domain for $\mathcal{H}^{\text {rd }} \quad 199$

Appendix B. Serre's half-canonical invariant 199

B.1. Half-canonical classes from odd ramification 200

B.2. Coordinates from $\theta$ functions 200

$\begin{array}{lll}\text { B.3. } \theta \text { functions along the moduli space } & 201\end{array}$

B.4. Monodromv. characteristics and Prvms 204

Appendix C. Covers and lifting invariants 207

C.1. The lifting invariant and Nielsen separation 207

C.2. Frattini central extensions 208

Appendix D. The Open Image Theorem 208

D.1. Interpreting and extending the Open Image Theorem 208

D.2. $p$-part of the outer monodromv group 209

D.3. Finding the Frobenius 210

Appendix E. Simple branching and $j$-awareness 212

E.1. More complicated Hurwitz spaces for non-p-perfect $G$ 0 212

E.2. Modular curve quotients from a Modular Tower 212

Appendix F. A representation difference between $\bar{M}_{4}$ on $\mathrm{Ni}_{1}^{+}$and $\mathrm{Ni}_{1}^{-} \quad 215$

Appendix G. Where are those regular realizations? 216

G.1. Reminder of the setup 216

G.2. From Chevallev realizations to the whole $p$-Frattini tower 217

$\begin{array}{ll}\text { References } & 218\end{array}$

LIST OF TABLES

$\begin{array}{lll}1 & \text { List } \mathrm{Ni}\left(A_{5} \cdot \mathbf{C}_{34}\right)^{\text {abs }} & 105\end{array}$

2 sh-Incidence Matrix for $\mathrm{Nin} \quad 112$

3 Constellation of spaces $\mathcal{H}\left(A_{n}, \mathbf{C}_{3 r}\right) 122$

$4 \mathrm{mps} 10 \times 10$ part of sh-Incidence Matrix for $O_{1}^{+} \quad 178$

5 The configuration of $\mathbb{P}^{1} \mathrm{~S}$ at a special fiber 210

\section{Overview, related fundamental groups and preliminaries}

Here is one corollary of a result from the early 1990s. There is an exact sequence ([FV92, discussed in [MM99, Cor. 6.14] and [Vö96 Cor. 10.30]):

$$
1 \rightarrow \tilde{F}_{\omega} \rightarrow G_{\mathbb{Q}} \rightarrow \prod_{n=2}^{\infty} S_{n} \rightarrow 1
$$

The group on the left is the profree group on a countable number of generators.

The group on the right is the direct product of the symmetric groups, one copy 
for each integer: (1.1) catches the absolute Galois group $G_{\mathbb{Q}}$ of $\mathbb{Q}$ between two known groups. Suppose a subfield $K$ of $\overline{\mathbb{Q}}$ has $G_{K}$ a projective (profinite) group. Further, FV92 conjectures: Then $G_{K}$ is pro-free (on countably many generators) if and only if $K$ is Hilbertian. The conjecture generalizes (1.1) and Shafarevich's conjecture that the cyclotomic closure of $\mathbb{Q}$ has a pro-free absolute Galois group.

So, (1.1) is a positive statement about the Inverse Galois Problem. Still, it works by maneuvering around the nonprojectiveness of $G_{\mathbb{Q}}$ as a projective profinite group. Modular Towers captures, in moduli space properties, implications of this nonprojective nature of $G_{\mathbb{Q}}$. Its stems from constructing from each finite group $G$, a projective profinite group $\tilde{G}$ whose quotients naturally entangle $G$ with many classical spaces: modular curves (2.8) and spaces of Prym varieties (B.4 and E.2, to mention just two special cases. This $\tilde{G}$ started its arithmetic geometry life on a different brand of problem [FJ86, Chap. 21].

Fri95a gave definitions, motivations and applications surrounding Modular Towers starting from the special case of modular curve towers. This paper continues that, with a direct attack on properties of higher Modular Tower levels. Our models for applications include the Open Image Theorem on modular curve towers in Ser68] (details in D.2). In Modular Tower's language this is the (four) dihedral group involution realizations case (Fri94] and [Fri95c have very elementary explanations). Modular Towers comes from profinite group theory attached to any finite group, a prime dividing its order and a choice of conjugacy classes in the group. This moduli approach uses a different type of group theory - modular representations - than the homogeneous space approach typical of modular curves or Siegel upper half space. Still, these spaces appear here with a more elementary look and a new set of applications.

It shows especially when we compare the monodromy groups of the tower levels with those for modular curves. A key property used in [Ser68: Levels of a modular curve tower attached to the prime $p(p \geq 5)$ have a projective sequence of Frattini covers as monodromy groups. Results similar to this should hold also for Modular Towers (Ques. D.1). That is part of our suggestion that there is an Open Image Theorem for Modular Towers. We first briefly summarize results; then set up preliminaries to explain them in detail. The name Harbater-Mumford representatives appears so often, it could well have been part of the title. Its precise definition is as a type of element in a Nielsen class. Geometrically its meaning for Hurwitz spaces is an especially detectable degeneration of curves in a family. The name derives from two papers: Ha84 and Mu72. 99.7 recognizes there are two uses made of $\mathrm{H}-\mathrm{M}$ reps. It suggests generalizations of these based on $p^{\prime}$ properties.

The tough question for Modular Towers is not if it is useful or connected well to the rest of mathematics. Rather, it is if its problems are sufficiently tractable for progress. By proving our Main Conjecture in convincing cases, with analytic detail often applied to modular curves, we assure rapid progress is possible. The Main Conjecture shows regular realizations of significant finite groups as Galois groups will not appear serendipitously. Indeed, the Hurwitz space approach gives meaning to considering where to look for them. Therefore, we have put a summary statement for those who ask:

So, where are those regular realizations $(\mathrm{G})$ ?

1.1. Quick summary of results. Four branch point Hurwitz families produce quotients of the upper half plane by finite index subgroups of $\mathrm{PSL}_{2}(\mathbb{Z})$. The 
Hurwitz monodromy group $H_{4}$ a group $\bar{M}_{4}$, identified with $\mathrm{PSL}_{2}(\mathbb{Z})$, as a natural quotient. The structure of a Modular Tower gives sequences of $j$-line covers formed by natural moduli problems. These are immensely more numerous than the special case of modular curve towers though they have both similarities to them and exotic new properties generally.

There is a Modular Tower for any finite group $G$, prime $p$ dividing $|G|$ and collection $\mathbf{C}$ of $(r \geq 3) p^{\prime}$ conjugacy classes. Then $G$ has a universal $p$-Frattini profinite group cover, the profinite limit of a sequence $\left\{G_{k}\right\}_{k=0}^{\infty}$ of $p$-extensions of $G=G_{0}$. Each level $k \geq 0$ has a moduli space $\mathcal{H}_{k}$ in the (reduced) Modular Tower. When $r=4$ all components of the $\mathcal{H}_{k}$ s are $j$-line covers. Then, reduced inner Hurwitz spaces generalize the modular curve sequence $\left\{Y_{1}\left(p^{k+1}\right)\right\}_{k=0}^{\infty}$. Analyzing the action of $H_{4}$ (and $\bar{M}_{4}$ ) on Nielsen classes attached to each Modular Tower level gives their detailed structure. If there is $k \geq 0$ with all $\mathcal{H}_{k}$ components of genus at least 2, then for each number field $K$ there is $k_{G_{0}, \mathbf{C}, K}$ with $\mathcal{H}_{k}(K)=\emptyset$ for $k \geq k_{G_{0}, \mathbf{C}, K}$ (Thm. 6.1).

Main Conjecture (FK97, Main Conj. 0.1], Prob. 1.1 86.1.2): For $r \geq 4, G$ centerless and $p$-perfect (3.6.1), and an explicit $k_{G_{0}, \mathbf{C}}^{\prime}=k_{0}^{\prime}$, all $\mathcal{H}_{k}$ components have general type if $k \geq k_{G_{0}, \mathbf{C}}^{\prime}$. So, if $r=4$, the genus of $\mathcal{H}_{k}$ components, $k \geq k_{0}^{\prime}$, exceeds 1. 3.4.2 reminds how finding regular realizations of the collection $\left\{G_{k}\right\}_{k=0}^{\infty}$ forces this problem on the levels of a Modular Tower. The methods of the paper pretty much restrict to the case $r=4$. We use $p=2$ and $G=A_{5}$ to find considerable about excluding $r=4$ for general $(G, p)$. 9.6 states the unsolved problems for $r=4$ framed by the genus computations of 8.1 The effect of increasing components in going to higher levels of a Modular Tower justifies concentration on analyzing the component structures at level 1 in our main examples.

For $G_{0}=A_{5}, p=2$ and $\mathbf{C}_{3^{4}}$, four 3-cycle conjugacy classes, $k_{0}^{\prime}=1$ works. Level $k=0$ has one (genus 0 ) component. Level $k=1$ is the moduli of Galois covers with group ${ }_{2}^{1} \tilde{A}_{5}=G_{1}$, a maximal Frattini extension of $A_{5}$ with exponent 2 kernel, and order 3 branching. A $\mathbb{Q}$ regular realization of $\left({ }_{2}^{1} \tilde{A}_{5}, \mathbf{C}_{3^{4}}\right)$ produces a $\mathbb{Q}$ point on level 1. Level 1 components $\left(\mathcal{H}_{1}^{+}\right.$and and $\mathcal{H}_{1}^{-}$, of genuses 12 and 9$)$ correspond to values of a spin cover invariant (as in [Fri95a, Part III] and Serre [Ser90b). Pairings on Modular Tower cusps appear in the symmetric sh-incidence matrix. Each component corresponds to a mapping class orbit on reduced Nielsen classes, whose notation is $\operatorname{Ni}\left({ }_{2}^{1} \tilde{A}_{5}, \mathbf{C}_{3^{4}}\right)\left(\mathrm{Ni}\left(G_{k} \mathbf{C}\right)\right.$ for the level $k$ Nielsen class generally).

Level 1 has one component of real points containing all Harbater-Mumford $(\mathrm{H}-$ $\mathrm{M})$ and near $H-M$ reps. (Thm. 8.19). The finitely many $\left(\mathrm{PGL}_{2}(\mathbb{C})=\mathrm{PSL}_{2}(\mathbb{C})\right.$ equivalence) $\left({ }_{2}^{1} \tilde{A}_{5}, \mathbf{C}_{3^{4}}\right)$ regular $\mathbb{Q}$ realizations are on the genus 12 component. Our computation for real points emphasizes that cusps also interpret as elements of a Nielsen class. This combines geometry with group computations. H-M cusps are real points on the reduced moduli space representing total degeneracy of the moduli of curve covers upon approach to the cusp. The near H-M cusps, also real, are a Modular Tower phenomenon. The corresponding cusp at one level below in the tower, is an H-M rep. Above level 0, H-M and near H-M cusps are the only real cusps. This holds for any Modular Tower when the prime $p$ is 2 .

Given $\alpha_{0} \in H^{2}\left(G_{0}, \mathbb{Z} / p\right)$ there is a universal sequence of nontrivial elements $\left\{\alpha_{k} \in H^{2}\left(G_{k}, \mathbb{Z} / p\right)\right\}_{k=0}^{\infty}$. Properties are in Prop. 9.6 This is a special case of [FK97, Schur multipliers results 3.3]. An embedding of a group $G$ in the alternating group $A_{n}(n \geq 4)$ is spin separating if the lift $\hat{G}$ to the spin cover $\operatorname{Spin}_{n}=\hat{A}_{n} \rightarrow A_{n}$ 
is nonsplit over $G$ (see Def. 9.11). Such a central extension represents an element $\alpha \in H^{2}(G, \mathbb{Z} / 2)$. Given an a priori $\alpha$, we also say the embedding of $G$ in $A_{n}$ is $\alpha$ spin separating. When $G_{0}=G$, this produces a sequence $\left\{\alpha_{k} \in H^{2}\left(G_{k}, \mathbb{Z} / 2\right)\right\}_{k=0}^{\infty}$ (as above for $p=2$ ). So, each $\alpha_{k}$ represents a central extension $T_{k}^{\prime} \rightarrow G_{k}$ with $\mathbb{Z} / 2$ as kernel. Finding spin separating representations for these extensions has applications that include identifying projective systems of components on certain Modular Tower levels. These conjecturally traces to the evenness and oddness of associated $\theta$-nulls on these components ( $\mathrm{B})$.

Prop. 9.8 produces spin separating representations of $G_{1}$ for which $\hat{G}_{1}=T_{1}^{\prime}$ is $\alpha_{1}$. Let $M$ be the kernel of $G_{1} \rightarrow A_{5}$ and use that $G_{1}$ factors through the spin cover $\mathrm{SL}_{2}(\mathbb{Z} / 5) \rightarrow A_{5}$. A special case of a general operating principle applies next. With $\boldsymbol{g} \in \mathrm{Ni}\left(A_{5}, \mathbf{C}_{3^{4}}\right)$ an H-M rep. at level 0, let $\bar{M}_{\boldsymbol{g}}$ be the subgroup of $\bar{M}_{4}$ stabilizing $\boldsymbol{g}$. This allows us (a significant special case of a general situation) to regard $\bar{M}_{\boldsymbol{g}}$ acting on $(a, b) \in M^{2}$ with both $a, b$ over $-1 \in \mathrm{SL}_{2}(\mathbb{Z} / 5)$ and lying in a certain linear subspace of $M \times M$. There are two orbits: $a$ and $b$ in the same $A_{5}$ orbit (action on $M$ ); and $a$ and $b$ in different $A_{5}$ orbits (Prop. 9.8). This gives information on all levels of the same tower, and toward the Main Conjecture when $r=4$ for all groups $G_{0}=G(9.6)$.

Finite covers of the $r$-punctured Riemann sphere $\mathbb{P}_{z}^{1}=\mathbb{C} \cup\{\infty\}$ have an attached group $G$ and unordered set of conjugacy classes $\mathbf{C}$ of $G$. The braid group approach to the Inverse Galois Problem combines moduli space geometry with finite group representations to find $(G, \mathbf{C})$ regular realizations as rational points on Hurwitz spaces. Fri99 includes an update on applying this to classical problems without obvious connection to the Inverse Galois Problem.

The history of the Inverse Galois Problem shows it is hard to find $\mathbb{Q}$ realizations of most finite groups $G$. Such a realization is a quotient of $\pi_{1}\left(U_{\boldsymbol{z}}, z_{0}\right): U_{z}$ is $\mathbb{P}_{z}^{1}$ with $r$ distinct points $z$ removed. To systematically investigate the diophantine difficulties we take large (maximal Frattini) quotients ${ }_{p} \tilde{G}$ of $\pi_{1}\left(U_{\boldsymbol{z}, z_{0}}\right)^{\text {alg }}$ covering $G$ instead of one finite group $G$. Modular Towers are moduli space sequences encoding quotients of $\pi_{1}\left(U_{\boldsymbol{z}}, z_{0}\right)$ isomorphic to ${ }_{p} \tilde{G}$ as $z$ varies. This generalizes classical modular curve sequences, and their traditional questions, through an Inverse Galois Problem formulation. The modular curve case starts with a dihedral group $D_{p}=\mathbb{Z}_{p} \times{ }^{s}\{ \pm 1\}$, and a natural projective limit of the dihedral groups $\left\{D_{p^{k+1}}\right\}_{k=0}^{\infty}$. In the generalization, the maximal $p$-Frattini (universal $p$-Frattini; 3.3 ) cover ${ }_{p} \tilde{G}$ of any finite group $G$ replaces $\mathbb{Z}_{p} \times^{s}\{ \pm 1\}$.

Levels of a Modular Tower correspond to characteristic quotients $\left\{G_{k}\right\}_{k=0}^{\infty}$ of ${ }_{p} \tilde{G}$, replacing $\left\{D_{p^{k+1}}\right\}_{k=0}^{\infty}$. Finding $\mathbf{C}$ regular realizations of the groups $\left\{G_{k}\right\}_{k=0}^{\infty}$ is to finding rational points on levels of a Modular Tower as finding regular involution realizations of $\left\{D_{p^{k+1}}\right\}_{k=0}^{\infty}$ is to finding rational points on the modular curves $\left\{X_{1}\left(p^{k+1}\right)\right\}$ (2.8.1). Though the Modular Tower definition supports many general properties of modular curves, when $r>4$ the moduli space levels are algebraic varieties of dimension $r-3>1$. By conjecture (under the $p$-perfect hypothesis) we expect a structured disappearance of rational points at high levels in generalization of modular curves. This holds by proof in many cases beyond those of modular curves from this paper.

Modular Towers joins number theory approaches to $G_{\mathbb{Q}}$ actions on one hand to the Inverse Galois Problem braid group approach. Profinite systems of points on Modular Towers reveal relations in the Lie group of $G_{\mathbb{Q}}$ attached to a $p$-adic 
representation. Yet, they benefit from the explicit tools of finite group theory (modular representations).

Our concentration is on Modular Towers for groups distinct from dihedral groups. Many have geometric properties not appearing for towers of modular curves. Especially they can have several connected components. The Hurwitz viewpoint applied to towers of modular curves shows how some points of general Modular Towers work. Examples: 2.8.1 on cusps of the modular curves $X_{0}\left(p^{k+1}\right)$ and $X_{1}\left(p^{k+1}\right)$; and $\$ 2.8 .3$ comparing the moduli problems from isogenies of elliptic curves with the Hurwitz space interpretation of these spaces. Each Modular Tower level maps naturally to a space of abelian varieties, given by the jacobians (with dimension inductively computed from $\mathrm{E}$ each far from simple) of the curve covers parametrized by the points of the moduli space. For example, level 1 of our $\left(A_{5}, \mathbf{C}_{3^{4}}, p=2\right)$ Modular Tower maps to a one-dimensional (nonconstant) space of Jacobians having dimension $1+2^{7}$. far is a Modular Tower from being a tower of modular curves.

1.2. Classical paths on $U_{z}$. Let $z=\left(z_{1}, \ldots, z_{r}\right)$ be distinct points (punctures on $\mathbb{P}_{z}^{1}$ ) and denote $\mathbb{P}_{z}^{1} \backslash z$ by $U_{z}$. Riemann's Existence Theorem produces $(G, \mathbf{C})$ covers. Classical generators come from paths, $P_{i}=\gamma_{i} \delta_{i} \gamma_{i}^{-1}, i=1, \ldots, r$, satisfying these conditions:

(1.2a) $\delta_{i}$ clockwise bounds a disc $\Delta_{i}$ around $z_{i}, \Delta_{i} \backslash\left\{z_{i}\right\} \subset U_{z}$;

(1.2b) $\gamma_{i}$ starts at $z_{0}$ and ends at some point on $\delta_{i}$; and

(1.2c) excluding their beginning and end points, $P_{i}$ and $P_{j}$ never meet if $i \neq j$.

Let $\bar{g}_{1}, \ldots, \bar{g}_{r}$ be the respective homotopy classes of $P_{1}, \ldots, P_{r}$. Use (1.2t] to attach an order of clockwise emanation to $P_{1}, \ldots, P_{r}$. Assume it is in the order of their subscript numbering. Then:

(1.3) $\bar{g}_{1} \bar{g}_{2} \ldots \bar{g}_{r}=1$ : The product-one condition.

A surjective homomorphism $\psi: \pi_{1}\left(U_{\boldsymbol{z}}, z_{0}\right) \rightarrow G$ produces a cover with monodromy group $G$. More precise data stipulates that a system of classical generators map to $\mathbf{C}$. Such a $\psi$ is a geometric $(G, \mathbf{C})$ representation.

If $\alpha$ is an automorphism of $\pi_{1}\left(U_{\boldsymbol{z}}, z_{0}\right)$, it sends generators to new generators, changing $\psi$ to $\psi \circ \alpha$. An inner automorphism of $\pi_{1}\left(U_{\boldsymbol{z}}, z_{0}\right)$ produces a cover equivalent to the old cover. We use moduli of covers, so equivalence two homomorphisms if they differ by an inner automorphism. Only automorphisms of $\pi_{1}\left(U_{\boldsymbol{z}}, z_{0}\right)$ from the Hurwitz monodromy group $H_{r}$ send classical generators to classical generators (2.4): possibly changing the intrinsic order of the paths). Such automorphisms arise by deforming $\left(\boldsymbol{z}, z_{0}\right)$. They permute the conjugacy classes of the $\bar{g}_{i} \mathrm{~s}$ in $\pi_{1}\left(U_{\boldsymbol{z}}, z_{0}\right)$. Given $\psi, \mathbf{C}$ is a well defined $H_{r}$ invariant.

Suppose $z_{0}$ and the unordered set $z$ have definition field $\mathbb{Q}$. Lem. 3.7 the Branch Cycle Lemma, gives a necessary (rational union) condition for an arithmetic $(G, \mathbf{C})($ over $\mathbb{Q})$ representation; $\psi$ factors through a representation of the arithmetic fundamental group ( $(3.2)$. Such a cover is a $(G, \mathbf{C})$ (regular) realization (over $\mathbb{Q}$ ).

For every finite group $G$ and prime $p$ dividing $|G|$, there is a universal $p$ Frattini (3.3) cover ${ }_{p} \tilde{G}$ of $G$. Generators of $G$ lift automatically to generators of any finite quotient of ${ }_{p} \tilde{G}$ factoring through $G$. This and other properties give all finite quotients of ${ }_{p} \tilde{G}$ a touching resemblance to $G$. A Modular Tower (\$4) is the system of moduli spaces encoding all $p$-Frattini $(G, \mathbf{C})$ covers of $\mathbb{P}_{z}^{1}(3.4 .2)$. Reducing by $\mathrm{PSL}_{2}(\mathbb{C})$ action gives a reduced Modular Tower. Let $Y_{1}\left(p^{k}\right)$ be the 
modular curve $X_{1}\left(p^{k}\right)$ without its cusps. With $G$ a dihedral group $D_{p}$ ( $p$ odd) and C four repetitions of the involution conjugacy class, the (reduced) Modular Tower is the curve sequence

$$
\cdots \rightarrow Y_{1}\left(p^{k+1}\right) \rightarrow Y_{1}\left(p^{k}\right) \rightarrow \cdots \rightarrow Y^{1}(p) \rightarrow \mathbb{P}_{j}^{1} \backslash\{\infty\} .
$$

Our main results compute properties of an $A_{5}$ Modular Tower attached to $\mathbf{C}_{3^{4}}$, four repetitions of elements of order 3 as associated conjugacy classes $\mathbf{C}_{3^{4}}$. 3 extends the general discussion of Modular Towers beyond Fri95a and [FK97.

1.3. Results of this paper. When $r=4$, reduced Modular Tower levels are curves covering the classical $j$-line. As they are upper half plane quotients by finite index subgroups of $\mathrm{SL}_{2}(\mathbb{Z})$, this case shows how reduced Modular Towers generalize properties of modular curves. The groups $H_{4}$ (Hurwitz monodromy group) and $\bar{M}_{4}$ (reduced mapping class group) from Thm. 2.9 apply to make reduced Modular Tower computations such as these.

(1.4a) Branch cycle descriptions and cusp data for these $j$-line covers (4.2).

(1.4b) A Klein 4-group $\mathcal{Q}^{\prime \prime}$ test for reduced Hurwitz spaces being $b$-fine or fine moduli spaces (Prop. 4.7).

The group $\mathcal{Q}^{\prime \prime}$ is a quotient of a normal quaternion subgroup of $H_{4}$. We use the term $b$ (irational)-fine to mean that excluding the locus over $j=0$ or 1 (or $\infty$ ), the reduced space is a fine moduli space. The geometric description of these groups in Prop. 2.3 presents arithmetic conclusions when the action of $\mathcal{Q}^{\prime \prime}$ on Nielsen classes is trivial. These are convenient for applications to the Inverse Galois Problem (see 2.3 .4 and Rem. 2.6.

The universal $p$-Frattini cover of a finite group $G$ lies behind the construction of Modular Towers. Any triple $(G, p, \mathbf{C})$ with $p$ a prime dividing $|G|$ and $\mathbf{C}$ some $p^{\prime}$ conjugacy classes in $G$ produces a tower of moduli spaces. $\$ 5$ is brief about the relevant group theory, relying on results from [FK97. Still, 5.1 reminds of the geometry of inaccessibility that makes Frattini covers intriguing. The moduli space phenomena in the case $G=A_{5}$ and $p=2$ interprets modular representation theory in the geometry of the particular Modular Tower.

1.3.1. 2-Frattini covers of $A_{5}$. 5 describes the characteristic quotients of the universal 2-Frattini cover of $A_{5}$. It produces the sequence of groups $\left\{G_{k}\right\}_{k=0}^{\infty}$ defining the levels of the Modular Tower of this paper where $G_{0}=A_{5}$. 6.4 uses $j$-line branch cycles (4.2) to draw diophantine conclusions about regular realizations. The main information comes from analyzing components of real points at level 0 and 1 of the $\left(A_{5}, \mathbf{C}_{3^{4}}\right)$ Modular Tower.

1.3.2. $\mathbb{R}$-cover and $\mathbb{R}$-Brauer points. If $\varphi: X \rightarrow \mathbb{P}_{z}^{1}$ is a (4 branch point) cover over a field $K$ in a given Nielsen class Ni (3.1.1), then it produces a $K$ point on the corresponding reduced Hurwitz space $\mathcal{H}^{\text {rd }}$ (Def. 3.26). Call this a $K$-cover point. The branch locus $z$ of a $K$-cover point gives a $K$ point on $\mathbb{P}_{j}^{1} \backslash\{\infty\}$. Detailed analysis of the case $K=\mathbb{R}$ is especially helpful when $p=2$. There are three kinds of $K$ points on $\mathcal{H}^{\text {rd }}$ :

(1.5a) $K$-cover points (with $K$ understood, call these cover points);

(1.5b) $K$-Brauer points (not $K$-cover points) given by $K$ covers $\varphi: X \rightarrow Y$ with $Y$ a conic in $\mathbb{P}^{2}$ over $K$ (4.4.1); and

(1.5c) $K$ points that are neither $K$-cover or $K$ - Brauer points. 
When $\mathcal{H}^{\text {rd }}$ is a fine (resp. b-fine) moduli space (1.4.1), there are only $K$-cover and $K$-Brauer points (resp. excluding points over $j=0$ or 1 ). 7 interprets how cusps attach to orbits of an element $\gamma_{\infty}$ in the Hurwitz monodromy group quotient we call $\bar{M}_{4}$. It also explains H-M and near H-M representatives (of a Nielsen class). These correspond to cusps at the end of components of $\mathbb{R}$ points on levels $k \geq 1$ of our main Modular Tower. These (eight) $\mathbb{R}$ points are exactly the $\mathbb{R}$-cover points at level $k=1$ of the $\left(A_{5}, \mathbf{C}_{3^{4}}\right)$ Modular Tower lying above any $j_{0} \in(1, \infty) \subset \mathbb{P}_{j}^{1} \backslash\{\infty\}$.

There are also eight $\mathbb{R}$-Brauer points, complements of $\mathrm{H}-\mathrm{M}$ and near $\mathrm{H}-\mathrm{M}$ reps. (7.2.1). This gives the full set of $\mathbb{R}$ points on the genus 12 component of $\mathcal{H}\left(G_{1}, \mathbf{C}_{3^{4}}\right)^{\mathrm{in}, \mathrm{rd}}=\mathcal{H}_{1}$ we label $\mathcal{H}_{1}^{+}$. Prop. 8.19 shows there is only one component of $\mathbb{R}$ points on $\mathcal{H}_{1}$. So, $\mathcal{H}_{1}^{+}$contains all $\mathbb{Q}$ points at level 1 .

The $k$ th level $(k \geq 1), \mathcal{H}_{k}^{\text {rd }}, k \geq 1$, of this reduced Modular Tower has a component $\mathcal{H}_{k}^{\mathrm{rd},+}$ with both H-M reps. and near H-M reps. (Prop. 8.12). There is a nontrivial central Frattini extension $T_{k}^{\prime} \rightarrow G_{k}$ (Prop. 9.6). Near H-M reps. also correspond to $T_{k}^{\prime}$ covers whose field of moduli over $\mathbb{R}$ is $\mathbb{R}$, though $\mathbb{R}$ is not a field of definition. There are also $\mathbb{R}$-Brauer points on $\mathcal{H}_{k}^{\mathrm{rd},+}$ (Prop. 6.8). The production of an infinite number of significantly different examples of these types of points from one moduli problem is a general application of Modular Towers.

Real points on $\mathcal{H}\left(A_{5}, \mathbf{C}_{3^{4}}\right)^{\text {abs,rd }}$ (the absolute reduced Hurwitz space; equivalence classes of degree 5 covers $)$ and the inner reduced space $\mathcal{H}\left(A_{5} \mathbf{C}_{3^{4}}\right)^{\text {in,rd }}$ are all cover points (Lem. 6.11 and Lem. 6.14). This is a special case of the general Prop. 2.3 when the group $\mathcal{Q}^{\prime \prime}$ acts trivially on Nielsen classes.

1.3.3. Analysis of level 1 and the Main Conjecture. 9 lists geometric properties of level 1 of the reduced $\left(A_{5}, \mathbf{C}_{3^{4}}\right)$ Modular Tower $(p=2)$.

Level 0 is an irreducible genus 0 curve with infinitely many $\mathbb{Q}$ points Fri90. A dense set of $\mathbb{Q}$ points produces a $\mathbb{Q}$ regular realization of $\left(A_{5}, \mathbf{C}_{3^{4}}\right)$; each gives a $\mathbb{Q}$ regular realization of the $A_{5}$ spin cover $\operatorname{Spin}_{5}$ which identifies with $\mathrm{SL}_{2}(\mathbb{Z} / 5)$ (Rem. 2.9.2). The level 1 group ${ }_{2}^{1} \tilde{A}_{5}=G_{1}$ covers $A_{5}=G_{0}$, factoring through the spin cover. Thm. 9.1 shows level 1 has two absolutely irreducible $\mathbb{Q}$ components of genuses 12 and 9 . These correspond to two $H_{4}$ orbits, $O_{1}^{+}$and $O_{1}^{-}$, in the Nielsen class $\mathrm{Ni}\left({ }_{2} \tilde{A}_{5}, \mathbf{C}_{3^{4}}\right)^{\text {in }}(3.1 .2)$.

Further, all $\mathbb{R}$ (and therefore $\mathbb{Q}$ ) points lie only in the genus 12 component, which has just one connected component of real points (Prop. 8.19). This starts with showing the $16 \mathrm{H}-\mathrm{M}$ and 16 near $\mathrm{H}-\mathrm{M}$ representatives in the (inner - not reduced; see 6.3 and (7.3) Nielsen class fall in one $H_{4}$ orbit. Together these account for the eight longest (length-20) cusp widths on the corresponding reduced Hurwitz space component.

Computations in 479 show how everything organizes around these cusps lying at the end of real components on $\mathcal{H}_{1}$. This separates two components of $\mathcal{H}_{1}^{\text {rd }}$ according to the cusps lying on each. The computer program $\underline{\mathbf{S}^{+} \mathbf{9 5}}$ helped guide our results (9.2), which include $\mathbf{S}^{+} \mathbf{9 5}$-less arguments accounting for all phenomena of this case. This reveals the group theoretic obstructions to proving the main conjecture (Prob. 1.1 for $r=4$ ). We still need Falting's Theorem to conclude there are only finitely many $\left(G_{1}, \mathbf{C}_{3^{4}}\right)$ realizations over $\mathbb{Q}$ (or over any number field).

Many Modular Towers should resemble this one, especially in the critical analysis of H-M and near H-M reps. Detailed forms, however, of the Main Conjecture are unlikely to be easy as we see from sketches of other examples in Ex. 9.2 and 
Ex. 9.3 H-M and near H-M reps. in these examples work for us, though they differ in detail from our main example.

1.3.4. Reduced equivalence classes. When $r=4$, analyzing the $\bar{M}_{4}=\mathrm{PSL}_{2}(\mathbb{Z})$ action on reduced Nielsen classes has a short summary (Prop. 4.4). Suppose $\boldsymbol{g}=$ $\left(g_{1}, g_{2}, g_{3}, g_{4}\right) \in G^{4}$ (where $G=G_{k}$ for some $k$ ) represents an element in a reduced Nielsen class. Then $\boldsymbol{g}$ satisfies the product-one condition: $g_{1} g_{2} g_{3} g_{4}=1$. We have the following actions.

(1.6a) The shift, $\gamma_{1}=$ sh of 2.7 maps $\left(g_{1}, g_{2}, g_{3}, g_{4}\right)$ to $\left(g_{2}, g_{3}, g_{4}, g_{1}\right)$.

(1.6b) The middle twist $q_{2}=\gamma_{\infty}$ maps $\boldsymbol{g}$ to $\left(g_{1}, g_{2} g_{3} g_{2}^{-1}, g_{2}, g_{4}\right)$.

Orbits of $\gamma_{\infty}$ correspond to cusps of a $j$-line cover. Each $\left\langle\mathbf{s h}, \gamma_{\infty}\right\rangle=\bar{M}_{4}$ (a mapping class group quotient) orbit corresponds to a cover of the $j$-line. 2.10 introduces the (symmetric) sh-incidence matrix summarizing a pairing using sh on $\gamma_{\infty}$ orbits. This matrix shows the $\bar{M}_{4}$ orbit structure of the genus 12 component of $\mathcal{H}_{1}^{\text {rd }}$ (8.5). This orbit contains all H-M (or near H-M) orbits and their shifts (Def. 8.11).

This illustrates analyzing the $\bar{M}_{4}$ and $\gamma_{\infty}$ orbits on reduced Nielsen classes, including arithmetic properties of cusps. Since components are moduli spaces this means analyzing degeneration of objects in the moduli space on approach (over $\mathbb{R}$ or $\mathbb{Q}_{p} ;$ Fri96] to the cusps. It generalizes analysis of elliptic curve degeneration in approaching a cusp of a modular curve. These are moduli spaces of curves. Here as in 1.4.8 Modular Towers involve nilpotent fundamental groups that detect degeneration of curves, not just their Jacobians. 2.10.2 shows the idea of shincidence works for general $r$. It is a powerful tool for simplifying the computation of braid orbits on Nielsen classes, though we haven't had time to explore its geometry interpretations for general $r$.

1.3.5. Inverse Galois Problem and obstructed components. Up to w-equivalence (3.11b) there are but finitely many four branch point $\mathbb{Q}$ realizations of $\left(\frac{1}{2} \tilde{A}_{5}, \mathbf{C}_{3^{4}}\right)$, a diophantine result (Thm. 9.1). Should any exist, they correspond to rational points on the genus 12 (level 1) component of the Modular Tower. From Lem. 7.5] $\mathcal{Q}^{\prime \prime}$ acts faithfully at level 1 . So, $\mathbb{Q}$ points on $\mathcal{H}_{1}^{\text {rd }}$ correspond either to $\mathbb{Q}$ regular realizations of the representation cover for the Schur multiplier for ${ }_{2}^{1} \tilde{A}_{5}$ ( $\mathbb{Q}$-cover points) or to $\mathbb{Q}$-Brauer points (Lem. 4.11). Which is a diophantine subtlety.

Only the genus 12 orbit $O_{1}^{+}$is unobstructed for the big $H_{r}$ invariant $\nu\left(O_{1}^{+}\right)$ of [Fri95a, Def. 3.11] (9.3). The component corresponding to $O_{1}^{-}$is obstructed FK97, §0.C]. The general meaning of obstructed - applied to a component (at level, say, $k)$ - is that it has nothing above it at level $k+1$. This applies either to the geometric moduli space component or to the $H_{r}$ orbit on a Nielsen class associated to it. Prop. 9.5 shows $\nu\left(O_{i}\right), i=1,2$, distinguishes the components. That is, $\nu\left(O_{1}^{-}\right)$doesn't contain 1 and the next levels of the Modular Tower have nothing over it. The $\nu$ invariant separates most Modular Tower components in this paper. A more general idea of lifting invariant appears in $[$ The more general idea is easier to understand, though not so efficient for actually separating components.

1.3.6. Clifford algebras and Serre's invariant. Ser90b interpreted a case of this, for alternating groups. The ingredients included a Clifford algebra, and halfcanonical classes ( $\theta$-characteristics). Serre's invariant is the special case of $₫$ Cusing pullbacks of subgroups of alternating groups to their spin covers. It appears nontrivially at level 0 of each $A_{n}$ Modular Tower with $p=2$ and 3-cycle conjugacy classes (Table 3 Prop. 5.11] Fri95a, Ex. III.12]). 
The phrase spin separating representation refers to presenting a group inside the alternating group so it has a nonsplit central extension through pull back to the spin cover (Def. 9.11). 9.4 uses a Clifford algebra to produce spin separating representations of $G_{1}={ }_{2}^{1} \tilde{A}_{5}$. $\mathrm{B}$ outlines the potential $\theta$-nulls (giving automorphic functions on the Modular Tower levels) coming from spin separating representations (details in the expansion of [Fri96]).

This explains part of this paper's most mysterious phenomenon: The two $\bar{M}_{4}$ orbits on $\mathrm{Ni}\left({ }_{2}^{1} \tilde{A}_{5}, \mathbf{C}_{3^{4}}\right)^{\text {in,rd }}$ have the same image groups, and degrees. This is despite their being different $\bar{M}_{4}$ representations distinguished just by the number and length of their $\gamma_{\infty}$ orbits. Appearance of the Clifford algebra dominates details of our study of this Modular Tower level 1 through component separation.

We haven't yet shown a similar separated components phenomenon happens beyond level 1 of our main Modular Tower (see 8.5). Still, 4.3.2 relates the subtly different geometry of the (real) cusps associated with H-M reps. versus near H$\mathrm{M}$ reps. to the phenomenon of fields of moduli versus field of definitions attached to realizing Frattini central extensions. This Schur multiplier result (Prop. 8.12) happens at all levels of the Modular Tower (certain to generalize to most Modular Towers). This is a weaker form of the geometry of spin separation and an example of how Frattini central extensions (Schur multipliers) of perfect groups affect the geometry of all levels of any Modular Tower.

The two level 1 representations of $\bar{M}_{4}$, with their relation to spin separating representations has ingredients like those in GKS99. So, 9 E uses this analogy to find a precise measure of the difference between the two representations.

1.4. General results and divergences with modular curves. Let $K$ be a field. A point on a Modular Tower is a projective system of points $\left\{\boldsymbol{p}_{k}\right\}_{k+0}^{\infty}$ : $\boldsymbol{p}_{k+1}$ on the $k+1$ st level maps to $\boldsymbol{p}_{k}$ at level $k$. Similarly, define points on reduced Modular Towers. If all $\boldsymbol{p}_{k} \mathrm{~s}$ have definition field $K$, then this sequence defines a $K$ point. Suppose $K$ is a number field (more generally, $K \leq \overline{\mathbb{Q}}$ has an infinite number of places with finite residue class field). Then, Thm. 6.1 implies a Modular Tower (of inner Hurwitz spaces) has no $K$ points.

Problem 1.1 (Main Problem on Modular Towers). Assume ( $G, \mathbf{C}, p)$ is data for a Modular Tower with $G$ centerless and $p$-perfect (Def. 3.18). For $k$ large, show all components of a Modular Tower at level $k$ have general type. Let $K$ be a number field. Find explicit large $k$ so the $k$ th level of a Modular Tower of inner Hurwitz spaces contains no $K$ points ([FK97]; see 3.4.2).

1.4.1. Fine moduli. The hypotheses on $(G, \mathbf{C}, p)$ say that $G$ has no $\mathbb{Z} / p$ quotient. The centerless condition ensures a Modular Tower of inner Hurwitz spaces consists of fine moduli spaces (Prop. 3.21). Our Main Example $\left(A_{5}, \mathbf{C}_{3^{4}}, p=2\right)$ has all inner levels fine moduli spaces. Its reduced spaces are also, if $k \geq 1$ (Lem. 7.5). Level 0, however, of this (inner and reduced) Modular Tower is not a fine or b-fine moduli space (2.6.6).

1.4.2. Modular curve-like aspects. Suppose $G=P \times{ }^{s} H$ where $H$ is a $p^{\prime}$-group (2.1.3) acting through automorphisms on a $p$-group $P$. Then, ${ }_{p} \tilde{G}$ is $\tilde{P} \times{ }^{s} H$ with $\tilde{P}$ the pro-free pro- $p$ group on the minimal number of generators for $P$ (Rem. 5.2 or FJ86 Chap. 21]). Refer to this as $p$-split data for a Modular Tower.

EXAmple 1.2 ( $H$ acting on a lattice $L)$. Assume a finite group $H$ acts on $L=\mathbb{Z}^{n}$ (possibly the integers of a number field). For each prime $p$ not dividing 
$|H|$, form $H_{p}=L / p L \times{ }^{s} H$. Let $\tilde{F}_{p, n}$ be the pro-free pro- $p$ group of rank $n$ and let $\mathbf{C}$ be conjugacy classes in $H$. Form the Modular Tower for $\left(H_{p}, \mathbf{C}, p\right)$, based on the characteristic $p$-Frattini quotients of ${ }_{p} \tilde{H}_{p}=\tilde{F}_{p, n} \times{ }^{s} H$ (directly from those of $\tilde{F}_{p, n}$ ).

This has a modular curve-like property: You can vary $p$. Modular curves are the case $n=1, H=\mathbb{Z} / 2$ with $\mathbf{C}$ four repetitions of the nontrivial element of $H$. Whenever $n=1$, the analysis is similar to modular curves. Compare the case $n=1, H=\mathbb{Z} / 3, \mathbf{C}$ two repetitions each of the conjugacy classes of $\pm 1 \in \mathbb{Z} / 3$ and $p \equiv 1 \bmod 3$ with the harder case where $n=2, p=2$ in Ex. 9.2

EXAMPLE 1.3 ( $G$ any simple group). Characteristic ${ }_{p} \tilde{G}$ quotients $\left\{G_{k}\right\}_{k=0}^{\infty}$ are perfect and centerless (Prop. 3.21). For each $k \geq 1$, the natural map $G_{k+1} \rightarrow G_{k}$ maps through the exponent $p$ part of the universal central extension $\hat{G}_{k}$ of $G$.

Modular Towers for Ex. 1.3 depart from towers of moduli spaces in the literature. This paper emphasizes new group and geometry phenomena amid comfortable similarities to modular curves even for the many Modular Towers covering the classical $j$-line. This paper aims to show, when $r=4$, the genus of reduced Modular Tower components grows as the levels rise.

Modular curve-like aspects in Ex. 1.3 guide finding information about cusp behavior. The phenomenon of obstructed components, appearing in our main example, is the most non-modular curve-like aspect of general Modular Towers. Suppose in Ex. 1.2 for a given $p, H$ acts irreducibly on $L / p L$. Then, the Nielsen class $\mathrm{Ni}\left(H_{p}, \mathbf{C}\right)$ is nonempty (Lemma 5.18). Deciding in Ex. 1.2 if there are obstructed components $\left(H_{r}\right.$ orbits $)$ at high levels above a level $0 H_{r}$ orbit seems difficult.

When $r=4$, this reflects on the genuses of the corresponding $j$-line covers. Even when $n=2, p=2, H=\mathbb{Z} / 3$, and $\mathbf{C}$ consists of several copies of the two nontrivial conjugacy classes of $\mathbb{Z} / 3$, obstructed components appear at all level $k$ for $r$ suitably large (dependent on $k$ ). This special case of [FV91, Conway-Parker App.] uses the nontrivial Schur multiplier appearing at each level (Prop. 9.6 generalizing FK97 §4]). The case $G_{0}=(\mathbb{Z} / 2 \times \mathbb{Z} / 2) \times{ }^{s} \mathbb{Z} / 3=A_{4}\left(\leq A_{5}\right)$ has elements like our main example, because their universal 2-Frattini covers are close (\$5.2). Yet, for $\mathbf{C}$ consisting of $r=4$ ( $r$ not large) 3- cycle conjugacy classes, the early levels of the Modular Towers for $A_{4}$ and $A_{5}(p=2)$ are not at all alike (Ex. 9.2).

These calculations collect the key points for considering a full proof of the Main Modular Tower Conjecture when $r=4$. Using this paper, Fri96 Main Theorem] will show what to expect of all Modular Tower levels from $A_{n}$ and $r(\geq n-1)$ 3 -cycles as conjugacy classes.

1.4.3. Immediate extensions when $r=4$. A full result on $A_{5}, p=2$ and $r=4$ would contribute to the following questions on the Inverse Galois Problem.

(1.7a) Does any other reduced $A_{5}$ Modular Tower have $\mathbb{Q}$ points at level 1?

(1.7b) Is there any set of four conjugacy classes $\mathbf{C}$ for ${ }_{2}^{1} \tilde{A}_{5}$ where the reduced inner Hurwitz space contains infinitely many (any?) $\mathbb{Q}$ points?

An example best illustrates the serious points about Question (1.76). Let $\mathrm{C}_{5}^{+}$be the conjugacy class of (12345), $\mathrm{C}_{5}^{-}$the conjugacy class of (13524) (with $\mathrm{C}_{3}$ the conjugacy class of 3-cycles).

From the Branch Cycle Lemma, the only positive possibilities for (1.7b) are Modular Towers for $A_{5}$ with $\mathbf{C}=\left(\mathrm{C}_{3}, \mathrm{C}_{3}, \mathrm{C}_{5}^{+}, C_{5}^{-}\right)$or $\left(\mathrm{C}_{5}^{+}, \mathrm{C}_{5}^{+}, \mathrm{C}_{5}^{-}, \mathrm{C}_{5}^{-}\right)$. For both, the level 0 Modular Tower has two components, with exactly one obstructed (nothing above it at level 1 ). The respective unobstructed $H_{4}$ orbits contain the 
following representatives:

a) $((254),(453),(12345),(21534))$

b) $((12345),(54321),(21435),(53412))$.

Prop. 5.11] explains this and why only the (1.8) Modular Tower has real points at level 1. Rem. 5.12 explains the one mystery left on answering (1.70), about genus 1 curves at level 1 of the reduced Modular Tower for $\left(\mathrm{C}_{5}^{+}, \mathrm{C}_{5}^{+}, \mathrm{C}_{5}^{-}, \mathrm{C}_{5}^{-}\right)$.

Modular Towers use $p^{\prime}$ conjugacy classes. If we allow conjugacy classes where $p$ divides the elements' orders, then Hurwitz spaces attached to $G_{k}$ and these classes must have $r$ large if the components are to have definition field $\mathbb{Q}$ (Thm. 6.1). There appear, however, to be nontrivial cases of $\mathbb{Q}$ components for $r=4$ and ${ }_{2}^{1} \tilde{A}_{5}$ in (1.7b) using conjugacy classes of order 4 . Completing a yes answer to the following question requires completing these cases.

Problem 1.4. Are there only finitely many ${ }_{2}^{1} \tilde{A}_{5}$ realizations (over $\mathbb{Q}$; up to w-equivalence) with at most four branch points?

For, however, the group ${ }_{2}^{2} \tilde{A}_{5}$, if $r=4$, the finitely many possible realizations can only fall on level 2 of the Modular Tower for $\left(A_{5}, \mathbf{C}_{3^{4}}\right)$ or for (1.8)

1.4.4. Extending results to $r \geq 5$. Mes90 produced $\mathbb{Q}$ covers in the Nielsen class $\operatorname{Ni}\left(A_{n}, \mathbf{C}_{3^{n-1}}\right)$ when $n \geq 5$ is odd (see also [Ser92 §9.3]). Fri96 Thm. 1] shows there is exactly one component here. So, the results combine to show $\mathbb{Q}$ points are dense in $\mathcal{H}\left(A_{n}, \mathbf{C}_{3^{n-1}}\right)^{\text {in }}$ : There are many $\left(A_{n}, \mathbf{C}_{3^{n-1}}\right)$ realizations over $\mathbb{Q}$ that produce $\operatorname{Spin}_{n}$ realizations.

Problem 1.5. Does Thm. 9.1 generalize to say $\mathcal{H}\left({ }_{2}^{1} \tilde{A}_{n}, \mathbf{C}_{3^{n-1}}\right)^{\mathrm{in}, \mathrm{rd}}(\mathbb{Q})$ is finite for $n \geq 5$ odd?

For $n$ even this follows from Fri96 Thm. 1] showing $\mathcal{H}\left({ }_{2}^{1} \tilde{A}_{n}, \mathbf{C}_{3^{n-1}}\right)^{\text {in,rd }}$ is empty. Falting's Theorem Fal83 works when $n=5$ because the moduli spaces are curves. There is no replacement yet for Falting's Theorem for $n \geq 6$.

Here is a special case of the problem that would produce positive results for the Inverse Galois Problem.

Problem 1.6. Is $\mathcal{H}\left({ }_{2}^{k} \tilde{A}_{n}, \mathbf{C}_{3^{r}}\right)^{\text {in,rd }}(\mathbb{Q}) \neq \emptyset$ for $r$ large (dependent on $\left.n, k\right)$.

Ex. 3.17 describes the complete set of components for $\mathcal{H}\left({ }_{2}^{1} \tilde{A}_{n}, \mathbf{C}_{3^{r}}\right)^{\text {in,rd }}$ (see Table 21). The procedure of Fri96 Thm. 1] uses the many embeddings of $A_{k}$ in $A_{n}$ for $n \geq k$. Any such embedding extends to an embedding of the level $k$ characteristic 2-Frattini cover of $A_{k}$ into the level $k$ characteristic 2-Frattini cover of $A_{n}$ (special case of Lem. 5.1). So, Thm. 9.3 describing the two components of $\mathcal{H}\left({ }_{2}^{1} \tilde{A}_{5}, \mathbf{C}_{3^{4}}\right)^{\text {in,rd }}$ immediately gives information on components of $\mathcal{H}\left({ }_{2}^{1} \tilde{A}_{n}, \mathbf{C}_{3^{r}}\right)^{\text {in,rd }}$ for all $r \geq n-1$ and $n \geq 5$. [FV91, Conway-Parker App.] proves for $n, k$ fixed and $r$ large each element of the Schur multiplier of ${ }_{2}^{k} \tilde{A}_{n}$ determines a component. This is a special case of a general result, though here we are precise about the components for all values of $r$. Combining this with 9.6 hints at describing components for higher levels of Modular Towers for all alternating groups.

There are many $j$-line covers. It is significant to find simple invariants distinguishing Modular Towers from general quotients of the upper half plane by a subgroup of $\mathrm{PSL}_{2}(\mathbb{Z})$. As this paper and its consequents shows, they have far more in common with modular curves than does a general such quotient. The structure of a symmetric integral sh-incidence matrix makes such a distinction, effectively 
capturing complicated data about Modular Tower levels. Example: It displays each connected component (Lem. 2.26). The cusp pairing for $r=4$ extends to the case $r \geq 5$ (2.10.2).

1.4.5. Significance of cusps. Thm. 9.1 results from information about the level 1 cusps of this Modular Tower. Most interesting are cusps attached to H-M representatives. [Fri95a $\S I I I . F]$ explains the moduli manifestation of total degeneration around such a cusp. The compactification is through equivalence classes of specialization sequences. Especially short specialization sequences detect H-M reps. from total degeneration. This is a very different compactification from that of the stable compactification theorem cover versions, like the log structures We01 uses. Specialization sequences require no extension of the base field. So they are compatible with absolute Galois group actions. H-M reps. give the type of moduli degeneration useful for a tangential basepoint (language of Ihara-Matsumoto IM95 and Nakamura $\mathbf{N a 9 8}$ ). These also use compactifications not quite like stable compactification (see [Fri95a p. 163, Comments C.5]). Likely for $r=4$ there is not a huge difference between these compactifications, though that is unlikely for $r \geq 5$.

For Modular Towers over $\mathbb{Q}$ with $p=2$ and $r=4$ (or higher) consider pull back to the configuration space $\mathbb{P}^{4} \backslash D_{4}$ of branch points of these covers. Then, a real point on $\mathcal{H}_{k}^{\text {in }}$ with $k \geq 1$ corresponds to a cover with complex conjugate pairs of branch points (Thm. 6.1). So, one must recast relations on the image of $G_{\mathbb{Q}}$ coming from the Grothendieck-Teichmüller group. More appropriate for this situation than four real points is complex conjugate pairs of branch points (compare with [ha91 p. 107]). For other problems one would want configurations with a complex conjugate pair and two real points of branch points (as in 6.4.1). This adapts for $G_{\mathbb{Q}}$ acting on quotients of the profinite completion of $H_{4}$ corresponding to projective limits of the monodromy groups of monodromy group cover from Modular Towers with $p=2$.

Here we connect to $\mathbf{B F 9 8}$ at level 0. An example result gives exactly three degree five $\left(A_{5}, \mathbf{C}_{3^{4}}\right)$ covers of $\mathbb{P}_{z}^{1}$ (up to w- equivalence (3.1b) $)$, over $\mathbb{Q}$ with branch points in $\mathbb{Q}$. There are three non-cusp points in the order 12 group generated by the cusps on the absolute reduced space attached to $\left(A_{5}, \mathbf{C}_{3^{4}}\right)$. These three covers correspond to those three non-cusp points. Mazur's explicit bound on torsion points shows, among all $\left(A_{5}, \mathbf{C}_{3^{4}}\right)$ realizations (over $\mathbb{Q}$ ), exactly three w-equivalence classes can have rational branch points (BF98). A result of Serre (Prop. 5.9] Ser90a) allows computing cusp widths in the $\left({ }_{2}^{1} A_{5}, \mathbf{C}_{3^{4}}\right)$ case. This includes the length-20 cusps attached to H-M and near H-M representatives. Computing the genus of curves at all levels of a Modular Tower depends on such formulas.

1.4.6. Characterizing divergences with modular curves. We show many phenomena for Modular Tower levels of simple groups not appearing in modular curves. There is a precise dividing line between two types of Modular Towers, with modular curves a model for one type. Fri95c told the story of how modular curves are essentially the moduli for dihedral group realizations with four involution conjugacy classes. The absolute (resp. inner) Hurwitz spaces correspond to the curves $X_{0}\left(p^{k+1}\right)$ (resp. $X_{1}\left(p^{k+1}\right)$ ). The case of general $r \geq 4$ is about Hurwitz spaces associated to modular curve like covers of the moduli of hyperelliptic curves of genus $g=\frac{r-2}{2}$. A generalization of this situation would include $G_{0}=b Z / p^{t} \times{ }^{s} A$ with $A$ a(n abelian) subgroup of $\left(\mathbb{Z} / p^{t}\right)^{*}$ (acting naturally on $G_{0}$ ) and a collection of conjugacy classes $\mathbf{C}$ whose elements generate $A$. For, however, inner Hurwitz 
spaces to be over $\mathbb{Q}, \mathbf{C}$ must be a rational union of conjugacy classes (trivial case of the branch cycle argument). This puts a lower bound on $r$. For example, if $A$ is cyclic and order $d$, then $\mathbf{C}$ must contain a minimum of $\varphi(d)$ conjugacy classes. That is why one rarely sees these spaces in the classical context, though they share with modular curves the attribute of having 1-dimensional $p$-Frattini modules.

For a $p$-perfect centerless group, the characteristic $p$-Frattini $G_{0}$ module $M_{0}$ has dimension 1 if and only if $\mathbf{1}_{G_{k}}$ never appears in the Loewy layers of $M_{k}$ for any $k$ (5.2.2 and 55.7 . Appearance of those $\mathbf{1}_{G_{k}}$ s could mean obstructed components, uncertain location of cusps in components and related moduli interpretations of spaces for covers whose field of moduli is not a field of definition of representing points of the levels of a Modular Tower. These useful geometric phenomena, present Modular Towers as a new tool for investigating still untouched mysteries. That is our major theme. Further, given $k$, if the conjugacy classes in $\mathbf{C}$ repeat often enough (as a function of $k$ ), FV91 App.] implies these complications will occur at level $k$ of the Modular Tower. A big mystery is whether they occur at infinitely many levels of any Modular Tower for a $p$-perfect centerless group.

1.4.7. The Open Image Theorem and $j$-awareness. $\S \mathbb{D}$ formulates how to extend part of Serre's Open Image Theorem Ser72 (for just the prime $p$ ) to Modular Towers. This extension would be a tool for finding a precise lower bound on $k$ for which higher levels of a Modular Tower have no rational points. Ex. 1.2 is appropriate for generalizations of Mazur-Merel.

Problem 1.7. Consider $\mathcal{H}\left(L / p^{k} L \times{ }^{s} H, \mathbf{C}\right)^{\text {in,rd }}=\mathcal{H}_{L / p^{k} L \times{ }^{s} H, \mathbf{C}}$ with $p$ running over primes not dividing $|H|$ and integers $k \geq 0$. Find $m(H, \mathbf{C})$ so $\mathcal{H}_{L / p^{k} L \times{ }^{s} H, \mathbf{C}}$ has general type if $p+k$ exceeds $m(H, \mathbf{C})$. If $r=4$, and $d \geq 1$, find explicit $m(H, \mathbf{C}, d)^{\prime}$ with $\mathcal{H}_{L / p^{k} L x^{s} H, \mathbf{C}}(K)$ empty for $k+p>m(G, \mathbf{C}, d)^{\prime}$ and $[K: \mathbb{Q}] \leq d$.

Mazur-Merel is the case $H=\mathbb{Z} / 2=\{ \pm 1\}$ and $L=\mathbb{Z}$ (-1 acting by multiplication), $r=4$ and classes all the nontrivial element in $H$.

We continue with the case $r=4$. What is the analog of Serre's result for nonintegral $j_{0} \in \mathbb{P}_{j}^{1} \backslash\{\infty\}$. We interpret that to say, for $j_{0} \in \overline{\mathbb{Q}}$ not an algebraic integer, the action of $G_{\mathbb{Q}\left(j_{0}\right)}$ on projective systems of points on $\left\{X_{1}\left(p^{k+1}\right)\right\}_{k=0}^{\infty}$ lying over $j_{0}$ has an open orbit. The ingredients in our generalizing statement (still a conjecture) is a Modular Tower meaning to $j_{0}$ being suitably ( $\ell$-adically) close to $\infty$ and the Ihara-Matsumoto-Wewers approach to using tangential base points. One topic we couldn't resist was what we call $j$-awareness. It would be trivial to generalize the Open Image Theorem if each appropriate Modular Tower was close to being a tower of modular curves. That would mean there is some cover $Y \rightarrow \mathbb{P}_{j}^{1}$ so the pullback of a tower of modular curves dominates the levels of the given Modular Tower. Checking this out on the main examples of this paper (with $p=2$ ) repeatedly called for analyzing Prym varieties (Ex. 3.27 and $\$ \mathrm{E})$.

1.4.8. Developing a p-adic theory. We compare our approach to $\mathbb{R}$ points with that of Moc96 p. 978]. Recall the complex conjugate of a complex manifold. Suppose $\left\{\left(U_{\alpha}, \varphi_{\alpha}\right)\right\}_{\alpha \in I}$ is an atlas for a complex manifold $X$. For simplicity, assume $X$ is a 1-dimensional complex manifold. Create a new manifold by composing each $\varphi_{\alpha}: U_{\alpha} \rightarrow \mathbb{C}$ with complex conjugation. Call the resulting map $\varphi_{\alpha}^{*}$.

Lemma 1.8. The atlas $\left\{\left(U_{\alpha}, \varphi_{\alpha}^{*}\right)\right\}_{\alpha \in I}$ is a new complex manifold structure $X^{*}$ on the set $X$. 
Proof. Denote by $\bar{z}_{\alpha}(x)$ the value of $\varphi_{\alpha}^{*}(x)$. Compare the transition functions $\varphi_{\beta}^{*} \circ\left(\varphi_{\alpha}^{*}\right)^{-1}\left(\bar{z}_{\alpha}\right)=\bar{z}_{\beta}$ with the function $\varphi_{\beta} \circ \varphi_{\alpha}^{-1}\left(z_{\alpha}\right)$ as $\bar{z}_{\alpha}$ varies over the complex conjugate of $z_{\alpha}$ running over $\varphi_{\alpha}\left(U_{\alpha} \cap U_{\beta}\right)$. The effect of the former is this:

$$
\bar{z}_{\alpha} \mapsto z_{\alpha} \mapsto \varphi_{\alpha}^{-1}\left(z_{\alpha}\right) \mapsto \varphi_{\beta}\left(\varphi_{\alpha}^{-1}\left(z_{\alpha}\right)\right)=z_{\beta} \mapsto \bar{z}_{\beta} .
$$

Suppose $f$ is a local expression of the transition function $\varphi_{\beta} \circ \varphi_{\alpha}^{-1}\left(z_{\alpha}\right)$ as a power series (about the origin) in $z_{\alpha}$. Then, the power series expressions for $\varphi_{\beta}^{*} \circ\left(\varphi_{\alpha}^{*}\right)^{-1}\left(\bar{z}_{\alpha}\right)$ comes by applying complex conjugation to the coefficients of $f$, and so the resulting function is analytic in $\bar{z}_{\alpha}$.

Definition 1.9. A complex manifold $X$ has an $\mathbb{R}$ structure if $X^{*}$ is analytically isomorphic to $X$.

Apply this to a cover $\varphi: X \rightarrow \mathbb{P}_{z}^{1}$ using the complex structure from the $z$-sphere, as in Prop. 6.3 You can also use the complex structure from uniformization by the upper half plane $\mathbb{H}$ as in Moc96 p. 978]. Keep in mind: $X^{*}$ is the same set as $X$. For $x \in X$, there is a natural map $x \mapsto x^{*} \in X^{*}$ where we regard $x^{*}$ as the complex conjugate of $x$, though in this formulation $x^{*}$ is the same point on the set $X$. Also, the lower half plane $\mathbb{H}^{*}$ naturally uniformizes $X^{*}$. An $\mathbb{R}$ structure induces $\psi: X^{*} \rightarrow X$, which in turn induces $\tilde{\psi}: \mathbb{H}^{*} \rightarrow \mathbb{H}$. Of course, $\psi$ induces $\psi^{*}: X \rightarrow X^{*}$. Mochizuki makes simplifying assumptions: There is $x^{*}$ with $\varphi\left(x^{*}\right)=x$ and $\psi^{*} \circ \psi$ the identity map.

Take $\mathcal{E}(X, x)^{\text {alg }}$ to be all meromorphic algebraic functions in a neighborhood of $x$ that extend analytically along each path in $X$ (3.2.1). Then, $\mathcal{E}(X, x)^{\text {alg }} / \mathbb{R}(z)$ is Galois with profinite group $\pi_{1}(X, x)^{\text {alg, }, \mathbb{R}}$ forming a split sequence $\pi_{1}(X, x)^{\text {alg }} \leq$ $\pi_{1}(X, x)^{\text {alg, }, \mathbb{R}} \rightarrow G(\mathbb{C} / \mathbb{R})$ (as in Prop. 3.4). The virtues of this Gu67 inspired approach are these.

(1.9a) A splitting of $G(\mathbb{C} / \mathbb{R})$ given by $\tilde{\psi} \circ C$, an anti-holomorphic involution $\left(\begin{array}{ll}a & b \\ c & d\end{array}\right) \in G L_{2}^{-}$(determinant -1 ) acting on $\tau \in \mathbb{H}$ by $\tau \mapsto \frac{a \tau+b}{c \tau+d}$.

$(1.9 \mathrm{~b})$ So, $\pi_{1}(X, x)^{\text {alg, }, \mathbb{R}}$ acts through the matrix group $\mathrm{GL}_{2}(\mathbb{R})$ extending $\rho$ : $\pi_{1}(X, x)^{\text {alg }} \rightarrow \mathrm{PSL}_{2}(\mathbb{R})$.

(1.9c) Extend $\rho$ by $\mathrm{PSL}_{2}(\mathbb{R}) \rightarrow \mathrm{PSL}_{2}(\mathbb{C})$ to consider the $\mathbb{P}_{\tau}^{1}$ bundle over $X$ with a natural flat connection (algebraic from Serre's GAGA).

Mochizuki calls this uniformization construction the canonical indigenous bundle. Compare to our Prop. 6.3 with several complex conjugation operators $\hat{\kappa}$, corresponding to a type of degenerate behavior at a cusp (and the location of a set of branch points over $\mathbb{R}$ on $\left.\mathbb{P}_{z}^{1}\right)$. His simplifying assumption $\left(\varphi\left(x^{*}\right)=x\right)$ applied to Modular Towers when $p=2$ would reduce to considering $X$ that appear as H-M reps. (say, in Cor. 6.7), just one case we must treat in describing the full real locus for a result like Prop. 8.19 on a Modular Tower level.

Mochizuki calls his prime $p$, though in our context it would be $\ell$ prime to $\left|G=G_{0}\right|$, since we use $p$ as a prime for the construction of a Modular Tower. His construction is for any family of curves over any base. It extends to work for $\ell$-adic uniformization giving a notion of $\ell$-ordinary points on a family of curves over $\mathbb{Q}_{\ell}$. The Modular Tower goal would be to detect ordinary $\mathbb{Q}_{\ell}($ with $(\ell,|G|)=1$ ) points on a Modular Tower, generalizing (from $G_{0}$ a dihedral group) ordinary elliptic curves over $\operatorname{Spec}\left(\mathbb{Z}_{\ell}\right)$ Moc96 p. 1089]. Only the genus 12 component at level 1 of the $\left(A_{5}, \mathbf{C}_{3^{4}}\right)$ Modular Tower supports totally degenerate cusps, associated with the 
name Harbater-Mumford representatives (6). An analysis that combines Harbater patching and Mumford's theory of total $\ell$-adic degeneration shows most points on such components will exhibit ordinary $\ell$-adic behavior. Nilpotent fundamental groups enter directly in all Modular Tower definitions.

Wewers We01 uses the degeneration behavior type (a tangential basepoint) to consider $\ell$-adic $((\ell,|G|)=1)$ information for level 0 of our main Modular Tower. His goal is to detect fields of moduli versus fields of definition (following [DDE00). 4.3 .2 explains why the geometry of H-M reps. and near H-M reps. should have an $\ell$-adic analog at all levels of our $A_{5}$ Modular Tower. His analysis is also a tool for our approach to generalizing Serre's Open Image Theorem (D.3).

\section{Presenting $H_{4}$ as an extension}

2.1. Notation and the groups $B_{r}, H_{r}$ and $M_{r}$. Denote the Artin Braid group on $r$ strings by $B_{r}$. It has generators $Q_{1}, \ldots, Q_{r-1}$ satisfying these relations:

$$
\begin{aligned}
Q_{i} Q_{i+1} Q_{i}=Q_{i+1} Q_{i} Q_{i+1}, & i=1, \ldots, r-2 ; \text { and } \\
Q_{i} Q_{j}=Q_{j} Q_{i}, & 1 \leq i<j-1 \leq r-1 .
\end{aligned}
$$

2.1.1. $B_{r}$ and $H_{r}$ as automorphisms. We start from Bohnenblust Boh47] (or Mar45). Let $F_{r}$ be the free group of rank $r$ on generators $\left\{\bar{\sigma}_{1}, \ldots, \bar{\sigma}_{r}\right\}$, and denote $\operatorname{Aut}\left(F_{r}\right)$ by $\bar{A}_{r}$. Then $B_{r}$ embeds in $\bar{A}_{r}$ via this right action of $Q_{i}$ :

$$
\left(\bar{\sigma}_{1}, \ldots, \bar{\sigma}_{r}\right) Q_{i}=\left(\bar{\sigma}_{1}, \ldots, \bar{\sigma}_{i-1}, \bar{\sigma}_{i} \bar{\sigma}_{i+1} \bar{\sigma}_{i}^{-1}, \bar{\sigma}_{i}, \bar{\sigma}_{i+2}, \ldots, \bar{\sigma}_{r}\right)
$$

Also, $B_{r}$ consists of automorphisms of $F_{r}$ fixing $\bar{\sigma}_{1} \cdots \bar{\sigma}_{r}$ and mapping $\bar{\sigma}_{1}, \ldots, \bar{\sigma}_{r}$ to permutations of conjugates of these generators. Let $G_{r}$ be the quotient of $F_{r}$ by the relation $\Pi(\overline{\boldsymbol{\sigma}})=\bar{\sigma}_{1} \cdots \bar{\sigma}_{r}=1$.

The Hurwitz monodromy group $H_{r}$ is the quotient of $B_{r}$ by adding the relation

$$
Q_{1} Q_{2} \cdots Q_{r-1} Q_{r-1} \cdots Q_{1}=1
$$

It is also the fundamental group of an open subset of $\mathbb{P}^{r}(2.2)$.

Denote inner automorphisms of a group $G$ by $\operatorname{Inn}(G)$. Then, $H_{r}$ induces automorphisms of $G_{r}$ mapping the images of $\bar{\sigma}_{1}, \ldots, \bar{\sigma}_{r}$ to permutations of conjugates of these generators, modulo $\operatorname{Inn}\left(G_{r}\right)$.

If $G \leq H$, denote the normalizer of $G$ in $H$ by $N_{H}(G)$. The next lemma is obvious. Suppose $\psi: G_{r} \rightarrow G$. Define an induced action of $H_{r}$ on the collection of such $\psi$ s by $\left(\psi\left(\bar{\sigma}_{1}, \ldots, \bar{\sigma}_{r}\right)\right) Q \stackrel{\text { def }}{=} \psi\left(\left(\bar{\sigma}_{1}, \ldots, \bar{\sigma}_{r}\right) Q\right)$.

LEMMA 2.1. The action of (2.3) commutes with conjugation on an r-tuple by an element of $F_{r}$. For $G \leq S_{n}$ suppose $\psi: G_{r} \rightarrow G$. The induced action of $H_{r}$ on $\psi$ commutes with conjugation by $N_{S_{n}}(G)$.

2.1.2. A presentation of $M_{r}$. The mapping class group $M_{r}$ is the quotient by $\operatorname{Inn}\left(G_{r}\right)$ of automorphisms of $G_{r}$ mapping $\bar{\sigma}_{1}, \ldots, \bar{\sigma}_{r}$ to permutations of conjugates. This maps $B_{r}$ to $M_{r}$ factoring through $H_{r}$. Further, $M_{r}$ is the quotient of $B_{r}$ by the following relations ( $\mathbf{K M S 6 6} \S 3.7]$ or $\mathbf{M a 3 4})$ :

$$
\begin{array}{r}
\tau_{1}=\left(Q_{r-1} Q_{r-2} \cdots Q_{2}\right)^{r-1}, \tau_{2}=Q_{1}^{-2}\left(Q_{r-1} \cdots Q_{3}\right)^{r-2}, \ldots, \tau_{\ell+1}= \\
\left(Q_{\ell} \cdots Q_{1}\right)^{-\ell-1}\left(Q_{r-1} \cdots Q_{\ell+2}\right)^{r-\ell-1}, \ldots, \tau_{r-1}=\left(Q_{r-2} \cdots Q_{1}\right)^{1-r} \\
\text { and } \tau=\left(Q_{r-1} \cdots Q_{1}\right)^{r} .
\end{array}
$$


This complicated presentation is oblivious to the map $H_{r} \rightarrow M_{r}$ dominating this paper. Using it conceptualizes the $B_{r} \rightarrow M_{r}$ kernel, $N_{r}=\operatorname{ker}\left(B_{r} \rightarrow M_{r}\right)$. The switch of emphasis shows in Prop. 9.21. With $Q_{1} Q_{2} Q_{3}^{2} Q_{2} Q_{1}=D=R_{1}$ write generators of $N_{r}$ as follows:

$$
R_{1}, R_{2}=Q_{1}^{-1} R_{1} Q_{1}, R_{3}=Q_{2}^{-1} R_{2} Q_{2}, R_{4}=Q_{3}^{-1} R_{3} Q_{3} \text { and }\left(Q_{1} Q_{2} Q_{3}\right)^{4} .
$$

Thm. 2.9 presents both $M_{4}$ and $N_{4}$ memorably. An appearance of $N_{4}$ in 9.5 offers a place for spin separation to explain similarities of the two $M_{4}$ orbits in level 1 of our main example.

2.1.3. Notation for fundamental groups. The Riemann sphere uniformized by the variable $z$ is $\mathbb{P}_{z}^{1}=\mathbb{C} \cup\{\infty\}$. Relation (2.4) comes from the geometry of $r$ distinct points on $\mathbb{P}_{z}^{1}$. We sometimes drop the notation for uniformizing by $z$. Similarly, copies of $\mathbb{A}^{r}$ (affine $r$-space) and $\mathbb{P}^{r}$ (projective $r$-space) come equipped with coordinates suitable for a diagram:

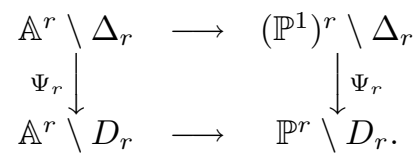

The upper left copy of $\mathbb{A}^{r}=\mathbb{A}_{\boldsymbol{z}}^{r}$ has coordinates $\left(z_{1}, \ldots, z_{r}\right)$. Then, $\Delta_{r}$ is the fat diagonal of $r$-tuples with two or more of the $z_{i}$ s equal. Embed $\mathbb{A}_{z}^{r}$ in $\left(\mathbb{P}_{z}^{1}\right)^{r}$ by $\left(z_{1}, \ldots, z_{r}\right) \mapsto\left(z_{1}, \ldots, z_{r}\right)$. In $\left(\mathbb{P}_{z}^{1}\right)^{r}$ the $z_{i}$ s may take on the value $\infty$. Regard $\Psi_{r}$ as the quotient action of $S_{r}$ permuting the coordinates of $\left(\mathbb{P}^{1}\right)^{r}$. As with $B_{r}$, put this action on the right.

Thus, the lower copy of $\mathbb{A}^{r}=\mathbb{A}_{x}^{r}$ has coordinates $\left(x_{1}, \ldots, x_{r}\right)$, with $x_{i}$ equal to $(-1)^{i}$ times the $i$ th symmetric function in $\left(z_{1}, \ldots, z_{r}\right)$. Regard this as giving a monic polynomial of degree $r$ in $z$ (with zeros $\left(z_{1}, \ldots, z_{r}\right)$ ). Then, complete the commutative diagram by taking $\mathbb{P}^{r}$ as all monic (nonzero) polynomials of degree at most $r$. The map $\Psi_{r}$ on the right sends each $r$-tuple $\left(z_{1}, \ldots, z_{r}\right)$ to $\prod_{i=1}^{r}\left(z-z_{i}\right)$. When $z_{i}=\infty$ replace the factor $\left(z-z_{i}\right)$ by 1 . The image of $\Delta_{r}$ is the discriminant locus of polynomials with two or more equal zeros. For coordinates around $z_{i}=\infty$, regard a monic polynomial of degree $t<r$ as having $r-t$ zeros at $\infty$.

This interprets $U_{r}=\mathbb{P}^{r} \backslash D_{r}$ as the space of $r$ distinct unordered points in $\mathbb{P}^{1}$, the image of $\left(\mathbb{P}^{1}\right)^{r} \backslash \Delta_{r}=U^{r}$. Thus, $\Psi_{r}: U^{r} \rightarrow U_{r}$ is an unramified Galois cover with group $S_{r}$. The alternating group of degree $r$ is $A_{r}$. The dihedral group of degree $r$ (and order $2 r$ ) is $D_{r}$. Denote the $p$-adic integers by $\mathbb{Z}_{p}$, with $\mathbb{Q}_{p}$ for its quotient field. Suppose $p$ is a prime and $\mathrm{C}$ is a conjugacy class in a group $G$. Call $G$ a $p^{\prime}$ group if $(|G|, p)=1$. Call $\mathrm{C}$ a $p^{\prime}$-conjugacy class (or a $p^{\prime}$ class) if elements in $\mathrm{C}$ have orders prime to $p$. This applies to conjugacy classes in profinite groups.

Each Hurwitz space (our main moduli spaces) comes from a finite group $G$, a set of conjugacy classes $\mathbf{C}$ in $G$ and an equivalence relation on the corresponding Nielsen class. 3.1 .2 reviews this definition while enhancing traditional equivalences in Fri95a, FV91, MM99 and Vö96.

2.2. Geometrically interpreting $M_{4}$ and $\bar{M}_{4}$. The fundamental group of $\mathbb{A}^{r} \backslash D_{r}$ (resp. $\mathbb{P}^{r} \backslash D_{r}$ ) is the Artin braid group $B_{r}$ (resp. the Hurwitz monodromy group $H_{r}$ ) ([Fri77, §4], MM99 Chap. III], Vö96 Chap. 10]). Embedding $\mathbb{A}^{r}$ into $\mathbb{P}^{r}$ in (2.6) gives the lower row surjective homomorphism from $B_{r}$ to $H_{r}$. The result is a commutative diagram of fundamental groups induced from a geometric commutative diagram. 
Fundamental groups in the (2.6) upper row are the straight (or pure) Artin braid and Hurwitz monodromy groups. That is, $S H_{r}=\pi_{1}\left(\left(\mathbb{P}^{1}\right)^{r} \backslash \Delta_{r}, \boldsymbol{x}_{0}\right)$ is the kernel of $\Psi_{r}^{*}: H_{r} \rightarrow S_{r}$ mapping $Q_{i}$ to $(i i+1), i=1, \ldots, r$. It increases precision to use capitals for generators $Q_{1}, \ldots, Q_{r-1}$ of $B_{r}$ and small letters for their images $q_{1}, \ldots, q_{r-1}$ in $H_{r}$.

2.2.1. A cover corresponding to $M_{r}$. Consider $z_{0}$, an $r$-tuple of distinct points in $\mathbb{P}_{z}^{1}$. Let $P_{1}, \ldots, P_{r}$ be classical generators of $\pi_{1}\left(U_{\boldsymbol{z}_{0}}, z_{0}\right)$ as in 1.2 Suppose $\tilde{U}_{r}$ is the universal covering space of $U_{r}$. The fiber $\tilde{U}_{r, z_{0}}$ over $z_{0}$ consists of isotopy classes of classical generators on $U_{z_{0}}$; it is a homogeneous space for $H_{r}$. Use the isotopy class of $P_{1}, \ldots, P_{r}$ as a designated point $u_{0}$ in the fiber, so each $u \in \tilde{U}_{r, \boldsymbol{z}_{0}}$ is $\left(u_{0}\right) q$ for some $q \in H_{r}$. The corresponding isotopy takes a base point for classical generators for $u_{0}$ to a basepoint for classical generators for $u$. Further, a path in $\tilde{U}_{r}$ from $u$ to $u^{\prime}$ induces a canonical isomorphism $\mu_{u^{\prime}, u}: \pi_{1}\left(U_{z_{0}}, z_{u}\right) \rightarrow \pi_{1}\left(U_{z_{0}}, z_{u^{\prime}}\right)$ : $z_{u}$ (resp. $z_{u^{\prime}}$ ) is the basepoint for classical generators for $u$ (resp. $u^{\prime}$ ).

Proposition 2.2. Equivalence two points $u, u^{\prime} \in \tilde{U}_{r, z_{0}}$ if some representing isomorphism $\mu_{u^{\prime}, u}$ (with $z_{u}=z_{u^{\prime}}$ ) induces an inner automorphism on $\pi_{1}\left(U_{z_{0}}, z_{u}\right)$. This equivalence gives the fiber over $\boldsymbol{z}_{0}$ for an unramified cover $V_{r} \rightarrow U_{r}$ corresponding to $M_{r}$.

Prop. 2.2 interprets $V_{r}$ as isotopy classes of (orientation preserving) diffeomorphisms of $U_{\boldsymbol{z}_{0}}$. Classes here means to mod out by diffeomorphisms deforming (through diffeomorphisms on $U_{\boldsymbol{z}_{0}}$ ) to the identity map. Then, $H_{r}$ acts through equivalence classes of orientation preserving diffeomorphisms on $U_{\boldsymbol{z}_{0}}$. This gives a natural map from it to $M_{r}: V_{r} \rightarrow U_{r}$ has group $M_{r}$.

2.2.2. Adding $\mathrm{PSL}_{2}(\mathbb{C})$ action. Equivalence $U_{z_{0}}$ and its image under $\mathrm{PSL}_{2}(\mathbb{C})$. Elements of $\mathrm{PSL}_{2}(\mathbb{C})$ that fix $z_{0}$ may equivalence some isotopy classes of diffeomorphisms not previously equivalenced in $V_{r}$. Map these new equivalence classes to $U_{r} / \mathrm{SL}_{2}(\mathbb{C})=J_{r}$ (3.7) giving a ramified cover with group $\bar{M}_{r}$. When $r=4$ : $J_{4}=\mathbb{P}_{j}^{1} \backslash\{\infty\}$ has trivial fundamental group while $M_{4}$ is far from trivial. There is a precise Teichmüller space $\operatorname{Teich}\left(U_{z_{0}}\right)$ for this situation. It is isotopy classes of orientation preserving diffeomorphisms $\varphi: U_{z_{0}} \rightarrow U_{z}$ with $z \in U_{r}$. Here $\varphi$ is equivalent to $\varphi^{\prime}: U_{z_{0}} \rightarrow U_{\boldsymbol{z}^{\prime}}$ if $\varphi^{\prime} \circ \varphi^{-1}$ is isotopic to an analytic map $U_{\boldsymbol{z}} \rightarrow U_{\boldsymbol{z}^{\prime}}$ $\left(\right.$ from $\mathrm{PSL}_{2}(\mathbb{C})$ ).

Then $J_{r}$, the quotient of this Teichmüller space (a ball by a famous theorem) by $M_{r}$, is a moduli space. For all $r \geq 4$, we interpret it as the moduli of $r$ branch point covers of $\mathbb{P}_{z}^{1}$ modulo $\mathrm{PSL}_{2}(\mathbb{C})$ (see the proof of Prop. 4.4). For $r=2 g+2$ it would typically be considered the moduli of hyperelliptic curves of genus $g$.

The following proposition geometrically interprets the group $\bar{M}_{4}$ appearing in Thm. 2.9. As elsewhere (see $\$ 6.4 .1$ and Lem. 6.5), change the $j$ variable linearly so 0 and 1 are the locus of ramification of the degree six cover $\mathbb{P}_{\lambda}^{1} \rightarrow \mathbb{P}_{j}^{1}$. DF99, Prop. 6.5] gave an approximation to Prop. 2.3 (as in Prop. 4.2) sufficient for that paper. Its proof comprises 22.3

Proposition 2.3. When $r=4$, Thm. 2.9 shows $\left(q_{1} q_{3}^{-1}\right)^{2}$ is the center of $H_{4}$. Then, $H_{4} /\left\langle\left(q_{1} q_{3}^{-1}\right)^{2}\right\rangle=M_{4}$. Two points $v_{1}, v_{2} \in V_{r}$, respectively over $\boldsymbol{z}_{1}$ and $\boldsymbol{z}_{2}$, induce an isomorphism $\mu_{v_{1}, v_{2}}: \pi_{1}\left(U_{z_{2}}\right) \rightarrow \pi_{1}\left(U_{z_{1}}\right)$. Equivalence $v_{1}$ and $v_{2}$ if some $\alpha \in \mathrm{PSL}_{2}(\mathbb{C})$ taking $\boldsymbol{z}_{1}$ to $\boldsymbol{z}_{2}$ induces $\alpha^{*}: \pi_{1}\left(U_{\boldsymbol{z}_{1}}\right) \rightarrow \pi_{1}\left(U_{\boldsymbol{z}_{2}}\right)$ inverse to $\mu_{v_{1}, v_{2}}$. These equivalence classes form a space $V_{4}^{\text {rd }}$, inducing $\psi_{4}^{\text {rd }}: V_{4}^{\text {rd }} \rightarrow \mathbb{P}_{j}^{1} \backslash\{\infty\}$ an 
analytic (ramified) cover with automorphism group $\bar{M}_{4}=H_{4} / \mathcal{Q}$ (Thm. 2.9). The group $\mathcal{Q} /\left\langle\left(q_{1} q_{3}^{-1}\right)^{2}\right\rangle=\mathcal{Q}^{\prime \prime}$ is a Klein 4-group.

All points in the cover $\psi_{4}^{\text {rd }}$ over $j=0$ ramify of order 3. All points over $j=1$ ramify of order 2. Any Hurwitz space cover $\mathcal{H} \rightarrow U_{4}$ induces a (ramified) cover $\mathcal{H}^{\mathrm{rd}} \rightarrow \mathbb{P}_{j}^{1} \backslash\{\infty\}$ through a permutation representation of $\bar{M}_{4}$.

Assume $\mathcal{H} \rightarrow U_{4}$ and $\boldsymbol{z} \in U_{4}$ have definition field $K$ and $\boldsymbol{z} \mapsto j_{\boldsymbol{z}} \in \mathbb{P}_{j}^{1} \backslash\{0,1, \infty\}$. Suppose $\mathcal{Q}^{\prime \prime}$ acts trivially on a Nielsen class. Then, each geometric point of the fiber $\mathcal{H}_{z}$ on $\mathcal{H}$ over $\boldsymbol{z}$ goes one-one to the fiber $\mathcal{H}_{j_{z}}^{\mathrm{rd}}$ with both points having exactly the same fields of definition over $K$.

2.3. Proof of Prop. 2.3. Relate the homotopy classes of classical generators for $u^{\prime}$ to those for $u$ through applying an element $Q_{u^{\prime}, u} \in H_{r}$ (Fri77 §4] or Vö96. $\S 10.1 .7])$. The action is that given by (2.3). The map $\mu_{u^{\prime}, u}$ explicitly makes this identification with an element of $H_{r}$.

From Lem. 2.1] if $Q_{u^{\prime}, u}$ acts as an inner automorphism, then it must be in the center of $H_{r}$. Apply Thm. [2.9] $\left(q_{1} q_{3}^{-1}\right)^{2}$ is in the center of $H_{4}$ and acts as an inner automorphism of $F_{4}$. Further, since $\left(q_{1} q_{3}^{-1}\right)^{2}$ generates the whole center of $H_{4}$, $H_{4} /\left\langle\left(q_{1} q_{3}^{-1}\right)^{2}\right\rangle=M_{4}$.

2.3.1. How $\mathrm{PSL}_{2}(\mathbb{C})$ acts on $\pi_{1}\left(U_{\boldsymbol{z}}\right)$. Now consider the equivalence from composing equivalence classes defining $V_{r}$ with equivalence of maps on fundamental groups of $\pi_{1}\left(U_{\boldsymbol{z}}\right)$ from elements of $\mathrm{PSL}_{2}(\mathbb{C})$. This lies over the equivalence classes for the action of $\mathrm{PSL}_{2}(\mathbb{C})$ on $U_{r}$. The $\mathrm{PSL}_{2}(\mathbb{C})$ equivalence class of $\boldsymbol{z}$ has a representative of form $(0,1, \infty, \lambda)$. For $\lambda$ not lying over $j=0$ or 1 , we show the elements $H_{\lambda}$ fixing the set $z$ form a Klein 4-group. These computations undoubtedly occur in the literature, so we give only an outline.

First: $H_{\lambda}$ contains a Klein 4-group. Example: The $\alpha \in \mathrm{PSL}_{2}(\mathbb{C})$ switching 0 and 1 , and switching $\infty$ and $\lambda$ is $\frac{-z+1}{-z / \lambda+1}$.

Second: To see that no $\alpha \in \mathrm{PSL}_{2}(\mathbb{C})$ acts like a 4-cycle on the support of $z$ unless $\lambda$ is special, assume with no loss,

$$
\alpha(0)=1, \alpha(1)=\infty, \alpha(\infty)=\lambda, \alpha(\lambda)=0 .
$$

Then, $\alpha(z)=\frac{\lambda z-1}{z-1}$. From the last condition $\lambda^{2}=1$ or $\lambda=-1$.

Third: To see that no $\alpha \in \mathrm{PSL}_{2}(\mathbb{C})$ acts like a 3-cycle on the support of $\boldsymbol{z}$ unless $\lambda$ is special, assume $\alpha$ is one of the permutations of $\{0,1, \infty\}$ and $\lambda$ is an outside fixed point of $\alpha$. Example: $\alpha(z)=\frac{z-1}{z}$ also fixes the two roots of $z^{2}-z+1$, which are sixth roots of 1 .

These computations show for each $j \in \mathbb{P}_{j}^{1} \backslash\{0,1, \infty\}$, the fiber of equivalence classes is the same as the fiber of $V_{4}^{\text {rd }}$ over $z$ modulo the faithful action of a Klein 4-group. Over $j=0$ the fiber is the same as the fiber of $V_{4}^{\text {rd }}$ over $z$ modulo the faithful action of a group isomorphic to $A_{4}$, and over $j=1$ it is the fiber over $z$ modulo the action of a group isomorphic to $D_{4}$, the dihedral group of order 8 .

2.3.2. Identifying the Klein 4-group in $M_{4}$. Complete the identification of the group of $V_{4}^{\text {rd }} \rightarrow \mathbb{P}_{j}^{1} \backslash\{\infty\}$ with $\bar{M}_{4}$ from Thm. 2.9] This only requires $\mathcal{Q} /\left\langle\left(q_{1} q_{3}^{-1}\right)^{2}\right\rangle=$ $\mathcal{Q}^{\prime \prime}$ to induce on $\pi_{1}\left(U_{\boldsymbol{z}}\right)$ the Klein 4 - group in $\mathrm{PSL}_{2}(\mathbb{C})$ that sits in $H_{\boldsymbol{z}}$ above: Those switching the support of $\boldsymbol{z}$ in pairs.

This could be a case-by-case identification. Since, however, Thm. 2.9 shows $\mathcal{Q}$ is the minimal normal subgroup of $H_{4}$ containing $q_{1} q_{3}^{-1}$, it suffices to achieve $q_{1} q_{3}^{-1}$ through an element of $\mathrm{PSL}_{2}(\mathbb{C})$. It is convenient to take $\boldsymbol{z}=\{0, \infty,+1,-1\}$, though this $H_{\boldsymbol{z}}$ is larger than a Klein 4 -group. 
Crucial to the proof of DF99 Prop. 6.5] is $\mu: z \mapsto-1 / z \in \mathrm{PSL}_{2}(\mathbb{C})$ and its effect on covers $\varphi: X \rightarrow \mathbb{P}_{z}^{1}$ in a Nielsen class branched over $z=\{0, \infty,+1,-1\}$. Under any reduced equivalence of covers in this paper, $\varphi$ and $\mu \circ \varphi$ are equivalent. The argument uses a set of paths $\lambda_{0}, \lambda_{\infty}, \lambda_{+1}, \lambda_{-1}$ based at $i$. These give classical generators (1.2) of $\pi_{1}\left(U_{z}, i\right)$ with product $\lambda_{0} \lambda_{\infty} \lambda_{+1} \lambda_{-1}$ homotopic to 1 .

The effect of $\mu^{-1}$ (up to homotopy) is to switch $\lambda_{0}$ and $\lambda_{\infty}$, and to map $\lambda_{+1}$ to a path $\lambda^{\prime}$ for which $\lambda_{0} \lambda^{\prime}$ is homotopic to $\lambda_{-1} \lambda_{0}$. Conjugating the resulting collection of paths by $\lambda_{0}$ gives the effect of $\mu^{-1}$ as follows. It takes a representative $\boldsymbol{g}$ of the Nielsen class of $\varphi$ to $(\boldsymbol{g}) q_{1} q_{3}^{-1}$ conjugated by $g_{1}$. This fills in DF99 Prop. 6.5] and corrects a typo giving $\mu(z)$ as $1 / z$ (which doesn't fix $i$ ).

2.3.3. The mapping from $V_{r}^{\text {rd }}$ to reduced Hurwitz spaces. The points of a Hurwitz space $\mathcal{H}$ over $z \in U_{r}$ correspond to equivalence classes of homomorphisms from $\pi_{1}\left(U_{z}\right) \rightarrow G$ where $G$ is a finite group attached to the Hurwitz space. While $\mathcal{H}$ may have several connected components, easily reduce to constructing a map from $V_{r}^{\text {rd }}$ to any one of them. Start with a base point $v_{0} \in V_{r}^{\text {rd }}$ over $z$ and a base point $\boldsymbol{p} \in \mathcal{H}$ over $\boldsymbol{z}$. Then, relative to the classical generators given by $v_{0}, \boldsymbol{p}$ corresponds to a specific homomorphism $\psi: \pi_{1}\left(U_{z}\right) \rightarrow G$ up to inner automorphism or some stronger equivalence (3.1.2). Any other point $v^{\prime} \in V_{r}$ lying over $\boldsymbol{z}^{\prime}$ comes with an isomorphism from $\pi_{1}\left(U_{\boldsymbol{z}}\right) \rightarrow \pi_{1}\left(U_{\boldsymbol{z}^{\prime}}\right)$.

This isomorphism takes classical generators of $\pi_{1}\left(U_{\boldsymbol{z}}\right)$ to classical generators of $\pi_{1}\left(U_{\boldsymbol{z}^{\prime}}\right)$. Relative to this new set of generators, form the same homomorphism into $G$. Interpret as a canonically given homomorphism $\pi_{1}\left(U_{\boldsymbol{z}^{\prime}}\right) \rightarrow G$. This homomorphism determines the image of $v$ in $\mathcal{H}$ and an unramified cover $V_{r} \rightarrow \mathcal{H}$. This map respects equivalence classes modulo $\mathrm{PSL}_{2}(\mathbb{C})$ action. So it produces $V_{r}^{\text {rd }} \rightarrow \mathcal{H}^{\text {rd }}$.

2.3.4. $\mathcal{Q}^{\prime \prime}$ action when $\mathcal{H}^{\text {rd }}$ has genus 0. For $r \geq 5$, the analog of $\mathcal{Q}^{\prime \prime}$ is trivial (6.4.4. Then, if a component $\mathcal{H}_{*}^{\text {rd }}$ of $\mathcal{H}^{\text {rd }}$ has a dense set of rational points, it is automatically true for $\mathcal{H}_{*}$.

REMARK 2.4 (If $\mathcal{Q}^{\prime \prime}$ acts faithfully: I). Assume $r=4, \mathcal{Q}^{\prime \prime}$ acts faithfully and $K=\mathbb{R}$. If $\mathcal{H}^{\text {in }}$ or $\mathcal{H}^{\text {abs }}$ is a fine moduli space (over $\mathbb{R}$ ), then its $\mathbb{R}$ points correspond to equivalence classes of covers represented by an actual cover over $\mathbb{R}$. Prop. 6.3. efficiently tests for $\mathbb{R}$ points on $\mathcal{H}^{\text {rd }}$ being cover or Brauer points (Cor. 6.7).

Remark 2.5 (If $\mathcal{Q}^{\prime \prime}$ acts faithfully: II). Suppose $r=4$ and a component $\mathcal{H}_{*}^{\text {rd }}$ of $\mathcal{H}^{\text {rd }}$ (image of a component $\mathcal{H}_{*}$ of the Hurwitz space $\mathcal{H}$ ) has genus 0 and dense $K$ points. If any conjugacy class in $\mathbf{C}$ is $K$ rational and distinct from the others, then Lem. 4.11 shows $\mathcal{H}_{*}(K)$ is also dense in $\mathcal{H}_{*}$. This, however, leaves many cases: all four conjugacy classes are distinct, none $K$ rational; or each conjugacy class appears at least twice in $\mathbf{C}$. Further, $\mathcal{Q}^{\prime \prime}$ can act neither faithfully, nor trivially, on Nielsen classes through $\mathbb{Z} / 2$ (see Ex. 9.2). As in Lem. 4.11 it is useful in all cases to distinguish between $K$-cover and $K$-Brauer points on $\mathcal{H}^{\text {rd }}$.

For the Inverse Galois Problem, $r=4$ is special for the possibility of direct computations, especially when the simplest case of Braid rigidity (for $r=3$ ) does not apply. So, Prop. 2.3 is valuable for applications to it. Here is an example long in the literature.

EXAmple 2.6 ( $\operatorname{Spin}_{5}$ realizations). The Hurwitz space $\overline{\mathcal{H}}\left(A_{5}, \mathbf{C}_{3^{4}}\right)^{\text {in,rd }}$ (over $\mathbb{Q})$ has genus 0 and a rational point $[$ Fri90 $\S 3]$. From Ex. 8.5 $\mathcal{Q}^{\prime \prime}$ acts trivially. Prop. 2.3 shows $\mathcal{H}\left(A_{5}, \mathbf{C}_{3^{4}}\right)^{\text {in }}$ has a dense set of $\mathbb{Q}$ points. Lem. 6.11 and Lem. 6.14 illustrate this phenomena. So, even though the reduced Hurwitz space is not a fine 
moduli space, its $K$ points are still realized by $K$-covers. This also happens when the reduced spaces are modular curves (as in the moduli dilemma of 2.8.2).

It is more subtle to test when $\boldsymbol{p} \in \mathcal{H}\left(A_{5}, \mathbf{C}_{3^{4}}\right)^{\text {in,rd }}(\mathbb{Q})$ gives a $\operatorname{Spin}_{5}$ realization. Any $\boldsymbol{p}$ produces a cover sequence $Y_{\boldsymbol{p}} \rightarrow X_{\boldsymbol{p}} \rightarrow \mathbb{P}_{z}^{1}$ with $Y=Y_{\boldsymbol{p}} \rightarrow \mathbb{P}_{z}^{1}$ geometrically Galois with group $\operatorname{Spin}_{5}$ having the following two properties.

(2.7a) $X=X_{\boldsymbol{p}} \rightarrow \mathbb{P}_{z}^{1}$ is an $A_{5}$ cover over $\mathbb{Q}$.

(2.7b) $Y \rightarrow X$ is unramified: from the unique $H_{4}$ orbit (2.9.3) on $\mathrm{Ni}\left(A_{5}, \mathbf{C}_{3^{4}}\right)$ having lifting invariant 1 (it contains an H-M rep. or from Prop. [5.9).

If $Y \rightarrow X$ is over $\mathbb{Q}$, this gives a regular $\operatorname{Spin}_{5}$ realization. [Ser92 100] summarizes a sufficient condition for this when the branch cycles have odd order (as here). It is that $X$ has a $\mathbb{Q}$ point.

See this directly by applying Lem. 3.15 to $Y \rightarrow X$. The resulting cover $Y^{\prime} \rightarrow X$ has degree 2 , is therefore Galois, and $Y^{\prime} \rightarrow \mathbb{P}_{z}^{1}$ produces the $\operatorname{Spin}_{5}$ realization. Yet, $X=X_{\boldsymbol{p}}$ won't have a $\mathbb{Q}$ point for all $\mathbb{Q}$ points $\boldsymbol{p}$. This fails even if we replace $\mathbb{Q}$ by $\mathbb{R}$. Those $A_{5}$ covers with a nontrivial complex conjugation operator don't achieve a $\operatorname{Spin}_{5}$ realization; those with a trivial conjugation operator do. See Lem. 6.11 and 6.14 to see both happen.

This is a special case of Prop. 6.8 The same phenomenon occurs at all levels of the $A_{5}$ Modular Tower for $p=2$. That is, there are real points achieving regular realization of a nontrivial central extension of $G_{k}$ (H-M reps.), and real points that don't (near H-M reps.; see Prop. 8.12).

2.3.5. Isotopy classes of diffeomorphisms. An elliptic curve over $K$ (genus 1 with a $K$ point) is a degree two cover of the sphere over $K$, ramified at four distinct unordered points $\boldsymbol{z}$. The equivalence class of $\boldsymbol{z}$ modulo the action of $\mathrm{PSL}_{2}(\mathbb{C})$ determines the isomorphism class of the elliptic curve. Isotopy classes of (orientation preserving) diffeomorphisms of a complex torus $X$ identifies with $\mathrm{SL}_{2}(\mathbb{Z})$, appearing from its action on $H^{1}(X, \mathbb{Z})$.

Isotopy classes of diffeomorphisms of $U_{\boldsymbol{z}_{0}}$ (as elements in $\bar{M}_{4}=\mathrm{PSL}_{2}(\mathbb{Z})$ : Prop. 2.3) relate to diffeomorphisms of the torus attached to $z_{0}$. The difference? The complex torus has a chosen origin (a canonical rational point) preserved by diffeomorphisms; there is no chosen point in $z$. This appears in mapping a space of involution realizations of dihedral groups to a modular curve (Fri78; the most elementary example from 2.8 .3 .

\subsection{Statement of the main presentation theorem.}

2.4.1. Presenting $H_{3}$. Basics about $H_{3}$ expressed in $q_{1}$ and $q_{2}: q_{1} q_{2}=\tau_{1}$ and $q_{1} q_{2} q_{1}=\tau_{2}$ generate $H_{3}$. From (2.4), $q_{1}^{2}=q_{2}^{-2}$. Further:

$$
\tau_{1}^{3}=q_{1} q_{2}\left(q_{1} q_{2} q_{1}\right) q_{2}=q_{1} q_{2}\left(q_{2} q_{1} q_{2}\right) q_{2}=q_{2}^{2}=q_{1}^{2}=\tau_{2}^{2} .
$$

This identifies $\mathrm{H}_{3}$.

Definition 2.7. Characterize the dicyclic (or quaternion) group $Q_{4 n}$ of order $4 n$ as having generators $\tau_{1}, \tau_{2}$ with $\operatorname{ord}\left(\tau_{1}\right)=2 n, \operatorname{ord}\left(\tau_{2}\right)=4, \tau_{2}^{-1} \tau_{1} \tau_{2}=\tau_{1}^{-1}$ and $\tau_{2}^{2}=\tau_{1}^{n}$. [St94, p. 43] has a memorable matrix representation of $Q_{4 n}$ in $\mathrm{GL}_{4}(\mathbb{R})$ : $\tau_{1}=\left(\begin{array}{cc}R_{2 \pi / n} & 0_{2} \\ 0_{2} & R_{2 \pi / n}\end{array}\right)$ and $\tau_{2}=\left(\begin{array}{cc}0_{2} & \mu \\ -\mu & 0_{2}\end{array}\right)$ with $R_{\theta}$ the rotation in 2-space through the angle $\theta, 0_{2}$ the $2 \times 2$ zero matrix and $\mu=\left(\begin{array}{cc}1 & 0 \\ 0 & -1\end{array}\right)$. For $n=3$ :

(2.8a) $\operatorname{ord}\left(\tau_{1}\right)=6$ and $\operatorname{ord}\left(\tau_{2}\right)=4$; and 
(2.8b) $H_{3}$ is the dicyclic group of order 12 .

Lemma 2.8. For each integer $k, \tau_{2} \tau_{1}^{k}$ in $Q_{4 n}$ has order $4: \tau_{2} \tau_{1}^{k} \tau_{2} \tau_{1}^{k}=\tau_{2}^{2}$. For $n=2^{t}$, every proper subgroup of $Q_{4 n}$ contains $\tau_{2}^{2}$, and $Q_{4 n} /\left\langle\tau_{2}^{2}\right\rangle$ is the dihedral group $D_{n}$ of order $2 n$. If $u \mid n$, then $Q_{4 u}=\left\langle\tau_{1}^{\frac{n}{u}}, \tau_{2}\right\rangle \leq Q_{4 n}$.

2.4.2. Notation for computations in $H_{4}$. From this point $r=4$. We give a convenient presentation of $H_{4}$ and various subgroups and quotients. Computations in both $H_{4}$ and in $M_{4}$ use the letters $q_{1}, q_{2}, q_{3}$ for the images of $Q_{1}, Q_{2}, Q_{3}$ in either. Distinguishing between their images in these two groups requires care about the context. Here is how we intend to do that.

When $r=4$, the extra relations for $M_{4}$ (beyond those for $B_{4}$ ) are

$$
\tau_{1}=\left(Q_{3} Q_{2}\right)^{3}=1, \tau_{2}=Q_{1}^{-2} Q_{3}^{2}=1, \tau_{3}=\left(Q_{2} Q_{1}\right)^{-3}=1
$$

and $\tau=\left(Q_{3} Q_{2} Q_{1}\right)^{4}=1$. Lemma 2.10 shows adding these relations to $B_{4}$ is equivalent to adding $q_{1}^{2} q_{3}^{-2}=1$ to $H_{4}$. This produces new equations:

$$
q_{1} q_{2} q_{1}^{2} q_{2} q_{1}=\left(q_{1} q_{2} q_{1}\right)^{2}=\left(q_{1} q_{2}\right)^{3}=1 .
$$

Then, $\gamma_{0}=q_{1} q_{2}, \gamma_{1}=q_{1} q_{2} q_{1}$ and $\gamma_{\infty}=q_{2}$ satisfy

$$
\gamma_{0} \gamma_{1} \gamma_{\infty}=1
$$

The relation $q_{1} q_{3}^{-1}=1$ is not automatic from (2.9). Crucial, however, is how $M_{4}$ acts on reduced Nielsen classes (4.2). This action does factor through the relation $q_{1} q_{3}^{-1}=1$ (3.7 and Prop. 3.28). Therefore it factors through the induced quotient $H_{4} / \mathcal{Q}=\bar{M}_{4}$ of Thm. 2.9 from $H_{4}$ acting on Nielsen classes (Prop. 4.4). The upper half plane appears as a classical ramified Galois cover of the $j$-line minus $\infty$. The elements $\gamma_{0}$ and $\gamma_{1}$ in $\bar{M}_{4}$ generate the local monodromy of this cover around 0 and 1 (4.2).

Sometimes $q_{2} \in H_{4}$ acts significantly different when viewed in $\bar{M}_{4}$. On these occasions denote it $\gamma_{\infty}$ : representing the local monodromy action corresponding to the cusp $\infty$ of the $j$-line (3.7). Similarly, denote $q_{1} q_{2} q_{3}$ as sh: the shift. From the above, sh and $\gamma_{1}$ are the same in $\bar{M}_{4}($ 2.7).

TheOrem 2.9. Let $\mathcal{Q}=\left\langle\left(q_{1} q_{2} q_{3}\right)^{2}, q_{1} q_{3}^{-1}\right\rangle$. The following hold.

(2.11a) $H_{4}$ has one nontrivial involution $z=\left(q_{1} q_{3}^{-1}\right)^{2}$ generating its center.

(2.11b) $\mathcal{Q} \triangleleft H_{4}$ and $\mathcal{Q}$ is the quaternion group $Q_{8}$ (Lem. [2.8).

(2.11c) $H_{4} / \mathcal{Q}=\bar{M}_{4} \cong \mathrm{PSL}_{2}(\mathbb{Z})$.

(2.11d) Exactly two conjugacy classes of $H_{4}$ subgroups $U_{1}=\left\langle q_{1}, q_{2}\right\rangle$ and $U_{2}=$ $\left\langle q_{2}, q_{3}\right\rangle$ (both containing $\langle z\rangle$ ) are isomorphic to $\mathrm{SL}_{2}(\mathbb{Z})$.

From (2.11b), $\mathcal{Q}$ is the smallest normal subgroup of $H_{4}$ containing either $\left(q_{1} q_{2} q_{3}\right)^{2}$ or $q_{1} q_{3}^{-1}$. So, from (2.9), $M_{4}=H_{4} /\langle z\rangle$ and $\bar{M}_{4}=\left\langle\gamma_{0}, \gamma_{1}\right\rangle=\left\langle\gamma_{1}, \gamma_{\infty}\right\rangle$ is $M_{4}$ modulo the relation $q_{1}=q_{3}$.

2.5. Start of Theorem 2.9 proof. Use 2.1 generators $\bar{\sigma}_{1}, \ldots, \bar{\sigma}_{4}$ of $F_{4}$, etc. Since $r=4$, use $A$ for $\bar{A}_{4}$. As above, reserve the symbols $Q_{1}, Q_{2}, Q_{3}$ for the generators of the $B_{4}$, and $q_{1}, q_{2}, q_{3}$ for their images in $H_{4}$. For convenience, arrange 
the $Q \mathrm{~s}$ action in a table. The $i$ th column has $Q_{i}$ acting on the 4 -tuple of $\bar{\sigma} \mathrm{s}$ :

$\begin{array}{cccc} & Q_{1} & Q_{2} & Q_{3} \\ \bar{\sigma}_{1} & \bar{\sigma}_{1} \bar{\sigma}_{2} \bar{\sigma}_{1}^{-1} & \bar{\sigma}_{1} & \bar{\sigma}_{1} \\ \bar{\sigma}_{2} & \bar{\sigma}_{1} & \bar{\sigma}_{2} \bar{\sigma}_{3} \bar{\sigma}_{2}^{-1} & \bar{\sigma}_{2} \\ \bar{\sigma}_{3} & \bar{\sigma}_{3} & \bar{\sigma}_{2} & \bar{\sigma}_{3} \bar{\sigma}_{4} \bar{\sigma}_{3}^{-1} \\ \bar{\sigma}_{4} & \bar{\sigma}_{4} & \bar{\sigma}_{4} & \bar{\sigma}_{3} .\end{array}$

Let $i: F_{4} \rightarrow A$ map $\bar{\sigma} \in F$ to the inner automorphism it induces. Initially we work with the image of $B_{4}$ in $A$ where $i\left(F_{4}\right)$ and $B_{4}$ commute. Denote $Q_{1} Q_{2} Q_{3}^{2} Q_{2} Q_{1}$ by $D$ : it maps $\bar{\sigma}$ to

$$
\begin{aligned}
& \left(\bar{\sigma}_{1} \bar{\sigma}_{2} \bar{\sigma}_{1}^{-1}, \bar{\sigma}_{1} \bar{\sigma}_{3} \bar{\sigma}_{1}^{-1}, \bar{\sigma}_{1} \bar{\sigma}_{4} \bar{\sigma}_{1}^{-1}, \bar{\sigma}_{1}\right) Q_{3} Q_{2} Q_{1}= \\
& \left(\bar{\sigma}_{1} \bar{\sigma}_{2} \bar{\sigma}_{3} \bar{\sigma}_{4} \bar{\sigma}_{1} \bar{\sigma}_{4}^{-1} \bar{\sigma}_{3}^{-1} \bar{\sigma}_{2}^{-1} \bar{\sigma}_{1}^{-1}, \bar{\sigma}_{1} \bar{\sigma}_{2} \bar{\sigma}_{1}^{-1}, \bar{\sigma}_{1} \bar{\sigma}_{3} \bar{\sigma}_{1}^{-1}, \bar{\sigma}_{1} \bar{\sigma}_{4} \bar{\sigma}_{1}^{-1}\right) .
\end{aligned}
$$

Further Notation: Let $G_{4}$ be the quotient of $F_{4}$ by the relation

$$
\Pi(\overline{\boldsymbol{\sigma}})=\bar{\sigma}_{1} \bar{\sigma}_{2} \bar{\sigma}_{3} \bar{\sigma}_{4}=1 .
$$

While $G_{4}$ is a free group on 3 generators, $H_{4}$ computations require $\bar{\sigma}_{1}, \bar{\sigma}_{2}, \bar{\sigma}_{3}, \bar{\sigma}_{4}$ to appear symmetrically. So, we use this quotient presentation. Let $\operatorname{Aut}\left(G_{4}\right)$ be the automorphisms of $G_{4}$. Then $\operatorname{Inn}\left(G_{4}\right)$ is the normal subgroup of inner automorphisms of $G_{4}$. Recall: The image of $D$, and all its conjugates, in $H_{4}$ is trivial.

LEMma 2.10. Both $\left(Q_{1} Q_{2} Q_{3}\right)^{4}$ and $\left(Q_{3} Q_{2} Q_{1}\right)^{4}$ map $\overline{\boldsymbol{\sigma}}$ to

$$
\bar{\sigma}_{1} \bar{\sigma}_{2} \bar{\sigma}_{3} \bar{\sigma}_{4} \overline{\boldsymbol{\sigma}} \bar{\sigma}_{4}^{-1} \bar{\sigma}_{3}^{-1} \bar{\sigma}_{2}^{-1} \bar{\sigma}_{1}^{-1}=(\overline{\boldsymbol{\sigma}}) i\left(\bar{\sigma}_{4}^{-1} \bar{\sigma}_{3}^{-1} \bar{\sigma}_{2}^{-1} \bar{\sigma}_{1}^{-1}\right) .
$$

Action of $B_{4}$ on $F_{4}$ induces $\alpha_{4}: B_{4} \rightarrow \operatorname{Aut}\left(G_{4}\right)$ with the center $\left\langle\left(Q_{1} Q_{2} Q_{3}\right)^{4}\right\rangle$ of $B_{4}$ generating the kernel. This induces $\mu_{4}: H_{4} \rightarrow \operatorname{Aut}\left(G_{4}\right) / \operatorname{Inn}\left(G_{4}\right)$. The kernel of $B_{4} \rightarrow M_{4}$ is the direct product of the free group $K_{4}^{*}=\left\langle\left(Q_{3} Q_{2}\right)^{3}, Q_{1}^{-2} Q_{3}^{2},\left(Q_{2} Q_{1}\right)^{-3}\right\rangle$ and $\left\langle\left(Q_{1} Q_{2} Q_{3}\right)^{4}\right\rangle$. Above, $Z=i\left(\bar{\sigma}_{1} \bar{\sigma}_{2} \bar{\sigma}_{3} \bar{\sigma}_{4}\right)$ is identical to an element of $B_{4}$. Consider the image $z$ of $Z$ in $H_{4}$ :

$$
\left(q_{1} q_{2} q_{3}\right)^{4} z=1=\left(q_{3} q_{2} q_{1}\right)^{4} z=q_{1}^{2} q_{3}^{-2} z \text { and } z^{2}=1 .
$$

In particular, combining this with (2.9) identifies $M_{4}$ with the image of $\mathrm{H}_{4}$ in $\operatorname{Aut}\left(G_{4}\right) / \operatorname{Inn}\left(G_{4}\right)$. So, the image of $z$ in $M_{4}$ is 1 .

Proof. The map $i$ induces $i: G_{4} \rightarrow \operatorname{Inn}\left(G_{4}\right)$. Action of $B_{4}$ preserves $\Pi(\overline{\boldsymbol{\sigma}})$. Thus, it induces a homomorphism of $B_{4}$ into $\operatorname{Aut}\left(G_{4}\right)$ where $D$ goes to the automorphism $i\left(\bar{\sigma}_{1}^{-1}\right), \overline{\boldsymbol{\sigma}} \mapsto \bar{\sigma}_{1} \overline{\boldsymbol{\sigma}} \bar{\sigma}_{1}^{-1}$ (as in (2.12)). Conclude: Modulo inner automorphisms of $G_{4}, D$ acts trivially, producing the desired homomorphism

$$
H_{4} \rightarrow \operatorname{Aut}\left(G_{4}\right) / \operatorname{Inn}\left(G_{4}\right) .
$$

Now consider the explicit formulas. Most of the calculation is in (2.12): $Q_{1} Q_{2} Q_{3}$ cycles entries of $\overline{\boldsymbol{\sigma}}$ back 1 , and conjugates all entries by the first entry's inverse. So $\left(Q_{1} Q_{2} Q_{3}\right)^{4}$ leaves entries of $\overline{\boldsymbol{\sigma}}$ untouched except for conjugating them by

$$
\left(\bar{\sigma}_{1} \bar{\sigma}_{2} \bar{\sigma}_{3} \bar{\sigma}_{4} \bar{\sigma}_{3}^{-1} \bar{\sigma}_{2}^{-1} \bar{\sigma}_{1}^{-1} \bar{\sigma}_{1} \bar{\sigma}_{2} \bar{\sigma}_{3} \bar{\sigma}_{2}^{-1} \bar{\sigma}_{1}^{-1} \bar{\sigma}_{1} \bar{\sigma}_{2} \bar{\sigma}_{1}^{-1} \bar{\sigma}_{1}\right)^{-1}=\left(\bar{\sigma}_{1} \bar{\sigma}_{2} \bar{\sigma}_{3} \bar{\sigma}_{4}\right)^{-1} \text {. }
$$

Also, $Q_{3} Q_{2} Q_{1}$ cycles the entries of $\bar{\sigma}$ forward 1 . The new first entry is the old 4 th entry conjugated by the inverse of the product of the old first three entries. Thus, $\left(Q_{1} Q_{2} Q_{3}\right)^{4}$ and $\left(Q_{3} Q_{2} Q_{1}\right)^{4}$ act the same. Add that $D$ maps to 1 in $H_{4}$ to see

$$
1=\left(q_{1} q_{2} q_{3}\right)^{4}\left(q_{3} q_{2} q_{1}\right)^{4}=\left(q_{1} q_{2} q_{3}\right)^{8}=z^{-2} .
$$


Let $Q^{\prime}=Q_{1} Q_{2} Q_{3}$. Extending the calculation above gives the next list:

$$
\begin{gathered}
1=\left(Q^{\prime}\right)^{-1} D Q^{\prime}\left(Q_{1} Q_{2}\right)^{3} i\left(\bar{\sigma}_{1} \bar{\sigma}_{2} \bar{\sigma}_{3} \bar{\sigma}_{4}\right) ; \\
1=\left(Q^{\prime} Q_{1} Q_{3}^{-1}\right)^{2}\left(Q^{\prime}\right)^{-1} D Q^{\prime} i\left(\bar{\sigma}_{1} \bar{\sigma}_{2} \bar{\sigma}_{3} \bar{\sigma}_{4}\right) ; \\
1=\left(Q^{\prime}\right)^{4} i\left(\bar{\sigma}_{1} \bar{\sigma}_{2} \bar{\sigma}_{3} \bar{\sigma}_{4}\right) ; \text { and } \\
1=\left(Q_{1} Q_{3}^{-1}\right)^{2}\left(Q^{\prime}\right)^{-1} D\left(Q_{1} Q_{2} Q_{3}\right)\left(Q_{1} Q_{2} Q_{3}\right)^{-2} D\left(Q_{1} Q_{2} Q_{3}\right)^{2} i\left(\bar{\sigma}_{1} \bar{\sigma}_{2} \bar{\sigma}_{3} \bar{\sigma}_{4}\right) .
\end{gathered}
$$

Add the relation $Q_{1} Q_{3}=Q_{3} Q_{1}$ to deduce, in order, these relations in $H_{4}$ :
a) $\left(q_{1} q_{2}\right)^{3} z=1$;
b) $\left(q_{1} q_{2} q_{1}\right)^{2} z=1$;
c) $\left(q_{1} q_{2} q_{3}\right)^{4} z=1$;
d) $\left(q_{1} q_{3}^{-1}\right)^{2} z=1$; e) $\quad q_{1} q_{3}=q_{3} q_{1}$.

So, the image of $z$ in $M_{4}$ is 1 .

The kernel of $\alpha_{4}$ contains elements of $B_{4}$ inducing inner automorphisms commuting with conjugation by $\bar{\sigma}_{1} \bar{\sigma}_{2} \bar{\sigma}_{3} \bar{\sigma}_{4}=c$. Since $c$ generates conjugations commuting with $c,\left\langle\left(Q_{1} Q_{2} Q_{3}\right)^{4}\right\rangle$ generates the kernel of $\alpha_{4}$.

Generators of $K_{4}^{*}$ act on $G_{4}$ : Respectively, $\left(Q_{3} Q_{2}\right)^{3}, Q_{1}^{-2} Q_{3}^{2},\left(Q_{2} Q_{1}\right)^{-3}$ induce conjugation by $g_{4}, g_{1} g_{2}, g_{1}$. These conjugations on $G_{4}$ form a free group. So $K_{4}^{*}$ is a free group on these generators.

The remainder of the proof of Thm. 2.9] is in $\$ 2.6$

2.6. Properties of $\mathcal{Q}$; combinatorics of (2.11). First we show $\mathcal{Q}$ is a normal subgroup isomorphic to $Q_{8}$. Then we list combinatorics contributing to (2.11).

2.6.1. $z$ is the involution in $Q_{16}$. From (2.13 $\left.\mathrm{d}\right), z$ is a word in $q_{1}$ and $q_{3}$. So, 2.13. shows $z$ commutes with $q_{1}$ and $q_{3}$. From

$$
q_{2}\left(q_{1} q_{2}\right)^{3}=\left(q_{1} q_{2} q_{1}\right)\left(q_{1} q_{2}\right)^{2}=\left(q_{1} q_{2}\right)^{3} q_{2},
$$

$q_{2}$ commutes with $\left(q_{1} q_{2}\right)^{3}$. Apply (2.13): $z$ commutes with $q_{i}, i=1,2,3$. Thus, Lemma 2.10] shows $z$ is a central involution. 22.6 .6 shows $z \neq 1$ and illustrates its significance. 2.6.4 reformulates property (2.11] :

(2.14) $z$ is the only involution of $H_{4}$.

Consider $\mathcal{Q}^{\prime}=\left\langle q_{1} q_{2} q_{3}, q_{1} q_{3}^{-1}\right\rangle$. Set $\alpha=q_{1} q_{2} q_{3}, \beta=q_{1} q_{2}$ and $\gamma=q_{1} q_{3}^{-1}$. These generators simplify presenting $H_{4}$.

LEMma 2.11. Rewrite $(2.13-\mathrm{d})$ using $\alpha, \beta$ and $\gamma$ to give

$$
\text { a) }(\beta)^{3}=z \text {; b) }(\alpha \gamma)^{2}=z \text {; c) }(\alpha)^{4}=z \text {; and d) }(\gamma)^{2}=z \text {. }
$$

Further, $H_{4}=\langle\alpha, \beta, \gamma\rangle$. Since $z$ is a central involution, $(2.15 \mathrm{~b}-\mathrm{d})$ show $\mathcal{Q}^{\prime}=\langle\alpha, \gamma\rangle$ is $Q_{16}$ and $\mathcal{Q}^{\prime} \bmod z$ is the dihedral group of order 8 (Lem. [2.8).

2.6.2. $\mathcal{Q}=\left\langle\alpha^{2}, \gamma\right\rangle \triangleleft H_{4}$ and $\mathrm{Cusp}_{4}$. From relations for $B_{4}$,

$$
\alpha^{2} \gamma=q_{1} q_{2} q_{3} q_{1} q_{2} q_{1}=q_{1} q_{2} q_{3} q_{2} q_{1} q_{2}=q_{1} q_{3} q_{2} q_{3} q_{1} q_{2}=q_{3} \alpha^{2} q_{3}^{-1}
$$

and $q_{3}(\gamma) q_{3}^{-1}=\gamma$. Thus $q_{3}$ normalizes $\mathcal{Q}$. Since $q_{1} q_{3}^{-1} \in \mathcal{Q}, q_{1}$ also normalizes $\mathcal{Q}$. We now show $q_{2}$ does also. Apply (2.13) in the form $q_{2}^{-1} q_{1}^{-1} q_{2}^{-1}=z q_{1} q_{2} q_{1}$ to get

$$
q_{2}^{-1} \gamma^{-1} q_{2}=q_{2}^{-1} q_{1}^{-1} q_{2}^{-1} q_{2} q_{3} q_{2}=z\left(q_{1} q_{2} q_{1}\right) q_{3} q_{2} q_{3}=z \alpha^{2} .
$$

Also, since $z$ is a central involution, conjugate by $q_{2}^{-1}$ to get $q_{2} \alpha^{2} q_{2}^{-1}=z \gamma^{-1}$.

Definition 2.12 (Cusp group). With $\mathcal{Q}^{\prime \prime}=\mathcal{Q} /\langle z\rangle$, denote the subgroup

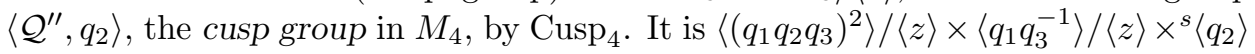
with $q_{2}$ switching the two factors on the copy of the Klein $K_{4}$. 
Nontrivial calculations with $\mathrm{Cusp}_{4}$ figure in computing the genus of reduced Hurwitz space components (see 7.2 .3 ).

2.6.3. Proof of (2.11i). From (2.9), the image of $q_{1} q_{2}$ and $q_{1} q_{2} q_{1}$ give elements of respective orders 3 and 2 generating $H_{4} / \mathcal{Q}$. A well-known abstract characterization of $\mathrm{PSL}_{2}(\mathbb{Z})$ is as the free product of elements of order 3 and 2. Further, $\mathrm{SL}_{2}(\mathbb{Z})$ is a free amalgamation of cyclic groups of order 6 and 4 along their common subgroups of order 2 Ser77]. Thus, $H_{4} / \mathcal{Q}$ is isomorphic to a quotient of $\mathrm{PSL}_{2}(\mathbb{Z})$. The proceeding arguments, however, have derived a presentation for $H_{4}$, and so for $H_{4} / \mathcal{Q}$. Thus $2.11 \mathrm{ic}$ ) holds.

2.6.4. Conclude properties (2.11). 2.6.2 and 2.6.3 give (2.11b) and (2.110). In any quotient of the group $\langle a, b\rangle$ where $a 2=b^{3}=1$, all involutions are conjugate to $a$. Thus, the only possible involutions in $H_{4}$ are in $\mathcal{Q}$, and this contains just one. From (2.13) (expression c)), $q_{1} q_{2} q_{1}$ has order 4. This gives 2.117it). It remains to discuss $z$ being nontrivial (as above) and (2.11]).

From (2.11 1 ), each subgroup of $H_{4}$ containing (a copy of) $\mathrm{SL}_{2}(\mathbb{Z})$ contains $z$. Thus, there is a one-one correspondence between subgroups $G$ of $H_{4}$ isomorphic to $\mathrm{SL}_{2}(\mathbb{Z})$ and subgroups of $H_{4} /\langle z\rangle$ isomorphic to $\mathrm{PSL}_{2}(\mathbb{Z})$ via $G \rightarrow G /\langle z\rangle$. So it suffices to show $H_{4} /\langle z\rangle$ contains precisely two conjugacy classes of subgroups isomorphic to $\mathrm{PSL}_{2}(\mathbb{Z})$. (These join together by the relation $q_{1} q_{3}^{-1}=1$.)

Expression (2.9) identifies two such groups, the images of $\left\langle q_{1}, q_{2}\right\rangle$ and $\left\langle q_{2}, q_{3}\right\rangle$. Consider $\mathcal{Q} /\langle z\rangle \stackrel{\text { def }}{=} \mathcal{Q}^{\prime \prime}, \beta\langle z\rangle \stackrel{\text { def }}{=} \beta^{\prime \prime}$ and $\alpha \gamma\langle z\rangle \stackrel{\text { def }}{=} \alpha^{\prime \prime}$. Note that $\mathcal{Q}^{\prime \prime}$ is a Klein 4-group. Denote the group $\left\langle\beta^{\prime \prime}, \alpha^{\prime \prime}\right\rangle$ by $\Gamma_{1}^{\prime}$. It is isomorphic to $\operatorname{PSL}_{2}(\mathbb{Z})$ and a complement to $\mathcal{Q}^{\prime \prime}$ in $H_{4} /\langle z\rangle$. Let $C$ be the centralizer, $\operatorname{Cen}_{\Gamma_{1}^{\prime}}\left(\mathcal{Q}^{\prime \prime}\right)$, of $\mathcal{Q}^{\prime \prime}$ (regard all as subgroups of $H_{4} /\langle z\rangle$ ). From (2.15), the quotient $\Gamma_{1}^{\prime} /{ }^{\prime} C$ is isomorphic to $S_{3} \cong$ $\operatorname{Aut}\left(\mathcal{Q}^{\prime \prime}\right)$. This identifies $C$ with a well-known rank 2 free subgroup of $\operatorname{PSL}_{2}(\mathbb{Z})$. Also, $\mathcal{Q}^{\prime \prime}$ is absolutely irreducible as an $\mathbb{F}_{2}\left[\Gamma_{1}^{\prime}\right]$ module.

Complements of $\mathcal{Q}^{\prime \prime}$ in $H_{4} /\langle z\rangle$ correspond to elements of $H^{1}\left(\Gamma_{1}^{\prime}, \mathcal{Q}^{\prime \prime}\right)$ Nor62, p. 244]. Conclude (2.11] if this cohomology group has order 2. Let $B$ be the largest elementary abelian 2- group quotient of the rank 2 free group $\operatorname{Cen}_{\Gamma_{1}^{\prime}}\left(\mathcal{Q}^{\prime \prime}\right)$. Then $B$ and $\mathcal{Q}^{\prime \prime}$ are isomorphic as $\mathbb{F}_{2}\left[\Gamma_{1}^{\prime}\right]$ modules. For any group $G$ with normal subgroup $H$ acting on a module $M$, there is an exact sequence [AW67, p. 100]:

$$
0 \rightarrow H^{1}\left(G / H, M^{H}\right) \stackrel{\mathrm{inf}}{\longrightarrow} H^{1}(G, M) \stackrel{\text { res }}{\longrightarrow} H^{1}(H, M)^{G} \rightarrow H^{2}\left(G / H, M^{H}\right) .
$$

Apply this with $M=\mathcal{Q}^{\prime \prime}, G=\Gamma_{1}^{\prime}$ and $H=\operatorname{Cen}_{\Gamma_{1}^{\prime}}\left(\mathcal{Q}^{\prime \prime}\right)$, so $G / H=S_{3}$. Restrict to a 2-Sylow $P_{2}$ of $S_{3}$. As in 9.4.5 if $H^{*}\left(P_{2}, M^{H}\right)$ is trivial, so is $H^{*}\left(G / H, M^{H}\right)$.

The action of $P_{2}$ on $\mathcal{Q}^{\prime \prime}$ is the regular representation on two copies of $P_{2}$, so $\mathcal{Q}^{\prime \prime}$ is a free $P_{2}$ module and the cohomology groups $H^{1}(G, M)$ and $H^{2}(G, M)$ are trivial. Conclude: $H^{1}\left(\Gamma_{1}^{\prime}, \mathcal{Q}^{\prime \prime}\right)=\operatorname{Hom}\left(B, \mathcal{Q}^{\prime \prime}\right)^{S_{3}}=\mathbb{Z} / 2$.

2.6.5. Comments on $q_{1} q_{3}^{-1} \neq 1$ in $M_{4}$. The nontrivial action of $q_{1} q_{3}^{-1}$ appears in many Nielsen classes. Especially in the action of $\mathcal{Q}^{\prime \prime}=\mathcal{Q} /\langle z\rangle$ on $\operatorname{Ni}\left(G_{k}, \mathbf{C}_{3^{4}}\right)$, $k \geq 1$ : all orbits have length four on $\operatorname{Ni}\left({ }_{2} \tilde{A}_{5}, \mathbf{C}_{3^{4}}\right)^{\text {in }}$ (Lem. 7.5). We make much, however, of the trivial action of $q_{1} q_{3}^{-1}$ when $k=0$.

Branch cycles for the $j$-line cover from the Nielsen class $\mathrm{Ni}\left(A_{5}, \mathbf{C}_{3^{4}}\right)$ abs and $\mathrm{Ni}\left(A_{5}, \mathbf{C}_{3^{4}}\right)^{\text {in }}$ are in Fri99 (7.7)]. Conjugate by $S_{5}$ to assume $\boldsymbol{g}$ representatives for $\operatorname{Ni}\left(A_{5}, \mathbf{C}_{3^{4}}\right)$ abs have $g_{1}=(123)$. Further conjugation by elements of $\left\langle g_{1},(45)\right\rangle$ gives the $\left(g_{2}, g_{3}, g_{4}\right)$ entries of absolute Nielsen class representatives (Table 1).

Then, $\mathcal{Q}$ acts trivially on everything in Table 1 Compute:

$$
Q_{1}=(25364)(798), \quad Q_{2}=(14985)(367) \text { and } Q_{3}=(25364)(798) \text {. }
$$


TABle 1. List $\operatorname{Ni}\left(A_{5}, \mathbf{C}_{3^{4}}\right)^{\text {abs }}$

${ }_{1} \boldsymbol{g}$ : ((132), (145), (154)); ${ }_{2} \boldsymbol{g}:((145),(154),(132)) ;{ }_{3} \boldsymbol{g}$ : ((145), (215), (243));

${ }_{4} \boldsymbol{g}:((145),(321),(354)) ;{ }_{5} \boldsymbol{g}:((145),(432),(415)) ;{ }_{6} \boldsymbol{g}:((145),(543),(521)) ;$

${ }_{7} \boldsymbol{g}$ : ((214), (245), (532)); ${ }_{8} \boldsymbol{g}$ : ((214), (325), (543)); ${ }_{9} \boldsymbol{g}$ : ((214), (435), (24 5)).

Even on $\operatorname{Ni}\left(A_{5}, \mathbf{C}_{3^{4}}\right)^{\text {in }}, \mathcal{Q}$ is trivial: $q_{1} q_{3}^{-1}$ maps $\boldsymbol{g}=((123),(321),(145),(541))$ to $\boldsymbol{g}^{*}=((321),(123),(541),(145))$, conjugate by $(23)(45)$ to $\boldsymbol{g}$.

2.6.6. Comments on $\left(q_{1} q_{3}^{-1}\right)^{2} \neq 1$. From Lem. 2.10 $z=1$ in the $M_{4}$ quotient. Acting on an inner Nielsen class (3.1.2 conjugating by $G$ ), $z$ will always be trivial. The following self-contained computation shows it is nontrivial in $H_{4}$ (compare with GB68 quoted in Bi75).

Suppose $z=1$ in $H_{4}$. Apply this to

$$
1=q_{1} q_{2} q_{3}^{2} q_{2} q_{1}=q_{1} q_{2} q_{1}^{2} q_{2} q_{1}=\left(q_{1} q_{2}\right)^{3} .
$$

Project $\left(\mathbb{P}_{z}^{1}\right)^{4} \backslash \Delta_{4} \rightarrow\left(\mathbb{P}_{z}^{1}\right)^{3} \backslash \Delta_{3}$ onto the first three factors (as in (2.6) ). Then, $\left(q_{1} q_{2}\right)^{3}$ goes to $\left(q_{1}^{\prime} q_{2}^{\prime}\right)^{3}$ with $q_{1}^{\prime}, q_{2}^{\prime}$ the generators of $H_{3}$. This implies $\left(q_{1}^{\prime} q_{2}^{\prime}\right)^{3}=1$ contrary to $\operatorname{ord}\left(q_{1}^{\prime} q_{2}^{\prime}\right)=6$ in (2.8).

Consider $\boldsymbol{g} \in \mathrm{Ni}(G, \mathbf{C}) / N^{\prime}$ with $N^{\prime}$ a group from 33.5 and its corresponding curve cover $\varphi_{\boldsymbol{g}}: X_{\boldsymbol{g}} \rightarrow \mathbb{P}_{z}^{1}$. Denote the subgroup of $H_{4}$ fixing $\boldsymbol{g}$ by $H_{\boldsymbol{g}}$. Fri89 $\left.\S 3.1\right]$ produces an explicit monodromy action of $H_{\boldsymbol{g}}$ on $H_{1}\left(X_{\boldsymbol{g}}, \mathbb{Z}\right)$. We ask if $z$ appears nontrivially in a structure from a Nielsen class, as in the situation of 2.6.7

Problem 2.13. Since $z$ fixes each element of the inner Nielsen classes, when is it nontrivial on each representative cover of the corresponding Hurwitz space? What are its \pm 1 eigenspaces on the first homology of curves in the family?

2.6.7. Finite quotients of $H_{4}$ where $z$ does not vanish. Add the relations $q_{i}^{4}=1$, $i=1,2,3$, to $H_{4}$.

Proposition 2.14. Let $G_{4}^{*}$ be the quotient of $H_{4}$ by the relations $q_{i}^{4}=1$, $i=1,2,3$. Denote the quotient of $\bar{M}_{4}$ by the (image of) the relations $q_{i}^{4}=1$, $i=1,2,3$, by $\bar{M}_{4}^{*}$. Then, $G_{4}^{*}$ is a (finite) nonsplit extension of $S_{4}=\bar{M}_{4}^{*}$ by $\mathcal{Q}$.

Proof. The image of $a_{12}=q_{1}^{2}, a_{13}=q_{1}^{-1} q_{2}^{2} q_{1}$ and $a_{14}=q_{1}^{-1} q_{2}^{-1} q_{3}^{2} q_{2} q_{1}$ in $\bar{M}_{4}$ identify with classical generators of $\mathbb{P}_{\lambda}^{1} \backslash\{0,1, \infty\}$ (3.7). Each $a_{1 j}$ has image an involution $\bar{a}_{1 j}$ in $\bar{M}_{4}^{*}$. The product-one condition, $\bar{a}_{12} \bar{a}_{13} \bar{a}_{14}=1$ implies these elements generate a Klein 4-group $K_{4}$ (Lem. 2.8). The group $\operatorname{Aut}\left(\mathbb{P}_{\lambda}^{1} / \mathbb{P}_{j}^{1}\right)$ is $S_{3}$ (in its regular representation). To check how $S_{3}$ acts on the $K_{4}$, note the element of order 3 (image of $\gamma_{0}=q_{1} q_{2}$ ) acts nontrivially on $K_{4}$ (by conjugation). Here is the effect (in $\bar{M}_{4}^{*}$ ) of conjugating $q_{1}^{2}$ by $q_{1} q_{2}$ :

$$
q_{1} q_{2}\left(q_{1}^{2}\right)\left(q_{1} q_{2}\right)^{-1}=\left(q_{1} q_{2} q_{1}\right)\left(q_{1} q_{2}^{3} q_{1}^{-1}\right)=q_{1}^{-1}\left(q_{1} q_{2}\right)^{3}\left(q_{2}^{2} q_{1}^{-1}\right)=q_{1}^{-1} q_{2}^{2} q_{1}^{-1} .
$$

Since $q_{1}^{-1} q_{2}^{2} q_{1}^{-1}=q_{1}^{-1} q_{2}^{2} q_{1}^{3}=a_{13} a_{12}$, the action is nontrivial and $\bar{M}_{4}^{*}$ is $S_{4}$.

To see these relations don't kill $z$, verify the normalizing action of $H_{4}$ on $\mathcal{Q}$ gives $q_{i}^{4}$ acting trivially, $i=1,2,3$. Use conjugation by $q_{i}$ as in $\$ 2.6 .2$

Finally, to see it is nonsplit, consider the element $q_{1} q_{2} q_{3} \in H_{4}$ whose image in $\bar{M}_{4}^{*}$ is $q_{1} q_{2} q_{1}$ (image of $\gamma_{1}$ ) of order 2. A splitting of $S_{4}$ would give a lift of this to an element of order 2, contrary to Lem. 2.11 giving no such element in $\mathcal{Q}^{\prime}$. 
2.7. The shift $\gamma_{1}=q_{1} q_{2} q_{1}$ and the middle twist $\gamma_{\infty}=q_{2}$. The action of $\gamma_{1}=q_{1} q_{2} q_{1}$ (from Prop. 4.4) on $\mathrm{Ni}(G, \mathbf{C})^{\text {in,rd }}$ is the same as $q_{1} q_{2} q_{3}$. It sends $\left(g_{1}, g_{2}, g_{3}, g_{4}\right)$ to $\left(g_{2}, g_{3}, g_{4}, g_{1}\right)$ modulo conjugation by $g_{1}$. Sometimes we want $\mathbf{s h}=$ $q_{1} q_{2} q_{3}$, the shift, instead of $\gamma_{1}$, on $\operatorname{Ni}(G, \mathbf{C})^{\text {in }}$. Here also, use $\mathbf{s h}$.

LEMMA 2.15. The (middle) twist $\gamma_{\infty}$ and $\mathbf{s h}\left(\right.$ on $\mathrm{Ni}(G, \mathbf{C})^{\text {in,rd })}$ entwine by

$$
\mathbf{s h} \gamma_{\infty} \mathbf{s h}=\gamma_{\infty}^{-1} \operatorname{sh} \gamma_{\infty}^{-1}
$$

Knowing $\mathbf{s h}$ is an involution and (2.18) holds gives a presentation of $\bar{M}_{4}=\left\langle\mathbf{s h}, \gamma_{\infty}\right\rangle$.

2.7.1. Twist action on products of two conjugacy classes. Let $G$ be a finite group with conjugacy classes $\left(\mathrm{C}_{1}^{\prime}, \mathrm{C}_{2}^{\prime}, \mathrm{C}_{3}^{\prime}\right)=\mathbf{C}^{\prime}$. Consider:

$$
S\left(\mathbf{C}^{\prime}\right)=\left\{\left(g_{1}, g_{2}\right) \mid g_{i} \in \mathrm{C}_{i}, i=1,2, \text { with } g_{1} g_{2} \in \mathrm{C}_{3}\right\} .
$$

Consider the twist $\gamma$ mapping $\left(g_{1}, g_{2}\right) \in S\left(\mathbf{C}^{\prime}\right)$ to $\left(g_{1} g_{2} g_{1}^{-1}, g_{1}\right) \in S\left(\mathbf{C}^{\prime \prime}\right)$ with $\mathrm{C}^{\prime \prime}=\left(\mathrm{C}_{2}^{\prime}, \mathrm{C}_{1}^{\prime}, \mathrm{C}_{3}^{\prime}\right)$.

LEMmA 2.16. For $g_{3} \in \mathrm{C}_{3}^{\prime}$ let $S\left(\mathbf{C}^{\prime}\right)_{g_{3}}$ be those $\left(g_{1}, g_{2}\right) \in S\left(\mathbf{C}^{\prime}\right)$ with $g_{1} g_{2}=g_{3}$. Then, $\gamma$ maps one-one from $S\left(\mathbf{C}^{\prime}\right)_{g_{3}}$ to $S\left(\mathbf{C}^{\prime \prime}\right)_{g_{3}}$. Also, $\left|\mathbf{C}_{3}^{\prime}\right|\left|S\left(\mathbf{C}^{\prime}\right)_{g_{3}}\right|=\left|S\left(\mathbf{C}^{\prime}\right)\right|$.

The structure constant formula $(\$ 2.7 .2)$ calculates $\left|S\left(\mathbf{C}^{\prime}\right)\right|$ using complex representations of $G$. Calculations in $\$ 7$ compute the length of $\gamma$ orbits on $S\left(\mathbf{C}^{\prime}\right)_{g_{3}}$. For $g_{1}, g_{2}$ in a group, denote the centralizer of $\left\langle g_{1}, g_{2}\right\rangle$ by $Z\left(g_{1}, g_{2}\right)$.

Proposition 2.17. Assume $g_{1} \neq g_{2}$. Let $\left(g_{1}, g_{2}\right) \in S\left(\mathbf{C}^{\prime}\right) g_{3}$. The orbit of $\gamma^{2}$ containing $\left(g_{1}, g_{2}\right)$ is $\left(g_{3}^{j} g_{1} g_{3}^{-j}, g_{3}^{j} g_{2} g_{3}^{-j}\right), j=0, \ldots$, ord $\left(g_{3}\right)-1$. So, the orbit of $\gamma^{2}$ has length

$$
\begin{gathered}
\operatorname{ord}\left(g_{3}\right) /\left|\left\langle g_{3}\right\rangle \cap Z\left(g_{1}, g_{2}\right)\right| \stackrel{\text { def }}{=} o\left(g_{1}, g_{2}\right) . \\
\text { Let } x=\left(g_{1} g_{2}\right)^{(o-1) / 2} \text { and } y=\left(g_{2} g_{1}\right)^{(o-1) / 2} \text {, so } g_{1} y=x g_{1} \text { and } y g_{2}=g_{2} x \text {. Assume: }
\end{gathered}
$$

(2.19) $o\left(g_{1}, g_{2}\right)=o$ is odd; and $y g_{2}$ has order 2 .

Then, the orbit of $\gamma$ has length $o\left(g_{1}, g_{2}\right)$. Otherwise, the orbit of $\gamma$ containing $\left(g_{1}, g_{2}\right)$ has length $2 o\left(g_{1}, g_{2}\right)$.

Proof. For $t$ an integer,

$$
\left(g_{1}, g_{2}\right) \gamma^{2 t}=\left(g_{3}^{t} g_{1} g_{3}^{-t}, g_{3}^{t} g_{2} g_{3}^{-t}\right) \text { and }\left(g_{1}, g_{2}\right) \gamma^{2 t+1}=\left(g_{3}^{t} g_{1} g_{2} g_{1}^{-1} g_{3}^{-t}, g_{3}^{t} g_{1} g_{1} g_{1}^{-1} g_{3}^{-t}\right) \text {. }
$$

The minimal $t$ with $\left(g_{1}, g_{2}\right) \gamma^{2 t}=\left(g_{1}, g_{2}\right)$ is $o\left(g_{1}, g_{2}\right)$. Further, the minimal $j$ with $\left(g_{1}, g_{2}\right) \gamma^{j}=\left(g_{1}, g_{2}\right)$ divides any other integer with this property. So $j \mid 2 o\left(g_{1}, g_{2}\right)$ and if $j$ is odd, $j \mid o\left(g_{1}, g_{2}\right)$.

From the above, if the orbit of $\gamma$ does not have length $2 o\left(g_{1}, g_{2}\right)$, it has length $o\left(g_{1}, g_{2}\right)$. Use the notation around (2.19). The expressions $g_{1} y=x g_{1}$ and $y g_{2}=g_{2} x$ are tautologies. If $o$ is odd, then $\left(g_{1}, g_{2}\right) q_{2}^{o}=x\left(g_{1}, g_{2}\right) q_{2} x^{-1}$. Assume this equals $\left(g_{1}, g_{2}\right)$, which is true if and only if $x g_{1}=g_{2} x=y g_{2}$. The expression $\left(g_{1} g_{2}\right)^{o}=1$ and $x g_{1} y g_{2}=1$ are equivalent. Conclude $\left(y g_{2}\right)^{2}=1$. So long as the order of $y g_{2}$ is not 1, this shows (2.19) holds. If, however, $y g_{2}=x g_{1}=g_{2} x=g_{1} y=1$, then $g_{1}=g_{2}$, contrary to hypothesis.

This reversible argument shows the converse: $\left(g_{1}, g_{2}\right) q_{2}^{o}=\left(g_{1}, g_{2}\right)$ follows from (2.19). This concludes the proof. 
Example $2.18\left(Z\left(g_{1}, g_{2}\right)\right.$ in Prop. 2.17). Prop. 2.17 applies to compute the $q_{2}$ orbit length of $\boldsymbol{g} \in \operatorname{Ni}(G, \mathbf{C})$ where $r=4$ and $G$ is centerless (see Lem. 2.19). With $\boldsymbol{g}=\left(g_{1}, g_{2}, g_{3}, g_{4}\right)$, replace $\left(g_{1}, g_{2}\right)$ in the proposition by the entries $\left(g_{2}, g_{3}\right)$. To give examples where $\left\langle g_{3}\right\rangle \cap Z\left(g_{1}, g_{2}\right) \neq\{1\}$, suppose $V=\left\langle g_{2}, g_{3}\right\rangle$ is a vector space and $A$ is an endomorphism fixing no nontrivial element of $V$. Entries of $\left(A g_{2}^{-1}, g_{2}, g_{3}, g_{3}^{-1} A^{-1}\right)$ generate a centerless group.

2.7.2. Products of conjugacy classes. The structure constant formula for $\mathbb{Z}[G]$ (for example, Ser92 \$7.2]) shows the result of multiplying elements from conjugacy classes. Let $m$ be a function constant on conjugacy classes $\mathrm{C}_{1}, \ldots, \mathrm{C}_{r}$ and $g \in G$. Let the common value of $m$ on $\mathrm{C}_{i}$ be $m\left(\mathrm{C}_{i}\right)$. Denote $\sum_{i=1}^{r} \sum_{u_{i} \in C_{i}} m\left(u_{1} \cdots u_{r} g\right)$ by $I(m ; \mathbf{C}, g)$. If $m=\chi$ is an irreducible character of $G$,

$$
I(\chi ; \mathbf{C}, g)=\sum_{i=1}^{r} \sum_{u_{i} \in C_{i}} \chi\left(u_{1} \cdots u_{r} g\right)=\chi(g) \prod_{i=1}^{r} \chi\left(u_{i}\right) / \chi(1)^{r} .
$$

List the irreducible complex characters, $\chi_{1}, \ldots, \chi_{s}$, of $G$. Write $m=m_{i} \chi_{i}$. Then, $I(m ; \mathbf{C}, g)=\sum_{i} m_{i} I\left(\chi_{i} ; \mathbf{C}, g\right)$. Consider $\psi_{G}=\frac{1}{|G|} \sum_{i=1}^{s} \chi_{i}(1) \chi_{i}: 1$ at $1_{G}$ and 0 otherwise. So, $I\left(\psi_{G} ; \mathbf{C}, g\right)$ counts solutions of $u_{1}, \ldots, u_{r} g=1$ with $u_{i} \in \mathrm{C}_{i}$ :

$$
N\left(\mathrm{C}_{1}, \ldots, \mathrm{C}_{r}, g\right)=|G|^{r-1} \sum_{i=1}^{s} \prod_{j=1}^{r} \chi_{i}\left(\mathrm{C}_{j}\right) \chi_{i}(g) .
$$

2.8. General context with modular curve illustrations. We do many computations of $q_{2}$ orbits on absolute, inner and reduced Nielsen classes. For general $r$ an obvious, but valuable, listing of $Q_{i}$ orbits appears in the next lemma.

Lemma 2.19. Let $\boldsymbol{g} \in \mathrm{Ni}(G, \mathbf{C})$ be a Nielsen class representative. With $\mu=$ $g_{i} g_{i+1}$, the orbit of $Q_{i}$ on $\boldsymbol{g}$ is the collection

$$
(\boldsymbol{g}) q_{i}^{k}= \begin{cases}\left(g_{1}, \ldots, g_{i-1}, \mu^{l} g_{i} \mu^{-l}, \mu^{l} g_{i+1} \mu^{-l}, g_{i+2}, \ldots\right) & \text { for } k=2 l \\ \left(g_{1}, \ldots, g_{i-1}, \mu^{l} g_{i} g_{i+1} g_{i}^{-1} \mu^{-l}, \mu^{l} g_{i} \mu^{-l}, g_{i+2}, \ldots\right) & \text { for } k=1+2 l .\end{cases}
$$

Producing modular curves from Hurwitz spaces illustrates modding out by various groups of automorphisms of $G$ on $\operatorname{Ni}(G, \mathbf{C})$ (Fri90 §2] or [Fri78]).

2.8.1. Modular curve cusps from a Hurwitz viewpoint. The Nielsen class in this case is for s-equivalence (3.5) of dihedral involution realizations.

Regard $D_{p^{k+1}}=\mathbb{Z} / p^{k+1} \times s\{ \pm 1\}$, the dihedral group of order $2 \cdot p^{k+1}$ with $p$ an odd prime, as $2 \times 2$ matrices. Start with absolute Nielsen classes (3.5.1) from the degree $p^{k+1}$ representation of $D_{p^{k+1}}$. Take $\mathrm{C}_{2}$ the conjugacy class of $\left(\begin{array}{cc}-1 & 0 \\ 0 & 1\end{array}\right)$; and $T: D_{p^{k+1}} \rightarrow S_{p^{k+1}}$ the standard representation. The upper-right element $b \in \mathbb{Z} / p^{k+1}$ determines elements of $\mathrm{C}_{2}$. Let $N_{k}$ be the normalizer in $S_{p^{k+1}}$ of $D_{p^{k+1}}$.

List $\mathrm{Ni}\left(D_{p^{k+1}}, \mathbf{C}_{2^{4}}, T\right)^{\text {abs }}=\mathrm{Ni}_{k}^{\text {abs }}$ as $\left(b_{1}, b_{2}, b_{3}, b_{4}\right) \in\left(\mathbb{Z} / p^{k+1}\right)^{4}$ modulo $N_{k}$. Count these using representatives with these properties:

(2.20a) $b_{1}=0, b_{2}-b_{3}+b_{4}=0$; and

(2.20b) $b_{2}=1$ or $p \mid b_{2}$ and $b_{3}=1$.

Then: $\left|\mathrm{Ni}_{k}^{\text {abs }}\right|=p^{k+1}+p^{k}$. Modding out only by $D_{p^{k+1}}$ - instead of $N_{k}$ - gives $\left|\mathrm{Ni}_{k}^{\text {in }}\right|=\left(p^{k+1}+p^{k}\right) \varphi\left(p^{k+1}\right) / 2$ with $\varphi$ the Euler $\varphi$-function.

Renormalize: Use $b_{2}-b_{3}=a p^{u}$ with $(a, p)=1$ in place of $b_{2}=1$; conjugate by $\left(\begin{array}{cc}\alpha & 0 \\ 0 & 1\end{array}\right)$ to take $a=1$. This allows further conjugation with $\alpha \equiv 1 \bmod p^{k+1-u}$. 
We pose some computations as an exercise. The superscript ${ }^{\text {rd }}$ means to add the quotient action of $\mathcal{Q}^{\prime \prime}$.

(2.21a) Show $\left|\mathrm{Ni}_{k}^{\text {abs, rd }}\right|=\left|\mathrm{Ni}_{k}^{\text {abs }}\right|$ and $\left|\mathrm{Ni}_{k}^{\mathrm{in}, \mathrm{rd}}\right|=\left|\mathrm{Ni}_{k}^{\mathrm{in}}\right|: \mathcal{Q}^{\prime \prime}$ action is trivial. Hint: Use H-M rep. $b_{2}=0, b_{3}=1=b_{4}$.

(2.21b) Find a length 1 (resp. $\left.p^{k+1}\right) q_{2}$ orbit on $\mathrm{Ni}_{k}^{\text {abs,rd }}:\left(b_{2}, b_{3}\right)=(1,1)$ (resp. $\left.\left(b_{2}, b_{3}\right)=(0,1)\right)$.

(2.21c) For $1 \leq u \leq k$ count $q_{2}$ orbits on $\mathrm{Ni}_{k}^{\text {abs,rd }}$ with $b_{2}-b_{3}=p^{u}$.

Hints on (2.2T): Let ${ }_{u} \boldsymbol{g}$ have $b_{2}=1$ and $b_{3}=1-p^{u}$. Use:

(2.22) $\quad\left({ }_{u} \boldsymbol{g}\right) q_{2}^{l}$ corresponds to the pair $\left(b_{2}, b_{3}\right)=\left(1+l p^{u}, 1+(l-1) p^{u}\right)$.

Compute the minimal $l$ so the 2nd entry of $\left({ }_{u} \boldsymbol{g}\right) q_{2}^{l}$ equals $\left(\begin{array}{cc}-1 & 1 \\ 0 & 1\end{array}\right), 1 \leq l \leq p^{k+1-u}$. Complete the set of $b_{2}$ s: prime to $p$, modulo $p^{u}$ up to multiplication by $H_{u}=\{\alpha \mid$ $\left.\alpha \equiv 1 \bmod p^{k+1-u}\right\}$. List $q_{2}$ orbits on $\left|\mathrm{Ni}_{k}^{\mathrm{abs}}\right|$.

LEMMA 2.20. Let $S_{u} \leq H_{u}$ be the subgroup stabilizing (2.22). Then, $\left|S_{u}\right|=$ $p^{\min (u, k+1-u)}$ and this gives a $q_{2}$ orbit of length $p^{k+1-u} / p^{\min (u, k+1-u)}$. Each $u$, $1 \leq u \leq k$ contributes $\varphi\left(p^{u}\right) /\left|H_{u}\right|$ orbits of $q_{2}$ of this length.

A traditional way to compute $X_{0}\left(p^{k+1}\right)$ cusp data is in Sh94 p. 25]. Note: In (2.21b), an $H-M$ rep. (from $\left(b_{2}, b_{3}\right)=(0,1)$, see g6) gives the longest $q_{2}$ orbit. Its shift gives the shortest $q_{2}$ orbit. This is the exact analog of Prop. 7.9

2.8.2. Modular curves from 2.8.1. Any $\boldsymbol{p} \in \mathcal{H}_{k}^{\text {abs }}$ represents a cover in the Nielsen class $\mathrm{Ni}_{k}^{\text {abs }}: \varphi_{\boldsymbol{p}}: X_{\boldsymbol{p}}=X \rightarrow \mathbb{P}_{z}^{1}$. Its four branch cycles all have order two, each fixing just one integer in the representation. This gives $g\left(X_{\boldsymbol{p}}\right)=0$ from Riemann-Hurwitz (as in Fri90 $\S 2]$ or Fri78]). The geometric (over $\mathbb{C}$ ) Galois closure of $\varphi_{\boldsymbol{p}}$ is a nondiagonal component $\hat{X}_{\boldsymbol{p}}$ of the $p^{k+1}$ times fiber product $X_{\mathbb{P}_{z}^{1}}^{\left(p^{k+1}\right)}$ (\$3.1.3 has the fiber product construction): $g\left(\hat{X}_{\boldsymbol{p}}\right)=1$. The copy of $\mathbb{Z} / p^{k+1}$ is the (unique) $p$-Sylow of the automorphism group of $\hat{X}_{\boldsymbol{p}} \rightarrow \mathbb{P}_{z}^{1}$. Let $\operatorname{Pic}^{0}\left(\hat{X}_{\boldsymbol{p}}\right)$ be the elliptic curve of degree 0 divisor classes on $\hat{X}_{\boldsymbol{p}}$.

Let $Y_{0}\left(p^{k+1}\right)$ be $X_{0}\left(p^{k+1}\right)$ without its cusps (points over $j=\infty$ ). Points of $Y_{0}\left(p^{k+1}\right)$ are elliptic curve isogeny classes $E \rightarrow E^{\prime}$ with kernel isomorphic to $\mathbb{Z} / p^{k+1}$. So, $\boldsymbol{p}$ gives a point in $Y_{0}\left(p^{k+1}\right): \operatorname{Pic}^{0}\left(\hat{X}_{\boldsymbol{p}}\right)=E \mapsto E /\left(\mathbb{Z} / p^{k+1}\right)=E^{\prime}$.

Produce $\mathcal{H}_{k}^{\text {in }} \rightarrow Y_{1}\left(p^{k+1}\right)$ : If $\boldsymbol{p}^{*} \in \mathcal{H}_{k}^{\text {in }}$ lies over $\boldsymbol{p} \in \mathcal{H}_{k}^{\text {abs }}$, then $\varphi_{\boldsymbol{p}^{*}}$ identifies $\operatorname{Aut}\left(\hat{X}_{\boldsymbol{p}} / \mathbb{P}_{z}^{1}\right)$ with $D_{p^{k+1}}$. Pick a generator $e \in \mathbb{Z} / p^{k+1}$. Let $\boldsymbol{o}_{E}$ be the origin on $E$. This gives a $p$-division point on $E$ : $e \mapsto e\left(\boldsymbol{o}_{E}\right)$. For the Hurwitz interpretation of $Y\left(p^{k+1}\right)$ add markings to the Nielsen classes BF82.

2.8.3. Moduli dilemma relating $\mathcal{H}\left(D_{p^{k+1}}, \mathbf{C}_{2^{4}}\right)^{\text {in,rd }}$ to $Y_{1}\left(p^{k+1}\right)$. 2.8.2 produces a $\mathbb{Q}$ rational isomorphism of $\mathcal{H}\left(D_{p^{k+1}}, \mathbf{C}_{2^{4}}\right)^{\text {in,rd }}$ and $Y_{1}\left(p^{k+1}\right)$. Their moduli problems, however, are different.

Points of $Y_{1}\left(p^{k+1}\right)$ correspond to pairs $(E, e)$ with $E$ an elliptic curve and $e$ a $p^{k+1}$ division point. Since $Y_{1}\left(p^{k+1}\right)$ is a fine moduli space, a $K$ point $\boldsymbol{p} \in Y_{1}\left(p^{k+1}\right)$ automatically produces the pair $\left(E_{\boldsymbol{p}}, e_{\boldsymbol{p}}\right)$ over $K$. This gives $E_{\boldsymbol{p}} \rightarrow E_{\boldsymbol{p}} /\left\langle-1, e_{\boldsymbol{p}}\right\rangle:-1$ is the unique involution of $E_{\boldsymbol{p}}$ fixing the origin; and $e_{\boldsymbol{p}}$ is shorthand for translating points on $E_{\boldsymbol{p}}$ by $e_{\boldsymbol{p}}$.

By contrast, $\mathcal{H}\left(D_{p^{k+1}}, \mathbf{C}_{2^{4}}\right)^{\text {in,rd }}=\mathcal{H}_{k}^{\text {rd }}$ is the moduli space of genus 1 Galois covers of $\mathbb{P}^{1}$ with these further properties.

(2.23a) The covering group has an isomorphism with $D_{p^{k+1}}$, up to conjugation.

(2.23b) Branch cycles for the cover are in $\mathbf{C}_{2^{4}}$. 
(2.23c) Covers $\hat{\varphi}: \hat{X} \rightarrow \mathbb{P}_{z}^{1}$ and $\alpha \circ \hat{\varphi}: \hat{X} \rightarrow \mathbb{P}_{z}^{1}$ for $\alpha \in \mathrm{PSL}_{2}(\mathbb{C})$ are equivalent if pullback by $\alpha$ respects the isomorphism $G\left(\hat{X} / \mathbb{P}_{z}^{1}\right)$ to $D_{p^{k+1}}$ (in 3.5.2).

In (2.21 b-fine) moduli space (Prop. 4.7). We explain further. Let $U_{j}=\mathbb{P}_{j}^{1} \backslash\{\infty\}$.

Let $\Phi: \mathcal{T} \rightarrow S$ be a smooth family of curves with an attached algebraic map $\Psi: S \rightarrow U_{j}$. Denote the fiber of $\Phi$ over $s \in S$ by $\mathcal{T}_{s}$. Suppose $D_{p^{k+1}}$ acts as a group scheme on $\mathcal{T}$ preserving each fiber $\mathcal{T}_{s}$. Write this as $\Gamma: D_{p^{k+1}} \times \mathcal{T} \rightarrow \mathcal{T}$. Then, form the quotient map $\varphi_{s}: \mathcal{T}_{s} \mapsto \mathcal{T}_{s} / D_{p^{k+1}}$. Assume for $s \in S(\mathbb{C})$ :

(2.24) An isomorphism of $\mathcal{T}_{s} / D_{p^{k+1}}$ with $\mathbb{P}_{z}^{1}$ presents $\varphi_{s}$ in the inner Nielsen class of $\left(D_{p^{k+1}}, \mathbf{C}_{2^{4}}\right)$ with branch points in the equivalence class $\Psi(s)$.

By assumption, $\Phi$ induces a natural map $\psi: S \rightarrow \mathcal{H}_{k}^{\text {rd }}$. If $\mathcal{H}_{k}^{\text {rd }}$ were a fine moduli space, the family $\Phi$ would pull back from a unique total family on $\mathcal{H}_{k}^{\text {rd }}$. Though it isn't, there is at least one total family on $\mathcal{H}_{k}^{\text {rd }}$ (identified with $Y_{1}\left(p^{k+1}\right)$ ).

Proposition 2.21. For some total family $\Phi^{*}: \mathcal{T}^{*} \rightarrow Y_{1}\left(p^{k+1}\right)$ :

(2.25a) $D_{p^{k+1}}$ acts on the total space commuting with $\Phi^{*}$; and

(2.25b) the fiber over $\boldsymbol{p} \in Y_{1}\left(p^{k+1}\right)$ is $E_{\boldsymbol{p}}$ (notation above) with restriction of $D_{p^{k+1}}$ producing the cover $E_{\boldsymbol{p}} \rightarrow E_{\boldsymbol{p}} /\left\langle-1, e_{\boldsymbol{p}}\right\rangle$.

Any $K$ point on $\mathcal{H}_{k}^{\mathrm{rd}}$ produces a $D_{p^{k+1}}$ regular realization in the inner Nielsen class of $\left(D_{p^{k}+1}, \mathbf{C}_{2^{4}}\right)$.

Proof. It only remains to show the last statement. Prop.2.3 gives this because 2.21国) shows $\mathcal{Q}^{\prime \prime}$ acts trivially on the Nielsen class. A classical argument, however, suffices in this case by contrast to 2.3.4

Given $\boldsymbol{p} \in \mathcal{H}_{k}^{k}(K)=Y_{1}\left(p^{k+1}\right)(K)$, consider the $K$ cover $E_{\boldsymbol{p}} \rightarrow E_{\boldsymbol{p}} /\left\langle-1, e_{\boldsymbol{p}}\right\rangle$. If $E_{\boldsymbol{p}} /\left\langle-1, e_{\boldsymbol{p}}\right\rangle$ is isomorphic to $\mathbb{P}_{z}^{1}$ over $K$, this gives a regular $D_{p^{k+1}}$ realization in the inner Nielsen class of $\left(D_{p^{k}+1}, \mathbf{C}_{2^{4}}\right)$. Such an isomorphism occurs if and only if $E_{\boldsymbol{p}} /\left\langle-1, e_{\boldsymbol{p}}\right\rangle$ has a $K$ point. The image from origin in $E_{\boldsymbol{p}}$ is such a $K$ point.

Suppose $\mathcal{H}_{k}^{k}$ were a fine (resp. b-fine) moduli space. Then the family $\Phi$ (resp. off of $j=0$ or 1 ) would be the pullback of this family. There would then be a section $\gamma: S \rightarrow \mathcal{T}$ for $\Phi$ from pulling back the section for the origin on the fibers of $\mathcal{T}^{*} \rightarrow Y_{1}\left(p^{k+1}\right)$. That section would have the same field of definition as does $(\Phi, \mathcal{T}, \Psi, \Gamma)$. The structural maps, including $\Gamma$, have field of definition $\mathbb{Q}$. We don't assume the genus zero curve $\mathcal{T}_{s} / D_{p^{k+1}}$ is isomorphic to $\mathbb{P}_{z}^{1}$ over $\mathbb{Q}(s)$; this would give a $\mathbb{Q}(s)$ regular realization $\mathcal{T}_{s} \rightarrow \mathbb{P}_{z}^{1}$ of $D_{p^{k+1}}$. It is exactly in this way the next proposition says the family $\mathcal{T}^{*} \rightarrow Y_{1}\left(p^{k+1}\right)$ is special.

2.8.4. $D_{p^{k+1}}$ involution covers of forms of $\mathbb{P}^{1}$. Prop. 2.21 would be most satisfying if there was a diophantine consequence for $\mathcal{H}_{k}^{\text {rd }}$ not being a fine (or b-fine) moduli space. Best of all would be values of $s$ with $\mathcal{T}_{s} / D_{p^{k+1}}$ not isomorphic over $\mathbb{Q}(s)$ to $\mathbb{P}_{z}^{1}$. For example, suppose $K$ is a $p$-adic field and $E$ is an elliptic curve over $K$ with a $p^{k+1}$ division point $e$. From this form $\psi_{e}: E \rightarrow E /\langle-1, e\rangle$ as in the Prop. 2.21 proof. Let $\mu$ be the $G_{K}$ module of all roots of 1 and let $E^{*}$ be the dual abelian variety to $E$. Tate has a theory (Ta58, [Ser96 p. 93]) identifying homogeneous spaces for $E$ with $\operatorname{Hom}_{G_{K}}\left(H^{0}\left(G_{K}, E^{*}\right), \mu\right)$.

We suggest a project. Let $C$ be a nontrivial homogeneous space for $E$, and $C^{(2)}$ its 2-fold symmetric product. Map positive divisors on $C$ of degree 2 to divisor classes as $s^{(2)}: C^{(2)} \rightarrow \operatorname{Pic}(C)^{(2)}$. Fibers of $s^{(2)}$ are forms of $\mathbb{P}^{1}$ over $K$ with a 
degree 2 map from $C$. Assume further that $\operatorname{Pic}(C)^{(2)}$ has $K$ points; it is isomorphic to $E$. Fri75] shows there is a $K$-fiber $Y$ of $s^{(2)}$ not isomorphic to $\mathbb{P}^{1}$ over $K$.

Problem 2.22. Assume $j(E) \neq 0$ or 1 (the special values for our normalized $j$ ). Does $s^{(2)}$ have a $D_{p^{k+1}}$ invariant $K$ fiber $Y$ not isomorphic to $\mathbb{P}_{K}^{1}$ ?

A positive solution gives a sequence $C \rightarrow Y \stackrel{\alpha}{\longrightarrow} Y$ (with $\alpha$ a degree $p^{k+1}$ map) that is a $D_{p^{k+1}}$ involution realization over $\bar{K}$ ( $\alpha$ agrees with 6.5.2). Despite Châtelet's interest in Brauer-Severi varieties and homogeneous spaces for elliptic curves (Rem. 6.13) he seems not to have come across this problem.

2.9. $p$-lifting property and using sh-incidence. 2.9.1 looks at $\gamma_{\infty}$ orbits. 2.9 .3 applies to $\mathrm{Ni}\left(A_{5}, \mathbf{C}_{3^{4}}\right)^{\text {in,rd }}$ a preferred rubric for computing $\bar{M}_{4}$ orbits.

2.9.1. Orbits for $\gamma$ related to $\operatorname{Ni}\left(A_{5}, \mathbf{C}_{3^{4}}\right)^{\text {in }}$. Let $\gamma$ be the twist operator of

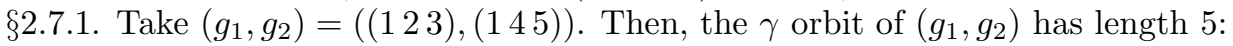

$$
(12345)^{2}(123)(123)(321)(12345)^{-2}=(145) \text {. }
$$

Similarly, take $\left(g_{1}, g_{2}\right)=((123),(134))$. On this element $\gamma$ produces an orbit of length 3: $(124)(123)(123)(321)(421)=(134)$.

2.9.2. The p-lifting property. The following lemma from FK97, Lifting Lemma 4.1] appears often in our computations.

Lemma 2.23. For any finite group $G=G_{0}$, let $G_{1}$ be the first characteristic quotient of the universal p-Frattini cover ${ }_{p} \tilde{G}$ of $G_{0}$ (5.2). Suppose $g \in G_{0}$ has order divisible by $p$. Then, any lift of $g$ to $G_{1}$ has order $p$ times the order of $g$.

Given $G=G_{0}$, an extension $\psi: H \rightarrow G$ has the $p$-lifting property if the conclusion of Lem. 2.23 holds (for $p$ ). Often a much smaller cover than $G_{1} \rightarrow G_{0}$ will suffice for the $p$-lifting property. For example, $A_{5}=\mathrm{PSL}_{2}(\mathbb{Z} / 5)$ has one conjugacy class of elements of order 2. That lifts to an element of order 4 in $\hat{A}_{5}=\mathrm{SL}_{2}(\mathbb{Z} / 5)$.

Lemma 2.24. Let $g_{i}, i=1,2$, be distinct order 2 elements in $A_{5}$. Denote lifts of them to $\hat{A}_{5}$ by $\hat{g}_{i}, i=1,2$. Then, $\left\langle\hat{g}_{1}, \hat{g}_{2}\right\rangle$ is a quaternion group.

Consider $\hat{A}_{n}$, the Spin cover of $A_{n}$. Write any $g \in A_{n}$ of order $2(n \geq 6)$ as a product $g_{1} g_{2}$ of two commuting elements $g_{1}, g_{2}$ of order 2 with the $g_{i} s$ having lifts $\hat{g}_{1}, \hat{g}_{2}$ of order 4. Then, $\left\langle\hat{g}_{1}, \hat{g}_{2}\right\rangle$ is isomorphic to $\mathbb{Z} / 2 \times \mathbb{Z} / 4$ if and only if $\hat{g}_{1} \hat{g}_{2}$ has order 2 (otherwise it is isomorphic to $Q_{8}$ ). Further, $\hat{g}_{1} \hat{g}_{2}$ has order 4 if and only if $g_{1} g_{2}$ is a product of $2 s$ transpositions, with $s$ odd.

PRoof. The opening statement follows because the quaternion group of order 8 is only group of order 8 with a Klein 4-group as quotient having all its elements lifting to have order 4. The last statement is a variant on that using Prop. 5.10]

Let $G_{1}$ be ${ }_{2} \tilde{A}_{5} /$ ker $_{1}$ for $p=2$, the first characteristic quotient of the universal 2-Frattini cover of $A_{5}$ (5.2). Suppose $\left(g_{1}^{\prime}, g_{2}^{\prime}\right)$ is a lift of $\left(g_{1}, g_{2}\right)$ to elements of order 3 in $G_{1}$ in either of these cases. Then, $\gamma$ on $\left(g_{1}^{\prime}, g_{2}^{\prime}\right)$ gives orbits of length $\operatorname{2ord}\left(g_{1}^{\prime} g_{2}^{\prime}\right)$. For example, let $\alpha=(12345)^{2}(123)=(24)(35)$. Suppose the orbit has length ord $\left(g_{1}^{\prime} g_{2}^{\prime}\right)$. Then, some lift of $\alpha$ to $\alpha^{\prime} \in G_{1}$ conjugates $g_{1}^{\prime}$ to $g_{2}^{\prime}$ and $g_{2}^{\prime}$ to $g_{1}^{\prime}$. Such an element, however, would have order 2. Since 2 divides the order $\alpha$, this contradicts Lemma $2.23 \alpha^{\prime}$ has order 2 times the order of $\alpha$. Similarly for $\alpha=(124)(123)=(13)(24)$. 
We don't know how to compute ord $\left(g_{1}^{\prime} g_{2}^{\prime}\right)$ in general as in Lemma 2.23 When $G_{0}$ is a subgroup of an alternating group and $p=2$, Prop. 5.9 contributes considerably. Here is an immediate example. 5.5 uses it on indirect examples.

Lemma 2.25. Take $G_{0}=A_{5}$ and $p=2$. Suppose $\left(g_{2}^{\prime}, g_{3}^{\prime}\right)$ is a pair of elements of order 3 lifting to $G_{1}$ either $\left(g_{2}, g_{3}\right)=((123),(145))$ or $\left(g_{2}, g_{3}\right)=((123),(134))$. Then, $\operatorname{ord}\left(g_{2}^{\prime} g_{3}^{\prime}\right)=2 \operatorname{ord}\left(g_{2} g_{3}\right)$. Further, assume $\boldsymbol{g}=\left(g_{1}, g_{2}, g_{3}, g_{4}\right)$ is in Table 1 Then, if $\operatorname{ord}\left(g_{2} g_{3}\right)=3$ or 5 , the conclusion holds for $\operatorname{ord}\left(g_{2}^{\prime} g_{3}^{\prime}\right)$.

2.9.3. Generating orbits from $H-M$ reps. for $\mathrm{Ni}\left(A_{5}, \mathbf{C}_{3^{4}}\right)^{\mathrm{in}, \mathrm{rd}}$. Improve Table to get a complete orbit for $\bar{M}_{4}=\left\langle\mathbf{s h}, \gamma_{\infty}\right\rangle$ on $\mathrm{Ni}\left(A_{5}, \mathbf{C}_{3^{4}}\right)^{\text {in,rd }}$ in 2-steps.

First: List $\gamma_{\infty}$ orbits for the H-M reps.

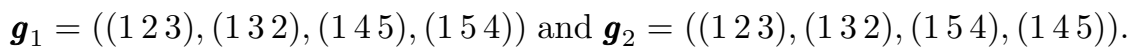

Here are $\left(\boldsymbol{g}_{1}\right) \gamma_{\infty}^{j}, j=1,2,3,4$ :

$$
(\bullet,(245),(132), \bullet),(\bullet,(513),(245), \bullet),(\bullet,(324),(513), \bullet),(\bullet,(451),(324), \bullet) .
$$

A - means repeat that position from the previous element. Conjugate these by (45) for list $\left(\boldsymbol{g}_{2}\right) \gamma_{\infty}^{j}, j=2,3,4,5$. The middle product (order) of a 4 -tuple $\boldsymbol{g}$ is $u=(\boldsymbol{g}) \stackrel{\text { def }}{=} \operatorname{ord}\left(g_{2} g_{3}\right)$.

Second: List elements $\boldsymbol{g}^{\prime}=(\boldsymbol{g})$ sh where $\boldsymbol{g}$ is in 2.26) and $\mathbf{m p}\left(\boldsymbol{g}^{\prime}\right)=1\left(\boldsymbol{g}=\boldsymbol{g}_{1}\right)$ or $3\left(\left(\boldsymbol{g}_{1}\right) \gamma_{\infty}^{j}, j=2,3\right)$ and close under $\left\langle\gamma_{\infty}\right\rangle$ action. Those with $\mathbf{m p}\left(\boldsymbol{g}^{\prime}\right)=1$ are $\left(\boldsymbol{g}_{1}\right) \mathbf{s h}$ and $\left(\left(\boldsymbol{g}_{1}\right) \mathbf{s h}\right) \gamma_{\infty}$. Those with $\mathbf{m p}\left(\boldsymbol{g}^{\prime}\right)=3$ are in $\left(\left(\boldsymbol{g}_{1}\right) \gamma_{\infty}^{j}\right) \mathbf{s h}\left\langle\gamma_{\infty}\right\rangle, j=2,3$ :

$$
\begin{aligned}
& ((513),(245),(154),(123)),(\bullet,(214),(245), \bullet),(\bullet,(415),(214), \bullet) \\
& ((324),(513),(154),(123)),(\bullet,(345),(513), \bullet),(\bullet,(541),(345), \bullet) .
\end{aligned}
$$

Note: Each row of (2.27) contains two elements which sh maps to each of the type $(5,5)$ orbits (5 is the order of both the middle twist and $\gamma_{\infty}$ orbits; as in $\$ 7.2 .1$ ).

Example: Let $\boldsymbol{g}^{*}$ be $(\bullet,(214),(245), \bullet)$ as above. Then,

$$
\left(\boldsymbol{g}^{*}\right) \mathbf{s h}^{-1}=((123),(513),(214),(245)),
$$

so $(123)\left(\boldsymbol{g}^{*}\right) \mathbf{s h}^{-1}(321)$ is in the $\gamma_{\infty}$ orbit of $\boldsymbol{g}_{2}$. The 3 rd elements of each (2.27) row map to each other by sh. Conclude: Two steps produce a list of 4-tuples of 3 -cycles (modulo conjugation by $A_{5}$ ) closed under sh and $\gamma_{\infty}$.

2.10. The sh-incidence matrix. For a general reduced Nielsen class, list $\gamma_{\infty}$ orbits as $O_{1}, \ldots, O_{n}$. The sh-incidence matrix $A(G, \mathbf{C})$ has $(i, j)$ term $\left|\left(O_{i}\right) \mathbf{s h} \cap O_{j}\right|$.

2.10.1. The sh-incidence matrix when $r=4$. Since $\mathbf{s h}$ has order two on reduced Nielsen classes, this is a symmetric matrix. Equivalence $n \times n$ matrices $A$ and $T A^{\mathrm{t}} T$ running over permutation matrices $T$ ( ${ }^{\mathrm{t}} T$ is its transpose) associated to elements of $S_{n}$. List $\gamma_{\infty}$ orbits as

$$
O_{1,1}, \ldots, O_{1, t_{1}}, O_{2,1}, \ldots, O_{2, t_{2}}, \ldots, O_{u}, 1, \ldots, O_{u}, t_{u}
$$

corresponding to $\bar{M}_{4}$ orbits. Choose $T$ to assume $A(G, \mathbf{C})$ is arranged in blocks along the diagonal.

Lemma 2.26. If $A_{j}$ is the $j$ th block of $A(G, \mathbf{C})$, then $A_{j}$ doesn't break into smaller blocks. So, $\bar{M}_{4}$ orbits form irreducible blocks in the $\mathbf{s h}$-incidence matrix.

Proof. With no loss assume one $\bar{M}_{4}$ orbit and two blocks, with orbit listings as $O_{1}, \ldots, O_{k}, O_{k+1}, \ldots, O_{t}$. As, however, there is one orbit, for some $j \leq k$, $\left|\left(O_{i}\right) \mathbf{s h} \cap O_{j}\right| \neq 0$ for some $i>k$. This contradicts there being two blocks. 
In practice it is difficult to list the $\gamma_{\infty}$ orbits. So, we start with the H-M reps., apply sh, then complete the $\gamma_{\infty}$ orbits and check $\left|\left(O_{i}\right) \mathbf{s h} \cap O_{j}\right|$. Sometimes we'll then be done. The case $\left(A_{5}, \mathbf{C}_{3^{4}}\right)$ illustrates this. Denote (as above) the $\gamma_{\infty}$ orbits of $\boldsymbol{g}_{1}$ and $\boldsymbol{g}_{2}$ by $O(5,5 ; 1)$ and $O(5,5 ; 2) ; \gamma_{\infty}$ orbits of

$$
((513),(245),(154),(123)) \text { and }((324),(513),(154),(123))
$$

by $O(3,3 ; 1)$ and $O(3,3 ; 2)$; and of $\left(\boldsymbol{g}_{1}\right) \mathbf{s h}$ by $O(1,2)$.

TABLE 2. sh-Incidence Matrix for $\mathrm{Ni}_{0}$

\begin{tabular}{|c|c|c|c|c|c|}
\hline Orbit & $O(5,5 ; 1)$ & $O(5,5 ; 2)$ & $O(3,3 ; 1)$ & $O(3,3 ; 2)$ & $O(1,2)$ \\
\hline$O(5,5 ; 1)$ & 0 & 2 & 1 & 1 & 1 \\
$O(5,5 ; 2)$ & 2 & 0 & 1 & 1 & 1 \\
$O(3,3 ; 1)$ & 1 & 1 & 0 & 1 & 0 \\
$O(3,3 ; 2)$ & 1 & 1 & 1 & 0 & 0 \\
$O(1,2)$ & 1 & 1 & 0 & 0 & 0 \\
\hline
\end{tabular}

2.10.2. The sh-incidence matrix for general $r$. For general $r$, denote $q_{1} \cdots q_{r-1}$ at the Hurwitz monodromy level to be the shift $\mathbf{s h}_{r}$, so $\mathbf{s h}_{4}$ is what we call the shift above. Ideas for $r=4$ generalize to indicate cusp geometry for general $r$.

The element $\mathbf{s h}_{r}$ plays the role of a shift in two ways. Consider an $r$-tuple $\overline{\boldsymbol{\sigma}}=\left(\bar{\sigma}_{1}, \ldots, \bar{\sigma}_{r}\right)$ of free generators of $F_{r}$. The effect of $\mathbf{s h}_{r}$ on $\overline{\boldsymbol{\sigma}}$ is to give

$$
(\overline{\boldsymbol{\sigma}}) q_{1} \cdots q_{r-1}=\left(\bar{\sigma}_{1} \bar{\sigma}_{2} \bar{\sigma}_{1}^{-1}, \ldots, \bar{\sigma}_{1} \bar{\sigma}_{r} \bar{\sigma}_{1}^{-1}, \bar{\sigma}_{1} \bar{\sigma}_{1} \bar{\sigma}_{1}^{-1}\right)
$$

In specializing to a Nielsen class the effect of $\mathbf{s h}_{r}$ is to the shift the Nielsen class representative entries by 1 . Iterate this $r$ times to see the effect of $\mathbf{s h}_{r}^{r}$ is conjugation on $\boldsymbol{\sigma}$ by the product $\bar{\sigma}_{1} \cdots \bar{\sigma}_{r}$ of these generators.

Such a conjugation commutes with the action of the braid group. So we have an interesting interpretation for the action of conjugating by $\mathbf{s h}_{r}$ on the generators $q_{1}, \ldots, q_{r}$. Define $q_{0}$ to be $\mathbf{s h}_{r}^{-1} q_{1} \mathbf{s h}_{r}$. Then, conjugation by $\mathbf{s h}_{r}$ on the left of the array $\left(q_{0}, q_{1}, \ldots, q_{r-2}, q_{r-1}\right)$ maps its entries to

$$
\begin{aligned}
\mathbf{s h}_{r}\left(q_{0}, \ldots, q_{r-1}\right) \mathbf{s h}_{r}^{-1} & =\left(q_{1}, q_{1} q_{2} q_{1} q_{2}^{-1} q_{1}^{-1}, q_{1} q_{2} q_{3} q_{2} q_{3}^{-1} q_{2}^{-1} q_{1}^{-1}, \ldots\right) \\
& =\left(q_{1}, q_{2}, \ldots, q_{r-1}, q_{0}\right) .
\end{aligned}
$$

To see the effect of conjugation of $\mathbf{s h}_{r}$ on $q_{r-1}$ use that $\mathbf{s h}_{r}^{r}$ is in the center of $H_{r}$ (or of $B_{r}$ ). Then, $q_{0}=\mathbf{s h}_{r}^{r}\left(q_{0}\right) \mathbf{s h}_{r}^{-r}=\mathbf{s h}_{r} q_{r-1} \mathbf{s h}_{r}^{-1}$.

Denote $q_{r-1} q_{r-2} \cdots q_{1}$ by $\mathbf{s h}_{r}^{\prime}$. Notice $\left(\mathbf{s h}_{r}^{\prime}\right)^{r}$ has exactly the same effect on $\boldsymbol{\sigma}$ as does $\mathbf{s h}_{r}^{r}$. In $H_{r}$ use that $q_{1} \cdots q_{r} q_{r} \cdots q_{1}=1$ to $\operatorname{see} \mathbf{s h}_{r}^{r}\left(\mathbf{s h}_{r}^{\prime}\right)^{r}=1$, so $\mathbf{s h}_{r}^{r}=z$ has its square equal to 1 . When $r=4$ the group $M_{4}$ is exactly $H_{r} /\left\langle\mathbf{s h}_{4}^{4}\right\rangle=H_{r} /\langle z\rangle$. An especially handy description of $z$ in this case is $q_{1} q_{3}^{-1}$. In general there is a $\mathbf{s h}_{r}$-incidence matrix. As in the case $r=4$, it suffices to choose the image of $q_{v}$ in $\bar{M}_{r}$ for some value of $v$. It doesn't make any difference which $v$, though for $r=4$ it was convenient to take $v=2$. Call the resulting element $\gamma_{\infty}$. List the $\gamma_{\infty}$ reduced orbits as $O_{1}, \ldots, O_{t}$ and define $A(G, \mathbf{C})$ to be the matrix with $(i, j)$ term $\left|\left(O_{i}\right) \mathbf{s h}_{r} \cap O_{j}\right|$. For general $r$ it won't be symmetric.

\section{Nielsen classes and the Inverse Galois Problem}

Let $G$ be a centerless transitive subgroup of $S_{n}$. Assume $\mathbf{C}$ is $r \geq 3$ conjugacy classes of $G$. This data produces several canonical moduli spaces. Four occur often: 
inner and absolute (adding a permutation representation on $G$ ) Hurwitz spaces and their reduced versions (quotients by a natural $\mathrm{PSL}_{2}(\mathbb{C})$ action). We review their definitions and relation to $H_{r}$. When $r=4$, the reduced versions are curves: upper half plane quotients by finite index subgroups of $\operatorname{PSL}_{2}(\mathbb{Z})$ covering the $j$-line and ramified at traditional points.

3.1. Equivalences on covers of $\mathbb{P}_{z}^{1}$. Consider covers $\varphi_{i}: X_{i} \rightarrow \mathbb{P}_{z}^{1}, i=1,2$. There are two natural equivalences between isomorphisms $\alpha: X_{1} \rightarrow X_{2}$.

(3.1a) Call $\alpha$ an s-equivalence if $\varphi_{2} \circ \alpha=\varphi_{1}$.

(3.1b) Call $\alpha$ a $w$-equivalence if there exists $\beta \in \mathrm{PSL}_{2}(\mathbb{C})$ with $\varphi_{2} \circ \alpha=\beta \circ \varphi_{1}$.

Definition 3.1. In the notation above, call $\varphi_{1}$ and $\varphi_{2}$ s-equivalent (resp. wequivalent) if (3.1. (1. (resp. (3.11D) holds for some $\alpha$.

Moduli spaces formed from equivalence (3.17 tion. The quotient is a moduli space for equivalence (3.11b. Let $\mathcal{H}^{\text {rd }}$ be one of these reduced moduli spaces. If $r=4$, its projective completion gives a ramified cover $\bar{\psi}: \overline{\mathcal{H}}^{\text {rd }} \rightarrow \mathbb{P}_{j}^{1}$. We refine DF99 Prop. 6.5] for computing a branch cycle description of $\bar{\psi}$. This clarifies that a geometric equivalence from a Klein 4-group comes exactly from the group labeled $\mathcal{Q}^{\prime \prime}=\mathcal{Q} /\langle z\rangle$ in 2.6 .4

3.1.1. Setup for Nielsen classes. Consider any smooth connected family of $r$ (fixed) branch point covers of $\mathbb{P}_{z}^{1}$. Denote the degree of the covers in the family by $n$. It has this attached data:

(3.2a) an associated group $G$;

(3.2b) a permutation representation $T: G \rightarrow S_{n}$; and

(3.2c) a set of conjugacy classes $\mathbf{C}=\left(\mathrm{C}_{1}, \ldots, \mathrm{C}_{r}\right)$ of $G$.

Denote the subgroup of $S_{n}$ normalizing $G$ and permuting the conjugacy classes C by $N_{S_{n}}(G, \mathbf{C})=N_{T}(G, \mathbf{C})$. If a cover $\varphi: X \rightarrow \mathbb{P}_{z}^{1}$ in this family has definition field $K$, then the Galois closure of the cover has Galois (arithmetic monodromy) group a subgroup of $N_{S_{n}}(G, \mathbf{C})$. Below use the notation $G(T, 1)$ for the elements $g \in G$ with (1)T( $g)=1: G(T, 1)$ is the stabilizer of 1 in the representation.

3.1.2. Nielsen class and $H_{r}$ action. Relate covers with data $(G, \mathbf{C})$ to homomorphisms of fundamental groups using the associated Nielsen class:

$$
\mathrm{Ni}=\mathrm{Ni}(G, \mathbf{C})=\left\{\boldsymbol{g}=\left(g_{1}, \ldots, g_{r}\right) \mid g_{1} \cdots g_{r}=1,\langle\boldsymbol{g}\rangle=G \text { and } \boldsymbol{g} \in \mathbf{C}\right\} .
$$

Writing $\boldsymbol{g} \in \mathbf{C}$ means the conjugacy classes of the $g_{i}$ s in $G$ are, in some possibly rearranged order, the same (with multiplicity) as those in $\mathbf{C}$.

Suppose given $\boldsymbol{z}, r$ branch points, and a choice $\overline{\boldsymbol{g}}$ of classical generators for $\pi_{1}\left(U_{\boldsymbol{z}}, z_{0}\right)$ (1.2). Then, $\operatorname{Ni}(G, \mathbf{C})$ lists all homomorphisms from $\pi_{1}\left(U_{\boldsymbol{z}}, z_{0}\right)$ to $G$ giving a cover with branch points $z$ associated to $(G, \mathbf{C})$. A Galois cover (over $\mathbb{Q}$ ) with attached data $(G, \mathbf{C})$ is a $(G, \mathbf{C})$ realization, suppressing $\mathbb{Q}$. Analogous results for any characteristic 0 field (including non-Galois covers) have easy formulations as in Fri77, Thm. 5.1].

Denote the subgroup of automorphisms Aut $(G)$ of $G$ permuting elements in $\mathbf{C}$ by $\operatorname{Aut}(G, \mathbf{C})$. An $\alpha \in \operatorname{Aut}(G, \mathbf{C})$ respects the multiplicity of a conjugacy class in C. It acts on $\mathrm{Ni}(G, \mathbf{C})$ by

$$
\left.(\boldsymbol{g}) \alpha=\left(\left(g_{1}\right) \alpha, \ldots,\left(g_{r}\right) \alpha\right)\right) .
$$

Given $N^{*} \leq \operatorname{Aut}(G)$ form $\operatorname{Ni}(G, \mathbf{C}) / N^{*}$ in the obvious way. 
Suppose $T: G \rightarrow S_{n}$ is a permutation representation. Let $N^{\prime}$ be a subgroup of $N_{T}(G, \mathbf{C})$ (3.1.1). Conjugation on $G$ gives a homomorphism $N^{\prime} \rightarrow \operatorname{Aut}(G, \mathbf{C})$. This is an isomorphism into if and only if $N^{\prime}$ contains no nontrivial centralizer of $G$. Abusing notation, denote the quotient of $N^{\prime}$ action by $\operatorname{Ni}(G, \mathbf{C}) / N^{\prime}$.

The braid group $B_{r}$ acts from $Q_{1}, \ldots, Q_{r-1}$ acting on $\left(\bar{g}_{1}, \ldots, \bar{g}_{r}\right) \in \operatorname{Ni}(G, \mathbf{C})$ as in (2.3). If $N^{\prime} \leq N_{T}(G, \mathbf{C})$ contains $G$, this $B_{r}$ action induces an $H_{r}=\pi_{1}\left(U_{r}\right)$ action (2.1.3). Assume $G \leq N^{\prime}$. Permutation representations of fundamental groups produce covers. For this $H_{r}$ action, denote the (Hurwitz space) cover of $U_{r}$ by $\mathcal{H}_{N^{\prime}}=\mathcal{H}(G, \mathbf{C})_{N^{\prime}}$.

3.1.3. Galois closure and permutation representations. Use notation from 3.1 .1 for a cover $\varphi: X \rightarrow \mathbb{P}_{z}^{1}$ of degree $n=\operatorname{deg}(\varphi)$ with $(G, \mathbf{C})$ and a permutation representation $T_{\varphi}=T: G \rightarrow S_{n}$ attached.

The Galois closure of $\varphi$ over any defining field for $(X, \varphi)$ has a geometric formulation. Take the fiber product $X^{(n)} \stackrel{\text { def }}{=} X_{\varphi}^{(n)} \stackrel{\text { def }}{=} X_{\mathbb{P}_{z}^{1}}^{(n)}$ of $\varphi, n$ times. Points on $X^{(n)}$ consist of $n$-tuples $\left(x_{1}, \ldots, x_{n}\right)$ of points on $X$ satisfying $\varphi\left(x_{1}\right)=\varphi\left(x_{2}\right)=$ $\cdots=\varphi\left(x_{n}\right)$. This variety will be singular around $n$-tuples where $x_{i}$ and $x_{j}$ are both ramified through $\varphi$. Replace $X^{(n)}$ by its normalization to make it now a nonsingular cover. Retain the notation $\varphi^{(n)}: X^{(n)} \rightarrow \mathbb{P}_{z}^{1}$. Then, $X^{(n)}$ has components where at least two of the coordinates are identical. These form the fat diagonal.

Remove components of this fat diagonal to give $X^{*}$. Over the algebraic closure $X^{*}$ has as many components as $\left(S_{n}: G\right)$. List one of these components over $\bar{K}$ as $X^{\dagger}$. The stabilizer in $S_{n}$ of $X^{\dagger}$ is a conjugate of $G$. Normalize by choosing $X^{\dagger}$ so the stabilizer is actually $G$. Now, choose any $K$ component $\hat{X}$ of $X^{*}$ containing $X^{\dagger}$. Then, $\hat{\varphi}: \hat{X} \rightarrow \mathbb{P}_{z}^{1}$ is Galois (over $K$ ) with group $\hat{G} \leq N_{S_{n}}(G, \mathbf{C}$ ) having $G$ as a normal subgroup. Also, $\hat{\varphi}$ has the same conjugacy classes $\mathbf{C}$ attached to the branch points $z$ and it factors through $\varphi$ (project on any coordinate of $X^{(n)}$ ). The Galois cover $\hat{X} \rightarrow X$ has group $\hat{G}(1)=\hat{G}(T, 1)$ where $T$ is the coset representation of $G$ on $G(1)$. The following is elementary Fri77. Lem. 2.1].

LEMMA 3.2. The centralizer of $G$ in $N_{S_{n}}(G, \mathbf{C})$ induces the automorphisms of $X$ that commute with $\varphi_{T}$.

Consider any permutation representation $T^{\prime}: G \rightarrow S_{n^{\prime}}$. This provides $\varphi_{T^{\prime}}$ : $X_{T^{\prime}} \rightarrow \mathbb{P}_{z}^{1} ; X_{T^{\prime}}$ is the quotient $\hat{X} / G\left(T^{\prime}, 1\right)$ (with $G\left(T^{\prime}, 1\right)$ as in 3.1 .1 ).

3.2. Motivation from the Inverse Galois Problem. The regular version of the Inverse Galois Problem asks if every finite $G$ is the group of a cover of $U_{\boldsymbol{z}}$, for some integer $r$, with equations for its automorphisms and the cover over $\mathbb{Q}$. Call such an $r$-branch point realization over $\mathbb{Q}$. To find $r, z$ and such a cover needs structure. Form the profinite completion $\pi_{1}\left(U_{\boldsymbol{z}}, z_{0}\right)^{\text {alg }}$ of $\pi_{1}\left(U_{\boldsymbol{z}}, z_{0}\right)$ with respect to all its finite index subgroups. Suppose $z_{0} \in \mathbb{Q} \cup\{\infty\}$ and $z$ is a $G_{\mathbb{Q}}$ stable set. There is a natural short exact sequence

$$
1 \rightarrow \pi_{1}\left(U_{\boldsymbol{z}}, z_{0}\right)^{\text {alg }} \rightarrow \pi_{1}\left(U_{\boldsymbol{z}}, z_{0}\right)^{\text {ar }} \rightarrow G_{\mathbb{Q}} \rightarrow 1 \text { ([Ser92 p. 57] or Prop. 3.4) }
$$

For an analog substituting $K$ for $\mathbb{Q}$, replace $G_{\mathbb{Q}}$ by $G_{K}$ in (3.3).

3.2.1. Grothendieck's $G_{\mathbb{Q}}$ action. Let $\mathcal{E}\left(U_{\boldsymbol{z}}, z_{0}\right)^{\text {alg }}$ be all meromorphic algebraic functions in a neighborhood of $z_{0}$ that extend analytically along each path in $U_{\boldsymbol{z}}$. Regard them as in the Laurent series $\mathcal{L}_{z_{0}}$ about $z_{0}$. Each is meromorphic in a disk about $z_{0}$ having the nearest point of $z$ on its boundary. These are the extensible functions in $U_{\boldsymbol{z}}$. To each such $f$ associate a definition field $K=K_{f}$. This has 
generators (over $\mathbb{Q}$ ) the ratios of coefficients of any (nonzero) polynomial $F(z, w)$ in $z$ and $w$ with $F(z, f(z)) \equiv 0$ near $z_{0}$. The field $K(z, f)$ that $f$ generates over $K(z)$ is the field of meromorphic functions of a cover $\bar{X} \rightarrow \mathbb{P}_{z}^{1}$ over $K$ (restricting to an unramified cover $\left.X=X_{f} \rightarrow U_{z}\right)$.

Suppose $\psi: X \rightarrow U_{z}$ is a finite (unramified) cover. Then, $\psi$ is s- equivalent to $\psi_{f}: X_{f} \rightarrow U_{\boldsymbol{z}}$ for some $f$ with the following properties.

(3.4a) $K_{f}$ is in the algebraic closure of $\mathbb{Q}\left(z_{0}, \boldsymbol{z}\right)$.

(3.4b) $f$ and each analytic continuation of it (around a closed path based at $z_{0}$ ) have nonzero differential at $z_{0}$.

So, these analytic continuations have $n=\operatorname{deg}\left(\psi_{f}\right)$ distinct values at $z_{0}$. Their power series around $z_{0}$ have coefficients in the algebraic closure of $\mathbb{Q}\left(z_{0}, \boldsymbol{z}\right)$.

With no loss, when $z_{0}$ and $z$ are over $\mathbb{Q}$, assume $\mathcal{E}\left(U_{\boldsymbol{z}}, z_{0}\right)^{\text {alg }}$ consists of power series around $z_{0}$ with coefficients in $\overline{\mathbb{Q}}$. There is an ordering on elements of $\mathcal{E}\left(U_{\boldsymbol{z}}, z_{0}\right)^{\text {alg: }} f \leq g$ if $\overline{\mathbb{Q}}(z, g) \supset \overline{\mathbb{Q}}(z, f)$. Analytic continuation over a path, as an operation on $\overline{\mathbb{Q}}(z, g)$, determines the path's analytic continuation on $\overline{\mathbb{Q}}(z, f)$. So, paths acting on these equivalence classes respect this ordering. Each equivalence class represents a specific function field, with all functions in it expanded around $z_{0}$. It is the exact data one expects from a cover and a point on the cover over $z_{0}$. This action by paths in $U_{\boldsymbol{z}}$ based at $z_{0}$ on the points above $z_{0}$ respects the action on points of a higher covering. For $\gamma \in \pi_{1}\left(U_{\boldsymbol{z}}, z_{0}\right)$, let $f_{\gamma}$ be the analytic continuation of $f$ to the endpoint of $\gamma$.

Definition 3.3. Define how $\sigma \in G_{\mathbb{Q}}$ acts on $\gamma \in \pi_{1}\left(U_{\boldsymbol{z}}, z_{0}\right)$ by how it acts on extensible algebraic functions: $f \mapsto f_{\sigma^{-1} \circ \gamma \circ \sigma}=f_{\gamma^{\sigma}}$. In words: Apply $\sigma^{-1}$ to the coefficients of $f$, continue $f$ around $\gamma$ and then apply $\sigma$ to the coefficients of the result. The effect of $\gamma$ on algebraic functions determines it. This defines $\gamma^{\sigma}$.

Unless $\sigma$ is complex conjugation, no actual path gives $\gamma^{\sigma}$. With our right action of $G_{\mathbb{Q}}$ replacing a left action, the following is compatible with [Iha91, p. 103].

Proposition 3.4. Fiber compatible action on a projective system of points $\mathbb{P}_{z_{0}}$ determines paths in $\pi_{1}\left(U_{\boldsymbol{z}}, z_{0}\right)$. Then, $\left\{\gamma^{\sigma}\right\}_{\gamma \in \pi_{1}\left(U_{\boldsymbol{z}}, z_{0}\right), \sigma \in G_{\mathbb{Q}}}$ also acts on $\mathbb{P}_{z_{0}}$.

Define $\pi_{1}^{\mathrm{alg}}$ as the projective completion of this action from finite Galois covers. Then, $\pi_{1}^{\text {alg }}$ is the projective limit of $\pi_{1}$ quotients from all normal subgroups of finite index. The previous action of $\sigma \in G_{\mathbb{Q}}$ on $\pi_{1}\left(U_{\boldsymbol{z}}, z_{0}\right)$ extends to $\pi_{1}^{\text {alg }}$, giving a splitting of the sequence (3.3).

3.2.2. The Branch Cycle Lemma over $\mathbb{Q}$. The Branch Cycle Lemma (Fri77. before Thm. 5.1] or the case in Lem. 3.7) gives a necessary condition for a $(G, \mathbf{C})$ realization (over $\mathbb{Q}$ ) through a quotient of $\pi_{1}\left(U_{\boldsymbol{z}}, z_{0}\right)^{\text {alg }}$. Let $\overline{\boldsymbol{g}}$ be classical generators of $\pi_{1}\left(U_{\boldsymbol{z}}, z_{0}\right)^{\text {alg }}\left(\Phi_{1.2}\right)$. Any homomorphism $\psi: \pi_{1}\left(U_{\boldsymbol{z}}, z_{0}\right)^{\text {alg }} \rightarrow G$ produces a geometrically Galois cover $\varphi_{\psi}: X_{\psi} \rightarrow \mathbb{P}_{z}^{1}$. Though construction of $\varphi_{\psi}$ is canonical, it depends on the base point. Remove this by equivalencing homomorphisms $\psi$ differing by conjugation of $\pi_{1}\left(U_{\boldsymbol{z}}, z_{0}\right)^{\text {alg }}$. \$3.5 explains this and related equivalences.

For $g \in G$ and $k \in \hat{\mathbb{Z}}$ (a profinite integer), $k$ acts on $g$ by putting it to the $k$ th power. Then, invertible elements $n \in \hat{\mathbb{Z}}^{*}$ map $g$ to another generator of $\langle g\rangle$.

Definition 3.5. Call a set of conjugacy classes $\mathbf{C}$ in $G$ a rational union if $\mathbf{C}^{n}=\mathbf{C}$ (both sides counted with multiplicity) for all $n \in \hat{\mathbb{Z}}^{*}$. Given conjugacy classes $\mathbf{C}$, there is a natural rationalization $\mathbf{C}^{\prime}$ : The minimal rational collection of conjugacy classes containing $\mathbf{C}$. 
Example 3.6. Suppose $C_{1}, C_{2}$ and $C_{3}$ are respectively the conjugacy classes of the 5 -cycles in $A_{5}$ given by $g_{1}=(12345), g_{2}=(13524)$ and $g_{1}$ again. Then, $C_{1}, C_{2}, C_{3}$ is not a rational union because the conjugacy class of $g_{1}$ appears with multiplicity 2 , while its square appears only with multiplicity 1 . The collection $\mathbf{C}^{\prime}=\left(C_{1}, C_{2}, C_{1}, C_{2}\right)$ is its rationalization.

The image $\boldsymbol{g}$ of $\overline{\boldsymbol{g}}$ gives conjugacy classes $\mathbf{C}$ in $G$, with $\mathrm{C}_{i}$ attached to $z_{i}$, $i=1, \ldots, r$. Suppose the following:

(3.5) $\varphi_{\psi}: X_{\psi} \rightarrow \mathbb{P}_{z}^{1}$ (with its automorphisms) has equations over $\mathbb{Q}$.

Lemma 3.7 (Branch Cycle Lemma). If (3.5) holds then $\boldsymbol{z}$ is a $\mathbb{Q}$ set. A necessary and sufficient condition for (3.5) is that $\psi$ extends to a homomorphism $\psi: \pi_{1}\left(U_{\boldsymbol{z}, z_{0}}\right)^{\text {ar }} \rightarrow G$ (as in (3.3) ). For an analog with any $K$ of characteristic 0 (including $\mathbb{R}$ or $\left.\mathbb{Q}_{p}\right)$ replace $G_{\mathbb{Q}}$ with $G_{K}$ in the definition of $\pi_{1}\left(U_{\boldsymbol{z}^{\prime}, z_{0}}\right)^{\text {ar }}$.

Assume $\sigma \in G_{K}$ maps to $n_{\sigma} \in \hat{\mathbb{Z}}^{*}=G\left(\mathbb{Q}^{\text {cyc }} / \mathbb{Q}\right)$ and $\pi \in S_{r}$ satisfies $z_{i}^{\sigma}=z_{(i) \pi}$. Then, 3.5 implies

$$
C_{(i) \pi}^{n_{\sigma}}=C_{i}, i=1, \ldots, r .
$$

So, when $K=\mathbb{Q}$ and (3.5) holds, $\mathbf{C}$ is a rational union (Def. 3.5).

Prop. 5.11 illustrates using Lem. 3.7 to answer question (1.71

3.2.3. Using Lem. 3.7 over a general field. Suppose $\varphi: X \rightarrow \mathbb{P}_{z}^{1}$ over $\mathbb{Q}$ has degree $n$ with attached $(G, \mathbf{C})$. Then, $\varphi$ produces a permutation representation $G \leq S_{n}$. Sometimes the goal is to find $(X, \varphi)$ over $\mathbb{Q}$, with less concern for the definition field of the Galois closure of the cover (as in DF99 and Fri99]). The necessary condition for this requires a choice of Galois closure group $\hat{G}$ between $G$ and $N_{S_{n}}(G, \mathbf{C})$ as in 3.5 For each such $\hat{G}$, replace (3.6) by

$$
g_{\sigma} C_{(i) \pi}^{n_{\sigma}} g_{\sigma}^{-1}=C_{i}, i=1, \ldots, r, \text { for some } g_{\sigma} \in \hat{G} .
$$

A general Branch Cycle Lemma, including for non-Galois covers is in Fri77. Thm. 5.1]. $K=\mathbb{Q}_{p}$, it pays to stipulate orbits of $G_{K}$ on the branch points precisely. For a general $K$, assume $G_{K}$ acts on the support of $z$ as $\mu: G_{K} \rightarrow S_{r}$. Regard $G\left(K^{\text {cyc }} / K\right)$ as a subgroup of $\hat{\mathbb{Z}}^{*}$. Consider:

$$
\begin{array}{cl}
H_{G, \mathbf{C}, \hat{G}, \mu}=\left\{\sigma \in G_{K} \mid g_{\sigma} C_{(i) \mu(\sigma)}^{n_{\sigma}} g_{\sigma}^{-1}=\right. & \left.C_{i}, i=1, \ldots, r, \text { for some } g_{\sigma} \in \hat{G}\right\} ; \\
\text { and } H_{G, \mathbf{C}, \hat{G}}=\left\{n^{\prime} \in G\left(K^{\mathrm{cyc}} / K\right)\right. & \mid g_{n^{\prime}} C_{(i) \pi}^{n^{\prime}} g_{n^{\prime}}^{-1}=C_{i}, i=1, \ldots, r, \\
& \text { for some } \left.g_{n^{\prime}} \in \hat{G} \text { and } \pi \in S_{r}\right\} .
\end{array}
$$

Let $\varphi: X \rightarrow \mathbb{P}_{z}^{1}$ be in the Nielsen class $\operatorname{Ni}(G, \mathbf{C}, T)$ have definition field $L \geq K$. Call $\varphi$ attached to $(K, \boldsymbol{z}, G, \hat{G}, \mathbf{C}, \mu)$ if its branch points $z$ are a $K$ set with the given action $\mu$ and its arithmetic monodromy group over $L$ is a subgroup of $\hat{G}$. Then, the fixed field $K_{G, \mathbf{C}, G, \hat{G}, \mu}$ of $H_{G, \mathbf{C}, \hat{G}, \mu}$ is a subfield of $L$ (similar to [DF90 §1.2]). When $G=\hat{G}$ drop the appearance of $\hat{G}$. Example: $K_{G, \mathbf{C}, G, \hat{G}, \mu}$ becomes $K_{G, \mathbf{C}, G, \mu}$.

3.3. Frattini group covers. The history of the Inverse Galois Problem shows it is hard to find $r$ and $\boldsymbol{z}$ producing $\mathbb{Q}$ realizations. To systematically investigate the diophantine nature of these difficulties we take large (maximal Frattini) quotients of $\pi_{1}\left(U_{\boldsymbol{z}, z_{0}}\right)^{\text {alg }}$, rather than finite groups.

Start with a finite group $G$. Call $\mu: H \rightarrow G$ a Frattini cover if for any subgroup $H^{*} \leq H, \mu\left(H^{*}\right)=G$ implies $H^{*}=H$. This exactly translates the cover property from 95.1 There is a (uni) versal Frattini group $\tilde{G}$ covering $G$ [FJ86 Chap. 20]. We 
review properties of $\tilde{G}$ by analogy with the universal Frattini cover of the dihedral group $D_{p}$ ( $p$ odd).

3.3.1. Using $G=D_{p}=\mathbb{Z} / p \times{ }^{s}\{ \pm 1\}$ as a model. Consider

$$
\varphi_{p}:{ }_{p} \tilde{D}_{p}=\mathbb{Z}_{p} \times{ }^{s}\{ \pm 1\} \rightarrow D_{p} \text { and } \varphi_{2}:{ }_{2} \tilde{D}_{p}=\mathbb{Z} / p \times{ }^{s} \mathbb{Z}_{2} \rightarrow D_{p}
$$

as pieces of the universal Frattini profinite cover of $D_{p}$. Patch these as a fiber product over $D_{p}$ :

$$
\tilde{D}_{p}=\left\{\left(g_{1}, g_{2}\right) \in{ }_{2} \tilde{D}_{p} \times{ }_{p} \tilde{D}_{p} \mid \varphi_{2}\left(g_{1}\right)=\varphi_{p}\left(g_{2}\right)\right\} .
$$

This generalizes: For each prime $p, p|| G \mid$, there is a universal $p$-Frattini cover $\psi_{p}:{ }_{p} \tilde{G} \rightarrow G$ with these properties [Fri95a Part II].

(3.8a) $\tilde{G}$ is the fiber product of ${ }_{p} \tilde{G}$ (over $G$ ) over $p$ primes dividing $|G|$.

(3.8b) Both $\operatorname{ker}\left(\psi_{p}\right)$ and a $p$-Sylow of ${ }_{p} \tilde{G}$ are pro-free pro- $p$ groups, and ${ }_{p} \tilde{G}$ is the minimal profinite cover of $G$ with this property.

(3.8c) ${ }_{p} \tilde{G}$ has a characteristic sequence of finite quotients $\left\{G_{k}\right\}_{k=0}^{\infty}$.

(3.8d) Each $p^{\prime}$-conjugacy class of $G$ lifts uniquely to a $p^{\prime}$ class of ${ }_{p} \tilde{G}$.

(3.8e) If $H \leq G_{k}$ has image in $G$ all of $G$, then $H=G_{k}$.

To simplify notation we suppress the appearance of $p$ in forming the characteristic sequence $\left\{G_{k}\right\}_{k=0}^{\infty}$. Denote $\operatorname{ker}\left(\psi_{p}\right)$ by $\operatorname{ker}_{0}$. For any pro- $p$ group, the Frattini subgroup is the closed subgroup that commutators and $p$ th powers generate. Let ker $_{1}$ be the Frattini subgroup of ker $_{0}$. Continue inductively to form $\operatorname{ker}_{k}$ as the Frattini subgroup of $\operatorname{ker}_{k-1}$. Then, $G_{k}={ }_{p} \tilde{G} / \mathrm{ker}_{k}$. Use of modular representation theory throughout this paper is from the action of $G_{k}$ on $\operatorname{ker}_{k} / \operatorname{ker}_{k+1} \stackrel{\text { def }}{=} M_{k}$, a natural $\mathbb{Z} / p\left[G_{k}\right]$ module. Rem. 5.2 notes some points about this.

We occasionally use $G_{K}$ with $K$ a field for the absolute Galois group of $K$. The context and use of capital letters for fields in that rare event should cause no confusion with this $G_{k}$ notation.

3.3.2. p-projectivity. Let $\psi: H \rightarrow G$ be a cover of profinite groups with the kernel of $\psi$ a pro- $p$ group. Such a cover is p-projective if for any cover $\psi^{\prime}: H^{\prime} \rightarrow G$ with kernel a pro- $p$ group, there is a homomorphism $\alpha: H \rightarrow H^{\prime}$ with $\psi^{\prime} \circ \alpha=\psi$.

Definition 3.8. With the same setup as above and $k$ a positive integer, we say $\psi$ is a $(p, k)$-projective cover if for any $\psi^{\prime}$ with kernel a $p$-group of exponent $p^{k}$, there exists $\alpha: H \rightarrow H^{\prime}$ with $\psi^{\prime} \circ \alpha=\psi$.

The next proposition captures the most significant property in the list (3.8). Equivalent to (3.810) is that ${ }_{p} \tilde{G}$ is $p$-projective.

Proposition 3.9. The group ${ }_{p} \tilde{G}$ is the minimal p-projective cover of $G$. Also, $G_{k}$ (with its morphism to $G=G_{0}$ is the minimal $(p, k)$-projective cover of $G$.

An analogous definition without reference to covers is of $p$-projective. That would be a profinite group $H$ such that any cover $\psi: H \rightarrow G$ is $p$-projective. Many fields relevant to arithmetic and algebraic geometry have absolute Galois groups that are $p$-projective (call the field itself $p$-projective). In particular that includes function fields over algebraically closed fields, and the PAC fields from [FJ86, that play a role in the exact sequence for $G_{\mathbb{Q}}$ in (1.1).

Further, many function fields have geometric fundamental groups that are $p$ projective. If $C$ is an algebraic curve (affine or projective) over an algebraically closed field of characteristic $p$, then its (profinite) fundamental group is $p$-projective. 
This $p$-projective result is due to Grothendieck, though there are many recent revisitings of this topic (for example [Ja99]). We know fundamental groups of projective curves in 0 characteristic, though not in positive characteristic; $p$-projectivity is a very good hint why not. The $p^{\prime}$ quotient of such fundamental groups (affine or projective) are like their characteristic 0 counterparts from Grothendieck's enhancement of Riemann's existence theorem (see 4.4.31). Non $p^{\prime}$ quotients are not, though we know the group is $p$ projective. Clearly the group is not pro-free for you can't put those two properties together in a pro-free group. We don't know how to characterize profinite groups that do put those properties together.

3.3.3. Is ker $_{0}$ characteristic? General automorphisms of ${ }_{p} \tilde{G}$ may not preserve ker $_{0}$. That is, the phrase characteristic quotients of ${ }_{p} \tilde{G}$ doesn't imply ker $_{0}$ is a characteristic subgroup of ${ }_{p} \tilde{G}$. Still, the following easy lemma often applies.

Lemma 3.10. If $\operatorname{ker}_{0}$ is a characteristic subgroup of the $p$-Sylow of ${ }_{p} \tilde{G}$, then by definition all $\operatorname{ker}_{k} s$ are characteristic subgroups. This holds for the universal $p$-Frattini cover of $D_{p}$. If all p-Sylows of $G$ intersect in $\{1\}$ (as when $G$ is a simple group), then $\operatorname{ker}_{0}$ is the intersection of all p-Sylows of ${ }_{p} \tilde{G}$. So, $\operatorname{ker}_{0}$ is also characteristic: All automorphisms of ${ }_{p} \tilde{G}$ preserve the intersection of the $p$-Sylows.

REMark 3.11 (Checking when ker $_{0}$ is characteristic). Suppose $P_{p}^{\prime}$ is a $p$-Sylow of ${ }_{p} \tilde{G}$. Since it is a pro-free pro- $p$ group, its characteristic subgroups appear from the filtrations by the $p^{u}$ th power subgroups and the lower central series. In principle this allows determining if ker $_{0}$ is a characteristic subgroup of $P_{p}^{\prime}$.

3.4. One prime at a time. The fiber product characterization of (3.8) allows dealing with one $p$-Frattini cover ${ }_{p} \tilde{G}$ of $G$ at a time.

3.4.1. Realization of ${ }_{p} \tilde{G}$ quotients. Fix $G$ and $p$. Here is a naive diophantine goal referencing the groups $G_{k}$ of (3.810).

Problem 3.12. For each $k$ find the following:

(3.9a) $r_{k}$ distinct points $\boldsymbol{z}_{k}$ (possibly varying with $k$ ); and

(3.9b) $\pi_{1}\left(U_{\boldsymbol{z}_{k}}, z_{0}\right)^{\text {alg }} \rightarrow G_{k} \rightarrow 1$ factoring through $\pi_{1}\left(U_{\boldsymbol{z}_{k}}, z_{0}\right)^{\mathrm{ar}} \rightarrow G_{k}$.

The Branch Cycle Lemma limits conjugacy classes $\mathbf{C}_{k}$ to satisfy (3.6).

Assume $\mathbf{C}$ consists of $p^{\prime}$-conjugacy classes. Suppose $G^{*}$ is simultaneously a quotient of ${ }_{p} \tilde{G}$ factoring through $G$ and of $\pi_{1}\left(U_{z_{k}}, z_{0}\right)^{\text {alg }}$. Call the corresponding cover of $\mathbb{P}_{z}^{1}$ a $p$-Frattini $(G, \mathbf{C})$ cover if classical generators $(\$ 1.2)$ of $\pi_{1}\left(U_{\boldsymbol{z}_{k}}, z_{0}\right)^{\text {alg }}$ also map to C. According to 3.810), if $(G, \mathbf{C})$ passes the Branch Cycle Lemma test, then so does $\left(G_{k}, \mathbf{C}\right)$ for all $k$. This illustrates the groups $G_{k}$ are similar. The guiding question asks this.

Question 3.13. Will all the $G_{k}$ fall to the Inverse Galois Problem with a bound, independent of $k$, on $r_{k}$ in Prob. 3.12?

3.4.2. Possible $(\boldsymbol{z}, r)$ and the Main Conjecture. A Yes! to Question 3.13 is equivalent to the following (Thm. 6.1 also with any number field $K$ replacing $\mathbb{Q}$ ).

(3.10) There is a rational set of $p^{\prime}$ classes $\mathbf{C}$ (with cardinality $r$ for some $r$ ) so (3.12) holds with $\mathbf{C}=\mathbf{C}_{k}$ (and $r_{k}=r$ ) for all $k \geq 0$.

So, with no loss, $z_{k}$ has exactly $r$ points in its support for all $k$.

The result is exactly the same if we replace $\mathbb{Q}$ by any number field $K$, which we now do. As in [FK97, Main Conj. 0.1] (or Prob. 1.1), if $G$ is p-perfect and 
centerless we conjecture the answer is No!. The Hurwitz spaces $\mathcal{H}\left(G_{k}, \mathbf{C}\right)^{\text {in }}$ for the inner $(G, \mathbf{C})$ Modular Tower of covers up to inner equivalence (3.5.2) generalize the modular curves $X_{1}\left(p^{k+1}\right)$. Assume there exists $r^{\prime}$ with $r_{k} \leq r^{\prime}$ for all positive integers $k$, and let $\mathbf{C}$ be the $p^{\prime}$ conjugacy class in (3.10). Then, Prop. 3.21 shows the $p$-perfect and centerless hypothesis on $G_{0}=G$ guarantees (3.12) is equivalent to producing $K$ points on each level $\mathcal{H}\left(G_{k}, \mathbf{C}\right)^{\text {in }}$ of the inner $(G, \mathbf{C})$ Modular Tower. Given $(G, p)$ with $p|| G \mid$, denote the set of integers $r$ where there is an $r$-tuple of $p^{\prime}$ conjugacy classes in $G$ with a $\mathbb{Q}$ point on every level of the $(G, \mathbf{C}, p)$ Modular Tower

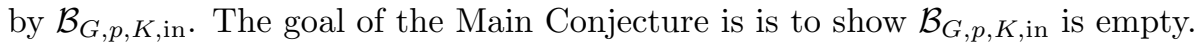

The methods of the paper pretty much restrict to showing $\mathcal{B}_{G, p, K \text {,in }}$ does not contain $r=3$ or 4 . When $r=4, p=2$ and $G=A_{5}$, we succeed in showing this, and in finding considerable about excluding $r=4$ for general $(G, p)$. 9.6 states the unsolved problems left from 8 in excluding $r=4$ from $\mathcal{B}_{G, p, K \text {,in. }}$. One sees there the effect of increasing components in going to higher levels of a Modular Tower. This justifies concentration on analyzing the component structures at level 1 in our main examples. A rubric for including all Modular Tower levels would regard $\mathbf{C}$ as ${ }_{p} \tilde{G}$ conjugacy classes. This produces the Nielsen class $\left.\operatorname{Ni}_{p} \tilde{G}, \mathbf{C}\right)$.

3.4.3. Relating the Main Conjecture to present diophantine literature. For Modular Towers defined by absolute equivalence, rational point conclusions at high levels are likely weaker. For absolute equivalence there is an analog set $\mathcal{B}_{G, p, K \text {,abs. As }}$ with the modular curves $X_{0}\left(p^{k+1}\right)$, the case $G=D_{p}$, many times the answer may also be No rational points at high levels! To get closer to a problem in [FKVo99, let $U \leq \operatorname{ker}_{0}$ be a normal subgroup of ${ }_{p} \tilde{G}$. An example would be $U=\left(\operatorname{ker}_{0}, \operatorname{ker}_{0}\right)$ as in 4.4.3. Let $G_{k, U}$ be ${ }_{p} \tilde{G} / \operatorname{ker}_{k} \cdot U$. Then, instead of the full Modular Tower (for absolute or inner equivalence), consider $\left\{\mathcal{H}\left(G_{k, U}, \mathbf{C}\right)^{\text {abs }}\right\}_{k=0}^{\infty}$ (or the inner case). We regard this as a quotient Modular Tower. Similarly, consider values $r$ in $\mathcal{B}_{G, p, U, K \text {,abs }}$ (or $\mathcal{B}_{G, p, U, K, \text { in }}$ ). The phrase Thompson tuples is from [FKVo99 Thm. 4.16].

Example 3.14 (Thompson tuples and challenges to the Main Conjecture). Consider the groups $\mathrm{PGL}_{n}(p)$ with $p$ a prime. To find regular realizations of this group, take $r=n+1$, and what Völklein calls Thompson triples. These produce regular realizations with $r$ branch points, where the attendant Hurwitz space is a nearly trivial cover of $\mathbb{P}^{r} \backslash D_{r}$. This is surprising, generalizing Belyi tuples. It works only for Chevalley groups using extremely special conjugacy classes. The concept that gives the realizations they call weak rigidity.

FKVo99 Thm. 4.5] describes the data that displays Thompson tuples in $\mathrm{GL}_{n}(q)$. Let $\mathbf{C}$ be the corresponding conjugacy class for these, and let $N_{\mathbf{C}}$ be the least common multiple of orders of element in $\mathbf{C}$. They consider such Thompson tuples where $n$ and $N_{\mathbf{C}}=N$ are fixed, yet there is an infinite set $S_{n, N}$ of corresponding primes $p$ (FKVo99 Lem. 4.7]; the $\mathbf{C}$ changes with $p$, though we suppress the extra notation). The following points are no contradiction to our Main Conjecture. Still, they reflect on it with $r=n+1$.

(3.11a) There is a value of $\boldsymbol{z}_{0} \in \mathbb{P}^{r} \backslash D_{r}$ and a finite extension $K_{n, N}$ of $\mathbb{Q}$ with $\mathrm{PGL}_{n}(p)$ regular realizations over $K_{n, N}$ for all $p \in S_{n, N}$.

(3.11b) There is also a cyclic cover $\varphi: Y \rightarrow \mathbb{P}_{z}^{1}$, over $K_{n, N}$, ramified at $\boldsymbol{z}_{0}$, so pullback of the regular realizations of (ail) over $Y$ are unramified Galois (over $K_{n, N}$ ) covers of $Y$. 
Consequence: Using that special point $\boldsymbol{z}_{0} \in \mathbb{P}^{r} \backslash D_{r}$, [FKVo99 Thm. 4.8] creates a curve $Y$ over a number field whose geometric fundamental group has an infinite quotient that is a projective limit of Galois unramified covers over the number field. One of the authors suggested to replace $\mathrm{PGL}_{n}(p)$, $p$ varying, with $\mathrm{PGL}_{n}\left(p^{t}\right)$ (for suitable $p$ ) with $t$ varying. Analogous to the above, there would be $S_{n, N, p}$ consisting of values of $t$ with corresponding realizations of $\mathrm{PGL}_{n}\left(p^{t}\right)$. It appears an argument similar to $\mathbf{F K V 0 9 9}$, would then produce $\mathbf{C}$, a collection of $p^{\prime}$ conjugacy classes, a number field $K_{n, N, p}$ and a point $z_{0} \in \mathbb{P}^{r} \backslash D_{r}$ with the following properties.

(3.12a) There is a projective system of covers

$$
\cdots \rightarrow \hat{X}_{k+1} \rightarrow \hat{X}_{k} \rightarrow \cdots \rightarrow \mathbb{P}_{z}^{1}:
$$

$$
\hat{X}_{0} \rightarrow \mathbb{P}_{z}^{1} \text { has branch points } \boldsymbol{z}_{0} \text { and } \hat{X}_{k+1} \rightarrow \hat{X}_{k} \text { is unramified, } k \geq 0 \text {. }
$$

(3.12b) A point of $\mathcal{H}\left(\mathrm{PGL}_{n}\left(p^{k+1}\right), \mathbf{C}\right)^{\text {in }}\left(K_{n, N, p}\right), k \geq 0$, gives $\hat{X}_{k+1} \rightarrow \mathbb{P}_{z}^{1}$.

Vö01 offers a systematic approach through the BC Functor to discussing projective systems of Chevalley groups, and so one may consider the whole apparatus as a challenge to the Main Conjecture. We conclude, however, by noting why (3.12) does not contradict it. It is true that $\mathrm{PGL}_{n}\left(p^{k+1}\right) \rightarrow \mathrm{PGL}_{n}(p)$ is a $p$ Frattini cover, if $n \geq 2, p$ is odd, $(p, n)=1$ and if $p=3, n>2$ [ö95a \$]. The group $\mathrm{PGL}_{n}\left(\mathbb{Z}_{p}\right)$, however, is not the universal $p$-Frattini cover of $\mathrm{PGL}_{n}(p)$ : The kernel to $\mathrm{PGL}_{n}\left(\mathbb{Z}_{p}\right) \rightarrow \mathrm{PGL}_{n}(p)$ is not a pro- free pro- $p$ group as is the kernel of ${ }_{p} \widetilde{\mathrm{PGL}_{n}}(p) \rightarrow \mathrm{PGL}_{n}(p)$. Even the rank of the latter is larger than that of $\operatorname{ker}\left(\mathrm{PGL}_{n}\left(\mathbb{Z}_{p}\right) \rightarrow \mathrm{PGL}_{n}(p)\right)$. Example: $\operatorname{ker}\left(\mathrm{PGL}_{2}\left(\mathbb{Z}_{5}\right) \rightarrow \mathrm{PGL}_{2}(5)\right)$ has rank 3, while the kernel of ${ }_{5} \widetilde{\mathrm{PGL}_{2}}(5) \rightarrow \mathrm{PGL}_{n}(5)$ has rank 6 [Fri95a, Rem. 2.10].

3.5. Refined s-equivalence from $N_{S_{n}}(G, \mathbf{C})$. Make a choice $\overline{\boldsymbol{g}}$ of classical generators for $\pi_{1}\left(U_{\boldsymbol{z}}, z_{0}\right)$ (1.2). Let $T: G \rightarrow S_{n}$ be a permutation representation. Any surjective homomorphism $\psi: \pi_{1}\left(U_{z}, z_{0}\right) \rightarrow G$ produces a degree $\operatorname{deg}(T)$ cover $X_{\psi} \rightarrow \mathbb{P}_{z}^{1}$ canonically. Points of $X_{\psi}$ are homotopy classes of paths $\gamma:[0,1] \rightarrow U_{\boldsymbol{z}}$ with $\gamma(0)=z_{0}$ modulo this relation: $\gamma^{\prime}:[0,1] \rightarrow U_{z}$ and $\gamma$ are equivalent if

(3.13) $\gamma(1)=\gamma^{\prime}(1)$ and $\psi\left(\gamma^{\prime} \gamma^{-1}\right) \in G(T, 1)$.

To be canonical requires the covering space have a base point. For this take the constant path from $z_{0}$.

3.5.1. Interpreting equivalence classes of Galois covers. To avoid running into a base point $z_{0}$, deforming $z$ requires moving $z_{0}$. So the cover is independent of $z_{0}$ only up to inner automorphism. As in 3.1.1 consider any group $N^{\prime}$ between $G$ and $N_{T}(G, \mathbf{C})$. We now explain different Hurwitz spaces as equivalence classes of covers corresponding to points $\boldsymbol{p} \in \mathcal{H}_{N^{\prime}}$. The results we quote in 3.5 .2 and 3.5 .4 are refinements of [FV91] suitable for this paper. Throughout we equivalence only $(G, \mathbf{C})$ covers, with $G$ and $\mathbf{C}$ fixed.

3.5.2. Case: $N^{\prime}=G$ and inner equivalence. The structure on $\mathcal{H}_{G}$ is the moduli space of inner equivalence classes, denoted $\mathcal{H}^{\text {in }}$. Interpret $\boldsymbol{p} \in \mathcal{H}_{G}=\mathcal{H}^{\text {in }}$ as an equivalence class of pairs:

$$
\left(\varphi: X \rightarrow \mathbb{P}_{z}^{1}, G\left(X / \mathbb{P}_{z}^{1}\right) \stackrel{\alpha}{\longrightarrow} G\right)
$$

with $G\left(X / \mathbb{P}_{z}^{1}\right)$ the automorphisms, of a Galois cover and $\alpha$ a group isomorphism. Then, $\left(\varphi^{\prime}: X^{\prime} \rightarrow \mathbb{P}_{z}^{1}, G\left(X^{\prime} / \mathbb{P}_{z}^{1}\right) \stackrel{\alpha^{\prime}}{\longrightarrow} G\right)$ is equivalent to (3.14) if a continuous oneone map $\psi: X \rightarrow X^{\prime}$ induces the latter from the former. Example of an equivalent pair: Let $X=X^{\prime}$ and compose $\alpha$ with conjugation by $g \in G$ to get $\alpha^{\prime}$. Composing $\alpha$, however, with an outer automorphism of $G$ gives a new equivalence class of pairs. 
3.5.3. Further explanation of automorphisms. For $\boldsymbol{g} \in \mathrm{Ni}(G, \mathbf{C}) / G$, automorphisms of $X / \mathbb{P}_{z}^{1}$ identify with the centralizer of $G$ in $N_{R}(G, \mathbf{C})$ (Lem. 3.2) with $R$ the regular representation. A map between two pairs as in (3.14) is unique if and only if $G$ has no center. This is equivalent to there being a unique total family $\mathcal{T}_{G, \mathbf{C}}^{\text {in }}=\mathcal{T}^{\text {in }} \rightarrow \mathcal{H}^{\text {in }} \times \mathbb{P}_{z}^{1}$. For $\boldsymbol{p} \in \mathcal{H}^{\text {in }}$, points $\mathcal{T}_{\boldsymbol{p}}^{\text {in }}$ over $\boldsymbol{p} \times \mathbb{P}_{z}^{1}$ form a Galois cover of $\mathbb{P}_{z}^{1}$ with group $G$ representing $\boldsymbol{p}$. This makes $\mathcal{H}^{\text {in }}$ a fine moduli space. Then, the (minimal) definition field of $\mathcal{T}_{\boldsymbol{p}}^{\text {in }} \rightarrow \mathbb{P}_{z}^{1}$ and the automorphisms given by $G$ is $\mathbb{Q}(\boldsymbol{p})$, generated over $\mathbb{Q}$ by coordinates of $\boldsymbol{p}$ FV91. The total family may exist even if $G$ has a center, though it won't be unique.

Lemma 3.15. Even if $G$ has a center, for any point $\boldsymbol{p} \in \mathcal{H}^{\text {in }}$, there is a cover $\varphi_{\boldsymbol{p}}: Y_{\boldsymbol{p}} \rightarrow \mathbb{P}_{z}^{1}$ with definition field $\mathbb{Q}(\boldsymbol{p})$. It may not, however, be Galois over $\mathbb{Q}(\boldsymbol{p})$.

More generally, suppose $\varphi: Y \rightarrow X$ is a Galois cover of nonsingular projective curves with $X$ over $K$ and $x_{0} \in X(K)$ is unramified in $Y$. Assume $\varphi^{\sigma}: Y^{\sigma} \rightarrow X$ is s-equivalent to $\varphi$ for each $\sigma \in G_{K}$. Then there exists $\varphi^{\prime}: Y^{\prime} \rightarrow X$ over $K$, s-equivalent to $\varphi$ (over $\bar{K}$ ) with a rational point over $x_{0}$.

Proof. A version of this is in CoH85. Consider any Galois cover $\varphi: Y \rightarrow \mathbb{P}_{z}^{1}$ (over some algebraic closure $\bar{K}$ of $K=\mathbb{Q}(\boldsymbol{p})$ ) in the equivalence class of $\boldsymbol{p}$. Then, choose any $y \in Y$ over $z_{0} \in \mathbb{Q}$ unramified in $Y$. From the moduli property, for $\sigma \in G_{K}$, there is an isomorphism $\psi_{\sigma}: Y \rightarrow Y^{\sigma}$ commuting with the maps to $\mathbb{P}_{z}^{1}$. Compose such a map with the unique automorphism of the Galois cover assuring $\psi_{\sigma}$ takes $y$ to $y_{\sigma}$.

Apply Weil's cocycle condition to $(Y, y)$ for an equivalent pair $\left(\varphi^{\prime}: Y^{\prime} \rightarrow \mathbb{P}_{z}^{1}, y^{\prime}\right)$ over $\mathbb{Q}(\boldsymbol{p})$ We56. We may, however, lose the automorphisms: $\varphi^{\prime}: Y^{\prime} \rightarrow \mathbb{P}_{z}^{1}$ defines a cover in $\mathcal{H}_{N_{R}(G, \mathbf{C})}$ where $R$ is the regular representation of $G$. The cover, however, is special, for it has a rational point $y^{\prime}$ over $z_{0}$.

The proof works with a general curve replacing $\mathbb{P}_{z}^{1}$. With $\mathbb{A}^{r} \backslash D_{r}$ replacing $U_{r}$ and $z_{0}=\infty$, this construction works uniformly to give a fine moduli space of geometrically Galois covers with a point over $\infty$.

3.5.4. Absolute equivalence: $N^{\prime}=N_{S_{n}}(G, \mathbf{C})$. For $G \leq N^{\prime} \leq N_{S_{n}}(G, \mathbf{C}), H_{r}$ action on $\operatorname{Ni}(G, \mathbf{C}) / N^{\prime}$ gives $\mathcal{H}_{N^{\prime}}$ (as in 13.1 .1.1). Refer back to $\$ 3.1 .3$ for the notation for Galois closure of a cover. Given $\varphi: X \rightarrow \mathbb{P}_{z}^{1}$, we chose $X^{\dagger}$ to be a geometric Galois closure of $\varphi$. This depended on choosing a coset of $G$ in $N_{S_{n}}(G, \mathbf{C})$. A particular choice determined an isomorphism of $G$ with $\operatorname{Aut}\left(X^{\dagger} / \mathbb{P}_{z}^{1}\right)$.

A point $\boldsymbol{p} \in \mathcal{H}_{N^{\prime}}$ corresponds to a cover $\varphi: X \rightarrow \mathbb{P}^{1}$ up to a choice of $X^{\dagger}$ determined by a coset of $N^{\prime}$ in $N_{S_{n}}(G, \mathbf{C})$. Formally: $\left(\varphi^{\prime}: Y \rightarrow \mathbb{P}_{z}^{1}, G\left(Y^{\dagger} / \mathbb{P}_{z}^{1}\right) \stackrel{\beta}{\longrightarrow} G\right)$ is $N^{\prime}$ - equivalent to the corresponding expression for $\left(X, X^{\dagger}\right)$ if some continuous one-one map $\psi: X \rightarrow Y$ induces the latter up to conjugation of $\beta$ by $N^{\prime}$ from the former. This generalizes inner equivalence, the case $N^{\prime}=G$.

When $N^{\prime}=N_{S_{n}}(G, \mathbf{C})$ denote $\mathcal{H}(G, \mathbf{C})_{N^{\prime}}$ by $\mathcal{H}(G, \mathbf{C})^{\text {abs }}$, absolute equivalence classes of covers $X \rightarrow \mathbb{P}_{z}^{1}$ with associated permutation representation $T$. From 3.1 .3 this requires no choice in $X^{\dagger}$. So, two covers are $N_{S_{n}}(G, \mathbf{C})$-equivalent if there is a map between them commuting with their maps to $\mathbb{P}_{z}^{1}$.

Assume $G$ has no center, so $\mathcal{H}^{\text {in }}(G, \mathbf{C})$ has a unique total representing family. Then, $H_{r}$ acting on $\mathrm{Ni} / G$ and $\mathrm{Ni} / N^{\prime}$ produces $\Psi_{N^{\prime}}^{\text {in }}: \mathcal{H}^{\text {in }}(G, \mathbf{C})=\mathcal{H}^{\text {in }} \rightarrow \mathcal{H}_{N^{\prime}}$. Rem. 3.2 .3 gives the cyclotomic field $K_{G, \mathbf{C}, N^{\prime}}\left(\right.$ resp. $\left.K_{G, \mathbf{C}}\right)$ as the definition field of $\mathcal{H}_{N^{\prime}}\left(\operatorname{resp} . \mathcal{H}^{\text {in }}\right.$ and $\left.\Psi_{N^{\prime}}^{\text {in }}\right)$. 
If $\mathbf{C}$ is a rational union of conjugacy classes, this field is $\mathbb{Q}$. The following interprets the main technical result of [FV91. Recall previous notation for the fibers of a family $\Phi: \mathcal{T} \rightarrow \mathcal{H} \times \mathbb{P}_{z}^{1}$ (or $\operatorname{pr}_{2} \circ \Phi: \mathcal{T} \rightarrow \mathcal{H}$ ). If $\boldsymbol{p} \in \mathcal{H}$, then $\mathcal{T}_{\boldsymbol{p}}$ is the set of points of $\mathcal{T}$ over $\boldsymbol{p} \times \mathbb{P}_{z}^{1}$ and $\varphi_{\boldsymbol{p}}: \mathcal{T}_{\boldsymbol{p}} \rightarrow \mathbb{P}_{z}^{1}$ is restriction of $\Phi$.

TheOREM 3.16. Suppose $G$ has no nontrivial centralizer in $N^{\prime}$. Then there are total representing families $\Phi_{N^{\prime}}: \mathcal{T}_{N^{\prime}} \rightarrow \mathcal{H}_{N^{\prime}} \times \mathbb{P}^{1}$ and $\Phi^{\text {in }}: \mathcal{T}^{\text {in }} \rightarrow \mathcal{H}^{\text {in }} \times \mathbb{P}^{1}$. For $\boldsymbol{p} \in \mathcal{H}_{N^{\prime}}$ and $\hat{\boldsymbol{p}} \in \mathcal{H}^{\text {in }}$ over $\boldsymbol{p}$, the covers $\varphi_{\hat{\boldsymbol{p}}}^{\text {in }}: \mathcal{T}_{\hat{\boldsymbol{p}}}^{\text {in }} \rightarrow \mathbb{P}_{z}^{1}$ and $\varphi_{N^{\prime}, \boldsymbol{p}}: \mathcal{T}_{N^{\prime}, \boldsymbol{p}} \rightarrow \mathbb{P}_{z}^{1}$ corresponding to these points have the following properties.

$(3.15 \mathrm{a}) \varphi_{\hat{\boldsymbol{p}}}^{\text {in }}\left(\right.$ resp. $\left.\varphi_{N^{\prime}, \boldsymbol{p}}\right)$ has field of definition $K_{G, \mathbf{C}}(\hat{\boldsymbol{p}})$ (resp. $\left.K_{G, \mathbf{C}, N^{\prime}}(\boldsymbol{p})\right)$.

(3.15b) $\varphi_{\hat{\boldsymbol{p}}}^{\text {in }}$ is an absolutely irreducible component of a $K_{G, \mathbf{C}, N^{\prime}}(\boldsymbol{p})$ (arithmetic) component of the Galois closure of $\varphi_{N^{\prime}, p}$ (via the 3.1 .3 construction).

(3.15c) $K_{G, \mathbf{C}}(\hat{\boldsymbol{p}}) / K_{G, \mathbf{C}, N^{\prime}}(\boldsymbol{p})$ is a Galois extension with group naturally isomorphic to a subgroup of $N^{\prime} / G$.

EXAMPLE 3.17 ( $A_{n}$ and 3-cycles). Let $T_{n}$ be the (standard) representation of $A_{n}, n \geq 5$. [Fri96 Thm. 1] lists the complete set of inner and absolute Hurwitz space components at level 0 of the $\left(A_{n}, \mathbf{C}_{3^{r}}\right)$ Modular Tower. Table 3 displays these for inner spaces (the result is nontrivially the same for absolute spaces). All these components have definition field $\mathbb{Q}$. Locations in this diagram have an attached integer pair $(n, r)$. In each case the $\oplus$ inner component $\mathcal{H}_{n, r}^{\text {in, } \oplus}$ maps to the absolute $\oplus$ component $\mathcal{H}_{n, r}^{\text {abs, }} \oplus$ by a degree 2 (Galois) map with group identified with $S_{n} / A_{n}$. Similarly for the corresponding $\otimes$ components. In Table 3 the notation for components corresponds to lifting invariant values as in Prop. [5.9 (or $\mathrm{C}$, specifically in (C.1的). The genus at $(n, r)$ of a degree $n$ cover is $g=r-n+1$.

TABle 3 . Constellation of spaces $\mathcal{H}\left(A_{n}, \mathbf{C}_{3^{r}}\right)$

\begin{tabular}{|c|c|c|c|c|c|c|}
\hline$\stackrel{g \geq 1}{\longrightarrow}$ & $\otimes \oplus$ & $\otimes \oplus$ & $\ldots$ & $\otimes \oplus$ & $\otimes \oplus$ & $\stackrel{1 \leq g}{\longleftarrow}$ \\
\hline$\stackrel{g=0}{\longrightarrow}$ & $\otimes$ & $\oplus$ & $\ldots$ & $\otimes$ & $\oplus$ & $\stackrel{0=g}{\longleftarrow}$ \\
\hline$n \geq 4$ & $n=4$ & $n=5$ & $\ldots$ & $n$ even & $n$ odd & $4 \leq n$ \\
\hline
\end{tabular}

3.6. $p$-perfect groups and fine moduli. Start with any equivalence between covers of $\mathbb{P}_{z}^{1}$. The Hurwitz space representing these equivalences is a fine moduli space if it has a unique total family representing the equivalence classes of its points. Prop. 3.21 considers only Hurwitz spaces of type $\mathcal{H}(G, \mathbf{C})^{\text {abs }}$ or $\mathcal{H}(G, \mathbf{C})^{\text {in }}$.

3.6.1. p-perfect groups. For any $G$ module $A$, consider a group extension $\varphi$ : $\hat{G} \rightarrow G$ with $\operatorname{ker}(\varphi)=A$ and the lifted conjugation action of $G$ on $A$ is that given. Then, $H^{1}(G, A)$ corresponds to automorphisms of $\hat{G}$ trivial on $G$ and $A$ modulo automorphisms induced by conjugation by $A$ Nor62 p. 239]. Suppose $A=\mathbb{Z} / p$, with $G$ acting trivially. Then, $H^{1}(G, A)$ is just the homomorphisms of $G$ into $A$.

Definition 3.18 ( $p$-perfect groups). For $p$ a prime, a group $G$ is $p$-perfect if it has no $\mathbb{Z} / p$ quotient. That is, $H^{1}(G, \mathbb{Z} / p)$ is trivial.

Let $U_{p}$ be the $p$ part of the Schur multiplier of $G$ (this may be trivial). That $G$ is $p$-perfect interprets as $G$ having a central extension ${ }_{p} \hat{G}$ with this property. 
$U_{p}=\operatorname{ker}\left({ }_{p} \hat{G} \rightarrow G\right)$ and ${ }_{p} \hat{G}$ is universal for central extensions of $G$ with $p$-group kernel.

Prop. 9.17 illustrates the necessity of this condition.

Any finite group has a centerless cover FV91. No cover, however, of $G$ can be $p$-perfect, unless $G$ is. Here is another characterization of $p$-perfect.

Lemma 3.19. The $p^{\prime}$ elements in $G$ generate if and only $G$ is p-perfect.

Proof. Let $H$ be the (normal) subgroup of $G$ generated by its $p^{\prime}$ elements. If $H$ is a proper subgroup of $G$, then $G / H$ is a nontrivial $p$-group, and any $p$-group has a $\mathbb{Z} / p$ quotient. Conversely, given $\varphi: G \rightarrow \mathbb{Z} / p$, the kernel of $\varphi$ contains all the $p^{\prime}$ elements of $G$.

Note: Perfect groups are exactly those $G$ that are $p$-perfect for every prime dividing $|G|$. Let $\left\{G_{k}\right\}_{k=0}^{\infty}$ be the characteristic quotients of ${ }_{p} \tilde{G}$, the universal $p$ Frattini cover of $G_{0}=G$ and $M_{k}=\operatorname{ker}\left(G_{k+1} \rightarrow G_{k}\right)$ as in (3.8). Prop. 3.21 uses notation from the Loewy display of $M_{k}$ as a $G_{k}$ module (55.2.2).

EXAMPLE 3.20 (Centers in quotients of $p$-perfect centerless group). Suppose $G_{0}$ is $p$-perfect, centerless, and has a nontrivial $p$ part in its Schur multiplier. Let $G_{1}$ be the first characteristic Frattini cover of $G_{0}$ as in (3.8id). Then the canonical map $G_{1} \rightarrow G_{0}$ factors through a nontrivial central extension of $G_{0}$.

Further, this automatically replicates at all levels. For all $k, G_{k}$ is $p$-perfect and centerless (Prop. 3.21) and $G_{k+1} \rightarrow G_{k}$ factors through a nontrivial central extension of $G_{k}$ (Prop. 9.6). Subexample: The universal exponent 2-Frattini extension $G_{1}$ of $A_{n}=G_{0}$ factors through the spin cover of $\hat{A}_{n}$.

3.6.2. Fine moduli for a Modular Tower. The next proposition is a characterization for all levels of a Modular Tower having fine moduli.

Proposition 3.21. Suppose a Hurwitz space is of type $\mathcal{H}(G, \mathbf{C})^{\text {abs }}$ with associated permutation representation $T$. It is a fine moduli space if $T: G \rightarrow S_{n}$ has image with no centralizer in $S_{n}$ (see 3.8.2). A Hurwitz space of type $\mathcal{H}(G, \mathbf{C})^{\mathrm{in}}$ is a fine moduli space if $G$ has no center.

Assume $G_{0}=G$ is a centerless p-perfect group. Then, for each $k$, so is $G_{k}$ : $\mathbf{1}_{G_{k}}$ does not appear at the far left of the Loewy display of $M_{k}$. Let $\mathbf{C}$ be a set of $p^{\prime}$ classes of $G$. Then, the Hurwitz spaces $\left\{\mathcal{H}\left(G_{k}, \mathbf{C}\right)^{\mathrm{in}}\right\}_{k=0}^{\infty}$ are all fine moduli spaces.

Proof. The first part is a subset of Thm. 3.16. Assume $G_{0}=G$ is centerless and $p$-perfect. We inductively show $G_{k}$ also has these properties for all $k$.

[Fri95a Lem. 3.6] shows $G_{k+1}$ is centerless if the following hold.

(3.17a) $G_{k}$ has no center.

(3.17b) $\operatorname{ker}_{k} / \operatorname{ker}_{k+1}$ has no $G_{k}$ subquotient of Loewy type $\mathbf{1} \rightarrow \mathbf{1}$.

The module in 3.17 ) is distinct from $\mathbf{1} \oplus \mathbf{1}$. It comes from a nontrivial representation of $G_{k}$ of form: $g \in G \mapsto\left(\begin{array}{cc}1 & a_{g} \\ 0 & 1\end{array}\right)$. The map $g \in G_{k} \mapsto a_{g}$ is a homomorphism of $G_{k}$ into the $\mathbb{Z} / p$. By hypothesis this doesn't exist.

That leaves showing $G_{k+1}$ has no quotient isomorphic to $\mathbb{Z} / p$, assuming $G_{k}$ is centerless and has no such quotient. Suppose $\varphi: G_{k+1} \rightarrow \mathbb{Z} / p$ is surjective with kernel $K$. Consider the map from $K$ to $G_{k}$ induced by the canonical map $G_{k+1} \rightarrow G_{k}$. This is a Frattini cover. So, $K$ is not onto $G_{k}$. Then, $K$ has image an index $p$ normal subgroup of $G_{k}$. This is contrary to our assumptions. 
Finally, since $G_{k}$ is centerless, $G_{k+1}$ has a center if and only if $G_{k}$ stabilizes some nontrivial element of $M_{k}$; if and only if $\mathbf{1}_{G_{k}}$ appears to the far left in the Loewy display of $M_{k}$ ).

3.6.3. Ordering branch points. We comment on a Hurwitz space topic that arises in DF90b. Let $H$ be a subgroup of $S_{r}$. Use the diagram of (2.6) for $\Psi_{r}:\left(\mathbb{P}^{1}\right)^{r} \backslash \Delta_{r} \rightarrow \mathbb{P}^{r} \backslash D_{r}$. So, $H$ defines a quotient $\left.U^{H}=\left(\mathbb{P}_{z}^{1}\right)^{r} \backslash \Delta_{r}\right) / H$ : $\Psi_{H}: U^{r} \rightarrow U^{H}$ is then the canonical map. A component $\mathcal{H}_{1}$ of a Hurwitz space $\mathcal{H}$ has an $H$-ordering on its branch points if $\Psi: \mathcal{H}^{\prime} \rightarrow \mathbb{P}^{r} \backslash D_{r}$ factors through $U^{H}$. Up to whatever equivalence defines the moduli problem for $\mathcal{H}$, this means for any cover $\varphi: \boldsymbol{p}: X_{\boldsymbol{p}} \rightarrow \mathbb{P}_{z}^{1}$ representing the equivalence class of $\boldsymbol{p} \in \mathcal{H}^{\prime}$, the effect of $G_{\mathbb{Q} \boldsymbol{p}}$ on orderings $\left(z_{1}, \ldots, z_{r}\right)$ of the branch points $z_{\boldsymbol{p}}$ of $\varphi_{\boldsymbol{p}}$ is conjugate to a subgroup of $H$. The notion depends only on the conjugacy class of $H$ in $S_{r}$. This topic arises naturally in considering the Branch Cycle Lemma (see \$3.2.3).

Definition 3.22. For $H \leq S_{r}$, and $\mathcal{H}_{1}$ a component of a Hurwitz space $\mathcal{H}$, let $\mathcal{H}^{\prime}$ be a component of the fiber product $\mathcal{H}_{1} \times_{\mathbb{P}^{r}} U^{H}$. Such an $\mathcal{H}^{\prime}$ is an $H$-ordering (of the branch points) of $\mathcal{H}_{1}$. When $H=\{1\}$ this is the traditional meaning of an ordering the branch points.

There is a simple Nielsen class interpretation for $\mathcal{H}_{1}$ having an $H$-ordering. Let $O$ be the $H_{r}$ orbit on $\mathrm{Ni}(G, \mathbf{C}) / N$ (as in 3.1.1) corresponding to $\mathcal{H}_{1}$. We also say, an $H$-ordering of $O$. Recall $\Psi_{r}^{*}: H_{r} \rightarrow S_{r}$ from $\$ 2.2$

LEMMA 3.23. For $\boldsymbol{g} \in O$, let $H_{\boldsymbol{g}}$ be the subgroup of $H_{r}$ stabilizing the equivalence class of $\boldsymbol{g}$. Then, there is an $H$-ordering of $O$ if and only if some conjugate of $\Psi_{r}^{*}\left(H_{\boldsymbol{g}}\right) \leq S_{r}$ is in $H$.

Proof. Suppose $\mathcal{H}_{1} \rightarrow U_{r}$ factors through $U^{r} / H \rightarrow U_{r}$. Then a point $\boldsymbol{p} \in \mathcal{H}_{1}$ has image $\boldsymbol{u} \in U^{r} / H$. The (geometric) decomposition group for $\boldsymbol{u}$ in the cover $U^{r} \rightarrow U^{r} / H$ is a subgroup of $H$. It must contain the image $\Psi_{r}^{*}\left(H_{\boldsymbol{g}}\right)$ since $H_{\boldsymbol{g}}$ is a subgroup of the fundamental group of $U^{r} / H$. The argument is reversible.

ExAmple 3.24 (Pairs of conjugacy classes). Suppose $r=4, \mathrm{C}_{1}=\mathrm{C}_{2}, \mathrm{C}_{3}=\mathrm{C}_{4}$, $N=G$ and $\mathrm{C}_{1} \neq \mathrm{C}_{3}$. Then, the minimal (up to conjugacy in $S_{4}$ ) group for which there is an $H$-ordering of an $H_{4}$ orbit on $\mathrm{Ni}(G, \mathbf{C})^{\text {in }}$ is $H=\langle(12),(34)\rangle$. If, however, $\mathrm{C}_{1}$ and $\mathrm{C}_{2}$ are conjugate in a group $N$ between $G$ and $N_{S_{n}}(G)$, then the minimal $H^{\prime}$-ordering of an $H_{4}$ orbit on $\mathrm{Ni}(G, \mathbf{C}) / N$ is $H^{\prime}=\langle(12),(34),(13)(24)\rangle$.

3.7. $\mathrm{SL}_{2}(\mathbb{C})$ action on Hurwitz spaces and covers of $\Lambda_{r}$ and $J_{r}$. Use the notation from $\$ 2.1 .3$ Denote $\left(\mathbb{P}^{1}\right)^{r} \backslash \Delta_{r}$ by $U^{r}$ and $\mathbb{P}^{r} \backslash D_{r}$ by $U_{r}$. Let $N^{\prime}$ be one of the groups from 3.5.1 Action of $H_{r}$ on $\mathrm{Ni}(G, \mathbf{C}) / N^{\prime}$ produces an unramified cover $\Psi_{G, \mathbf{C}}: \mathcal{H}(G, \mathbf{C}) \rightarrow U_{r}(2.3)$. Pull this cover back to $U^{r}=\left(\mathbb{P}^{1}\right)^{r} \backslash \Delta_{r}$ as the fiber product $\mathcal{H}(G, \mathbf{C})^{\prime}=\mathcal{H}(G, \mathbf{C}) \times_{U_{r}} U^{r}$.

3.7.1. Quotient by $\mathrm{PSL}_{2}(\mathbb{C})$. Consider $\mathrm{PSL}_{2}(\mathbb{C})$ acting diagonally on $r$ copies of $\mathbb{P}_{z}^{1}$. For $\alpha \in \mathrm{PSL}_{2}(\mathbb{C})$ and $\boldsymbol{z} \in U^{r}, \alpha(\boldsymbol{z}) \mapsto\left(\alpha\left(z_{1}\right), \ldots, \alpha\left(z_{r}\right)\right)$. This action commutes with $S_{r}$ permuting coordinates; put $\mathrm{PSL}_{2}(\mathbb{C})$ on the left. So, $\mathrm{PSL}_{2}(\mathbb{C}) \backslash U^{r}=\Lambda_{r}$ generalizes the $\lambda$-line minus $\{0,1, \infty\}$ from modular curves. Also, $\mathrm{PSL}_{2}(\mathbb{C}) \backslash U_{r}=J_{r}$ generalizes the $j$-line minus $\infty$. Both spaces have complex dimension $r-3$.

LEMMA 3.25. For any strong equivalence of covers (from 3.5.1 including absolute or inner equivalence), composing $\varphi: X \rightarrow \mathbb{P}_{z}^{1}$ with $\alpha \in \mathrm{PSL}_{2}(\mathbb{C})$ preserves 
the Nielsen class. Further, the equivalence classes of $\varphi$ and $\alpha \circ \varphi$ lie in the same component of the corresponding Hurwitz space.

Proof. A Nielsen class is given by $(G, \mathbf{C}, T)$ with $T: G \rightarrow S_{n}$ a faithful permutation representation. The equivalence depends on some subgroup of $S_{n}$, containing $G$ and normalizing it. A cover in the Nielsen class is of degree $n$ and has $T$ the natural permutation representation associated to it. When, it is an inner class, we also attach an isomorphism between the group of the cover and the group $G$. The monodromy groups of the covers $\varphi$ and $\alpha \circ \varphi$ are exactly the same. If $z$ are the branch points for $\varphi$, then $\alpha(\boldsymbol{z})$ are the branch points of $\alpha \circ \varphi$, with $\alpha\left(z_{i}\right)$ having the conjugacy class $\mathrm{C}_{i}$ attached to it. This shows $\alpha$ preserves Nielsen classes.

The action of $\alpha \in \mathrm{PSL}_{2}(\mathbb{C})$ mapping on s-equivalence classes of covers in a Nielsen class is continuous. Given $\varphi: X \rightarrow \mathbb{P}_{z}^{1}$, map $\alpha \in \mathrm{PSL}_{2}(\mathbb{C})$ to the sequivalence class of $\alpha \circ \varphi$. Since $\mathrm{PSL}_{2}(\mathbb{C})$ is connected, the $\mathrm{PSL}_{2}(\mathbb{C})$ orbit of $\varphi$ lies in one connected component of the Hurwitz space. The orbit contains $\varphi$; the component is that of $\varphi$.

Definition 3.26 (Reduced Hurwitz spaces). DF99 §6.2] shows $\mathrm{PSL}_{2}(\mathbb{C})$ action extends to $\mathcal{H}_{N^{\prime}}$ and to $\mathcal{H}^{\text {in }}$. This produces affine schemes $\mathcal{H}_{N^{\prime}} / \mathrm{PSL}_{2}(\mathbb{C})$ and $\mathcal{H}^{\text {in }} / \mathrm{PSL}_{2}(\mathbb{C})$ covering (usually ramified) $J_{r}$. These are reduced Hurwitz spaces.

3.7.2. The $j$ and $\lambda$-lines. Take $r=4$. Four unordered distinct points, $z \in U_{4}$, are the branch points of a unique degree two cover $E_{\boldsymbol{z}} \rightarrow \mathbb{P}_{z}^{1}$. With the right choice of inhomogeneous coordinate on $J_{4}$, the image of $z$ in $J_{4}$ is the classical elliptic curve $j$-invariant. Take the elliptic curve to be degree 0 divisor classes on $E_{\boldsymbol{z}}: \operatorname{Pic}^{0}\left(E_{\boldsymbol{z}}\right)$. Identify $J_{4}$ with $\mathbb{P}_{j}^{1} \backslash\{\infty\}$.

Suppose $r=4$ and $\psi^{\text {rd }}: \mathcal{H}^{\text {rd }} \rightarrow \mathbb{P}_{j}^{1} \backslash\{\infty\}$ is a reduced Hurwitz space cover. Assume also that a general point $\boldsymbol{p} \in \mathcal{H}^{\text {rd }}$ corresponds to the equivalence class of a cover $X_{p} \rightarrow \mathbb{P}_{z}^{1}$ whose Galois closure maps surjectively to the elliptic curve with invariant $j=\psi^{\text {rd }}(\boldsymbol{p})$. We say $\mathcal{H}^{\text {rd }}$ is $j$-aware. Many Hurwitz spaces are $j$-aware.

EXAmple 3.27 (Branch cycles in $S_{n} \backslash A_{n}$ and $j$-awareness). Assume each class in $\mathbf{C}$ is in $S_{n} \backslash A_{n}$. Let $\boldsymbol{p} \in \mathcal{H}(G, \mathbf{C})^{\text {in,rd }}$ lie over $\boldsymbol{z}$. The regular representation of $G$ gives a map $G \rightarrow S_{|G|}$. The cover $\varphi_{\boldsymbol{p}}: X_{\boldsymbol{p}} \rightarrow \mathbb{P}_{z}^{1}$ naturally factors through $E_{z} \rightarrow \mathbb{P}_{z}^{1}$ : Quotient $X_{\boldsymbol{p}}$ by $G \cap A_{|G|}$. (This works for any even $r ; E_{\boldsymbol{z}}$ is then hyperelliptic.)

Suppose $r=4$ and $\boldsymbol{g} \in \operatorname{Ni}(G, \mathbf{C})$ with $G \leq A_{n}$. Choose $h_{1}, h_{2} \in S_{n} \backslash A_{n}$. Then, $\left(h_{1} g_{1}, g_{2} h_{2}, h_{2}^{-1} g_{3}, g_{4} h_{1}^{-1}\right)$ satisfies the product-one condition. It produces a Nielsen class (for some new group) with moduli problem directly recognizing the $j$-line as parameterizing elliptic curves.

In summary we have the following DF99 Prop. 6.3]. Further remarks on $j$-awareness appear in $\underline{\mathrm{E}}$

Proposition 3.28. The (unramified) cover $\Psi_{4}: U^{4} \rightarrow U_{4}$ modulo $\mathrm{PSL}_{2}(\mathbb{C})$ produces the classical (ramified) map $\psi: \mathbb{P}_{\lambda}^{1} \backslash\{0,1, \infty\} \rightarrow \mathbb{P}_{j}^{1} \backslash\{\infty\}$. This extends to a (ramified) cover $\mathcal{H}_{N^{\prime}} / \mathrm{PSL}_{2}(\mathbb{C}) \rightarrow \mathbb{P}_{j}^{1} \backslash\{\infty\}$.

Prop. 4.4 gives the precise action of $\bar{M}_{4}$ on reduced Nielsen classes. This produces a branch cycle description of the cover $\mathcal{H}_{N^{\prime}} / \mathrm{PSL}_{2}(\mathbb{C}) \rightarrow \mathbb{P}_{j}^{1} \backslash\{\infty\}$. Many computations of this paper depend on this.

Similar to 3.6.3 Let $H$ be a subgroup of $S_{3}$ (the group of the Galois cover $\left.\mathbb{P}_{\lambda}^{1} \rightarrow \mathbb{P}_{j}^{1}\right)$. A component $\mathcal{H}_{1}^{\text {rd }}$ of a reduced Hurwitz space $\mathcal{H}^{\text {rd }}$ has an $H$-ordering on 
its branch points if $\Psi: \mathcal{H}_{1}^{\text {rd }} \rightarrow \mathbb{P}_{j}^{1} \backslash\{\infty\}$ factors through $\mathbb{P}_{\lambda}^{1} / H$. Up to the reduced equivalence defining the moduli problem for $\mathcal{H}^{\text {rd }}$, for any cover $\varphi: p: X_{p} \rightarrow \mathbb{P}_{z}^{1}$ representing the reduced equivalence class of $\boldsymbol{p} \in \mathcal{H}^{\prime}$, the effect of $G_{\mathbb{Q}(\boldsymbol{p})}$ on the orderings $\left(z_{1}, z_{2}, z_{3}, z_{4}\right)$, of $\boldsymbol{z}_{\boldsymbol{p}}$ of $\varphi_{\boldsymbol{p}}$ is conjugate to a subgroup of $H$. $\mathrm{E} .2 .1$ is an application of the next lemma.

Lemma 3.29. Suppose $H \leq S_{4}$, and for $r=4$ a component $\mathcal{H}_{1}$ of a Hurwitz space $\mathcal{H}$ has an $H$-ordering (as in 3.6.3) of its branch points. Then, the corresponding reduced Hurwitz space component $\mathcal{H}_{1}^{\text {rd }}$ has an $H /\left(H \cap K_{4}\right)$ ordering of its branch points. Particularly, if $H \leq K_{4}$, then $\mathcal{H}_{1}^{\text {rd }} \rightarrow \mathbb{P}_{j}^{1} \backslash\{\infty\}$ factors through the natural map $\mathbb{P}_{\lambda}^{1} \rightarrow \mathbb{P}_{j}^{1}$.

Proof. The argument of 2.3 .2 shows how to identify the group of the cover $\mathbb{P}_{\lambda}^{1} \rightarrow \mathbb{P}_{j}^{1}$ with the action of $S_{4}$ on the points of $\boldsymbol{z}$ modulo a Klein 4-group.

EXAMPLE 3.30 (Pairs of conjugacy classes continued). As in Ex. 3.24 assume $r=4, \mathrm{C}_{1}=\mathrm{C}_{2}, \mathrm{C}_{3}=\mathrm{C}_{4}, N=G, \mathrm{C}_{1} \neq \mathrm{C}_{3}$, and $\mathrm{C}_{1}$ and $\mathrm{C}_{2}$ are conjugate in a group $N$ between $G$ and $N_{S_{n}}(G)$. Then, the minimal groups with the reduced Nielsen classes $\operatorname{Ni}(G, \mathbf{C})^{\mathrm{in}, \mathrm{rd}}$ and $\operatorname{Ni}(G, \mathbf{C})^{\mathrm{rd}} / N$ having an ordering of the branch points are the same:

$\langle(12),(34)\rangle /\langle(12),(34)\rangle \cap K_{4}=\langle(12),(34),(13)(24)\rangle /\langle(12),(34),(13)(24)\rangle \cap K_{4}$.

3.8. Monodromy groups of $\psi: \mathcal{H} \rightarrow U_{r}$ and $\psi^{\text {rd }}: \mathcal{H}^{\text {rd }} \rightarrow J_{r}$. Let $\mathcal{H}^{\text {in }}$ (resp. $\mathcal{H}^{\text {in,rd }}$ ) be a component of a Hurwitz (resp. reduced Hurwitz) space covering $U_{r}$ (resp. $J_{r}$ ). The geometric monodromy (Galois closure) groups $G_{\psi} \leq S_{\operatorname{deg} \psi}$ and $G_{\psi^{\mathrm{rd}}} \leq S_{\operatorname{deg} \psi^{\mathrm{rd}}}$ of these covers are invariants of the component (and of the Nielsen class) describing this cover.

We call attention to delicate points useful outside the area of this paper for investigating rational points on these spaces. Example: Formula (3.20) for how complex conjugation acts on the branch cycle description of a reduced Hurwitz space may not determine $c$. Recognition, however, of how to locate one H-M rep. gives the correct determination.

3.8.1. Paths for computing complex conjugation. Apply complex conjugation directly to paths representing $q_{1}, \ldots, q_{r-1}$, the braid generators of $H_{r}$. For example, suppose $\boldsymbol{z}$ consists of real points $z_{1}<z_{2}<\cdots<z_{r} \in \mathbb{P}_{z}^{1}(\mathbb{R})$ arranged around the real circle. As in [Fri95a App. C and App. D] let $B_{i}$ be a clockwise circle on $\mathbb{P}_{z}^{1}$ with a marked diameter on the real axis having $z_{i}$ and $z_{i+1}$ as endpoints. (One of these has $z_{r}$ and $z_{1}$ at the endpoints of the directed diameter.) Parametrize the top of $B_{i}$ with $t \mapsto B_{i}^{+}(t)$ on $[0,1]$, so $B_{i}^{+}(0)=z_{i}$ and $B_{i}^{+}(1)=z_{i+1}$. Similarly, parametrize the bottom of $B_{i}$ with $t \mapsto B_{i}^{-}(t)$ on $[0,1]$ so $B_{i}^{-}(0)=z_{i+1}$ and $B_{i}^{-}(1)=z_{i}$. Consider the path

$$
t \mapsto\left(z_{1}, \ldots, z_{i-1}, B_{i}^{+}(t), B_{i}^{-}(t), z_{i+2}, \ldots, z_{r}\right), t \in[0,1] .
$$

The range of (3.18) in $\mathbb{P}^{r} \backslash D_{r}$ represents the braid group generator $q_{i}^{-1}$. The inverse of path (3.18) is

$$
t \mapsto\left(z_{1}, \ldots, z_{i-1}, \bar{B}_{i}^{-}(t), \bar{B}_{i}^{+}(t), z_{i+2}, \ldots, z_{r}\right), t \in[0,1]
$$

where the notation $\bar{B}$ indicates complex conjugation applied to the coordinate. 
Apply this when $r=4$ to compute complex conjugation on the branch cycles for reduced Hurwitz covers of the $j$-line. Use

$$
\gamma_{0}=q_{1} q_{2}, \gamma_{1}=q_{1} q_{2} q_{1}, \gamma_{\infty}=q_{2}
$$

for paths in $\mathbb{P}_{j}^{1} \backslash\{0,1, \infty\}$, images by $\mathrm{PSL}_{2}(\mathbb{C})$ reduction from the paths above.

Lemma 3.31. Let $r=4$ and $j_{0} \in \mathbb{P}_{j}^{1}(\mathbb{R})$ on the interval $(1, \infty)$. Assume $\bar{\psi}$ : $\overline{\mathcal{H}}^{\text {rd }} \rightarrow \mathbb{P}_{j}^{1}$ is the cover from an absolutely irreducible component of a reduced Hurwitz with $\bar{\psi}$ over $\mathbb{R}$. Let $G_{\bar{\psi}}$ be its geometric monodromy group and take $N=\operatorname{deg}(\bar{\psi})$. Then, an involution $c \in N_{S_{N}}\left(G_{\bar{\psi}}\right)$ gives the effect of complex conjugation on the points of $\overline{\mathcal{H}}^{\text {rd }}$ lying over $j_{0}$.

Suppose $\left(\gamma_{0}^{\prime}, \gamma_{1}^{\prime}, \gamma_{\infty}^{\prime}\right)$ are the branch cycles from Prop. 4.4 from the action on reduced Nielsen classes. Then,

$$
c \gamma_{1} c=\gamma_{1} \text { and } c \gamma_{\infty} c=\gamma_{\infty}^{-1} .
$$

Proof. The hypotheses are for the situation of a 3-branch point real cover of the sphere. The element $c$ will be independent of the value of $j_{0} \in(1, \infty)$. Since $j_{0}$ is the image of $\boldsymbol{z}$ with $z_{1}<z_{2}<\cdots<z_{r} \in \mathbb{P}_{z}^{1}(\mathbb{R})$, Lem. 6.5 says $j_{0} \in(1, \infty)$. The formula for computing complex conjugation is the special case of Prop. 6.3 where $r=3$ and all branch points are real.

The effect of complex conjugation on the $q_{i}$ s take them to their inverse. This induces the effect of taking $q_{1} q_{2} q_{1}$ to its inverse $q_{1}^{-1} q_{2}^{-1} q_{1}^{-1}$. The effect, however, of this image element on reduced Nielsen classes is an element of order 2. Since the permutation effect of complex conjugation is to conjugate by an involution $c$, this gives the formula $c \gamma_{1} c=\gamma_{1}$. Similarly, $c \gamma_{\infty} c=\gamma_{\infty}^{-1}$.

3.8.2. Elements of $N_{S_{\operatorname{deg}\left(\psi^{\mathrm{rd}}\right)}}\left(G_{\psi^{\mathrm{rd}}}\right)$ that centralize $G_{\psi^{\mathrm{rd}}}$. Suppose $g_{1}, \ldots, g_{r}$ are generators of a group $G$, and $c \in G \leq S_{n}$ is an involution. Let $\operatorname{Cen}_{S_{n}}(G)$ be the centralizer of $G$ in $S_{n}$.

PRINCIPLE 3.32 (Centralizer Principle). If $\mathrm{Cen}_{S_{n}}(G)$ contains no involutions, then conjugation by $c$ on $g_{1}, \ldots, g_{r}$ determines it. Assume $G$ is transitive. Then, with no assumptions on $\operatorname{Cen}_{S_{n}}(G)$, if $c$ fixes 1 , then its conjugation effect on $g_{1}, \ldots, g_{r}$ determines $c$.

Suppose a (n irreducible) cover $\varphi: X \rightarrow Y$ over a field $K$ with $n=\operatorname{deg}(\varphi)$ has monodromy group $G_{*} \leq S_{n}$ over $K$. Then, the group $\operatorname{Aut}(\varphi)_{K}$ of $K$ automorphisms of $X$ commuting with $\varphi$ identifies with $\operatorname{Cen}_{S_{n}}\left(G_{*}\right)$.

Proof. Suppose $\operatorname{Cen}_{S_{n}}(G)$ contains no involutions. Let $c, c^{\prime}$ be two involutions with the same conjugation effect on $g_{1}, \ldots, g_{r}$. Then $c c^{\prime}$ is an involution that centralizes $G$, and so it is trivial.

Now assume nothing about $\operatorname{Cen}_{S_{n}}(G)$, that $c$ and $c^{\prime}$ have the same effect on $g_{1}, \ldots, g_{r}$, both fix 1 and $\mathrm{G}$ is transitive. Then, $c c^{\prime} \stackrel{\text { def }}{=} c^{*} \in \operatorname{Cen}_{S_{n}}(G)$ fixes 1 . From transitivity, for any $i \in\{1, \ldots, n\}$, there is $g \in G$ with $(1) g=i$. Conclude: $(i) c^{*}=(1) g c^{*}=(1) c^{*} g=(1) g=i$, and $c^{*}$ is the identify.

The statement on Aut $(\varphi)_{K}$ comes from identifying it with $N_{G_{*}}\left(G_{*}(1)\right) / G_{*}(1)$. List the right cosets $G_{*}(1) g_{1}, \ldots, G_{*}(1) g_{n}$ of $G_{*}(1)$ in $G_{*}$. Elements of $G_{*}$ that permute these by left multiplication on these cosets are in $N_{G_{*}}\left(G_{*}(1)\right)$. Those acting trivially are in $G_{*}(1)$. Left action commutes with the right action of $G_{*}$, thus producing elements in $\operatorname{Cen}_{S_{n}}\left(G_{*}\right)$. [Fri77, Lem. 2.1] has complete details. 
3.8.3. Outer automorphisms of $G$ and computing $c$ using (3.20). 6.4 uses (3.20) to detect all real points on $\mathcal{H}\left(A_{5}, \mathbf{C}_{3^{4}}\right)^{\text {abs,rd }}$ and $\mathcal{H}\left(A_{5}, \mathbf{C}_{3^{4}}\right)^{\text {in,rd }}=\mathcal{H}_{0}^{\text {in,rd }}$. Then, 8.6 does the same for the space $\mathcal{H}\left(G_{1}, \mathbf{C}_{3^{4}}\right)^{\text {in, rd }}$ which is level 1 of the reduced Modular Tower for $\left(A_{5}, \mathbf{C}_{3^{4}}\right)$. Lem. 7.1 shows the arithmetic (resp. geometric) monodromy group $\hat{G}_{0 \text {,in }}$ (resp. $G_{0, \text { in }}$ ) of $\overline{\mathcal{H}}_{0}^{\text {in,rd }} \rightarrow \mathbb{P}_{j}^{1}$ over $\mathbb{R}($ resp. $\mathbb{C}$ ) is $\mathbb{Z} / 2$ ? $S_{9}$ (resp. $\mathbb{Z} / 2$ ? $\left.A_{9}\right)$ in $S_{18}$. Further, $\operatorname{Cen}_{S_{18}}\left(G_{0 \text {,in }}\right)$ is isomorphic to $\mathbb{Z} / 2$. Here it identifies with the element $(1, \ldots, 1) \in(\mathbb{Z} / 2)^{9}$ generating the center of $G_{0, \text { in }}$. Therefore, (3.20) does not determine the complex conjugation $c$.

We now show this complication is common, occurring at all levels of many Modular Towers. 8.6 however, gives a satisfying remedy for it. Recall the normalizer $N_{S_{n}}(G, \mathbf{C})$ from 3.1 .3 .

Proposition 3.33. Let $G \leq S_{n}$ be a transitive subgroup and $\mathbf{C}$ a collection of conjugacy classes from $G$. Use this permutation representation for absolute Nielsen classes. Let $H=N_{S_{n}}(G, \mathbf{C}) / G$. Suppose $\mathcal{H}^{\prime \prime}$ (resp. $\left.\mathcal{H}^{\prime}\right)$ is an absolutely irreducible

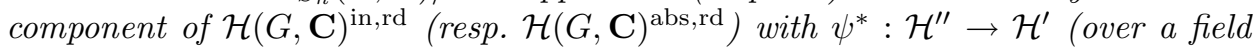
$K)$ from the natural map $\psi: \mathcal{H}(G, \mathbf{C})^{\mathrm{in}, \mathrm{rd}} \rightarrow \mathcal{H}(G, \mathbf{C})^{\text {abs,rd }}$. Then, $\psi^{*}$ is Galois with group $H_{\psi^{*}}$ a subgroup of $H$.

Denote the monodromy group of $\psi^{\prime \prime}: \mathcal{H}^{\prime \prime} \rightarrow J_{r}($ over $K)$ by $G_{\psi^{\prime \prime}}$. Then the centralizer of $G_{\psi^{\prime \prime}}$ in its natural permutation representation of degree $\operatorname{deg}\left(\psi^{\prime \prime}\right)$ contains a subgroup isomorphic to $H_{\psi}$.

Proof. Once we know that $\psi$ is Galois and $H_{\psi}$ is a subgroup of $H$, the centralizer statement follows from Princ. 3.32 That identification is in [FV91, §2.1].

EXAMPLE 3.34 (The outer automorphism of $A_{n}$ ). Consider the map of Hurwitz spaces $\mathcal{H}^{\text {in,rd }}\left(A_{n}, \mathbf{C}_{3^{r}}\right) \rightarrow \mathcal{H}^{\text {abs,rd }}\left(A_{n}, \mathbf{C}_{3^{r}}\right)(r \geq n-1)$ from Ex. 3.17 As noted there, each component of $\mathcal{H}^{\text {in,rd }}\left(A_{n}, \mathbf{C}_{3^{r}}\right)$ maps by a degree 2 map to a component of $\mathcal{H}^{\text {abs,rd }}\left(A_{n}, \mathbf{C}_{3^{r}}\right)$. This gives cases when complex conjugation $c$ on the monodromy group of the cover does not determine its action on the fibers of a Hurwitz (resp. reduced Hurwitz) space over $U_{r}\left(\operatorname{resp} . J_{r}\right)$.

3.8.4. Using $H$-M reps. to determine c. Often at level 0 , it is easy to compute elements that look like complex conjugation operators (as in Prop. 3.33) by inspection. A few tricks, however, are needed if (3.20) does not determine the effect $c$ of complex conjugation on a $j$-line cover.

The simplest remedy is to identify an $\mathbb{R}$-cover point. Suppose all branch points $z$ of a cover $\varphi: X \rightarrow \mathbb{P}_{z}^{1}$ are real. We may choose whatever paths we desire on $U_{\boldsymbol{z}}$ to compute Nielsen representatives of covers. For the next lemma, choose them to detect covers defined over $\mathbb{R}$ with $z$ as real branch points using the $\hat{\kappa}$ in Prop. 6.3. Call these the $\hat{\kappa}_{\boldsymbol{z}}$-paths. For any set $\boldsymbol{z} \in U_{r}(\mathbb{R})$ there are such $\hat{\kappa}_{\boldsymbol{z}}$-paths. Let $\operatorname{Ni}\left(\hat{\kappa}_{\boldsymbol{z}}\right)$ be branch cycles in $\operatorname{Ni}(G, \mathbf{C})^{\text {in }}$ for covers passing the test of Prop. 6.3

Lemma 3.35. Suppose $j_{0} \in(1, \infty)$ is the image of $\boldsymbol{p} \in \mathcal{H}(G, \mathbf{C})^{\mathrm{in}}(\mathbb{R})$ corresponding to a cover $\varphi: X \rightarrow \mathbb{P}_{z}^{1}$ branched over $z$ consisting of four real points on $\mathbb{P}_{z}^{1}$. Then, the complex conjugation operator $c$ for the cover fixes the reduced Nielsen classes coming from any elements of $\mathrm{Ni}\left(\hat{\kappa}_{\boldsymbol{z}}\right)$.

ExAmple 3.36 (Cont. Ex. 3.34 $c$ for $\mathcal{H}\left(A_{5}, \mathbf{C}_{3^{4}}\right)^{\text {in,rd }}=\mathcal{H}_{0}^{\text {in,rd }}$ ). Consider $\bar{\psi}: \overline{\mathcal{H}}_{0}^{\text {in,rd }} \rightarrow \mathbb{P}_{j}^{1}$. It has definition field $\mathbb{Q}$. 6.5 .3 computes $c$ for it. This cover has 
geometric (resp. arithmetic monodromy) $G_{\bar{\psi}}\left(\right.$ resp. $\left.\hat{G}_{\bar{\psi}}\right)$ in $S_{18}$ and

$$
c^{\dagger}=(110)(211)(312)(413)(514)(615)(716)(817)(918)
$$

generates $N_{S_{18}}\left(\hat{G}_{\bar{\psi}}\right)$ (6.5.3). The criterion of Prop. 6.3 gives two possible complex conjugation operators corresponding to $j_{0} \in(1, \infty)$, that labeled $c_{1, \infty}$ in (6.13) and $c^{\dagger} c_{1, \infty}$. Only, however, the former fixes an H-M rep. (represented by the integers 1 and 10) as is necessary from Lem. 3.35.

Using that the real components form a 1-dimensional manifold often is effective to handle the intervals outside $j \in(1, \infty)$. 6.5 .3 illustrates this.

At levels beyond the first it is usually prohibitive to produce the complex conjugation operators directly from the monodromy of reduced Hurwitz space covers. For example, $\mathbf{\mathbf { S } ^ { + } \mathbf { 9 5 }}$ couldn't do it for level 1 of our main example. Yet, with reasonable computation ability with the group $G_{k}$ of the level, it suffices to check what $\hat{\kappa}$ (for four real branch points) does to the elements of $\mathrm{Ni}\left(G_{k}, \mathbf{C}\right)^{\mathrm{in}, \mathrm{rd}}=\mathrm{Ni}\left(G_{k}, \mathbf{C}\right)^{\text {in }}$ $\bmod \mathcal{Q}^{\prime \prime}$. 88.6 illustrates by showing the genus 12 component of $\mathcal{H}\left(G_{1}, \mathbf{C}_{3^{4}}\right)^{\text {in }}$ has one component of real points, while the genus 9 component has no real points.

\section{Moduli and reduced Modular Towers}

Consider Hurwitz spaces $\mathcal{H}\left(G_{k}, \mathbf{C}\right)^{\text {in }}$ attached to $\left\{\left(G_{k}, \mathbf{C}\right)\right\}_{k=0}^{\infty}$ as in Prop. 3.21] This is the Modular Tower for $\left(G=G_{0}, \mathbf{C}, p\right)$ (or for $\left({ }_{p} \tilde{G}, \mathbf{C}\right)$ [Fri95a, Part III].

4.1. Reduced Modular Towers. Reduce elements of $\mathrm{Ni}\left(G_{k+1}, \mathbf{C}\right)^{\text {in }}$ modulo the kernel of $G_{k+1} \rightarrow G_{k}$. This induces $\mathcal{H}\left(G_{k+1}, \mathbf{C}\right)^{\mathrm{in}} \rightarrow \mathcal{H}\left(G_{k}, \mathbf{C}\right)^{\mathrm{in}}$. The $\mathrm{PSL}_{2}(\mathbb{C})$ action is compatible with these maps. This produces the sequence for the reduced Modular Tower for $\left({ }_{p} \tilde{G}, \mathbf{C}\right)$ :

$$
\cdots \rightarrow \mathcal{H}\left(G_{k+1}, \mathbf{C}\right)^{\mathrm{in}, \mathrm{rd}} \rightarrow \mathcal{H}\left(G_{k}, \mathbf{C}\right)^{\mathrm{in}, \mathrm{rd}} \rightarrow \cdots \rightarrow \mathcal{H}\left(G_{0}, \mathbf{C}\right)^{\mathrm{in}, \mathrm{rd}} \rightarrow J_{r} .
$$

Definition 4.1. Call a sequence of representations $\left\{T_{k}: G_{k} \rightarrow S_{n_{k}}\right\}_{k=0}^{\infty}$ compatible if $G_{k+1}(1)$ goes to a conjugate of $G_{k}(1)$ by the canonical map $G_{k+1} \rightarrow G_{k}$.

A sequence of absolute Hurwitz spaces requires a compatible system of representations [Fri95a, Part III]. Regular representations of each group $G_{k}$ give one example. Another example appears when $\left(\left|G_{0}(1)\right|, p\right)=1$. Apply Schur-Zassenhaus to the inverse image of $G_{0}(1)$ in $G_{k}$ to conclude $G_{0}(1)$ embeds compatibly in all the $G_{k}$ s. Take $T_{k}$ the action of $G_{k}$ on $G_{0}(1)$ cosets. Example: With $G_{k}=D_{p^{k+1}}$ in its standard representation with $p$ odd, $G_{0}(1)$ is cyclic of order 2 2.8.1).

Quotient by $\mathrm{PSL}_{2}(\mathbb{C})$ to produce

$$
\begin{aligned}
\cdots \rightarrow \mathcal{H}\left(G_{k+1}, \mathbf{C}, T_{k+1}\right)^{\mathrm{abs}, \mathrm{rd}} & \rightarrow \mathcal{H}\left(G_{k}, \mathbf{C}, T_{k}\right)^{\mathrm{abs}, \mathrm{rd}} \rightarrow \\
& \cdots \rightarrow \mathcal{H}\left(G_{0}, \mathbf{C}, T_{0}\right)^{\mathrm{abs}, \mathrm{rd}} \rightarrow J_{r} .
\end{aligned}
$$

We suppress the appearance of $T_{k}$ when the representation is obvious.

REMARK 4.2 (Sequences of other equivalences). We don't know example sequences of representations $\left\{T_{k}: G_{k} \rightarrow S_{n_{k}}\right\}_{k=0}^{\infty}$ compatible for the characteristic quotients of ${ }_{p} \tilde{G}$. For example, suppose $G_{0}=A_{5}$ and $p=2$, and $G_{0}(1)$ includes the whole 2-Sylow of $A_{5}$. Then, Prop. [5.6 shows $G_{k}(1)$ must be the pullback of $G_{0}(1)$ in $G_{k}$. Such an example (giving not faithful representations) is useless for most purposes. The faithful representations of $G_{1}$ in Prop. 9.14 have $G_{1}(1)$ lying 
over $G_{0}(1)$, a group with 2-Sylow of order 2 . These representations give spin separation (Def. 9.11). We suspect there are extending compatible $G_{k}(1)$, one for each $k$, giving spin separation at all levels, though we haven't found them yet.

Compatible sequences of permutation representations, suggest considering compatible sequences of subgroups $N_{k}^{\prime} \leq N_{S_{n_{k}}}\left(G_{k}, \mathbf{C}\right)$. Compatibility would require that $N_{k+1}^{\prime}$ map to $N_{S_{n_{k}}}\left(G_{k}, \mathbf{C}\right)$. The next lemma notes this is not automatic.

LEMMA 4.3. To induce an action on the cosets of $G_{k}(1)$ in $G_{k}$ requires knowing $N_{k+1}^{\prime}$ normalizes the kernel of $G_{k+1} \rightarrow G_{k}$. This holds automatically if $\operatorname{ker}_{0}$ is a characteristic subgroup of ${ }_{p} \tilde{G}$ : a common event (see Lem. [3.10).

4.2. $j$-line covers when $r=4$. Let $\mathcal{H}_{O^{\prime}}$ correspond to an $H_{4}$ orbit $O^{\prime}$ in its action on $\mathcal{H}(G, \mathbf{C})^{\text {abs }}$ (resp. on $\mathcal{H}(G, \mathbf{C})^{\text {in }}$ ). Thus, $\mathcal{H}_{O^{\prime}}$ is an absolutely irreducible component of a Hurwitz space $\mathcal{H}(G, \mathbf{C}$ ) (over some number field $K$ ), equivalence classes of $r=4$ branch point covers. Prop. 3.28 produces a finite cover

$$
\beta_{4}^{\mathrm{rd}}\left(O^{\prime}\right): \mathcal{H}_{O^{\prime}} / P S L_{2}(\mathbb{C})=\mathcal{H}_{O^{\prime}}^{\mathrm{rd}} \rightarrow \mathbb{P}_{j}^{1} \backslash\{\infty\}
$$

Complete this to a cover $\bar{\beta}^{\text {rd }}\left(O^{\prime}\right): \overline{\mathcal{H}}_{O^{\prime}}^{\text {rd }} \rightarrow \mathbb{P}_{z}^{1}$.

Let $q_{1}, q_{2}, q_{3}$ be the images of $Q_{1}, Q_{2}, Q_{3}\left(\right.$ or $\left.q_{1}, q_{2}, q_{3} \in H_{4}\right)$ in $M_{4}=H_{4} / Q$ as in (2.11). Form one further equivalence on $\mathrm{Ni}(G, \mathbf{C})^{\text {abs }}$ (or $\mathrm{Ni}(G, \mathbf{C})^{\text {in }}$ ). Recall: For $\boldsymbol{g} \in \mathrm{Ni}(G, \mathbf{C}), g_{1} g_{2} g_{3} g_{4}=1$. For $\boldsymbol{g} \in \mathrm{Ni}(G, \mathbf{C}), Q_{1} Q_{3}^{-1}$ has this effect:

$$
\boldsymbol{g} \mapsto\left(g_{1} g_{2} g_{1}^{-1}, g_{1}, g_{4}, g_{4}^{-1} g_{3} g_{4}\right)
$$

Similarly, with $\alpha=Q_{1} Q_{2} Q_{3}, \alpha^{2}$ has this effect:

$$
\boldsymbol{g} \mapsto\left(g_{2}, g_{3}, g_{4}, g_{1}\right) Q_{1} Q_{2} Q_{3} \mapsto\left(g_{3}, g_{4}, g_{1}, g_{2}\right) .
$$

DF99 Prop. 6.5] used the normal subgroup of $H_{4}$ that $Q_{1} Q_{3}^{-1}$ generates acting on $\mathrm{Ni}(G, \mathbf{C})$. It simplifies computations to make these observations from Thm. 2.9]

(4.5a) The action of $\mathcal{Q}$ on $\mathrm{Ni}(G, \mathbf{C})$ factors through the Klein 4-group $\mathcal{Q}^{\prime \prime}$.

(4.5b) $\mathcal{Q}$ is the minimal normal subgroup of $H_{4}$ containing either $\alpha^{2}$ or $Q_{1} Q_{3}^{-1}$. Denote the $\mathcal{Q}^{\prime \prime}=\left\langle Q_{1} Q_{3}^{-1}, \alpha^{2}\right\rangle$ orbits on $\mathrm{Ni}(G, \mathbf{C})$ by $\mathrm{Ni}(G, \mathbf{C})^{\mathrm{rd}}$ : reduced classes. Apply (2.5): Action of $H_{4}$ on $\mathrm{Ni}(G, \mathbf{C}) / N^{\prime}$ (as in 3.5) induces $H_{4} / Q$ acting on $\operatorname{Ni}(G, \mathbf{C})^{\text {rd }} / N^{\prime}$. For $\mathrm{Ni}(G, \mathbf{C})^{\text {abs }}$ (resp. $\mathrm{Ni}(G, \mathbf{C})^{\text {in }}$ ) there is the quotient set $\mathrm{Ni}(G, \mathbf{C})^{\text {abs,rd }}\left(\right.$ resp. $\left.\mathrm{Ni}(G, \mathbf{C})^{\text {in,rd }}\right)$. Continue using $q_{1}$ and $q_{2}$ for $Q_{1}$ and $Q_{2}$ acting on $\mathrm{Ni}(G, \mathbf{C})^{\text {rd }}$. Then, DF99 Prop. 6.5] computes branch cycles for $\bar{\beta}^{\text {rd }}\left(O^{\prime}\right)$.

Proposition 4.4 ( $j$-line branch cycles). Consider $\gamma_{0}=q_{1} q_{2}$ and $\gamma_{1}=q_{1} q_{2} q_{1}$, generators of $\bar{M}_{4}$, with relations $\gamma_{0}^{3}=\gamma_{1}^{2}=1: \bar{M}_{4} \equiv \mathrm{PSL}_{2}(\mathbb{Z})$ (2.9). Further, with $\gamma_{\infty}=q_{2}$, the product-one condition $\gamma_{0} \gamma_{1} \gamma_{\infty}=1$ holds (2.10).

Then, $\bar{M}_{4}$ orbits on $\mathrm{Ni}(G, \mathbf{C})^{\text {abs, rd }}$ (resp. $\mathrm{Ni}(G, \mathbf{C})^{\mathrm{in}, \mathrm{rd}}$ ) correspond one-one to $H_{4}$ orbits on $\mathrm{Ni}(G, \mathbf{C})^{\text {abs }}$ (resp. $\left.\mathrm{Ni}(G, \mathbf{C})^{\mathrm{in}}\right)$. Let $O^{\prime}$ be the orbit in the discussion above. Let $\gamma_{0}^{\prime}, \gamma_{1}^{\prime}$ and $\gamma_{\infty}^{\prime}$ be respective actions of $\gamma_{0}, \gamma_{1}$ and $\bar{q}_{2}=\gamma_{\infty}$ on the image of $O^{\prime}$ in $\mathrm{Ni}(G, \mathbf{C})^{\text {abs, rd }}$ (resp. $\left.\mathrm{Ni}(G, \mathbf{C})^{\text {in }}\right)$. Then $\left(\gamma_{0}^{\prime}, \gamma_{1}^{\prime}, \gamma_{\infty}^{\prime}\right)$ is a branch cycle description of the cover $\bar{\beta}^{\text {rd }}\left(O^{\prime}\right)$.

Comments on the proof. Suppose $Q \in H_{r}$ gets killed in the $\mathrm{PSL}_{2}(\mathbb{C})$ quotient of all moduli spaces of $r$ branch point covers. This happens if for every $\boldsymbol{z} \in U_{r}$, there exists $\alpha \in \mathrm{PSL}_{2}(\mathbb{C})$ fixing $\boldsymbol{z}$ and inducing on $\pi_{1}\left(U_{\boldsymbol{z}}\right)$ the same effect (modulo inner automorphisms) as does $Q$. Prop. 2.3 identifies the group $\mathcal{Q}$ as the group of such $Q$, and $\bar{M}_{4}$ as the quotient of $M_{4}$ by a Klein 4 -group, $\mathcal{Q}^{\prime \prime}=\mathcal{Q} /\left\langle\left(q_{1} q_{3}^{-1}\right)^{2}\right\rangle$. 
4.3. Reduced moduli spaces: b-fine and fine. Let $\mathcal{Q}^{\prime \prime}=\mathcal{Q} /\langle z\rangle$ as in Thm. 2.9 We start with results assuring some kind of fine moduli condition for reduced Hurwitz spaces. 4.3 .2 then illustrates why we cannot escape considering situations where it does not hold.

4.3.1. Fine moduli. We interpret the phrase fine (resp. b-fine) reduced Hurwitz space for inner equivalence (absolute equivalence is similar, Rem. 4.8).

Let $\mathcal{H}^{\text {rd }}$ be the reduced space for inner equivalence on Nielsen classes of covers attached to $(G, \mathbf{C})$. Consider any smooth family $\Phi: \mathcal{T} \rightarrow S$ of curves with an analytic map $\Psi: S \rightarrow J_{r}$. Denote the fiber of $\Phi$ over $s \in S$ by $\mathcal{T}_{s}$. Assume $G$ acts as a group scheme on $\mathcal{T}$ preserving each fiber $\mathcal{T}_{s}: \Gamma: G \times \mathcal{T} \rightarrow \mathcal{T}$. For the quotient $\varphi_{s}: \mathcal{T}_{s} \mapsto \mathcal{T}_{s} / G$, assume for $s \in S(\mathbb{C})$ :

(4.6) An isomorphism of $\mathcal{T}_{s} / G$ with $\mathbb{P}_{z}^{1}$ presents $\varphi_{s}$ in $\mathrm{Ni}(G, \mathbf{C})^{\mathrm{in}}$ with branch points in the equivalence class $\Psi(s)$.

By assumption this induces $\psi: S \rightarrow \mathcal{H}^{\text {rd }}: \mathcal{H}^{\text {rd }}$ is a target for such maps. The quotient $\mathcal{T} / G$ is a geometric $\mathbb{P}^{1}$ bundle over $S$. 4.4 .1 briefly discusses the obstruction to fibers being $\mathbb{P}^{1}$ over their definition field.

Definition 4.5 (Fine reduced moduli). Call $\mathcal{H}^{\text {rd }}$ a fine moduli space (has fine moduli) if for every such family, there is a unique family $\mathcal{T}^{\text {rd }} \rightarrow \mathcal{H}^{\text {rd }}$ satisfying (4.6) inducing $\mathcal{T}$ by pullback from $\psi$.

Definition 4.6 (b-Fine reduced moduli). Consider $U_{j}^{\prime}=\mathbb{P}_{j}^{1} \backslash\{0,1, \infty\}$ and $\mathcal{H}_{U_{j}^{\prime}}^{\text {rd }}$, the restriction of $\mathcal{H}^{\text {rd }}$ over $U_{j}^{\prime}$. The weaker notion b-fine is that $\mathcal{T}$ restricted to $\psi^{*}\left(\mathcal{H}_{U_{j}^{\prime}}^{\text {rd }}\right)$ is the pullback by $\psi$ of $\mathcal{T}^{\text {rd }}$ restricted to $\mathcal{H}_{U_{j}^{\prime}}^{\text {rd }}$.

Each notion applies separately to any component of $\mathcal{H}^{\text {rd }}$. Further, there is an obvious generalization to $r>4$, though we will not be able to be so precise about testing for it. The action of $\mathcal{Q}^{\prime \prime}$ on Nielsen classes gives an if and only if test for a reduced Hurwitz space being a b-fine moduli space (Prop.4.7 for inner equivalence, Rem. 4.8 for absolute equivalence). The $k$ th level of the $\left(A_{5}, \mathbf{C}_{3^{4}}, p=2\right)$ Modular Tower passes this test for b-fine moduli, $k \geq 1$ (Prop. [7.9); even for fine moduli (Ex. 8.5 and Lem. 8.11). It is not even b-fine for level $k=0$.

Proposition 4.7. Let $\mathcal{H}$ be a Hurwitz space with inner Nielsen class $\operatorname{Ni}(G, \mathbf{C})$. A component $\mathcal{H}_{*}^{\text {rd }}$ of the reduced space $\mathcal{H}^{\text {rd }}$ has fine moduli if and only if there is a unique total space $\Phi_{*}: \mathcal{T}_{*}^{\text {rd }} \rightarrow \mathcal{H}_{*}^{\text {rd }}$ with a $G$ action on $\mathcal{T}_{*}^{\text {rd }}$ satisfying (4.6) when $S=\mathcal{H}_{*}^{\text {rd }}$. For $\boldsymbol{p} \in \mathcal{H}_{*}^{\mathrm{rd}}(K), \varphi_{\boldsymbol{p}}: \mathcal{T}_{*, \boldsymbol{p}} \mapsto \mathcal{T}_{*, \boldsymbol{p}} / G$ has definition field $K$.

Assume $r=4$ and $\mathcal{H}$ has fine moduli (as in Prop. [3.21). Let $O$ be an $H_{4}$ orbit on the Nielsen class corresponding to a component $\mathcal{H}_{*}$ of $\mathcal{H}$ with $\mathcal{H}_{*}^{\text {rd }}$ its image in $\mathcal{H}^{\text {rd }}$. Then, $\mathcal{Q}^{\prime \prime}$ orbits on $O$ have length 4 if and only if $\mathcal{H}_{*}^{\text {rd }}$ has b-fine moduli. Further, assuming b-fine moduli, $\mathcal{H}_{*}^{\text {rd }}$ has fine moduli if and only if all its points over $j=0$ and $j=1$ ramify (Prop. 4.4; $\gamma_{0}^{\prime}$ and $\gamma_{1}^{\prime}$ have no fixed points).

If $r \geq 5$, and $\mathcal{H}$ has fine moduli, then all components of $\mathcal{H}^{\text {rd }}$ have $b$-fine moduli.

Suppose $\mathcal{T}_{*, \boldsymbol{p}}$ has a $K$ divisor with odd degree image in $\mathcal{T}_{*, \boldsymbol{p}} / G$. (Examples: a branch point conjugacy class is $K$ rational and distinct from other branch cycle conjugacy classes; or $K$ is a finite field.) Then, $\mathcal{T}_{*, \boldsymbol{p}} / G$ is $K$ isomorphic to $\mathbb{P}_{z}^{1}$.

Proof. The field of definition statement follows from $\varphi_{\boldsymbol{p}}$ defined over $K$. Then, the divisor hypothesis produces an odd degree $K$ divisor on $\mathcal{T}_{*, \boldsymbol{p}} / G$. Since $\mathcal{T}_{*, \boldsymbol{p}} / G$ has genus 0 and an odd degree $K$ divisor, it is $K$ isomorphic to $\mathbb{P}_{z}^{1}$. If $K$ is a finite 
field, a homogeneous space for any Brauer-Severi variety always has a rational point (the Brauer group of a finite field being trivial Ser96 p. 126]).

Let $r=4$. Let $\boldsymbol{g} \in O$ and $\boldsymbol{g}^{\prime}$ be in the $\mathcal{Q}^{\prime \prime}$ orbit of $\boldsymbol{g}$. Consider a given set of branch points $\boldsymbol{z}$ and classical generators $\overline{\boldsymbol{g}}$ of $\pi_{1}\left(U_{\boldsymbol{z}}, z_{0}\right)$. Assume the image of $\boldsymbol{z}$ in $\mathbb{P}_{j}^{1}$ is different from 0 or 1 . Denote by $\varphi_{\boldsymbol{g}}: X_{\boldsymbol{g}} \rightarrow \mathbb{P}_{z}^{1}$ and $\varphi_{\boldsymbol{g}^{\prime}}: X_{\boldsymbol{g}^{\prime}} \rightarrow \mathbb{P}_{z}^{1}$ the covers from the homomorphisms sending $\overline{\boldsymbol{g}}$ respectively to $\boldsymbol{g}$ and to $\boldsymbol{g}^{\prime}$. Suppose for some $\alpha \in \mathrm{PSL}_{2}(\mathbb{C})$ there exists an isomorphism $\psi: X_{\boldsymbol{g}} \rightarrow X_{\boldsymbol{g}^{\prime}}$ for which $\alpha \circ \varphi_{\boldsymbol{g}}=\varphi_{\boldsymbol{g}^{\prime}} \circ \psi$. As in 2.3 .1 and $2.3 .2 \alpha$ lies in a Klein 4-group identified with $\mathcal{Q}^{\prime \prime}$. The b-fine hypotheses implies $\psi$ is unique.

So, the subgroup of $\mathrm{PSL}_{2}(\mathbb{C})$ fixing $z \in U_{4}$ extends to a faithful action of $\mathcal{Q}^{\prime \prime}$ on $\mathcal{T}$ over $\mathcal{H} \times U_{j}^{\prime}$. Quotient action gives the total space representing w-equivalence classes of covers for points of $\mathcal{H}_{*, U_{j}^{\prime}}^{\text {rd }}$. The fine moduli hypothesis say $\alpha$ fixed on $z$ over $j=0$ or 1 extends to $\mathcal{H}_{*}$ without fixed points over that value of $\boldsymbol{z}$.

For $r \geq 5$, the b-fine property follows from 6.4 .4

REMARK 4.8 (Absolute equivalence). Let $\mathcal{H}^{\text {rd }}$ be the reduced space for absolute equivalence on Nielsen classes of covers attached to $(G, \mathbf{C}, T)$. Consider any smooth family $\mathcal{T} \stackrel{\Phi}{\longrightarrow} \mathcal{Y} \stackrel{\pi}{\longrightarrow} S$ with an analytic map $\Psi: S \rightarrow J_{r}$ with $\pi$ a (geometric) $\mathbb{P}^{1}$ bundle. Assume also the fiber $\mathcal{T}_{s} \rightarrow \mathcal{Y}_{s}$ over $s \in S$ satisfies this:

(4.7) An isomorphism of $\mathcal{Y}_{s}$ with $\mathbb{P}_{z}^{1}$ presents $\varphi_{s}$ in $\mathrm{Ni}(G, \mathbf{C}, T)^{\text {in }}$ with branch points in the equivalence class $\Psi(s)$.

The analogs of b-fine and fine moduli are clear, and the proof and conclusion of Prop. 4.7 hold with little adjustment.

4.3.2. The geometry of not having fine moduli. Dilemma: $\mathcal{H}\left(D_{p^{k+1}}, \mathbf{C}_{2^{4}}\right)^{\mathrm{in}, \mathrm{rd}}$ is $X_{1}\left(p^{k+1}\right)$ without its cusps. The former space, however, isn't a fine (even b-fine) moduli space according to Prop. 4.7 Yet, it is classical the latter is a fine moduli space. Resolution: It is for elliptic curves with a $p^{k+1}$ division point, though not for (inner) w-equivalence of dihedral group Galois covers (2.8.3).

REMARK 4.9 (Serre's criterion). An observation of Serre says an automorphism of an elliptic curve fixing a $p^{k+1} \geq 3$ division point must be the identity. This assures $X_{1}\left(p^{k+1}\right)$ is a fine moduli space. An appropriate generalization is to decide for $r=4$, when $\gamma_{0}$ and $\gamma_{1}$ have no fixed points on the level $k$ Nielsen classes $\mathrm{Ni}\left(G_{k}, \mathbf{C}\right)^{\mathrm{in}, \mathrm{rd}}$ for a Modular Tower, when $k$ is large (see 9.6).

Definition 4.10 (Field of moduli). Let $\varphi: X \rightarrow \mathbb{P}_{z}^{1}$ represent a cover in a Nielsen class $\mathrm{Ni}$ for inner or absolute equivalence. Assume it has definition field in the algebraic closure of a field $K$ (assume char. 0 for simplicity). Apply each $\sigma \in G_{K}$ to $\varphi$, denoting the result $\varphi^{\sigma}$. Let $G_{K, \varphi}$ be the collection of $\sigma$ for which $\varphi^{\sigma}$ is equivalent to $\varphi$. The field of moduli, $K_{\varphi}$ is the fixed field of $G_{K, \varphi}$. For reduced equivalence, we have a corresponding field $K_{\varphi, \text { rd }}$.

Some circumstances might use a cover both as an inner cover, and as an absolute cover. Reflecting this the notation would be $K_{\varphi \text {,in }} \supset K_{\varphi \text {,abs }}$. Having a fine moduli space for the Inverse Galois Problem assures for $\boldsymbol{p} \in \mathcal{H}(G, \mathbf{C})^{\text {in }}\left(\right.$ or $\mathcal{H}(G, \mathbf{C})^{\text {abs }}$ or $\mathcal{H}(G, \mathbf{C})^{\mathrm{in}, \mathrm{rd}}$, etc. $)$ there is a cover representing that point over $\mathbb{Q}(\boldsymbol{p})$.

In both the inner case (when $G$ has a center), or the absolute case (when $\operatorname{Cen}_{S_{n}}(G)$ is nontrivial), there has been work to make Hurwitz spaces useful. Modular Towers include all information about Frattini central extensions, as in Ex. 3.20 Lem. 3.15reminds of the Harbater-Coombes argument: In the inner case, even with 
a center, there is an absolute cover over $\mathbb{Q}(\boldsymbol{p})$. This representing cover, however, may have no automorphisms over $\mathbb{Q}(\boldsymbol{p})$. The extension of this result to any base (in place of a point) does not hold [DDE00 §5].

This recognizes existence of a family over $\mathcal{H}$ and existence of a representing cover over $\boldsymbol{p} \in \mathcal{H}$ as part of the same problem, though changing the base makes a difference. Both problems suit the language of stacks or gerbes (as in first version of this question [Fri77, §4]). Deb01, §2] gives an exposition on the gerbe approach of DDE00 and attempts to compute the obstructions to these problems.

We01 uses an approach like $\$$ relating cusps and complex conjugation operators. Consider again our main example $\mathcal{H}\left(G_{k}, \mathbf{C}_{3^{4}}\right)^{\text {in }}, k \geq 0$. There is a nontrivial central Frattini extension $T_{k}^{\prime} \rightarrow G_{k}$. Then, $\mathcal{H}\left(G_{k}, \mathbf{C}_{3^{4}}\right)^{\text {in }}$ contains, among its components, the components of $\mathcal{H}\left(T_{k}^{\prime}, \mathbf{C}_{3^{4}}\right)^{\text {in }}$ (the moduli for inner covers with group $T_{k}^{\prime}$ ). For each $k \geq 1$, Prop. 6.8 notes there are two kinds of real points on $\mathcal{H}\left(G_{k}, \mathbf{C}_{3^{4}}\right)^{\text {in }}$ : those for H-M reps. and those for near H-M reps. H-M rep. points in $\mathcal{H}\left(T_{k}^{\prime}, \mathbf{C}_{3^{4}}\right)^{\text {in }}(\mathbb{R})$ have representing covers over $\mathbb{R}$. Near H-M rep. points in $\mathcal{H}\left(T_{k}^{\prime}, \mathbf{C}_{3^{4}}\right)^{\text {in }}$ have no representing cover over $\mathbb{R}$ : for these points the field of moduli is not a field of definition. This interprets as different degeneration at cusps on $\mathcal{H}\left(G_{k}, \mathbf{C}_{3^{4}}\right)^{\text {in,rd }}$ attached to H-M reps. from those for near H-M reps.

The phenomena above also happens at $k=0$, though there are no near H-M reps. there. We01 starts there to explain a $p$-adic theory. IM95 provides a Hurwitz space context for the Drinfeld-Ihara-Grothendieck relations (that apply to elements of the absolute Galois group; call these DIG relations). The approach was through tangential base points. This is the We01 approach, though he does not take the exact same tangential base points. For example, he often uses complex conjugate pairs of branch points, while they always used sets of real branch points. Ihara's use of the DIG relations has been primarily to describe the Lie algebra of the absolute Galois group acting through various pronilpotent braid groups, especially on the 3 punctured $\lambda$-line. [Fri95a App. C] proposed Modular Towers, though a profinite construction, as suitably like finite representations of the fundamental group to see the DIG relations at a finite level. There is an analogy with Ihara in that modular curves are close to considerations about the $\lambda$-line, we know no direct phenomenon for modular curves suggesting the DIG relations. Still, modular curves are just one case of Modular Towers.

In Prop. 8.12 the Modular Tower attached to $A_{5}$ and four 3- cycles produces a system of Serre obstruction situations from covers of $A_{5}$, typical for a Modular Tower. For $\left(\ell,\left|A_{5}\right|\right), \ell$-adic points on these Modular Towers levels should have a similar tangential base point (cusp geometry) analysis to the near H-M and H-M reps. over $\mathbb{R}$. We expect the H-M rep. analysis appplied to $\ell$-adic fields to give a tower of $T_{k}^{\prime}$ realizations. The near H-M rep. analysis, done $\ell$-adically adds even more interest. We expect these to provide examples at each level $k$, over $\ell$-adic fields of a function field extension with group a central Frattini extension of $G_{k}$ whose field of moduli is not a field of definition. Further, this geometry should reveal the DIG relations on actual covers, instead of as a Lie algebra relation. Though there are some points that must be handled to do this, the computations of Cor. 9.7 for level 1 of this $A_{5}$ Modular Tower should give a precise analog of We01 Prop. 2.17] (for level 0).

4.4. Points on a Modular Tower. As in 4.3 let $\mathcal{H}$ be a Hurwitz space and $\mathcal{H}^{\text {rd }}$ its reduced version. We assume $\mathcal{H}^{\text {rd }}$ has (at least) b-fine moduli (for inner or 
absolute equivalence). For $K$ a field, a $K$ point on $\mathcal{H}$ produces a $K$ point on $\mathcal{H}^{\text {rd }}$. 4.4 .1 interprets the subtlety of the converse. Then, 4.4 .2 applies this to points on a Modular Tower. 4.4 .3 and $₫ \mathbb{D}$ formulate Serre's Open Image Theorem for $\overline{\mathbb{Q}}$ points on a Modular Tower.

4.4.1. $\mathrm{PSL}_{2}$ cocycles. Use the setup for inner Hurwitz spaces in (Prop. 4.7). Suppose $\mathcal{H}^{\text {rd }}$ is a fine (resp. b-fine) moduli space, and $\boldsymbol{p}^{\text {rd }} \in \mathcal{H}^{\text {rd }}(K)$ (resp. also, doesn't lie over $j=0$ or 1$)$. To simplify notation, refer to the total family over $\mathcal{H}^{\text {rd }}$ (resp. $\mathcal{H}_{U_{j}^{\prime}}^{\text {rd }}$ ) as $\mathcal{T}$. To simplify further, also assume fine moduli, for the adjustments to b-fine are obvious. The (geometric) $\mathbb{P}_{z}^{1}$ bundle $\mathcal{T}^{\text {rd }} / G$ is algebraic from Serre's GAGA. We may even cover $\mathcal{H}^{\text {rd }}$ with $K$ affine sets $\left\{W_{i}\right\}_{i \in I}$ so the restriction of $\mathcal{T}^{\text {rd }} / G$ over $W_{i}$ is a conic bundle in $\mathbb{P}^{2} \times W_{i}$. That is, for each $\boldsymbol{p}^{\text {rd }} \in W_{i}$, the fiber over $\boldsymbol{p}^{\text {rd }}$ is a conic in $\mathbb{P}^{2}$.

We drop the subscript referring to $\boldsymbol{p}^{\text {rd }}$. This gives the reduced class of a cover $\varphi: X \rightarrow Y=\mathcal{T}_{\boldsymbol{p}^{\text {rd }}}^{\text {rd }} / G$ over $K$, with group $G$, in the Nielsen class. To simplify, Then, if $Y$ has a $K$ point, $\varphi$ is a $K$-cover (of $\mathbb{P}_{z}^{1}$ ) in the Nielsen class. Compatible with definitions of $\$ 1.3 .2$ call each $\boldsymbol{p}^{\text {rd }}$ a $K$-cover point (the structure of the Nielsen class is over $K)$. Otherwise, $Y$ is isomorphic to a conic over $K$. When $Y$ has no $K$ point, call $\boldsymbol{p}^{\text {rd }}$ a $K$-Brauer point (of $\mathcal{H}^{\text {rd }}(K)$ ).

The conic attached to a point $p \in \mathcal{H}^{\text {rd }}(\bar{K})$ defines an element of the group of 2-torsion elements in the Brauer group $B_{2}(K(\boldsymbol{p}))$ of the field $K(\boldsymbol{p})$. Any $K$ component $\mathcal{H}_{*}^{\text {rd }}$ of the Hurwitz space defines an element of $B_{2}\left(K\left(\mathcal{H}_{*}^{\text {rd }}\right)\right)$ by the same argument for a generic point of $\mathcal{H}_{*}^{\text {rd }}$. When the closure of $\mathcal{H}_{*}^{\text {rd }}$ is isomorphic to $\mathbb{P}_{w}^{1}$, regard the conic bundle as an element $b\left(\mathcal{H}_{*}^{\mathrm{rd}}\right) \in B r_{2}(K(w))$. This case arises often in the Inverse Galois Problem. Given a rational function $g\left(w^{\prime}\right)$ in a new variable $w^{\prime}$, consider $K(w)$ as a subfield of $K\left(w^{\prime}\right)$ by setting $w=g\left(w^{\prime}\right)$. This induces $B_{2}(K(w)) \rightarrow B_{2}\left(K\left(w^{\prime}\right)\right)$. [Ser96 p. 114-116] discusses (what is in our notation) if for some choice of $g$ the image of $b\left(\mathcal{H}_{*}^{\text {rd }}\right)$ in $B_{2}\left(K\left(w^{\prime}\right)\right)$ vanishes. There is a natural notion of poles of $b\left(\mathcal{H}_{*}^{\mathrm{rd}}\right)$, and Mes94 shows that if there are at most four poles, then such a $g$ exists. As in Prop. 2.3] when $\mathcal{Q}^{\prime \prime}$ acts trivially on Nielsen classes, we can take $g$ of degree 1 , regarding it as a section for the $j$-invariant. So, in the next lemma we emphasize the crucial case when $\mathcal{Q}^{\prime \prime}$ acts faithfully on the Nielsen classes for the component $\mathcal{H}_{*}$.

LEMma 4.11 (Reduced cocycle Lemma). Let $\mathcal{H}$ be a fine moduli space for the Nielsen class. Assume $\mathcal{H}_{*}^{\mathrm{rd}}$, a component of $\mathcal{H}^{\mathrm{rd}}$, is a fine moduli space and $y \in$ $Y(\bar{K})=Y_{\boldsymbol{p}^{\mathrm{rd}}}(\bar{K})$ as above for $\boldsymbol{p}^{\mathrm{rd}} \in \mathcal{H}_{*}^{\mathrm{rd}}$. This produces a cover $\varphi_{y}: X \rightarrow \mathbb{P}_{z}^{1}$ over $K(y)$ and a unique cocycle class in $H^{1}\left(G_{K}, \mathrm{PSL}_{2}(\mathbb{C})\right)$. These fit in a cocycle of Nielsen class covers. The cocycle class is trivial if and only if $Y$ is isomorphic to $\mathbb{P}_{z}^{1}$ over $K$. In turn this holds if and only if $Y$ has an odd degree $K$ divisor.

Conversely, given a cocycle of $(G, \mathbf{C})$ Nielsen class covers attached to $X$, there is $K$ cover $\varphi: X \rightarrow Y$, which over $\bar{K}$ is in the Nielsen class.

Suppose $Y$ has a $K$ point. Let $j_{0}$ be the image in $J_{r}$ of $\boldsymbol{p}^{\mathrm{rd}}$. Then, there is a one-one association between $K$ points in the fiber $U_{r, j_{0}}$ of $U_{r} \rightarrow J_{r}$ and $K$ points $\boldsymbol{p} \in \mathcal{H}$ over $\boldsymbol{p}^{\mathrm{rd}}$.

Proof. The linear system $\mathcal{L}_{y}$ attached to $y$ gives an isomorphism of the genus 0 curve $Y$ with $\mathbb{P}_{z}^{1}: \mu_{y}: Y \rightarrow \mathbb{P}_{z}^{1}$. Take $\varphi_{y}$ to be the composition of $\varphi$ and this isomorphism. Apply each $\sigma \in G_{K}$ to $\varphi_{y}$ to get ${ }^{\sigma} \varphi_{y}: X \rightarrow \mathbb{P}_{z}^{1}$. The isomorphism here is that given by replacing $y$ by $\sigma(y)$. Since $\mathcal{H}^{\text {rd }}$ is a fine moduli space, there 
is a unique $\alpha_{\sigma} \in \mathrm{PSL}_{2}(\bar{K})$ and $\psi_{\sigma}: X \rightarrow X$ satisfying $\alpha_{\sigma} \circ \varphi_{y}={ }^{\sigma} \varphi_{y} \circ \psi_{\sigma}$. The cocycle condition follows from the uniqueness conditions. Call this data a cocycle of Nielsen class covers attached to $X$. It is standard the cocycle is trivial if and only if $Y$ has a $K$ point. Since $Y$ has genus 0 , this is equivalent to $Y$ having a degree one $K$ divisor. Since the canonical class on $Y$ is a degree -2 class over $K$, this is equivalent to $Y$ having an odd degree $K$ divisor.

Now suppose we have such a cocycle of Nielsen class covers. This produces $\varphi$ as $\mu_{y}^{-1} \circ \varphi_{y}$. We have only to check $\varphi$ is well-defined, independent of $\sigma \in G_{K}$. The cocycle condition guarantees this.

Finally, if there is a $K$ point on $Y$, this gives $\boldsymbol{p} \in \mathcal{H}$ lying over $\boldsymbol{p}^{\mathrm{rd}}$. Composing the cover $\varphi_{\boldsymbol{p}}: X_{\boldsymbol{p}} \rightarrow \mathbb{P}_{z}^{1}$ with elements of $\mathrm{PSL}_{2}(K)$ gives the correspondence between the $K$ points of $U_{r, j_{0}}$ and the $K$ points of $\mathcal{H}$ over $\boldsymbol{p}^{\text {rd }}$.

ExAmPle 4.12. 2.8.2 considers the Nielsen class is $\mathrm{Ni}\left(D_{p}, \mathbf{C}_{2^{4}}\right)$. Then, a point $\boldsymbol{p}^{\mathrm{rd}} \in \mathcal{H}^{\mathrm{rd}}(K)$ has a $K$ point of $\mathcal{H}$ over it. This is because an elliptic curve isogeny $E \rightarrow E^{\prime}$ over $K$ represents $\boldsymbol{p}^{\text {rd }}$. The quotient of $E^{\prime}$ by $\langle-1\rangle$ has a rational point from the image of the rational point on $E^{\prime}$. This gives $E \rightarrow \mathbb{P}_{z}^{1}$ representing $\boldsymbol{p} \in \mathcal{H}(K)$ lying over $\boldsymbol{p}^{\text {rd }}$.

REMARK 4.13. Suppose $\mathcal{H}^{\text {rd }}$ is not a fine moduli space. We can still ask which $\boldsymbol{p} \in \mathcal{H}^{\text {rd }}$ are $K$-cover points or $K$-Brauer points as in Rem. 2.4 and Rem. 2.5]

4.4.2. Projective systems of points. Let $\left\{\boldsymbol{p}_{k}^{\text {rd }} \in \mathcal{H}\left(G_{k}, \mathbf{C}\right)^{\text {rd }}\right\}_{k=0}^{\infty}$ be a projective system of points on a reduced Modular Tower. Call this a point on a reduced Modular Tower. Suppose $\mathcal{H}_{k}^{\text {rd }}\left(\boldsymbol{p}^{\text {rd }}\right)$ is the absolutely irreducible component containing $\boldsymbol{p}_{k}^{\text {rd }}$. Then $\mathcal{H}^{\mathrm{rd}}\left(\boldsymbol{p}^{\mathrm{rd}}\right)=\left\{\mathcal{H}_{k}^{\mathrm{rd}}\left(\boldsymbol{p}^{\mathrm{rd}}\right)\right\}_{k=0}^{\infty}$ is a projective sequence of algebraic varieties. This works with $\boldsymbol{p}$ a projective system of points on Hurwitz spaces $\mathcal{H}(\boldsymbol{p})=\left\{\mathcal{H}_{k}(\boldsymbol{p})\right\}_{k=0}^{\infty}$ rather than reduced Hurwitz spaces.

PRINCIPLE 4.14. Components of $\mathcal{H}^{\mathrm{rd}}$ are manifolds and moduli spaces. Conclude: $\boldsymbol{p}_{k}^{\mathrm{rd}}$ being a $K_{k}$ point implies $K_{k}$ contains a definition field for $\mathcal{H}_{k}^{\mathrm{rd}}\left(\boldsymbol{p}^{\mathrm{rd}}\right)$.

Assume $\boldsymbol{p}_{0} \in \mathcal{H}_{0}$ lies over the level 0 point $\boldsymbol{p}_{0}^{\text {rd }}$ of $\boldsymbol{p}^{\text {rd }}$. Then, this produces a point $\boldsymbol{p}$ on the Modular Tower with its level 0 point equal $\boldsymbol{p}_{0}$.

For $W$ any algebraic variety, and $w_{0} \in W$, denote the pro- $p$ completion of $\pi_{1}\left(W, w_{0}\right)$ by $\pi_{1}\left(W, w_{0}\right)^{(p)}$. It is the closure of $\pi_{1}\left(W, w_{0}\right)$ in the diagonal of the product of all finite $p$-group quotients of $\pi_{1}\left(W, w_{0}\right)$. When considering homomorphisms involving it, defined up to conjugation by an element of this group, with no loss drop the $w_{0}$ decoration.

We concentrate now on inner Hurwitz spaces. For $z_{0} \in K$, not in $\boldsymbol{z}$, consider classical generators $\overline{\boldsymbol{g}}$ for $\pi_{1}\left(U_{\boldsymbol{z}}, z_{0}\right)$ (\$1.2). Let $\left\{\boldsymbol{p}_{k}^{\mathrm{rd}} \in \mathcal{H}\left(G_{k}, \mathbf{C}\right)^{\mathrm{in}}\right\}_{k=0}^{\infty}$ be a projective system of points over $\boldsymbol{p}_{0} \in \mathcal{H}\left(G_{k}, \mathbf{C}\right)^{\text {in }}$ with $X_{\boldsymbol{p}_{k}} \rightarrow \mathbb{P}_{z}^{1}$ a representing cover. Any projective system $\left\{x_{k} \in X_{\boldsymbol{p}_{k}}\right\}_{k=0}^{\infty}$ of points over $z_{0}$ gives a compatible system of homomorphisms $\psi_{k}: \pi_{1}\left(U_{\boldsymbol{z}}, z_{0}\right)^{\text {alg }} \rightarrow G_{k}$ factoring through $\pi_{1}\left(U_{\boldsymbol{z}}, z_{0}\right)^{\text {ar }}$ (3.3). This produces $\tilde{\psi} \in \operatorname{Hom}\left(\pi_{1}\left(U_{\boldsymbol{z}}, z_{0}\right)^{\mathrm{alg}},{ }_{p} \tilde{G}\right) /{ }_{p} \tilde{G}$ with $\overline{\boldsymbol{g}}$ mapping into $\mathbf{C}$ (3.4.2), not depending on $\left\{x_{k}\right\}_{k=0}^{\infty}$.

Restriction of $\tilde{\psi}$ to the kernel of $\psi_{0}$ factors through $\pi_{1}\left(X_{\boldsymbol{p}_{0}}\right)^{(p)}$ (Thm. 6.1 as in 3.4.2). Factor $\pi_{1}\left(U_{\boldsymbol{z}}, z_{0}\right)$ by the kernel of this map, and denote the result by $M_{\boldsymbol{p}_{0}}$.

Proposition 4.15. The group $M_{p_{0}}$ fits in a natural exact sequence

$$
1 \rightarrow \pi_{1}\left(X_{\boldsymbol{p}_{0}}\right)^{(p)} \rightarrow M_{\boldsymbol{p}_{0}} \rightarrow G_{0} \rightarrow 1 \text {. }
$$


Keep the notation $\overline{\boldsymbol{g}}$ for the image of $\overline{\boldsymbol{g}}$ in $M_{\boldsymbol{p}_{0}}$. Points on the Modular Tower for $(G, \mathbf{C})$ over $\boldsymbol{p}_{0} \in \mathcal{H}(G, \mathbf{C})^{\text {in }}$ correspond one-one with elements of $\operatorname{Hom}\left(M_{\boldsymbol{p}_{0}},{ }_{p} \tilde{G}\right) /{ }_{p} \tilde{G}$ mapping $\overline{\boldsymbol{g}}$ into $\mathbf{C}$. In turn these correspond with elements of $\left.\operatorname{Ni}_{p} \tilde{G}, \mathbf{C}\right)^{\mathrm{in}}$. Then, the action of $H_{r}$ on $\overline{\boldsymbol{g}}$ induces an action of $H_{r}$ on $\mathrm{Ni}_{p}(\tilde{G}, \mathbf{C})^{\mathrm{in}}$.

Let $\boldsymbol{p} \in \mathcal{H}$, a point on the projective sequence of components $\left\{\mathcal{H}_{k}\right\}_{k=0}^{\infty}$ containing

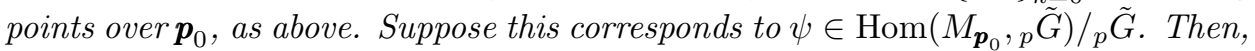
the collection of points $\boldsymbol{p}^{\prime} \in \mathcal{H}$ above $\boldsymbol{p}_{0}$ correspond to an $H_{r}$ orbit of $\boldsymbol{p}$.

4.4.3. A Grassman like object. Let $g_{0}=g$ be the genus of $X_{\boldsymbol{p}_{0}}$. For $\pi_{1}\left(X_{\boldsymbol{p}_{0}}\right)$, too, there is a notion of classical generators. These are topological generators, $\boldsymbol{a}=\left(a_{1}, \ldots, a_{g}\right)$ and $\boldsymbol{b}=\left(b_{1}, \ldots, b_{g}\right)$, satisfying these properties.

(4.8a) The only relation in $\pi_{1}$ is the commutator product $\prod_{i=1}^{g}\left[a_{i}, b_{i}\right]=1$.

(4.8b) In $H_{1}\left(X_{\boldsymbol{p}_{0}}, \mathbb{Z}\right)$, the cup product pairing maps $\left(a_{i}, a_{j}\right)$ to $0,\left(b_{i}, b_{j}\right)$ to 0 , and $\left(a_{i}, b_{j}\right)$ to $\delta_{i j}$ (Kronecker $\delta$ function) for all $i$ and $j$.

Let $\pi_{1}\left(X_{\boldsymbol{p}_{0}}\right)^{(p)}=\pi_{1}^{(p)}$. As in Prop. 4.15 consider the collection $F_{\boldsymbol{p}_{0}}$ of homomorphisms $\tilde{\psi}: M_{\boldsymbol{p}_{0}} \rightarrow{ }_{p} \tilde{G}$ (up to inner action) having this property:

(4.9) $\tilde{\psi}$ induces the identity map $M_{\boldsymbol{p}_{0}} / \pi_{1}^{(p)} \rightarrow{ }_{p} \tilde{G} / \operatorname{ker}_{0}$.

Since ${ }_{p} \tilde{G} \rightarrow G_{0}$ is a Frattini cover, such a $\tilde{\psi}$ is necessarily surjective. Denote the surjective homomorphisms in $\operatorname{Hom}\left(\pi_{1}^{(p)}, \operatorname{ker}_{0}\right)$ by $\operatorname{Hom}^{*}\left(\pi_{1}^{(p)}, \operatorname{ker}_{0}\right)$. Thus, $F_{\boldsymbol{p}_{0}}$ maps into this space. Also, let $\mathbb{T}_{p}\left(X_{\boldsymbol{p}_{0}}\right)=\mathbb{T}_{p}=\pi_{1}^{(p)} /\left[\pi_{1}^{(p)}, \pi_{1}^{(p)}\right]$ denote the (dual of the) Tate module of $X_{\boldsymbol{p}_{0}}$.

Proposition 4.16. Let $K$ be a subfield of $\overline{\mathbb{Q}}$. Assume $\boldsymbol{p}_{0} \in \mathcal{H}\left(G_{0}, \mathbf{C}\right)(K)$ and $\mathcal{H}\left(G_{0}, \mathbf{C}\right)$ has fine moduli. Then, $G_{K}$ acts naturally on $\operatorname{Hom}^{*}\left(\pi_{1}^{(p)}, \operatorname{ker}_{0}\right) / \operatorname{ker}_{0}$. This permutes elements of $F_{\boldsymbol{p}_{0}}$, compatible (according to 3.2.1) with acting on coordinates of points $\boldsymbol{p} \in \mathcal{H}$. It induces a $G_{K}$ action on $\operatorname{Hom}^{*}\left(\mathbb{T}_{p}, \operatorname{ker}_{0} /\left(\operatorname{ker}_{0}, \operatorname{ker}_{0}\right)\right)$.

Proof. The action of $G_{K}$ on $\pi_{1}\left(X_{\boldsymbol{p}_{0}}\right)$ alg (3.2.1) induces an action on any closed subgroup of $\pi_{1}\left(X_{\boldsymbol{p}_{0}}\right)^{\text {alg }}$. For $\sigma \in G_{K}$ denote its fixed field by $K_{\sigma}$. Let $\boldsymbol{p} \in \mathcal{H}$, so it defines a homomorphism $\psi_{\boldsymbol{p}}: \pi_{1}\left(X_{\boldsymbol{p}_{0}}\right)^{\text {alg }} \rightarrow{ }_{p} \tilde{G}$. Then, $\boldsymbol{p}$ is a projective system of points over $K_{\sigma}$ of $\sigma$ if and only if $\sigma$ normalizes the kernel of $\psi_{\boldsymbol{p}}$, and the induced action of $\sigma$ on the quotient is trivial. Since levels of the Modular Tower are fine moduli spaces, this produces the desired sequence of Galois covers over $K_{\sigma}$.

The argument reverses. Further, $\sigma$ acts on $\pi_{1}^{(p)} /\left(\pi_{1}^{(p)}, \pi_{1}^{(p)}\right)$, a characteristic subgroup of $\pi_{1}^{(p)}$ (on which $G_{K}$ acts by hypothesis). The image of $\psi_{\boldsymbol{p}}$ in ${ }_{p} \tilde{G}$ is into $\left(\operatorname{ker}_{0}, \operatorname{ker}_{0}\right)$. This induces a $G_{K}$ action on $\operatorname{Hom}^{*}\left(\mathbb{T}_{p}, \operatorname{ker}_{0} /\left(\operatorname{ker}_{0}, \operatorname{ker}_{0}\right)\right)$.

Thm. 6.1 says $G_{K}$ has no fixed points on $F_{\boldsymbol{p}_{0}}$. More generally, there are no $K_{\sigma}$ points $\boldsymbol{p} \in \mathcal{H}$ if $\sigma$ induces the Frobenius on $\mathbb{T}_{\ell}$ for some prime $\ell$ not dividing $\left|G_{0}\right|$. This topic continues in $\leqq$

\section{Group theory of an $A_{5}$ Modular Tower}

A Modular Tower has levels corresponding to a sequence of groups

$$
\cdots \rightarrow G_{k+1} \rightarrow G_{k} \rightarrow \cdots \rightarrow G_{0} \text { (Prop. 3.21). }
$$

If $G_{0}$ is centerless and $p$-perfect, each $G_{k}$ is a centerless (Prop. 3.21) Frattini extension of $G_{0}$ with $p$-group as kernel. Frattini extensions of perfect groups are 
perfect: Commutators of the covering group generate the image, so they generate the covering group.

$\$ 5.1$ recounts the geometry behind a Frattini cover. $\$ 5.2$ extends the discussion of FJ86 Chap. 20] on the universal $p$-Frattini cover of $G_{0}$, starting with the case when its $p$-Sylow is normal. 5.3 describes the groups $G_{k}$ when $G_{0}=A_{5}$. [Fri95a, Part II] describes some aspects of the universal Frattini cover $\tilde{A}_{5}$ of $A_{5}$, especially the ranks of the Universal $p$-Frattini kernels. Then, $\tilde{A}_{5}$ has three pieces ${ }_{2} \tilde{A}_{5},{ }_{3} \tilde{A}_{5}$ and ${ }_{5} \tilde{A}_{5}$, one for each prime $p$ dividing $\left|A_{5}\right|$ (3.3). We use [FK97, Frattini Principle 2.3] to enhance and simplify properties of the characteristic modules $M_{k}$ $\left(\operatorname{ker}_{k} / \operatorname{ker}_{k+1} \stackrel{\text { def }}{=} M_{k}\right.$ in 3.3 .1$)$ of ${ }_{2} \tilde{A}_{5}:{ }_{p} \tilde{G}$ when $G=A_{5}$ and $p=2$.

5 5.5 shows only two Modular Towers for $A_{5}, p=2$ and $r=4$ have $\mathbb{Q}$ points at level 1. Then, 6 explains H-M and near H-M representatives. When $p=2$, these describe connected components of real points on levels 1 and above of a Modular Tower. As a prelude for 996.4 uses $j$-line branch cycles (4.2) for diophantine conclusions about regular realizations. This describes real point components on level 0 of the $\left(A_{5}, \mathbf{C}_{3^{4}}\right)$ Modular Tower. This paper uses properties of $M_{1}$ as a $G_{1}$ module.

5.1. Frattini curve covers. Let $\varphi: X \rightarrow Z$ be a Galois cover with group $G$, and let $\psi: Y \rightarrow X$ be a cover for which $\varphi \circ \psi$ is Galois with group $G^{*}$. Call $\varphi \circ \psi$ a Frattini extension of $\varphi$ if the following holds. For any sequence $Y \rightarrow W \rightarrow Z$, of (not necessarily Galois) covers with $W \neq Z$, there is always a proper cover of $Z$ through which both $W \rightarrow Z$ and $X \rightarrow Z$ factor. A Frattini extension of $\varphi$ has no differentials and functions that are pullbacks from covers disjoint from $\varphi$.

For a field theoretic restatement of the property let $K \subset \hat{L} \subset \hat{M}$ be a chain of fields with $\hat{M} / K$ (resp. $\hat{L} / K$ ) Galois with group $G^{*}$ (resp. $G$ ). This is a Frattini chain if the only subfield $K \leq T \leq \hat{M}$ for which $T \cap \hat{L}=K$, is $T=K$. Denote by res* $^{*} G^{*} \rightarrow G$ the natural map.

Let $T=\hat{M}^{H}$ be the fixed field of a subgroup $H$ of $G^{*}$. Then, $T \cap \hat{L}=K$ is equivalent to res* $H \rightarrow G$. Hint: $T \cap \hat{L}=K$ allows extending any automorphism of $\hat{L}$ to $T \cdot \hat{L}$ to be the identity on $T$. The group theoretic restatement is that $H \leq G^{*}$ and res* $(H)=G$ implies $H=G^{*}:$ res* $^{*} G^{*} \rightarrow G$ is a Frattini cover. A Frattini cover $G^{*} \rightarrow G$ always has a nilpotent kernel.

\subsection{The normalizer of a $p$-Sylow and Loewy display.}

5.2.1. Starting with a $p$-Sylow. Let $H=N_{G}\left(P_{p}\right)$ be the normalizer of a $p$-Sylow $P_{p}$ of any finite group $G$. Apply Schur-Zassenhaus to write $H$ as $P_{p} \times{ }^{s} H^{*}$ with $H^{*}$ having order prime to $p$. Let ${ }_{p} \tilde{F}_{t}$ be the pro-free pro- $p$ group on $t$ generators, with $t$ the rank (minimal number of generators) of $P_{p}$. Then, the universal $p$-Frattini cover of $H$ is ${ }_{p} \tilde{F}_{t} \times{ }^{s} H^{*}$; extend $H^{*}$ acting on $P_{p}$ to ${ }_{p} \tilde{F}_{t}$ through the map $\psi:{ }_{p} \tilde{F}_{t} \rightarrow P_{p}$ as in Remark 5.2

For any group $G, \operatorname{ker}_{0}(G)$ is the pro-free pro- $p$ kernel of ${ }_{p} \tilde{G} \rightarrow G$. Commutators and $p$ th powers in $\operatorname{ker}_{0}$ generate $\operatorname{ker}_{1}(G)$, the Frattini subgroup of $\operatorname{ker}_{0}(G)$. Iterating this produces $\operatorname{ker}_{k}(G) \leq \operatorname{ker}_{k-1}(G)$. Let $\operatorname{ker}_{0}(G) / \operatorname{ker}_{1}(G)=M(G)$ be the first characteristic quotient of the universal $p$-Frattini cover of $G$. Then $M(G)$ is a $\mathbb{Z} / p[G]$ module and $G_{1}={ }_{p} \tilde{G} / \operatorname{ker}_{1}$ is a Frattini extension of $G$ by $M(G)$.

LemMA 5.1. Let $W$ be any subgroup of $G$. Then, ${ }_{p} \tilde{W}$ embeds in ${ }_{p} \tilde{G}$. Further, for each $k \geq 0, W_{k}$ naturally embeds in $G_{k}$. If $\operatorname{rk}(M(W))=\operatorname{rk}(M(G))$, then ${ }_{p} \tilde{W}$ 
appears from the universal p-Frattini cover $\tilde{\varphi}:{ }_{p} \tilde{G}$ as $\tilde{\varphi}^{-1}(W)$. For $H=N_{G}\left(P_{p}\right)$ this applies if $\operatorname{rk}\left(P_{p}\right)=\operatorname{rk}(M(G))$.

Proof. A $p$-Sylow of ${ }_{p} \tilde{G}$ contains a $p$-Sylow of $\tilde{\varphi}^{-1}(W)$. So, the latter is profree. [FJ86 Prop. 20.33] characterizes ${ }_{p} \tilde{W}$ as the minimal cover of $W$ with pro-free $p$-Sylow. So, there is a natural map $\gamma_{W}: \tilde{\varphi}^{-1}(W) \rightarrow{ }_{p} \tilde{W}$ commuting with the map to $W$. As ${ }_{p} \tilde{W}$ is a $p$-Frattini cover of $W$ the map is surjective. Since the natural map $\tilde{\varphi}^{-1}(W) \rightarrow W$ has a pro- $p$ group as kernel, the natural map ${ }_{p} \tilde{W} \rightarrow W$ produces $\psi_{W}:{ }_{p} \tilde{W} \rightarrow \tilde{\varphi}^{-1}(W)$ commuting with the projections to $W$.

The composition $\gamma_{W} \circ \psi_{W}$ (commuting with the projections to $W$ ) is an endomorphism of ${ }_{p} \tilde{W}$. The image of $\gamma_{W} \circ \psi_{W}$ is a closed subgroup of ${ }_{p} \tilde{W}$ mapping surjectively to $W$. So, from the Frattini property, $\gamma_{W} \circ \psi_{W}$ is onto. An onto endomorphism of finitely generated profinite groups is an isomorphism [FJ86. Prop. 15.3]. In particular, $\psi_{W}$ is an injection. The characteristic quotients have maps between them induced by $\psi_{W}$, and so $W_{k}$ injects into $G_{k}$, inducing an injection of $\operatorname{ker}_{k}(W) / \operatorname{ker}_{k+1}(W) \rightarrow \operatorname{ker}_{k}(W) / \operatorname{ker}_{k+1}(W)$. If (for $k=0$ ), $M(W)$ and $M(G)$ have the same dimension, they are isomorphic. As these groups characterize $\operatorname{ker}_{0}(W)$ and $\operatorname{ker}_{0}(G)$, that implies they are equal. This gives an isomorphism of $\operatorname{ker}_{0}(H)$ and $\operatorname{ker}_{0}(G)$ in the special case.

REMARK 5.2 (Extending $H^{*}$ ). Since ${ }_{p} \tilde{F}_{t}$ is a pro-free pro- $p$ group, it is easy to create a profinite group $\bar{H}^{*}$ whose action on ${ }_{p} \tilde{F}_{t}$ extends $H^{*}$ on $P_{p}$. Any $h \in H^{*}$ lifts to an automorphism $\bar{h}$ of ${ }_{p} \tilde{F}_{t}$ commuting with the map to $P_{p}$. The (profinite) automorphism group of ${ }_{p} \tilde{F}_{t}$ is the projective limit of automorphism groups of finite group quotients (by finite index characteristic subgroups) of ${ }_{p} \tilde{F}_{t}$.

Let $\left\{h_{i} \mid i \in I\right\}$ be any generators of $H^{*}$. Choose $\bar{H}^{*}$ to be the closure of $\left\langle\bar{h}_{i} \mid i \in I\right\rangle$ in the automorphism group of ${ }_{p} \tilde{F}_{t}$. By construction it maps surjectively to $H^{*}$. The problem is to split off a copy of $H^{*}$. Automorphisms of ${ }_{p} \tilde{F}_{t}$ trivial on its Frattini quotient have $p$-power order Ha63 Thm. 12.2.2: Philip Hall]. The kernel from $\bar{H}^{*} \rightarrow H^{*}$ will be trivial on the Frattini quotient of ${ }_{p} \tilde{F}_{t}$ (which equals the Frattini quotient of $P_{p}$ ). So Schur-Zassenhaus (for profinite groups, [FJ86. Chp. 10]) always allows splitting off a copy of $H^{*}$ in $\bar{H}^{*}$. Since, however, it depends on $\psi$, it is an art to do this explicitly. Again by Schur-Zassenhaus, this copy of $H^{*}$ is unique up to conjugacy.

5.2.2. Appearance of $1_{G_{k}}$ in $M_{k}$. That $\operatorname{ker}_{k} / \operatorname{ker}_{k+1} \stackrel{\text { def }}{=} M_{k}$ in 3.3 .1 is a $\mathbb{Z} / p\left[G_{k}\right]$ module appears in using modular representation theory at each level of a Modular Tower. Significantly, $M_{k}$ is not a $G_{k-1}$ module (though it may have nontrivial quotients that are). For each $k$, however, $M_{k}$ is an $H^{*}$ module through this lifted action. Let $P_{p, k}$ be a $p$-Sylow of $G_{k}$ and $M_{k}\left(P_{p, k}\right)\left(\operatorname{resp} . M_{k}\left(P_{p, k} \times{ }^{s} H^{*}\right)\right.$ the restriction of $M_{k}$ to $P_{p, k}$ (resp. the normalizer of $P_{p, k}$ in $G_{k}$ ).

Recall the Loewy display of composition factors for a $G$ module $N$. It derives from the radical submodules of $N$,

$$
1 \leq N_{t} \leq N_{t-1} \leq \cdots \leq N_{1} \leq N_{0}=N:
$$

$N_{i}$ is the minimal $G$ submodule of $N_{i-1}$ with $N_{i-1} / N_{i}$ semi-simple. The display consists of writing the simple module summands of $N_{i-1} / N_{i}$ at the $i-1$ position with arrows indicating relations between modules at different levels. 
Warning! A Loewy display is not a sequence of module homomorphisms; it indicates the relation between modules at different levels in the Krull decomposition. We write this display right to left, compatible with the quotients from an exact sequence, instead of top to bottom (as group theorists often do). A Loewy display of all information on subquotients of a module often requires several arrows between layers; there may be several arrows from a simple level $i$ module toward level $i-1$ modules (see the examples of Prop. [5.7). Note: Such a display uniquely determines a module.

The Loewy display of $M_{k}\left(P_{p, k}\right)$ consists of copies of $\mathbf{1}_{P_{p, k}}$, the only simple $p$ group module. Jenning's Theorem [Ben91, p. 87] is an efficient tool to figure the dimension of the Loewy layers of $M_{k}\left(P_{p, k}\right)$. (The proof of Prop.9.17has an example of its use.) Further, since the action of $H^{*}$ respects this construction, it efficiently reveals how $H^{*}$ acts on the Poincaré-Witt basis of the $P_{p, k}$ group ring's universal enveloping algebra. The inductive detection of $\mathbf{1}_{H^{*}}$ on Loewy layers of $M_{k}\left(P_{p, k}\right)$ often comes through $\mathbf{1}_{H^{*}}$ appearing from a previous Loewy layer, or from tensors products of representations from previous Loewy layers (like a representation and its complex conjugate appearing juxtaposed). The following argument (a collaboration with D. Semmen) for the $p$-split case shows $\mathbf{1}_{H^{*}}$ usually appears quickly unless the $p$-Sylow is cyclic. Princ. 5.13 already shows $\mathbf{1}_{G}$ appears at infinitely many levels if $G$ has a center.

Proposition 5.3 ( $p$-split case). Let $G=P \times{ }^{s} H$ be centerless, with $P$ a $p$ group and $(|H|, p)=1$. If $P$ is not cyclic, $\mathbf{1}_{G}$ appears infinitely often. Otherwise, $M_{k}(G)$ is the same cyclic $G$ module for each $k$. So, $1_{G}$ does not appear.

Proof. By replacing $P$ by $P / \Phi(P)$, with $\Phi(P)$ its Frattini subgroup, form $P / \varphi(P) \times{ }^{s} H=G^{*}$. The universal $p$-Frattini cover of $P / \varphi(P) \times{ }^{s} H$ is the same as that of $G$. So, Princ. 5.13 shows $1_{G^{*}}$ occurring at some level for $G^{*}$ implies it occurs at infinitely many levels for $G$. So, for our question, with no loss take $G=G^{*}$ to assume $P$ is an elementary $p$-group and a $\mathbb{Z} / p[G]$ module.

Then, with $P_{\mathbf{1}}$ the projective indecomposable for $\mathbf{1}_{P}$, consider it as an $H$ (therefore a $G)$ module. Since $((G: P), p)=1$, Higman's criterion says it is the projective indecomposable for $\mathbf{1}_{G}$ Ben91 p. 64]. Its most natural display might by $\mathbb{Z} / p[G]\left(\sum_{h \in H} h\right)$. In its Loewy display $\mathbf{1}_{G}$ appears to the far right. Write the next Loewy layer as $S_{1} \oplus \cdots \oplus S_{t}$, with the $S_{i}$ s irreducible $H(G)$ modules.

Forming $P_{1}^{*}$ (* is the $\mathbb{Z} / p$ dual) returns $P_{\mathbf{1}}$. So the dual of the $v$ th socle layer of $P_{\mathbf{1}}$ is the $v$ th Loewy layer of $P_{\mathbf{1}}$. Conclude that to the far left there is $\mathbf{1}_{G}$ (1st socle layer) with $S_{1}^{*} \oplus \cdots \oplus S_{t}^{*}$ immediately to the right of that (2nd socle layer). Now we apply that $P$ is an elementary $p$-group. The $P$ module $P_{1}$ is the group ring $\mathbb{Z} / p[P]$. Let $x_{1}, \ldots, x_{m}$ be a basis for $P$ and identify $P$ in the vector space $\mathbb{Z} / p[P]$ as the space spanned by $\left\{x_{1}-1, \ldots x_{m}-1\right\}$. The action of $H$ on $\mathbb{Z} / p[P]$ preserves this space modulo the second power of the augmentation ideal of $\mathbb{Z} / p[G]$. Conclude: $P \equiv S_{1} \oplus \cdots \oplus S_{t}$ as $H$ (or $G$ ) modules.

As in Prop. 9.17 use the notation of [Fri95a Part II]. So, the kernel $\Omega(1, G)$ of $P_{\mathbf{1}} \rightarrow \mathbf{1}_{G}$ starts with $S_{1} \oplus \cdots \oplus S_{t}$. Now consider a minimal projective $P_{\Omega(1, G)}$ mapping surjectively to $\Omega(1, G)$. Higman's criterion again implies the $G$ module $P_{\mathbf{1}_{G}} \otimes_{G}\left(S_{1} \oplus \cdots S_{t}\right)$, being projective for $P$, is also projective for $G$. The Loewy layers of $T=P_{\mathbf{1}_{G}} \otimes_{G}\left(S_{1} \oplus \cdots S_{t}\right)$ are just the Loewy layers of $P_{\mathbf{1}_{H}}$ tensored over $\mathbb{Z} / p[H]$ with $S_{1} \oplus \cdots S_{t}$ modules. Reason: a Loewy layer of $P_{\mathbf{1}_{G}}$ tensored with 
$S_{1} \oplus \cdots S_{t}$ is semisimple. Therefore $T=P_{\Omega(1, G)}$. Then,

$$
M(G)=M_{0}(G) \equiv \Omega(2, G)=\operatorname{ker}(T \rightarrow \Omega(1, G))
$$

modulo projective summands (as in Fri95a, Projective Indecomposable Lem. 2.3], called $\Omega^{2}\left(\mathbf{1}_{G}\right)$ there). The 2 nd socle layer of $\Omega(2, G)$ is $\left(S_{1}^{*} \oplus \cdots \oplus S_{t}^{*}\right) \otimes_{G}\left(S_{1} \oplus \cdots \oplus S_{t}\right)$ with one copy of $1_{G}$ removed (from the end of $\Omega(1, G)$. The module $S_{i} \otimes_{G} S_{i}^{*}=$ $S_{i} \otimes_{H} S_{i}^{*}$ has exactly one appearance of $\mathbf{1}_{H}$ for each absolutely irreducible factor in $S_{i} \otimes_{\mathbb{Z} / p} \overline{\mathbb{Z}} / p$ (Maschke's Theorem; since $P$ acts trivially on $S_{i}$, the same is true of $\left.\mathbf{1}_{G}\right)$. Thus, $\mathbf{1}_{G}$ appears in the Loewy display of $\Omega(2, G)$ as a $G$ module unless $t=1$, and $S_{1}$ is absolutely irreducible.

Now suppose $t=1$. Instead of looking at $M_{0}(P)=M_{0}(G)$, look at $M_{1}(P)$ as an $H$ module. By our hypotheses, $P$ is not a cyclic module. Therefore, a computation of the rank of $M_{1}(P)$ comes directly from Schreier's formula for ranks of subgroups of pro-free groups (see $\mathrm{E}$.2.1). The rank of $M_{k}$, as $k$ increases, exceeds the degree of any irreducible $H$ module. Replace $P$ with a suitable $M_{k}$ to revert to the case $P$ is not absolutely irreducible. This completes showing the appearance of $\mathbf{1}_{G}$.

The structure constant formula $(\$ 2.7 .2)$ can detect $\mathbf{1}_{H^{*}}$ appearing here, as $\left(\left|H^{*}\right|, p\right)$ means it applies in characteristic $p$. This topic continues in 55.7

5.3. Producing ${ }_{2} \tilde{A}_{5}$. Lemma $\left[5.1\right.$ hypotheses imply $M(H)$ is a $\mathbb{F}_{p}[G]$ module.

5.3.1. Converse to Lemma 5.1. In the proof of Lemma 5.1] $\gamma_{H}: \tilde{\varphi}^{-1}(H) \rightarrow{ }_{p} \tilde{H}$ induces a surjective $H$ module map $\gamma_{H}^{\prime}: M(G) \rightarrow M(H)$.

Proposition 5.4. Suppose $H=N_{G}\left(P_{p}\right)$ ( $P_{p}$ a p-Sylow of $G$ ) and $M(H)$ is a $G$ module extending the $H$ action so the following holds.

(5.1) There is an extension $G_{1}^{*} \rightarrow G$ with kernel $M(H)$ so the pullback of $H$ in $G_{1}^{*}$ is the natural quotient ${ }_{p} \tilde{H} / \operatorname{ker}_{1}(H)$.

Then, the Lemma [5.1 conclusion holds. This applies with $p=2$ and $A_{5}=G$.

Proof. The hypothesis says the morphism $\gamma_{H}^{\prime}$, a priori an $H$ module homomorphism, is actually a $G$ module homomorphism. Here is why. Suppose $G^{\dagger}$ is a proper subgroup of $G_{1}^{*}$ mapping surjectively to $G$. Then, the pullback $H^{*}$ of $H$ in $G^{\dagger}$ is a proper subgroup of the pullback of $H$ in $G_{1}^{*}$. Further, $H^{*}$ maps surjectively to $H$, contrary to ${ }_{p} \tilde{H} / \operatorname{ker}_{1}(H) \rightarrow H$ being a Frattini cover. Conclude that $G_{1}^{*} \rightarrow G$ is also a Frattini cover. So, there is a natural map from $G_{1}={ }_{p} \tilde{G} / \operatorname{ker}_{1}(G) \rightarrow G_{1}^{*}$ inducing a surjective $G$ module homomorphism $M(G) \rightarrow M(H)$.

Since ${ }_{p} \tilde{H} / \operatorname{ker}_{1}(H)$ is the 1 st characteristic quotient of ${ }_{p} \tilde{H}$, universal for covers with elementary $p$-group kernel, there is an $H$ module splitting of $M(G) \rightarrow M(H)$. Higman's Theorem Ben91 p. 64, Prop. 3.6.4] says, since $((G: H), p)=1$, an $H$ splitting of this $G$ map gives a $G$ splitting. This is contrary to $M(G)$ being an indecomposable $G$ module unless this is an isomorphism ([Ben91, p. 11, Exec. 1] or FK97, Indecom. Lem. 2.4]).

Suppose a $p$-Sylow $P_{p}$ of $G$ has this property: Either

$$
g P_{p} g^{-1}=P_{p} \text { or } g P_{p} g^{-1} \cap P_{p}=\{1\}
$$

for each $g \in G$. Ben91, Cor. 3.6.19] shows

$$
H^{2}(G, M(H)) \stackrel{\text { rest }}{\longrightarrow} H^{2}(H, M(H))
$$


is an isomorphism (so both have dimension 1) guaranteeing (5.1). This holds for $A_{5}$ and its 2-Sylows since they are distinguished by which integer from $\{1, \ldots, 5\}$ each element in the 2-Sylow fixes.

Remark 5.5 (Cases and extensions of Prop.5.4). Suppose $G_{k}, k \geq 1$ is a higher characteristic quotient of ${ }_{p} \tilde{G}$. Let $P_{p, k}$ be a $p$-Sylow of $G_{k}$. Then, the hypotheses of Prop. 5.4 automatically hold.

Even when $k=0$, given $P_{p}$, suppose $H_{1} \leq G$ contains $H=N_{G}\left(P_{p}\right)$ and is maximal for this property.

(5.2) For some extension $H_{1}^{*} \rightarrow H$ with kernel $M(H)$, the pullback of $H$ in $H_{1}^{*}$ is the natural quotient ${ }_{p} \tilde{H} / \operatorname{ker}_{1}(H)$.

As in [Fri95a, Rem. 2.10], let $\operatorname{Ind}(M(H))_{H_{1}}^{G}$ be the $G$ module induced from $H_{1}$ acting on $M(H)$. Apply Shapiro's Lemma Ben91, Cor. 2.8.4]: $H^{2}\left(H_{1}, M(H)\right)=$ $H^{2}\left(G, \operatorname{Ind}(M(H))_{H_{1}}^{G}\right)$. So there is an extension of $G$ with kernel $\operatorname{Ind}(M)_{H_{1}}^{G}$ whose pullback over $H_{1}$ has $H_{1}^{*}$ as a quotient. Fr02 uses $\operatorname{Ind}(M(H))_{H_{1}}^{G}$ to produce the characteristic $p$-Frattini module $M_{0}=M(G)$ from the $p$-split case using indecomposability of $M_{0}(G)$ [FK97, Indecom. Lem. 2.4].

5.3.2. Producing ${ }_{2}^{1} \tilde{A}_{5}$. As in Fri95a Part II], producing ${ }_{p} \tilde{A}_{5}$ for $p$ either 3 or 5 requires using the $G$ module induced from the $H$ module $M(H)$ (generalizing Prop. 5.4). Prop. 5.4 applies immediately to $A_{5}$ when $p=2$.

Proposition 5.6. Let $H=A_{4}=K_{4} \times^{s} \mathbb{Z} / 3$. Then, $M(H)$ identifies with the $\mathbb{Z} / 2[H]$ module generated by the six cosets of a $\mathbb{Z} / 2$ in $A_{4}$, modulo the module generated by the sum of the cosets. Any $D_{5}$ in $A_{5}$ has a unique $\mathbb{Z} / 2$ lying in $A_{4}$. So, the action of $A_{4}$ on $\mathbb{Z} / 2$ cosets extends to an $A_{5}$ action on cosets of a dihedral group. Thus, Prop. 5.4 gives ${ }_{2}^{1} \tilde{A}_{5}$ as an extension of $A_{5}$ by $\operatorname{ker}_{0}(H)$.

Proof. Suppose ${ }_{p} \tilde{F}_{t}$ is pro-free pro- $p$ group on $t$ generators. Let $\psi:{ }_{p} \tilde{F}_{t} \rightarrow P_{p}$ be a surjective homomorphism, with $P_{p}$ any (finite) $p$ group. Schreier's construction gives explicit generators of the kernel of $\psi$ [FJ86 \$15.6]. Apply this with $t=2$ and $P_{p}=K_{4}$, the Klein 4 -group and $\operatorname{ker}_{0}=\operatorname{ker}(\psi)$. Let $\bar{\alpha}$ be a generator of $\mathbb{Z} / 3$. For $u$ and $v$ generators of ${ }_{p} \tilde{F}_{2}$ let $\bar{\alpha}$ act on ${ }_{p} \tilde{F}_{2}$ by mapping $(u, v)$ to $\left(v^{-1}, v^{-1} u\right)$.

Use $S=\left\{1, u, v^{-1}, u^{-1} v\right\}$ as coset representatives for $\operatorname{ker}_{0}$ in ${ }_{p} \tilde{F}_{2}$. Form the set $V$ of elements in ker $_{0}$ having the form $t u s^{-1}$ or $t v s^{-1}$ with $s, t \in S$. Toss from $V$ those that equal 1. Now consider the images of $\bar{\alpha}$ and $\bar{\alpha}^{2}$ on $u u=u^{2}=m_{1} \in V$. This produces $v^{-1} v^{-1}=m_{2}$ and $u^{-1} v u^{-1} v=m_{3}$.

Consider $\bar{\alpha}$ on $m_{6}=u\left(v^{-1}\right)^{2} u^{-1}$. Recall: Modulo ker ${ }_{1}$ any two elements in ker $_{0}$ commute. Apply this to get

$$
v^{-1}\left(u^{-1} v\right)^{2} v=\left(v^{-1} u^{-1} v\right)\left(u^{-1}\right) v^{2} \quad \bmod \operatorname{ker}_{1}=v u^{-1} v u^{-1}=m_{5} .
$$

Apply $\bar{\alpha}$ again to get $v^{-1} u^{2} v=m_{4}$. The action of $K_{4} \times^{s} \mathbb{Z} / 3=A_{4}$ on the $m_{i}$ s is the same as the action on the six cosets of an element of order 2 .

Denote a commutator of two elements $w_{1}, w_{2}$ by $\left(w_{1}, w_{2}\right)$. Modulo ker $_{1}$ there are relations among the $m_{i} \mathrm{~s}: m_{1} m_{2} m_{3}=(u, v) \bmod \operatorname{ker}_{1} ;$ and $m_{4} m_{5} m_{6}=$ $(u, v)^{-1} \bmod \mathrm{ker}_{1}$. So, the product of the $m_{i} \mathrm{~s}$ is 1 . The proof follows from associating a $\mathbb{Z} / 2$ in $A_{4}$ with a dihedral in $A_{5}$ as in the statement of the proposition.

5.3.3. Summary of ${ }_{2}^{1} \tilde{A}_{5}$ properties. We finish a self-contained treatment of much of [Fri95a Part II]. The first characteristic quotient of the universal 2-Frattini cover of $A_{5}$ is a (nonsplit) extension of $\mathrm{SL}_{2}(\mathbb{Z} / 5)$ by an irreducible module $\mathrm{V}$ using 
$A_{5} \cong \mathrm{PSL}_{2}(\mathbb{Z} / 5) \cong \mathrm{PSL}_{2}\left(\mathbb{F}_{4}\right)$. Prop. [5.6 shows this as follows. As previously, let $M\left(A_{5}\right)$ be the kernel of ${ }_{2}^{1} \tilde{A}_{5} \rightarrow A_{5}$. Sums of $D_{5}$ cosets, $\sum_{i=1}^{6} a_{i} T_{i}$, represent its elements. The augmentation map sends such an element to the sum $\sum_{i=1}^{6} a_{i}$.

Let $V$ be the 4-dimensional kernel of the augmentation map. Nonzero elements of $V$ have representatives $\sum_{i=1}^{6} a_{i} T_{i}$ with two of the $a_{i}$ s nonzero. Let $\mathbb{Z} / 3$ be the subgroup $\langle\bar{\alpha}\rangle \leq A_{5}$ in the proof of Prop. 5.6. The action of $\mathbb{Z} / 3$ on $V$ is two copies of the 2-dimensional irreducible of $\mathbb{Z} / 3$. So, no element of $V$ centralizes $\mathbb{Z} / 3$ and the centralizer of $\mathbb{Z} / 3$ in $M=M\left(A_{5}\right)$ is a $\mathbb{Z} / 2$. Denote ${ }_{2}^{1} \tilde{A}_{5}$ by $G_{1}$. Let $P_{1}$ be a $p$-Sylow of $G_{1}$ with $P_{0}$, a Klein 4-group, its image in $A_{5}=G_{0}$. Denote the module for restriction of $M$ to a subgroup $H \leq A_{5}$ by $M_{H}$.

Corollary 5.7. Besides the origin there are three conjugacy classes (orbits for $A_{5}$ action) in $M$. These are (15) elements of $V \backslash\{0\} ; 10$ representing sums $\sum_{i=1}^{6} a_{i} T_{i}$ with exactly three $a_{i} s$ nonzero (in $M \backslash V$ ); and six representing sums $\sum_{i=1}^{6} a_{i} T_{i}$ with exactly one $a_{i}$ nonzero (in $M \backslash V$ ). Call the second set $M_{3}^{\prime}$ and the third $M_{5}^{\prime}$. Elements of $M_{3}^{\prime}$ (resp. $M_{5}^{\prime}$ ) are exactly those in $M \backslash\{0\}$ some 3-cycle (resp. 5-cycle) stabilizes. Correspond to each the respective 3 or 5-Sylow of $A_{5}$ that stabilizes it.

The action of $P_{0}$ on $M$ has a module presentation

$$
0 \rightarrow J\left(P_{0}\right) \stackrel{\text { def }}{=} \mathbf{1} \oplus \mathbf{1} \rightarrow M\left(A_{5}\right)_{P_{0}} \rightarrow \mathbf{1} \oplus \mathbf{1} \oplus \mathbf{1}=M / J\left(P_{0}\right) \rightarrow 0 .
$$

Extending this action to $A_{4}$ gives a Loewy display

$$
0 \rightarrow U_{3} \rightarrow U_{3} \oplus \mathbf{1}
$$

with $U_{3}$ the two dimensional $A_{4}$ module on which $\mathbb{Z} / 3$ acts irreducibly. Restrict $A_{4}$ to $M$ for an exact sequence $0 \rightarrow J\left(A_{4}\right) \rightarrow M\left(A_{5}\right)_{A_{4}} \rightarrow \mathbf{1} \rightarrow 0$. The Loewy display of $J\left(A_{4}\right)$ (resp. $\left.\left\langle 1_{H}+3_{H}+5_{H}, J\left(P_{0}\right)\right\rangle\right)$ is $U_{3} \rightarrow U_{3}$ (resp. $U_{3} \rightarrow 1$ ).

If $g \in G_{1}$ has order 3 or 5 and $m \in M$ then, giving mgm and stipulating $m \in M \backslash V$ determines $m$. Suppose $\alpha \in G_{1}$ has order 4. Then, the centralizer $Z_{\alpha}$ of $\alpha$ on $M$ has dimension 3 and on $V$ has dimension $2, \alpha^{2} \in M \backslash V$ and $Z_{\alpha}$ contains two elements each from $M_{3}^{\prime}$ and from $M_{5}^{\prime}$.

Proof. The action of $A_{5}$ preserves cosets. So, orbits for conjugation by $A_{5}$ are clear if $V \backslash\{0\}, M_{3}^{\prime}$ and $M_{5}^{\prime}$ each consist of one $A_{5}$ orbit. This follows from triple transitivity of $A_{5}$ in the standard representation.

The exact sequence (5.3) comes from writing the right cosets of $H=\langle(12)(34)\rangle$ in $A_{4}$. Use $1_{H}=H, 2_{H}=\{(13)(24),(23)(14)\}, 3_{H}=\{(123),(134)\}, 4_{H}=$ $\{(432),(142)\}, 5_{H}=\{(132),(234)\}$ and $6_{H}=\{(124),(314)\}$. Then, $J\left(P_{0}\right)$ has generators $1_{H}+2_{H}$ and $3_{H}+4_{H}$. Images of $1_{H}, 3_{H}$ and $5_{H}$ generate $M / J\left(P_{0}\right)$.

Consider $\alpha \in G_{1}$ of order 4 . To be explicit assume $\alpha=\alpha_{12}$ lifts (12)(34). Then, $\alpha$ fixes $1_{H}, 2_{H}$ and $3_{H}+4_{H}$, so it acts like $\beta_{\alpha}=(34)(56)$ on the cosets. The centralizer of $\alpha_{12}$ in $M\left(A_{5}\right)$ is therefore

$$
U=\left\langle\left(x_{1}, x_{2}, a_{1}, a_{1}, a_{2}, a_{2}\right) \mid a_{1}, a_{2}, x_{1}, x_{2} \in \mathbb{Z} / 2\right\rangle /\langle(1,1,1,1,1,1)\rangle .
$$

An element in $U$ is in $M \backslash V$ if and only if $x_{1} \neq x_{2}$. Note: $\alpha$ fixes $1_{H}, 2_{h} \in M_{5}^{\prime}$. Sum both with $3_{H}+4_{H}$ to get two elements in $M_{3}^{\prime}$. The observation that $\alpha^{2} \in M \backslash V$ is a special case of Prop. 5.10

Now consider the sequence (5.4). Write $A_{4}$ as $K_{4} \times^{s}\langle\beta\rangle$ with $\beta=(123)$. First: $J\left(P_{0}\right)=\mathbf{1} \oplus \mathbf{1}$ is an $\langle\beta\rangle$ module. As $\beta$ sends $1_{H}+2_{H}$ to $3_{H}+4_{H}$, it preserves the leftmost layer of the Loewy display. In the quotient $\mathbf{1} \oplus \mathbf{1} \oplus \mathbf{1}=M / J\left(P_{0}\right)$, 
$1_{H}$ and $3_{H}$ generate a copy of $U_{3}$. Then, $A_{4}$ fixes $1_{H}+3_{H}+5_{H} \bmod J\left(P_{0}\right)$ and $\left\langle 1_{H}+3_{H}, 3_{H}+5_{H}\right\rangle$ generates a copy of $U_{3}$.

The module $V_{A_{4}}$ has Loewy display $U_{3} \rightarrow U_{3}$. If there is a subquotient of $M\left(A_{5}\right)_{A_{4}}$ having a Loewy display $U_{3} \rightarrow 1$, it must be $T=\left\langle 1_{H}+3_{H}+5_{H}, J\left(P_{0}\right)\right\rangle$. Clearly this is invariant under $\mathbb{Z} / 3$. The action of (13)(24) is to take $1_{H}+3_{H}+5_{H}$ to $2_{H}+4_{H}+5_{H}$. Since $1_{H}+3_{H}+5_{H}$ and $2_{H}+4_{H}+5_{H}$ differ by an element of $J\left(P_{0}\right)$, this shows $T$ is invariant under $\langle(123),(13)(24)\rangle=A_{4}$.

Remark 5.8 (A $Q_{8}$ version of Prop. 5.6). We take advantage of the special coset aspect of module $M\left(A_{5}\right)$ throughout the study of our main example. The seed, however, is an explicit presentation of $M\left(P_{p}\right)$ as a $P_{p}$ module, with $P_{p}$ a $p$ Sylow of $G$. The proof of Prop. 9.17 shows, with illustration from $Q_{8}$ (versus $K_{4}$ above) an effective way to compute the analog of (5.3).

5.4. Cusps and a theorem of Serre. As with modular curves, the Prop.4.4 branch cycle description tells much about the reduced cover of the $j$-line, especially about its points over $\infty \in \mathbb{P}_{j}^{1}$, the cusps. Cusp widths are the lengths of these orbits. An analysis of possible cusp widths starts with a general group computation. We refer to computations in 2.7 .1

5.4.1. Lifting odd order elements. Suppose $g \in S_{n}$ has odd order (so necessarily $\left.g \in A_{n}\right)$. Write $g$ as a product $\prod_{i=1}^{t} \beta_{i}$ of disjoint cycles with $\beta_{i}$ of length $s_{i}$, $i=1, \ldots, t$. Define $\omega(g)$ to be $\sum_{i=1}^{t}\left(s_{i}^{2}-1\right) / 8$. Serre notes: $\omega(g) \equiv 0 \bmod 2$ if and only if $\prod_{i=1}^{t} s_{i} \equiv \pm 1 \bmod 8$. Let $\operatorname{Spin}_{n} \rightarrow A_{n}$ be the universal central exponent 2 extension of $A_{n}$. Regard the kernel of this map as $\{ \pm 1\}$.

Proposition 5.9 ([Ser90a $)$. Assume entries of $\boldsymbol{g}=\left(g_{1}, \ldots, g_{r}\right)$ (in $\left.A_{n}\right)$ have odd order. Lift to $\operatorname{Spin}_{n}$ the entries of $\boldsymbol{g}$, preserving their respective orders. Denote the lifted r-tuple by $\boldsymbol{g}^{*}$. Suppose the following hold.

(5.5a) Product-one condition: $\prod_{i=1}^{r} g_{i}=1$.

(5.5b) Transitivity: $\langle\boldsymbol{g}\rangle$ is a transitive subgroup of $A_{n}$.

(5.5c) Genus 0 condition: $\sum_{i=1}^{r} \operatorname{ind}\left(g_{i}\right)=2(n-1)$.

Then, $s(\boldsymbol{g})=\prod_{i=1}^{r} g_{i}^{*}=(-1)^{\sum_{i=1}^{r} \omega\left(g_{i}\right)}$.

The transitivity hypothesis in Prop. 5.9 is not serious. Restrict $\boldsymbol{g}$ to each orbit of $\langle\boldsymbol{g}\rangle$. If the genus 0 condition applies in each such restriction, then $s(\boldsymbol{g})$ is the product of the $s$ values appearing in each restriction. The genus 0 condition, however, is very serious. Examples of $\$ 5.5$ or [Fri95a Ex. 3.12] show how to use Prop. 5.9 to go beyond the genus 0 condition.

A preliminary example! Take $\boldsymbol{g}=((123),(123),(123))=\left(g_{1}, g_{1}, g_{1}\right)$. With $n=3$, the lifting invariant $s(\boldsymbol{g})$ is clearly $+1: \boldsymbol{g}^{*}=\left(g_{1}^{*}, g_{1}^{*}, g_{1}^{*}\right)$ with $g_{1}^{*}$ of order 3. The conclusion, however, of Prop. 5.9 seems to be $s\left(\boldsymbol{g}^{*}\right)=(-1)^{3}$. It, however, doesn't apply because the genus 0 condition doesn't hold.

5.5 .2 and Princ. 5.15 have cases of this computation without the genus 0 hypothesis; still by using Prop. [5.9

5.4.2. Lifting even order elements. Suppose $g \in G$ has even order, and $\hat{G} \rightarrow G$ is a central extension with kernel an elementary abelian 2- group. Let $\hat{g} \in G$ be a lift of $g$ to $\hat{G}$. Then, the order of $\hat{g}$ is independent of the choice of the lift. The following applies the technique of proof from Prop. 5.9 to analyze the orders of lifts of elements of order 2 to $\hat{A}_{n}$. We compare this to an analog question with $G_{1}$, the first characteristic quotient of ${ }_{2} \tilde{A}_{5}$ replacing $A_{n}$ (Lem. 9.10). 
Proposition 5.10. Assume $n \geq 4$, and $g \in A_{n}$ of order 2 is a product of $2 \mathrm{~s}$ disjoint 2-cycles. Any lift $\hat{g} \in \hat{A}_{n}$ of $g$ has order 4 if $s$ is odd and 2 if $s$ is even.

Proof. We review the Clifford algebra setup used in Ser90a. Let $C_{n}$ be the Clifford algebra on $\mathbb{R}^{n}$ with generators $x_{1}, \ldots, x_{n}$ subject to relations

$$
x_{i}^{2}=1,1 \leq i \leq n \text {, and } x_{i} x_{j}=-x_{j} x_{i} \text { if } i \neq j .
$$

In the Clifford algebra, write $[i j]=\frac{1}{\sqrt{2}}\left(x_{i}-x_{j}\right)$. Then, $[i j]^{2}=1$ and $[i j]=-[j i]$. The collection of $[i j]$ under multiplication generate a subgroup $\hat{S}_{n}$. Characterization: It is the central nonsplit extension of $S_{n}$ whose restriction to transpositions splits, and whose restriction to products of two disjoint transpositions is nontrivial Ser92 p. 97]. The map $\hat{S}_{n} \rightarrow S_{n}$ appears from $[i j] \mapsto(i j)$.

So, $\hat{A}_{n}=A_{n} \times\{ \pm 1\}$ if $n \leq 3$. That $\hat{A}_{n} \rightarrow A_{n}$ is nontrivial if $n \geq 4$ shows from lifts of certain elements of order 2. Example: $(12)(34)$ lifts to have order 4 :

$$
\left(\frac{1}{\sqrt{2}}\left(x_{1}-x_{2}\right) \frac{1}{\sqrt{2}}\left(x_{3}-x_{4}\right)\right)^{2}=-[12]^{2}[34]^{2}=-1 .
$$

Of course the order of a lift is conjugacy class invariant. Similarly, with $n \geq 8$,

$$
([12][34] \ldots[s-1 s])^{2}=(-1)^{2(s-2)}([12][34])^{2}([56] \ldots[s-1 s])^{2} .
$$

By induction, the result is $(-1)^{s}:[12][34] \ldots[s-1 s]$ has order $2^{1+\frac{1-(-1)^{s}}{2}}$.

5.5. Further Modular Towers for $A_{5}, p=2$ and $r=4$. Prop. 5.11 gives a partial answer to (1.7 about existence of rational points at level 1 of other $A_{5}$ Modular Towers. There are three $2^{\prime}$ classes: the two classes of 5-cycles, $\mathrm{C}_{5}^{+}$ (the class of (12345)) and $\mathrm{C}_{5}^{-}$(the class of (13524)) and 3-cycles. From the Branch Cycle Lemma, excluding $\mathbf{C}_{3^{4}}$, to give a Galois cover over $\mathbb{Q}$ with $r=4$ requires $\mathbf{C}$ be $\mathbf{C}_{5_{+} 5_{-} 3^{2}}=\left(\mathrm{C}_{5}^{+}, \mathrm{C}_{5}^{-}, \mathrm{C}_{3}, \mathrm{C}_{3}\right)$ or $\mathbf{C}_{5_{+}^{2} 5_{-}^{2}}=\left(\mathrm{C}_{5}^{+}, \mathrm{C}_{5}^{-}, \mathrm{C}_{5}^{+}, \mathrm{C}_{5}^{-}\right)$. Both give Modular Towers with two components at level 0 , one obstructed (nothing above it at level 1), one not.

Proposition 5.11. The only Modular Towers for $A_{5}, r=4$ and $p=2$ with possible $\mathbb{Q}$ components at level 1 (for realizations of $\frac{1}{2} \tilde{A}_{5}$ ) have $\mathbf{C}=\mathbf{C}_{3^{4}}, \mathbf{C}_{5_{+} 5_{-} 3^{2}}$ or $\mathbf{C}_{5_{+}^{2} 5_{-}^{2}}$. Each of the latter two has exactly two level 0 components, one obstructed, one not. There are no $\mathbb{R}($ so, no $\mathbb{Q})$ points at level 1 of the $\mathbf{C}_{5_{+} 5_{-} 3^{2}}$ Modular Tower.

The next four subsections prove this by applying a lift to $\mathrm{SL}_{2}(\mathbb{Z} / 5)$ in the two new cases. For computational reasons, start with $\mathbf{C}_{5_{+} 5_{-} 3^{2}}$.

Remark 5.12. Ex. 9.3 notes the two genus 1 components at level 1 on the Modular Tower for $\left(A_{5}, \mathbf{C}_{5_{+}^{2} 5_{-}^{2}}\right)$ over the level 0 unobstructed component. If have definition field $\mathbb{Q}$, they might have infinitely many ${ }_{2}^{1} \tilde{A}_{5}$ realizations over $\mathbb{Q}$. This is the only hope for infinitely many (up to $\mathrm{PSL}_{2}(\mathbb{C})$ equivalence) $\mathbb{Q}$ regular realizations of ${ }_{2}^{1} \tilde{A}_{5}$ with $r=4$. Applying 99.6 to this to see there will be at most finitely many realizations ${ }_{2}^{2} \tilde{A}_{5}$ for $r=4$.

5.5.1. Setup for Prop. 5.11. Suppose $\boldsymbol{g} \in \mathrm{Ni}\left(A_{5}, \mathbf{C}_{5_{+} 5_{-} 3^{2}}\right)$ is in the $H_{4}$ orbit $O_{\boldsymbol{g}}$. Then, there is $\boldsymbol{g}^{*} \in \operatorname{Ni}\left({ }_{2}^{1} \tilde{A}_{5}, \mathbf{C}_{5_{+} 5_{-} 3^{2}}\right)$ over $\boldsymbol{g}$ if and only if there is something from $\mathrm{Ni}\left({ }_{2} \tilde{A}_{5}, \mathbf{C}_{5_{+} 5_{-} 3^{2}}\right)$ over each element of $O_{\boldsymbol{g}}$. If this holds, call $O_{\boldsymbol{g}}$ unobstructed (1.3.5. We list inner Nielsen classes $\mathrm{Ni}^{\text {in }}$ for $\mathbf{C}_{5_{+} 5_{-} 3^{2}}$ restricting to pieces where 
it is easy to demonstrate they lie in one $H_{4}$ orbit. With no loss use representatives for $\boldsymbol{g} \in \mathrm{Ni}^{\text {in }}$ with $g_{1}=\left(\begin{array}{l}1 \\ 2\end{array} 345\right) \in \mathrm{C}_{5}^{+}$and $g_{2} \in \mathrm{C}_{5}^{-}$.

Conjugate the collection of 5 -cycles by $g_{1}$. There are four length one and four length five orbits. Two length five orbits of $g_{1}, U_{1}$ and $U_{2}$, are 5 - cycles in $\mathrm{C}_{5}^{-}$. There are two cases:

(5.6a) $g_{2}=g_{1}^{2}$ or $g_{1}^{3}, g_{3}$ and $g_{4}$ have 1 in their common support; or

(5.6b) $g_{2}$ is a representative from $U_{1}$ or $U_{2}$.

Here are $g_{2}$ representatives for $(5.60)$ :

$$
g_{2,1}=(21345) \text { and } g_{2,2}=(12543) .
$$

5.5.2. Separating obstructed and unobstructed $\mathrm{H}_{4}$ orbits. We show the orbit of $\boldsymbol{g}$ satisfying (5.61) is obstructed. Example: $\boldsymbol{g}=((12345),(13524),(351),(241))$. Suppose $\boldsymbol{g}^{\prime} \in \operatorname{Ni}\left({ }_{2}^{1} \tilde{A}_{5}, \mathbf{C}_{5_{+} 5_{-} 3^{2}}\right)$ lies over $\boldsymbol{g}$. Consider the image $\boldsymbol{g}^{*}$ in $\mathrm{SL}_{2}(\mathbb{Z} / 5)$ of $\boldsymbol{g}^{\prime}$, as in proof of Lem. [8.9] Denote the product of the entries of $\boldsymbol{g}^{*}$ by $s(\boldsymbol{g})$. Then, $g_{2}^{*}=\left(g_{1}^{*}\right)^{2}$. The product-one condition, $s(\boldsymbol{g})=1$, holds for $\boldsymbol{g}^{*}$ if and only if it holds

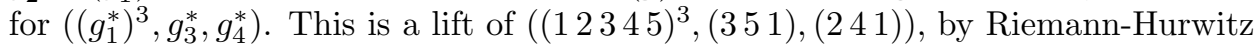
a branch cycle description of a genus 0 cover.

Apply Prop. [5.9] Entries of $\left(\left(g_{1}^{*}\right)^{3}, g_{3}^{*}, g_{4}^{*}\right)$ have product

$$
(-1)^{\frac{25-1}{8}+2\left(\frac{9-1}{8}\right)}=-1 \in \operatorname{Spin}_{5} \text {. }
$$

The product-one condition doesn't hold for $\boldsymbol{g}^{*}$. So, it can't hold in ${ }_{2}^{1} \tilde{A}_{5}$. This is necessary for a Nielsen class element, concluding the proof of obstruction.

Now consider which elements of (5.61) are obstructed. Check:

(5.7a) $g_{1} g_{2,1}=(24)(35)$ limits $\left(g_{3}, g_{4}\right)$ to be conjugates of $((432),(532))$ by the Klein four group centralizer of $(24)(35)$.

(5.7b) $g_{1} g_{2,2}=(152)$ limits $\left(g_{3}, g_{4}\right)$ to be one of two types:

- conjugates of ((532), (231)) by the centralizer of (152); or

- both are in $\langle(152)\rangle$.

As above, consider the unique lift $\boldsymbol{g}^{*}$ to $\mathrm{SL}_{2}(\mathbb{Z} / 5)$ of $\boldsymbol{g}$ that could be an image from $\boldsymbol{g}^{\prime} \in \mathrm{Ni}\left({ }_{2}^{1} \tilde{A}_{5}, \mathbf{C}_{5_{+} 5_{-} 3^{2}}\right)$ for the second of (5.7b). Since the lifts are unique, $g_{4}^{*} \in\left\langle g_{3}^{*}\right\rangle$. Typically: $\boldsymbol{g}^{*}$ would lift

$$
\boldsymbol{g}^{\dagger}=((12345),(12543),(152),(152)) .
$$

Apply Prop. [5.9 to ((12543), (152), (345)): $s((12543),(152),(345))=-1$.

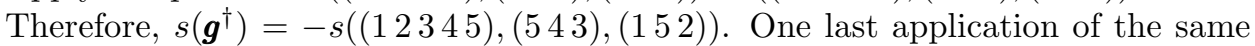
computation shows $s\left(\boldsymbol{g}^{\dagger}\right)=(-1)^{2}$. To conclude $\boldsymbol{g}^{\dagger}$ is unobstructed, use the following from [FK97 §3]. The Loewy display of $\operatorname{ker}_{k} / \operatorname{ker}_{k+1}$ refers to the $G_{k+1}$ action in the $p$-Frattini cover $G_{k+1} \rightarrow G_{k}$.

PRINCIPLE 5.13. Obstruction can occur from level $k$ to level $k+1$ in a Modular Tower only where $\mathbf{1}_{G_{k}}$ appears in the Loewy display of $\operatorname{ker}_{k} / \operatorname{ker}_{k+1}=M_{k}$. Translate this to say there is a (Frattini cover) sequence $G_{k+1} \rightarrow U_{k} \rightarrow W_{k} \rightarrow G_{k}$ with $W_{k}$ and $\operatorname{ker}\left(U_{k} \rightarrow W_{k}\right)$ is a trivial $W_{k}$ module. The appearance of $\mathbf{1}_{G_{k}}$ for any value of $k$ implies that $\mathbf{1}_{G_{k^{\prime}}}$ appears in $M_{k^{\prime}}$ for finitely many $k^{\prime}$. If $G_{0}$ is p-perfect and centerless, then so is $W_{k}$ (argument of Prop. 3.21).

Proof. Everything has an explanation already except the automatic appearance of $1_{G_{k^{\prime}}}$ in $M_{k^{\prime}}$ for finitely many $k^{\prime}$ given its appearance at level $k$. Apply Prop. 9.6 with $W_{k}$ replacing $G_{k}$. Then, use that the universal $p$-Frattini cover of 
both $W_{k}$ and $G_{k}$ is the same as that of $G_{0}$. So, the characteristic quotients for the universal $p$-Frattini of $W_{k}$ (in place of $G_{0}$ ) are cofinal in the projective system $\left\{G_{k}\right\}_{k=0}^{\infty}$. Therefore the simple modules (including copies of the identity representation) appearing in the analog for $W_{k}$ of the modules $M_{k}\left(G_{0}\right)$ also appear as simple modules for a cofinal collection from $\left\{G_{k}\right\}_{k=0}^{\infty}$.

Since $1_{A_{5}}$ appears only at the head (in the $A_{5}$ Schur multiplier) of $\operatorname{ker}_{0} / \mathrm{ker}_{1}$ (\$5.2), $\boldsymbol{g}^{\dagger}$ passes the lifting test to $\mathrm{SL}_{2}(\mathbb{Z} / 5)$ exactly if there is $\boldsymbol{g}^{\prime} \in \mathrm{Ni}_{1}$ over it.

5.5.3. Conclusion for $\mathbf{C}_{5_{+} 5_{-} 3^{2}}$. The principle from $\$ 5.5 .2$ is the following, where $s(\boldsymbol{g})$ is as in Prop. 5.9

Principle 5.14. Suppose for $\boldsymbol{g} \in A_{n}^{r}$, the product-one condition holds and there exists $i, j$ with $g_{i}$ a 3-cycle, $g_{j}$ a 5-cycle and their product (in either order) is a 3-cycle. To compute $s(\boldsymbol{g})$ we may assume $i=1, j=2$, and $s(\boldsymbol{g})=$ $-s\left(g_{1} g_{2}, g_{3}, \ldots, g_{r}\right)$. If $n=5, s(\boldsymbol{g})=1$ if and only if $\boldsymbol{g}$ is unobstructed.

Proof. With no loss assume $i<j$. The exists $Q \in H_{r}$ braiding $\boldsymbol{g}$ to $\dot{\boldsymbol{g}}$ in the same Nielsen class with $\dot{g}_{1}=g_{i}$ and $\dot{g}_{j}=g_{2}$. From [Fri95a Part III], $s(\boldsymbol{g})=s((\boldsymbol{g}) Q)$. (More generally, $\nu((\boldsymbol{g}) Q)=\nu(\boldsymbol{g})$ where $\nu$ is the big braid invariant of a Nielsen class.) Apply the argument of $\$ 5.5 .2$ to $\left(\dot{g}_{1}, \dot{g}_{2},\left(\dot{g}_{1}, \dot{g}_{2}\right)^{-1}\right)$ to reduce computing $s(\boldsymbol{g})$ to computing $-s\left(\dot{g}_{1} \dot{g}_{2}, \dot{g}_{3}, \ldots, \dot{g}_{r}\right)$.

Consider a case of (5.7ti) $: \boldsymbol{g}=((12345),(21345),(432),(532))$. Princ. 5.14 applies to $i=1$ and $i=3$. The product $g_{1} g_{3}=(145)$ is a 3 -cycle. Thus, $s(\boldsymbol{g})=-s\left(\boldsymbol{g}^{\dagger}\right)$ where $\boldsymbol{g}^{\dagger}$ is a 3 -tuple consisting of two 3-cycles and a 5-cycle. As previously, $s\left(\boldsymbol{g}^{\dagger}\right)=-1$ and $s(\boldsymbol{g})=1$. Since $n=5, \boldsymbol{g}$ is unobstructed.

Finally, consider the first case of (5.7D). Note: The genus 0 hypothesis of Prop. 5.9 doesn't hold.

PRINCIPLE 5.15. For each ordering of the conjugacy classes $\mathbf{C}_{5_{+} 5_{-} 3}$, the nielsen class $\mathrm{Ni}\left(A_{5}, \mathbf{C}_{5_{+} 5_{-}}\right)$in has exactly one element, for a total of six elements. All representatives $\boldsymbol{g}$ have $s(\boldsymbol{g})=1$.

Proof. That there is only one $\boldsymbol{g} \in \mathrm{Ni}\left(A_{5}, \mathbf{C}_{5_{+} 5_{-} 3}\right)^{\text {in }}$ with $g_{1} \in \mathrm{C}_{5}^{+}, g_{2} \in \mathrm{C}_{5}^{-}$ and $g_{3} \in \mathrm{C}_{3}$ follows from the data in (5.7). The computation $s(\boldsymbol{g})=1$ comes from $s\left(\boldsymbol{g}^{\dagger}\right)=1$ in (5.8).

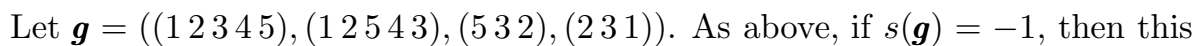
is obstructed. Princ. 5.15 shows $s(\boldsymbol{g})=s((152),(532),(231))$. Since the entries satisfy the genus 0 hypothesis, Prop. 5.9 gives this value as -1 .

Thm. 6.1 shows level 1 of the Modular Tower for $\left({ }_{2}^{1} \tilde{A}_{5}, \mathbf{C}_{5_{+} 5_{-} 3^{2}}\right)$ has no real points; that would require $\mathrm{H}-\mathrm{M}$ reps. at level 0 (see (6.1)). It has none, for the inverse of an element of $\mathrm{C}_{5}^{+}$is in $\mathrm{C}_{5}^{+}$.

5.5.4. The case $\mathbf{C}_{5_{+}^{2} 5_{-}^{2}}$. This brings a new issue. There are H-M representatives: ((12345),(54321),(21345),(54312)). So some elements are unobstructed. For an obstructed element in the Nielsen class use $\boldsymbol{g}^{\prime} \in \operatorname{Ni}\left(A_{5}, \mathbf{C}_{5_{+}^{3}}\right)$, three repetitions of the $C_{5}^{+}$conjugacy class. (Note: (5.7) shows $\mathrm{Ni}\left(A_{5}, \mathbf{C}_{5_{+}^{2} 5_{-}}\right)$is empty.) Write the first entry $g_{1}^{\prime}$ as $h^{2}$. Take $g_{1}^{*}=g_{2}^{*}=h, g_{3}^{*}=g_{2}^{\prime}$, and $g_{4}^{*}=g_{3}^{\prime}$ to produce $\boldsymbol{g}^{*} \in \mathrm{Ni}\left(A_{5}, \mathbf{C}_{5_{+}^{2} 5_{-}^{2}}\right)$. Clearly $s\left(\boldsymbol{g}^{*}\right)=s\left(\boldsymbol{g}^{\prime}\right)$. Example:

$$
\boldsymbol{g}^{\prime}=((12345),(35142),(45231)) \text {. }
$$

The next principle gives an obstructed element in $\mathrm{Ni}\left(A_{5}, \mathbf{C}_{5_{+}^{2} 5_{-}^{2}}\right)$. 
PRINCIPLE 5.16. The Nielsen class $\operatorname{Ni}\left(A_{5}, \mathbf{C}_{5_{+}^{3}}\right)^{\text {in }}$ has one element. For $\boldsymbol{g}$ in this class, $s(\boldsymbol{g})=-1$.

Proof. Write $\boldsymbol{g}=\left(g_{1}, g_{2}, g_{3}\right)$ with $g_{1}=(12345)$ and $g_{2}$ a conjugate of $g_{1}$ not in $\left\langle g_{1}\right\rangle$. Choose $g_{2}$ in one of the orbits $U_{3}$ or $U_{4}$ (for conjugation by $g_{1}$; 5.5.1) in $C_{5}^{+}$. If we choose $U_{3}$ to be the orbit of $g_{2}^{\prime}$ (in $\boldsymbol{g}^{\prime}$ ), then conjugate by $\left\langle g_{1}\right\rangle$ to see $g_{3}^{\prime}$ is also in $U_{3}$. The other $\left\langle g_{1}\right\rangle$ orbit of 5-cycles, represented by (24153), doesn't give an element in the Nielsen class: The product (12345)(24153) is a 3-cycle.

Calculate $s\left(\boldsymbol{g}^{\prime}\right)$ by considering $\boldsymbol{g}^{\prime \prime}=\left((123), \boldsymbol{g}^{\prime},(321)\right)$. Then, $s\left(\boldsymbol{g}^{\prime \prime}\right)=s\left(\boldsymbol{g}^{\prime}\right)$. Use Princ. [5.15] then Princ. [5.14 to see $s\left(\boldsymbol{g}^{\prime \prime}\right)$ equals

$$
s((13245),(35142),(45231),(321))=-s((13245),(35142),(451)) .
$$

One last application of Princ. [5.15 now shows $s(\boldsymbol{g})=-1$.

Remark 5.17. Noting the position of a 3-cycle gives $\left|\operatorname{Ni}\left(A_{5}, \mathbf{C}_{5_{+}^{2} 3}\right)^{\text {in }}\right|=3$. A representative for the $H_{3}$ orbit is ((12345),(124),(32541)). Put (321) on the left and (123) on the right as in Princ. 5.15. Conclude its lifting invariant is - 1.

5.6. Modular Towers from $p$-split data. Let $G_{0}$ be $(\mathbb{Z} / p)^{u} \times{ }^{s} H$ where $H$ is a $p^{\prime}$-group acting irreducibly (and nontrivially) through $\mathrm{GL}_{u}(\mathbb{Z} / p)$. This presents $G_{0}$ as a primitive affine group where $(\mathbb{Z} / p)^{u}=M$ are the letters of the permutation representation. As in Ex. [1.2 ${ }_{p} \tilde{G}$ is ${ }_{p} \tilde{F}_{u} \times{ }^{s} H$ with ${ }_{p} \tilde{F}_{u}$ the pro-free pro- $p$ group on $u$ generators. (Action of $H$ extends to ${ }_{p} \tilde{F}_{u}$ - Remark [5.2 - uniquely up to conjugacy. Still, it will rarely be easy to find.) Let $G_{k}$ be the $k$ th characteristic quotient of ${ }_{p} \tilde{G}, k \geq 0$.

Assume given $p^{\prime}$ conjugacy classes $\mathbf{C}$ in $H$. The next lemma shows how to find lifts of an element $\boldsymbol{g} \in \mathrm{Ni}(H, \mathbf{C})$ to $\boldsymbol{g}^{*} \in \mathrm{Ni}\left(G_{0}, \mathbf{C}\right)$, ensuring $\mathrm{Ni}\left(G_{0}, \mathbf{C}\right)$ is nonempty.

Lemma 5.18. As above, let $\boldsymbol{g}^{*} \in \mathbf{C}$ have $g_{i}^{*}=\left(m_{i}, g_{i}\right)$ with $m_{i} \in M, i=$ $1, \ldots, r$. Then, $\boldsymbol{g}^{*} \in \mathrm{Ni}\left(G_{0}, \mathbf{C}\right)$ if and only if

(5.9a) there is no $m \in M$ with $m-m^{g_{i}^{-1}}=m_{i}, i=1, \ldots, r$, and

(5.9b) $m_{1}+m_{2}^{g_{1}^{-1}}+\cdots+m_{r}^{g_{1}^{-1} \cdots g_{r-1}^{-1}}=0$.

So, there is a unique solution for $m_{r}$ given any elements $m_{1}, \ldots, m_{r-1}$.

Proof. Condition (5.9D) is the product one condition for $\boldsymbol{g}^{*}$. Since $M$ is an irreducible $H$ module, given $m_{1}, \ldots, m_{r-1}$, there is a unique solution for $m_{r}$. From the same argument, $\left\langle\boldsymbol{g}^{*}\right\rangle=G_{0}$ if and only if the projection of $\left\langle\boldsymbol{g}^{*}\right\rangle$ onto $H$ has no kernel; otherwise the kernel is all of $M$ since it is a nontrivial $H$ invariant submodule of $M$. Conclude, $\left\langle\boldsymbol{g}^{*}\right\rangle$ is a splitting of $H$ into $G_{0}$. From Schur-Zassenhaus, it is conjugate to the canonical copy of $H$ in $G_{0}$. Condition (5.97) is exactly the computation for that.

Levels 0 and 1 of the $\left(A_{4}, \mathbf{C}_{3_{+}^{2} 3_{-}^{2}}, p=2\right)$ Modular Tower (Ex. 9.2) show the $p$-split situation, for $u>1$, is a serious challenge for our Main Conjecture.

5.7. Criteria for $G_{k}$ faithful on $M_{k}$ and appearance of $1_{G_{k}}$. These general comments on the universal $p$-Frattini cover appear here for reference in [Fr01]. Let $O_{p^{\prime}}(G)$ be the maximal $p^{\prime}$ normal subgroup of any finite group $G$.

Lemma 5.19. Let $H$ be any $p^{\prime}$ subgroup of $G$ and let $M_{0}(H)$ be restriction of $G$ on $M_{0}$ to $H$. Then, this action extends to an action of $H$ on $\operatorname{ker}_{0}$, unique up 
to conjugation inside ${ }_{p} \tilde{G}$. Such an $H$ acts trivially on $\operatorname{ker}_{0}$ if and only if it acts trivially on $M_{0}(H)$. If $H=O_{p^{\prime}}(G)$, then this action is trivial.

Proof. The extending action of $H$ to $\operatorname{ker}_{0}$ is a special case of Rem. 5.2 applied to $M_{0}(H) \times{ }^{s} H$. The extending group $\operatorname{ker}_{0} \times{ }^{s} H$ is the universal $p$-Frattini of $M_{0}(H) \times{ }^{s} H$. If the action on $M_{0}(H)$ is trivial, then the trivial extension of $H$ to ker $_{0}$ is an extending action.

Consider the case $H=O_{p^{\prime}}(G)$. We have the natural short exact sequence $1 \rightarrow O_{p^{\prime}}(G) \rightarrow{ }_{p} \tilde{G} \rightarrow{ }_{p} G \widetilde{O_{p^{\prime}}}(G) \rightarrow 1$. Form the (group) fiber product

$$
{ }_{p} \tilde{G}^{\dagger} \stackrel{\text { def }}{=}{ }_{p} G / \widetilde{O_{p^{\prime}}}(G) \times_{G / O_{p^{\prime}}(G)} G .
$$

By the basic property of the $p$-Frattini cover, ${ }_{p} G \widetilde{O_{p^{\prime}}(G)}$ is the minimal $p$-projective cover of $G / O_{p^{\prime}}(G)$. Since $G \rightarrow G / O_{p^{\prime}}(G)$ has $p^{\prime}$ kernel, ${ }_{p} \tilde{G}^{\dagger}$ is the minimal $p$ projective cover of $G$. So, by Prop. [3.9] it is ${ }_{p} \tilde{G}$.

Warning! Each $p^{\prime}$ subgroup $H$ of $G$ does have its action extend to $\operatorname{ker}_{0}$, and that extending action is unique up conjugacy. Assuming a group is $p$-perfect then means we can extend the action of generators on ker $_{0}$. Those actions, however, won't fit together to have $G$ act on $\operatorname{ker}_{0}$ (as in $\S \mathbf{5 . 2 . 2}$ ).

In Prop. 5.3 we found many explicit appearances of $\mathbf{1}_{G_{k}}$ in $M_{k}$ in the $p$-split case, excluding the case that $M_{0}$ is cyclic (with nontrivial $G_{k}$ ) action. The following result, basically from [GS78, characterizes when $G_{0}$ is faithful on $M_{0}$. It is an ingredient in Thm. 5.21] giving an asymptotic formula for the multiplicity of appearance of any simple $G_{0}$ module in $M_{k}$ for large $k$.

TheOrem 5.20. Suppose the dimension of $M_{0}$ exceeds 1. Then, $G_{k} / O_{p^{\prime}}\left(G_{0}\right)$ acts faithfully on $M_{k}$, for all $k \geq 0$.

Proof. Once we know the rank of $M_{0}$ exceeds 1 , then so does the rank of $G_{k}$ on $M_{k}$ for all $k$. Since $O_{p^{\prime}}\left(G_{0}\right)$ is a $p^{\prime}$ normal subgroup, there is a splitting of the pullback of $O_{p^{\prime}}\left(G_{0}\right)$ in ${ }_{p} \tilde{G}$. That identifies $O_{p^{\prime}}\left(G_{0}\right)$ as the maximal normal $p^{\prime}$ subgroup of $G_{k}$ for each $k$. Given the Griess-Schmid result for $k=0$ therefore gives it for all $k$.

With the previous notation, assume $\mathbf{1}_{G_{k}}$ appears in the Loewy display of $M_{k}$. Princ. 5.13 shows $\mathbf{1}_{G_{k^{\prime}}}$ appears (even where) in the Loewy display for infinitely many integers $k^{\prime} \geq k$. Thm. [5.21] is in $\mathbf{S e 0 4 b}$. As a special case it shows that $\mathbf{1}_{G_{k}}$ appears with an explicit positive density in $M_{k}$ for $k$ large. The result is effective, though for small values of $k$ it is subtle to predict the appearance of $\mathbf{1}_{G_{k}}$. Further, it is imprecise on the place in the Loewy display of the appearance of the $\mathbf{1}_{G_{k}} \mathrm{~s}$. So, Princ. 5.13 and the very effective $p$-split case remain valuable.

Recall: Over an algebraically closed field the set of simple $G_{0}$ modules has the same cardinality as the set of $p^{\prime}$ conjugacy classes. Let $S$ be any simple $G_{0}$ module. Let $K$ be an algebraically closed field and retain the notation $M_{k}$ after tensoring with $K$. We use $\left\langle S, M_{k}\right\rangle$, and related compatible notation, for the total multiplicity of $S$ in all Loewy layers of the $G_{k}$ module $M_{k}$.

Theorem 5.21 (Semmen). The condition $\operatorname{dim}_{K}\left(M_{0}\right) \neq 1$ is equivalent to

$$
\lim _{n \mapsto \infty} \frac{\left\langle S, M_{k}\right\rangle}{\operatorname{dim}_{K}\left(M_{k}\right)}=\frac{\left\langle S, K\left[G / O_{p^{\prime}}(G)\right]\right\rangle}{\operatorname{dim}_{K}\left(K\left[G / O_{p^{\prime}}(G)\right]\right)} .
$$


REMARK 5.22 (Griess-Schmid characterization that $\operatorname{dim}_{\mathbb{Z} / p}\left(M_{0}\right)=1$ ). Let $O_{p}(G)$ be the maximal normal $p$-subgroup of $G$. Recall that a group $H$ is $p$ supersolvable if $G / O_{p}\left(G / O_{p^{\prime}}(G)\right)$ is abelian of exponent dividing $p-1$. GS78. has the following characterization of $\operatorname{dim}_{\mathbb{Z} / p}\left(M_{0}\right)=1$ : $\mathrm{G}$ is a $p$-supersolvable group with a cyclic $p$-Sylow. Specifically, if $G_{0}$ is simple, then $\operatorname{dim}_{\mathbb{Z} / p}\left(M_{0}\right) \neq 1$.

\section{Real points on Hurwitz spaces}

Investigations of modular curves often analyze behavior of functions near cusps. Degeneration behavior of the curve covers associated to points on Hurwitz spaces, as the points approach the cusps, hints at diophantine properties. This section illustrates with detailed analysis of the easiest case: Degeneration behavior of real points on Hurwitz and reduced Hurwitz spaces. So doing, it points to special cusps named for this degeneration. The word cusp implies some compactification of the Hurwitz space. Therefore, after generalities on Hurwitz spaces for any $r$, we concentrate on cusp behavior of reduced Hurwitz spaces for the case $r=4$. Then the spaces are curves, and the compactification behavior stays within the confines of this paper. In Modular Tower higher levels for $p=2$, real points on Hurwitz spaces at those levels associate to branch cycles we call H-M and near H-M reps. Cusps at the end of the corresponding components of real points then inherit the $\mathrm{H}-\mathrm{M}$ and near H-M moniker.

6.1. Diophantine properties of a Modular Tower. In Thm. 6.1 $\mathcal{H}_{k}$ is level $k$ of a Modular Tower of inner Hurwitz spaces. In assuming $G_{0}$ is centerless and $p$-perfect (Def. 3.18), these are fine moduli spaces (Prop. 3.21). As a diophantine corollary, over any field $K$ with $\left(\operatorname{char}(K),\left|G_{0}\right|\right)=1$ (see Fri95a Reduction Hypothesis E.3] or We98), $\boldsymbol{p} \in \mathcal{H}_{k}(K)$ if and only if some cover (in the inner equivalence class) associated to $p$ has definition field $K$.

6.1.1. $H-M$ and near $H-M$ representatives. A Harbater-Mumford (H-M) representative of Nielsen class $\operatorname{Ni}(G, \mathbf{C})$ is an $r$-tuple $\boldsymbol{g} \in \mathbf{C}$ with the following property:

(6.1) $g_{2 i-1}=g_{2 i}^{-1}, i=1, \ldots, s$, with $r=2 s$.

Call a component of $\mathcal{H}(G, \mathbf{C})^{\text {in }}$ an H-M component if it corresponds to an $H_{r}$ orbit of an H-M rep(resentative).

The definition of a near $H-M$ representative applies to any Modular Tower at level $k \geq 1$ if $p=2$, and $r=2 s$ is even. It uses an operator $\hat{\kappa}$ on Nielsen classes, the special case $r_{1}=0$ for the operator $\hat{\kappa}$ in (6.8). Define the effect of $\hat{\kappa}$ on $\boldsymbol{g} \in \operatorname{Ni}(G, \mathbf{C})$ by consecutively listing entries of $\hat{\kappa}(\boldsymbol{g})$. List entries of $\boldsymbol{g}$ as $\left(g_{1,1}, g_{1,2}, \ldots, g_{s, 1}, g_{s, 2}\right)$. As in (6.8), denote $g_{j, 1} g_{j, 2}$ by $g_{j, .}$.

$$
\begin{aligned}
\hat{\kappa}(\boldsymbol{g})_{1,1} & =\left(g_{2, .} \cdots g_{s, .}\right)^{-1} g_{1,2}^{-1}\left(g_{2, .} \cdots g_{s, .}\right) \\
\hat{\kappa}(\boldsymbol{g})_{1,2} & =\left(g_{2, .} \cdots g_{s, .}\right)^{-1} g_{1,1}^{-1}\left(g_{2, .} \cdots g_{s, .}\right) \\
& \cdots, \\
\hat{\kappa}(\boldsymbol{g})_{s, 1} & =g_{s, 2}^{-1}, \hat{\kappa}(\boldsymbol{g})_{s, 2}=g_{s, 1}^{-1} .
\end{aligned}
$$

Then, $\boldsymbol{g}^{*} \in \mathrm{Ni}\left(G_{k}, \mathbf{C}\right)^{\text {in }}, k \geq 1$, is a near H-M rep. if the following hold.

(6.3a) $\boldsymbol{g}^{*}$ isn't an H-M representative.

(6.3b) There exists an involution $c \in G_{k}$ satisfying $c \boldsymbol{g}^{*} c=\hat{\kappa}\left(\boldsymbol{g}^{*}\right)$.

(6.3c) $\boldsymbol{g}^{*} \bmod \operatorname{ker}_{k-1}$ is an H-M representative. 
6.1.2. A general diophantine statement about Modular Towers. We use $\$ 6.3$ for direct calculation of real points on reduced Hurwitz spaces. Reference to $\mathbf{C}_{k}$ means conjugacy classes varying with $k$, not necessarily consisting of $p^{\prime}$ classes. For any prime $\ell$, let $\mathbb{Q}^{(\ell)}$ denote the field with all $\ell^{n}$ th roots of $1, n=1,2, \ldots$, adjoined. If $Y \subset \mathcal{H}_{0}$, denote the complement of $Y$ pulled back to $\mathcal{H}_{k}$ by $\mathcal{H}_{k} \backslash Y$.

TheOREM 6.1. Assume $G=G_{0}$ is centerless and p-perfect. Fix $r^{*}$ and a subfield $K \leq \overline{\mathbb{Q}}$. Suppose there are $\left(G_{k}, \mathbf{C}_{k}\right)$ realizations (over $K$ ) with $r_{k} \leq r^{*}$ conjugacy classes in $G_{k}$ as the entries of $\mathbf{C}_{k}$, for each $k \geq 0$.

(6.4) Assume $\left[\mathbb{Q}^{(p)} \cap K: \mathbb{Q}\right]<\infty$.

Then, the only possibility for such $K$ realizations is there exists $r$, with $r \leq r^{*}, p^{\prime}$ classes $\mathbf{C}$ with $\left(G_{k}, \mathbf{C}\right)$ realizations (over $K$ ) for all $k$.

Assume there is a prime $\ell,\left(\ell,\left|G_{0}\right|\right)=1$, satisfying the following.

(6.5) At some place $P$ of $K$ over $\ell$ the residue class field $F_{P}$ is finite. Then, reduction of $\mathcal{H}_{k}$ at $P$ has no $F_{P}$ points for $k$ large.

Suppose there is a proper algebraic subset $Y \subset \mathcal{H}_{0}^{\mathrm{rd}}$ with this property.

(6.6) $\mathcal{H}_{k}^{\mathrm{rd}} \backslash Y(K)$ is finite for each $k$.

Then, for some $k_{K, G_{0}, \mathbf{C}}, \mathcal{H}_{k}^{\mathrm{rd}} \backslash Y(K)$ is empty for $k \geq k_{K, G_{0}, \mathbf{C}}$. Assume $r=4$ and all components of $\mathcal{H}_{k^{\prime}}^{\mathrm{rd}}$ have genus at least 2 for some $k^{\prime} \geq 0$. Then, there is $k_{K, G_{0}, \mathbf{C}}>0$ with no $\left(G_{k}, \mathbf{C}\right)$ realizations over $K$ for $k \geq k_{K, G_{0}, \mathbf{C}}$.

Assume $p=2$ and $K \subset \mathbb{R}$. Then all $\left(G_{k}, \mathbf{C}\right)$ realizations over $K$ for $k \geq 1$ appear in components of $\mathcal{H}_{k}$ for $H_{r}$ orbits containing $H$ - $M$ or near $H$ - $M$ reps. (This holds even replacing $\mathbf{C}$ by $\mathbf{C}_{k}$ varying with $\left.k.\right) \quad A \boldsymbol{p} \in \mathcal{H}_{k}(\mathbb{R})$ corresponds to a $\left(G_{k}, \mathbf{C}\right)$ realization over $\mathbb{R}$. A connected component of $\mathcal{H}_{k}(\mathbb{R})$ associated to an $H-M$ rep. has a connected component of $\mathcal{H}_{k+1}(\mathbb{R})$ above it. A connected component of $\mathcal{H}_{k}(\mathbb{R})$ for a near $H-M$ rep. has nothing in $\mathcal{H}_{k+1}(\mathbb{R})$ above it.

Explicit formulas (as in (8.1), Lem. 8.2 and give us confidence in deciding FK97, Main Conj. 0.1] when $r=4$. If the FK97, Main Conj. 0.1] conjecture is correct, that $\mathcal{H}_{k}^{\text {rd }}$ has no rational points for $k$ large, then $\mathbb{Q}$ realizations of $G_{k}$ require increasingly large sets of conjugacy classes as $k$ grows. This is subtler than information from the Branch Cycle Lemma.

4.4 .2 succinctly interprets points on a Modular Tower as a statement on quotients of a fundamental group. Thm. 6.1 says if $p=2$, real points on a Modular Tower (versus points at various levels) appear only on projective sequences of H-M components. In 9.1 we see this gives a tool for progress on the Main Conjecture.

The proof of Thm. 6.1 appears in two subsections. \$6.2 gives the argument bounding points at high levels on a Modular Tower of inner Hurwitz spaces. 6.3 gives details on $\mathbb{R}$ points. Note: Hurwitz spaces $\mathcal{H}_{k}$ are affine varieties. The theorem says nothing about rational points on (any) closure of $\mathcal{H}_{k}$ including the boundary.

6.2. Bounding levels with $K$ points. [FK97. Thm. 4.4] gives the first statement of Thm. 6.1. Bounding $k$ with $\left(G, \mathbf{C}_{k}\right)$ having $K$ realizations and $\mathbf{C}_{k}$ not consisting of $p^{\prime}$ classes requires only the Branch Cycle Lemma. This is effective, though dependent on data about ${ }_{p} \tilde{G}$ for a result referencing only $r^{*}, K$ and $G_{0}$.

Consider any suitable Modular Tower for inner equivalence where $G=G_{0}$ is $p$-perfect and centerless. Then, the inner Hurwitz spaces are fine moduli spaces (Prop. 3.21). Also, they and their reduced versions have good reduction modulo any prime $\ell$, if $(\ell,|G|)=1$ ([Fri95a p. 167] or with more details We98]). Use

$$
\cdots \rightarrow \mathcal{H}_{k}^{\mathrm{rd}} \rightarrow \mathcal{H}_{k-1}^{\mathrm{rd}} \rightarrow \cdots \rightarrow \mathbb{P}_{j}^{1} \backslash \infty
$$


for the sequence of reduced spaces. Assume each $\mathcal{H}_{k}^{\text {rd }}$ has a $K$ point. Let $P$ be a prime of $K$ over $\ell$ with residue class field $F_{P}$.

Suppose, contrary to the conclusion of the theorem, $\mathcal{H}_{k}^{\text {rd }}$ has an $F_{P}$ rational point for each integer $k$. The set $\mathcal{H}_{k}^{\mathrm{rd}}\left(F_{P}\right)$ is finite for each $k$. So, these finite sets form a nonempty projective system. Conclude: There is a projective sequence of points $\boldsymbol{p}^{\text {rd }}$ on them. Let $F^{\prime}$ be a finite extension of $F_{P}$ over which the point $\boldsymbol{p}_{0}^{\text {rd }}$ produces an inner cover $X_{\boldsymbol{p}_{0}} \rightarrow \mathbb{P}_{z}^{1}$ in the Nielsen class corresponding to $\boldsymbol{p}_{0}$ on the level 0 Hurwitz space lying over $\boldsymbol{p}_{0}^{\mathrm{rd}}$. Apply Princ. 4.14 when $K$ is a finite field. That translates to a projective system of $\left(G_{k}, \mathbf{C}\right)$ regular realizations with definition field $F^{\prime}$. With no loss, take this finite extension to be $F_{P}$.

Let $T_{p}\left(X_{\boldsymbol{p}_{0}}\right)=T_{p}$ be the Tate module for $X_{\boldsymbol{p}_{0}}$ formed by taking an abelian quotient of the Modular Tower as in Prop.4.16. Suppose the kernel of the universal $p$-Frattini cover has rank $u$. For a general Modular Tower this implies the Frobenius for $F_{P}$ acts trivially on a rank $u$ quotient of $T_{p}$. Contradiction: The Frobenius has eigenvalues of absolute value $\left|F_{P}\right|^{1 / 2}$. This contradiction shows there cannot be $F_{P}$ points on the reduced space $\mathcal{H}_{k}^{\text {rd }}$ for all $k$.

Now assume (6.6) holds. There are finitely many points on $\mathcal{H}_{k}^{\text {rd }} \backslash Y(K)$. So, the argument above shows that either $\mathcal{H}_{k}^{\text {rd }} \backslash Y(K)$ is empty for some value of $k$ or there is a projective system of $K$ points. Taking a suitably large prime $\ell$ allows reducing the covers for the projective system modulo $\ell$, and the proceeding argument completes the proof of this part of the theorem.

REMARK 6.2 (Thm. 6.1 and absolute classes). The argument of Thm. 6.1 does not hold for a Modular Tower of absolute Hurwitz spaces. Modular curves give an example of this. Consider a fixed prime $p$, and all of the curves $Y_{0}\left(p^{k+1}\right)(\$ 2.8 .2)$ as in a Modular Tower. Let $\ell \neq p$ or 2 be a prime, and reduce modulo $\ell$.

The following facts are in Rob73 Chap. 3]. There are many supersingular elliptic curves in characteristic $\ell$, roughly $\ell / 12$ of them. Further, all have field of definition $K=\mathbb{F}_{\ell^{2}}$. Characterize a supersingular curve (over $\overline{\mathbb{F}}_{\ell}$ ) by its having no $\ell$ division points. So, any curve isogenous to it, say by a cyclic degree $p^{k+1}$ isogeny, is also supersingular. Since both curves have definition field $K$, the isogeny has definition field $K$ (though it is not a Galois cover over $K$ ). This gives a large number of points on $X_{0}\left(p^{k+1}\right)$, as many as can be expected for $k$ large. This is Ihara's first example for producing many points on high genus curves over a finite field of square order. Ihara uses Shimura curves to do the same over finite fields of order $\ell^{2 t}$ for $t>1$. (Iha00 has an exposition on this result's scattered literature.) The Shimura curves he uses are compact families of abelian varieties, unlike levels of a Modular Tower which have cusps.

6.3. Remainder of Thm. 6.1 proof. We apply DF90 using the pattern of [Fri95a App. C and App. D]. This explicitly described real points on any of the fine moduli Hurwitz spaces $\mathcal{H}$ appearing in this paper.

6.3.1. Connected components of $\mathcal{H}(\mathbb{R})$. Let $\varphi: X \rightarrow \mathbb{P}_{z}^{1}$ be a cover over $\mathbb{R}$, branched over $\boldsymbol{z}$, with $z_{0} \in \mathbb{R}$. Specific classical generators of $\pi_{1}\left(U_{\boldsymbol{z}}, z_{0}\right)$ produce an explicit uniformization of any real points on $X$ over $z_{0}$. This uses a complex conjugation operator $\hat{\kappa}$ from $(6.8$ on Nielsen classes. It includes giving a combinatorial restatement for $X$ having definition field $\mathbb{R}$. So, fixed points of this complex conjugation operator produce points in $\mathcal{H}(\mathbb{R})$. With a fine moduli assumption these are exactly the points of $\mathcal{H}(\mathbb{R})$. The process works efficiently for unramified Frattini 
extensions of the covers $X \rightarrow \mathbb{P}^{1}$ appearing in an inner family (using the language of 5.17. So, this gives a useful description of real points on a Modular Tower.

6.3.2. Points in $z$ over $\mathbb{R}$. The approach falls into cases from how the support of $z$ behaves under complex conjugation. As $z$ has definition field $\mathbb{R}$, it has $s_{1}$ complex conjugate pairs and $r_{1}=r-2 s_{1}$ real points. To simplify, call the latter $z_{1}, \ldots, z_{r_{1}}$, arranged left to right on the real line and the former, $z_{r_{1}+1}, \ldots, z_{r}$. With no loss (see 96.4 assume $z_{0}, z_{1}, \ldots, z_{r_{1}}$ appear in that order on the real line (circle on the Riemann sphere). Then, DF90 produces paths based at $z_{0}$,

$$
P_{1}, P_{2}, \ldots, P_{r_{1}}, P_{r_{1}+1,1}, P_{r_{1}+1,2}, \ldots, P_{r_{1}+r_{2}, 1}, P_{r_{1}+r_{2}, 2},
$$

with explicit complex conjugation action $c$ on these paths and on points over $z_{0}$.

Represent $c$ as $c_{\boldsymbol{z}}=c_{z_{0}, \boldsymbol{z}}$, by its permutation effect on points over $z_{0}$. With these conditions this description is uniform in $z_{0}$. Under $c$ the paths $P_{i}$ go to a new set of paths, $P_{i}^{\prime}, i=1, \ldots, r$. From DF90: If $P$ and $P^{\prime}$ are one of the $s_{1}$ pairs of paths, then $c$ sends $P$ (resp. $\left.P^{\prime}\right)$ to a conjugate of $\left(P^{\prime}\right)^{-1}$ (resp. $\left.P^{-1}\right)$. Branch cycles for the complex conjugate cover $\varphi^{c_{z}}: X^{c_{z}} \rightarrow \mathbb{P}_{z}^{1}$ relative to the $P_{i}^{\prime}$ s are also g. A formula (dependent on the particular paths) computes branch cycles for $\varphi^{c_{z}}$ relative to the $P_{i}$ s: Call this $\hat{\kappa}(\boldsymbol{g})=\hat{\kappa}_{z}(\boldsymbol{g})$ (as in (6.8)). If $\varphi^{c_{z}}$ is s-equivalent to $\varphi$ (Def. 3.1), then $c_{\boldsymbol{g}}$ conjugates the last branch cycle description to $\boldsymbol{g}$.

6.3.3. Formula defining $c_{\boldsymbol{z}}=c \in N^{\prime}$. Assume $G \leq N^{\prime} \leq N_{T}(G, \mathbf{C})$ as in $\$ 3.5 .1$. Prop. 6.3 produces for each $\boldsymbol{g} \in \mathrm{Ni}(G, \mathbf{C}) / N^{\prime}$ a test for a connected component of $\mathcal{H}_{N^{\prime}}(\mathbb{R})$. Let $\varphi_{\boldsymbol{g}}: X_{\boldsymbol{g}} \rightarrow \mathbb{P}_{z}^{1}$ be a cover with branch cycle description $\boldsymbol{g}$ (relative to the chosen classical generators for $\pi_{1}\left(U_{\boldsymbol{z}}, z_{0}\right)$ ). The test succeeds if some component has a point corresponding to $\varphi_{\boldsymbol{g}}$. The basic formula (6.8) is from [DF90, Thm. 4.4].

Proposition 6.3. Suppose $\boldsymbol{g} \in \operatorname{Ni}(G, \mathbf{C}) / N^{\prime}$. Then, $\boldsymbol{g}$ represents $\boldsymbol{p}_{\boldsymbol{g}} \in \mathcal{H}_{N^{\prime}}$

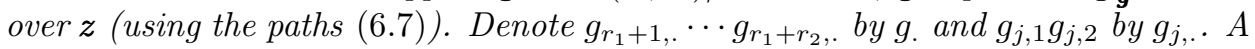
connected component of $\mathcal{H}_{N^{\prime}}(\mathbb{R})$ goes through $\boldsymbol{p}_{\boldsymbol{g}}$ if for some $c=c_{\boldsymbol{g}} \in N^{\prime}, c \boldsymbol{g} c=\hat{\kappa}(\boldsymbol{g})$ with $\hat{\kappa}_{\boldsymbol{z}}=\hat{\kappa}$ as follows. For such a $c_{\boldsymbol{g}}, c_{\boldsymbol{g}}^{2}$ centralizes $G$.

$$
\begin{aligned}
\hat{\kappa}(\boldsymbol{g})_{1} & =g_{1}^{-1}, \\
\hat{\kappa}(\boldsymbol{g})_{2} & =\left(g_{3} \cdots g_{r_{1}} g_{.}\right)^{-1} g_{2}^{-1}\left(g_{3} \cdots g_{r_{1}} g .\right), \ldots, \\
\hat{\kappa}(\boldsymbol{g})_{r_{1}-1} & =\left(g_{r_{1}} g\right)^{-1} g_{r_{1}-1}^{-1} g_{r_{1}} g_{.}, \\
\hat{\kappa}(\boldsymbol{g})_{r_{1}} & =g^{-1} g_{r_{1}}^{-1} g_{.} \\
\hat{\kappa}(\boldsymbol{g})_{r_{1}+1,1} & =\left(g_{r_{1}+2, .} \cdots g_{r_{1}+r_{2}, .}\right)^{-1} g_{r_{1}+1,2}^{-1}\left(g_{r_{1}+2, .} \cdots g_{r_{1}+r_{2}, .}\right), \\
\hat{\kappa}(\boldsymbol{g})_{r_{1}+1,2} & =\left(g_{r_{1}+2, .} \cdots g_{r_{1}+r_{2}, .}\right)^{-1} g_{r_{1}+1,1}^{-1}\left(g_{r_{1}+2, .} \cdots g_{r_{1}+r_{2}, .}\right), \ldots, \\
\hat{\kappa}(\boldsymbol{g})_{r_{1}+r_{2}, 1} & =g_{r_{1}+r_{2}, 2}^{-1}, \quad \hat{\kappa}(\boldsymbol{g})_{r_{1}+r_{2}, 2}=g_{r_{1}+r_{2}, 1}^{-1} .
\end{aligned}
$$

If a cover over $\mathbb{R}$ represents $\boldsymbol{p}_{\boldsymbol{g}} \in \mathcal{H}_{N^{\prime}}$ (using the paths (6.7)), then some involution $c_{\boldsymbol{g}}$ satisfies $c_{\boldsymbol{g}} \boldsymbol{g} c_{\boldsymbol{g}}=\hat{\kappa}(\boldsymbol{g})$. If $\mathcal{H}_{N^{\prime}}$ is a fine moduli space, such an involution $c_{\boldsymbol{g}}$ exists if and only if a connected component of $\mathcal{H}_{N^{\prime}}(\mathbb{R})$ goes through $\boldsymbol{p}_{\boldsymbol{g}}$.

Comments on The PROOF. The operator $\hat{\kappa}$ acts as an involution: $\hat{\kappa}^{2}(\boldsymbol{g})=\boldsymbol{g}$ and $\hat{\kappa}$ commutes with conjugation by $c_{\boldsymbol{g}}$. So $c_{\boldsymbol{g}}^{2}$ acts trivially on $\boldsymbol{g}$. The condition for $\mathcal{H}_{N^{\prime}}$ to have fine moduli is that the centralizer of $G$ in $N^{\prime}$ is trivial (Thm. 3.16).

Remark 6.4 (When $c_{\boldsymbol{g}}$ is an involution). Prop. 6.8 includes an archetype example for an inner Hurwitz family with group $T^{\prime}$ having a nontrivial center: It is a Frattini central extension of a centerless group $G$. If $\boldsymbol{g}^{\prime}$ is branch cycles for a $T^{\prime}$ 
cover, we can usually decide if $c_{\boldsymbol{g}^{\prime}}$ is an involution. So, we can decide if there is a cover over $\mathbb{R}$ realizing $\boldsymbol{p}_{\boldsymbol{g}^{\prime}}$.

6.3.4. When $G=G_{k}$ is the level $k \geq 1$ group. Suppose $\boldsymbol{p} \in \mathcal{H}\left(G_{k}, \mathbf{C}\right)^{\mathrm{in}}(\mathbb{R})$ lies over $z$ and $z_{0}$ is a real base point relative to which (6.8) is a branch cycle description of $\varphi_{\boldsymbol{p}}: X_{\boldsymbol{p}} \rightarrow \mathbb{P}_{z}^{1}$. Assume:

(6.9) Complex conjugation $c_{\boldsymbol{p}}=c$ is trivial, and $r_{1}=0$ in 6.8.

Then, the branch cycle description for $\varphi_{\boldsymbol{p}}: X_{p} \rightarrow \mathbb{P}_{z}^{1}$ is an H-M representative. This is in the $H_{r}$ orbit on $\operatorname{Ni}\left(G_{k}, \mathbf{C}\right)$ corresponding to the component of $\mathcal{H}\left(G_{k}, \mathbf{C}\right)^{\text {in }}$ containing $\boldsymbol{p}$. To complete the proof of Thm. 6.1 requires showing two things:

(6.10a) (6.9) is equivalent to having an $\mathrm{H}-\mathrm{M}$ representative.

(6.10b) If $k \geq 1$ and $c \boldsymbol{g} c=\hat{\kappa}(\boldsymbol{g})$ with $c \neq 1$, then $\boldsymbol{g}$ is a near H-M representative.

First assume $r_{1}>0$. Suppose $c=c_{z_{0}}$ is the identity. Then (6.8) says $g_{1}=g_{1}^{-1}$, or $g_{1}$ has order 2. This can't hold if this conjugacy class is $2^{\prime}$. It also can't hold if $k \geq 1$. The reason is this: The hypotheses remain the same for the reduction of $\boldsymbol{g}$ modulo ker $_{k-1}$. Apply Lem. 2.23 Any lift to $G_{k}$ of an element of order divisible by 2 in $G_{k-1}$ increases its order. So, $g_{1}$ can't have order 2 .

Let $c^{\prime}$ be complex conjugation at level 0 (reduction modulo ker $_{0}$ ). The same applies to it. So, $c^{\prime}$ has order two (not 1 ) if $r_{1}>0$. For inner classes, $c^{\prime} \in G_{0}$. Apply Lem. 2.23 again so $c$ has order exceeding that of $c^{\prime}$. This contradicts that $c$, a complex conjugation operator, has order 2 and is a lift of $c^{\prime}$. Now we know $r_{1}=0$. Further, this shows $c^{\prime}$ is the identity.

This argument applies in going from level $k$ to level $k+1, k \geq 0$. Two possibilities happen. Assume $\boldsymbol{g}^{\prime} \in \mathrm{Ni}\left(G_{k}, \mathbf{C}\right)^{\text {in }}$ lies below $\boldsymbol{g} \in \mathrm{Ni}\left(G_{k+1}, \mathbf{C}\right)^{\text {in }}$, where $\boldsymbol{g}$ defines a connected component of real points on $\mathcal{H}\left(G_{k+1}, \mathbf{C}\right)^{\text {in }}$. Let $c \in G_{k+1}\left(\right.$ resp. $\left.c^{\prime}\right)$ be the complex conjugation operator associated with $\boldsymbol{g}$ (resp. $\boldsymbol{g}^{\prime}$ ). Then: Either

(6.11a) $c$ is the identity and $\boldsymbol{g}$ is an H-M representative; or

(6.11b) $\bullet c^{\prime}$ is the identity, $\boldsymbol{g}^{\prime}$ is an H-M rep.,

- $c \in \operatorname{ker}_{k} / \operatorname{ker}_{k+1}$ is not the identity, and

- nothing in $\mathcal{H}\left(G_{k+2}, \mathbf{C}\right)^{\mathrm{in}}(\mathbb{R})$ is over the $\mathbb{R}$ component corresponding to $\boldsymbol{g}$ in $\mathcal{H}\left(G_{k+1}, \mathbf{C}\right)^{\mathrm{in}}$.

Near H-M representatives (satisfying (6.11b) ) occur at level $k \geq 1$ of the Modular Tower for $\left(A_{5}, \mathbf{C}_{3^{4}}\right)$ (\$7.3.3 especially Prop. 7.11).

6.4. Real points when $r=4$. 6.4 .1 gives an elementary lemma about $j$ values of cover points over $\mathbb{R}$ on a Hurwitz space. Then, 6.4 .2 shows how real components over critical intervals fit together.

6.4.1. $j$ values of cover points over $\mathbb{R}$. We note the $j$ invariant separates four branch point covers over $\mathbb{R}$ according to a configuration of their branch points.

Lemma 6.5. Suppose $\varphi: X \rightarrow \mathbb{P}_{z}^{1}$ is a four branch point cover over $\mathbb{R}$ with either 0 or 4 real branch points. Then, the corresponding $j$ value under the representative of $\varphi$ on a reduced Hurwitz space is in the interval $(1,+\infty)$ along the real line.

If $\varphi$ has, instead, two complex conjugate and two real branch points, then the corresponding $j$ value is in the interval $(-\infty, 1)$.

PROOF. Recall the cross ratio of distinct points $z_{1}, \ldots, z_{4}: \lambda_{\boldsymbol{z}}=\frac{\left(z_{1}-z_{3}\right)\left(z_{2}-z_{4}\right)}{\left(z_{2}-z_{3}\right)\left(z_{1}-z_{4}\right)}$. The basics are in Ah79 p. 79]. Four points in complex conjugate pairs (or on the real line) lie on a circle and the cross ratio is real. The cross-ratio is invariant 
under a transform of the points $z$ by $\alpha \in \mathrm{PGL}_{2}(\mathbb{C})$. Since there is an $\alpha \in \mathrm{PGL}_{2}(\mathbb{C})$ that takes two complex conjugate pairs of points to four points in the reals, with no loss assume $\boldsymbol{z}$ has either two or four real points in its support. For these cases apply $\beta \in \mathrm{PSL}_{2}(\mathbb{R})$ to assume $0=z_{1}$ and $\infty=z_{2}$. Then, $\lambda_{\boldsymbol{z}}=\frac{z_{4}}{z_{3}}$.

In the former case $\lambda_{z}$ runs over the unit circle (excluding 1) and in the latter case over all real numbers (excluding 0,1 and $\infty$ ). The $j_{\boldsymbol{z}}$ value corresponding to $\lambda_{z}$ is $J_{4}(\lambda)=\frac{4}{27} \frac{\left(1-\lambda_{z}+\lambda_{z}^{2}\right)^{3}}{\left.\lambda_{z}^{2}\left(1-\lambda_{z}\right)^{2}\right)}$ with Ah79 p. 282]. (Classically this is without the $\frac{4}{27}$. We chose it so the ramified $j$-values are $0,1, \infty$.)

For $\lambda_{z} \in \mathbb{R} \backslash\{0,1\}$ the connected range of $j_{z}$ includes large positive values and is bounded away from 0 . So the range of $j_{\boldsymbol{z}}$ for real $\lambda_{\boldsymbol{z}}$ is $(1, \infty)$. For $\lambda_{\boldsymbol{z}}=e^{2 \pi i \theta}=\lambda(\theta)$ in the unit circle (minus 1 ), the range of $j_{z}$ includes both sides of 0 . Also, for $\theta$ close to 1 , the numerator of $j_{z}$ is positive and bounded, while the denominator is approximately $(i \theta)^{2}$. Therefore the range is the interval $(-\infty, 1)$.

6.4.2. Pairings of real point components. Suppose $\psi: \overline{\mathcal{H}}^{\mathrm{rd}} \rightarrow \mathbb{P}_{j}^{1}$ is a reduced Hurwitz space cover defined over $\mathbb{R}$. Lem. 6.5 shows the intervals $(-\infty, 1)$ and $(1,+\infty)$ on $\mathbb{P}_{j}^{1}$ lie under real points on $\mathcal{H}$ (the original Hurwitz space) coming from covers over $\mathbb{R}$ with two different styles of branch points. The interval $(-\infty, 1)$ goes through $0 \in \mathbb{P}_{j}^{1}$, though this is a branch point for the cover $\psi$. This is because ramification over 0 has order 3 (or 1), and a unique 3rd root of 1 (or -1) is real.

The same simple observation gives the next dessins d'enfant type lemma. Denote by $S_{(1, \infty)}\left(\right.$ resp. $\left.S_{(-\infty, 1)}\right)$ the real points of $\mathcal{H}^{\text {rd }}$ over the interval $(1, \infty)$ (resp. $(-\infty, 1))$ of $\mathbb{P}_{j}^{1}(\mathbb{R})$. The closure of each component $C$ of $S_{(1, \infty)}$ has endpoints $\boldsymbol{p}_{1}$ over 1 and $\boldsymbol{p}_{\infty}$ over $\infty$. Let $e_{1}(C)=e_{1}\left(\right.$ resp. $\left.e_{\infty}(C)=e_{\infty}\right)$ be the ramification order of $\boldsymbol{p}_{1}$ (resp. $\boldsymbol{p}_{\infty}$ ) over 1 (resp. $\infty$ ). Note: $e_{1}=1$ or 2 . The same attachment of endpoints applies to components of $S_{(-\infty, 1)}$.

Lemma 6.6. Suppose for a given $C$ in $S_{(1, \infty)}, e_{1}(C)$ (resp. $\left.e_{\infty}(C)\right)$ is odd. Then there is a unique $C^{\prime}$ in $S_{(-\infty, 1)}$ with $e_{1}\left(C^{\prime}\right)=e_{1}(C)$ (resp. $e_{\infty}\left(C^{\prime}\right)=e_{\infty}(C)$ ).

If $e_{1}(C)$ (resp. $e_{\infty}(C)$ ) is even, then there is a unique $C^{\prime}$ in $S_{(1, \infty)}$ with $e_{1}\left(C^{\prime}\right)=e_{1}(C)$ (resp. $\left.e_{\infty}\left(C^{\prime}\right)=e_{\infty}(C)\right)$. So, no $C^{\prime \prime}$ in $S_{(-\infty, 1)}$ has $e_{1}\left(C^{\prime \prime}\right)=e_{1}(C)$ (resp. $\left.e_{\infty}\left(C^{\prime \prime}\right)=e_{\infty}(C)\right)$.

Proof. Suppose $t$ is a local uniformizing parameter for $\overline{\mathcal{H}}^{\text {rd }}$ in a neighborhood of $\boldsymbol{p}_{\infty}$. The argument for $\boldsymbol{p}_{1}$ is the same, so we do only the former case. In local analytic coordinates over $\mathbb{R}$, choose $t$ so $t^{e_{\infty}}=1 / j$ and there is a parametrization of the neighborhood of $\boldsymbol{p}_{\infty}$ using power series in $t$ with real coefficients. If $e_{\infty}$ is odd, then real points around $t=0$ map one-one to real points around $j=\infty$. If $e_{\infty}$ is even, then real points around $t=0$ map two-one to the positive number side of $j=\infty$ (no points falling on the negative side of $j=\infty$ ). That interprets the lemma's statement in local coordinates.

6.4.3. $\mathbb{R}$ points on reduced Hurwitz spaces. Assume $r=4$. Prop. 2.3 shows, if $\mathcal{Q}^{\prime \prime}$ acts trivially on Nielsen classes, $\mathbb{R}$-cover points produce all the points on a reduced Hurwitz space $\mathcal{H}^{\text {rd }}=\mathcal{H}(G, \mathbf{C})^{\text {rd }}$ (with any equivalence on Nielsen classes) except possibly in the fibers of $\mathcal{H}^{\text {rd }}$ lying over $j=0$ or 1 . Our next result extends Prop. 6.3 by combining it with Lem. 4.11 to consider any $\mathcal{Q}^{\prime \prime}$ action.

Corollary 6.7. Assume, as above, $j \in(1, \infty)$ (resp. $(-\infty, 0)$ or $(0,1)$ ) and $\hat{\kappa}$ is the operator of Prop. 6.3 for complex conjugate pairs of points $\boldsymbol{z}$ (resp. a complex 
conjugate point and two real points). Let $\boldsymbol{z} \in U_{4}(\mathbb{R})$ lie over $j$. Let $\boldsymbol{g}_{1}, \ldots, \boldsymbol{g}_{t}$ be a listing of representatives from the equivalence classes of Nielsen classes. Then, $\mathbb{R}$ points of $\mathcal{H}^{\mathrm{rd}}$ over $j$ correspond one-one with reduced equivalence classes $\bmod \mathcal{Q}^{\prime \prime}$ that upon containing $\boldsymbol{g}_{i}$ also contain $\hat{\kappa}\left(\boldsymbol{g}_{i}\right)$.

Suppose $\mathcal{H}$ has fine moduli, and $\mathcal{H}^{\mathrm{rd}}$ has b-fine (resp. fine) moduli (Prop. 4.7). Then $\boldsymbol{p} \in \mathcal{H}^{\mathrm{rd}}(\mathbb{R})$ over $j \in(-\infty, 1) \cup(1,+\infty)$ (resp. $(-\infty,+\infty)$ ) is either an $\mathbb{R}$-cover point, or an $\mathbb{R}$-Brauer point. When $\mathcal{H}$ is an inner Hurwitz space, and $X_{\boldsymbol{p}} \rightarrow \mathbb{P}_{z}^{1}$ corresponds to $\boldsymbol{p}$, then $X_{\boldsymbol{p}}(\mathbb{R}) \neq \emptyset$ if and only if $\boldsymbol{p}$ corresponds to an $H$ - $M$ rep.

This result applies to the main Modular Tower of this paper.

Proposition 6.8. Each level $\mathcal{H}_{k}^{\mathrm{rd}}=\mathcal{H}\left(G_{k}, \mathbf{C}_{3^{4}}\right)^{\mathrm{in}, \mathrm{rd}}$ of the $\left(A_{5}, \mathbf{C}_{3^{4}}, p=2\right)$ Modular Tower has an absolutely irreducible component $\mathcal{H}_{k, *}^{\mathrm{rd}}$ with both $H$ - $M$ and near $H$-M reps. Each point of $\mathcal{H}_{k, *}^{\mathrm{rd}}$ of either type produces $\mathbb{R}$-cover points in the Nielsen class. Suppose $\boldsymbol{g}$ is a branch cycle description of such a cover with respect to $\boldsymbol{z}$ having complex conjugate pairs of branch points. Then, the complement $(\boldsymbol{g}) \mathbf{c m}$ of $\boldsymbol{g}$ (Def. 7.4) corresponds to an $\mathbb{R}$-Brauer point of $\mathcal{H}_{k, *}^{\mathrm{rd}}$.

Consider the nontrivial central Frattini extension $T_{k}^{\prime} \rightarrow G_{k}$ from Cor. 9.7. The natural one-one (not necessarily onto) map $\mathcal{H}\left(T_{k}^{\prime}, \mathbf{C}_{3^{4}}\right)^{\mathrm{in}, \mathrm{rd}} \rightarrow \mathcal{H}\left(G_{k}, \mathbf{C}_{3^{4}}\right)^{\mathrm{in}, \mathrm{rd}}$ gives a component of $\mathcal{H}\left(T_{k}^{\prime}, \mathbf{C}_{3^{4}}\right)^{\mathrm{in}, \mathrm{rd}}$ isomorphic (equivalent as covers of $\mathbb{P}_{j}^{1}$ ) to $\mathcal{H}_{k, *}^{\mathrm{rd}}$. An $\mathbb{R}$-cover point $\boldsymbol{p} \in \mathcal{H}_{k, *}^{\mathrm{rd}}$ for an $H$-M (resp. near $H$-M) rep. in the Nielsen class $\mathrm{Ni}\left(G_{k}, \mathbf{C}_{3^{4}}\right)$ corresponds to a cover in $\mathrm{Ni}\left(T_{k}^{\prime}, \mathbf{C}_{3^{4}}\right)$ with minimal field of definition $\mathbb{R}$ (resp. $\mathbb{C})$. So, an $\mathbb{R}$-cover point for a near $H$ - $M$ rep. (for $G_{k}$ ) corresponds to a $T_{k}^{\prime}$ cover with field of moduli $\mathbb{R}$, but minimal definition field $\mathbb{C}$.

Proof. Prop. 7.11 gives the component containing both $\mathrm{H}-\mathrm{M}$ and near $\mathrm{H}-$ $\mathrm{M}$ reps. The statement on $T_{k}^{\prime}$ is from Prop. 8.12 Nontriviality of $T_{k}^{\prime}$ is from the discussion prior to Cor. 9.7 an inductive consequence of Prop. 9.6] and that $T_{0}^{\prime}=\mathbb{Z} / 2=\operatorname{ker}\left(\operatorname{Spin}_{5} \rightarrow A_{5}\right)$.

The complex conjugation operator for a near H-M rep. in this case gives conjugation by an element whose lift to $R_{k}^{\prime}$ has order 4 . From this, Prop. 6.3 shows a near H-M $\boldsymbol{p} \in \mathcal{H}_{k, *}(\mathbb{R})$ (regarding it in $\mathcal{H}\left(T_{k}^{\prime}, \mathbf{C}_{3^{4}}\right)^{\text {in }}$ ) has no cover realizing it over $\mathbb{R}$. If, however, $\boldsymbol{p}$ corresponds to an H-M rep., then regarding it as the inner class of an $T_{k}^{\prime}$ cover, it has a trivial complex conjugation operator.

So, we have $X \rightarrow \mathbb{P}_{z}^{1}$ with group $G_{k}$ over $\mathbb{R}$ and geometrically $Y \rightarrow X \rightarrow \mathbb{P}_{z}^{1}$ ( $Y \rightarrow X$ unramified) with field of moduli $\mathbb{R}$, and the group of $Y \rightarrow \mathbb{P}_{z}^{1}$ is $T_{k}^{\prime}$. Apply Lem. 3.15 to $Y \rightarrow X$; the resulting cover $Y^{\prime} \rightarrow X$ has degree 2 . It is therefore Galois, and $Y^{\prime} \rightarrow \mathbb{P}_{z}^{1}$ produces the $T_{k}^{\prime}$ realization over $\mathbb{R}$.

REMARK 6.9 (Change conjugate pairs $\boldsymbol{z}$ to all real points). Assume $\boldsymbol{z}$ has two conjugate pairs of points. Choose $\beta \in \mathrm{PSL}_{2}(\mathbb{C})$ to map $z$ to $\boldsymbol{z}^{\prime}$, four points in $\mathbb{R}$. If $\varphi: X \rightarrow \mathbb{P}_{z}^{1}$ is a cover over $\mathbb{R}$ with branch points $z$, then $\beta \circ \varphi: X \rightarrow \mathbb{P}_{z}^{1}$ is in the reduced equivalence class of $\varphi$. It is not, however, a cover over $\mathbb{R}$ as it stands, because $\mathbb{R}$ is not a field of definition of $\beta \circ \varphi$. Sometimes, however, $\beta \circ \varphi$ is strong equivalent (so it has the same branch points $\boldsymbol{z}^{\prime}$ ) to a cover over $\mathbb{R}$. An example arising in this paper is the absolute and inner reduced equivalence classes for $\mathrm{Ni}\left(A_{5}, \mathbf{C}_{3^{4}}\right)$. Consider the cover with H-M description $\boldsymbol{g}=((123),(132),(145),(154))$ and $\boldsymbol{z}$ as branch points. With a base point $z_{0} \in \mathbb{P}_{z}^{1}(\mathbb{R})$, the effect of complex conjugation from Prop. 6.3 is the identity. The points on $X$ over $z_{0}$ are all real. 
Transform the paths giving $\boldsymbol{g}$ above by $\beta$. This gives the same branch cycle description of $\beta \circ \varphi$ with respect to these new paths. Since, however, $\boldsymbol{z}^{\prime}$ consists of four real points, use the corresponding complex conjugation operator. As $\beta\left(z_{0}\right)$ may not be real, this may require adjustment in getting a base point $z_{0}^{\prime} \in \mathbb{P}_{z}^{1} \backslash \beta \boldsymbol{z}$. Still, with this choice, $c_{1}=(23)(45)$ relative to $\beta$ transformed paths gives complex conjugation on the points above $z_{0}^{\prime}$. Thus, the cover (either degree 5 or the Galois closure of the degree 5 cover) is equivalent to one over $\mathbb{R}$. This might happen whenever $\mathcal{Q}^{\prime \prime}$ is not faithful on a Nielsen class, especially one with an H-M rep., $\boldsymbol{g}=\left(g_{1}, g_{1}^{-1}, g_{2}, g_{2}^{-1}\right)$. Then, $(\boldsymbol{g}) q_{1} q_{3}^{-1}=\left(g_{1}^{-1}, g_{1}, g_{2}^{-1}, g_{2}\right)$ and $h \boldsymbol{g} h^{-1}=(b g) q_{1} q_{3}^{-1}$ is equivalent to the phenomenon above. For, however, H-M reps. in $\operatorname{Ni}\left(G_{1}, \mathbf{C}_{3^{4}}\right)$, there is no such $h$. So the corresponding cover with real branch points is not equivalent to a cover over $\mathbb{R}$.

6.4.4. How it works for $r \geq 5$. Suppose $r \geq 5$ and $\boldsymbol{z} \in U_{r}$ is suitably general (lying off an explicit Zariski closed subset of $U_{r}$, dependent on $r$ ). Then, there is no nonidentity $\alpha \in \mathrm{PSL}_{2}(\mathbb{C})$ with $\alpha(\boldsymbol{z})=\boldsymbol{z}$. The argument of 2.3 .1 for (fixed $r$ ), shows the set of $\boldsymbol{z}$, with $\alpha$ acting on it as an element of order exceeding 3, is a Zariski closed subset. If $\alpha$ has order 3 , assume with no loss $\left\{z_{1}, z_{2}, z_{3}\right\}$ is $\{0,1, \infty\}$. This reduces the problem to noting there are only two other specific fixed points and no other cycles of order 3 (2.3.1). We show only a proper Zariski closed subset of $z$ is fixed by some involution. With no loss, consider four values $0,1, \infty, z_{4}$ transposed in pairs. This determines $\alpha$, so other elements of $z$, either fixed or transposed in pairs, are special. Denote this exceptional subset by $J_{r, \text { fix }}$.

Proposition 6.10. Suppose $r \geq 5, \boldsymbol{z} \in U_{r}(K)$ and $\mathcal{H}$ is a Hurwitz space over $K$. Assume $\boldsymbol{z} \mapsto j^{\prime} \in J_{r} \backslash J_{r, \mathrm{fix}}$. Then, points of the fiber $\mathcal{H}_{z}$ go one-one to the points of the fiber $\mathcal{H}_{j^{\prime}}^{\text {rd }}$, so they have exactly the same fields of definition over $K$.

There is a proper algebraic subset $J_{r, \mathrm{br}}$ of $J_{r}$ so that if $j^{\prime} \in J_{r} \backslash J_{r, \mathrm{br}}$ is a $K$ point, then the fiber of $U_{r}$ over $j^{\prime}$ has $K$ points. For $j^{\prime} \in J_{r}(K) \backslash\left(J_{r, \text { br }} \cup J_{r, \text { fix }}\right)$, a $K$ point on $\mathcal{H}^{\text {rd }}$ over $j^{\prime}$ is the image of a $K$ point on $\mathcal{H}$ for some $\boldsymbol{z}$ mapping to $j^{\prime}$.

So, for $r \geq 5$, some problems for $r=4$ don't appear in comparing $\mathcal{H}^{\mathrm{rd}}(K)$ and $\mathcal{H}(K)$. Particularly, from Prop.4.7 if $\mathcal{H}$ is a fine moduli space, each component of $\mathcal{H}^{\text {rd }}$ is a b-fine moduli space; and avoiding $\boldsymbol{z}$ over $J_{r, \mathrm{br}} \cup J_{r, \text { fix }}$, we can lift $K$ points from $\mathcal{H}^{\text {rd }}$ to $\mathcal{H}$. Still, the points of $J_{r, \text { br }} \cup J_{r, \text { fix }}$, including the orbifold points of $J_{r, \text { fix }}$ require a refined analysis to extend the full version of Prop. 4.7 when $r=4$.

6.5. Level $0 \mathbb{R}$ points on the $\left(A_{5}, \mathbf{C}_{3^{4}}\right)$ Modular Tower. 4.2 produces a ramified cover $\psi_{0, \text { abs }}: \mathcal{H}_{0}^{\text {abs,rd }} \rightarrow \mathbb{P}_{j}^{1} \backslash\{\infty\}$ from $\bar{M}_{4}=H_{4} / \mathcal{Q}$ acting on reduced absolute Nielsen classes. Closure of this cover over the $j$-line gives $\bar{\varphi}^{\text {abs,rd }}: \overline{\mathcal{H}}_{0}^{\text {abs,rd }} \rightarrow \mathbb{P}_{j}^{1}$ with branch cycles $\left(\gamma_{0}^{\prime}, \gamma_{1}^{\prime}, \gamma_{\infty}^{\prime}\right)$ Fri99, $\left.\$ 7.4\right]$ :

$$
\gamma_{0}^{\prime}=(214)(378)(569), \gamma_{1}^{\prime}=(45)(39)(12)(86), \gamma_{\infty}^{\prime}=(14985)(367) .
$$

This is a cover with branch points $\{0,1, \infty\}$. We explicitly uniformize the real points on this cover, and on the inner version of this space, including the points over branch points. From Lem. 77.1, the geometric (resp. arithmetic) monodromy group of the cover is $A_{9}$ (resp. $S_{9}$ ). So, (3.20) uniquely determines the effect of complex conjugation. 
6.5.1. Absolute reduced Hurwitz space real points. For a reduced Hurwitz space (over $\mathbb{R}$ ) covering $\mathbb{P}_{j}^{1}$, there are three complex conjugation operators $c_{-\infty, 0}, c_{0,1}$ and $c_{1,+\infty}$ corresponding to the three intervals over which the cover is unramified. As in $6.4 .2 c_{-\infty, 0}, c_{0,1}$ will produce the same number of real points lying over a corresponding $j$ value. Apply (6.8). With $u_{1}, u_{2} \in\{0,1, \infty\}$, write the operator as $c_{u_{1}, u_{2}}$ (suppressing the \pm ). Its characterization is $c \gamma_{u_{i}}^{\prime} c=\left(\gamma_{u_{i}}^{\prime}\right)^{-1}, i=1,2$. As $\mathcal{Q}^{\prime \prime}$ acts trivially on $\mathrm{Ni}\left(A_{5}, \mathbf{C}_{3^{4}}\right)^{\text {abs }}$, Prop. 2.3 shows all elements of $\mathcal{H}_{0}^{\text {abs,rd }}(\mathbb{R})$ are $\mathbb{R}$-cover points. We see this directly in the next lemma.

LEMMA 6.11. All three points of $\mathcal{H}_{0}^{\mathrm{abs}, \mathrm{rd}}(\mathbb{R})$ lying over $j \in(1, \infty)$ are $\mathbb{R}$-cover points. The same is true for each point over the intervals $(-\infty, 0)$ and $(0,1)$.

Proof. From the above, $c_{\infty, 0}$ conjugates both $\gamma_{0}^{\prime}$ and $\gamma_{\infty}^{\prime}$ to their inverses. The unique element doing this is $c_{\infty, 0}=(14)(37)(59)$. So there are three real points above $j_{0} \in(-\infty, 0)$. Check: $c_{1, \infty}=(36)(89)(45)$ and $c_{0,1}=(38)(96)(12)$. Now we show cover points account for all $\mathbb{R}$ points on $\mathcal{H}_{0}^{\text {abs,rd }}$.

The fixed points of $c_{1, \infty}=(36)(89)(45)$ point to three covers in Table 1 labeled ${ }_{1} \boldsymbol{g}{ }_{2} \boldsymbol{g},{ }_{7} \boldsymbol{g}$. Each attaches (by the $\gamma_{\infty}$ operator) to a distinct cusp over $j=\infty$, of respective widths 5, 1 and 3 . That, however, comes from choosing classical generators of $\pi_{1}\left(U_{\boldsymbol{z}}, z_{0}\right)$ for some $\boldsymbol{z}$ and $z_{0}$. It doesn't show what the description of branch cycles will be for a set of paths on $U_{\boldsymbol{z}}$ supporting a complex conjugation operator of Prop. 6.3. Actual covers $\varphi: X \rightarrow \mathbb{P}_{z}^{1}$ having these real structures come from two types of $z$ s using the paths (6.7).

For $\boldsymbol{z}$ with real entries: $\boldsymbol{g}_{1}=((123),(132),(145),(154))$ has complex operator $c_{1}=(23)(45) ; \boldsymbol{g}_{2}=((123),(145),(154),(132))$ has $c_{2}=(13)(54)$ and $\boldsymbol{g}_{7}=((123),(214),(245),(532))$ has $c_{7}=(23)$.

6.5.2. General expectations for genus 0 covers. Let $K$ be a perfect field with $Y$ isomorphic to $\mathbb{P}_{z}^{1}$ over $\bar{K}$. For $X$ a projective nonsingular curve, assume $\varphi: X \rightarrow Y$ is over $K$. If $X$ has genus 0 , then $X$ defines an element $[X] \in H^{2}\left(G_{K}, \bar{K}^{*}\right)$ of order 2 Ser96 p. 126]. A simple formula relates these: $n[X]=[Y]$ with $n=\operatorname{deg}(\varphi)$ Fri75 Thm. 1]. Further, for any odd integer $n, K$ and $[Y] \in H^{2}\left(G_{K}, \bar{K}^{*}\right)$, there is such a $\varphi: X \rightarrow Y$ over $K$ with $X$ of genus 0 [Fri75 Thm. 2].

Problem 6.12. Given $[Y]$ and $n$ odd, which Nielsen classes of genus 0 curve covers have $\varphi: X \rightarrow Y$ over $K$ of degree $n$ in the Nielsen class?

Remark 6.13 (Chătalet's Work). See [CT88, p. 389] or Ch44, Châtalet's Thesis] for background on these comments. Châtalet knew that $X$ a Brauer-Severi variety of dimension $n$ defines a central simple algebra over $K$ of dimension $n^{2}$. We recognize this as $[X] \in H^{2}\left(G_{K}, \bar{K}^{*}\right)$. If $X(L) \neq \emptyset$ with $([L: K], n)=1$, then it possesses a $K$ point (from the corestriction-restriction sequence of Galois cohomology [AW67, p. 105]). Also, if $X_{K_{\boldsymbol{p}}}=Y_{K_{\boldsymbol{p}}}$ for each completion $\boldsymbol{p}$ of $K$, then Chătelet knew $X \equiv Y$. This gives the easy direction of [Fri75 Thm. 1] when their images in the completions of a field determine the Brauer-Severi variety. According to [T88, Weil We56 gave the modern Galois cohomology interpretation.

6.5.3. Inner reduced Hurwitz space real points. Now consider branch cycles for $\bar{\varphi}^{\text {in,rd }}: \overline{\mathcal{H}}^{\text {in,rd }} \rightarrow \mathbb{P}_{j}^{1}$ from inner classes $\operatorname{Ni}\left(A_{5} \mathbf{C}_{3^{4}}\right)^{\text {in }}$ (Fri99 $\left.\$ 7.10\right]$, Fri90]):

$$
\begin{aligned}
\gamma_{0}^{\prime \prime} & =(1132)(3717)(41110)(5159)(61814)(81216), \text { and } \\
\gamma_{1}^{\prime \prime} & =(111)(210)(39)(414)(513)(617)(716)(815)(1218) \\
\gamma_{\infty}^{\prime \prime} & =(211)(141885)(101391714)(31516)(1267) .
\end{aligned}
$$


For $j_{0} \in(1, \infty)$, inspection gives $c_{1, \infty}$ conjugating $\gamma_{1}^{\prime \prime}$ and $\gamma_{\infty}^{\prime \prime}$ to their inverses:

$$
c_{1, \infty}=(917)(716)(818)(1314)(1215)(63)(45) .
$$

The centralizer of the monodromy group ( $c^{\dagger}$ in Ex. 3.36) moves 1. To show $c_{1, \infty}$ is the correct complex conjugation, it suffices to check the complex conjugation operator for four real points fixes 1 and 10 (the H-M reps.).

Similarly, for $j_{0} \in(-\infty, 0)$, by inspection find

$$
c_{-\infty, 0}=(1013)(14)(914)(73)(1216)(112)(156)(518)
$$

conjugates $\gamma_{0}^{\prime \prime}$ and $\gamma_{\infty}^{\prime \prime}$ to their inverses. Note: $c_{1, \infty}$ fixes 2 and 11. If the complex conjugation operator for $(-\infty, 0)$ fixes either, the set of real points would not be a 1-dimensional manifold in the neighborhood of the index 2 branch point over $\infty$. As $c_{-\infty, 0} c^{\dagger}$ fixes 2 and $11, c_{-\infty, 0}$ is the correct complex conjugation operator.

Applying DF90, §4.1] gives the following information on points over $\infty$ cusps - in each cover. Again, since $\mathcal{Q}^{\prime \prime}$ acts trivially on $\mathrm{Ni}\left(A_{5}, \mathbf{C}_{3^{4}}\right)^{\text {in }}$, Prop. 2.3 shows all elements of $\mathcal{H}_{0}^{\text {abs,rd }}(\mathbb{R})$ are $\mathbb{R}$ - cover points. Our computations above confirm this directly. (Ex. 2.6 has more on using this example with $\operatorname{Spin}_{5}$ covers.)

Lemma 6.14. For each $j_{0} \in(1, \infty) \subset \mathbb{P}_{j}^{1}(\mathbb{R})$ (resp. $j_{0} \in(-\infty, 1)$ ) there are four (resp. two) points of $\mathcal{H}_{0}^{\mathrm{in}, \mathrm{rd}}(\mathbb{R})$. There are three $\mathbb{Q}$ points over $\infty$ in the cover $\bar{\varphi}^{\mathrm{abs}, \mathrm{rd}}$ of ramification indices 1, 3 and 5. Denote these $x_{1}, x_{3}, x_{5}$. Real points over $\infty$ in the cover $\bar{\varphi}^{\mathrm{in}, \mathrm{rd}}$ correspond to disjoint cycles in $\gamma_{\infty}^{\prime \prime}$ that conjugation by $c_{\mathrm{in}, \mathrm{rd}}$ maps to their inverses. These are the disjoint cycles (211), (141885) and (101391714). There are three real points over $\infty$, one over $x_{1}$ and two over $x_{5}$.

\section{Cusps on a Modular Tower}

This section and 99 describe level $k=1$ components of the $\left(A_{5}, \mathbf{C}_{3^{4}}\right)$ Modular Tower, $\mathcal{H}\left(G_{1}, \mathbf{C}_{3^{4}}\right)^{\text {in }}=\mathcal{H}_{1}$ and its reduced version $\mathcal{H}\left(G_{1}, \mathbf{C}_{3^{4}}\right)^{\text {in,rd }}=\mathcal{H}_{1}^{\text {rd }}$. As usual, $\mathbf{C}_{3^{4}}$ is four repetitions of the class of 3-cycles in $A_{5}$. For this noncongruence tower our detail is comparable to literature describing some specific modular curves.

The idea is to apply Thm. 6.1 to inspect those cusps at the end of real components on $\mathcal{H}_{1}$. This information about the cusps eventually separates the two components of $\mathcal{H}_{1}^{\text {rd }}$ according to the cusps lying in each. When $r=4, \mathcal{Q}^{\prime \prime}$ action produces the phenomenon $q_{2}$ orbit shortening affecting precise genus calculations. 77.2 .2 and 7.2 .3 develop lemmas anticipating the general case.

7.1. Fundamental domains and cusp data. Let $F$ be a standard fundamental domain for $\mathrm{PSL}_{2}(\mathbb{Z})$ on the upper half plane. Branch cycle data from Prop. 4.4 allows computing a fundamental domain for the subgroup of $\mathrm{PSL}_{2}(\mathbb{Z})$ defining $\psi^{\text {rd }}: \overline{\mathcal{H}}^{\text {rd }} \rightarrow \mathbb{P}_{j}^{1}$ (with compatible notation for inner and absolute reduced spaces). The element $\gamma_{0}$ (resp. $\gamma_{1}$ ) identifies with the standard element of order 3 (resp. order 2) in $\mathrm{PSL}_{2}(\mathbb{Z})$. The action of $\gamma_{0}$ and $\gamma_{1}$ on $\operatorname{Ni}\left(G, \mathbf{C}\right.$ ) ${ }^{\text {abs,rd }}$ (or $\operatorname{Ni}(G, \mathbf{C})^{\mathrm{in}, \mathrm{rd}}$ depending on the type of Hurwitz space) gives $\gamma_{0}^{\prime}$ and $\gamma_{1}^{\prime}$, generating a permutation group $\left\langle\gamma_{0}^{\prime}, \gamma_{1}^{\prime}\right\rangle$ whose orbits correspond to $j$-line covers.

For further reference, let $O$ be one of these orbits. Take $\Gamma=\Gamma(O)$ to be the subgroup of $\mathrm{PSL}_{2}(\mathbb{Z})$ stabilizing an element in $O$. A fundamental domain for a cover for the orbit $O$ comes from translating $F$ around by coset representatives for $\Gamma(O)$ in $\mathrm{PSL}_{2}(\mathbb{Z})$. 
7.1.1. Monodromy for $\overline{\mathcal{H}}\left(A_{5}, \mathbf{C}_{3^{4}}\right)^{\text {abs,rd }}$ and $\overline{\mathcal{H}}\left(A_{5}, \mathbf{C}_{3^{4}}\right)^{\text {in,rd }}$. We give the geometric and arithmetic monodromy of both $j$-line covers. Denote $\mathcal{H}\left(A_{5}, \mathbf{C}_{3^{4}}\right)^{\text {abs,rd }}$ by $\mathcal{H}_{0}^{\text {abs,rd }}$, and $\mathcal{H}\left(G_{k}, \mathbf{C}_{3^{4}}\right)^{\text {in,rd }}$ by $\mathcal{H}_{k}^{\text {in,rd }}$.

Consider any sequence of (separable) absolutely irreducible covers $X \rightarrow Y \rightarrow Z$ over a field $K$. Let $G_{Y}$ (resp. $G_{X}$ ) be the group of the galois closure of $Y \rightarrow Z$ (resp. $X \rightarrow Y$ ) over $K$. Then, the group $G=G_{X / Z}$ of the Galois closure (over $K$ ) of the cover $X \rightarrow Z$ is a subgroup of the wreath product $G_{X} \prec G_{Y}$. Further, it has the following properties Fri70 p. 51].

(7.1a) The projection of $G$ to $G_{Y}$ is surjective.

(7.1b) The kernel of $G \rightarrow G_{Y}$ contains a group isomorphic to $G_{X}$.

LEMma 7.1. The geometric monodromy $G_{\psi^{\mathrm{abs}, \mathrm{rd}}}=G_{0, \mathrm{abs}}$ of $\overline{\mathcal{H}}_{0}^{\mathrm{abs}, \mathrm{rd}} \rightarrow \mathbb{P}_{j}^{1}$ is $A_{9}$. Let $\alpha=\sqrt{-15}$. The arithmetic monodromy group $\hat{G}_{0, \text { abs }}$ is $S_{9}$. The two groups fit in a natural short exact sequence

$$
1 \rightarrow G_{0, \mathrm{abs}} \rightarrow \hat{G}_{0, \mathrm{abs}} \rightarrow G(\mathbb{Q}(\alpha) / \mathbb{Q}) \rightarrow 1 .
$$

The geometric monodromy group $G_{\psi^{\mathrm{in}, \mathrm{rd}}}=G_{0 \text {,in }}$ of $\psi^{\mathrm{in}, \mathrm{rd}}: \overline{\mathcal{H}}_{0}^{\mathrm{in}, \mathrm{rd}} \rightarrow \mathbb{P}_{j}^{1}$ is $\mathbb{Z} / 2 \imath A_{9} \stackrel{\text { def }}{=}(\mathbb{Z} / 2)^{9} \times{ }^{s} A_{9}$ with $A_{9}$ acting as permutation of the coordinates of $(\mathbb{Z} / 2)^{9}$. The arithmetic monodromy group $\hat{G}_{0, \text { in }}$ maps to $\hat{G}_{0, \text { abs }}$ producing the extension

$$
\left.1 \rightarrow G_{0, \text { in }} \rightarrow \hat{G}_{0, \text { in }} \rightarrow G(\mathbb{Q}(\alpha) / \mathbb{Q})\right) \rightarrow 1 .
$$

Proof. Use $\gamma_{\infty}^{\prime}$ in (6.12) to see that $G_{0, \text { abs }}$ contains a 5 -cycle, $\left(\gamma_{\infty}^{\prime}\right)^{3}$, so it must primitive. It also contains a 3 - cycle $\left(\gamma_{\infty}^{\prime}\right)^{5}$. A well-known argument says a primitive subgroup of $A_{n}$ containing a 3 -cycle is $A_{n}: G_{0, \text { abs }}=A_{9}$. Apply Fri95b, Prop. 2.1 and Irrational Cycle Lemma]. The gist: As the branch points of the cover are rational, the arithmetic monodromy group contains the character field of the conjugacy class of any element of form (1)(3)(5). Elements that are products of distinct odd disjoint cycle lengths, form two conjugacy classes in $A_{9}$. The outer automorphism of $A_{9}$ (conjugation by (12)) permutes these two conjugacy classes.

For $\psi^{\text {in,rd }}$, the degree 18 cover breaks into a chain of degree 9 and degree 2 covers. The largest possible group for the geometric closure is $\mathbb{Z} / 2 \imath A_{9}$ and for the arithmetic closure it is $\mathbb{Z} / 2 \imath S_{9}$. To complete the proof only requires showing the kernel of the geometric closure to $A_{9}$ contains one of the factors of $(\mathbb{Z} / 2)^{9}$. Since $\left(\gamma_{\infty}^{\prime \prime}\right)^{15}=(211)$ generates such a factor, we are done.

Example 7.2. To illustrate, consider (6.12) for $\operatorname{Ni}\left(A_{5}, \mathbf{C}_{3^{4}}\right)^{\text {abs }}$ (or 6.5.3) for $\left.\mathrm{Ni}\left(A_{5}, \mathbf{C}_{3^{4}}\right)^{\text {in }}\right)$. The representation has degree 9 (or 18). An explicit orbit comes as follows. Label 9 words $w\left(\gamma_{0}^{\prime}, \gamma_{1}^{\prime}\right)_{i}=w_{i}, i=1, \ldots, 9$, in the elements

$$
\gamma_{0}^{\prime}=(214)(378)(569) \text { and } \gamma_{1}^{\prime}=(21)(45)(68)(39) \text {, }
$$

so these words applied to 1 give the complete set $\{1,2, \ldots, 9\}$. That is, they are coset representatives. Example: $w_{1}$ the trivial word, $\gamma_{0}^{\prime}=w_{2},\left(\gamma_{0}^{\prime}\right)^{2}=w_{3}, w_{4}=\gamma_{0}^{\prime} \gamma_{1}^{\prime}$, $w_{5}=w_{4} \gamma_{0}^{\prime}, w_{6}=w_{5} \gamma_{0}^{\prime}, w_{7}=w_{6} \gamma_{1}^{\prime}, w_{8}=w_{7} \gamma_{0}^{\prime}, w_{9}=w_{8} \gamma_{0}^{\prime}$. Then,

$$
\begin{array}{lllll}
\text { (1) } w_{1}=1, & (1) w_{2}=4, & (1) w_{3}=2, & (1) w_{4}=5, & (1) w_{5}=6, \\
\text { (1) } w_{6}=9, & (1) w_{7}=3, & (1) w_{8}=7, & (1) w_{9}=8 .
\end{array}
$$

Finally, apply these, with $\gamma_{0}$ and $\gamma_{1}$ replacing $\gamma_{0}^{\prime}$ and $\gamma_{1}^{\prime}$ to $F$. Take the union of the images as a fundamental domain for the $j$-line cover. 
7.1.2. (2,3) generation. Every group with two generators, $\gamma_{0}^{\prime}, \gamma_{1}^{\prime}$, with respective orders 3 and 2 , is a quotient of $\mathrm{PSL}_{2}(\mathbb{Z})$. Sh01 calls these $(2,3)$ generated groups. Finding which such finite simple groups do so occur has been a problem with much literature for over 100 years. The groups $A_{n}$ have this property for $n \geq 9$, a result of Miller from 1901, and all simple $(2,3)$ generated groups are known Sh01. The group $A_{8}$ does not so occur, though $\mathrm{PSL}_{2}(\mathbb{Z} / 7)$ (a simple group) does give a primitive subgroup of $A_{8}$ that is $(2,3)$ generated. Though to us it is icing on the cake that the main example of this paper starts at the border of this old area, we consider it more evidence that there is a cake.

7.1.3. Fundamental domains from cusps. Let $\mathbb{R}_{\infty}=\mathbb{R} \cup\{\infty\}$. Recall: Cusps are equivalence classes for $\mathbb{Q} \cup\{\infty\}$ on $\mathbb{R}_{\infty}$ for the $\Gamma(O)$ action. These equivalence classes correspond to orbits of $\gamma_{\infty}^{\prime}$. For example, in (6.12), $\gamma_{\infty}^{\prime}=(14985)(367)$. So, there are three cusps, with widths (lengths) 1, 3 and 5. By contrast, $\Gamma_{0}(p)$, with $p$ a prime, has two cusps of widths 1 and $p$. With no loss, $\infty$ corresponds to the cycle of length 1 in $\gamma_{\infty}^{\prime}$. (Let the image of $F$ corresponding to the integer 2 to have $\infty$ as its cusp.) Then, there exist elements $g_{3}^{\prime}, g_{5}^{\prime} \in \mathrm{PSL}_{2}(\mathbb{Z})$ as follows:

$$
\begin{aligned}
& g_{3}^{\prime} \Gamma\left(g_{3}^{\prime}\right)^{-1} \cap\left\langle\left(\begin{array}{ll}
1 & 1 \\
0 & 1
\end{array}\right)\right\rangle=\left\langle\left(\begin{array}{ll}
1 & 3 \\
0 & 1
\end{array}\right)\right\rangle, \text { and } \\
& g_{5}^{\prime} \Gamma\left(g_{5}^{\prime}\right)^{-1} \cap\left\langle\left(\begin{array}{ll}
1 & 1 \\
0 & 1
\end{array}\right)\right\rangle=\left\langle\left(\begin{array}{ll}
1 & 5 \\
0 & 1
\end{array}\right)\right\rangle .
\end{aligned}
$$

To get explicit $g_{3}^{\prime}$ and $g_{5}^{\prime}$ from this calculation choose an expression in the $w \mathrm{~s}$ taking an integer in the respective 3 and 5 cycles to the integer 2 . The following summarizes this explicitness result.

Proposition 7.3. Start with any Nielsen class having $r=4$. Prop. 4.4 produces a fundamental domain (in the upper half plane) and basic data on the cusp points of the cover $\overline{\mathcal{H}}^{\mathrm{rd}} \rightarrow \mathbb{P}_{j}^{1}$ coming from that Nielsen class.

The main result of 9 shows $\mathcal{H}_{1}^{\text {rd }}$ has two absolutely irreducible ( $\mathbb{Q}$-curve) components, of respective genuses 12 and 9. The former component corresponds to a braid orbit $\left(\mathrm{O}_{1}^{+}\right.$below) on $\mathrm{Ni}_{1}$ containing all $\mathrm{H}-\mathrm{M}$ and near $\mathrm{H}-\mathrm{M}$ representatives (₫6). So, Thm. 6.1 implies only the genus 12 component contains $\mathbb{Q}$ points.

Branch cycles for these components come from applying the program $\mathbf{S}^{+} \mathbf{9 5}$ to compute $\gamma_{0}, \gamma_{1}$ on $\operatorname{Ni}\left({ }_{2}^{1} \tilde{A}_{5}, \mathbf{C}_{3^{4}}\right)^{\text {in,rd }}$. As in Prop. 4.4 this gives $\gamma_{0}^{\prime}, \gamma_{1}^{\prime}, \gamma_{\infty}^{\prime}$ for a cover from each $\bar{M}_{4}$ orbit. Especially important are the lengths of $\gamma_{\infty}^{\prime}$ orbits.

$\$$ A isolates an expected general structure of fundamental domains for higher levels of a Modular Tower. It is that the monodromy groups of the higher reduced levels over level 0 should be near $p$-groups (2-groups in our example).

7.2. Cusp notation. Denote the image of $q_{2} \in H_{4}$ in $\bar{M}_{4}$ by $\gamma_{\infty}$. Refined calculations differentiate between $q_{2}$ orbits on inner Nielsen classes $\mathrm{Ni}_{1}^{\text {in }}$ and $\gamma_{\infty}$ orbits on $\mathrm{Ni}_{1}^{\mathrm{rd}}=\mathrm{Ni}_{1}^{\mathrm{in}, \mathrm{rd}}$. The latter attach to cusps on $j$-line covers.

7.2.1. $\gamma_{\infty}$ and $q_{2}$ orbit notation. We often denote $\gamma_{\infty}$ orbits on $\mathrm{Ni}_{1}^{\text {rd }}$ by notation like $O(u, v ; a)$. If $\boldsymbol{g}=\left(g_{1}, g_{2}, g_{3}, g_{4}\right) \in O(u, v ; a)$, then $u=\mathbf{m p}(\boldsymbol{g}) \stackrel{\text { def }}{=} \operatorname{ord}\left(g_{2} g_{3}\right)$ is the middle product $(2.9 .3)$ of $\boldsymbol{g}$. Also, $v=\mathbf{w d}(\boldsymbol{g}) \stackrel{\text { def }}{=}|O(u, v ; a)|$ is the width of $\boldsymbol{g}$.

The decoration $a$ distinguishes orbits with specific $(u, v)$. If it is cumbersome, or we don't know $(u, v)$, a briefer notation suffices. Given $\boldsymbol{g} \in O(u, v ; a)$, refer to its orbit type as $(u, v)=(\mathbf{m p}(\boldsymbol{g}), \mathbf{w d}(\boldsymbol{g}))$. When the context is clear, use $(u, v)$ as 
the type of a $q_{2}$ orbit on $\mathrm{Ni}_{1}^{\text {in }}$. The $q_{2}$ orbit type of $\boldsymbol{g} \in \mathrm{Ni}_{1}^{\text {in }}$ may be different from its $\gamma_{\infty}$ orbit type if $\mathcal{Q}^{\prime \prime}$ orbit shortening reduces the value of $v$ (\$7.2.3).

Consider $\boldsymbol{g} \in \mathrm{Ni}_{1}^{\text {in }}$ lifting $\boldsymbol{g}^{\prime} \in \mathrm{Ni}_{0}^{\text {in }}$ with $\mathbf{m p}\left(\boldsymbol{g}^{\prime}\right)=3$ or 5 . According to Lem. 2.25 $\boldsymbol{g}$ has a complementary element $(\boldsymbol{g}) q_{2}^{\mathbf{m p}(\boldsymbol{g}) / 2}$. This is the unique element in the $\gamma_{\infty}$ orbit of $\boldsymbol{g}$, distinct from $\boldsymbol{g}$, that maps to $\boldsymbol{g}^{\prime}$. Denote this by $(\boldsymbol{g}) \mathbf{c m}$.

Definition 7.4 (More complements). Prop.7.11 generalizes the case $k=1$ to define $(\boldsymbol{g}) \mathbf{c m}$ as $(\boldsymbol{g}) q_{2}^{\mathrm{mp}(\boldsymbol{g}) / 2}$ for $\boldsymbol{g} \in \mathrm{Ni}_{k}\left(G_{k}, \mathbf{C}_{3^{4}}\right), k \geq 1$. 9.6 generalizes further.

7.2.2. $\mathcal{Q}^{\prime \prime}$ orbits. Thm. 2.9 says $\mathcal{Q} \triangleleft H_{4}$ acts on an inner Nielsen class through a Klein 4-group, $K_{4}=\mathcal{Q}^{\prime \prime}$.

As in Fri90 or [Fri99 $\S 7.10]$, the Nielsen class $\mathrm{Ni}_{0}=\mathrm{Ni}\left(A_{5}, \mathbf{C}_{3^{4}}\right)^{\text {in }}$ contains exactly 18 elements. Just two are H-M representatives. Branch cycles for these are

$$
\begin{aligned}
& \boldsymbol{g}_{\mathrm{hm}, 1}=((123),(321),(145),(541)) \text { and } \\
& \boldsymbol{g}_{\mathrm{hm}, 2}=\left(\left(\begin{array}{ll}
1 & 23
\end{array}\right),\left(\begin{array}{ll}
3 & 21
\end{array}\right),\left(\begin{array}{ll}
5 & 4
\end{array}\right),(145)\right) \text {. }
\end{aligned}
$$

These two are equivalent in the absolute Nielsen classes by (45) conjugation, an outer automorphism of $A_{5}$. Further, $H_{4}$ is transitive on $\mathrm{Ni}_{0}$. So, the number of lifts of any $\boldsymbol{g} \in \mathrm{Ni}_{0}$ to $\mathrm{Ni}_{1}$ is independent of the choice of $\boldsymbol{g}$.

We first show faithful action of $\mathcal{Q}^{\prime \prime}$ on $\mathrm{Ni}_{1}$. As usual, $\left\{G_{k}\right\}_{k=0}^{\infty}$ are the characteristic quotients of ${ }_{p} \tilde{G}$, with $\mathbf{C}$ a collection of $p^{\prime}$ conjugacy classes of $G_{0}=G$. Write $\mathcal{Q}^{*}=\left\langle Q_{1} Q_{3}^{-1},\left(Q_{1} Q_{2} Q_{3}\right)^{2}\right\rangle$ when we regard it as a subgroup of $B_{4}$. So, $\mathcal{Q}^{*}$ acts on $\operatorname{Ni}(G, \mathbf{C})$ (no equivalence by conjugation by $G$ ). The notation $\mathcal{Q}^{\prime \prime}$ means this Klein 4-group is acting on inner classes. Use $\operatorname{Stab}_{\mathcal{Q}^{\prime \prime}}(\boldsymbol{g})$ for the stabilizer in $\mathcal{Q}^{\prime \prime}$ of $\boldsymbol{g} \in \mathrm{Ni}^{\text {in }}$ (for absolute equivalence, mod out further by $G \leq N^{\prime} \leq S_{n}$ ). For any group $S$, express the orbit of $\boldsymbol{g}$ under $S$ as $(\boldsymbol{g}) S$.

Lemma 7.5. Let $\mathrm{Ni}(G, \mathbf{C})$ be a Nielsen class for $r=4$ with $G$ centerless. Then, $\boldsymbol{g} \in \operatorname{Ni}(G, \mathbf{C})$ is invariant under $\left(Q_{1} Q_{3}^{-1}\right)^{2} \in \mathcal{Q}^{*}$ if and only if $\boldsymbol{g}$ is an H-M rep. If $\boldsymbol{g}=\left(g_{1}, g_{1}^{-1}, g_{2}, g_{2}^{-1}\right)$ is an $H-M$ rep., then $\mathcal{Q}^{*}$ acts on $(\boldsymbol{g}) \mathcal{Q}^{*}$ as a quotient of the Klein 4-group $K_{4}$. The $K_{4}$ action is faithful unless $g_{1}$ and $g_{2}$ are involutions.

Let $\mathrm{Ni}_{k}$ be the kth level inner Nielsen class for any Modular Tower with $r=4$. Length of the $\mathcal{Q}^{\prime \prime}$ orbit on $\boldsymbol{g} \in \mathrm{Ni}_{k}$ depends only on the $H_{4}$ (or $\bar{M}_{4}$ ) orbit of $\boldsymbol{g}$. Faithful action of $\mathcal{Q}^{\prime \prime}$ on an $\mathrm{H}_{4}$ level $k$ orbit extends to faithful action on an $\mathrm{H}_{4}$ level $k+1$ orbit above it. Similarly, if $\operatorname{Stab}_{\mathcal{Q}^{\prime \prime}}\left((\boldsymbol{g})\left\langle q_{2}\right\rangle\right)=\{1\}$, then $\operatorname{Stab}_{\mathcal{Q}^{\prime \prime}}\left(\left(\boldsymbol{g}^{\prime}\right)\left\langle q_{2}\right\rangle\right)=$ $\{1\}$ for $\boldsymbol{g}^{\prime} \in \mathrm{Ni}_{k+1}$ above $\boldsymbol{g}$.

Assume $p=2$, and $\mathrm{Ni}_{k}, k \geq 1$, is the Nielsen class for centerless 2-perfect $G_{0}=G$. Let $O$ be an $H_{4}$ orbit on $\mathrm{Ni}_{k}$ containing an $H-M$ rep. Then all $\mathcal{Q}^{\prime \prime}$ orbits on $O$ have length four. $S o, \mathcal{H}_{O}^{\mathrm{rd}}$, the reduced component corresponding to $O$ has $b$-fine moduli (Prop. 4.7).

For $G_{0}=A_{5}, p=2$ and $\mathbf{C}=\mathbf{C}_{3^{4}}, \mathcal{Q}^{\prime \prime}$ is faithful on all $H_{4}$ orbits in $\mathrm{Ni}_{k}, k \geq 1$.

Proof. Since $(\boldsymbol{g})\left(Q_{1} Q_{3}^{-1}\right)^{2}$ is conjugation of $\boldsymbol{g}$ by $g_{1} g_{2}$, if $G$ is centerless, and $Q_{1} Q_{3}^{-1}$ fixes $\boldsymbol{g}$, then $g_{1} g_{2}=1$. So, $\boldsymbol{g}$ is an H-M rep. Squares of elements in $\mathcal{Q}^{*}$ act trivially on an H-M rep. This shows $\mathcal{Q}^{*}$ acts on the set $(\boldsymbol{g}) \mathcal{Q}^{*}$ as a quotient of $K_{4}$.

Assume $\boldsymbol{g}$ is an H-M rep. and $(\boldsymbol{g}) Q_{1} Q_{3}^{-1}=\boldsymbol{g}$. Then $g_{1}=g_{1}^{-1}$ and $g_{2}=g_{2}^{-1}$ : Both are involutions. Similarly, if $(\boldsymbol{g})\left(Q_{1} Q_{2} Q_{3}\right)^{2}=\boldsymbol{g}$, then $g_{2}=g_{1}$ and $G$ is cyclic (in particular abelian). Also, $(\boldsymbol{g}) Q_{1} Q_{3}^{-1}\left(Q_{1} Q_{2} Q_{3}\right)^{2}=\boldsymbol{g}$ implies $g_{2}=g_{1}^{-1}$, contradicting $G$ is centerless. 
Assume $\boldsymbol{g} \in \mathrm{Ni}_{k}$. As $\mathcal{Q}^{\prime \prime} \triangleleft M_{4}$ (Prop. 2.3), for $\boldsymbol{g} \in \mathrm{Ni}$,

$$
\operatorname{Stab}_{\mathcal{Q}^{\prime \prime}}(\boldsymbol{g})=Q^{-1} \operatorname{Stab}_{\mathcal{Q}^{\prime \prime}}((\boldsymbol{g}) Q) Q .
$$

So, $\left|(\boldsymbol{g}) \mathcal{Q}^{\prime \prime}\right|$ depends only on the $M_{4}$ orbit of $\boldsymbol{g}$. If $\boldsymbol{g} \in \mathrm{Ni}_{k}$, then $\operatorname{Stab}_{\mathcal{Q}^{\prime \prime}}(\boldsymbol{g}) \bmod$ $\operatorname{ker}_{k-1}=\operatorname{Stab}_{\mathcal{Q}^{\prime \prime}}\left(\boldsymbol{g} \bmod \operatorname{ker}_{k-1}\right)$. This gives the statement on faithful action at level $k-1$.

Now assume $p=2$ and $G_{0}$ is $p$-perfect and centerless, so these hypotheses apply at all levels (Prop. 3.21). With no loss, on the general statement on an $H_{4}$ orbit containing $\boldsymbol{g}=\left(g_{1}, g_{1}^{-1}, g_{2}, g_{2}^{-1}\right)$, assume $k=1$.

Suppose for $q \in \mathcal{Q}^{\prime \prime} \backslash\{1\},(\boldsymbol{g}) q=h \boldsymbol{g} h^{-1}$ for some $h \in G_{1}$. From the above, $h$ mod $\operatorname{ker}_{0}$ is not the identity. As $q^{2}$ acts trivially on $\boldsymbol{g},(\boldsymbol{g}) q^{2}=h^{2} \boldsymbol{g} h^{-2}$ with $h^{2} \in M_{0}=\operatorname{ker}_{0} / \operatorname{ker}_{1} \stackrel{\text { def }}{=} M \backslash\{0\}$ (Lem. [2.23). This contradicts $G_{1}$ being centerless.

Now assume $G_{0}=A_{5}$. We show $\mathcal{Q}^{\prime \prime}$ is faithful on any $H_{4}$ orbit $O$ in $\mathrm{Ni}_{1}$. Any orbit at level 1 has elements lying over any element of the unique orbit at level 0 . Anything in $\mathrm{Ni}_{1}$ above an H-M rep. $\bar{g} \in \mathrm{Ni}_{0}$ looks like $\boldsymbol{g}=\left(g_{1}, m g_{1}^{-1} m, n g_{2} n, g_{2}^{-1}\right)$ with $m, n \in M_{0}$, and some $g_{1}, g_{2}$ generating $G_{1}$. We handled when $m$ is in the centralizer of $g_{1}$, so assume it is not. Apply the previous argument when $k-1=0$ with $q=q_{1} q_{3}^{-1}$. Conclude: If $(\boldsymbol{g}) q=h \boldsymbol{g} h^{-1}$, then $h \in G_{1}$ lifts an element of $A_{5}$ having order 2. From Prop. 5.10 $h^{2} \in M \backslash V$. Compute the first two entries of (g) $q^{2}$ to be $\left(m m^{g_{1}^{-1}} g_{1} m m^{g_{1}^{-1}}, m^{g_{1}^{-1}} g_{1}^{-1} m^{g_{1}^{-1}}\right)$.

The remainder of the argument uses Cor. 5.7 Let $c \in M \backslash V$ be the generator of the centralizer of $g_{1}$. Multiply $m$ if necessary by $c$ to assume $m \in M \backslash V$. Then, $h^{2}=m m^{g_{1}^{-1}} c=m^{g_{1}^{-1}}$. Conclude $m=c$ contrary to our assumption. For other elements in $\mathcal{Q}^{\prime \prime} \backslash\{1\}$, the argument is similar, though no easier.

REMARK 7.6 (Lem. 7.5 modular curves and $p \neq 2$ ). Two involutions generate a dihedral group. So, $\mathcal{Q}^{*}$ stabilizing an element of $\mathrm{Ni}$ in Lem. 7.5 comes precisely from the Hurwitz version of modular curves in 2.8.1

Suppose $r=4, \mathrm{Ni}\left(G_{k}, \mathbf{C}\right)^{\mathrm{in}}=\mathrm{Ni}_{k}$ is a level $k \geq 1$ Nielsen class for $G_{0}=G$, centerless and $p$-perfect. Assume $O$, an $H_{4}$ orbit on $\mathrm{Ni}_{k}$, contains an $\mathrm{H}-\mathrm{M}$ rep. These are the hypotheses of Lem. 7.5 except $p \neq 2$. Then, there may be H-M reps. ${ }^{k} \boldsymbol{g} \in \mathrm{Ni}_{k}^{\text {in }}$ with

(7.3) $h_{k} \in G_{k}$ (an involution, or the identity if these are involutions) with $h_{k}{ }^{k} g_{i} h_{k}^{-1}={ }^{k} g_{i}^{-1}, i=1,2$ and all $k \geq 0$.

We don't know if $h_{k} \mathrm{~s}$ and ${ }^{k} \boldsymbol{g} \mathrm{s}$ as in (7.3) exist in this case: $G_{0}=A_{5}, p=5$, $\mathbf{C}=\mathbf{C}_{3^{4}}$ and ${ }^{k} g_{1},{ }^{k} g_{2} \in G_{k}$ lie over the pair ${ }^{0} g_{1}=(123),{ }^{0} g_{2}=(145)$.

7.2.3. $q_{2}$ orbit shortening and Cusp $_{4}$. Again $r=4$, with Nielsen classes of any type (though we continue to simplify notation by using inner classes). Slightly abusing the notation of 2.6.2 use $\alpha^{2}=\left(q_{1} q_{2} q_{3}\right)^{2}$ and $\gamma=q_{1} q_{3}^{-1}$ as generators of $\mathcal{Q}^{\prime \prime}$. Recall the $M_{4}$ subgroup $\operatorname{Cusp}_{4}=\left\langle\alpha^{2}\right\rangle \times\langle\gamma\rangle \times{ }^{s}\left\langle q_{2}\right\rangle$ with $q_{2}$ switching the two factors on the copy of $K_{4}$. In this subsection $O(u, v ; a)=O(u, v) \subset \mathrm{Ni}^{\text {in }}$ is a $q_{2}$ orbit with $u=\mathbf{m p}(\boldsymbol{g})$ for $\boldsymbol{g} \in \mathrm{Ni}^{\text {in }}$. Then, $v$ is the $q_{2}$ orbit length: If $\mathbf{m p}(\boldsymbol{g})$ is odd and (2.19) holds, then $v=u$, otherwise $v=2 \cdot u$.

For $\boldsymbol{g} \in O(u, v), \operatorname{Stab}_{\mathcal{Q}^{\prime \prime}}(O(u, v))$ is the subgroup of $\mathcal{Q}^{\prime \prime}$ stabilizing $O(u, v)$ :

$\operatorname{Stab}_{\mathcal{Q}^{\prime \prime}}(O(u, v)) / \operatorname{Stab}_{\mathcal{Q}^{\prime \prime}}(\boldsymbol{g})$ with cardinality $\left|(\boldsymbol{g}) \mathcal{Q}^{\prime \prime} \cap O(u, v)\right|$.

Denote the set of $\mathcal{Q}^{\prime \prime}$ orbits on $(\boldsymbol{g}) \operatorname{Cusp}_{4}$ by $(\boldsymbol{g}) \mathrm{Cusp}_{4}^{*}=(O(u, v)) \mathrm{Cusp}_{4}^{*}$. We speak of the $\gamma_{\infty}$ orbit type of $(O(u, v))$ Cusp. $_{4}^{*}$. 
Lemma 7.7. Suppose $0<k<v$ and $(\boldsymbol{g}) \alpha^{2} \gamma=(\boldsymbol{g}) q_{2}^{k}$; or $(\boldsymbol{g}) \alpha^{2}=(\boldsymbol{g}) q_{2}^{k}$ (or with $\gamma$ replacing $\left.\alpha^{2}\right)$ and $k$ is even. Then $k=v / 2$.

Suppose $(\boldsymbol{g}) \gamma=(\boldsymbol{g}) q_{2}^{k}$ with $k$ odd. Then, $\operatorname{Stab}_{\mathcal{Q}^{\prime \prime}}(O(u, v))=\mathcal{Q}^{\prime \prime}, k=v / 4$ and $(\boldsymbol{g}) \alpha^{2}=(\boldsymbol{g}) q_{2}^{-k}$ and $(\boldsymbol{g}) \alpha^{2} \gamma=(\boldsymbol{g}) q_{2}^{v / 2}$.

In all cases $(O(u, v))$ Cusp $_{4}^{*}$ is a $\gamma_{\infty}$ orbit of type $\left(u, v /\left|(\boldsymbol{g}) \mathcal{Q}^{\prime \prime} \cap O(u, v)\right|\right)$.

Proof. We do all computations on inner Nielsen classes $\boldsymbol{g} \in \mathrm{Ni}^{\text {in }}$. Suppose $0<k<v$ and $(\boldsymbol{g}) \alpha^{2} \gamma=(\boldsymbol{g}) q_{2}^{k}$ or $(\boldsymbol{g}) \alpha^{2}=(\boldsymbol{g}) q_{2}^{k}$ (or with $\gamma$ replacing $\alpha^{2}$ ) and $k$ is even. As $\mathcal{Q}^{\prime \prime}$ commutes with even powers of $q_{2}$, this gives $\boldsymbol{g}=(\boldsymbol{g}) q_{2}^{2 k}: k=v / 2$.

Suppose $(\boldsymbol{g}) \gamma=(\boldsymbol{g}) q_{2}^{k}$ with $k$ odd. Apply $\gamma$ to both sides. From $\$ 2.6 .2$ conclude

$$
\boldsymbol{g}=(\boldsymbol{g}) q_{2}^{k} \gamma q_{2}^{-k} q_{2}^{k}=(\boldsymbol{g}) \alpha^{2} q_{2}^{k} .
$$

So, $(\boldsymbol{g}) q_{2}^{-k}=(\boldsymbol{g}) \alpha^{2}$. Apply $q_{2}$ to both sides of $(\boldsymbol{g}) \gamma=(\boldsymbol{g}) q_{2}^{k}$. This gives

$$
(\boldsymbol{g}) q_{2}\left(q_{2}^{-1} \gamma q_{2}\right)=(\boldsymbol{g}) \alpha^{2}=\left((\boldsymbol{g}) q_{2}\right) q_{2}^{k} .
$$

Inductively, this shows $\operatorname{Stab}_{\mathcal{Q}^{\prime \prime}}(O(u, v))=\mathcal{Q}^{\prime \prime}$. So,

$$
\text { (g) } \gamma \alpha^{2}=(\boldsymbol{g}) q_{2}^{k} \alpha^{2} q_{2}^{-k} q_{2}^{k}=(\boldsymbol{g}) \gamma q_{2}^{k}=q_{2}^{2 k},
$$

and $(\boldsymbol{g}) \alpha^{2}=(\boldsymbol{g}) q_{2}^{-k}$, or $(\boldsymbol{g}) \alpha^{2} \gamma=(\boldsymbol{g}) q_{2}^{v / 2}$.

REMARK 7.8 (Shortening types). When $\left|\operatorname{Stab}_{\mathcal{Q}^{\prime \prime}}(\boldsymbol{g})\right|=2$, the $\gamma_{\infty}$ orbit type of $(O(u, v)) \operatorname{Cusp}_{4}^{*}$ is $(u, v / 2)$ if $\operatorname{Stab}_{\mathcal{Q}^{\prime \prime}}(O(u, v))=\mathcal{Q}^{\prime \prime}$ (two-shortening); of type $(u, v)$ otherwise. When $\operatorname{Stab}_{\mathcal{Q}^{\prime \prime}}(O(u, v))=\{1\}$, the $\gamma_{\infty}$ orbit type of $(O(u, v))$ Cusp $_{4}^{*}$ is $(u, v)$ (no shortening).

Assume $\operatorname{Stab}_{\mathcal{Q}^{\prime \prime}}(\boldsymbol{g})=\{1\}$. When $\operatorname{Stab}_{\mathcal{Q}^{\prime \prime}}(O(u, v))=\mathcal{Q}^{\prime \prime}$, the $\gamma_{\infty}$ orbit type of $(O(u, v)) \operatorname{Cusp}_{4}^{*}$ is $(u, v / 4)$ (total- shortening). This is equivalent to $(\boldsymbol{g}) \gamma=(\boldsymbol{g}) q_{2}^{\ell}$ with $\ell=v / 4$ odd. When $\left|\operatorname{Stab}_{\mathcal{Q}^{\prime \prime}}(O(u, v))\right|=\langle q\rangle=2$, the $\gamma_{\infty}$ orbit type of $(O(u, v)) \mathrm{Cusp}_{4}^{*}$ is $(u, v / 2)$ (two-shortening; then $(\boldsymbol{g}) q=(\boldsymbol{g}) q_{2}^{v / 2}$ with $v$ even).

7.3. $\mathbf{H}-\mathbf{M}$ and near $\mathbf{H}-\mathbf{M}$ reps. in $\mathrm{Ni}\left(G_{1}, \mathbf{C}_{3^{4}}\right)$. The subsections of this section together comprise the complete the proof of Prop.7.9 the main goal of this section. The first two subsections describe the H-M reps. Then 7.3 .3 describes how the H-M reps. produce the near H-M reps. Denote $\operatorname{ker}\left(G_{1} \rightarrow A_{5}\right)$ by $M$ and $\operatorname{ker}\left(G_{1} \rightarrow \mathrm{SL}_{2}(\mathbb{Z} / 5)\right)$ by $V$.

Proposition 7.9. There are 2304 elements in $\mathrm{Ni}_{1}=\mathrm{Ni}\left({ }_{2}^{1} \tilde{A}_{5}, \mathbf{C}_{3^{4}}\right)^{\mathrm{in}}$. Exactly 16 are $H-M$ reps.; 16 others are near $H-M$ reps. (as in (6.3)). The $q_{2}$ orbit of an $H-M$ (resp. near $H-M$ ) rep. contains exactly one $H-M$ (resp. near $H-M$ ) rep. All orbits of $\mathcal{Q}^{\prime \prime}$ on $\mathrm{Ni}_{1}^{\text {in }}$ have length four, and $\mathcal{Q}^{\prime \prime}$ maps $H-M$ (resp. near $H-M$ ) reps. among themselves. In particular, $\gamma_{\infty}$ orbits in $\mathrm{Ni}_{1}^{\mathrm{in}, \mathrm{rd}}$ containing either $H-M$ or near $H$-M reps. have type $(10,20)$.

From Lem. 7.5, there are four orbits of $\gamma_{\infty}$ on $\mathrm{Ni}_{1}^{\mathrm{rd}}$ containing $H$-M (resp. near $H-M)$ reps. giving eight $\gamma_{\infty}$ orbits on $\mathrm{Ni}_{1}^{\text {rd }}$ containing $H-M$ or near $H-M$ reps.

7.3.1. Lifting from $\mathrm{Ni}\left(A_{5}, \mathbf{C}_{3^{4}}\right)=\mathrm{Ni}_{0}$. Choose $\boldsymbol{g}^{*} \in \mathrm{Ni}_{1}$ by lifting $(123)=g_{1}$ and $(145)=g_{2}$ to $g_{1}^{*}$ and $g_{2}^{*}$ of order 3 . Take $\boldsymbol{g}^{*}=\left(g_{1}^{*},\left(g_{1}^{*}\right)^{-1}, g_{2}^{*},\left(g_{2}^{*}\right)^{-1}\right)$. As $H_{4}$ is transitive on $\mathrm{Ni}_{0}$, it suffices to count lifts of $\left(g_{1}, g_{1}^{-1}, g_{2}, g_{2}^{-1}\right)=\boldsymbol{g}$. Multiply by $18=\left|\mathrm{Ni}\left(A_{5}, \mathbf{C}_{3^{4}}\right)^{\text {in }}\right|$ to count elements in $\mathrm{Ni}\left({ }_{2}^{1} \tilde{A}_{5}, \mathbf{C}_{3^{4}}\right)^{\text {in }}=\mathrm{Ni}_{1}$. Use notation from 55.3.3. Let $\tilde{g}_{1}$ and $\tilde{g}_{2}$ be the unique order 3 lifts to $\mathrm{SL}_{2}(\mathbb{Z} / 5)$ of $g_{1}$ and $g_{2}$.

Lifts of $\boldsymbol{g}$ to $\mathrm{Ni}_{1}$ correspond exactly to lifts of $\left(\tilde{g}_{1},\left(\tilde{g}_{1}\right)^{-1}, \tilde{g}_{2},\left(\tilde{g}_{2}\right)^{-1}\right)$ to $\mathrm{Ni}_{1}$. Count these by counting conjugates of an element of order 3 by the kernel $V$ from 
$G_{1} \rightarrow \mathrm{SL}_{2}\left(\mathbb{Z} / 5\right.$ ). If $v \in V$ and $g$ has order 3, then $v g v=g v^{g} v$ (as in 7.3.3). As $V$ is an irreducible $A_{5}$ module (Cor. 5.7), the set $\{g v\}_{v \in V}$ gives the complete set of conjugates of $g$ by $M$.

Three entries of a Nielsen class 4-tuple determine the 4 th from the product-one condition by rewriting entries $g v^{g} v$. Divide by inner automorphisms from the kernel from $G_{1} \rightarrow A_{5}$. As $G_{1}$ is centerless (Prop. 3.21), there are $2^{7}=2^{12} / 2^{5}$ such lifts of $\tilde{\boldsymbol{g}}$ to $\mathrm{Ni}_{1}$. This gives $2^{7} \cdot 18=2304$ total inner Nielsen classes.

7.3.2. Counting $H-M$ lifts of any $H-M$ rep. of $\mathrm{Ni}\left(A_{5}, \mathbf{C}_{3^{4}}\right)$. Continue using $\boldsymbol{g}^{*} \in$ $\mathrm{Ni}_{1}$. Fix any H-M representative mapping to $\boldsymbol{g} \in \mathrm{Ni}_{0}$. Modulo inner action of $G_{1}$, select representatives with $g_{1}^{*},\left(g_{1}^{*}\right)^{-1}$ in the first two positions. Other H-M representatives come from conjugating $\left(g_{2}^{*},\left(g_{2}^{*}\right)^{-1}\right)$ by $\operatorname{ker}_{0} / \operatorname{ker}_{1}=M\left(A_{5}\right)$. As in 7.3 .1 or Cor. 5.7 take lifts by conjugating $g_{2}^{*}$ by elements of $V$. Or should we choose, by conjugating $g_{2}^{*}$ by elements of $M \backslash V$. The centralizer of $\left\langle g_{1}^{*}\right\rangle$ in $M\left(A_{5}\right)$ is a $\mathbb{Z} / 2$ acting nontrivially on $g_{2}^{*}$. This cuts from 16 to 8 the inner classes that are H-M representatives and lifts of $\left(g_{1}, g_{1}^{-1}, g_{2}, g_{2}^{-1}\right)$. This concludes the part of Prop. 7.9 counting H-M representatives.

Since the $g_{1}$ and $g_{4}$ positions determine an H-M rep. $\boldsymbol{g}$, the $q_{2}$ orbit of $\boldsymbol{g}$ can contain only one H-M rep. By inspection, $\mathcal{Q}^{\prime \prime}$ maps H-M reps. to H-M reps.

7.3.3. Near $H-M$ reps. in $\mathrm{Ni}\left({ }_{2}^{k} \tilde{A}_{5}, \mathbf{C}_{3^{4}}\right), k \geq 1$. Unless otherwise said, this subsection is about inner and inner reduced Nielsen classes. The definition of near $\mathrm{H}-\mathrm{M}$ reps. is in (6.3). A modular representation observation produces near H-M reps. $\boldsymbol{g}^{*} \in \mathrm{Ni}\left({ }_{2}^{1} \tilde{A}_{5}, \mathbf{C}_{3^{4}}\right)$ by tweaking an H-M rep. $\boldsymbol{g}=\left(g_{1}, g_{1}^{-1}, g_{2}, g_{2}^{-1}\right) \in \mathrm{Ni}_{1}$.

For $m \in M\left(A_{5}\right)$ and $g \in{ }_{2}^{1} \tilde{A}_{5}$, the notation $m^{g}$ is shorthand for $g^{-1} m g$. This is the same as the right action of $g$ in $A_{5}$ acting on $m$. Let $\hat{\kappa}$ be the complex conjugation operator in (6.2) for $z_{1}, z_{2}$ and $z_{3}, z_{4}$ as complex conjugate pairs.

Lemma 7.10. Assume $\left\langle g_{1}, g_{2}\right\rangle={ }_{2}^{1} \tilde{A}_{5}$ and $g_{1}, g_{2} \in \mathrm{C}$. Then $g_{1} g_{2}$ has order 10 $\left(5\right.$ mod $\left.k_{0}\right)$. It fixes a unique nontrivial $c=\left(g_{1} g_{2}\right)^{5} \in M\left(A_{5}\right)$ (Cor. 5.7).

Let $d, e \in M\left(A_{5}\right)$. Then $\boldsymbol{g}^{*}=\left(g_{1}^{-1}, d g_{1} d, e g_{2} e, g_{2}^{-1}\right) \in \mathrm{Ni}_{1}^{\text {in }}$ if and only if $d^{g_{1}} d e e^{g_{2}^{-1}}=1$. In this case, the $q_{2}$ orbit of $\boldsymbol{g}^{*}$ in $\mathrm{Ni}_{1}^{\text {in }}$ has length 20 .

Proof. That $g_{1} g_{2}$ has order 10 is a special case of Lemma 8.9 An element of order 5 in $A_{5}$ acts on $M\left(A_{5}\right)$ by right multiplying cosets of a $D_{5}$ in $A_{5}$ (Prop. [5.6). So $g_{1} g_{2}$ fixes one nontrivial element. It must be $\left(g_{1} g_{2}\right)^{5}$.

That $\boldsymbol{g}^{*}$ satisfies the product-one condition is exactly that $d^{g_{1}} d e e^{g_{2}^{-1}}=1$. Since $\left\langle g_{1}^{-1}, g_{2}^{-1}\right\rangle={ }_{2}^{1} \tilde{A}_{5}$ has no center, what a conjugation on $\boldsymbol{g}^{*}$ does to the 1 st and 4 th elements determines it. Therefore, the $q_{2}$ orbit of $\boldsymbol{g}^{*}$ has length 2 times the order of $d g_{1} d e g_{2} e$ : The orbit has length 20 .

Let $G_{k}$ be the $k$ th characteristic quotient of ${ }_{2} \tilde{A}_{5}$. We use the following proposition in generality. Its special case with $g_{1}, g_{2} \in{ }_{2}^{1} \tilde{A}_{5}$ appears in precise calculations for level 1 of this Modular Tower. The symbol $\hat{\kappa}$ in Prop. 17.11 is the complex conjugation operator from Prop. 6.3 from complex conjugate pairs of branch points.

Proposition 7.11. Suppose $g_{1}, g_{2} \in G_{k}$ lie over $g_{1}^{\prime}, g_{2}^{\prime} \in A_{5}$ with $g_{1}^{\prime} g_{2}^{\prime}$ having order $y=3$ or 5 . Then, $g_{1} g_{2}$ has order $y \cdot 2^{k}$.

If $y=5$, let $c=\left(g_{1} g_{2}\right)^{5 \cdot 2^{k-1}}$. Then,

$$
\boldsymbol{g}^{*}=\left(g_{1}, c^{g_{2}^{-1}} g_{1}^{-1} c^{g_{2}^{-1}}, c g_{2} c, g_{2}^{-1}\right) \in \mathrm{Ni}_{k}^{\mathrm{in}}
$$


satisfies the product-one condition and $c \boldsymbol{g}^{*} c=\hat{\kappa}\left(\boldsymbol{g}^{*}\right)$ (so is a near $H$-M rep. from (6.3) at level $k$ ). For $k \geq 1, H$ - $M$ reps. give Galois covers $\varphi: X \rightarrow \mathbb{P}_{z}^{1}\left(\right.$ in $\left.\mathrm{Ni}_{k}^{\text {in }}\right)$ over $\mathbb{R}$ with branch points $\boldsymbol{z}$ in complex conjugate pairs and $X(\mathbb{R}) \neq \emptyset$. For near $H-M$ reps. there are such covers $\varphi$ over $\mathbb{R}$, but $X(\mathbb{R})=\emptyset$.

When $k=1, c$ and $c^{g_{2}^{-1}}$ are in the $A_{5}$ orbit labeled $M_{5}^{\prime}$ in Cor. 5.7 Conversely, given $\boldsymbol{g}^{*}$ satisfying $c \boldsymbol{g}^{*} c=\hat{\kappa}\left(\boldsymbol{g}^{*}\right)$, an $H-M$ rep. $\boldsymbol{g}$ exists giving $\boldsymbol{g}^{*}$ by (17.4).

Proof. That (7.4) holds is a simple check. The order of the product $g_{1} g_{2}$ is from Lem. 8.9 Apply Prop. 6.3 to an H-M rep. cover $\varphi: X \rightarrow \mathbb{P}_{z}^{1}$ with respect to the $\hat{\kappa}_{0}$ operator for two pairs of complex conjugate branch points to compute the effect of complex conjugation over $z_{0} \in \mathbb{P}_{z}^{1}(\mathbb{R})$. It is given by $c_{z_{0}}$ equal the identity. So all points on $X$ over $z_{0}$ are real. For a near H-M rep. the effect of complex conjugation is given by $c$ as in the statement of the proposition. So, $c_{z_{0}}$ moves all points over $z_{0}$. There are no real points on $X$.

Now assume $k=1$ and $\boldsymbol{g}^{*}$ satisfies $c \boldsymbol{g}^{*} c=\hat{\kappa}\left(\boldsymbol{g}^{*}\right)$ (automatically associated with $\hat{\kappa}$ for $z_{1}, z_{2}$ and $z_{3}, z_{4}$ as complex conjugate pairs). Write $\hat{\kappa}\left(\boldsymbol{g}^{*}\right)$ :

$$
\left(c c^{g^{-1}} v_{1} g_{1} v_{1} c c^{g_{2}^{-1}}, c c^{g_{2}^{-1}} g_{1}^{-1} c c^{g_{2}^{-1}}, g_{2}, c g_{2}^{-1} c\right) \text {. }
$$

From Cor. [5.7 assume $v_{1}, c \in M\left(A_{5}\right) \backslash V$. Apply $c \boldsymbol{g}^{*} c=\hat{\kappa}\left(\boldsymbol{g}^{*}\right)$ to see

$$
\boldsymbol{g}^{*}=\left(g_{1}, v_{1} g_{1}^{-1} v_{1}, c g_{2} c, g_{2}^{-1}\right)=\left(g_{1}, g_{1}^{-1} v_{1}^{g_{1}^{-1}} v_{1}, c c^{g_{2}^{-1}} g_{2}, g_{2}^{-1}\right) .
$$

The product-one condition for $\boldsymbol{g}^{*}$ is equivalent to $v_{1}^{g_{1}^{-1}} v_{1}=c c^{g_{2}^{-1}}$. Then, $c \boldsymbol{g}^{*} c=\hat{\kappa}\left(\boldsymbol{g}^{*}\right)$ gives four conditions according to the entries of $c \boldsymbol{g}^{*} c$, with the last two automatic. The second gives

$$
c v_{1} g_{1}^{-1} v_{1} c=c c^{g_{2}^{-1}} g_{1}^{-1} c c^{g_{2}^{-1}}
$$

showing $v_{1}=c^{g_{2}^{-1}}$ (Cor. [5.7): the first condition is automatic. The product-one condition says $c=c^{g_{1} g_{2}}: c=\left(g_{1} g_{2}\right)^{5}$ generates the centralizer of $g_{1} g_{2}$.

To conclude proving Prop. 7.9 requires two points.

- A unique near H-M rep. is in the $q_{2}$ orbit of a near H-M rep.

- $\mathcal{Q}^{\prime \prime}$ is stable on the set of near H-M reps.

As with H-M reps. the 1st and 4th positions determine them. So the former is clear. Apply $q_{1} q_{3}^{-1}$ to $\boldsymbol{g}^{*}=\left(g_{1}, c^{g_{2}^{-1}} g_{1}^{-1} c^{g_{2}^{-1}}, c g_{2} c, g_{2}^{-1}\right)$ to get

$$
\left(c g_{1}^{-1} c, g_{1}, g_{2}^{-1}, c^{g_{2}^{-1}} g_{2} c^{g_{2}^{-1}}\right) \text {. }
$$

Write $g_{1}^{\prime}=c g_{1}^{-1} c$ and $c^{g_{2}^{-1}} g_{2} c_{2}^{g_{2}^{-1}}=\left(g_{2}^{\prime}\right)^{-1}$, and compute that $c^{\prime}=c^{g_{2}^{-1}}$ centralizes $g_{1}^{\prime} g_{2}^{\prime}$. Thus, $q_{1} q_{3}^{-1}$ maps $\boldsymbol{g}^{*}$ to a near H-M representative. Similarly for $\left(q_{1} q_{2} q_{3}\right)^{2}$.

\section{Cusp widths and the genus of components}

Assume $r=4$, and $\operatorname{Ni}(G, \mathbf{C})$ is a Nielsen class. Distinguishing between absolute and inner Nielsen classes is cumbersome, though computations using them both are invaluable (as in Thm. 3.16) and similar (add the action of some group $N^{\prime}$ as in 33.5). For simplicity, assume inner Nielsen classes so the decoration $\operatorname{Ni}(G, \mathbf{C})^{\text {rd }}$ means reduced inner classes (unless said otherwise).

Following two preliminary subsections, for the $\left(A_{5}, \mathbf{C}_{3^{4}}, p=2\right)$ Modular Tower this section lists cusps of a given width from $\gamma_{\infty}$ action on $\mathrm{Ni}_{1}^{\text {in,rd }}=\mathrm{Ni}_{1}^{\text {rd }}$. Possible widths are $2,4,6,8,10,12$ and 20 . The aim is to relate all $\gamma_{\infty}$ orbits to orbits of 
width 20 (especially to H-M reps.). Lem. 7.5 and Prop.7.9 report precisely on H-M and near H-M cusps. Prop.9.8 gives the spin separation ingredient that establishes the distribution of cusps between two $M_{4}$ orbits $O_{1}^{+}$and $O_{1}^{-}$on $\mathrm{Ni}_{1}^{\mathrm{in}, \mathrm{rd}}$.

When no further ramification occurs from level $k=0$ to level 1 over the elliptic points $j=0$ and $j=1$, the genus of level 1 components comes just from the story of $\gamma_{\infty}$ and $\left\langle\mathbf{s h}, \gamma_{\infty}\right\rangle=\bar{M}_{4}$ orbits. 8 8.1 observations on this continue in $\$ 9.6$ has subtle conjugation computations for $\mathcal{Q}^{\prime \prime}$ shortening of $q_{2}$ orbits to $\gamma_{\infty}$ orbits.

8.1. Orbit genus and fixed points of $\gamma_{0}$ and $\gamma_{1}$. For much of this subsection, the prime $p$ is arbitrary. Suppose $O$ is an orbit of $\bar{M}_{4}$ acting on $\operatorname{Ni}(G, \mathbf{C})^{\mathrm{rd}}$. Use 97.2 .1 notation for $\gamma_{\infty}$ orbits: For $\boldsymbol{g} \in O(u, v ; a), u=\mathbf{m p}(\boldsymbol{g})$ and $v=\mathbf{w d}(\boldsymbol{g})$. Let $\mathrm{t}_{O}\left(\gamma_{i}\right)$ be the number of fixed points of $\gamma_{i}$ on $O, i=1,2$. From Prop. 4.4. the Riemann-Hurwitz formula gives the genus $g_{O}$ of the reduced Hurwitz space component $\mathcal{H}_{O}^{\text {rd: }}$

$$
2\left(|O|+g_{O}-1\right)=\frac{2\left(|O|-\mathrm{t}_{O}\left(\gamma_{0}\right)\right)}{3}+\frac{|O|-\mathrm{t}_{O}\left(\gamma_{1}\right)}{2}+\sum_{O(u, v ; a) \subset O} v-1 .
$$

Use this to rephrase for $r=4$ our Main Problem 1.1 on Modular Towers. For $G$ centerless and $p$-perfect, show for each $\bar{M}_{4}$ orbit $O$ on $\mathrm{Ni}\left(G_{k}, \mathbf{C}\right)^{\mathrm{rd}}=\mathrm{Ni}_{k}^{\mathrm{rd}}, g_{O} \geq 2$ if $k$ is large. If this holds, we say $k$ is in the hyperbolic range. Thm. 6.1 says this implies there are no $K$ points ( $K$ a number field) on $\mathcal{H}\left(G_{k}, \mathbf{C}\right)^{\text {rd }}$ if $k$ is large (possibly larger than the beginning of the hyperbolic range).

Assume $O_{k}$ (resp. $\left.O_{k+1}\right)$ is a $\bar{M}_{4}$ orbit in $\mathrm{Ni}_{k}^{\text {rd }}\left(\right.$ resp. $\mathrm{Ni}_{k+1}^{\text {rd }}$ over $\left.O_{k}\right)$. So, cusps of $\mathcal{H}_{O_{k+1}}^{\mathrm{rd}}$ lie over cusps of $\mathcal{H}_{O_{k}}^{\mathrm{rd}}: \gamma_{\infty}$ orbits on $O_{k+1}$ lie over $\gamma_{\infty}$ orbits on $O_{k}$.

8.1.1. Two helpful assumptions.

(8.2a) $\mathrm{t}_{O_{k}}\left(\gamma_{0}\right)+\mathrm{t}_{O_{k}}\left(\gamma_{1}\right)=0$; and for $\boldsymbol{g}^{\prime} \in O_{k+1}$ over $\boldsymbol{g} \in O_{k}$,

(8.2b) if $\left(\boldsymbol{g}^{\prime}\right) \mathbf{s h}$ not an H-M rep., then $\mathbf{m p}\left(\boldsymbol{g}^{\prime}\right)=p \cdot \mathbf{m} \mathbf{p}(\boldsymbol{g})$.

The condition $\left(\boldsymbol{g}^{\prime}\right) \mathbf{s h}$ not an H-M rep. in 8.20 is equivalent to $\boldsymbol{m p}\left(\boldsymbol{g}^{\prime}\right) \neq 1$, so is absolutely necessary. For a $\gamma_{\infty}$ orbit $O\left(u^{\prime}, v^{\prime} ; a^{\prime}\right) \subset O_{k+1}$ use the notation $O(u, v ; a) \subset O_{k}$ for cusps of $O_{k}$ under $O\left(u^{\prime}, v^{\prime} ; a^{\prime}\right)$.

Lemma 8.1. If (8.21a) holds (for $O_{k}$ ), then it holds with $O_{k+1}$ replacing $O_{k}$. For $\mathrm{Ni}_{0}^{\mathrm{rd}}=\mathrm{Ni}\left(A_{5}, \mathbf{C}_{3^{4}}\right)^{\mathrm{in}, \mathrm{rd}}(p=2)$, both (8.2) and $8.2 \mathrm{~b}$ ) hold for any $\bar{M}_{4}$ orbit $O^{\prime} \subset \mathrm{Ni}_{k}^{\mathrm{rd}}, k \geq 1$.

Proof. Suppose $\boldsymbol{g} \in \mathrm{Ni}_{k+1}^{\mathrm{in}}$ and $(\boldsymbol{g}) Q_{1} Q_{2} Q_{1}=\alpha(\boldsymbol{g}) Q^{\prime} \alpha^{-1}$ for some $\alpha \in G_{k}$ and $Q^{\prime} \in \mathcal{Q}^{\prime \prime}$. Reduce all expressions modulo $\operatorname{ker}_{k}$ to conclude $\gamma_{1}$ fixes $\boldsymbol{g}$ mod $\operatorname{ker}_{k}$. This is a contradiction. The same argument works for $\gamma_{0}$.

By inspection (6.5.3) shows (8.27) holds for $\left(A_{5}, \mathbf{C}_{3^{4}}, p=2\right)$, and so at all levels in this $\left(A_{5}, \mathbf{C}_{3^{4}}\right)$ Modular Tower. If $\mathbf{m p}(\boldsymbol{g})=3$ or 5 , (8.21b) follows from the opening statement of Prop. 17.11] The only other possibility is that $(\boldsymbol{g}) \mathbf{s h}$ is an H-M rep., but $\left(\boldsymbol{g}^{\prime}\right) \mathbf{s h}$ above it is not. Lem. 8.9 says if $p \mid \mathbf{m p}\left(\boldsymbol{g}_{k}\right)$, then $\mathbf{m p}\left(\boldsymbol{g}_{k+1}\right)=p \cdot \mathbf{m p}\left(\boldsymbol{g}_{k}\right)$ for $\boldsymbol{g}_{k+1}$ over $\boldsymbol{g}_{k}$. That completes the proof.

Assume at level $k$ of a Modular Tower there are still components of genus 0 or 1. Lem. 8.2 inspects the contribution of cusp ramification toward the genus of components at level $k+1$. Three phenomena play a role in this contribution for each cusp in going from level $k$ to level $k+1$.

(8.3a) Detecting if condition (2.19) in Prop. 2.17 changes. 
(8.3b) Deciding if there is a multiplication by $p$ factor as in 8.2b).

(8.3c) Computing $q_{2}$ orbit shortening changes from one level to another. As above, $O_{k}$ is a $\bar{M}_{4}$ orbit in $\mathrm{Ni}_{k}^{\mathrm{rd}} ; O_{k+1}$ is a $\bar{M}_{4}$ orbit in $\mathrm{Ni}_{k+1}^{\mathrm{rd}}$ above it.

Lemma 8.2. For $\boldsymbol{g}_{k} \in O(u, v ; a)$, assume $Z\left(g_{2}, g_{3}\right) \cap\left\langle g_{2} g_{3}\right\rangle=\{1\}$ so the $q_{2}$ orbit type is $(u, 2 u)$ unless $u$ is odd and (2.19) holds (Prop. 2.17). For each $\gamma_{\infty}$ orbit $O(u, v ; a) \subset O_{k}$ let $\alpha(u, v ; a)=2$ in the former case, 1 in the latter. If $\alpha(u, v ; a)=$ 2 , then $\alpha\left(u^{\prime}, v^{\prime} ; a^{\prime}\right)=2$ for $O\left(u^{\prime}, v^{\prime} ; a^{\prime}\right)$ over $O(u, v ; a)$. Let ind $\left(u^{\prime}, v^{\prime} ; a^{\prime}\right)$ be the index of ramification of $O\left(u^{\prime}, v^{\prime} ; a^{\prime}\right)$ over $O(u, v ; a)$. Also, let $\beta\left(u^{\prime}, v^{\prime} ; a^{\prime}\right)=\frac{u^{\prime}}{u}(1$ or $p$; automatically $p$ if $p \mid u$ from Lem. 2.23). Depending on the amount of $q_{2}$ orbit shortening (7.2.3), let $\mu(u, v ; a)=1$ (no shortening), 2 (two-shortening) or 4 (total shortening). Then,

$$
\operatorname{ind}\left(u^{\prime}, v^{\prime} ; a^{\prime}\right)=\frac{\alpha\left(u^{\prime}, v^{\prime} ; a^{\prime}\right) \beta\left(u^{\prime}, v^{\prime} ; a^{\prime}\right) \mu(u, v ; a)}{\alpha(u, v ; a) \mu\left(u^{\prime}, v^{\prime} ; a^{\prime}\right)} .
$$

Suppose $g_{O_{k}}=1$. Then $g_{O_{k+1}} \geq 2$ if for some $\gamma_{\infty}$ orbit $O\left(u^{\prime}, v^{\prime} ; a^{\prime}\right) \subset O_{k+1}$ over $O(u, v ; a) \subset O_{k}, \operatorname{ind}\left(u^{\prime}, v^{\prime} ; a^{\prime}\right)>1$.

Suppose $g_{O_{k}}=0$. Then,

$$
2\left(\frac{\left|O_{k+1}\right|}{\left|O_{k}\right|}+g_{O_{k+1}}-1\right) \geq \sum_{O\left(u^{\prime}, v^{\prime} ; a^{\prime}\right) \subset O_{k+1}} \operatorname{ind}\left(u^{\prime}, v^{\prime} ; a^{\prime}\right)-1 .
$$

If $\mathrm{t}_{O_{k}}\left(\gamma_{0}\right)+\mathrm{t}_{O_{k}}\left(\gamma_{1}\right)=0$, then equality holds in (8.5).

Proof. The references explain most of this lemma. Given $O\left(u^{\prime}, v^{\prime} ; a^{\prime}\right)$ over $O(u, v ; a)$ the ramification index of the respective cusps is exactly $\frac{v^{\prime}}{v}$ which by previous comments is ind $\left(u^{\prime}, v^{\prime} ; a^{\prime}\right)$ as in (8.4). It is well-known that if a $X \rightarrow Y$ is a covering of projective nonsingular curves with the lower curve of genus 1 , then the genus of $X$ is 1 if and only if the cover is unramified. Formula 8.5 expresses the Riemann-Hurwitz formula applies to the relative curve covering $\mathcal{H}_{O_{k}+1}^{\mathrm{rd}} \rightarrow \mathcal{H}_{O_{k}}^{\mathrm{rd}}$, when the latter has genus 0 .

We use Lem. 8.2 to show what goes into computing the genus of the two $\mathcal{H}\left(G_{1}, \mathbf{C}_{3^{4}}\right)^{\text {rd }}$ components.

Corollary 8.3. There are two orbits $O_{1}^{+}$and $O_{1}^{-}$of $\bar{M}_{4}$ on $\mathrm{Ni}\left(G_{1}, \mathbf{C}_{3^{4}}\right)^{\mathrm{rd}}$, each of degree 16 over the unique $\bar{M}_{4}$ orbit $\mathrm{Ni}_{0}$. The genus of the orbit $O_{1}^{+}$containing $H$ - $M$ reps. is 12. The other orbit $O_{1}^{-}$has genus 9.

Proof. Apply (8.5) to $O_{1}^{+}$. From Prop. 8.12 one $\bar{M}_{4}$ orbit on $\mathrm{Ni}_{1}^{\text {rd }}$ contains all H-M and near H-M reps. Call this orbit $O_{1}^{+}$. Prop. 9.8 says there are exactly eight $\gamma_{\infty}$ orbits with the following properties:

(8.6a) 5 divides $\mathbf{m p}$;

(8.6b) they are in the $\bar{M}_{4}$ orbit of a near H-M rep.

Prop. 7.9] says the $\gamma_{\infty}$ cusp type of an H-M or near H-M rep. is $(10,20)$. So, a cusp of $O_{1}^{+}$lying over an $\mathrm{H}-\mathrm{M}$ rep. of $\mathrm{Ni}_{0}^{\text {rd }}$ has ramification index 4 . Let $\overline{\mathcal{H}}_{1}^{+} \rightarrow \mathbb{P}_{j}^{1}$ be the component of $\overline{\mathcal{H}}_{1}^{\text {rd }}$ corresponding to $O_{1}^{+}$. Conclude: Each cusp (eight total) of $O_{1}^{+}$with $\mathbf{m p}$ divisible by 5 has ramification index 4 over the cusp below them at level 0 . Together they contribute $2 \cdot 4 \cdot 3=24$ to the right side of (8.5).

Similarly, consider cusps of $O_{1}^{+}$over cusps of $\mathrm{Ni}_{0}^{\text {rd }}$ with mp equal 3. Prop. 8.13 gives a similar conclusion about cusps at level 0 with middle product 3 . Cusps 
above them on $O_{1}^{+}$have ramification index 4 ( $\mathcal{Q}^{\prime \prime}$ does not shorten them). They also contribute $2 \cdot 3 \cdot 4=24$ to the right side of (8.5).

Now apply Prop. 8.6 for the contribution of cusps on $O_{1}^{+}$over sh applied to level $0 \mathrm{H}-\mathrm{M}$ reps. This contributes 2 for the shift of $\mathrm{H}-\mathrm{M}$ reps. and $2 \cdot 2$ for the others to the right side of (8.5). So, the right side of (8.5) is 54. The expression $2\left(16+g_{O_{1}^{+}}-1\right)=4 \cdot 3 \cdot 4+2 \cdot 3$ gives $g_{O_{1}^{+}}=12$.

Let $O_{1}^{-}$be the collection of cusps at level 1 not in $O_{1}^{+}$. First assume they all lie in one $\bar{M}_{4}$ component for the computation of the genus of this orbit. From Prop. 8.13 there are eight cusps in $O_{1}^{-}$with width 6 , and four with width 12 . Similarly, there eight cusps in $O_{1}^{-}$with width 10, and four with width 20. Finally, Lem. 8.17 gives 8 type $(2,4) \gamma_{\infty}$ orbits in $O_{1}^{-}$. To complete the calculation above for $g_{O_{1}^{-}}$, list respective contributions to the right of 8.5 : Type $(2,4)$ contribute 8 ; type $(6,6)$ contribute 8 ; type $(6,12)$ contribute $4 \cdot 3$; type $(10,10)$ contribute 8 and type $(10,20)$ contribute $4 \cdot 3$. So, $2\left(16+g_{O_{1}^{-}}-1\right)=48$ gives $g_{O_{1}^{-}}=9$.

Since it is true at level 0 , it is also true at level 1 that every $\boldsymbol{g}$ not in $O_{1}^{+}$is in the $\bar{M}_{4}$ orbit of an element $\boldsymbol{g}^{\prime}$ with $5 \mid \mathbf{m p}\left(\boldsymbol{g}^{\prime}\right)$. From Prop. 8.13 (and its notation) any cusp of $L_{20}$ (resp. $L_{10}$ ) connects to each cusp of $L_{6}$ (resp. $L_{12}$ ). So, to prove all elements of $O_{1}^{-}$lie in one $\bar{M}_{4}$, it suffices to connect some cusp of $L_{6}$ (resp. $L_{20}$ ) to some cusp of $L_{12}$ (resp. $L_{10}$ ). This shows a component $\overline{\mathcal{H}}^{\prime}$ containing a cusp of $O_{1}^{-}$has degree sixteen over a cusp at level 0 with $\mathbf{m p}=3$. So $\overline{\mathcal{H}}^{\prime} \rightarrow \overline{\mathcal{H}}_{0}^{\text {rd }}$ has degree sixteen everywhere. For the degree over every cusp to be 16 forces including all cusps in $O_{1}^{-}$. To join something in $L_{10}$ to something in $L_{20}$ consider a $\gamma_{\infty}$ type $(2,4)$ orbit in $O_{1}^{-}$. A representative for such an orbit has $\boldsymbol{g}=\left(g_{2}^{-1}, g_{1}, a g_{1}^{-1} a, b g_{2} b\right)$. Lem. 8.17 shows each type $(2,4) \gamma_{\infty}$ orbit in $O_{1}^{-}$is such an element. This concludes the proof of the corollary.

8.1.2. The mystery of $\gamma_{0}$ and $\gamma_{1}$ fixed points. Consider orbits of length one for $\gamma_{0}$ and $\gamma_{1}$ (of respective orders 3 and 2) acting on $\operatorname{Ni}(G, \mathbf{C})^{\text {rd }}$.

Suppse $G \leq N^{\prime} \leq S_{n}$ is a situation for absolute equivalence, as in 3.5.4 Knowing the length one orbits tells what is the contribution of $\gamma_{0}$ and $\gamma_{1}$ to the genus of components of $\mathcal{H}(G, \mathbf{C})^{\text {rd }} / N^{\prime}$ in (8.1). Consider $\gamma_{1} ; \gamma_{0}$ is similar.

Let $\boldsymbol{z}=\{1,-1, i,-i\}$ be a set of branch points representing the elliptic point for $\gamma_{1}$. Then $\alpha: z \mapsto i z \in \mathrm{PSL}_{2}(\mathbb{C})$ cycles the set $z$. A fixed set of classical generators of $\pi_{1}\left(U_{\boldsymbol{z}}, z_{0}\right)$ produces a list of covers (up to $N^{\prime}$-equivalence) $\varphi_{i}: X_{i} \rightarrow \mathbb{P}_{z}^{1}, i=1, \ldots, t$. Composing the $\varphi_{i}$ s with $\alpha$ permutes them. For some choice of classical generators, this action is $q_{1} q_{2} q_{1}$ modulo the action of $\mathcal{Q}^{\prime \prime}$.

Problem 8.4 (Elliptic fixed points). Generalize Rem. 4.9 to the general case of inner reduced Hurwitz spaces when $r=4$. Further, if $\mathcal{H}\left(G_{k}, \mathbf{C}\right)^{\text {in,rd }}$ is a Modular Tower, with $p$-perfect and centerless $G_{0}$, when can there be a projective system of Nielsen classes fixed by $\gamma_{i}$ for $i=0$ or 1 ?

The Modular Tower version of this question makes sense for any $r$ applied to the orbifold points in $J_{r}$ : When can a Modular Tower have a projective system of Nielsen class representatives fixed by a nontrivial element in $\bar{M}_{r}$ associated with an orbifold stabilizer?

EXAmple 8.5 (Action on $\mathrm{Ni}\left(A_{5}, \mathbf{C}_{3^{4}}\right)$ ). According to $7.1 .1 \mathcal{Q}^{\prime \prime}$ acts trivially on the list of Table 1 (2.6.6). Note: $\gamma_{1}$ fixes ${ }_{7} \boldsymbol{g}$ from $\mathrm{Ni}\left(A_{5}, \mathbf{C}_{3^{4}}\right)^{\text {abs }}$. Yet, $\gamma_{1}$ fixes no item of $\operatorname{Ni}\left(A_{5}, \mathbf{C}_{3^{4}}\right)^{\text {in }}$. Since $\mathcal{Q}^{\prime \prime}$ acts trivially, this means, from the list 
$\varphi_{i}: X_{i} \rightarrow \mathbb{P}_{z}^{1}, i=1, \ldots, 9$, of degree 5 covers, exactly one of $\alpha \circ \varphi_{i}$ is equivalent to $\varphi_{i}$. Here is a fact about the list: Suppose an item from it has two 3-cycles (not necessarily consecutive) with exactly two integers of common support. Up to conjugation by $S_{5}$ these 3 -cycles are ((123), (2 14)) where the common support integers ( 1 and 2 here) appear in opposite order in the second 3-cycle. Further, for ${ }_{7} \boldsymbol{g}$, this is true for all consecutive pairs of 3-cycles, especially including the 4 th and 1st, taken in that order. Now, apply $\gamma_{1}$ as the shift of $\$ 2.7$

8.2. Subtle conjugations. Conjugation by an element of $G_{1}$ is determined by its action on two generators $g_{1}, g_{2}$ of order 3 . Several computations require the precise effect of that conjugation given the generators and what the conjugation does modulo $\mathrm{ker}_{0}$. Use the notation of Lem. 7.7 for elements of $\mathcal{Q}^{\prime \prime}$. For $\boldsymbol{g} \in \mathrm{Ni}_{1}^{\text {in }}$, let $O_{\boldsymbol{g}}$ be its $q_{2}$ orbit. Let $\delta \in \mathcal{Q}^{\prime \prime} \backslash 0$. If $(\boldsymbol{g}) \delta \in O_{\boldsymbol{g}}$, we say $\delta$ shortens $\boldsymbol{g}$ (as in 7.2 .3 ). Variants: $\mathcal{Q}^{\prime \prime}$ shortens $\boldsymbol{g}$ or $\mathcal{Q}^{\prime \prime}$ shortens $O_{\boldsymbol{g}}$. Use $\boldsymbol{h}_{+}=((123),(132),(145),(154))$ and $\boldsymbol{h}_{-}=((123),(132),(154),(145))$ as representatives of the two H-M reps. in $\mathrm{Ni}_{0}^{\text {in }}$ 7.2.1 has notation for the type of orbits.

Proposition 8.6. Let $g_{1}, g_{2} \in G_{1}$ be order 3 lifts of (123), (145) $\in A_{5}$. Denote (23)(45), (24)(35) and (25)(34) respectively by $\alpha_{23}, \alpha_{24}$ and $\alpha_{25}$. Each $\boldsymbol{g} \in \mathrm{Ni}_{1}^{\text {in }}$ with $\mathbf{m p}(\boldsymbol{g})=2$ has the form $\boldsymbol{g}=\left(g_{1}, g_{2}, c g_{2}^{-1} c, d g_{1}^{-1} d\right)$ with $c, d \in M\left(A_{5}\right)$. (So, $c^{g_{2}^{-1}} c=d d^{g_{1}}$.) If $(\boldsymbol{g}) q_{1} q_{3}^{-1}$ (resp. $\left.(\boldsymbol{g})\left(q_{1} q_{2} q_{3}\right)^{2}, q_{3}\left(q_{1} q_{2} q_{3}\right)^{2} q_{3}^{-1}\right)$ is conjugate to (g) $q_{2}^{2}$, then the conjugation is by a lift of $(123) \alpha_{24}$ (resp. $\left.\alpha_{25},(145)^{-1} \alpha_{23}\right)$.

Suppose $\boldsymbol{g}^{\prime}$ is a near $H-M$ rep. Then, $\mathcal{Q}^{\prime \prime}$ shortens $\boldsymbol{g}=\left(\left(\boldsymbol{g}^{\prime}\right) \mathbf{c m}\right) \mathbf{s h}$. Thus, shifted complements of near $H-M$ reps. fall into two pairs of $\gamma_{\infty}$ type (2,2) orbits. All other $\boldsymbol{g} s$ with $\mathbf{m p}(\boldsymbol{g})=2$ fall into type $(2,4) \gamma_{\infty}$ orbits.

Three subsections cover the proof of Prop. 8.6. The first establishes the relevance of $\alpha_{2 j}, j=3,4,5$, to the existence of appropriate conjugations. 88.2 .2 shows neither $q_{1} q_{3}^{-1}$ nor $q_{3}\left(q_{1} q_{2} q_{3}\right)^{2} q_{3}^{-1}$ shorten any $\boldsymbol{g}$ with $\mathbf{m p}(\boldsymbol{g})=2$. 8.2 .3 shows $(\boldsymbol{g})\left(q_{1} q_{2} q_{3}\right)^{2}$ shortens the complement of a near H-M rep. As this is an even power of $q_{2}$, Rem. 7.8 notes this suffices to determine exactly the shortening type: It is two-shortening.

8.2.1. Preliminaries. With $c, d \in M, \boldsymbol{g}=\left(g_{1}, g_{2}, c g_{2}^{-1} c, d g_{1}^{-1} d\right) \in \mathrm{Ni}_{1}^{\mathrm{in}}$ as above:

$$
(\boldsymbol{g}) q_{2}^{2}=\left(g_{1}, c c^{g_{2}^{-1}} g_{2} c^{g_{2}^{-1}} c, c^{g_{2}^{-1}} g_{2}^{-1} c^{g_{2}^{-1}}, d g_{1}^{-1} d\right) .
$$

Then, $g_{1}^{-1}(\boldsymbol{g}) q_{1} q_{3}^{-1} g_{1}=\left(g_{2}, g_{1}, d^{g_{1}} g_{1}^{-1} d^{g_{1}}, d^{g_{1}} d c g_{2}^{-1} d^{g_{1}} d c\right)$. If this is conjugate to (8.7), it is by a lift of an $A_{5}$ conjugation switching (123) and (145). The element $\alpha_{24}$ gives this conjugation.

Consider if $(\boldsymbol{g})\left(q_{1} q_{2} q_{3}\right)^{2}$ is conjugate to (8.7). Such a conjugation is by a lift of an $A_{5}$ conjugation mapping (123) to (154) and (145) to (132). Thus, the conjugation is a lift of $\alpha_{25}$.

Now consider if $g_{2}(\boldsymbol{g}) q_{3}\left(q_{1} q_{2} q_{3}\right)^{2} q_{3}^{-1} g_{2}^{-1}=\left(d^{g_{1}} g_{1}^{-1} d^{g_{1}}, c^{g_{2}^{-1}} g_{2}^{-1} c^{g_{2}^{-1}}, g_{2}, g_{1}\right)$ is conjugate to $(\boldsymbol{g}) q_{2}^{2}$. If so, it is by a lift of an $A_{5}$ conjugation mapping each of $(123)$ and $(145)$ to their inverses. The element $\alpha_{23}$ gives this conjugation.

8.2.2. $q_{1} q_{3}^{-1}$ shortens no $\boldsymbol{g}$ with $\mathbf{m p}(\boldsymbol{g})=2$. Suppose $g \in G$ normalizes a subgroup $H$. Denote the centralizer of $g$ in $H$ by $Z_{H}(g)$. Denote $Z_{M}\left(g_{i}\right)$ by $\left\langle m_{i}\right\rangle$, $i=1,2$. From the last statement in Cor. 5.7 with no loss $\left(g_{1}, g_{2}, c g_{2}^{-1} c, d g_{1}^{-1} d\right)$ uniquely determines $c$ and $d$ knowing also $c, d \in M\left(A_{5}\right) \backslash V$. Suppose $g_{1}^{-1}(\boldsymbol{g}) q_{1} q_{3}^{-1} g_{1}$ 
is conjugate to (8.7). Then some lift $\alpha$ of $\alpha_{24}$ conjugates as follows:

$$
\begin{aligned}
& g_{1} \mapsto g_{2}, c^{g_{2}^{-1}} g_{2}^{-1} c^{g_{2}^{-1}} \mapsto d^{g_{1}} g_{1}^{-1} d^{g_{1}}, \\
& c c^{g_{2}^{-1}} g_{2} c^{g_{2}^{-1}} c \mapsto g_{1} \text { and } d g_{1}^{-1} d \mapsto d^{g_{1}} d c g_{2}^{-1} d^{g_{1}} d c .
\end{aligned}
$$

The 1st, 2nd and 3rd expressions give the effect of conjugating $\alpha^{2} \in M\left(A_{5}\right) \backslash V$ (Lemma 2.23) on $g_{1}$ two ways:

$$
\alpha^{2}=c^{g_{2}^{-1} \alpha} d^{g_{1}} m_{1}=\left(c c^{g^{-1}}\right)^{\alpha} m_{1} .
$$

Conclude $d^{g_{1}}=c^{\alpha}$. Similarly, figure the effect of $\alpha^{2}$ conjugating $g_{2}$ :

$$
\alpha^{2}=\left(d^{g_{1}} c^{g_{2}^{-1} \alpha}\right)^{\alpha} d^{\alpha} d^{g_{1}} d c m_{2}=\left(d^{g_{1}} c^{g_{2}^{-1} \alpha}\right)^{\alpha} m_{2} .
$$

Now we show $\alpha$ satisfying (8.8) does not exist.

From (8.10) and the product one condition $\left(c^{g_{2}^{-1}} c=d d^{g_{1}}\right) c^{g_{2}^{-1}}=d^{\alpha}$ and

(8.11) $d^{\alpha} c=d^{g_{1}} d=c^{\alpha} d$, an expression invariant under $\alpha$.

From (8.9) and (8.10), $m_{1}=m_{2}$. This is false: The nontrivial element $m_{2}$ would centralize $G_{1}=\left\langle g_{1}, g_{2}\right\rangle$. As $G_{1}$ has no center (Prop. 3.21), $\alpha$ doesn't exist.

8.2.3. $\left(q_{1} q_{2} q_{3}\right)^{2}$ shortens the shift of a near H-M rep. complement. The following shows there is a natural braid taking the reduced class of a complement of a near H-M rep. over $\boldsymbol{h}_{+}$to one over $\boldsymbol{h}_{-}$. So, for reduced classes, complements of near H-M reps. are similar to a pair of H-M reps. $\boldsymbol{g}$ and $\left((\boldsymbol{g}) q_{1}\right.$ naturally paired as being over $\boldsymbol{h}_{+}$and $\boldsymbol{h}_{-}$.

Suppose $(\boldsymbol{g})\left(q_{1} q_{2} q_{3}\right)^{2}$ is conjugate to (8.7). A lift $\alpha$ of $\alpha_{25}$ conjugates as follows:

$$
\begin{aligned}
& g_{1} \mapsto c g_{2}^{-1} c, \quad c^{g_{2}^{-1}} g_{2}^{-1} c^{g_{2}^{-1}} \mapsto g_{1}, \\
& c c^{g_{2}^{-1}} g_{2} c^{g_{2}^{-1}} c \mapsto d g_{1}^{-1} d \quad \text { and } d g_{1}^{-1} d \mapsto g_{2} .
\end{aligned}
$$

Here are the analogs of (8.9) and (8.10):

$$
\alpha^{2}=c^{g_{2}^{-1} \alpha} c^{\alpha} m_{1}=c^{g_{2}^{-1} \alpha} d m_{1} \text { and } \alpha^{2}=c^{g_{2}^{-1}} c m_{2}=c^{g_{2}^{-1}} d^{\alpha} m_{2} .
$$

Conjugate the latter by $\alpha$ to conclude $m_{1}=m_{2}^{\alpha}$ and $c^{\alpha}=d$ together are equivalent to $(\boldsymbol{g})\left(q_{1} q_{2} q_{3}\right)^{2}$ shortening $\boldsymbol{g}$. The expression $m_{1}=m_{2}^{\alpha}$ is automatic from $g_{1}^{\alpha}=$ $c g_{2}^{-1} c$ and conjugating $m_{1} m_{1}^{g_{1}}=1$ by $\alpha$.

As in the proof of Cor. [5.7 compute the effect of $\alpha_{25}$ on $1_{H}, \ldots, 6_{H}$. By explicit computation $g_{1}$ acts as $\beta_{g_{1}}=\left(1_{H} 3_{H} 5_{H}\right)\left(2_{H} 4_{H} 6_{H}\right)=(135)(246)$. Similarly, $g_{2}$ acts as $\beta_{g_{2}}=(132)(456)$. Finally, $\alpha_{25}$ acts as $\beta_{\alpha}=\left(3_{H} 1_{H}\right)\left(2_{H} 5_{H}\right)=(31)(25)$. The remaining list of possible $c$ values is in the following lemma.

LEMMA 8.7. With $d=c^{\alpha_{25}},\left(q_{1} q_{2} q_{3}\right)^{2}$ shortens the $\gamma_{\infty}$ orbit of $\boldsymbol{g}$ if and only if $u=c^{g_{2}^{-1} \alpha_{25}} c^{\alpha_{25}} m_{1}$ is invariant under $\alpha_{25}$. Given a value of $u$ (or $c$ ) with this property, all others arise by running over all possible lifts of $\alpha_{25}$.

PROOF. There is an orbit shortening for $\left(q_{1} q_{2} q_{3}\right)^{2}$ only if there is an $\alpha$ (lifting $\left.\alpha_{25}\right)$ with $\alpha^{2}=u$. This implies $u$ is invariant under $\alpha_{25}$. Given one such lift $\alpha$, Cor. [5.7 shows you get all others by multiplying this $u$ by $m m^{\alpha_{25}}$ as $m$ runs over $M$. Given a lift $\alpha$ giving $u$, multiplying $\alpha$ by $m$ produces $u m m^{\alpha}=(m \alpha)^{2}$.

Producing one such $\alpha$ is the final step. The Cor. 5.7 proof gives explicit action of $g_{1}, g_{2}, \alpha_{25}$ on the cosets of the $D_{5}=\langle(13425),(12)(34)\rangle$. Respectively:

$$
\beta_{g_{1}}=(135)(246), \beta_{g_{2}}=(132)(456), \beta_{\alpha_{25}}=(31)(25) .
$$


Consider a near H-M rep. as in (7.4):

$$
\boldsymbol{g}^{\prime}=\left(g_{1}^{\prime},\left(c^{\prime}\right)^{\left(g_{2}^{\prime}\right)^{-1}}\left(g_{1}^{\prime}\right)^{-1}\left(c^{\prime}\right)^{\left(g_{2}^{\prime}\right)^{-1}}, c^{\prime} g_{2}^{\prime} c^{\prime},\left(g_{2}^{\prime}\right)^{-1}\right) \in \mathrm{Ni}_{1}^{\text {in }},
$$

with $c^{\prime}$ centralizing $g_{1}^{\prime} g_{2}^{\prime}$. For reasons coming up, we take $g_{1}^{\prime}=g_{2}$, a lift of $(145)$ and $\left(g_{2}^{\prime}\right)^{-1}=g_{1}$, a lift of (123).

Let $t$ be the centralizer of $\left(g_{1}^{\prime}\right)^{-1} g_{2}^{\prime}=\left(g_{1} g_{2}\right)^{-1}$. Then, compute the shift of the complement of $\boldsymbol{g}^{\prime}$ to get

$$
\boldsymbol{g}=\left(g_{1}, g_{2}, t\left(c^{\prime}\right)^{g_{1}} m_{2} g_{2}^{-1} m_{2}\left(c^{\prime}\right)^{g_{1}} t, t c^{\prime} m_{1} g_{1}^{-1} m_{1} c^{\prime} t\right)
$$

with $c=t\left(c^{\prime}\right)^{g_{1}} m_{2}$ and $d=t c^{\prime} m_{1}$ and $c^{\prime}$ centralizing $g_{2} g_{1}^{-1}$.

Since $m_{2}^{\alpha}=m_{1}$, the desired shortening amounts to showing $t^{\alpha}\left(c^{\prime}\right)^{g_{1} \alpha}=t c^{\prime}$ and (using that by definition $\left.m_{2}^{g_{2}^{-1}}=m_{2}\right) c c^{g_{2}^{-1}} m_{2}=t t^{g_{2}^{-1}}\left(c^{\prime}\right)^{g_{1}} c^{\prime} m_{2}$ centralizes $\alpha$. Explicit computations are reassuring: $\beta_{g_{1}} \beta_{g_{2}}=(12536)$ and so $t=(0,0,0,1,0,0)$. Similarly, $\beta_{g_{2}} \beta_{g_{1}}^{-1}=(25436)$, so $c^{\prime}=(1,0,0,0,0,0)$. Thus, $t^{\alpha}=t$ and $\left(c^{\prime}\right)^{g_{1} \alpha}=c^{\prime}$. The other check works as easily.

8.3. Length two and four cusp widths. From 88.2 .2 and 88.2 .3 there is a concise relation between $\gamma_{\infty}$ orbits of length two and H-M and near H-M reps. As previously, use $u$ for $\mathbf{m p}(\boldsymbol{g})=\operatorname{ord}\left(g_{2} g_{3}\right)$.

8.3.1. Types of width 2 and 4 cusps. Continue the notation from 8.2 for $\boldsymbol{h}_{+}$, $\boldsymbol{h}_{-}$and the types of cusps.

Proposition 8.8. There are 16 total $\boldsymbol{g} \in \mathrm{Ni}_{1}^{\text {in }}$ with $u=1$; all have $(\boldsymbol{g}) \mathbf{s h}$ an $H-M$ rep. This gives two $\gamma_{\infty}$ orbits $O(1,2 ; 1)$ and $O(1,2 ; 2)$ of type $(1,2)$.

Sixteen total $\boldsymbol{g} \in \mathrm{Ni}_{1}^{\mathrm{in}}$ have these properties:

(8.14a) $g_{2} g_{3}$ has order $u=2$; and

(8.14b) $\boldsymbol{g}$ is in a $\gamma_{\infty}$ orbit of type $(2,2)$.

Such $\boldsymbol{g}$ have $(\boldsymbol{g}) \mathbf{s h}$ a complement of a near $H$-M rep. These account for the $\gamma_{\infty}$ orbits $O(2,2 ; 1)$ and $O(2,2 ; 2)$ of type $(2,2)$, giving all width 2 cusps.

There are $16 \cdot 16$ total $\boldsymbol{g} \in \mathrm{Ni}_{1}^{\mathrm{in}}$ with $g_{2} g_{3}$ having $u=2$. From these there are $14 q_{2}$ orbits in $\mathrm{Ni}_{1}^{\mathrm{in}, \mathrm{rd}}$ of type $(2,4)$. All $q_{2}$ orbits $O$ in this proposition have $(O) \mathbf{s h}$ modulo $\mathrm{ker}_{0}$, mapping surjectively to $\left\{\boldsymbol{h}_{+}, \boldsymbol{h}_{-}\right\}$. All $H-M$ and near $H-M$ reps. lie in one $M_{4}$ orbit containing the width two cusps.

Proof. Each $\boldsymbol{g}$ with $\mathbf{m p}(\boldsymbol{g})=1$ is the shift of an H-M rep. $\left(h_{1}, h_{1}^{-1}, h_{2}, h_{2}^{-1}\right)$. Further, applying $q_{2}$ to such a $g$ gives another such element. By inspection $\mathcal{Q}^{\prime \prime}$ is stable on this set. So, according to Prop. 7.9] the shift applied to H-M reps. contributes a total of two length 2 orbits for $q_{2}$ on reduced classes.

Now, consider the case $\boldsymbol{g}$ has $u=2: g_{2} g_{3} \in \operatorname{ker}_{0} / \operatorname{ker}_{1}=M\left(A_{5}\right) \backslash\{0\}$. With no loss, $g_{1}=(123)$ and $g_{4}=(321) \bmod$ ker $_{0}$. Further, assume $g_{2}=(145)$ or (154) mod ker 0 . Fix $g_{1}$. There are $2^{3}$ choices of $g_{2}$, all lifts of $g_{2} \bmod \operatorname{ker}_{0}$ modulo conjugation by the centralizer of $g_{1}$. Then, there are $2^{4}$ lifts of $g_{4} \bmod k_{0}$, now determining $g_{3}$.

So, there are $16 \cdot 16$ total $\boldsymbol{g} \in \mathrm{Ni}_{1}^{\text {in }}$ with $g_{2} g_{3}$ having $u=2$. Since $\mathcal{Q}^{\prime \prime}$ acts faithfully (Prop. (7.9), there are $16 \cdot 4$ elements of $\mathrm{Ni}_{1}^{\text {in,rd }}$ in $q_{2}$ orbits of length 2 or 4. 8.3 .3 completes showing all H-M and near H-M reps. fall in one $M_{4}$ orbit. 
8.3.2. Cusp widths containing $H$-M reps. Lem. 2.19 and Lem. 8.9 explain the eight length 20 orbits of $\gamma_{\infty}$ on $\mathrm{Ni}\left(A_{5}, \mathbf{C}_{3^{4}}\right)^{\text {in,rd }}$ containing $\mathrm{H}-\mathrm{M}$ and near $\mathrm{H}-\mathrm{M}$ reps. Part of the next lemma applies to any Modular Tower with $r=4$.

Lemma 8.9. Let $G_{k}$ be the kth characteristic quotient of ${ }_{2} \tilde{A}_{5}$. For an $H$-M rep. $\boldsymbol{g}=\left(g_{1}, g_{1}^{-1}, g_{2}, g_{2}^{-1}\right) \in \mathrm{Ni}\left(G_{k}, \mathbf{C}_{3^{4}}\right)^{\text {in }}, \mathbf{m p}(\boldsymbol{g})$ is $2^{k} \cdot 5$. The $q_{2}$ orbit of of $\boldsymbol{g}$ has length $2^{k+1} \cdot 5$. Further, only one $H-M$ rep. is in a given $q_{2}$ orbit.

Generally, let $\left\{\boldsymbol{g}_{k}\right\}_{k=0}^{\infty}$ be a projective system of Nielsen class representatives in the $\left(A_{5}, \mathbf{C}_{3^{4}}, p=2\right)$ Modular Tower. If $\mathbf{m p}\left(\boldsymbol{g}_{0}\right) \neq 1$, then $\mathbf{m p}\left(\boldsymbol{g}_{k}\right)=2^{k} \mathbf{m p}\left(\boldsymbol{g}_{0}\right)$, $k \geq 0$. For $\boldsymbol{g} \in \mathrm{Ni}\left(G_{1}, \mathbf{C}_{3^{4}}\right)^{\text {in }}$ with $\mathbf{m p}(\boldsymbol{g}) \neq 1$, its $q_{2}$ orbit has length 4, 12 or 20.

Let $\mathrm{Ni}\left(G_{k}, \mathbf{C}\right)$ be the $k$ th level Nielsen class in any Modular Tower (any $p$ ). For $\tilde{\boldsymbol{g}}=\left\{\boldsymbol{g}_{k}\right\}_{k=0}^{\infty}$, a projective system of Nielsen class representatives, either:

(8.15a) $\boldsymbol{m p}\left(\boldsymbol{g}_{k}\right)=\mathbf{m p}\left(\boldsymbol{g}_{0}\right)$ for all $k$; or

(8.15b) some smallest $k_{0} \stackrel{\text { def }}{=} \operatorname{jmp}(\tilde{\boldsymbol{g}})$ satisfies $p \mid \mathbf{m p}\left(\boldsymbol{g}_{k_{0}}\right)$ and

$$
\mathbf{m p}\left(\boldsymbol{g}_{k}\right)=p^{k-k_{0}} \mathbf{m p}\left(\boldsymbol{g}_{k_{0}}\right), k \geq k_{0} .
$$

Proof. We show the conclusion of (8.15) first. Consider the projective system $\tilde{\boldsymbol{g}}=\left\{\boldsymbol{g}_{k}\right\}_{k=0}^{\infty}$ in the Modular Tower for $\left(G_{0}, \mathbf{C}, p\right)$. Let $g_{2, k}, g_{3, k}$ be the second and 3 rd entries of $\boldsymbol{g}_{k}$. Suppose $k$ exists so $p \mid g_{2, k} g_{3, k}$. By assumption, $g_{2, k+1} g_{3, k+1}$ is a lift of $g_{2, k} g_{3, k}$ to $G_{k+1}$. Thus, Lem. 2.23 says $\mathbf{m p}\left(\boldsymbol{g}_{k+1}\right)=p \mathbf{m p}\left(\boldsymbol{g}_{k}\right)$. Inductively apply this for the conclusion of the lemma.

For $\boldsymbol{g} \in \mathrm{Ni}\left(A_{5}, \mathbf{C}_{3^{4}}\right)$ the value of $\mathbf{m p}(\boldsymbol{g})$ is either 1,3 or 5 . If $\mathbf{m p}(\boldsymbol{g})=1$, then $\boldsymbol{g}$ is an H-M rep. If $\mathbf{m p}(\boldsymbol{g})=3$, then $\left\langle g_{2}, g_{3}\right\rangle=A_{4}$, and the 3-tuple $\left(g_{2}, g_{3},\left(g_{2} g_{3}\right)^{-1}\right)$ satisfies the product-one and genus $0(2(4+g-1)=6$ implies $g=0)$ conditions of Prop. 5.9] Conclude that $s\left(g_{2}, g_{3},\left(g_{2} g_{3}\right)^{-1}\right)=-1$. Equivalently: If $\left(\hat{g}_{2}, \hat{g}_{3}\right)$ are the lifts of $\left(g_{2}, g_{3}\right)$ to $\operatorname{Spin}_{4}$, then $\operatorname{ord}\left(\hat{g}_{2} \hat{g}_{3}\right)=6$.

Similarly, if $\mathbf{m p}(\boldsymbol{g})=5$, then $\left\langle g_{2}, g_{3}\right\rangle=A_{5}$, the genus 0 condition holds, and the conclusion from Prop. [5.9] is that $\hat{g}_{2} \hat{g}_{3}$ has order 10. From Lemma 2.19] if $2 \mid \mathbf{m p}(\boldsymbol{g})$, then the length of the orbit of $q_{2}$ on $\boldsymbol{g}$ is twice ord $\left(g_{1}^{-1} g_{2}\right)$ with $\boldsymbol{g}$ written as a perturbation of an H-M rep. $\left(g_{1}, a g_{1}^{-1} a, b g_{2} b, g_{2}^{-1}\right), a, b \in M$.

REMARK 8.10. When $r=4, \mathbf{m p}(\boldsymbol{g})=1$ is equivalent to $(\boldsymbol{g}) \mathbf{s h}$ is an H-M rep. Suppose $\tilde{\boldsymbol{g}}$ is a projective system of Nielsen class representatives as in Lem. 8.9 with $\boldsymbol{m p}\left(\boldsymbol{g}_{0}\right)=1$ and $p=2$. Then, $\operatorname{jmp}(\tilde{\boldsymbol{g}})$ is the smallest integer $k_{0}$ with $\left(\boldsymbol{g}_{k_{0}}\right) \mathbf{s h}$ not an H-M rep. If all the $\boldsymbol{g}_{k} \mathrm{~s}$ are H-M reps., then $\operatorname{jmp}(\tilde{\boldsymbol{g}})=\infty$.

Definition 8.11. If $\boldsymbol{g}$ is an H-M (or near H-M) rep., refer to its $\gamma_{\infty}$ orbit as an H-M (or near H-M) rep. orbit. Call a $\gamma_{\infty}$ orbit of an element in $(O)$ sh with $O$ an H-M (or near H-M) orbit the shift of an H-M (or near H-M) orbit.

8.3.3. $\bar{M}_{4}$ braids $H$-M to near $H$-M reps. Prop. 7.11 braids a near H-M rep.,

$$
\boldsymbol{g}^{*}=\left(g_{1}, c^{g_{2}^{-1}} g_{1}^{-1} c^{g_{2}^{-1}}, c g_{2} c, g_{2}^{-1}\right),
$$

to the H-M rep. $\left(g_{1}, g_{1}^{-1}, g_{2}, g_{2}^{-1}\right)$. That $\boldsymbol{g}^{*}$ satisfies the product one condition is equivalent to $c$ centralizes $g_{1} g_{2}$. As in 3.3.1 denote $\operatorname{ker}_{k} / \operatorname{ker}_{k+1}$ by $M_{k}$ and $M_{0}=M\left(A_{5}\right)$. The central extension $T_{k}^{\prime} \rightarrow G_{k}$ appears above Cor. 9.7 Its characterization is that $\mathbb{Z} / 2=\operatorname{ker}\left(T_{k}^{\prime} \rightarrow G_{k}\right)$ and any lift to $T_{k}^{\prime}$ of the nontrivial element in $\operatorname{ker}\left(\operatorname{Spin}_{5} \rightarrow A_{5}\right)$ has order $2^{k+1}$.

Proposition 8.12. Suppose $\boldsymbol{g}^{\prime}=\left(g_{1}, \bar{c} g_{1}^{-1} \bar{c}, \bar{c} g_{2} \bar{c}, g_{2}^{-1}\right)$ with $\bar{c} \in M\left(A_{5}\right)$ (and $g_{1}^{-1} g_{2}$ of order 5). Then $\boldsymbol{g}^{\prime}$ satisfies the product one condition if and only if $\bar{c}$ 
centralizes $g_{1}^{-1} g_{2}$. If $\bar{c} \neq 1$, it is $\left(g_{1}^{-1} g_{2}\right)^{5}$. Then, $\left(\boldsymbol{g}^{\prime}\right) q_{2}^{10}=\left(\boldsymbol{g}^{\prime}\right) \mathbf{c m}$ is the H-M rep. $\left(g_{1}, g_{1}^{-1}, g_{2}, g_{2}^{-1}\right)$. Also, $\left(\boldsymbol{g}^{*}\right) q_{1}^{-1} q_{2}^{10}=\left(\boldsymbol{g}^{*}\right) q_{1}^{-1} \mathbf{c m}$ is a different $H-M$ rep. lying over the same $H-M$ rep. at level 0 as does $\left(\boldsymbol{g}^{\prime}\right) \mathbf{c m}$

Then, if $\boldsymbol{g}$ is an $H-M$ rep., $(\boldsymbol{g}) \mathbf{c m} \gamma_{\infty}^{-1} \mathbf{s h} \gamma_{\infty}^{-1}$ represents the reduced class of a near $H$-M rep. Each type (2,2) cusp has Nielsen class representatives consisting of $\mathbf{s h}$ applied to elements lying over either $\boldsymbol{h}_{+}$or $\boldsymbol{h}_{-}$. Conclude: All near $H-M$ reps. and elements in width 2 cusps fall in one $M_{4}$ orbit.

More generally, consider $g_{1}^{\dagger}, g_{2}^{\dagger} \in G_{k}$, and suppose the image of $\left(g_{1}^{\dagger}\right)^{-1} g_{2}^{\dagger}$ in $A_{5}$ has order 5. So, $\left(g_{1}^{\dagger}\right)^{-1} g_{2}^{\dagger}$ has order $5 \cdot 2^{k}$ (Lem. 8.9) and $\left(\left(g_{1}^{\dagger}\right)^{-1} g_{2}^{\dagger}\right)^{5 \cdot 2^{k-1}}=c^{\dagger}$ has order 2. Further, any lift of $c^{\dagger}$ in the cover $T_{k}^{\prime} \rightarrow G_{k}$ has order 4 . There is a braid from $\left(g_{1}^{\dagger},\left(g_{1}^{\dagger}\right)^{-1}, g_{2}^{\dagger},\left(g_{2}^{\dagger}\right)^{-1}\right)$ to a near $H$-M rep. $\boldsymbol{g}^{\prime \prime}$. Complex conjugation $\hat{\kappa}$ (for two pairs of complex conjugate branch points) applied to $\boldsymbol{g}^{\prime \prime}$ gives $c^{\prime \prime} \boldsymbol{g}^{\prime \prime} c^{\prime \prime}$ with $c^{\prime \prime}$ lifting to $T_{k}^{\prime}$ to have order 4 .

Proof. The statement on $\boldsymbol{g}^{\prime}$ comes to noting the product-one condition is equivalent to $\bar{c}^{g_{1}^{-1}} \bar{c} \bar{c} \bar{c}^{g_{2}^{-1}}=\bar{c}^{g_{1}^{-1}} \bar{c}^{g_{2}^{-1}}=1$. Apply $g_{2}$ to both sides to see $g_{1}^{-1} g_{2}$ fixes $\bar{c}$. Prop. 7.11 shows $\bar{c}$ is the unique nontrivial element of $M\left(A_{5}\right)$ in $\left\langle g_{1}^{-1} g_{2}\right\rangle$. Lem. 2.19] shows $q_{2}^{10}$ braids $\boldsymbol{g}^{\prime}$ to $\left(g_{1}, g_{1}^{-1}, g_{2}, g_{2}^{-1}\right)$.

Apply $q_{1}^{-1}$ to $\boldsymbol{g}^{*}$ to get

$$
\left(c^{g_{2}^{-1}} g_{1}^{-1} c^{g_{2}^{-1}}, c^{g_{2}^{-1} g_{1}^{-1}} c^{g_{2}^{-1}} g_{1} c^{g_{2}^{-1}} c^{g_{2}^{-1} g_{1}^{-1}}, c g_{2} c, g_{2}^{-1}\right)
$$

Use $c^{g_{1} g_{2}}=c$ to rewrite this as $\left(g_{1}^{\prime}, c\left(g_{1}^{\prime}\right)^{-1} c, c g_{2} c, g_{2}^{-1}\right)=\boldsymbol{g}^{\prime \prime}$ with $g_{1}^{\prime}=c^{g_{2}^{-1}} g_{1}^{-1} c^{g_{2}^{-1}}$. Apply the first part of this lemma with $\bar{c}=c$ : An element of $H_{4}$ takes $\boldsymbol{g}^{\prime \prime}$ to the $\mathrm{H}-\mathrm{M}$ representative $\left(g_{1}^{\prime},\left(g_{1}^{\prime}\right)^{-1}, g_{2}, g_{2}^{-1}\right)$.

As in Lem. 8.9] $c=\left(g_{1} g_{2}\right)^{5} \in M\left(A_{5}\right) \backslash\{0\}$ centralizes $g_{1} g_{2}$. Use that $c$ and its conjugates don't centralize $g_{1}$ (Cor. [5.7) to see

$$
\left(g_{1}^{\prime},\left(g_{1}^{\prime}\right)^{-1}, g_{2}, g_{2}^{-1}\right) q_{1}=\left(\left(g_{1}^{\prime}\right)^{-1}, g_{1}^{\prime}, g_{2}, g_{2}^{-1}\right)
$$

and $\left(g_{1}, g_{1}^{-1}, g_{2}, g_{2}^{-1}\right)$ give two $\mathrm{H}-\mathrm{M}$ reps. at level 1 in one $H_{4}$ orbit lying over the same level $0 \mathrm{H}-\mathrm{M}$ rep.

We already know how to braid from $\boldsymbol{h}_{+}$to $\boldsymbol{h}_{-}$. Since, there are only two H-M reps. over $\boldsymbol{h}_{+}$, it suffices that we have this braid from one to the other. So, 8.2 .3 gives the braiding between all the width two cusps. The last expression relating $\mathrm{H}-\mathrm{M}$ reps. to complements of near H-M reps. comes from writing $q_{1}$ as

$$
q_{2}^{-1}\left(q_{1} q_{2} q_{1}\right) q_{2}^{-1}=q_{2}^{-1}\left(q_{2} q_{1} q_{2}\right) q_{2}^{-1}
$$

to express this in standard generators of $\bar{M}_{4}$.

The relation between H-M reps. $\boldsymbol{g}$ and near H-M reps. given by $(\boldsymbol{g}) \mathbf{c m} \gamma_{\infty}^{-1} \mathbf{s h} \gamma_{\infty}^{-1}$ works at any level (with $p=2$ ). Prop. 7.11 has already established that near H-M reps. have the complex conjugation properties stated here. The element $c^{\dagger}$ has the form $\left(\left(g_{1}^{\dagger}\right)^{-1} g_{2}^{\dagger}\right)^{5 \cdot 2^{k-1}}$. Lift it to $T_{k}^{\prime}$ by lifting $g_{1}^{\dagger}$ and $g_{2}^{\dagger}$ to (respectively) $g_{1}$ and $g_{2}$ of order 3 in $T_{k}^{\prime}$. Then form $\left.\left(g_{1}\right)^{-1} g_{2}\right)^{5 \cdot 2^{k-1}}$. Let $h=\left(\left(g_{1}\right)^{-1} g_{2}\right)^{5}$. The characterizing property of $T_{k}^{\prime}$ implies $h$ has order $2^{k+1}$ or $h^{2^{k-1}}$ has order 4 . So, any lift of $c^{\dagger}$ to $T_{k}^{\prime}$ has order 4 . Since $c^{\prime \prime}$ is just a conjugate of $c^{\dagger}$, it applies to $c^{\prime \prime}$ as well.

By now it is clear H-M reps. figure in many computations. Denote the collection of these by $S_{\mathrm{HM}}$.

8.4. Cusp widths of length 6 and 10. The effect of $q_{2}^{2}$ on $\boldsymbol{g} \in \mathrm{Ni}_{1}^{\mathrm{in}}$ is conjugation of $\left(g_{2}, g_{3}\right)$ by $g_{2} g_{3}$ (Lem. 2.19) leaving $g_{1}$ and $g_{4}$ fixed. 
8.4.1. $\gamma_{\infty}$ orbits of $\boldsymbol{g} \in \mathrm{Ni}_{1}^{\mathrm{in}, \mathrm{rd}}$ with length six. A $\gamma_{\infty}$ orbit of length six implies a length three orbit of $\gamma_{\infty}^{2}$ on $\boldsymbol{g}$. The group $H=\left\langle g_{1}, g_{4}\right\rangle$ is the pullback in $G_{1}={ }_{2}^{1} \tilde{A}_{5}$ of a copy of $A_{4}$ in $A_{5}$ (Table 1 items 3,6,7 or one of the conjugates of these by (45)). So, $H$ has no nontrivial centralizer in $G_{1}$. Conclude: Three divides the order of $g_{2} g_{3}$, and with $v=\left(g_{2} g_{3}\right)^{3} \in M\left(A_{5}\right), \boldsymbol{g}_{v}=\left(g_{1}, v g_{2} v, v g_{3} v, g_{4}\right)=(\boldsymbol{g}) q_{2}^{6}$ does not equal $\boldsymbol{g}$ in $\mathrm{Ni}_{1}^{\text {in }}$ (Prop. 5.9). Note: $v \in M\left(A_{5}\right) \backslash V$ generates the centralizer in $M\left(A_{5}\right)$ of $g_{2} g_{3}$.

So, the $\gamma_{\infty}$ orbit of $\boldsymbol{g} \in \mathrm{Ni}_{1}^{\text {in,rd }}$ has length six if and only if

(8.16) $\left(\boldsymbol{g}_{v}\right) Q^{\prime}$ is conjugate to $\boldsymbol{g}$ for some $Q^{\prime} \in \mathcal{Q}^{\prime \prime} \backslash\{0\}$.

Prop. [7.9] shows no element of $\mathcal{Q}^{\prime \prime} \backslash\{1\}$ fixes any inner classes.

Proposition 8.13. There are eight $\gamma_{\infty}$ orbits on $\mathrm{Ni}_{1}^{\mathrm{in}, \mathrm{rd}}$ of length six. Let $\boldsymbol{h} \in \mathrm{Ni}_{0}^{\mathrm{in}, \mathrm{rd}}$ be in a length three $\gamma_{\infty}$ orbit, $\boldsymbol{h}\left\langle\gamma_{\infty}\right\rangle$. Then there are exactly six length twelve and four length six $\gamma_{\infty}$ orbits on $\mathrm{Ni}_{1}^{\mathrm{in}, \mathrm{rd}}$ lying over $\boldsymbol{h}\left\langle\gamma_{\infty}\right\rangle$. They have these further properties. For each $H-M$ and near $H-M$ orbit $O^{\prime}$ :

(8.17a) four length twelve orbits $O$ over $\boldsymbol{h}\left\langle\gamma_{\infty}\right\rangle$ satisfy $\left|(O) \mathbf{s h} \cap O^{\prime}\right|=1$; and

(8.17b) the remaining length twelve or six over $\boldsymbol{h}\left\langle\gamma_{\infty}\right\rangle$ satisfy $\left|(O) \mathbf{s h} \cap O^{\prime}\right|=0$.

Denote the collection of length 6 orbits and those of length 12 not meeting $H-M$ or near $H-M$ reps., respectively by $L_{6}$ and $L_{12}$.

Similarly, there are eight $\gamma_{\infty}$ orbits on $\mathrm{Ni}_{1}^{\mathrm{in}, \mathrm{rd}}$ of length 10 and four of length 20 that are not $H-M$ or near $H-M$ orbits. Denote the former by $L_{10}$ and the latter by $L_{20}$. For $O \in L_{6}$ and $O^{\prime} \in L_{20}$ or for $O \in L_{12}$ and $O^{\prime} \in L_{10},\left|(O) \mathbf{s h} \cap O^{\prime}\right|=1$. This accounts for all entries in the sh-incidence matrix for orbits with 3 dividing $\mathbf{m p}$ paired with orbits with 5 dividing $\mathbf{m p}$.

Subsections 8.4 .2 and 8.4 .4 report on respective length six and twelve orbits to conclude the proof of Prop 8.13 The calculation shows that various sh-incidence intersections are 0 or 1 (at most 1 ). These incidence intersections divide according to how the orbits relate to H-M and near H-M reps. This is a consequence of the lifting invariant from Prop. 9.8. Calculations for (8.17) are similar to those in the last half of the statement. So, we only give details for the former.

8.4.2. Length six orbits over $\boldsymbol{h}\left\langle\gamma_{\infty}\right\rangle$. Use the previous notation for $\boldsymbol{g}_{v}$ with $\boldsymbol{g}$ lying over $\boldsymbol{h}\left\langle\gamma_{\infty}\right\rangle$. Then $\boldsymbol{g}\left\langle\gamma_{\infty}\right\rangle$ has length six exactly when $\left(\boldsymbol{g}_{v}\right) Q^{\prime}$ is inner equivalent to $\boldsymbol{g}$ for some $Q^{\prime} \in \mathcal{Q}^{\prime \prime}$. Modulo $\operatorname{ker}_{0}, \boldsymbol{g}=\boldsymbol{g}_{v}$. Since nontrivial elements of $\mathcal{Q}^{\prime \prime}$ have order 2, an element of order 4 must give any conjugation of $\boldsymbol{g}$ to $\left(\boldsymbol{g}_{v}\right) Q^{\prime}$.

The only possibility is that $\boldsymbol{g}$ is conjugate in $G_{1}$ to one of the following:

$$
\begin{aligned}
& \left(v g_{3} v, g_{4}, g_{1}, v g_{2} v\right), \quad\left(g_{1} v g_{2} v g_{1}^{-1}, g_{1}, g_{4}, g_{4}^{-1} v g_{3} v g_{4}\right) \text {, or } \\
& \left(g_{3} v g_{4} v g_{3}^{-1}, g_{3}, g_{2}, g_{2}^{-1} v g_{1} v g_{2}\right) \text {. }
\end{aligned}
$$

Try these by cases. Suppose $\alpha$ conjugates $\boldsymbol{g}$ to $\left(g_{1} v g_{2} v g_{1}^{-1}, g_{1}, g_{4}, g_{4}^{-1} v g_{3} v g_{4}\right)$. Then, $g_{2}=\alpha^{-2}\left(g_{2}\right) \alpha^{2}=g_{1} g_{2} g_{1}^{-1} \bmod \operatorname{ker}_{0}$. There is a contradiction: When $\mathbf{m p}(\boldsymbol{g})$ divides three, the images of $g_{1}$ and $g_{2}$ in $A_{5}$ don't commute (check Table $\mathbb{1}$ ).

If $\alpha$ conjugates $\boldsymbol{g}$ to $\left(g_{3} v g_{4} v g_{3}^{-1}, g_{3}, g_{2}, g_{2}^{-1} v g_{1} v g_{2}\right)$, then $\alpha^{2}$ commutes with $g_{2}$ and $g_{3}$. These generate the pullback of $A_{4}$ in $G_{1}$ which has no center (Cor. 5.7). This implies $\alpha^{2}=1$, contrary to $\alpha$ having order 4 .

This leaves the serious case. Consider if $\boldsymbol{g}$ is conjugate to

$$
\left(v g_{3} v, g_{4}, g_{1}, v g_{2} v\right)=\left(\boldsymbol{g}_{v}\right) Q^{\prime}, Q^{\prime}=\left(Q_{1} Q_{2} Q_{3}\right)^{2} .
$$


That is, for some $\alpha \in G_{1}$ :

$$
\alpha g_{1} \alpha^{-1}=v g_{3} v, \alpha g_{2} \alpha^{-1}=g_{4}, \alpha g_{3} \alpha^{-1}=g_{1} \text {, and } \alpha g_{4} \alpha^{-1}=v g_{2} v \text {. }
$$

Apply conjugation by $\alpha$ to these expressions with $v^{\prime}=v^{\alpha}$. Conclude: Conjugation by $\alpha^{2}$ on $\boldsymbol{g}$ gives $\left(v^{\prime} g_{1} v^{\prime}, v g_{2} v, v g_{3} v, v^{\prime} g_{4} v^{\prime}\right)$.

Opening arguments in 8.2.2 imply $\alpha^{2}=v^{\prime}=v$. Yet, $v$ generates the centralizer of $g_{2} g_{3}$. So, $H=\left\langle g_{2} g_{3}, \alpha\right\rangle$ stabilizes $v$. There are two cases. If the image of $H$ in $A_{5}$ is transitive on four letters, then the image is isomorphic to $A_{4}$ : its pullback in $G_{1}$ (as above) has no center. Otherwise, the image of $H$ in $A_{5}$ is isomorphic to $S_{3}$.

8.4.3. Counting length six orbits. 8.4 .2 gives the only allowable shape for elements $\boldsymbol{g} \in \mathrm{Ni}_{1}$ in a length six orbit of $\gamma_{\infty}$. We prove there are in total eight such orbits. To be explicit, assume $h_{2} h_{3} \equiv(142) \bmod \operatorname{ker}_{0}$ (with $\boldsymbol{h}={ }_{3} \boldsymbol{g}$ in Table (1). If $\boldsymbol{g} \in \mathbf{C}_{3^{4}}$ lies over $\boldsymbol{h}$, conjugating by $\alpha \equiv(12)(35)$ mod ker $_{0}$ has the effect of switching $h_{1}$ with $h_{3}$ (resp. $h_{2}$ with $h_{4}$ ).

Apply Lem. 8.7 to find $\alpha$ having an additional property: $\alpha^{2}$ generates the centralizer $\operatorname{Cen}_{M}\left(h_{2} h_{3}\right)$ of $h_{2} h_{3}$ in $M\left(A_{5}\right)$. Fix some $g_{1}$ in its conjugacy class, lifting $h_{1}$. Let $g_{2}$ be arbitrary in its conjugacy class lifting $h_{2}$. Define $g_{3}$ to be $g_{1}^{\alpha}$ and $g_{2}$ to be $g_{4}^{\alpha}$. The resulting $\boldsymbol{g}$ gives a $\gamma_{\infty}$ orbit of length six if $\Pi(\boldsymbol{g})=1$.

If $\Pi(\boldsymbol{g})=v^{\prime}$, then $s(\boldsymbol{h})=1$ (as in Prop. [5.9) implies $v^{\prime} \in V$. For $m \in M\left(A_{5}\right)$, $(\alpha m)^{2}=\alpha^{2}$ if and only if $m m^{\alpha}=1: \alpha$ fixes $m$. Conjugate $g_{4}$ by $u \in V\left(A_{5}\right)$ and replace $\alpha$ by $\alpha m=\alpha^{\prime}$ with $m^{\alpha}=m$ to construct $\boldsymbol{g}^{\prime}$ from $\boldsymbol{g}$. Compute $\Pi\left(\boldsymbol{g}^{\prime}\right)$ as

$$
g_{1} m\left(u g_{4} u g_{1}\right)^{\alpha} m u g_{4} u=g_{1} g_{4}^{\alpha}\left(u^{g_{4} g_{1}} u^{g_{1}}\right)^{\alpha} u u^{g_{4}^{-1}} m m^{\left(g_{4} g_{1}\right)^{\alpha}} g_{1}^{\alpha} g_{4} .
$$

Lemma 8.14. For each $m \in C e n_{M}(\alpha)$, there is a unique $u \in V$ giving product 1 in (8.18). Representatives $\boldsymbol{g}$ for distinct length six orbits of $\gamma_{\infty}$ over $\boldsymbol{h}$ correspond to the four elements $m \in V$ that centralize $\alpha$ (Cor. 5.7).

Proof. The first sentence follows if $u \in V \mapsto\left(u^{g_{4} g_{1}} u^{g_{1}}\right)^{\alpha} u u^{g_{4}^{-1}}$ is one-one. Consider $A=1+(234)+(12)(35)((123)+(241))(12)(35)$ as an element in the group ring $\mathbb{Z} / 2\left[A_{5}\right]$. It suffices to show $A$ is invertible on $V$.

Use notation from the proof of Lem. 8.7 $g_{1}, g_{2}$ and $\alpha_{25}$ on $D_{5}$ cosets act by $\beta_{g_{1}}=(135)(246), \beta_{g_{2}}=(132)(456)$ and $\beta_{\alpha_{25}}=(31)(25)$. Since $(12)(35)=$ $\left(g_{1} g_{2}\right)^{2}(25)(34)\left(g_{1} g_{2}\right)^{-2}$, compute $\beta_{\alpha}$ to be $\left(\beta_{g_{1}} \beta_{g_{2}}\right)^{2} \beta_{\alpha_{25}}\left(\beta_{g_{1}} \beta_{g_{2}}\right)^{-2}=(16)(23)$. Then $A$, in the group ring, acts on the six cosets generating $M\left(A_{5}\right)$ as follows:

$$
\beta_{A}=1+(541)(623)+(16)(23)((135)(246)+(164)(253))(16)(23)
$$

or as $1+(541)(623)+(134)(256)+(146)(235)$.

Recall $\sum_{i=1}^{6} i_{H}=0$ (in $\left.M\left(A_{5}\right)\right)$. Check $A$ on a basis for $V$ :

$1_{H}+2_{H} \mapsto 5_{H}+6_{H}, 2_{H}+3_{H} \mapsto 1_{H}+5_{H}, 3_{H}+4_{H} \mapsto 3_{H}+5_{H}, 4_{H}+5_{H} \mapsto 2_{H}+5_{H}$

The range vectors form a basis. So, $A$ is invertible on $V$. The remainder of the proof follows from the setup to this lemma.

8.4.4. Length twelve orbits over $\boldsymbol{h}\left\langle\gamma_{\infty}\right\rangle$. We apply the higher lifting invariant of Prop. 9.8 to compute the sh-incidence entries for H-M and near H-M orbits (Def. 8.11) and $\gamma_{\infty}$ orbits of elements with $\mathbf{m p}$ six.

LEMMA 8.15. Suppose $O_{\boldsymbol{g}}$ is the $\gamma_{\infty}$ orbit of $\boldsymbol{g} \in \mathrm{Ni}_{1}^{\mathrm{in}, \mathrm{rd}}$ where $\mathbf{m p}(\boldsymbol{g})=6$ and (g) sh is in the $\gamma_{\infty}$ orbit of an $H-M$ or near $H-M$ rep. Then, for each $\gamma_{\infty}$ orbit $O$ of an $H-M$ or near $H-M$ rep., $\left|\left(O_{\boldsymbol{g}}\right) \mathbf{s h} \cap O\right|=1$. Suppose $O^{\prime}$ is the $\gamma_{\infty}$ orbit of 
$\boldsymbol{g}^{\prime} \in \mathrm{Ni}_{1}^{\mathrm{in}, \mathrm{rd}}$ with $\mathbf{m p}\left(\boldsymbol{g}^{\prime}\right)=10$ with $O^{\prime}$ not the orbit of either an $H$-M or near $H-M$ rep. Then, $\left|\left(O_{\boldsymbol{g}}\right) \mathbf{s h} \cap O^{\prime}\right|=0$.

Proof. Suppose $\boldsymbol{g}$ is an H-M or near H-M rep., or a complement of one of these. Then, $\left((\boldsymbol{g}) \gamma_{\infty}^{j}\right) \mathbf{s h}$ has $\mathbf{m p}$ six if only if $j \equiv 2,3 \bmod 5$. So, for values of $j$ mod 20 , there are eight distinct elements $\left((\boldsymbol{g}) \gamma_{\infty}^{j}\right)$ sh with $\mathbf{m p}$ six. We show no two are on the same $\gamma_{\infty}$ orbit.

First consider level 0 from Table 1 and $\left(\left({ }_{1} \boldsymbol{g}\right) \gamma_{\infty}^{2}\right)$ sh and $\left(\left({ }_{1} \boldsymbol{g}\right) \gamma_{\infty}^{3}\right)$ sh. Conjugation by (45) takes one of the two $\gamma_{\infty}$ orbits to the other. So, it suffices to show $\left((\boldsymbol{g}) \gamma_{\infty}^{2+m 5}\right) \mathbf{s h}, m \in\{0,1,2,3\}$ are in distinct $\gamma_{\infty}$ orbits. That requires showing $\left((\boldsymbol{g}) \gamma_{\infty}^{2}\right)$ sh and $\left((\boldsymbol{g}) \gamma_{\infty}^{2+10}\right)$ sh are in distinct $\gamma_{\infty}$ orbits. This is saying the complements shift to distinct $\gamma_{\infty}$ orbits.

The computations are similar for H-M and near H-M reps., so we do the former only. For simplicity write $\boldsymbol{g}$ as $\left(g_{1}^{-1}, g_{1}, g_{2}, g_{2}^{-1}\right)$. With $u=g_{1} g_{2}$ and $m=u^{5}$, we show $\left(u g_{1} u^{-1}, u g_{2} u^{-1}, g_{2}^{-1}, g_{1}^{-1}\right)=\boldsymbol{g}^{\prime}$ and $\left(m u g_{1} u^{-1} m, m u g_{2} u^{-1} m, g_{2}^{-1}, g_{1}^{-1}\right)=\boldsymbol{g}^{\prime \prime}$ are in distinct $\gamma_{\infty}$ orbits. By reducing modulo modker ${ }_{0}$, the only possibility is $\left(\boldsymbol{g}^{\prime}\right) \gamma_{\infty}^{6}$ is in the same reduced equivalence class as $\boldsymbol{g}^{\prime \prime}$. Check easily this isn't so.

The level one lifting invariant of Prop.9.8 has value +1 on sh applied to the $\gamma_{\infty}$ orbits of elements $\left((\boldsymbol{g}) \gamma_{\infty}^{j}\right)$ sh with $\mathbf{m p}$ six described above. From Prop. 9.8 such an element $\boldsymbol{g}^{\prime}$ is in the $\bar{M}_{4}$ orbit of an H-M or near H-M rep. Prop. 9.8 also says that orbits $O^{\prime}$ in the statement of the lemma have lifting invariant -1 . Since the lifting invariant is an $\bar{M}_{4}$ invariant, they cannot intersect the set from sh applied to the $\gamma_{\infty}$ orbit of $\left((\boldsymbol{g}) \gamma_{\infty}^{j}\right)$ sh.

8.5. The sh-incidence matrix at level 1 . Table 2 has the sh-incidence matrix for the reduced Nielsen classes $\mathrm{Ni}\left(A_{5}, \mathbf{C}_{3^{4}}\right)^{\mathrm{in}, \mathrm{rd}}=\mathrm{Ni}_{0}$. We have all the data in place to compute the $\mathbf{s h}$-incidence matrix for $\mathrm{Ni}_{1}$ (replacing $G_{0}=A_{5}$ by $G_{1}$ ). As at level 0 , a 2 -stage process gives the block for orbit $O_{1}^{+}$: List $\mathrm{H}-\mathrm{M}$ and near H-M reps. and compute their $\gamma_{\infty}$ orbits; then, apply sh to these, and compute their $\gamma_{\infty}$ orbits. What results is closed under sh, so the process completes after two stages. Use the notation for $\boldsymbol{h}_{+}$and $\boldsymbol{h}_{-}$in the above discussion.

Label the type $(10,20) \gamma_{\infty}$ orbits as $O(10,20 ; j), j=1, \ldots, 4$ for those containing $\mathrm{H}-\mathrm{M}$ reps. and $O(10,20 ; j), j=5, \ldots, 8$ for those containing near H-M reps. Refinement: $O(10,20 ; j)$ with $j$ odd (resp. even) lies over the $\gamma_{\infty}$ orbit at level 0 containing $\boldsymbol{h}_{+}$(resp. $\left.\boldsymbol{h}_{-}\right)$. Further refinement: $\mid(O(1,2 ; 1) \mathbf{s h} \cap O(10,20 ; j) \mid=1$, $j=1,2 ; \mid(O(1,2 ; 2) \mathbf{s h} \cap O(10,20 ; j) \mid=1, j=3,4$. According to Prop. 8.6 complements of near H-M reps. produce $\mid(O(2,2 ; 1) \mathbf{s h} \cap O(10,20 ; j) \mid=1, j=5,6$; and $\mid(O(2,2 ; 2) \mathbf{s h} \cap O(10,20 ; j) \mid=1, j=7,8$.

Lemma 8.16. Two type (2,4) $\gamma_{\infty}$ orbits, $O(2,4 ; i), i=1,2$, contain $\mathbf{s h}$ applied to two near $H$-M reps. and two complements of $H$-M reps. So, sh applied to $O(2,4 ; i)$ covers $\left\{\boldsymbol{h}_{+}, \boldsymbol{h}_{-}\right\}$evenly, $i=1,2$.

Let $\boldsymbol{g}$ be an H-M rep. Let $\boldsymbol{g}^{\prime}$ be the near H-M rep. attached to $\boldsymbol{g}$ by Prop. 7.11. Let $\boldsymbol{g}^{\prime \prime}$ be the near H-M rep. attached to the H-M rep. $\left(((\boldsymbol{g}) \mathbf{s h}) q_{2}\right) \mathbf{s h}$. Then, $\left(\boldsymbol{g}^{\prime}\right) \mathbf{s h}$ is different from $\left(\left(\boldsymbol{g}^{\prime \prime}\right) \mathbf{s h}\right) q_{2}$.

Proof. Let $\boldsymbol{g}$ be an H-M rep. The relationship $(\boldsymbol{g}) \mathbf{c m} \gamma_{\infty}^{-1} \mathbf{s h} \gamma_{\infty}^{-1}$ already says $\gamma_{\infty}^{-1} \mathbf{s h} \gamma_{\infty}^{-1}$ takes the complement of an H-M rep. to a near H-M rep. Now use $\operatorname{sh} \gamma_{\infty}^{-1} \operatorname{sh} \gamma_{\infty}^{-1} \mathbf{s h}=\gamma_{\infty}$ in $\bar{M}_{4}$. Let $\boldsymbol{g}^{\prime}$ be $(\boldsymbol{g}) \mathbf{c m}$, and apply sh to it, to get

$$
\left(g^{\prime}\right) \operatorname{sh}\left(\operatorname{sh} \gamma_{\infty}^{-1} \operatorname{sh} \gamma_{\infty}^{-1} \operatorname{sh}\right)
$$


is the shift of a near H-M rep. This shows why a type $(2,4)$ orbit containing sh applied to a complement of an H-M rep. also contains sh applied to a near H-M rep. The remaining conclusions are similar.

As in Prop. 8.8 use $\boldsymbol{h}_{+}$and $\boldsymbol{h}_{-}$for the two H-M reps. in $\mathrm{Ni}_{0}^{\mathrm{in}}$.

Lemma 8.17. Beyond Lem. 8.16, there are four type $(2,4) \gamma_{\infty}$ orbits $O(2,4 ; j)$, $j=3, \ldots, 6$, with lifting invariant +1 (Prop. 9.8). For $j=3, \ldots, 6$,

$$
|(O(2,4 ; j)) \mathbf{s h} \cap O(10,20 ; k)|=0 \text { or } 1 .
$$

For $\boldsymbol{g}$ an H-M rep., $(\boldsymbol{g}) q_{2}^{5}$ and $\boldsymbol{g}$ lie over the same element of $\mathrm{Ni}_{0}^{\mathrm{rd}}$. Each of these $(O(2,4 ; j))$ sh sets contains exactly one reduced equivalence class of form $\left((\boldsymbol{g}) q_{2}^{5}\right) \mathbf{s h}$.

That leaves eight type $(2,4) \gamma_{\infty}$ orbits with lifting invariant -1 (from Prop. [9.8). A representative for such an orbit has $\boldsymbol{g}=\left(g_{2}^{-1}, g_{1}, a g_{1}^{-1} a, b g_{2} b\right)$. The shift applied to the $\gamma_{\infty}$ orbit of $\boldsymbol{g}$ intersects exactly two type $(10,20) \gamma_{\infty}$ orbits, with these covering $\left\{\boldsymbol{h}_{+}, \boldsymbol{h}_{-}\right\}$. This accounts for all 16 of the elements with shifts of type $(2,4)$ in the four $(10,20)$ orbits with lifting invariant -1 . Shifts of the remaining two elements from each $(2,4)$ orbit meet type $(10,10) \gamma_{\infty}$ orbits, covering $\left\{\boldsymbol{h}_{+}, \boldsymbol{h}_{-}\right\}$.

Proof. Let $\boldsymbol{g}$ be an H-M rep. of the form $\left(g_{1}^{-1}, g_{1}, g_{2}, g_{2}^{-1}\right)$. Then, $(\boldsymbol{g}) q_{2}^{5}$ and $\boldsymbol{g}$ lie over the same element of $\mathrm{Ni}_{0}^{\mathrm{rd}}$.

In each width $20 q_{2}$ orbit, there are four elements to which sh gives an element with $\mathbf{m p}(\boldsymbol{g})=1$ or 2 . Apply the shift to the remaining (from 16) elements in the orbits containing H-M and near H-M reps. that lie over $\boldsymbol{g} \in \mathrm{Ni}_{0}$ with $\mathbf{m p}((\boldsymbol{g}) \mathbf{s h})=$ 3 . There are $8 \times 8=64$ elements in the width 20 cusps with shifts of this type.

The remaining elements with $\mathbf{m p} 2$ must have lifting invariant -1 . A representative has the form $\boldsymbol{g}=\left(g_{2}^{-1}, g_{1}, a g_{1}^{-1} a, b g_{2} b\right)$ with $(a, b) \in M_{3}^{\prime} \times M_{5}^{\prime} \cup M_{5}^{\prime} \times M_{3}^{\prime}$ as in Prop. 9.8 With $\left\langle c_{1}\right\rangle$ the centralizer of $g_{1}$ in $M$, the $\gamma_{\infty}$ orbit of $\boldsymbol{g}$ includes

$$
\begin{aligned}
& (\boldsymbol{g}) q_{2}=\left(g_{2}^{-1}, a^{g_{1}^{-1}} g_{1}^{-1} a^{g_{1}^{-1}}, g_{1}, b g_{2} b\right), \\
& \boldsymbol{g}_{a}=\left(g_{2}^{-1}, c_{1} a a^{g_{1}^{-1}} g_{1} c_{1} a a^{g_{1}^{-1}}, a^{g_{1}^{-1}} g_{1}^{-1} a^{g_{1}^{-1}}, b g_{2} b\right), \operatorname{and}\left(\boldsymbol{g}_{a}\right) q_{2} .
\end{aligned}
$$

An analog the Lem. 8.14 proof shows that if $(\boldsymbol{g}) \mathbf{s h} \in L_{10}$, then $\left(\boldsymbol{g}_{a}\right) \mathbf{s h} \in L_{20}$. As there, this argument uses the special presentation of $M\left(A_{5}\right)$ from Cor. 5.7

Let $O$ be one of the $(6,12)$ type $\gamma_{\infty}$ orbits with lifting invariant +1 . From 88.4 .4 $|(O) \mathbf{s h} \cap O(10,20 ; j)|=1, j=1, \ldots, 8$. Further, $(O)$ sh hits two other $(6,12) \gamma_{\infty}$ orbits with multiplicity two. The argument is similar to that of Lem. 8.18 Use the $\mathbf{s h} \gamma_{\infty}$ sh operator applied to $\boldsymbol{g}$ with $\mathbf{m p}$ six with $(\boldsymbol{g}) \gamma_{\infty}$ also having $\mathbf{m p}$ six. This gives the $8 \times 12=96$ elements in $(6,12)$ type orbits with lifting invariant +1 .

The only unaccounted nonzero entries in the sh-incidence matrix for orbit $O_{1}^{+}$have the form $\mid(O)$ sh $\cap O^{\prime} \mid$ with $O$ and $O^{\prime}$ running over $\mathrm{H}-\mathrm{M}$ and near $\mathrm{H}-$ $\mathrm{M}$ orbits. From symmetry, it suffices to fix $O$ an H-M rep. orbit, and account for the intersections as $O^{\prime}$ varies.

Lemma 8.18. As $O^{\prime}$ runs over $H-M$ and near $H$ - $M$ orbits, $\sum_{O^{\prime}}\left|(O) \operatorname{sh} \cap O^{\prime}\right|=8$. If $\boldsymbol{g}$ is an $H-M$ rep., then the $H-M$ orbit $O_{\boldsymbol{g}^{\prime}}$ of $\boldsymbol{g}^{\prime}=\left(((\boldsymbol{g}) \mathbf{s h}) \gamma_{\infty}\right)$ meets it with multiplicity four. Two near $H$-M orbits $O_{1}^{\prime}$ and $O_{2}^{\prime}$ meet $O$ with multiplicity two.

Proof. Lemmas 8.16 and 8.17account for all of the intersections of sh applied to orbits with $\mathbf{m p}=2$ with $O$. There are four such intersections. 8.4.4 shows that $\mathbf{s h}$ applied to orbits with $\mathbf{m p}=6$ contributes eight intersections with $O$. Since 
there are 20 elements in $O, \gamma_{\infty}$ orbits $O_{\boldsymbol{g}^{\prime}}, O_{1}^{\prime}, O_{2}^{\prime}$ account for all the remaining intersections with $O$. Now we show why $O_{\boldsymbol{g}^{\prime}}, O_{1}^{\prime}, O_{2}^{\prime}$ give the indicated intersections.

Such intersections lie above corresponding level 0 intersections. 2.7 gives the formula $\operatorname{sh} \gamma_{\infty} \operatorname{sh}=\gamma_{\infty}^{-1} \operatorname{sh} \gamma_{\infty}^{-1}$ acting on reduced inner Nielsen classes. Suppose $\boldsymbol{g} \in O$ lies over $\boldsymbol{h}_{+}$and $(\boldsymbol{g}) \mathbf{s h}$ has middle product 2 . For example, this would hold if $\boldsymbol{g}$ is an H-M rep. Then (Lem. 8.16), $\boldsymbol{g}^{\prime}=\left(((\boldsymbol{g}) \mathbf{s h}) \gamma_{\infty}\right)$ sh lies over $\boldsymbol{h}_{-}$, on an H-M or near H-M rep. orbit, and its shift also has middle product 2. So $\left(\left(\boldsymbol{g}^{\prime}\right) \gamma_{\infty}\right) \mathbf{s h}=(\boldsymbol{g}) \gamma_{\infty}^{-1}, \mathbf{s h}$ applied to $\left(\boldsymbol{g}^{\prime}\right) \gamma_{\infty}$ is an intersection of $O_{\boldsymbol{g}}$ and $\left(O_{\boldsymbol{g}^{\prime}}\right) \mathbf{s h}$. If we use instead $\mathbf{s h} \gamma_{\infty}^{-1} \mathbf{s h}=\gamma_{\infty} \mathbf{s h} \gamma_{\infty}$, this gives another intersection of $O_{\boldsymbol{g}}$ and $\left(O_{\boldsymbol{g}^{\prime}}\right) \mathbf{s h}:\left(\left(\boldsymbol{g}^{\prime}\right) \gamma_{\infty}^{-1}\right) \mathbf{s h}=(\boldsymbol{g}) \gamma_{\infty}$.

If $\boldsymbol{g}$ is the H-M rep. on $O$, then $\boldsymbol{g}^{\prime}$ is an H-M rep. Suppose

$$
\text { (g) } \gamma_{\infty}^{10}=\left(g_{1}^{-1}, d g_{1} d, d g_{2} d, g_{2}^{-1}\right)
$$

$\left(d=\left(g_{1} g_{2}\right)^{5}\right.$; the complement of an H-M rep.), then $\boldsymbol{g}^{\prime}$ is a near H-M rep. as given by Prop. 17.11. Similarly, replacing $\boldsymbol{g}$ by $(\boldsymbol{g}) \gamma_{\infty}^{15}$ and $(\boldsymbol{g}) \gamma_{\infty}^{5}$ replaces $\boldsymbol{g}^{\prime}$ by the complement of $\boldsymbol{g}^{\prime}$ and the other near H-M rep. over $\boldsymbol{g}_{-}$.

Lem. 8.18 fully accounts for the sh-incidence matrix restricted to the block corresponding to the pairings of $\gamma_{\infty}$ orbits in $\bar{M}_{4}$ orbit $O_{1}^{+}$having lifting invariant +1 . The $32 \times 32$ block of pairings of $\gamma_{\infty}$ orbits in $\bar{M}_{4}$ orbit $O_{1}^{-}$having lifting invariant -1 is more complicated. 9.5 .3 discusses why there may be a representation theory connection between the two orbits, though it would not be numerically simple. To conclude this section we illustrate the lemmas above with a contribution to the complete sh-incidence matrix at level 1 for having +1 lifting invariant.

From here to the end of this subsection, $\gamma_{\infty}$ orbits refer to suborbits of $O_{1}^{+}$. The part of the sh-incidence matrix pairing $O$ and $O^{\prime}$ with respective middle products 6 and 10 is significant. Still, this $8 \times 8$ block consists of just one's (Prop. 8.13). More interesting is the $8 \times 8$ block of intersections from $(10,20)$ type $\gamma_{\infty}$ orbits. For economy, replace $(10,20)$ as the $\gamma_{\infty}$ orbit type in Table 4 by the symbol $\dagger$.

TABLE $4 . \mathbf{m p s} 10 \times 10$ part of sh-Incidence Matrix for $O_{1}^{+}$

\begin{tabular}{|c|c|c|c|c|c|c|c|c|}
\hline Orbit & $O(\uparrow ; 1$ & $O\left(_{\uparrow} ; 2\right.$ & $O(+; 3)$ & $O(† ; 4)$ & $O(+; 5)$ & $O(\uparrow ; 6)$ & $O(+; 7)$ & $\overline{\partial(† ; 8)}$ \\
\hline$O(† ; 1)$ & 0 & 0 & 0 & 4 & 0 & 0 & 2 & 2 \\
\hline$O(+; 2)$ & 0 & 0 & 4 & 0 & 2 & 2 & 0 & 0 \\
\hline$O(+; 3)$ & 0 & 4 & 0 & 0 & 0 & 0 & 2 & 2 \\
\hline$O(+; 4)$ & 4 & 0 & 0 & 0 & 2 & 2 & 0 & 0 \\
\hline$O(\dagger ; 5)$ & 0 & 2 & 0 & 2 & 0 & 0 & 0 & 4 \\
\hline$O(\dagger ; 6)$ & 0 & 2 & 0 & 2 & 0 & 0 & 4 & 0 \\
\hline$O(+7)$ & 2 & 0 & 2 & 0 & 0 & 4 & 0 & 0 \\
\hline$O(+; 8)$ & 2 & 0 & 2 & 0 & 4 & 0 & 0 & 0 \\
\hline
\end{tabular}

8.6. Real points on level 1 of the $\left(A_{5}, \mathbf{C}_{3^{4}}\right)$ Modular Tower. We show the genus 12 component of $\mathcal{H}\left(G_{1}, \mathbf{C}_{3^{4}}\right)^{\text {in }}$ has one component of real points, while the genus 9 component has no real points.

We check the effect of $\hat{\kappa}$ at level 1 extending the use of the complex conjugation operator at level 0 as Lem. 6.11] reports. Note: $\hat{\kappa}^{2}$ acts trivially on $\boldsymbol{g}$ satisfying the product-one condition. For $Q \in \mathcal{Q}^{\prime \prime}$, while $Q^{2}$ acts trivially on a Nielsen class, it usually is a nontrivial conjugation dependent on the $\boldsymbol{g}$ representing a Nielsen class. 
First, check the effect over $j_{0} \in(1, \infty)$. Corresponding to $j_{0}$, we know there are eight cover points corresponding to the four H-M reps. and the four near H-M reps. lying on $\mathcal{H}_{1}^{\text {in,rd }}$ (Prop. [7.9) and the complex conjugation operator $\hat{\kappa}_{0}$. The Nielsen classes for the corresponding components all lie in distinct $\bar{M}_{4}$ orbits. For each, moreover, their complementary Nielsen class gives another point over $j_{0}$ from Lem.6.6. These 16 real components over the interval $(1, \infty)$ all lie on the component $\mathcal{H}_{1}^{+}$(as in Prop. 9.9). Denote the complete set of real points (corresponding to reduced Nielsen classes) lying on $\mathcal{H}_{1}^{\text {in,rd }}$ over $j_{0}$ by $\mathrm{Ni}_{j_{0}}$.

Proposition 8.19. For $j_{0} \in(1, \infty)$, elements of $\mathrm{Ni}_{j_{0}}$ lie on exactly 16 components of $\mathcal{H}_{1}^{+}(\mathbb{R})$ lying over $(1, \infty) \subset \mathbb{P}_{j}^{1}(\mathbb{R})$. The closure in $\overline{\mathcal{H}}_{1}^{+}(\mathbb{R})$ of these components consists of one connected component of real points. There are no $\mathbb{R}$ points on $\mathcal{H}_{1}^{\text {in,rd }}$ over $(-\infty, 1) \subset \mathbb{P}_{j}^{1}(\mathbb{R})$, and no $\mathbb{R}$ points at all on $\mathcal{H}_{1}^{-}$.

The proof of Prop. 8.19 takes up the next five subsections. Its arrangement starts with an equivalent equation defining a point on $\mathcal{H}_{1}^{\text {in,rd }}(\mathbb{R})$ for a reduced equivalence class of covers represented by a particular cover with branch points all in $\mathbb{R}$ or in complex conjugate pairs. Assume a representing cover has branch cycles $\boldsymbol{g} \in \operatorname{Ni}\left(G_{1}, \mathbf{C}_{3^{4}}\right)$. Then, $\hat{\kappa}(\boldsymbol{g})=h(\boldsymbol{g}) Q h^{-1}$ with $\hat{\kappa}$ the complex conjugation operator corresponding to the branch point configuration, $h \in G_{1}$ and $Q \in \mathcal{Q}^{\prime \prime}$. Also, mod $\operatorname{ker}_{0}, Q$ acts trivially modulo conjugation.

8.6.1. $\hat{\kappa}_{4}$ for four real branch points. The level 0 real point data for the absolute Hurwitz space appears at the end of the proof of Lem. 6.11.

For four points $\boldsymbol{z}$ in $\mathbb{R}: \boldsymbol{g}_{1}^{\prime}=((123),(132),(145),(154))$ has complex operator $c_{1}=(23)(45) ; \boldsymbol{g}_{2}^{\prime}=((123),(145),(154),(132))$ has $c_{2}=(13)(54)$. The involution $c_{1, \infty}=(36)(89)(45)$ acts on Nielsen classes in Table 1 revealing $\boldsymbol{g}_{1}^{\prime}$ and $\boldsymbol{g}_{2}^{\prime}$ as the first two items in that table. Since the complex conjugation operator for $\boldsymbol{g}_{7}^{\prime}$ is $(23)$, the Galois closure of that cover is not over $\mathbb{R}$. So, points corresponding to these branch cycle descriptions on the absolute space have no real points on the inner space above them.

6.5 .3 has the level 0 inner Hurwitz space data summarized in the complex conjugation operator from (6.13): $c_{1, \infty}=(917)(716)(818)(1314)(1215)(63)(45)$. The fixed points correspond to real points, with branch cycle descriptions $\boldsymbol{g}_{1}^{\prime}$ and $\boldsymbol{g}_{2}^{\prime}$ and their conjugates by (45).

Let $\hat{\kappa}_{4}$ be the complex conjugation operator for four real branch points. It suffices to handle the defining equation, when $\hat{\kappa}_{4}(\boldsymbol{g})=h(\boldsymbol{g}) Q h^{-1}$. With no loss, take $\boldsymbol{g} \bmod \mathrm{ker}_{0}$ equal either $\boldsymbol{g}_{1}^{\prime}$ or $\boldsymbol{g}_{2}^{\prime}$.

8.6.2. $\hat{\kappa}_{0}$ for complex conjugate pairs of branch points. First consider the case $\boldsymbol{g}_{1}^{\prime}$, which is an H-M rep. at level 0 . Here and below we use the normalization that writes $\boldsymbol{g}=\left(g_{1}, a g_{1}^{-1} a, b g_{2} b, g_{2}^{-1}\right)$, a perturbation of an H-M rep. With no loss $\left(g_{1}, g_{2}\right)$ lies over $((123),(145))$ or $((123),(154))$ with $a, b \in M \backslash V$. It is convenient to use past data by replacing $\hat{\kappa}_{4}$ by $\hat{\kappa}_{0}$, the complex conjugation operator for complex conjugate pairs of branch points. As in Rem. 6.9 this doesn't change the real points representing reduced classes.

Assume $\boldsymbol{g}$ covers $\left(\left(\begin{array}{l}2 \\ 2\end{array}\right),(123)^{-1},(145),(145)^{-1}\right)$. Double the number of components corresponding to using (154) in place of (145). Use the notation around Prop. 9.8 to distinguish the $\bar{M}_{4}$ orbit of $\boldsymbol{g}$ by whether $a$ and $b$ are in the same $G_{1}$ orbit (acting on $M \backslash V$ ). 
Suppose $\boldsymbol{g}$ corresponds to $(a, b)$ in different $G_{1}$ orbits. We run through nontrivial elements $Q \in \mathcal{Q}^{\prime \prime}$, showing that $\hat{\kappa}_{0}(\boldsymbol{g})=h(\boldsymbol{g}) Q h^{-1}$ is not possible for any $h \in G_{1}$. The product of 3 rd and 4 th entries of $\boldsymbol{g}$ is $v=b b^{g_{2}^{-1}}$. So, $\hat{\kappa}_{0}$ maps $\boldsymbol{g}$ to $\left(v a g_{1} a v, v g_{1}^{-1} v, g_{2}^{-1}, b g_{2} b\right)$. The components $\mathcal{H}_{1}^{+}$and $\mathcal{H}_{1}^{-}$have field of definition $\mathbb{Q}$ and so $\hat{\kappa}_{0}(\boldsymbol{g})$ gives branch cycles for a cover in $\mathcal{H}_{1}^{-}$. Prop. 9.9 implies it is attached to $\left(a^{\prime}, b\right)$ as a perturbation of an H-M rep. One case is easy: $Q=\left(q_{1} q_{2} q_{3}\right)^{2}$. On the right side these produce $\boldsymbol{g}^{*}$ conjugate to $\left(\left(b g_{2} b, g_{2}^{-1}, g_{1}, a g_{1}^{-1} a\right)\right.$. Though $\boldsymbol{g}^{*}$ is still a perturbation of an H-M rep., it corresponds to $\left(a^{*}, b^{*}\right)$ with $a^{*}$ (resp. $\left.b^{*}\right)$ in the opposite $G_{1}$ orbit to $a$ (resp. $b$ ). Similarly for $\left.Q=\left(q_{1} q_{2} q_{3}\right)^{2} q_{1} q_{3}^{-1}\right)$.

Yet, when $Q=q_{1} q_{3}^{-1},(\boldsymbol{g}) Q=\left(a g_{1}^{-1} a, g_{1}, g_{2}, b g_{2}^{-1} b\right)$. A conjugation of $(\boldsymbol{g}) Q$ that gives $\hat{\kappa}_{0}(\boldsymbol{g})$ is by a lift $\alpha$ of (23)(45) with square the centralizer $c_{2}$ of $g_{2}$ in $M \backslash V$, and $(\alpha v)^{2}$ the centralizer $c_{1}$ of $g_{1}$ in $M \backslash V$. The last is equivalent to $\alpha^{2} v^{\alpha} v=c_{1}$, or $c_{1}=v^{\alpha} v c_{2}$. Cor. 5.7notes that $\alpha$ fixes exactly four elements in $V$ and two elements of $M \backslash V$ in the conjugacy class $M_{3}^{\prime}$. They are $c_{1}$ and $c_{2}$. So, $v^{\alpha} v=c_{1} c_{2}$ is possible for exactly four elements in $V$. (The proof of Cor. 5.7 has explicit computations, for a different $\alpha$, corroborating this.) The expression $w^{g_{2}^{-1}} w=v$ then has a unique solution $w \in V$ from which we derive values of $b=c_{2} w$. We already know four values of $b$ that satisfy this. These correspond to the complements of $\mathrm{H}-\mathrm{M}$ and near H-M reps. lying above the real component of $\mathcal{H}_{0}$ (over $(1, \infty) \subset \mathbb{P}_{j}^{1}$ ) corresponding to the cover points with branch cycles ((123), (132),(145),(154)) and complex conjugation operator $\hat{\kappa}_{0}$. Thus, Prop. 7.9 shows for $(a, b)$ in different $G_{1}$ orbits on $M \backslash V, v^{\alpha} v=c_{1} c_{2}$ is not possible.

8.6.3. Action of $\mathcal{Q}^{\prime \prime}$ on $\boldsymbol{g}$ over $\boldsymbol{g}_{2}^{\prime}$. Adopt the idea of using a perturbation of an H-M rep. here by writing $\boldsymbol{g}=\left(a g_{1} a, b g_{2}^{-1} b, g_{2}, g_{1}^{-1}\right)$. The product-one condition gives $a^{g_{1}} a=b b^{g_{2}}$. We show $\hat{\kappa}_{4}(\boldsymbol{g})=h(\boldsymbol{g}) Q h^{-1}$ is impossible for $Q \in \mathcal{Q}^{\prime \prime}$. All computations are similar. We do just $Q=q_{1} q_{3}^{-1}$. This will complete that there are exactly 16 components of $\mathcal{H}^{\mathrm{in}, \mathrm{rd}}(\mathbb{R})$ over the $j$-interval $(1, \infty)$. Compute $\hat{\kappa}_{0}(\boldsymbol{g})$ :

$$
\begin{aligned}
& \left(a g_{1}^{-1} a, g_{1} g_{2}^{-1} b g_{2} b g_{2} g_{1}^{-1}, g_{1} g_{2}^{-1} g_{1}^{-1}, g_{1}\right) \\
= & h\left(a(a b)^{g_{1}^{-1}} g_{1} g_{2}^{-1} g_{1}^{-1} a(a b)^{g_{1}^{-1}}, a g_{1} a, g_{1}^{-1}, g_{1} g_{2} g_{1}^{-1}\right) h^{-1} .
\end{aligned}
$$

The effect of $h$ on the $3 \mathrm{rd}$ and 4 th entries shows $h^{2}$ is the identity. Since, however, $h$ is a lift of $(14)(35)$, it has order 4 (Lem. 2.23).

8.6.4. Connecting 16 components of $\mathcal{H}_{1}^{+}(\mathbb{R})$ over $(1, \infty) \subset \mathbb{P}_{j}^{1}$. Now we show these 16 components of real points close up to one connected component on $\overline{\mathcal{H}}_{1}^{+}$. That is, if we add the endpoints over $\infty$ (cusps 77.2 .1 ) and 1 to these components, they form one connected set. Branch cycles for an H-M rep. and its complement give real components on $\mathcal{H}_{1}^{+}$meeting (each pair) at a cusp of type $(10,20)$ (Prop. 7.9). The same is true for near H-M reps.

Apply sh to these to get another real component (over $(1, \infty)$ ). This is the result of reflection off the $j=1$ boundary of the entering real locus. Apply Prop. 8.6 to decipher the labeling of branch cycles to components on applying sh to the H-M, near H-M reps. and their complements. The result is a cusp of type $(2,2)$ or $(2,4)$. In the former case it reflects off the $j=\infty$ boundary to give another real component. In the latter case an application of $\gamma_{\infty}^{2}$ gives a real component reflection. Applying Prop. 8.6 to these reflections shows there is one connected component of real points.

8.6.5. $\mathcal{H}_{1}^{\text {in,rd }}(\mathbb{R})$ is empty over $j \in(-\infty, 1)$. To finish Prop. 8.19 we show there are no points on $\mathcal{H}_{1}^{\text {in,rd }}(\mathbb{R})$ over $j \in(-\infty, 1)$. According to Lem. 6.5 this is a check that $\hat{\kappa}_{2}(\boldsymbol{g})=h(\boldsymbol{g}) Q h^{-1}$ is impossible under the following conditions. 
(8.20a) $\hat{\kappa}_{2}$ is the complex conjugation operator for two real and a complex conjugate pair of branch points.

(8.20b) $h \in G_{1}$ and $Q \in \mathcal{Q}^{\prime \prime}$.

As in 8.6 .3 consider branch cycles for real points on $\mathcal{H}_{0}^{\text {in,rd }}$. Write $\hat{\kappa}_{2}(\boldsymbol{g})=$ $\left(g_{1}^{-1},\left(g_{3} g_{4}\right)^{-1} g_{2}^{-1} g_{3} g_{4}, g_{4}^{-1}, g_{3}^{-1}\right)$. Running through entries of Table 1 gives the existence of $h$ in $\hat{\kappa}\left(\boldsymbol{g}^{\prime}\right)=h \boldsymbol{g}^{\prime} h^{-1}$ with $\boldsymbol{g}^{\prime} \in \mathrm{Ni}_{0}$ only for ${ }_{1} \boldsymbol{g}$ with $h=(23){ }_{3} \boldsymbol{g}$ with $h=(13)(45)$ and ${ }_{9} \boldsymbol{g}$ with $h=(23)$. As in 88.6.1 only the real component corresponding to ${ }_{3} \boldsymbol{g}$ lies below real components of $\mathcal{H}_{0}^{\text {in,rd }}$. This is compatible with $c_{-\infty, 0}=(1013)(14)(914)(73)(1216)(112)(156)(518)$ from 6.5 .3 fixing two integers. It suffices to consider $\boldsymbol{g} \in \mathrm{Ni}_{1}^{\text {in,rd }}$ lying over ${ }_{3} \boldsymbol{g}=((123),(145),(215),(243))$. Write $\boldsymbol{g}$ as $\left(g_{1}, g_{2}, \beta g_{2}^{-1} \beta^{-1}, \beta g_{1}^{-1} \beta^{-1}\right)$ with $\beta=g_{1} g_{2}$ or a perturbation of this of the form $\left(g_{1}, g_{2}, a \beta g_{2}^{-1} \beta^{-1} a, b \beta g_{1}^{-1} \beta^{-1} b\right)$ with $a, b \in M \backslash V$. The calculation to show that for no $Q$ and $h$ can this give an $\mathbb{R}$ point on $\mathcal{H}_{1}^{\text {in,rd }}$ is similar to that of 8.6.3. We leave it to the reader.

\section{Completing level 1 of an $A_{5}$ Modular Tower}

Cor. 8.3 computes genuses of the two components $\mathcal{H}_{1}^{+}$and $\mathcal{H}_{1}^{-}$of level of the $\left(A_{5}, \mathbf{C}_{3^{4}}\right)$ Modular Tower. Prop. 8.19 shows only $\mathcal{H}_{1}^{+}$has real points (one component of them on $\left.\overline{\mathcal{H}}_{1}^{+}\right)$. 9.2 describes data we got from $\left[\mathbf{S}^{+} \mathbf{9 5}\right.$. Following that, all but the last subsection is group theory for the spin structure explanation that nails the two components in Cor. 8.3 The $\mathbf{S}^{+} \mathbf{9 5}$ data was reassuring at several stages. Still, it was crude compared to our final arguments. Despite the detail developed for our special case, there remains the mysterious similarity, amidst the differences, of the two level 1 orbits. 9.5 gives ideas for that appropriate for testing the speculative Lie algebra guess in $\mathbb{F}$ The paper (excluding appendices) concludes with lessons on computing (genuses of) components for all Modular Towers (9.6).

Orbits of $\gamma_{\infty}$ correspond to cusps of a $j$-line cover. Orbits of $\left\langle\gamma_{1}, \gamma_{\infty}\right\rangle$ correspond to components of the $j$-line cover. Finding discrete invariants of a Modular Tower means analyzing the $\left\langle\gamma_{1}, \gamma_{\infty}\right\rangle$ and $\gamma_{\infty}$ orbits on reduced Nielsen classes. That includes analyzing arithmetic properties of the cusps. Since components are moduli spaces this means analyzing degeneration of objects in the moduli space on approach (over $\mathbb{R}$ or $\mathbb{Q}_{p}$ ) to the cusps. This generalizes analysis of elliptic curve degeneration in approaching a cusp of a modular curve (Fri96 introduces using $\theta$ functions to make this analysis; $(\mathrm{B})$. Even for the one Modular Tower for $\left(A_{5}, \mathbf{C}_{3^{4}}\right)$, it would be a major event to prove the analog of Prop. 8.19 for $p$-adic points (see $\$ \mathrm{D}$ ).

9.1. Diophantine implications. Apply Falting's Theorem Fal83 to these genus 12 and 9 components to conclude the following from Thm. 6.1

THEOREM 9.1. There are only finitely many $\left(\frac{1}{2} \tilde{A}_{5}, \mathbf{C}_{3^{4}}\right)$ realizations over any number field $K$. For $k$ large there are no $\left(G_{k}, \mathbf{C}_{3^{4}}\right)$ realizations over $K$.

Example 9.2 (The $\left(A_{4}, C_{3^{4}}, p=2\right.$ ) Modular Tower). If $G_{k}$ is the level k characteristic quotient for ${ }_{2} \tilde{A}_{5}$, then the pullback of $A_{4} \leq A_{5}$ in $G_{k}$ is $G_{k}^{\prime}$, the level $k$ characteristic quotient of ${ }_{2} \tilde{A}_{4}$. The notation $\left(A_{4}, C_{3^{4}}\right)$ is ambiguous. There are two conjugacy classes of 3 -cycles in $A_{4}$. Compatible with Ex. 9.3 we mean each pair of conjugacy classes appears twice, or what we might label as $\left(A_{4}, C_{3_{+}^{2} 3_{-}^{2}}\right)$. From the Branch Cycle Lemma this is equivalent to the levels of the Modular Tower have definition field $\mathbb{Q}$. 
Level 0 is a special case of [Fri96] (Ex. 3.17). There are two components, $\mathcal{H}_{0}^{+}$ and $\mathcal{H}_{0}^{-}$, corresponding to two $\mathrm{H}_{4}$ orbits $\mathrm{O}_{0}^{+}$and $\mathrm{O}_{0}^{-}$of the lifting invariant from $A_{4}$ to $\operatorname{Spin}_{4}$ (analogous to Prop. 9.8). Note: $\mathcal{Q}^{\prime \prime}$ acts through $\mathbb{Z} / 2$ on these inner classes (E.2.1 has details). For absolute equivalence these spaces are families of genus 1 curves. It is a special case of [FKK01 that the map of each component to the moduli space of curves of genus 1 is generically surjective (see $[\mathrm{B}$ ). As in Prop. 9.8 there is nothing above the component $\mathcal{H}_{0}^{-}$at level 1.

Applying this paper as with the $A_{5}$ Modular Tower, the first author's thesis will show level 1 for $\left(A_{4}, C_{3^{4}}\right), p=2$ has six components. From 7.3 .1 some differences between this case and $\left(A_{5}, C_{3^{4}}\right)$ are clear. For $A_{4},\{g v\}_{v \in V}$ is not the complete set of conjugates of $g \in G_{1}^{\prime}$, though one can still choose conjugation by $v \in V$ or $m \in M \backslash V$. Also, there are level 0 cases where $\mathbf{m p}(\boldsymbol{g})=2$.

The same lifting invariant from Prop. 9.8 applies. At level 1 there are components that together comprise a moduli space $\mathcal{H}_{1}^{+}$, and another $\mathcal{H}_{1}^{-}$. Unlike, however, for $A_{5}$, each of these has several (three) connected (reduced, inner) components.

For $\mathcal{H}_{1}^{+}$the two H-M components have genus 1, and the 3rd, which contains near H-M reps., has genus 3. So, all these components have real points. Two distinct components of the inner classes at level 1 contain H-M reps. Further, the two orbits differ by an outer automorphism of ${ }_{2}^{1} \tilde{A}_{4}$. We don't know if the H-M rep. components are conjugate over $\mathbb{Q}$. They come from an outer automorphism (as in Thm. 3.16). So, if they are conjugate, it must be from an extension of $\mathbb{Q}$ in the Galois closures of the absolute covers in either one of these $\mathrm{H}-\mathrm{M}$ families.

For $\mathcal{H}_{1}^{-}$, there are no real points. It has two genus 0 components, with corresponding $\bar{M}_{4}$ orbits differing by an outer automorphism of ${ }_{2}^{1} \tilde{A}_{4}$ (different from that binding the two H-M components). The genus 0 components are conjugate over $\mathbb{Q}$ by the complex conjugation operator. The last component of $\mathcal{H}_{1}^{-}$has genus 3 .

Since the genus 0 components are on $\mathcal{H}_{1}^{-}$, they are obstructed: there is nothing above them at level 2. The Main Conjecture for this case (Prob. 1.1) therefore follows by assuring any component at level 2 above the genus $1 \mathrm{H}-\mathrm{M}$ components has genus at least 2. This, however, follows easily from Lem. 8.2 the situation where one of the orbits (at level $k=1$ ) has genus 1 . Use that at level 1, 2 divides all mp values, except for H-M reps. Then, use that an H-M rep. orbit contains elements with 3 dividing their $\mathbf{m p}$, so it contains non-H-M reps. above which are elements with larger mp.

Example 9.3 (Level 1 for $\left(A_{5}, \mathbf{C}_{5_{+}^{2} 5_{-}^{2}}, p=2\right)$ ). Prop. 5.11 discusses level 0 for this Modular Tower, while the level 1 components and genuses are exactly as for Ex. 9.2. Here automorphisms join the two H-M and two genus 0 orbits at level 1, generating the full group of ${ }_{2} \tilde{A}_{5}$ outer automorphisms. There are two puzzles.

(9.1a) Why are Exs. 9.3 and 9.2 so alike?

(9.1b) If the two H-M components in either case have definition field $\mathbb{Q}$ ?

9.2. Working with $\left[\mathbf{S}^{+} \mathbf{9 5}\right.$. We had $\left[\mathbf{S}^{+} \mathbf{9 5}\right.$ list each inner Nielsen class, computing the action of the $Q_{i}$ s as elements of $S_{2304}$. Let $O_{1}^{+}$and $O_{1}^{-}$be the braid orbits in $\{1, \ldots, 2304\}$. They each have cardinality 1152 . For $j=1,2$, mod out on $O_{j}$ by the action of $\mathcal{Q}^{\prime \prime}$. In each $O_{j}$ there are 288 orbits of $\mathcal{Q}^{\prime \prime}$, each of size 4. $\mathbf{S}^{+} \mathbf{9 5}$ automatically computes the action of $\bar{M}_{4}$ on $O_{j}$. This gives the branch cycles of both degree 288 covers of $\mathbb{P}_{j}^{1}$. $\mathbf{S}^{+} \mathbf{9 5}$ doesn't seem to have facility with split extensions, which meant we interpreted $G_{1}$ as embedded in $S_{n}$ for some integer 
$n$. We started with a representation of degree 192, and eventually used a degree 80 representation $T_{80}$ allowing $\mathbf{S}^{+} \mathbf{9 5}$ faster computation. In $A_{4}$ let $\alpha$ have order 3 , and let $K$ be a Klein 4-group in $\operatorname{ker}_{0} / \operatorname{ker}_{1}=M\left(A_{5}\right)$ on which $\alpha$ acts irreducibly. The group $K, \alpha$ and the centralizer of $\alpha$ generate has order 24 . Its cosets give $T_{80}$.

9.2.1. Branch cycle description on $O_{1}^{+}$. By inspection below:

(9.2a) $\gamma_{0}$ has ninety-six 3-cycles; $\gamma_{1}$, one hundred and forty-four 2-cycles; and (9.2b) $\gamma_{\infty}$, four 2-cycles, six 4-cycles, eight 12-cycles and eight 20-cycles.

From Riemann-Hurwitz, the genus $g\left(O_{1}^{+}\right)$of a cover with these branch cycles satisfies $2\left(288+g\left(O_{1}^{+}\right)-1\right)=96 \cdot 2+144+4+6 \cdot 3+8 \cdot 11+8 \cdot 19=598: g\left(O_{1}^{+}\right)=12$.

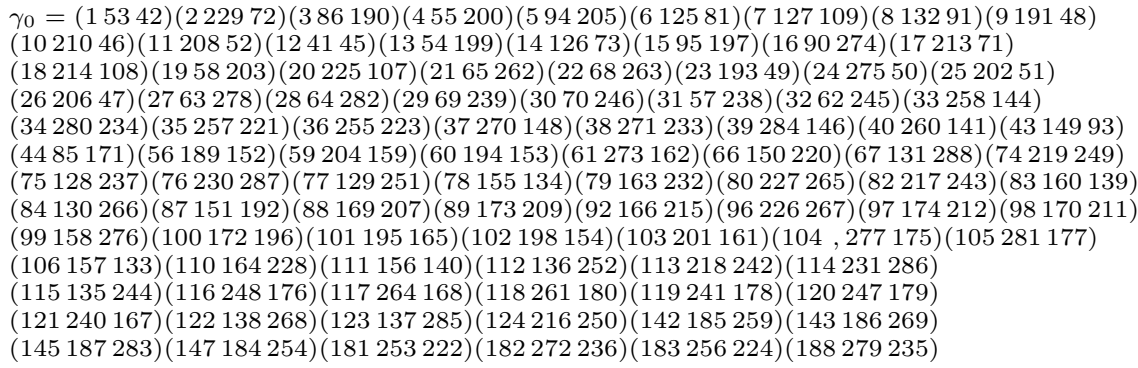

9.2.2. Branch Cycle Description on $O_{1}^{-}$. By inspection as above:

(9.3a) $\gamma_{0}$ has ninety-six 3-cycles; $\gamma_{1}$, one hundred and forty-four 2-cycles; and

(9.3b) $\gamma_{\infty}$, eight 4-cycles, eight 6-cycles, eight 10-cycles, four 12-cycles and four 20-cycles.

Riemann-Hurwitz gives $g\left(O_{1}^{-}\right): 2\left(288+g\left(O_{1}^{-}\right)-1\right)=96 \cdot 2+144+8 \cdot 3+8 \cdot 5+$ $8 \cdot 9+4 \cdot 11+4 \cdot 19=592$ or $g\left(O_{1}^{-}\right)=9$. 


\begin{abstract}
$\gamma_{0}=(112963)(2218172)(79237169)(5151142)(6131$ 150)(7 211 166)(89 216 165)(18 178 192)(9 33 143) $(10277174)(122155254)(12152187)(1340190)(14157182)(1517566)(16132146)(17177148)(37557)$ (19 196 242)(20 67 286)(21 271227$)(2264257)(23160241)(24164273)(2525288)(26158239)(2724886)$ (28 269 87)(29 287224$)(30247281)(31206135)(3291$ 139)(34 115 180)(35 94 184)(36 105 133)(37 116 179) (38 130 101)(39 236 121)(41 183 113)(42 138 108)(43 188 93)(44 255 96)(45 272 117)(46 235 207)(47 283 95) $(48280246)(49100221)(5080245)(519272)(52240124)(5381244)(5471259)(55214285)(58112278)$ (59 109 217)(60 213275$)(62176111)(68107222)(461134)(6974284)(70219260)(76171106)(78253198)$ (82 200205 )(83 210250$)(8498288)(85125270)(102156136)(90154$ 137)(97268 228)(99 229 161)(8 77 65) $(103141$ 145)(104 185 149)(110 153 181)(114 140191$)(118163262)(119276223)(120195261)(117356)$ (123251 234)(126265 225)(127 266226$)(128249282)(144202193)(147203186)(159256208)(162215258)$ (167 279 243)(168 231263$)(170220264)(173212$ 274)(189 204 194)(197 201232$)(199230238)(209267233)$
\end{abstract}

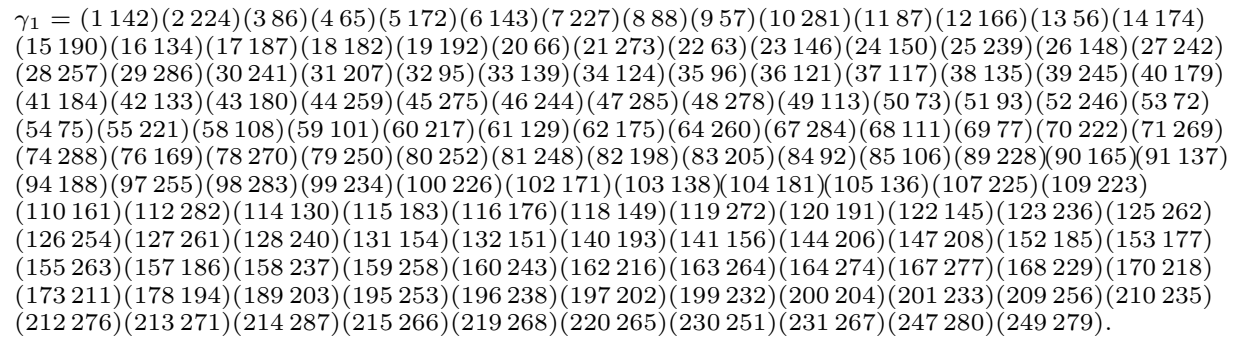

$\gamma_{\infty}=(11511661)(63391154)(154011662)(42105156103)(17152104153)(18157203194)$

(41 9443115$)(38206193114)(727160109276173)(1024748112249167)(2116421211945213)$

(99251199201267168)(106270198205250169)(28642199744 71)(2967749847214)

$(3016027912852280)(4772842017511122226022129)(9752599618411322128595139)$

(3623623416118114926285171 136)(56190662862241721426325787)

(32283845118835255228165137)(1917820482253120140202232238)

(2617711022926312214110276237)(25158792104681271962301233980)

(3123583200189147256233197144)(2 287551002661628926870107265170)

(32485392288698252501126954)(78125163220126155231209159215127195)

(52182641181851221127424131902162582081861427724323132)

(13732451211331082782461241809372244207135101217275117179)

(342402825813814525422568176372722235913019126122649183)

(571431502732271661871482398865134146241281174182192242 86).

From Lem. [7.9] there are $16 \mathrm{H}-\mathrm{M}$ representatives in $\mathrm{Ni}\left({ }_{2}^{1} \tilde{A}_{5}, \mathbf{C}_{3^{4}}\right)^{\text {in }}=\mathrm{Ni}_{1}$. 77.2 .2 shows all $\mathcal{Q}^{\prime \prime}$ orbits on $\mathrm{Ni}_{1}$ have length four.

RESUlt 9.4. $\mathbf{S}^{+} \mathbf{9 5}$ identifies the $H$-M representatives in $O_{1}^{+}$as corresponding to the integers $S_{H M}=\{41,42,43,44\}$ in the permutations $\gamma_{0}, \gamma_{1}$, and $\gamma_{\infty}$. Further, 41 and 44 lie over $((123),(132),(154),(145)) \in \mathrm{Ni}\left(A_{5}, \mathbf{C}_{3^{4}}\right)$ while 42 and 43 lie over ((123), (132),(145),(154)). Also, it also shows that $S_{n H M}$, the near H-M representatives satisfying (6.3), lie in this orbit.

9.3. Schur multipliers detect two $H_{4}$ orbits on $\mathrm{Ni}_{1}=\mathrm{Ni}\left(G_{1}, \mathbf{C}_{3^{4}}\right)$. Let $O$ be an $H_{4}$ orbit on a Nielsen class Ni. [Fri95a Part III] uses an invariant $\nu(O)$, the big invariant of $O$, a union of conjugacy classes in $\operatorname{ker}_{0}=\operatorname{ker}\left({ }_{p} \tilde{G} \rightarrow G\right)$. We adapt this for an $H_{4}$ orbit on $\mathrm{Ni}_{1}$. For $k \geq 1$ define

$$
\nu_{k}(O)=\left\{\Pi(\tilde{\boldsymbol{g}}) \mid \tilde{\boldsymbol{g}} \in G_{k+1}^{4} \cap \mathbf{C}_{3^{4}} \text { and } \tilde{\boldsymbol{g}} \bmod \operatorname{ker}_{1} \in O\right\} .
$$

We use a quotient invariant of $\nu_{1}(O)$. Prop. 9.6 with $p=2, k=1$ and $G_{1}={ }_{2}^{1} \tilde{A}_{5}$ produces $R=T_{1}^{\prime}$ with $\operatorname{ker}\left(R \rightarrow G_{1}\right)=\mathbb{Z} / 2$, and any lift of $m \in M \backslash V$ to $R$ has order 2 (Cor. 9.7).

Proposition 9.5. There are two $H_{4}$ orbits on $\mathrm{Ni}\left({ }_{2}^{1} \tilde{A}_{5}, \mathbf{C}_{3^{4}}\right)$. Denote these by $O_{i}$; use $\mathcal{H}_{O_{i}}$ for their corresponding Hurwitz space components, $i=1,2$. Then, $1 \in \nu_{1}\left(O_{1}^{+}\right)$, but $1 \neq \nu_{2}\left(O_{1}^{-}\right)$. Therefore, $\mathcal{H}_{O_{1}^{-}}$is an obstructed component, having 
nothing above it in the Modular Tower. Suppose $\boldsymbol{p} \in \mathcal{H}_{O_{1}^{+}}(K)$ is a $K$ point for any number field $K$. Then, this corresponds to a $K$ cover $\hat{\varphi}_{\boldsymbol{p}}: \hat{X}_{\boldsymbol{p}} \rightarrow \mathbb{P}_{z}^{1}$ with the following properties.

(9.4a) $\hat{\varphi}_{\boldsymbol{p}}$ is in the Nielsen class $\operatorname{Ni}\left({ }_{2}^{1} \tilde{A}_{5}, \mathbf{C}_{3^{4}}\right)$.

(9.4b) $\hat{\varphi}_{\boldsymbol{p}}$ extends to a $K$ sequence of covers $\hat{Y}_{\boldsymbol{p}} \stackrel{\hat{\psi}_{\boldsymbol{p}}}{\longrightarrow} \hat{X}_{\boldsymbol{p}} \stackrel{\hat{\varphi}_{\boldsymbol{p}}:}{\longrightarrow} \mathbb{P}_{z}^{1}$ with group $R$.

(9.4c) $\hat{\psi}_{\boldsymbol{p}}$ is unramified of degree 2.

Each $\boldsymbol{p} \in \mathcal{H}_{O_{1}^{-}}(K)$ satisfies (9.4in), but not the combination of (9.4b) and 9.4(a).

Prop. 9.8 gives the essential ingredient of the proof: The two orbits separate by the values of invariants called $s\left(O_{1}^{+}\right)$and $s\left(O_{1}^{-}\right)$, quotients of $\nu_{1}\left(O_{1}^{+}\right)$and $\nu_{1}\left(O_{1}^{-}\right)$.

9.3.1. Lifting invariants. Prop. 9.6 generalizes [FK97 §4]. Assume $G=G_{0}$ is $p$-perfect and centerless with $G_{k}$ the $k$ th characteristic quotient of ${ }_{p} \tilde{G}$. Let $\psi_{k}: R_{k} \rightarrow G_{k}$ be the universal exponent $p$ central extension of $G_{k}$; this exists from Def. 3.18 Write $R_{k}$ as ${ }_{p} \tilde{G} / \operatorname{ker}_{k}^{*}$, and the closure of $\left(\operatorname{ker}_{k}, \operatorname{ker}_{k}^{*}\right)\left(\operatorname{ker}_{k}^{*}\right)^{p}$ in $\operatorname{ker}_{k+1}$ as $\operatorname{ker}_{k+1}^{\prime}$. The canonical morphism $\varphi_{k+1, k}: G_{k+1} \rightarrow G_{k}$ factors through $\psi_{k}$.

Proposition 9.6. Denote $G_{k+1} / k^{\prime} r_{k+1}^{\prime}$ by $R_{k+1}^{\prime}$. Then, $G_{k+1}$ acts trivially on $\operatorname{ker}_{k+1} / \operatorname{ker}_{k+1}^{\prime}$. For $k \geq 0$, the $\mathbb{Z} / p$ module map of $\operatorname{ker}_{k} / \operatorname{ker}_{k}^{*}$ to $\operatorname{ker}_{k+1} / \operatorname{ker}_{k+1}^{\prime}$ by the pth power is injective. So, a lift of $m \in \operatorname{ker}\left(\varphi_{k+1, k}\right)$ to $\operatorname{ker}\left(R_{k+1}^{\prime} \rightarrow G_{k}\right)$ has order $p^{2}$ if and only if the image of $m$ in $\operatorname{ker}\left(R_{k} \rightarrow G_{k}\right)$ is nontrivial. This characterizes $R_{k+1}^{\prime}$ as a quotient of $R_{k+1}$.

Proof. Since $\psi_{k}$ is a Frattini cover with kernel of exponent $p, \varphi_{k+1, k}$ factors through $\psi_{k}$. There are two types of generators of $\operatorname{ker}_{k+1} / \operatorname{ker}_{k+1}^{\prime}:\left(v, v^{\prime}\right)$ and $v^{p}$ with $v, v^{\prime} \in \operatorname{ker}_{k}$. Let $g \in G_{k+2}$. By assumption, there are $h, h^{\prime} \in \operatorname{ker}_{k}^{*}$ with $g^{-1} v g=v h$ and $g^{-1} v^{\prime} g=v^{\prime} h^{\prime}$. From $v h=h v \bmod \operatorname{ker}_{k+1}^{\prime}$,

$$
g^{-1} v^{p} g=(v h)^{p} \equiv v^{p} h^{p} \bmod \operatorname{ker}_{k+1}^{\prime} \equiv v^{p}
$$

Similarly, $g^{-1}\left(v, v^{\prime}\right) g=\left(v h, v^{\prime} h^{\prime}\right) \equiv\left(v, v^{\prime}\right)\left(h, h^{\prime}\right) \bmod _{\operatorname{ker}_{k+1}^{\prime}} \equiv\left(v, v^{\prime}\right)$. Conclude: $G_{k+1}$ acts trivially on $\operatorname{ker}_{k+1} / \operatorname{ker}_{k+1}^{\prime}$.

Finally, suppose $m \in$ ker $_{k}$. Since $\operatorname{ker}_{k}$ is a pro-free, pro- $p$ group, $m^{p}$ mod $\operatorname{ker}_{k+1}^{\prime}$ is nontrivial if and only if $m$ is not in $\operatorname{ker}_{k}^{*}$. This translates the last sentence.

9.4 combines the next corollary with the computation of Prop. 5.10 Prop.9.6 with $p=2, k=0$ and $G_{1}={ }_{2}^{1} \tilde{A}_{5}$ produces $T_{0}^{\prime} \stackrel{\text { def }}{=} R_{0}^{\prime}$. In this case $T_{0}^{\prime} \rightarrow G_{0}=A_{5}$ is the spin cover of $A_{5}$. Use Prop. 5.10 to inductively define a quotient $T_{k}^{\prime}$ of $R_{k}^{\prime}$, $k=1,2, \ldots$ so that $\operatorname{ker}\left(T_{k}^{\prime} \rightarrow G_{k}\right)=\mathbb{Z} / 2$; all lifts of the nontrivial element of $\operatorname{ker}\left(T_{0}^{\prime} \rightarrow G_{0}\right)$ to $T_{k}^{\prime}$ have order $2^{k}$. Let $T_{1}^{\prime}=R$, so $\operatorname{ker}\left(R \rightarrow G_{1}\right)=\mathbb{Z} / 2$. As previously, $M\left(A_{5}\right)=M=\operatorname{ker}\left(G_{1} \rightarrow G_{0}\right)$ and $\hat{M}$ is the pullback of $M$ to $R$.

Corollary 9.7. Let $R_{1} \rightarrow G_{1}$ be the representation cover of $G_{1}$ and $U_{1}=$ $\operatorname{ker}\left(R_{1} \rightarrow G_{1}\right)$. Then, $U_{1}$ is a natural $G_{1}$ (acting trivially) quotient module of $\operatorname{ker}_{1} / \operatorname{ker}_{2}$. With $s_{1}(O)$ the image of $\nu_{1}(O)$ in $U_{1}, s_{1}(O)$ is a single element.

Let $\hat{a}_{i}$ be a lift of $a_{i} \in M \backslash V$ to $\hat{M}$ where $a_{i}^{g_{i}} \neq a_{i}, i=1,2$ and $a_{1} \neq a_{2}$. Then, each of $H_{a_{i}}=\left\langle\hat{a}_{i}^{g_{i}^{-1}}, \hat{a}_{i}\right\rangle, i=1,2$, and $H_{a_{1}, a_{2}}=\left\langle\hat{a}_{1}, \hat{a}_{2}\right\rangle$ is isomorphic to $\mathbb{Z} / 4 \times \mathbb{Z} / 2$.

Proof. Since $R_{1}$ is a Frattini cover of $G_{1}$ it is a quotient of ${ }_{2} \tilde{A}_{5}$. This defines $U_{1}$ as the maximal quotient of $\operatorname{ker}_{1} / \operatorname{ker}_{2}$ on which $G_{1}$ acts trivially. So, there is Frattini cover $\psi^{*}: G^{*} \rightarrow G_{1}$, a central extension of $G_{1}$ with kernel $U_{1}$. Each $2^{\prime}$ element of $G_{1}$ has a unique $2^{\prime}$ lift to $G^{*}$. 
Suppose $\tilde{\boldsymbol{g}} \in G_{2}^{4} \cap \mathbf{C}_{3^{4}}$ and $\tilde{\boldsymbol{g}}=\boldsymbol{g} \bmod \mathrm{ker}_{1} \in \mathrm{Ni}_{1}$. Then, the unique lifts of the entries of $\boldsymbol{g}$ to $\left(G^{*}\right)^{4} \cap \mathbf{C}_{3^{4}}$ determine the image of $\Pi(\tilde{\boldsymbol{g}})$ in $U_{1}$. Further, this element only depends on the $H_{4}$ orbit of $\boldsymbol{g}$.

The two given generators in the groups $H_{a_{i}}, i=1,2$, or $H_{a_{1}, a_{2}}$ have product of order 2 . The resulting groups have order 8 , the Klein 4 -group as a quotient and a pair of generators with one of order 4 and one of order 2 . Only the group $\mathbb{Z} / 4 \times \mathbb{Z} / 2$ has these properties: elements of order 4 don't generate the dihedral group, and the quaternion group's only element of order 2 is in its center.

9.3.2. Identifying lifting invariants from $G_{1}$ to $R$. The next proposition gives the key invariant separating the two orbits $O_{1}^{+}$and $O_{1}^{-}$. Together with the shincidence matrix of 8.5 it explains precisely the two $\bar{M}_{4}$ orbits on the reduced Nielsen class $\mathrm{Ni}\left(G_{1}, \mathbf{C}_{3^{4}}\right)^{\text {in,rd }}$.

Proposition 9.8. Let $\left(g_{1}, g_{1}^{-1}, g_{2}, g_{2}^{-1}\right)$ be an $H$ - $M$ representative. Consider

$$
\left(g_{1}, a g_{1}^{-1} a, b g_{2} b, g_{2}^{-1}\right)=\boldsymbol{g}^{*} .
$$

With no loss, assume $a, b \in M \backslash V$ (Cor. 5.7). Let $\hat{\boldsymbol{g}}^{*}$ have as entries the unique lifts of $\boldsymbol{g}^{*}$ entries to $\left(R, \mathbf{C}_{3^{4}}\right)$. Then, $\Pi\left(\hat{\boldsymbol{g}}^{*}\right)=s\left(\boldsymbol{g}^{*}\right)$ equals $\hat{a}^{g_{1}^{-1}} \hat{a} \hat{b} \hat{b}^{g_{2}^{-1}}$. With no loss, take $\boldsymbol{g}^{*}$ as a representative of a given $\bar{M}_{4}$ orbit $O$. Then, if $O=O_{1}^{+}$:

(9.5) $s\left(\boldsymbol{g}^{*}\right)=\hat{a}^{g_{1}^{-1}} \hat{a} \hat{b}^{g_{2}^{-1}}=+1 ;$ and $(a, b) \in M_{3}^{\prime} \times M_{3}^{\prime}$ or $M_{5}^{\prime} \times M_{5}^{\prime}$.

The corresponding statement for $\boldsymbol{g}^{*} \in O_{1}^{-}$is

(9.6) $\hat{a}^{g_{1}^{-1}} \hat{a} \hat{b} \hat{b}^{g_{2}^{-1}}=-1$, with $(a, b) \in M_{3}^{\prime} \times M_{5}^{\prime}$ or $M_{5}^{\prime} \times M_{3}^{\prime}$. $A \bar{M}_{4}$ orbit satisfying either (9.5) or (9.6) contains 8 reduced classes of such $g^{*} s$.

Proof. If $g \in G_{1}$ has order 3 , and $a \in M$, both $\hat{a} \hat{g} \hat{a}$ and $\widehat{a g a}$ are order 3 lifts to $R$ of aga. So, they are equal. The first computation is almost trivial:

$$
\hat{\boldsymbol{g}}^{*}=\left(\hat{g}_{1}, \hat{g}_{1}^{-1} \hat{a}^{g_{1}^{-1}} \hat{a}, \hat{b} \hat{b}^{g_{2}^{-1}} \hat{g}_{2}, \hat{g}_{2}^{-1}\right) .
$$

The lifting invariant is a braid invariant. The formulas of (9.5) and (9.6) come to computing $\hat{a}_{1}^{g_{1}^{-1}} \hat{a} \hat{b}^{g_{2}^{-1}} \hat{b}$ when without the hats it is 1 .

Each element in the pullback $\hat{V}$ of $V$ in $R$ has order 2. Therefore, $\hat{V}$ is an elementary 2-group and a $G_{1}$ module. Suppose $g \in G_{1}$ has order 3 . For $v \in V$, $\hat{v}^{g} \hat{v}$ is the trivial element if and only if $v=0$. So, as $v \in V$ varies, $\hat{v}^{g} \hat{v}$ (or $\hat{v}^{g^{-1}} \hat{v}$ ) runs over an $\mathbb{F}_{2}\left[G_{1}\right]$ module $V_{g}=V_{g^{-1}}$ of $R$ mapping to $V$ by the canonical map $\varphi_{1}: R \rightarrow G_{1}$. Further, $m \mapsto \hat{m}^{g} \hat{m}$ has the same image as $v$, where $m$ and $v$ differ by the element generating the centralizer of $g$. In particular, $M$ also maps to $V_{g}$ under this map.

Now suppose $V_{g_{1}} \neq V_{g_{2}}$. Then, there are $m_{1}, m_{2} \in M$ with $\hat{m}_{1}^{g_{1}^{-1}} \hat{m}_{1} \neq \hat{m}_{2}^{g_{2}^{-1}} \hat{m}_{2}$ having the same image in $V$. Conclude: There exists $\boldsymbol{g} \in \mathrm{Ni}_{1}$ with $s(\boldsymbol{g})=-1$ if and only if $V_{g_{1}} \neq V_{g_{2}}$.

If, however, $V_{g_{1}}=V_{g_{2}}=\tilde{V}$, then $\tilde{V}$ is a proper $\mathbb{F}_{2}\left[G_{1}\right]$ module of $\hat{V}$ mapping surjectively to $V$. So, as a $\mathbb{F}_{2}\left[G_{1}\right]$ module, $\hat{V}$ is the direct sum of $\tilde{V}$ and $\mathbf{1}$. Consider $\hat{M}=\varphi_{1}^{-1}(M) \leq R$. Cor. 9.7 shows this is a $\mathbb{Z} / 4\left[G_{1}\right]$ module. Then, $\hat{M} / \tilde{V}$ is a $\mathbb{Z} / 4\left[G_{1}\right]$ module of order 4 . Thus, it is either $\mathbb{Z} / 4$ or $\mathbb{F}_{2} \oplus \mathbb{F}_{2}=C_{1} \oplus C_{2}$.

Suppose $\hat{M} / \tilde{V}=\mathbb{Z} / 4$, and $G_{1}$ acts nontrivially here. This would force $G_{1}$ to have a nontrivial $\mathbb{Z} / 2$ quotient, a contradiction to $G_{1}$ being 2 -perfect. Now assume $\hat{M} / \tilde{V}=\mathbb{Z} / 4$ and $G_{1}$ acts trivially. Then, $\hat{M} / \tilde{V} \rightarrow R / \tilde{V} \rightarrow A_{5}$ is a central Frattini cover with kernel $\mathbb{Z} / 4$. Exclude this because the Schur multiplier of $A_{5}$ is $\mathbb{Z} / 2$. 
So $\hat{M} / \tilde{V}=C_{1} \oplus C_{2}$ holds. We may choose $C_{1}$ to map trivially and $C_{2}$ to map nontrivially by $\varphi_{1}$ to $M / V$. Thus, as an abelian group we can can write $\hat{M}$ as $C_{1} \oplus\left\langle C_{2}, \tilde{V}\right\rangle$. Since $\left\langle C_{2}, \tilde{V}\right\rangle / C_{1}$ maps surjectively to $M$ and has the same order, It is isomorphic to $M$. Contrary to how we formed $R$, each element in $\hat{M}$ would have order 2. This concludes showing $V_{g_{1}} \neq V_{g_{2}}$ : Both $\bar{M}_{4}$ orbits are nonempty.

We show there are eight values of $(a, b)$ with $\hat{a}^{g_{1}^{-1}} \hat{a} \hat{b}^{g_{2}^{-1}} \hat{b}=+1$ (resp. -1). Consider pairs $\left(\hat{v}_{1}, \hat{v}_{2}\right) \in V_{g_{1}} \times V_{g_{2}}$ with $\hat{v}_{1} \hat{v}_{2}$ over the trivial element in $V$. These form an $\mathbb{F}_{2}$ module $\hat{W}$ consisting of the two cosets of the submodule $\hat{U}$ where $\hat{v}_{1} \hat{v}_{2}=+1$. So, each of the two invariants contribute eight elements.

Now we show the distinguishing conditions of (9.5) and (9.6) hold.

(9.7a) If $\boldsymbol{g}^{*}=\left(g_{1}, a g_{1}^{-1} a, b g_{2} b, g_{2}^{-1}\right)$ is in an H-M rep. orbit, then

$$
(a, b) \in M_{3}^{\prime} \times M_{3}^{\prime} \cup M_{5}^{\prime} \times M_{5}^{\prime} \text {, so } s\left(\boldsymbol{g}^{*}\right)=+1 \text {. }
$$

(9.7b) Otherwise $(a, b) \in M_{3}^{\prime} \times M_{5}^{\prime} \cup M_{5}^{\prime} \times M_{3}^{\prime}$ and $s\left(\boldsymbol{g}^{*}\right)=-1$.

Toward the former, consider $(a, b)$ when $\boldsymbol{g}^{*}$ is an H-M, a near H-M rep. or a complement of such. Let $c_{i} \in M \backslash V$ centralize $g_{i}, i=1,2$. Then, $(a, b)=\left(c_{1}, c_{2}\right) \in$ $M_{3}^{\prime} \times M_{3}^{\prime}$. Let $d \in M \backslash\{0\}$ centralize $g_{1}^{-1} g_{2}$. For the complement $\boldsymbol{g}^{\prime}$ of $\boldsymbol{g}^{*}$, $\left(a^{\prime}, b^{\prime}\right)=(d, d) \in M_{5}^{\prime} \times M_{5}^{\prime}$.

Next, suppose $\boldsymbol{g}^{*}=\left(g_{1}, c^{g_{2}^{-1}} g_{1}^{-1} c^{g_{2}^{-1}}, c g_{2} c, g_{2}^{-1}\right)$ where $c \in M \backslash\{0\}$ centralizes $g_{1} g_{2}$. As in Prop. 7.11 this is a near H-M rep. Here $(a, b)=\left(c^{g_{2}^{-1}}, c\right) \in M_{5}^{\prime} \times M_{5}^{\prime}$. Let $\left(a^{\prime}, b^{\prime}\right)=\left(d c_{2}^{g_{2}^{-1}} c_{1}, d c c_{2}\right)$ be the $(a, b)$ for the complement $\boldsymbol{g}^{\prime}$ of $\boldsymbol{g}^{*}$. To show

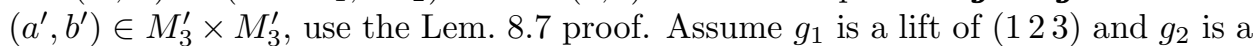
lift of $(145)$. As usual write $M$ as $(\mathbb{Z} / 2)^{6} /\langle(1,1,1,1,1,1)\rangle$ : $g_{1}$ (resp. $\left.g_{2}\right)$ permutes the coordinates as $\beta_{g_{1}}=(135)(246)$ (resp. $\beta_{g_{2}}=(132)(456)$ ).

Since $\beta_{g_{1}} \beta_{g_{2}}=(12536)$ (resp. $\left.\beta_{g_{1}^{-1}} \beta_{g_{2}}=(16524)\right), c=(0,0,0,1,0,0)$ and $d=(0,0,1,0,0,0)$. Also, $c_{1}=(1,0,1,0,1,0)$ and $c_{2}=(1,1,1,0,0,0)$. Then, $\left(d c^{g_{2}^{-1}} c_{1}, d c c_{2}\right)=((1,0,0,0,1,1),(1,1,0,1,0,0)) \in M_{3}^{\prime} \times M_{3}^{\prime}$ and $\left(a, a^{g_{1}^{-1}}, b, b^{g_{2}^{-1}}\right)$ is

$$
((1,0,0,0,1,1),(0,0,1,1,1,0),(1,1,0,1,0,0),(0,1,1,0,0,1)) .
$$

Let $U^{*} \leq V$ be the subgroup mapping isomorphically to $\hat{U}$ by the action of $\left(u_{1}, u_{2}\right) \in U^{*} \mapsto\left(\hat{u}_{1} \hat{u}_{1}^{g_{1}}, \hat{u}_{2} \hat{u}_{2}^{g_{2}}\right)$. To simplify exponents replace $u_{i}$ by $u_{i}^{g_{i}}$. Similarly, form $W^{*}$. The above shows that for all $\left(\hat{u}_{1}, \hat{u}_{2}\right) \in \hat{U}, c_{1} u_{1}$ and $c_{2} u_{2}$ are in the same conjugacy class of $M \backslash V$. To finish it suffices that the remaining eight pairs, with $\left(w_{1}, w_{2}\right) \in W^{*} \backslash U^{*},\left(c_{1} w_{1}, c_{2} w_{2}\right)$ are in different conjugacy classes in $M \backslash V$.

Do an explicit calculation using $\beta_{g_{1}}$ and $\beta_{g_{2}}, c_{1}$ and $c_{2}$. Elements in $V$ :

$$
\begin{array}{llll}
1_{v}=(1,1,0,0,0,0), & 2_{v}=(1,0,1,0,0,0), & 3_{v}=(1,0,0,1,0,0), & 4_{v}=(1,0,0,0,1,0), \\
5_{v}=(1,0,0,0,0,1), & 6_{v}=(0,1,1,0,0,0), & 7_{v}=(0,1,0,1,0,0), & 8_{v}=(0,1,0,0,1,0), \\
9_{v}=(0,1,0,0,0,1), & 10_{v}=(0,0,1,1,0,0), & 11_{v}=(0,0,1,0,1,0), & 12_{v}=(0,0,1,0,0,1), \\
13_{v}=(0,0,0,1,1,0), & 14_{v}=(0,0,0,1,0,1), & 15_{v}=(0,0,0,0,1,1) . &
\end{array}
$$

Apply $1+\beta_{g_{1}}$ (resp. $1+\beta_{g_{2}}$ ) to this list, to get two new lists $L_{i}, i=1,2$. For example, the 6 th item in $L_{1}$ (resp. the 10th item in List $\left.L_{2}\right)$ is $6_{v}+\left(6_{v}\right) \beta_{g_{1}}=$ $(1,0,0,0,0,1)=5_{v}\left(\operatorname{resp} .10_{v}+\left(10_{v}\right) \beta_{g_{2}}=(1,0,0,0,1)=5_{v}\right)$.

Treat these two lists as giving a permutation $P_{v}$ of $1_{v}, \ldots, 16_{v}$, where $1+\beta_{g_{i}}$ fixes $16_{v}=(0,0,0,0,0,0), i=1,2$. The rules for $P_{v}:\left(i_{v}\right) P_{v}=j_{v}$ if the ith element of $L_{1}$ equals the $j$ th element of $L_{2}$. The result:

$$
P_{v}=\left(16_{v}\right)\left(1_{v} 14_{v} 4_{v} 9_{v} 13_{v}\right)\left(2_{v} 12_{v} 11_{v} 6_{v} 10_{v}\right)\left(3_{v} 5_{v} 15_{v} 8_{v} 7_{v}\right) .
$$


The final step is to check the collection of pairs $\left(c_{1} i_{v}, c_{2} j_{v}\right) \in M \backslash V \times M \backslash V$ where $\left(i_{v}\right) P_{v}=j_{v}$. Eight of these pairs are in $M_{3}^{\prime} \times M_{5}^{\prime}$ or in $M_{5}^{\prime} \times M_{3}^{\prime}$. Examples: $\left(c_{1} 6_{v}, c_{2} 10_{v}\right) \in M_{3}^{\prime} \times M_{3}^{\prime}$ while $\left(c_{1} 5_{v}, c_{2} 15_{v}\right) \in M_{3}^{\prime} \times M_{5}^{\prime}$.

Proposition 9.9. There are two $\bar{M}_{4}$ orbits on $\mathrm{Ni}_{1}=\mathrm{Ni}\left(G_{1}, \mathbf{C}_{3^{4}}\right)^{\mathrm{in}, \mathrm{rd}}$. These correspond to two components $\mathcal{H}_{1}^{+}$and $\mathcal{H}_{1}^{-}$of $\mathcal{H}\left(G_{1}, \mathbf{C}_{3^{4}}\right)^{\mathrm{in}, \mathrm{rd}}=\mathcal{H}_{1}$, and in turn to the two values achieved by $s(\boldsymbol{g})$ as $\boldsymbol{g}$ runs over $\mathrm{Ni}_{1}$. Points on the genus 12 component $\mathcal{H}_{1}^{+}$have branch cycle descriptions $\boldsymbol{g}$ for which $s(\boldsymbol{g})=1$ (Prop. 9.5).

9.4. Spin separation and two $\bar{M}_{4}$ orbits on $\mathrm{Ni}_{1}^{\text {rd }}$. Alternating groups come with a standard faithful permutation representation. Some characteristic Frattini covers have faithful (less obvious) permutation representations. This subsection has our main illustration of spin separation (Def. 9.11). It is that the lifting invariant $s(O)$ for an $H_{4}$ orbit $O$ on $\mathrm{Ni}\left(G_{1}, \mathbf{C}_{3^{4}}\right)$ in (from $R \rightarrow G_{1}$ ) comes from the spin cover of $A_{40}$ (or of either $A_{60}$ or $A_{120}$ ).

9.4.1. Embedding groups in alternating groups. Suppose $k \geq 4$ and $T: G \rightarrow S_{k}$ is a permutation representation of a finite group $G$ of degree $k$. Assume the image of $G$ is in $A_{k}$, and let $\hat{A}_{k}$ be the central nonsplit extension of $A_{k}$ with kernel $\mathbb{Z} / 2$. Finally, let ${ }^{T} \hat{G}$ be the fiber product of $T$ and $\hat{A}_{k} \rightarrow A_{k}$.

Lemma 9.10. If elements of odd order generate $G$, then any permutation representation $T: G \rightarrow S_{k}$ has range in $A_{k}$. Also, ${ }^{T} \hat{G} \rightarrow G$ is nonsplit if some $g \in G$ of order 2 has a lift of order 4 in $^{T} \hat{G}$ (Prop. 5.10 applies; involution lifting property).

Proof. Elements of odd order in $S_{k}$ have a presentation as a product of an even number of disjoint 2-cycles, so they are always in $A_{k}$. If ${ }^{T} \hat{G}$ is split, then any element $g \in G$ has a lift $\hat{g} \in{ }^{T} \hat{G}$ having the same order. Since ${ }^{T} \hat{G} \rightarrow G$ is a central extension with order 2 kernel, the order of a lift of an element of even order is independent of the lift. This concludes the proof.

Let $G$ be a finite group, with $\mathbf{1}_{p}$ denoting the trivial $G$ module of dimension 1 over $\mathbb{Z} / p$. Consider $\alpha \in H^{2}\left(G, \mathbf{1}_{p}\right)$. This always defines a central extension of $G$, and it does so canonically if $G$ is $p$-perfect (Def. 3.18 and the following comments).

Definition 9.11. Suppose $p=2$. Call a faithful $T: G \rightarrow A_{k}$ an $\alpha$-spin separating representation if ${ }^{T} \hat{G}$ realizes $\alpha$.

When reference to $\alpha$ is clear refer to the representation as spin separating. In our application to $G_{1}$ the extension $\alpha$ is from Prop. 9.12 spin separating representations of $G_{1}$ interpret that ${ }^{T} \hat{G}_{1}$ is $R$. Even if each involution of $G$ lifts to an involution of ${ }^{T} \hat{G}$ (as in Lem. 9.10), ${ }^{T} \hat{G} \rightarrow G$ may still not split (9.4.5).

9.4.2. Embedding $G_{1}$ in alternating groups. Use the notation of Cor. 9.7

Proposition 9.12. For $T: G_{1} \rightarrow S_{k}$, a faithful permutation representation, $T\left(G_{1}\right) \leq A_{k}$. If a lift of $m \in M \backslash V$ to ${ }^{T} \hat{G}_{1}$ has order 4, then the following hold.

(9.8a) Each $m \in M \backslash V$ lifts to have order 4.

(9.8b) Each $v \in V$ lifts to have order 2.

(9.8c) ${ }^{T} \hat{G}_{1} \rightarrow G_{1}$ is the extension $R \rightarrow G_{1}$.

Therefore, with $a, b \in M \backslash V$, compute the lifting invariant $s(\boldsymbol{g})$ of the $\bar{M}_{4}$ orbit of

$$
\boldsymbol{g}=\left(g_{1}, a g_{1}^{-1} a, b g_{2} b, g_{2}^{-1}\right) \in \mathrm{Ni}\left(G_{1}, \mathbf{C}_{3^{4}}\right)
$$

(from Prop. 9.8) as ${ }^{T} \hat{a}^{g_{1}^{-1} T} \hat{a}^{T} \hat{b}^{T} \hat{b}^{g_{2}}$. 
Proof. Apply Lem. 9.10 Elements of order 3 generate $G_{1}$. Now suppose $m^{\prime} \in M \backslash V$ lifts to an element of order 4 . Then, the nonsplit central extension ${ }^{T} \hat{G}_{1}$ must be the quotient of the universal central extension of the perfect group $G_{1}$ that characterizes orders of lifts from $M$ in Prop. 9.6. The criterion for this is that $T\left(m^{\prime}\right)$ is a product of $2 l^{\prime}$ disjoint 2-cycles with $l^{\prime}$ odd (Prop. 5.10).

9.4.3. Spin separating representations of $G_{1}$. Consider $M=\operatorname{ker}\left(G_{1} \rightarrow A_{5}\right)$ as an $A_{5}$ module from Prop. [5.6. Let $H \leq G_{1}$, and denote the corresponding (right) coset representation by $T=T_{H}: G_{1} \rightarrow A_{k}$ with $k=\left(G_{1}: H\right)$. We list faithful spin separating representations $T_{H}$. This is equivalent to $m^{\prime} \in M \backslash V$ lifts to order 4 elements of ${ }^{T} \hat{G}_{1}$.

Prop. 5.10 characterizes this with two properties.

(9.9a) $H$ contains no nontrivial subgroup normal in $G_{1}$.

(9.9b) $m^{\prime}$ acting on $H$ cosets has $t$ fixed points with $k-t=4 l^{\prime}$ and $l^{\prime}$ odd.

A faithful representation of $G_{1}$ is not compatible (as in Def. 4.1) with the standard degree 5 representation on $A_{5}$. Reason: Any lift of $A_{4}$ (which contains a 2Sylow of $A_{5}$ ) contains a 2-Sylow of $G_{1}$. So, it does not give a faithful representation. Denote a 2 -Sylow of $G_{1}$ (resp. $\left.A_{5}\right)$ by $P_{1}$ (resp. $P_{0}$ ). Notation for the centralizer in $H$ of the set $S$ is $Z_{H}(S)$.

Lemma 9.13. Let $T: G \rightarrow S_{n}$ be any permutation representation with $H=H_{T}$ defining the cosets. For $h \in H$, denote by $U_{h} \leq G$ the subset of $G$ (not necessarily subgroup) whose inverses conjugate $h$ into $H$. Since multiplication by $H$ on the left maps $U_{h}$ into $U_{h}, U_{h}$ consists of right cosets of $H$. Use $\left(U_{h}: H\right)$ as the number of these. Then $T(h)$ fixes $\mathrm{t}(T(h))=\left(U_{h}: H\right)$ integers. Denote the orbit of $h$ under $U_{h}$ by $O$. Let $O_{1}, \ldots, O_{u}$ be the orbits of $H$ on $O$. Choose $h_{i} \in O_{i}, i=1, \ldots, u$. Then, $\mathrm{t}(T(h))=\sum_{i=1}^{u}\left(Z_{G}\left(h_{i}\right): Z_{H}\left(h_{i}\right)\right)$.

Suppose $G=G_{1}$ and $T_{H}$ is spin separating. Then, some $m^{\prime} \in M \backslash V$ is in $H$. Further, the 2-Sylow of $H$ has order at least $2^{4}$ and at most $2^{5}$.

Proof. If $H g h=H g$, then $g h(g)^{-1} \in H$, and $g \in U_{h}$. From our previous notation $g^{-1}$ conjugates $h$ into $H$. Right cosets of $H$ in $U_{h}$ are the $H$ cosets that $h$ fixes. Suppose $g_{1}^{-1}$ conjugates $h$ to $h_{i} \in O_{i}$. Running over $H g_{1}$ gives elements whose inverses conjugate $h$ into $O_{i}$. If $g_{2}^{-1}$ conjugates $h$ to $h_{i}$, then $s g_{1}=g_{2}$ with $s \in Z_{G_{1}}\left(h_{i}\right)$. So, the cosets with representatives conjugating $h$ to $h_{i}$ are in one-one correspondence with $Z_{G_{1}}\left(h_{i}\right) / Z_{H}\left(h_{i}\right)$.

The test for spin separation does not depend the choice of $m^{\prime} \in M \backslash V$. If $H$ contains no $m^{\prime} \in M \backslash V$, then $V$ contains the 2-Sylow of $H$ and $m^{\prime}$ fixes no cosets: $t=0$. This implies $k=4 l$ with $l$ odd. Also, the 2-Sylow of $H$ can't contain $V$, or else the representation won't be faithful. So the 2-Sylow $U$ of $H$ has order at most 8. This implies $k=\left(G_{1}: H\right)$ is divisible by $2^{4}$, a contradiction to (9.9b).

Now assume the 2-Sylow of $H$ has order at most 8 . As above, let $t$ be the number of cosets $m^{\prime} \in H \cap M \backslash V$ fixes. Then, $2^{4} \mid k$ and $k-4 l=t$ with $l$ odd, implies $t=4 t^{\prime}$ with $t^{\prime}$ odd. The centralizer of $m^{\prime}$ has a 2-Sylow of order $2^{6}$, and this centralizer is in $U_{m^{\prime}}$. This implies $8 \mid t$, a contradiction.

Now suppose the 2-Sylow $U$ of $H$ has order at least $2^{6}$. Assume first that $U$ surjects onto a 2-Sylow $P_{0}$ of $A_{5}$. Then $U$ is the whole 2-Sylow $P_{1}$ of $G_{1}$, for $P_{1} \rightarrow P_{0}$ is a Frattini cover. If, however, $U$ does not surject onto a 2-Sylow of $A_{5}$, then the kernel of the map has order $2^{5}$. So, $U$ contains $V$, and $T_{H}$ is not faithful. Conclude: $|U| \leq 2^{5}$. 
9.4.4. Degrees of spin separating representations of $G_{1}$. Prop. 9.14 lists properties of the spin separating representations of $G_{1}$ of degree 120. Prop. 9.15] does the same for the degree 60 and 40 representations.

Proposition 9.14. Let $\alpha^{\prime} \in P_{1}$ have order 4. Its image $\alpha \in A_{5}$ and $\left(\alpha^{\prime}\right)^{2}$ determine $\alpha^{\prime}$. Given $\alpha$, possible $\left(\alpha^{\prime}\right)^{2}$ are elements of $M\left(A_{5}\right) \backslash V$ fixed by $\alpha$.

Let $K \leq V$ be a Klein 4-group on which $\alpha^{\prime}$ acts. With $H_{\alpha^{\prime}, K}=H=\left\langle\alpha^{\prime}, K\right\rangle$, $T_{H}$ spin separates if and only if $\left|M_{3}^{\prime} \cap H\right|$ is odd: $\alpha^{\prime}$ is nontrivial on $K$.

If $\left|M_{3}^{\prime} \cap H\right|=1$ (resp. $\left|M_{5}^{\prime} \cap H\right|=1$ ), then $\left|M_{5}^{\prime} \cap H\right|=3$ (resp. $\left|M_{3}^{\prime} \cap H\right|=3$ ), $|V \backslash\{1\} \cap H|=3$. Further, corresponding to the two choices:

(9.10a) $\mathrm{t}\left(T_{H}(m)\right)=12$ (resp. $\mathrm{t}\left(T_{H}(m)\right)=36$ ) if $m \in M_{3}^{\prime}$;

(9.10b) $\mathrm{t}\left(T_{H}(m)\right)=60$ (resp. $\left.\mathrm{t}\left(T_{H}(m)\right)=20\right)$ if $m \in M_{5}^{\prime}$; and

(9.10c) $\mathrm{t}\left(T_{H}(v)\right)=24$ if $v \in V \backslash\{1\}$.

Four conjugacy classes of subgroups $H \leq G_{1}$ correspond to these choices. They give all spin separating representations of degree 120.

Suppose $\left|M_{3}^{\prime} \cap H\right|=1$. Then, $T_{H}$ acts imprimitively on ten sets of integers of cardinality 12. Each set consists of the integers fixed by an element of $M_{3}^{\prime}$. This induces the degree 10 representation of $A_{5}$ on ordered pairs of distinct integers from $\{1,2,3,4,5\}$. If $g \in G_{1}$ has order 10 , then $T_{H}(g)$ is a product of twelve 5-cycles and six 10-cycles. If $g \in G_{1}$ has order 6 , then $T_{H}(g)$ is a product of four 3-cycles and eighteen 6-cycles.

Proof. Use the notation for $\alpha^{\prime} \in P_{1}$ of order 4 in the statement. To be explicit assume - up to conjugacy $-\alpha^{\prime}$ lifts $\alpha_{24}=(24)(35)$. Given one lift $\alpha_{0}$, all others have the shape $m \alpha_{0}=\alpha^{\prime}$. Map $M \rightarrow V$ by $m \mapsto m m^{\alpha_{24}}=\psi(m)$. Cor. 5.7 says the kernel of $\psi$ has rank 3, so the image $\psi(M)$ of $\psi$ is a homogeneous space for the squares of lifts of $\alpha_{24}$. Conclude: $\alpha_{0}$ and $\left(\alpha^{\prime}\right)^{2}$ determine $\alpha^{\prime}$; and $\left(\alpha^{\prime}\right)^{2}$ runs over the subset $W_{24}$ of $M\left(A_{5}\right) \backslash V$ that $\alpha_{24}$ fixes.

Suppose $H$ has order 16 and $T_{H}$ is spin separating. Then, some $\alpha^{\prime} \in H$ has order $4, m^{\prime}=\left(\alpha^{\prime}\right)^{2} \in M \backslash V$ and $\alpha^{\prime}$ stabilizes $K=V \cap H$. Write $H_{\alpha^{\prime}, K}=H=$ $\left\langle K, \alpha^{\prime}\right\rangle$. Eight divides the degree of the representation. As in Lem. 9.13] spin separation is equivalent to $t=\left|U_{m^{\prime}}\right| /|H|$ being four times an odd number.

First case: Suppose $\alpha^{\prime}$ is trivial on the Klein 4-group $K$. Cor. 5.7 says $H$ contains exactly two conjugates of $m^{\prime}$, and all of $H$ centralizes $m^{\prime}$. Let $c$ be 3 (resp. 5) if $m^{\prime} \in M_{3}^{\prime}$ (resp. $m^{\prime} \in M_{5}^{\prime}$ ). So, $m^{\prime}$ fixes

$$
t=2 \cdot\left|Z_{G_{1}}\left(m^{\prime}\right)\right| /\left|Z_{H}\left(m^{\prime}\right)\right|=2 c \cdot 2^{6} / 2^{4}
$$

integers. Therefore, 8 divides $k-t$, and $T_{H}$ is not spin separating.

Second Case: Suppose $\alpha^{\prime}$ is nontrivial on $K$. If $\left(\alpha^{\prime}\right)^{2}=m^{\prime}$ is the only element in $H_{\alpha^{\prime}, K}$ in its conjugacy class, then the computation just concluded gives

$$
\mathrm{t}\left(T_{H}\left(m^{\prime}\right)\right)=\left|Z_{G_{1}}\left(m^{\prime}\right)\right| /\left|Z_{H}\left(m^{\prime}\right)\right|=c 2^{6} / 2^{4}=4 c .
$$

So, 4 exactly divides $k-t$ and $T_{H}$ is spin separating. If three elements in $H$ are conjugate to $\left(\alpha^{\prime}\right)^{2}=m^{\prime}$, then $\mathrm{t}\left(T_{H}\left(m^{\prime}\right)\right)=12 c$. Now suppose $v \in V \backslash\{1\}$. As $\alpha^{\prime}$ is nontrivial on $K$, conjugates (under $G_{1}$ ) of $v$ in $H$ fall in two $H$ orbits. Let $v^{\prime}$ be a representative of one of these orbits. Then, $Z_{G}\left(v^{\prime}\right) / Z_{H}\left(v^{\prime}\right)=4$, so $\left(U_{v^{\prime}}: H\right)=8$.

The argument is similar for 3 conjugates of $m^{\prime}$ in $H$. Now we count subgroups $H$, up to conjugacy in $G_{1}$, giving spin separating representations of degree 120 . 
Giving $H$ is equivalent to giving pairs $W_{24}^{*}=\left\{(w, m) \in W_{24} \times M\left(A_{5}\right) \backslash V\right\}$ with $m$ subject to these conditions.

$$
m^{\alpha_{24}} \neq m \text {, and }\left|M_{5}^{\prime} \cap\left\{w, m, m^{\alpha_{24}}, w m m^{\alpha_{24}}\right\}\right| \text { is odd. }
$$

Note: $m$ and $m^{\alpha_{24}}$ are in the same conjugacy class of $M \backslash V$; this asks exactly that $w$ and $w m m^{\alpha_{24}}$ are in different conjugacy classes of $M \backslash V$.

Count elements of $W_{24}^{*}$ from the proof of Cor. [5.7. With the cosets of $M\left(A_{5}\right)$ labeled as there, $\beta_{\alpha}=(16)(35)$ represents $\alpha_{24}$. Thus, its centralizer in $M\left(A_{5}\right)$ is

$$
U=\left\langle\left(a_{1}, x_{1}, a_{2}, x_{2}, a_{2}, a_{1}\right) \mid a_{1}, a_{2}, x_{1}, x_{2} \in \mathbb{Z} / 2\right\rangle /\langle(1,1,1,1,1,1)\rangle .
$$

Calculate: $W_{24}=\left\{\left(w_{1}, 0, w_{2}, 0, w_{2}, w_{1}\right) \mid w_{1}, w_{2} \in \mathbb{Z} / 2\right\}$. Uniquely determine a representative for $w$ by taking $x_{1}=1$ and $x_{2}=0$. The elements $w$ run over all choices $a_{1}, a_{2} \in \mathbb{Z} / 2^{2}$. So, $\left|W_{24}^{*}\right|$ equals the number of pairs $\left(a_{1}, 1, a_{2}, 0, a_{2}, a_{1}\right)$ and $\left(a_{1}+w_{1}, 1, a_{2}+w_{2}, 0, a_{2}+w_{2}, a_{1}+w_{1}\right)$ with one having three nonzero entries, and the other not. Given any pair $\left(a_{1}, a_{2}\right)$, there are two choices for $\left(w_{1}, w_{2}\right)$ satisfying this condition. Conjugate the set of groups $H_{w, m}$ attached to these choices by any lift of $(23)(45) \in A_{5}$ (centralizing $\alpha_{24}$ ). The result is a distinct subgroup. Conclude there are four such subgroups, falling in pairs according to $w \in M_{3}^{\prime}$ or $w \in M_{5}^{\prime}$.

Now, suppose $\left|M_{3}^{\prime} \cap H\right|=1$. As above, for $m \in M_{3}^{\prime}, T_{H}(m)$ fixes twelve integers. Running over the ten elements of $M_{3}^{\prime}$, transitivity of the representation guarantees some element of $M_{3}^{\prime}$ fixes any given integer in $\{1,2, \ldots, 120\}$. As $\left|M_{3}^{\prime}\right|=10$, these sets of fixed integers are disjoint, and they form a set of imprimitivity. With no loss assume $m \in H$, so $H \leq Z_{G_{1}}(m)$. Elements of $Z_{G_{1}}(m)=H^{*}$ are exactly those permuting the fixed integers for $T_{H}(m)$. Acting on $H^{*}$ cosets gives the degree 10 representation of $A_{5}$ on pairs of distinct integers from $\{1,2,3,4,5\}$.

Continue the hypothesis $\left|M_{3}^{\prime} \cap H\right|=1$. Suppose $g \in G_{1}$ has order 10 . Then, $g^{2}$ has order 5 , and $T_{H}(g)$ fixes no integers. On the other hand, $g^{5} \in M_{5}^{\prime}$. From above, $T_{H}\left(g^{5}\right)$ fixes exactly sixty integers. Conclude: $T_{H}(g)$ is a product of twelve 5 -cycles and six 10-cycles. For $g \in G_{1}$ of order 6 , similarly deduce $T_{H}(g)$ is a product of four 3-cycles and eighteen 6-cycles.

The following is similar to Prop. 9.14 We leave details to the reader.

Proposition 9.15. Continue Prop. 9.14 notation (for $\alpha^{\prime}$ ). Assume $\beta \in G_{1}$ has order three, $\left\langle\beta, \alpha^{\prime}\right\rangle$ has order $3 \cdot 2^{4}$ and the image of $\left\langle\beta, \alpha^{\prime}\right\rangle$ is an $S_{3} \leq A_{5}$. Then, with $H_{\alpha^{\prime}, \beta}=H^{\prime},\left|M_{3}^{\prime} \cap H^{\prime}\right|=1$ and $T_{H^{\prime}}$ spin separates. There are two conjugacy classes of such groups $H^{\prime}$ corresponding to these choices giving all spin separating representations of degree 40. If $m, m^{\prime} \in M_{3}^{\prime}$ are distinct, the four fixed integers of $T_{H^{\prime}}(m)$ are distinct from those fixed by $T_{H^{\prime}}\left(m^{\prime}\right)$.

Let $H$ be one of the groups with $T_{H}$ of degree 120, and let $\alpha^{*} \in V \backslash H$. Then, $H^{\prime \prime}=\left\langle H, \alpha^{*}\right\rangle \cap M_{3}^{\prime}$ has 4 or 6 elements. Exactly in the former case $T_{H^{\prime \prime}}$ spin separates. There are two conjugacy classes of such groups $H^{\prime \prime}$ corresponding to these choices giving all spin separating representations of degree 60.

Comments on the PROOF. If $\left|M_{3}^{\prime} \cap H\right|=1$, as in Prop. 9.14, the conditions on $\beta$ are that it centralizes this element of $M_{3}^{\prime}$ and it acts on $V \cap H$ in the standard Klein 4-group representation. For this case, $T_{H^{\prime}}(m)=4$ (resp. 20 and 8) if $m \in M_{3}^{\prime}$ (resp. $m \in M_{5}^{\prime}$ and $m \in V$ ).

Corollary 9.16. Prop.9.14 and Prop. 9.15 include the complete list of spin separating permutation representations of the $\bar{M}_{4}$ orbits on $\operatorname{Ni}\left(G_{1}, \mathbf{C}_{3^{4}}\right)^{\mathrm{in}, \mathrm{rd}}$. 
Proof. Assume for $H \leq G_{1}, T_{H}$ gives a faithful spin separation. According to Lem. 9.13 the 2-Sylow $U$ of $H$ has order $2^{4}$ or $2^{5}$ and it contains an element $m^{\prime}$. Prop. 9.14 lists all cases where $k=\left(G_{1}: H\right)$ is 120,40 or 60 . The only degrees left as dangling possibilities are where $H$ has order divisible by 5 or where it has order $2^{5} \cdot 3$. In the former case, since $H$ contains nontrivial elements of $V$, it must contain all of $V$. So it is not faithful. Similarly for the latter case: The action of an element of order 3 on $V \cap H$ forces this to be all of $V$.

9.4.5. Splitting versus the involution lifting property. Restriction of an element from $H^{2}(G, A)$ to $H^{2}\left(P_{p}, A\right)$ is injective if $P_{p}$ is the $p$-Sylow of $G$ AW67. p. 105]. Sometimes this allows taking $G$ to be a $p$-group. We use this and produce a 2-group extension $\psi: \hat{P} \rightarrow P$ with the following properties.

(9.12a) The kernel of $\psi$ is $\mathbb{Z} / 2$.

(9.12b) $\psi$ is nonsplit and spin separating: ${ }^{T} \hat{P}=\hat{P}$, pullback from an embedding $P \leq A_{n}$ for some $n$.

(9.12c) Each involution of $P$ lifts to an involution of $\hat{P}$.

The quaternion group presentation is in Def. 2.7 $Q_{4 n}$ of order $4 n$ has generators $\tau_{1}, \tau_{2}$ with $\operatorname{ord}\left(\tau_{1}\right)=2 n$, ord $\left(\tau_{2}\right)=4, \tau_{2}^{-1} \tau_{1} \tau_{2}=\tau_{1}^{-1}$ and $\tau_{2}^{2}=\tau_{1}^{n}$. Let $\hat{Q}_{4 n}$ be the group from dropping the condition $\tau_{2}^{2}=\tau_{1}^{n}$. Then, the only involution $\tau_{2}^{2}$ in $\hat{Q}_{4 n}$ lifts to an involution in $\hat{Q}_{4 n}$. This extension, however, does not split.

The calculations below apply for $n$ even to all the groups $\hat{Q}_{4 n}$. For simplicity we do the case $n=2$ and $Q_{8}$, using $\tau_{1}=a$ and $\tau_{2}=b ; \hat{Q}_{8}$ has generators $\hat{a}$ and $\hat{b}$, with relations $\hat{a}^{4}=\hat{b}^{4}=\hat{a}^{-1} \hat{b} \hat{a} \hat{b}=1$. For $n$ even, $Q_{4 n}$ doesn't have an extension satisfying (9.12), though $\hat{Q}_{4 n}$ does. (This observation should start with the Klein 4-group whose $Q_{8}$ extension in Lem. 2.24 is an example of spin separation.)

Proposition 9.17. The extension $\hat{P}=\hat{Q}_{8} \rightarrow P=Q_{8}$ has no spin separating extension. Still, $\hat{P}$ has an extension $\hat{\hat{P}}$ satisfying all properties of (9.12).

Proof. The group $Q_{8}$ has only one faithful transitive permutation representation as a subgroup of $S_{8}$. In this representation elements of order 4 have the shape (4)(4). So these generators are in $A_{8}: Q_{8} \leq A_{8}$. This is the only embedding we must test to check if $Q_{8}$ has a spin separating representation of any kind.

Form a central extension of $Q_{8}$ by mapping $\hat{a}$ to $a$ and $\hat{b}$ to $b$. In the extension, the square of $\hat{a}$ is cleaved away from the squares $\hat{b}^{2}=(\hat{a} \hat{b})^{2}$ (they remain equal). Let $1_{2}$ be the trivial $Q_{8}$ module. Recall: $Q_{8}$ has rank $e=2$ and order $n=8$. So, the 1st characteristic 2-Frattini module $M\left(Q_{8}\right)=\operatorname{ker}_{0}\left(Q_{8}\right) / \operatorname{ker}_{1}\left(Q_{8}\right)$ (notation of \$5.2.1) of $Q_{8}$ has dimension $9=1+(e-1) n$ [FJ86 $\left.\$ 15.6\right]$ : Schreier's formula for the number of generators of a subgroup of a free group of rank $e$ and index $n$. As a $Q_{8}$ module it is indecomposable [FK97, Indecom. Lem. 2.4]. The maximal quotient of it on which $Q_{8}$ acts trivially is $H^{2}\left(Q_{8}, \mathbf{1}_{2}\right)$.

We ouline our computation that $H^{2}\left(Q_{8}, \mathbf{1}_{2}\right)$ has dimension two. Use the notation of [Fri95a Part II]. The augmentation map gives $\mathbb{F}_{2}\left[Q_{8}\right] \rightarrow \mathbf{1}_{2}$. Denote its kernel by $\Omega(1)$. The version of Jenning's Theorem (Je41] or [Ben91 p. 89]) in Qu68 gives an effective tool for computing the Loewy layers of any $p$-group ring using the Poincaré-Witt basis of its universal enveloping algebra. The Loewy layer at the top of $\Omega(1)$ (the second radical layer in $\mathbb{F}_{2}\left[Q_{8}\right]$ ) is $\mathbf{1}_{2} \oplus \mathbf{1}_{2}$. The projective module $\mathbb{F}_{2}\left[Q_{8}\right] \oplus \mathbb{F}_{2}\left[Q_{8}\right]$ maps naturally and surjectively to $\Omega(1)$ extending the direct sum of two augmentation maps $\psi: \mathbb{F}_{2}\left[Q_{8}\right] \oplus \mathbb{F}_{2}\left[Q_{8}\right] \rightarrow \mathbf{1}_{2} \oplus \mathbf{1}_{2}$. The kernel 
of this map is $\Omega(2)=M\left(Q_{8}\right)$ [Fri95a Projective Indecomposable Lem. 2.3]. The explicit basis of $\mathbb{F}_{2}\left[Q_{8}\right]$ from the group elements produces a natural interpretation of $\psi$ as a matrix. Row reduce and compute explicitly a basis of $\Omega(2)$ from that of $\mathbb{F}_{2}\left[Q_{8}\right]$. This gives $\Omega(2)$ a $Q_{8}$ module structure. Rational canonical form of the matrix action $M_{a}$ of $a$ (resp. $b$ and $M_{b}$ ) on this module determines the maximal quotient on which $M_{a}$ (resp. $M_{b}$ ) acts like the identity matrix, giving the maximal quotient on which $Q_{8}$ acts like the identity. We let GAP do this computation.

All non-split extensions of $Q_{8}$ are isomorphic as groups though not as extensions. As in 3.6.1 the isomorphisms between them come from $H^{1}(G, \mathbb{Z} / 2)$ which is isomorphic to a Klein 4-group. Extensions correspond to which order 4 subgroup of $Q_{8}$ cleaves from the other order 4 subgroups.

The proof of Prop. 5.10 reviews the generators and relations for $\hat{S}_{n} \rightarrow S_{n}$. In that notation, symbols $[i j], 1 \leq i, j \leq n$ (in the multiplicative group of units in the Clifford algebra), generate subject to the relations $[i j]^{2}=1$ and $[i j]=-[j i]$. The map $\hat{S}_{n} \rightarrow S_{n}$ appears from $[i j] \mapsto(i j)$. Finally, $[i j][j k][i j]=[k i]$.

Like $Q_{8}, \hat{Q}_{8}$ has no non-trivial coreless subgroup. So, only its regular representation is faithful and its generators $\hat{a}$ and $\hat{b}$, of order 4, have the shape (4)(4)(4)(4); they are in $A_{16}$. Involutions in $\hat{Q}_{8}$ are the products of 8 disjoint two cycles. So Prop. 5.10 implies they lift to the same order on pullback in $\operatorname{Spin}_{16}$.

Now we show $\hat{Q}_{8}$ (the pullback of $\hat{Q}_{8}$ to $\operatorname{Spin}_{16}$ ) does not split off $\hat{Q}_{8}$. If the extension splits, the lifts would retain the relation $\hat{b}^{2}=(\hat{a} \hat{b})^{2}$. So, it suffices that the square of any lift of $\hat{b}$ differs from the square of any lift of $\hat{a} \hat{b}$. With no loss:

$$
\begin{aligned}
\hat{b} & =(15913)(261014)(371115)(481216), \text { and } \\
\hat{a} \hat{b} & =(16914)(271015)(381116)(451213) .
\end{aligned}
$$

Write each 4-cycle's lift to $\operatorname{Spin}_{16}$ as the product of three obvious generators. Example: (15913) lifts to [15][59][913]. Square the lifts of $\hat{b}$ and $\hat{a} \hat{b}$ using the relations from the previous paragraph. Then multiply the two squares together to get -1 , the generator of the kernel of $\operatorname{Spin}_{16} \rightarrow A_{16}$. This also shows -1 is in the Frattini subgroup, so the extension is Frattini, and therefore nonsplit.

The same procedure for $Q_{8}$ shows the squares of the lifts of $a, b$, and $a b$ are the same. Every non-split extension of $Q_{8}$ has one of these cleaved off from the rest. This shows the spin extension of $Q_{8}$ splits.

9.5. Understanding spin separation. Representations in Prop. 9.14 give spin separation of elements in $\mathrm{Ni}_{1}=\mathrm{Ni}\left({ }_{2}^{1} \tilde{A}_{5}, \mathbf{C}_{3^{4}}\right)^{\mathrm{in}, \mathrm{rd}}$. 9.5 .1 gives the lifting invariant that separates the two $\bar{M}_{4}$ orbits on $\mathrm{Ni}_{1}$. 9.5.2 and 9.5 .3 together suggest why the two orbits have the same $\bar{M}_{4}$ monodromy groups. 9.6 concludes the paper with lessons for similar computations at higher levels.

9.5.1. Explicit lifting invariants. First we characterize commuting pairs of elements of order 2 in $S_{n}$. Denote the elements of $S_{n}$ that are of products of $u$ disjoint 2-cycles by $C_{u}=C_{u, n}$. In the situation where $g \in C_{u}$ and $g^{\prime} \in C_{u^{\prime}}$, and $g$ and $g^{\prime}$ commute, we will identify the product. The most complicated standard situation is where $\left(g, g^{\prime}\right)$ is a $K 4$-pair:

(9.13) $g=\left(i_{1} i_{2}\right)\left(i_{3} i_{4}\right), g^{\prime}=\left(i_{1} i_{3}\right)\left(i_{2} i_{4}\right)$ and $g g^{\prime}=\left(i_{1} i_{4}\right)\left(i_{2} i_{3}\right)$.

Given $\left(g, g^{\prime}\right)$ as above, let $a\left(g, g^{\prime}\right)$ be the number of disjoint 2-cycles appearing in $g$ having no support in $g^{\prime}$. Note: $a\left(g, g^{\prime}\right)$ may not equal $a\left(g^{\prime}, g\right)$. Let $b\left(g, g^{\prime}\right)$ by the disjoint 2 -cycles appearing in both $g$ and $g^{\prime}$. Suppose $\left(h, h^{\prime}\right)$ is a $K 4$-pair, 
$g_{1}$ and $h$ (resp. $g_{1}^{\prime}$ and $h^{\prime}$ ) have no common support, and $g=g_{1} h$ and $g^{\prime}=g_{1}^{\prime} h^{\prime}$ commute. We say the $K 4$-pair $\left(h, h^{\prime}\right)$ appears in $\left(g, g^{\prime}\right)$. Finally, let $c\left(g, g^{\prime}\right)$ be the number of $K 4$-pairs appearing in $\left(g, g^{\prime}\right)$.

Lemma 9.18. Suppose $g \in C_{u}$ and $g^{\prime} \in C_{u^{\prime}}$ and $g g^{\prime}=g^{\prime} g$. Then, $g g^{\prime} \in C_{u^{\prime \prime}}$ with $a\left(g, g^{\prime}\right)+a\left(g^{\prime}, g\right)+2 c\left(g, g^{\prime}\right)=u^{\prime \prime}$.

ProOF. Write $g$ as $g_{a\left(g, g^{\prime}\right)} g_{b\left(g, g^{\prime}\right)} g_{1}$ with $g_{a\left(g, g^{\prime}\right)}$ (resp. $\left.g_{b\left(g, g^{\prime}\right)}\right)$ the disjoint cycles in $g$ that don't (resp. do) appear in $g^{\prime}$. Similarly, write $g^{\prime}$ as $g_{a\left(g, g^{\prime}\right)}^{\prime} g_{b\left(g, g^{\prime}\right)}^{\prime} g_{1}^{\prime}$. Since $g$ and $g^{\prime}$ commute, so do $g_{1}$ and $g_{1}^{\prime}$. Proving the lemma amounts to extracting a $K 4$-pair $\left(h, h^{\prime}\right)$ that appears in $\left(g_{1}, g_{1}^{\prime}\right)$. Then, an induction characterizes $\left(g_{1}, g_{1}^{\prime}\right)$ as given by products of disjoint $K 4$-pairs.

Suppose $i_{1}$ appears in a disjoint cycle $\left(i_{1} i_{2}\right)$ in $g_{1}$. By assumption, $i_{1}$ appears in a disjoint cycle $\left(i_{1} i_{3}\right)$ in $g_{1}^{\prime}$. Since we have extracted $g_{b\left(g, g^{\prime}\right)}, i_{3} \neq i_{2}$. Suppose $i_{3}$ is not in a disjoint cycle of $g_{1}$. Then, $g_{1} g_{1}^{\prime}$ applied to $i_{2}$ has the effect $i_{2} \mapsto i_{3} \mapsto i_{1}$. Thus, $g_{1} g_{1}^{\prime}$ has order larger than 2. So, contrary to a previous deduction, $g_{1}$ and $g_{1}^{\prime}$ don't commute.

Therefore, $i_{3}$ appears in a disjoint cycle $\left(i_{3} i_{4}\right)$ of $g_{1}$. Calculate: $g_{1} g_{1}^{\prime}$ applied to $i_{4}$ has the effect $i_{4} \mapsto i_{3} \mapsto i_{1}$. Now compute the effect of $g_{1}^{\prime} g_{1}$ on $i_{4}$ to conclude $g_{1}^{\prime}$ contains the disjoint cycle $\left(i_{2} i_{4}\right)$. That is, $\left(h, h^{\prime}\right)=\left(i_{1} i_{2}\right)\left(i_{3} i_{4}\right),\left(i_{1} i_{3}\right)\left(i_{2} i_{4}\right)$ appears in $\left(g, g^{\prime}\right)$.

Example 9.19 (Applying Lem. 9.18). Suppose $\left|M_{3}^{\prime} \cap H^{\prime}\right|=1$, as in Prop. 9.15. Suppose $m, m^{\prime} \in M_{3}^{\prime}$ are distinct. For this case, $\mathrm{t}\left(T_{H^{\prime}}(m)\right)=4=\mathrm{t} T_{H^{\prime}}\left(m^{\prime}\right)$ and $\mathrm{t}\left(T_{H^{\prime}}\left(m m^{\prime}\right)\right)=8: m, m^{\prime} \in C_{18}$ and $m m^{\prime} \in C_{16}$. Integers not in the support of $m$ appear in 2-cycles in $m^{\prime}$, and give $a\left(m, m^{\prime}\right)=a\left(m^{\prime}, m\right)=2$. Conclude $16=4+2 c\left(m, m^{\prime}\right): c\left(m, m^{\prime}\right)=6$. Use that $a\left(m, m^{\prime}\right)+b\left(m, m^{\prime}\right)+2 c\left(m, m^{\prime}\right)=18$ to conclude $b\left(m, m^{\prime}\right)=4$.

Proposition 9.20. Let $g \in A_{5}$ have order 5, and denote by $\hat{g}$ a lift of it to an element of order 10 in $G_{1}$. With $H^{\prime}$ giving the degree 40 representation used above, let $H^{*}$ be its image (isomorphic to $S_{3}$ ) in $A_{5}$. Then, we can speak of the 20 cosets of $T_{H^{\prime}}$ lying above the cosets $H^{*} g^{j}, j=0,1,2,3,4$, of $T_{H^{*}}$. Analogous to previous calculations, $T_{H^{\prime}}(\hat{g})$ consists of four 5-cycles and two 10-cycles. The integers moved (resp. fixed) by $T_{H^{\prime}}\left(\hat{g}^{5}\right)$ are in the support of the 10-cycles (resp. 5-cycles). Suppose $\hat{g}^{5} \in H^{\prime}$. Then, multiplying $\hat{g}$ on the right of these cosets gives four 5-cycles of $T_{H^{\prime}}(\hat{g})$. Two 10-cycles comprise the remaining action of $T_{H^{\prime}}(\hat{g})$. If $\hat{g}^{5} \notin H^{\prime}$, then the description of the 5-cycles and 10-cycles of $T_{H^{\prime}}(\hat{g})$ switches. If $m, m^{\prime} \in M_{5}^{\prime}$, $m \neq m^{\prime}$, then $a\left(m, m^{\prime}\right)=6, b\left(m, m^{\prime}\right)=0, c\left(m, m^{\prime}\right)=2$.

Proof. The number of integers in the support of 2-cycles from $T_{H^{\prime}}\left(\hat{g}^{5}\right)$ is the same as in the 10-cycles of $T_{H^{\prime}}(\hat{g})$. From Prop. 9.12 this means there are twenty such integers, and therefore two 10-cycles in $T_{H^{\prime}}(\hat{g})$. Since this element fixes no integers there are also four 5 -cycles in $T_{H^{\prime}}(\hat{g})$.

Without loss, take the 20 cosets of $T_{H^{\prime}}$ above the cosets $H^{*} g^{j}, j=0,1,2,3,4$, of $T_{H^{*}}$ to be $H^{\prime} m_{i} \hat{g}^{j}$, with $m_{0}=1, m_{i} \in M, i=1,2,3$, and $j=0, \ldots, 4$. The $m_{i} \mathrm{~s}$ are four representatives of $H^{\prime} \cap M$ cosets in $M$. First assume $\hat{g}^{5} \in H^{\prime}$. For each $i, \hat{g}$ cycles $H^{\prime} m_{i} \hat{g}^{j}, j=0, \ldots, 4$ (use that $\hat{g}^{5}$ commutes with $m_{i}$ ). The argument is the same if $\hat{g}^{5} \notin H^{\prime}$ : Write the distinct cosets above as $H^{\prime} m_{i} \hat{g}^{j}$ with $m_{0}=1$, $m_{i} \in M, i=1$ and $j=0, \ldots, 9$. 
Now inspect the relation between two elements of $M_{5}^{\prime}$. There are six elements in $M_{5}^{\prime}$. An element $g \in A_{5}$ of order 5 that stabilizes $m$ is transitive on the remaining five elements of $M_{5}^{\prime}$. Since $a\left(m, m^{\prime}\right)=a\left(m^{g},\left(m^{\prime}\right)^{g}\right), b\left(m, m^{\prime}\right)=b\left(m^{g},\left(m^{\prime}\right)^{g}\right)$ and $c\left(m, m^{\prime}\right)=c\left(m^{g},\left(m^{\prime}\right)^{g}\right)$, these values are independent of their arguments. Use that $a\left(m, m^{\prime}\right)+b\left(m, m^{\prime}\right)+2 c\left(m, m^{\prime}\right)=10$ and $a\left(m, m^{\prime}\right)+a\left(m^{\prime}, m\right)+2 c\left(m, m^{\prime}\right)=16$. Thus, $a\left(m, m^{\prime}\right)-b\left(m, m^{\prime}\right)=6$, and $b\left(m, m^{\prime}\right)=2,1$ or 0 .

If $T_{H^{\prime}}(m)$ and $T_{H^{\prime}}\left(m^{\prime}\right)$ have eight common fixed integers, then they also move eight common integers. So we show the former and conclude $b\left(m, m^{\prime}\right)=0$ and $c\left(m, m^{\prime}\right)=2$. With no loss, assume $m \in H^{\prime}$ and find the cosets from among $H^{\prime} m_{i} \hat{g}^{j}$ fixed by $m^{\prime}$. Since $m^{\prime} \neq m$, as $j$ varies, $\left(m^{\prime}\right)^{\hat{g}^{-j}}$ runs over all five other elements of $M_{5}^{\prime}$. Use $\hat{g}^{j} m^{\prime}=\left(m^{\prime}\right)^{\hat{g}^{-j}} \hat{g}^{j}$ to see $m^{\prime}$ fixes exactly two of these cosets as $j$ varies, and eight cosets in all.

9.5.2. $\bar{M}_{4}$ orbit mysteries. The two $\bar{M}_{4}$ orbits on $\mathrm{Ni}\left({ }_{2}^{1} \tilde{A}_{5}, \mathbf{C}_{3^{4}}\right)^{\text {in,rd }}$ have the same image groups, and degrees. This is despite their being considerably different as representations. They are clearly permutation inequivalent representations, or else $\gamma_{\infty}$ would have the same shape on each orbit. More strikingly, however, the permutation representations are inequivalent as representations (have different traces). The number of $\gamma_{\infty}$ orbits on each of the $\bar{M}_{4}$ orbits $O_{1}^{+}$and $O_{1}^{-}$(resp. 26 and $32 \gamma_{\infty}$ orbits) is different. This shows the two representations are different (see for example [Fri99 Lem. 5.3]) despite their similarities. $\$ \mathrm{E}$ speculates on representation theory (based on GKS99) that might explain this and also measure the difference between these two representations.

9.5.3. $B_{4}$ as evaluation homomorphisms. From Lem. 2.10, the direct product of the free group $K_{4}^{*}=\left\langle\left(Q_{3} Q_{2}\right)^{3}, Q_{1}^{-2} Q_{3}^{2},\left(Q_{2} Q_{1}\right)^{-3}\right\rangle$ and $\left\langle\left(Q_{1} Q_{2} Q_{3}\right)^{4}\right\rangle$ equals $N_{4} \stackrel{\text { def }}{=} \operatorname{ker}\left(B_{4} \rightarrow M_{4}\right)$. Denote $Q_{1} Q_{2} Q_{3}^{2} Q_{2} Q_{1}(D$ in (2.12) $)$ by $R_{1}$. The following presentation of $N_{4}$ is superior for our purposes. It easily follows from the notation and proof of Lemma 2.10 Recall: The effect of $R_{i}$ on $G_{4}$ is conjugation by $\bar{\sigma}_{i}$, $i=1, \ldots, 4$. We state this only for $r=4$, though it works for any value of $r$.

Proposition 9.21. These are generators of $N_{4}$ :

$$
\boldsymbol{R}=\left(R_{1}, R_{2}=Q_{1}^{-1} R_{1} Q_{1}, R_{3}=Q_{2}^{-1} R_{2} Q_{2}, R_{4}=Q_{3}^{-1} R_{3} Q_{3}\right) \text { and }\left(Q_{1} Q_{2} Q_{3}\right)^{4} .
$$

Any representative element $\boldsymbol{g}$ in a Nielsen class $\mathrm{Ni}(G, \mathbf{C})=$ Ni produces an evaluation homomorphism $\psi_{\boldsymbol{g}}: N_{4} \rightarrow G / \operatorname{Cen}(G)$ mapping $\prod_{i=1}^{4} R_{i}$ to 1 . Conversely, any homomorphism $\psi: N_{4} \rightarrow H$ mapping $\prod_{i=1}^{4} R_{i}$ to 1 produces an associated Nielsen class representative.

For $Q \in B_{4}$, act on $\psi_{\boldsymbol{g}}$ by applying evalution of $Q^{-1} \boldsymbol{R} Q$ to $\boldsymbol{g}$. An orbit on $\mathrm{Ni}$ is equivalent to a $B_{4}$ orbit on the homomorphisms $\psi_{\boldsymbol{g}}$ (up to conjugation by $G$ ).

For the computation of orbits of $H_{4}$, consider the induced action of $N_{4}$ on $G_{4}$. Denote images of $R_{1}, \ldots, R_{4}$ by $r_{1}, \ldots, r_{4}$, so these generate a free group with one relation $r_{1} r_{2} r_{3} r_{4}=1$. An obvious reformulation of Prop. 9.21 has a rephrasing in $H_{4}$ orbits using $\bar{N}_{4}=\left\langle r_{1}, \ldots, r_{4}\right\rangle$. Use the same notation $\psi_{\boldsymbol{g}}$ for the corresponding evaluation homomorphisms. Referring to the generators $r_{1}, \ldots, r_{4}$ allows us give the Nielsen class of a homomorphism by what conjugacy classes the generators hit.

Definition 9.22. Let $\mu$ be an automorphism of $\bar{N}_{4}$. We say $\mu$ preserves a Nielsen class $\mathrm{Ni}(G, \mathbf{C})=\mathrm{Ni}$ if $\psi_{\boldsymbol{g}} \circ \mu$ is in the same Nielsen class as $\psi_{\boldsymbol{g}}$. 
Lemma 9.23. If $\mu$ preserves the Nielsen class $\mathrm{Ni}$, then it permutes $H_{4}$ orbits. Any two $\mathrm{H}_{4}$ orbits under $\mu$ have the same length. Further, the monodromy groups (permutation groups for the action of $\mathrm{H}_{4}$ ) on these orbits will be the same.

Note: While the group on two $\mu$ orbits in Lem. 9.23 are the same, the permutation representations may be inequivalent. Denote the automorphism of $\bar{N}_{4}$ by $\left(r_{1}, r_{2}, r_{3}, r_{4}\right) \mapsto\left(r_{4}^{-1}, r_{3}^{-1}, r_{2}^{-1}, r_{1}^{-1}\right)$ by $\mu_{0}$.

Example 9.24 (Cont. Ex. 9.2). Among the six $\bar{M}_{4}$ orbits on $\mathrm{Ni}\left({ }_{2} \tilde{A}_{4}, \mathbf{C}_{3^{4}}\right)^{\text {in,rd }}$ ) the automorphism $\mu_{0}$ fixes four of them, but permutes the other two.

QUESTION 9.25. Is there a Nielsen class preserving automorphism interchanging the two $H_{4}$ orbits on $\operatorname{Ni}\left({ }_{2}^{1} \tilde{A}_{5}, \mathbf{C}_{3^{4}}\right)^{\text {in,rd? }}$

9.6. Inductive steps in going to higher Modular Tower levels. Explicit formulas (8.1) and Lem. 8.2) list properties of ${ }_{p} \tilde{G}$ characteristic quotients helpful to decide Prob. 1.1 and other properties of Modular Towers when $r=4$.

9.6.1. The starting point and Schur multipliers. By example, the paper establishes it is difficult to predict component genuses at level 0 of a Modular Tower. As in all our $A_{5}$ (and $A_{4}$ ) examples, genus 0 components are common. For many applications this is excellent; rational points from such a moduli space produce Galois realizations. So, establishing that at some level in the Modular Tower all components will have large genus requires exploiting the module representation theory of the universal $p$-Frattini cover. Whatever the genus of the components at level 0 , it is properties of the universal $p$-Frattini cover that force sufficient ramification at higher levels to have the genus go up (as in (8.1)).

Lem. 8.2 exploited the hypotheses (8.2), valid in the main Modular Tower $\left(A_{5}, \mathbf{C}_{3^{4}}, p=2\right)$. A more general hypothesis, with $\left\{O_{k}\right\}_{k=0}^{\infty}$ a projective system of $\bar{M}_{4}$ orbits, is the following:

(9.14) $\frac{\mathrm{t}_{O_{k}}\left(\gamma_{0}\right)+\mathrm{t}_{O_{k}}\left(\gamma_{1}\right)}{\left|O_{k}\right|} \mapsto 0$; and $\frac{I_{k}}{\left|O_{k}\right|} \mapsto 1$.

These two assumptions give a positive conclusion to Prob. 1.1 (when $r=4$ ) from Lem. 8.2 in that there is no bound on the genus of components corresponding to the projective system $\left\{O_{k}\right\}_{k=0}^{\infty}$.

Rem. 4.9 and Prob. 8.4 view fixed points of $\gamma_{0}$ and $\gamma_{1}$ on projective systems of Nielsen classes in a Modular Tower as generalizing a classical observation about fine moduli for the modular curve spaces $X_{1}\left(p^{k+1}\right)$.

Ex. 9.2 for $\left(A_{4}, \mathbf{C}_{3^{4}}, p=2\right)$ is a valuable test case. The genus situation from the view of Prob. 1.1 is even worse at level 0 and level 1 than for our main example. There are components, some with low genuses. Yet, the universal 2- Frattini situations produce the desired ramification.

9.6.2. Jumps in $\mathbf{m p}$ s. In all examples, perturbation of level $0 \mathrm{H}-\mathrm{M}$ reps. starts the story. Spin separating representations are the most intriguing element in structured description of Modular Towers levels, like listing real points in Prop. 8.19, Serre's Prop. [5.9 is indispensable, with $\$ 5.5$ using it indirectly even when the initial hypotheses don't apply. Still, this is only about the prime $p=2$, and only about universal 2-Frattini covers that have an initial relation with alternating groups.

An elementary approach to producing the desired ramification comes from the conjugacy class counting formula of 22.7 .2 Suppose $G_{k}$ is the $k$ th characteristic quotient of the universal $p$-Frattini cover of a finite $p$-perfect group $G=G_{0}$. Assume $g_{1}, g_{2} \in G_{k+1}$ have $p^{\prime}$ order. Princ. [5.13 reminds that obstruction (from FK97, 
$\S 3]$; as in 5.5 .2 occurs only when $\mathbf{1}_{G_{k}}$ appears in the Loewy display of $M_{k}=$ $\operatorname{ker}\left(G_{k+1} \rightarrow G_{k}\right): G_{k+1} \rightarrow U_{k} \rightarrow W_{k} \rightarrow G_{k}$ with $W_{k}$ centerless and $\operatorname{ker}\left(U_{k} \rightarrow W_{k}\right)$ the trivial $W_{k}$ module. With no loss for the question of concern, assume $g_{1}, g_{2} \in W_{k}$. Suppose also that $p$ does not divide the order of $g_{1} g_{2}$. Then, consider the triple of conjugacy classes $\mathrm{C}_{1}, \mathrm{C}_{2}, \mathrm{C}_{3}$ defined by $\left(g_{1}, g_{2},\left(g_{1} g_{2}\right)^{-1}\right)$. Consider (any) lifts $\hat{g}_{1}, \hat{g}_{2} \in U_{k}$. Let $U_{k}^{\prime}$ be the pullback of $\left\langle g_{1}, g_{2}\right\rangle$ in $U_{k}$. Asking if $p$ divides the order of $\hat{g}_{1} \hat{g}_{2}$ is equivalent to asking if element $\left.\left(g_{1}, g_{2},\left(g_{1} g_{2}\right)^{-1}\right)\right) \in \mathrm{Ni}\left(\left\langle g_{1}, g_{2}\right\rangle,\left(\mathrm{C}_{1}, \mathrm{C}_{2}, \mathrm{C}_{3}\right)\right)$ is obstructed in going to $\mathrm{Ni}\left(U_{k}^{\prime},\left(\mathrm{C}_{1}, \mathrm{C}_{2}, \mathrm{C}_{3}\right)\right)$. We continue this topic in 9.7.

9.6.3. sh-incidence, spin separation and the dominance of $H$ - $M$ reps. 8.5 found the data for computing the sh-incidence matrix for orbit $O_{1}^{+}$in two stages. Going to higher levels gives further reason to carefully organize information from $\mathcal{H}_{k+1}^{\mathrm{rd}} \rightarrow \mathcal{H}_{k}^{\mathrm{rd}}$. It makes sense to combine sh-incidence data and the use of RiemannHurwitz as in Lem. 8.2 .

Prop. 9.14 finds all degree 120 spin separating representations of $G_{1}$. That gave subgroups $H_{0} \leq A_{5}=G_{0}$ and $H_{1} \leq G_{1}$ with the natural map $G_{1} \rightarrow A_{5}$ inducing $H_{1} \rightarrow H_{0}$. Suppose we have a sequence $\left\{H_{k}\right\}_{k=0}^{\infty}$, with each $H_{k}$ inducing a spin separating representation of $G_{k}, k=0,1, \ldots$ Regard the projective limit $\lim _{\leftarrow k} H_{k}=\tilde{H}$ as giving the compatible system of spin separating representations of ${ }_{p} \tilde{G} 2$. The proof of Prop. 9.14 gives hints of the following sort. We can expect to find pro-2 $\tilde{H}$ containing a pro-cyclic subgroup $C$ with generator $\alpha \in{ }_{2} \tilde{G}$ where

(9.15) $\alpha^{2^{k+1}} \bmod \operatorname{ker}_{k} \in G_{k}$ is conjugate (in $G_{k}$ ) to no other element of $H_{k}$.

Lem. 8.17 and Lem. 8.14 has difficult arguments, respectively about shortening the respective type $(6,12)$ and type $(10,20) q_{2}$ orbits. These used the precise form of the level 1 characteristic module $M\left(A_{5}\right)$ from Cor.5.7 We have yet to capture the essence of $Q^{\prime \prime}$ shortening from $p$-group representations. Lem. [7.5 says an $H_{4}$ orbit containing an H-M rep., at level $k \geq 1$ when $p=2$, has faithful $\mathcal{Q}^{\prime \prime}$ action.

Note, too, the relation between near H-M and H-M reps. exploited in such places as Prop. 7.11] Further, the notion of complements also goes with H-M reps. Consider any Nielsen classes $\left\{\mathrm{Ni}_{k}=\mathrm{Ni}\left(G_{k}, \mathbf{C}\right)\right\}_{k=0}^{\infty}$ attached to a Modular Tower (for the prime $p$ ). Let $\left\{\boldsymbol{g}_{k}\right\}_{k=0}^{\infty}$ be a projective system of Nielsen class representatives. Suppose the conclusion of Prop. 17.11 holds for this system: $\mathbf{m p}\left(\boldsymbol{g}_{k+1}\right)=p \mathbf{m p}\left(\boldsymbol{g}_{k}\right)$ for $k \geq 0$. If $p=2$, Def. 7.4 would give $\left(\boldsymbol{g}_{k+1}\right) q_{2} \mathbf{m p ( \boldsymbol { g } _ { k + 1 } ) / 2}$ as the complement of $\boldsymbol{g}_{k+1}$ in $\mathrm{Ni}_{k+1}$. Both elements lie over the same element of $\mathrm{Ni}_{k}$. A similar notion with a general prime $p$ replacing 2 would give $p-1$ such elements as $\left(\boldsymbol{g}_{k+1}\right) q_{2}^{\mathbf{m p}\left(\boldsymbol{g}_{k+1}\right) / p},\left(\boldsymbol{g}_{k+1}\right) q_{2}^{2 \mathbf{m p}\left(\boldsymbol{g}_{k+1}\right) / p}, \ldots,\left(\boldsymbol{g}_{k+1}\right) q_{2}^{(p-1) \mathbf{m p}\left(\boldsymbol{g}_{k+1}\right) / p}$. These are examples of H-M reps. indicating a moduli (boundary) behavior that informs about the components in which they appear.

9.7. Generalizing the notion of $\mathbf{H}-\mathbf{M}$ reps. The following problem appears in 9.6 .2 in a special case. To start with assume ${ }_{p} \tilde{G}$ is the univeral $p$-Frattini cover of $G_{0}$, with notation as usual for the characteristic quotients. Let $\mathrm{C}_{1}, \mathrm{C}_{2}$ be $p^{\prime}$ conjugacy classes in $G_{0}$. Refer to $\left(g_{1}, g_{2}\right) \in G_{0}^{2}$ as $p$-divisible at level $k$ if all lifts $\left(\hat{g}_{1}, \hat{g}_{2}\right)$ to $G_{k}^{2} \cap\left(\mathrm{C}_{1}, \mathrm{C}_{2}\right)$ have $p$ dividing the order of $\hat{g}_{1} \hat{g}_{2}$. Refer to $\left(g_{1}, g_{2}\right)$ as $p$-divisible if it is $p$-divisible at some level $k$. If $\left\langle g_{1}, g_{2}\right\rangle$ is a $p^{\prime}$ group, then there is a lift of the whole group to $G_{k}$ for each $k$ (special case of Schur-Zassenhaus). That gives $p||\left\langle g_{1}, g_{2}\right\rangle \mid$ as necessary condition for $p$-divisibility. Is it sufficient?

Problem 9.26. Is $p$ dividing $\left|\left\langle g_{1}, g_{2}\right\rangle\right|$ sufficient for $\left(g_{1}, g_{2}\right)$ being $p$-divisible? 
The following says the $p$-divisible property holds for lifting to the universal $p$-Frattini cover of $G_{0}$ if and only if it holds for lifting to the universal $p$-Frattini cover of $\left\langle g_{1}, g_{2}\right\rangle$. Recall the universal $p$-Frattini cover of a $p^{\prime}$ group is just the group itself, so the lemma applies even if $\left\langle g_{1}, g_{2}\right\rangle=G^{1,2}$ is a $p^{\prime}$ group.

Lemma 9.27 (Local $p^{\prime}$-lift). That $\left(g_{1}, g_{2}\right)$ is not $p$-divisible is equivalent to their being no lift $\left(\hat{g}_{1}, \hat{g}_{2}\right)$ of $\left(g_{1}, g_{2}\right)$ to ${ }_{p} \tilde{G}^{2} \cap\left(\mathrm{C}_{1}, \mathrm{C}_{2}\right)$ with $\hat{g}_{1} \hat{g}_{2}$ having the same order as $g_{1} g_{2}$. Suppose $g_{1}, g_{2} \in G_{0}$ are $p^{\prime}$ elements. Let $G_{k}^{1,2}$ be the $k$ th characteristic quotient of the universal p-Frattini cover of $G^{1,2}$. Then, $\left(g_{1}, g_{2}\right)$ is p-divisible at level $k$ for lifts to $G_{k}$ if and only if it is p-divisible at level $k$ for lifts to $G_{k}^{1,2}$.

Proof. Suppose at each level $k$ there are lifts $\left(\hat{g}_{1, k}, \hat{g}_{2, k}\right)$ to $G_{k}$ as above, where $\hat{g}_{1, k}, \hat{g}_{2, k}$ has the same order as $g_{1} g_{2}$. The sets $L_{k}$ of such lifts in $G_{k}^{2}$ form a projective system of nonempty closed subsets. So, there is an element in the projective limit of the $A_{k}$ s giving the desired lift $\left(\hat{g}_{1}, \hat{g}_{2}\right) \in{ }_{p} \tilde{G}^{2}$. Now consider the relation between lifts to $G_{k}^{2}$ to that of lifts to $\left(G_{k}^{1,2}\right)^{2}$.

Apply Prop. 3.9 to the pullback $\tilde{G}^{*}$ of $\left\langle g_{1}, g_{2}\right\rangle$ in ${ }_{p} \tilde{G}_{0}$. Then, $\tilde{G}^{*}$ is a $p$-projective cover of $\left\langle g_{1}, g_{2}\right\rangle$. So, it maps surjectively to the $p$-Frattini cover of $\left\langle g_{1}, g_{2}\right\rangle$. A lifting $\left(\hat{g}_{1, k}, \hat{g}_{2, k}\right)$ to $G_{k}$, as in the statement where $\hat{g}_{1, k}, \hat{g}_{2, k}$ has the same order as $g_{1} g_{2}$, will map to such a lifting in the image. Conversely, the universal $p$-Frattini cover of $G^{1,2}$ has a map back through $\tilde{G}^{*}$ over $G_{1,2}$. Again, a lifting of the type in the lemma to $G_{k}^{1,2}$ produces one in $G_{k}$.

Example 9.28. Consider $\left(A_{5}, \mathbf{C}_{5_{+}^{2} 5^{2}}, p=2\right)$ at level 0. The two components separate by lifting invariant values. In the argument for Princ. 5.16] $\mathbf{C}_{5_{+} 5_{-3}}$ has lifting invariant +1 . This was a nontrivial case of nonobstruction for $r=3$. We still, however, don't know if in the $\left(A_{5}, \mathbf{C}_{5_{+} 5_{-} 3}, p=2\right)$ Modular Tower there is obstruction at some level. In the language here, is the following pair 2- divisible: ((1 2345$),(12543))$ ?

Reminder of the positive effect of obstructed components: They have nothing above them at higher levels, so that eliminates the component from consideration in the Main Conjecture for any value of $r$. In the problem here the negative effect of the appearance of $1 \mathrm{~s}$ is toward providing sufficient ramification over cusps in the Main Conjecture. Here is a projective version of it.

Problem 9.29 (Questions related to Prop. [5.9). Let $\tilde{\boldsymbol{g}}$ be a projective system of Nielsen class representatives as in Lem. 8.9 Characterize when $\operatorname{jmp}(\tilde{\boldsymbol{g}})=\infty$. What generalization of Serre's Prop. could deduce for a projective system above $\boldsymbol{g}_{0}$ what might be the values of $\operatorname{jmp}(\tilde{\boldsymbol{g}})$. Even if the genus 0 condition does not hold in Prop. [5.9, is there a general procedure for checking the possible values of the lifting invariant by coalescing branch cycles, as does $\$ 5.5$ to the case of genus 0 ?

The main effect of Harbater-Mumford representatives on our Main Conjecture has been to give lifts of pairs from level 0 to level $k$ where the order of the product does not change. This has the effect of giving projective systems of cusps on a Modular Tower with relative ramification of degree 1. For this property (depending on the outcome of Prob. 9.26), we suggest the natural generalization of H-M reps. is to consider elements $\boldsymbol{g} \in \operatorname{Ni}(G, \mathbf{C})$ with having the following form:

$$
\boldsymbol{g}=\left(g_{1}, \ldots, g_{s_{1}}, g_{s_{1}+1}, \ldots, g_{s_{1}+s_{2}}, \ldots, g_{s_{1}+s_{2}+\cdots+s_{t-1}+1}, \ldots, g_{s_{1}+s_{2}+\cdots+s_{t}}\right)
$$


(9.16)

where, $\left\langle g_{s_{1}+s_{2}+\cdots+s_{i}+1}, \ldots, g_{s_{1}+s_{2}+\cdots+s_{i+1}}\right\rangle$ is a $p^{\prime}$ group $, i=1, \ldots, t-1$, and for all $i, s_{i} \geq 2$.

EXAmple 9.30. Consider ${ }_{7} \boldsymbol{g},{ }_{8} \boldsymbol{g}$ and ${ }_{9} \boldsymbol{g}$ from Table 1 None are H-M reps. Yet, for $p=5$, each satisfies the $p^{\prime}$ property (9.16); $\left(g_{1}, g_{2}, g_{3}, g_{4}\right)$ has $\left\langle g_{1}, g_{2}\right\rangle$ and $\left\langle g_{3}, g_{4}\right\rangle$ isomorphic to $A_{4}$ (5 not dividing the order).

H-M reps. were simple enough to allude to them by just the shape of their Nielsen class representives. Some geometry applications of H-M reps. require not only that (9.16) holds. Suppose also for each $i,\left(g_{s_{1}+s_{2}+\cdots+s_{i}+1}, \ldots, g_{s_{1}+s_{2}+\cdots+s_{i+1}}\right)$ has entries with product 1, satisfying the genus 0 condition (on each orbit). Such situations aid in analyzing geometry behind components of reduced Hurwitz spaces.

As an example need for treating Prob. 9.26 note the difficulties in Lem. 77.5 in establishing that all $H_{4}$ orbits at level 1 of $\left(G_{1}, \mathbf{C}_{3^{4}}\right)$ have faithful $\mathcal{Q}^{\prime \prime}$ action. This used details from Cor. 5.7

\section{Appendix A. Coset description of a fundamental domain for $\mathcal{H}_{k}^{\text {rd }}$}

7.1 .3 suggests finding good cosets to go from fundamental a domain for level 0 of the $\left(A_{5}, \mathbf{C}_{3^{4}}, p=2\right)$ to a fundamental domain for level 1. This includes inspecting the monodromy groups $H_{k, 0}$ of the Galois closure of $\overline{\mathcal{H}}\left(G_{k}, \mathbf{C}_{3^{4}}\right) \rightarrow \overline{\mathcal{H}}\left(G_{0}, \mathbf{C}_{3^{4}}\right)$ for $k$ large. This will mostly be a 2 -group (for a general Modular Tower, mostly a $p$-group). When $k=1$ the map has degree 16 . The monodromy group, $H_{1,0}$ has these properties.

(A.1a) $\left|H_{1,0}\right|=192=2^{6} \cdot 3$ and its center has order 2 .

(A.1b) It has a subnormal series with abelian quotients: $T_{0} \triangleleft T_{1} \triangleleft T_{2} \triangleleft H_{1,0}$ with $\left|T_{2}\right|=2^{5} \cdot 3,\left|T_{1}\right|=2^{5}$ and $\left|T_{0}\right|=2$.

Note: $H_{1,0}$ is not entirely a 2-group, having a copy of $S_{3}$ at the top. This is from a $q \in \bar{M}_{4}$ inducing an automorphism on a 2-Sylow of $G_{1}$, though $q$ does not act as an automorphism of $G_{1}$ through its Nielsen class action. [Fr01 documents this very Galois-like (as in Rig96) and subtle phenomenon.

\section{Appendix B. Serre's half-canonical invariant}

For any a projective curve $X$, denote the divisor class of a divisor $D$ on $X$ by $[D]$. Our concern here is for divisors $D$ that are half-canonical: $[2 D]$ is the canonical class (of divisors of meromorphic differentials) on $X$. Consider the lifting invariant $s(\boldsymbol{g})$ for a cover $\varphi: X \rightarrow \mathbb{P}_{z}^{1}$ with a branch cycle description $\boldsymbol{g}$. Prop. 5.9 discusses the special case of the lifting invariant that $\mathbb{C}$ calls $s_{H, \mathbf{C}_{H}}(\boldsymbol{g})$. For that case, $H \rightarrow G$ comes from a spin separating representation and $\mathbf{C}_{H}$ is a lift of $2^{\prime}$ conjugacy classes. Particular cases are in Prop. 9.8 and Prop. 9.12 Much of our technical work is to establish the former proposition is a case of spin separation. For such a spin separating representation, under special hypotheses, [Ser90a, p. 479] gives a formula for computing $s(\boldsymbol{g})$, and $\$ 5.5$ gives examples of loosening the genus 0 hypothesis to make this computation. The formula of [Ser90b p. 548 and p. 550] ties the lifting invariant to an invariant of Riemann for describing many delicacies about Riemann surfaces. We explain its potential to create automorphic functions, produced by $\theta$-nulls, on certain Hurwitz space components. Such functions produced by natural moduli properties will reveal properties of the moduli space, especially about the nature of the cusps and divisors supported in the cusps. 
B.1. Half-canonical classes from odd ramification. Assume the cover $\varphi: X \rightarrow \mathbb{P}_{z}^{1}, \operatorname{deg}(\varphi)=n$ has odd order ramification. At each point $x^{\prime} \in X$, lying over $z^{\prime} \in \mathbb{P}_{z}^{1}$, express $\varphi$ locally as $t_{z^{\prime}}^{e}+z^{\prime}$ with $\left(z-z^{\prime}\right)^{1 / e}=t_{z^{\prime}}$ (if $z^{\prime}=\infty$ use $z^{-1 / e}=t^{\prime}$ ) with $e$ odd. As the multiplicity of zeros and poles of $d \varphi$ is independent of the local uniformizing paramater, the differential $d \varphi$ has all multiplicities of its zeros and poles even. Serre treats the case with a general curve $Y$ replacing $\mathbb{P}_{z}^{1}$. Then, the monodromy group of the cover may not be in $A_{n}$. That requires extra care describing the precise group cover $\hat{S}_{n}$ that one uses for computing the lifting invariant. Back to $Y=\mathbb{P}_{z}^{1}$.

Let $D_{\varphi}$ be the divisor $\frac{(d \varphi)}{2}$, the divisor of a half-canonical class. Replacing $\varphi$ by $\alpha \circ \varphi$ with $\alpha \in \mathrm{PSL}_{2}(\mathbb{C})$ replaces $D$ be a divisor linearly equivalent to $D$. The formula is $s(\boldsymbol{g})=(-1)^{\ell\left(D_{\varphi}\right)}(-1)^{\omega(\varphi)}$. Notations are as follows:

(B.1a) $\ell(D)$ is the dimension of the linear system of the divisor; and

(B.1b) $\omega(\varphi)$ is the sum over ramification indices $e_{x}$ of $\frac{e_{x}^{2}-1}{8}$ of ramified $x \in X$.

Formulas using ramification indices depend on the permutation representation giving a cover. For either of the Nielsen classes $\mathrm{Ni}\left(A_{5}, \mathbf{C}_{3^{4}}\right)$ abs or $\mathrm{Ni}\left(A_{4}, \mathbf{C}_{3_{+}^{2} 3_{-}^{2}}\right)^{\text {abs }}$ (absolute classes here mean for the standard representations of $A_{n}, n=4$ or 5), $\omega(\varphi)$ is even. So, $s(\boldsymbol{g})=(-1)^{\ell\left(D_{\varphi}\right)}$ (see $\underline{\mathrm{E} .2}$ ). This also holds for inner classes in this case. More significantly, it holds in replacing $G_{0}=A_{5}$ (resp. $A_{4}$ ) by $G_{k}$, the $k$ th characteristic quotient of the universal 2-Frattini cover of $G_{0}$, and the standard representation of $G_{0}$ by some spin separating representation $T_{k}$ of $G_{k}$.

For $\boldsymbol{p} \in \mathcal{H}^{\text {in }}$ and $(G, T)$ a group with a faithful representation, $G(T, 1)$ the stabilizer of 1 (3.1.1), we have always to keep in mind the relation of the Galois cover $\varphi_{\boldsymbol{p}}: \hat{X}_{\boldsymbol{p}} \rightarrow \mathbb{P}_{z}^{1}$ and the cover $\varphi_{\boldsymbol{p}, T}: \hat{X}_{\boldsymbol{p}} / G(T, 1) \rightarrow \mathbb{P}_{z}^{1}$ on the absolute space $\mathcal{H}^{\text {abs }}$. It is the latter we are attaching a half-canonical divisor class (and $\theta$ function below). Still, we suppress $T$ in the continuing discussion. Regard $\boldsymbol{p}$ as a point of $\mathcal{H}(G, \mathbf{C}, T)^{\text {abs,rd }}$. To simplify notation, refer to a cover associated with $\boldsymbol{p}$ as $X_{\boldsymbol{p}}$.

Proposition B.1 (Even $\theta$ nulls). Each representation $T: G_{1} \rightarrow A_{\operatorname{deg}(T)}$ from Prop. 9.14 and Prop. 9.15 provides an even $\theta$ function $\theta_{\boldsymbol{p}, 1}$ on the cover of degree $\operatorname{deg}(T)$ corresponding to a point $\boldsymbol{p} \in \mathcal{H}_{1}^{+} \subset \mathcal{H}_{1}^{\mathrm{in}, \mathrm{rd}}$. Similarly, there is an even $\theta$ function $\theta_{\boldsymbol{p}, 0}$ corresponding to the Galois cover $\varphi_{\boldsymbol{p}}$ with group $A_{5}$ attached to $\boldsymbol{p} \in \mathcal{H}_{0}^{\mathrm{in}, \mathrm{rd}}($ see 2.3 .4$)$.

B.2. Coordinates from $\theta$ functions. The final version of Fri96 gives normalizations of these theta functions (in their dependency on $\boldsymbol{p}$ ). That will refer to this exposition for its setup. Typically the normalization is to integrate the $\theta$ function with respect to a volume form, and set it equal to 1. Prop. B.1]implies the geometric realization of the unramified spin cover of $G$ comes from a $G$ invariant nontrivial 2-torsion point $u_{\boldsymbol{p}}$ on $\operatorname{Pic}\left(\hat{X}_{\boldsymbol{p}}\right)^{(0)}$; with this description $u_{\boldsymbol{p}}$ is unique. In some cases, the ratio $\left|\theta_{\boldsymbol{p}, 0}(0) / \theta_{\boldsymbol{p}, 0}\left(u_{\boldsymbol{p}}\right)\right|$ (or its $\log$ ) appears in a nontrivial measure of the distance from an $\mathrm{H}-\mathrm{M}$ cusp, so long as that ratio makes sense, the technical point of the duration of this section.

Let $\mathcal{M}_{g}$ be the moduli space of curves of genus $g$. Suppose $\mathcal{H}$ is a Hurwitz space of covers $\varphi: X \rightarrow \mathbb{P}_{z}^{1}$ having genus $g$. Then, let $[X] \in \mathcal{M}_{g}$ denote the isomorphism class of the curve. A corollary of one of the main theorems on moduli of curves is that the map $\boldsymbol{p} \in \mathcal{H} \mapsto\left[X_{\boldsymbol{p}}\right] \in \mathcal{M}_{g}$ is a morphism of quasi-projective varieties. 
For our applications, consider $\theta_{\boldsymbol{p}, 0}$ : An even function on the universal covering space $\widetilde{\operatorname{Pic}}\left(X_{\boldsymbol{p}}\right)^{(0)}$ of $\operatorname{Pic}\left(X_{\boldsymbol{p}}\right)^{(0)}$, the Jacobian (divisor classes of degree 0 ) of $X_{\boldsymbol{p}}$. Denote by $X_{p}^{(t)}$ the positive divisors on $X_{p}$ of degree $t \geq 1$ (different than in 33.1 .3 where it was a fiber product). Mu76 Lecture III] guided this, though detecting which bits of this construction depend only on $\boldsymbol{p}$ is less obvious.

We need a basis $\boldsymbol{\omega}=\left(\omega_{1}, \ldots, \omega_{g}\right)$ for global holomorphic differentials on $X_{\boldsymbol{p}}$ to see $\theta_{\boldsymbol{p}, 0}$ in its classical coordinates $\boldsymbol{w}=\left(w_{1}, \ldots, w_{g}\right)$. Choose a positive degree $g$ divisor $D^{\prime}=x_{1}^{\prime}, \ldots, x_{g}^{\prime}$. Then, consider any $g$-tuple of paths $\left(P_{1}, \ldots, P_{g}\right)$ on $X_{\boldsymbol{p}}$ where $P_{i}$ starts at $x_{i}^{\prime}$ (denote its endpoint $\left.x_{i}\right), i=1, \ldots, g$, so $D_{\boldsymbol{P}}=\sum_{i=1}^{g} x_{i} \in X_{\boldsymbol{p}}^{(g)}$ is a positive divisor of degree $g$. The Jacobi inversion theorem says the following about the map $\mu_{D^{\prime}, \boldsymbol{\omega}}: \boldsymbol{P}=\left(P_{1}, \ldots, P_{g}\right) \mapsto\left[D_{\boldsymbol{P}}-D^{\prime}\right] \in \operatorname{Pic}\left(X_{\boldsymbol{p}}\right)^{(0)}$.

(B.2a) $\mu_{D^{\prime}, \omega}$ is surjective.

(B.2b) It factors through $\boldsymbol{P} \rightarrow\left(\sum_{i=1}^{g} \int_{P_{i}} \omega_{1}, \ldots, \sum_{i=1}^{g} \omega_{g}\right) \in \mathbb{C}_{\boldsymbol{w}}$.

(B.2c) The induced map $\mathbb{C}_{\boldsymbol{w}} \rightarrow \operatorname{Pic}\left(X_{\boldsymbol{p}}\right)^{(0)}$ identifies $\mathbb{C}_{\boldsymbol{w}}$ with the universal covering space $\widetilde{\operatorname{Pic}}\left(X_{\boldsymbol{p}}\right)^{(0)}$.

This is the classical version of Jacobi Inversion Sp57, p. 281]. If we know $\operatorname{Pic}\left(X_{\boldsymbol{p}}\right)^{(0)}$ is a projective variety, then this complex analytic map $X_{\boldsymbol{p}}^{(g)} \rightarrow \operatorname{Pic}\left(X_{\boldsymbol{p}}\right)^{(0)}$ is algebraic (Chow's Lemma Mu66 p. 115] or Har77 Exer. 4.10, p. 107]). It is a birational morphism, identifying the two function fields. The differentials $\left(\omega_{1}, \ldots, \omega_{g}\right)$ identify with the differentials $\left(d w_{1}, \ldots, d w_{g}\right)=d \boldsymbol{w}$, which live on $\operatorname{Pic}\left(X_{\boldsymbol{p}}\right)^{(0)}$.

Further, for any integer $t$ and any divisor $D^{\prime \prime}$ of degree $t$, a similar map

$$
\mu_{D^{\prime \prime}, \boldsymbol{\omega}}: \boldsymbol{P}=\left(P_{1}, \ldots, P_{t}\right) \mapsto\left[D_{\boldsymbol{P}}-D^{\prime \prime}\right] \in \operatorname{Pic}\left(X_{\boldsymbol{p}}\right)^{(0)}
$$

factors through $X_{p}^{(t)}$. Taking $t=1$ and $D^{\prime \prime}=x_{0} \in X_{p}$ gives an embedding $\mu_{x_{0}, \boldsymbol{\omega}}: X_{\boldsymbol{p}} \rightarrow \operatorname{Pic}\left(X_{\boldsymbol{p}}\right)^{(0)}$ (assuming $g \geq 1$ ). Again by Chow's Lemma, this gives coordinates on $X_{p}$ compatible with those of $\operatorname{Pic}\left(X_{\boldsymbol{p}}\right)^{(0)}$. Recover the basis $\boldsymbol{\omega}$ as restriction of $d \boldsymbol{w}$ to $\mu_{x_{0}, \boldsymbol{\omega}}\left(X_{\boldsymbol{p}}\right)$. Finally, let $D_{0}$ be a positive divisor of degree $g-1$. Then, $\Theta_{D_{0}, \boldsymbol{\omega}}=\mu_{D_{0}, \boldsymbol{\omega}}\left(X_{\boldsymbol{p}}^{(g-1)}\right)$ is a divisor on $\operatorname{Pic}\left(X_{\boldsymbol{p}}\right)^{(0)}$.

Forming this data, however, seemed to require choices in $\boldsymbol{\omega}$ and the positive divisors. Under what circumstances can one use this to construct objects analytically varying in the coordinates of $\boldsymbol{p}$ (alone — not dependent on our choices)? We are looking for a global construction tightly tying the curves $X_{p}$ in the family to coordinates for $\operatorname{Pic}\left(X_{\boldsymbol{p}}\right)^{(0)}$. That is the topic for the rest of this section.

B.3. $\theta$ functions along the moduli space. Even the most relaxed conditions won't give explicit $\theta$ functions in classical coordinates from a uniform construction. Here are reasons.

(B.3a) A nontrivial family of curves will have nontrivial monodromy on the 1st homology (or cohomology) of its fibers.

(B.3b) Analytic coordinates in $\boldsymbol{p}$ for an embedding $\mu_{\boldsymbol{p}, t}: X_{\boldsymbol{p}}^{(t)} \rightarrow \operatorname{Pic}\left(X_{\boldsymbol{p}}\right)^{(0)}$ come from an analytic choice $\left[D_{\boldsymbol{p}, t}\right]$ of divisor class of degree $t$.

From (B.3tat), in nontrivial algebraic families it is impossible to get a uniform basis $\omega_{\boldsymbol{p}}$ of holomorphic differentials. Since $X_{\boldsymbol{p}}^{(t)}$ naturally embeds in $\operatorname{Pic}\left(X_{\boldsymbol{p}}\right)^{(t)}$, use $D \in X_{\boldsymbol{p}}^{(t)} \mapsto\left[D-D_{\boldsymbol{p}, t}\right] \in \operatorname{Pic}\left(X_{\boldsymbol{p}}\right)^{(0)}$ to give analytic coordinates in (B.3b). It is especially significant for $t=1$ and $t=g-1$. A $\left[D_{\boldsymbol{p}, t}\right]$ analytic in $\boldsymbol{p} \in \mathcal{H}$ need not have a representative divisor (positive or not) with coordinates in $\boldsymbol{p}$. The divisor 
class is sufficient. Finding, however, such an analytically varying divisor usually requires it appear from the map $\varphi_{\boldsymbol{p}}$ through ramification. That isn't a formal definition, though several illustrative situations occur in DF90b. An example of it for (B.3b) when $t=g-1$ is having an analytically varying half-canonical divisor class. In turn that produces a precise copy in $\operatorname{Pic}\left(X_{\boldsymbol{p}}\right)^{(0)}$ of the divisor $\Theta_{D_{\boldsymbol{p}, g-1}}$.

Let $h$ be any meromorphic function on $X_{\boldsymbol{p}}$ of degree $u$. Then, $h: X_{\boldsymbol{p}} \rightarrow \mathbb{P}_{v}^{1}$ has zeros $x_{1}^{0}, \ldots, x_{u}^{0}$ and poles $x_{1}^{\infty}, \ldots, x_{u}^{\infty}$.

B.3.1. Abel's Theorem motivates finding nondegenerate odd $\theta$-nulls. Suppose we have an embedding of $X_{\boldsymbol{p}}$ in $\operatorname{Pic}\left(X_{\boldsymbol{p}}\right)^{(0)}=J\left(X_{\boldsymbol{p}}\right)$. So, each zero $x_{i}^{0}$ and pole of $h$ produces a point in $J\left(X_{\boldsymbol{p}}\right)$. List these as $\boldsymbol{w}_{x_{i}^{0}}, \boldsymbol{w}_{x_{i}^{\infty}}, i=1, \ldots, u$. Yet, finding these $\boldsymbol{w}$ s doesn't require giving $h$. It only needs points $x_{1}^{0}, \ldots, x_{u}^{0}$ and $x_{1}^{\infty}, \ldots, x_{u}^{\infty}$ on $X_{\boldsymbol{p}}$ viewed as inside $J\left(X_{\boldsymbol{p}}\right)$. Define

$$
\left[D_{\boldsymbol{x}}\right]=\left[D\left(\boldsymbol{p}_{x_{i}^{0}}, \boldsymbol{w}_{x_{i}^{\infty}}, i=1, \ldots, u\right)\right]
$$

to be the sum of all the $\boldsymbol{w}_{x_{i}^{0}}$ s minus all the $\boldsymbol{w}_{x_{i}^{\infty}} \mathrm{s}$ on $J\left(X_{\boldsymbol{p}}\right)$. To say $\left[D_{\boldsymbol{x}}\right]$ is zero means it is the origin of $J\left(X_{\boldsymbol{p}}\right)$.

TheOREM B.2 (Abel's Theorem (generalized)). Existence of $h$ with these zeros and poles characterizes exactly when $[D]$ is zero.

If $h$ exists, consider the logarithmic derivative $d h / h$. This is a meromorphic differential of 3rd kind with pure imaginary periods. Even if $h$ doesn't exist, given the divisor $D_{x}$ above, the following always holds.

(B.4) There is a unique differential $\omega_{x}$ with residue divisor $D_{x}$ having pure imaginary periods.

Suppose $[D]$ is not zero, but $m[D]$ is zero on $J\left(X_{\boldsymbol{p}}\right)$ for some integer $m$. Then, repeating all the zeros and poles $m$ times produces a function $h$ on $X_{\boldsymbol{p}}$. The $m$ th root of $h$ defines a cyclic unramified degree $m$ cover $Y \rightarrow X_{\boldsymbol{p}}$.

B.3.2. Abel's Theorem and nondegenerate odd $\theta$ s. Riemann produced $\theta=\theta_{X_{\boldsymbol{p}}}$ functions to provide uniformizing coordinates for this construction. Torelli space $\mathcal{T}_{g}$ is a cover of $\mathcal{M}_{g}$. The points of $\mathcal{T}_{g}$ lying over $\left[X_{\boldsymbol{p}}\right]$ consists of a canonical basis for $H_{1}\left(X_{\boldsymbol{p}}, \mathbb{Z}\right)$ for which we borrow the notation of $\boldsymbol{a}=\left(a_{1}, \ldots, a_{g}\right)$ and $\boldsymbol{b}=\left(b_{1}, \ldots, b_{g}\right)$ generators from (4.8). See [Sh98, p. 151] for the full abelian variety context of the next statement.

Lemma B.3 (The Symplectic group). The fiber of $\mathcal{T}_{g}$ over $\left[X_{\boldsymbol{p}}\right]$ is a homogeneous space for $\mathrm{Sp}_{2 g}(\mathbb{Z})$ the group

$$
\left\{T=\left(\begin{array}{cc}
A & B \\
C & D
\end{array}\right) \mid A, B, C, D \in \mathbb{M}_{g}(\mathbb{Z}),{ }^{\mathrm{t}} T\left(\begin{array}{cc}
0_{g} & -I_{g} \\
I_{g} & 0_{g}
\end{array}\right) T=\left(\begin{array}{cc}
0_{g} & -I_{g} \\
I_{g} & 0_{g}
\end{array}\right)\right\}
$$

The standard normalization is to choose $\omega_{1}, \ldots, \omega_{g}=\boldsymbol{\omega}=\boldsymbol{\omega}_{\boldsymbol{a}}$ so the matrix $A_{\boldsymbol{\omega}_{\boldsymbol{a}}}$ with $(i, j)$ entry $\int_{a_{j}} \omega_{i}$ is the identity matrix. The matrix $B_{\boldsymbol{\omega}_{\boldsymbol{a}}}$ with $(i, j)$ entry $\int_{a_{j}} \omega_{i}$ is the period matrix. Then $B_{\boldsymbol{\omega}_{\boldsymbol{a}}, \boldsymbol{b}}$ is symmetric and has positive definite imaginary part (Riemann's bilinear relations: Sp57, p. 254]). Given this, there is a unique $\theta$ function $\theta=\theta_{\boldsymbol{p}, \boldsymbol{a}, \boldsymbol{b}}$ attached to $(\boldsymbol{a}, \boldsymbol{b})$.

Many mathematical items on $X_{\boldsymbol{p}}$ appear constructively from this. This includes functions and meromorphic differentials (with particular zeros and poles). This was a central goal in generalizing Abel's Theorem: To provide Abel(-Jacobi) 
constructions for a general Riemann surface. For the function $h$ it has this look:

$$
h(x)=\prod_{i=1}^{u} \theta\left(\int_{x_{i}^{0}}^{x} \boldsymbol{\omega}\right) / \prod_{i=1}^{u} \theta\left(\int_{x_{i}^{\infty}}^{x} \boldsymbol{\omega}\right) .
$$

In $\theta$ you see $g$ coordinates; the $i$ th entry is $\int_{x_{i}^{0}}^{x} \omega_{i}$, an integral over a path on $X_{\boldsymbol{p}}$. The integrals make sense up to integration around closed paths. So, they define a point in $J\left(X_{\boldsymbol{p}}\right)$.

Even if $h$ doesn't exist, the logarithmic differential of (B.5) does. It gives the third kind differential from (B.4). This gives the differential equation defining $\theta$ functions. In expressing $h$, replace $\int_{x_{i}^{\infty}}^{x} \boldsymbol{\omega}$ by a vector $\boldsymbol{w}$ in the universal covering space of the Jacobian. Form the logarithmic differential of it: $d \theta(\boldsymbol{w}) / \theta(\boldsymbol{w})$. To construct his $\theta$ functions, Riemann following Abel's case for cubic equations, imposed the condition that translations by periods would change the logarithmic differential only by addition of a constant. With $\nabla$ the gradient in $\boldsymbol{w}$,

(B.6) $\nabla(\nabla \theta(\boldsymbol{w}) / \theta(\boldsymbol{w}))$ is invariant under the lattice of periods.

The main point about $\theta$ functions (of 1st order for dimension $g$ ) is that there is essentially just one: $\theta(\boldsymbol{w}, B)$ where $B$ runs over symmetric $g \times g$ matrices with imaginary part positive definite. The $\Theta$ divisor determines $\theta(\boldsymbol{w}, B)$ for fixed $B$ up to a constant multiple, though we can always translate the $\Theta$ divisor on $\operatorname{Pic}\left(X_{\boldsymbol{p}}\right)^{(0)}$. The space of translated divisors is a homogeneous space for $\operatorname{Pic}\left(X_{\boldsymbol{p}}\right)^{(g-1)}$. In varying the $\theta$ function with $\boldsymbol{p}$, our concerns include that choice of constant, and also the analytic choice in $\boldsymbol{p}$ of $\left[D_{\boldsymbol{p}}\right]$ in $\operatorname{Pic}\left(X_{\boldsymbol{p}}\right)^{(g-1)}$. It depends on our goals (one element of depth in Riemann's results) what type of choice we want for $\left[D_{\boldsymbol{p}}\right]$. We illustrate with Riemann's original goal.

To represent all functions $h$ appropriately in (B.5), the following is necessary:

(B.7) $\theta$ must be an odd function so as to give 0 at $\boldsymbol{w}=\mathbf{0}$ with multiplicity 1 .

B.3.3. Fay's normalizations and Riemann's Theorem. Fay73 p. 1] makes a choice to replace the matrices $A=I_{g}$ and $B$ respectively by $2 \pi i I_{g}$ and $\Pi_{\boldsymbol{a}, \boldsymbol{b}}=2 \pi i B$ is symmetric and its real part is negative definite. This scaling doesn't change the isomorphism class of $\mathbb{C}_{\boldsymbol{w}}^{g} / L_{\boldsymbol{a}, \boldsymbol{b}}$ and it has numerous advantages. With this notation, and all these choices, rewrite Riemann's classical function $\theta(\boldsymbol{w}, B)$ as

$$
\theta\left(\boldsymbol{w}, \Pi_{\boldsymbol{a}, \boldsymbol{b}}\right)=\theta\left[\begin{array}{l}
\mathbf{0} \\
\mathbf{0}
\end{array}\right]\left(\boldsymbol{w}, \Pi_{\boldsymbol{a}, \boldsymbol{b}}\right)=\sum_{\boldsymbol{m} \in \mathbb{Z}^{g}} \exp \left(\frac{1}{2} \boldsymbol{m} \Pi_{\boldsymbol{a}, \boldsymbol{b}}{ }^{\mathrm{t}} \boldsymbol{m}+\boldsymbol{m}^{\mathrm{t}} \boldsymbol{w}\right) .
$$

One can see the difference in the formulas by contrasting with the notation of FaK01 Chap. 2]. The $2 \pi i$ factor appears in their expression for a $\theta$ function, and their version of the heat equation. [FaK01 only considers the case $g=1$. From our viewpoint, that is because they only consider moduli spaces of genus 1 curves where the $j$-invariant for a point $\boldsymbol{p}$ is the $j$-invariant of the curve it represents: These are modular curves. Their $\theta$-nulls are close to automorphic functions on these curves, and the relations among the $\theta$ - nulls with various characteristics are algebraic equations describing these curves. [FaK01 Chap. 2,§8]:

In fact, partial motivation for the results discussed so far is to better understand the multivariable case.

Even for $r=4$, Modular Towers forces relating moduli spaces of curves of infinitely many genuses parametrized by reduced 1-dimensional Hurwitz spaces. When $r=4$, the moduli spaces are upper half plane quotients. So comparing with $\theta$-nulls from 
modular curves is inevitable. Our period matrices $\Pi_{a, b}=\Pi_{a_{p}, b_{p}}$ for the curve $X_{p}$ are therefore (matrix) functions of $\tau \in \mathbb{H}$, rather than just being $\tau$.

Notice: Because a lattice is invariant under multiplication by $-1, \theta\left(\boldsymbol{w}, \Pi_{\boldsymbol{a}, \boldsymbol{b}}\right)$ is an even function of $\boldsymbol{w}$. This isn't the function that works in (B.5). Since the divisors for the $\theta$ functions differ only by a translation, giving an appropriate function for (B.5) comes by translating $\boldsymbol{w}$. Further, the even $\theta$ from Prop. B.1 for $g>1$, is not likely to be this one. As in $\$ 2.10$ use ${ }^{t} A$ for the transpose of a matrix.

Let $\boldsymbol{e}=2 \pi i \boldsymbol{\epsilon}+\boldsymbol{\delta} \Pi_{\boldsymbol{a}, \boldsymbol{b}}$ with $\boldsymbol{\epsilon}, \boldsymbol{\delta} \in \mathbb{R}^{g}$, represent an arbitrary point of $\mathbb{C}^{g}$. A special notation — given by characteristics — expresses the $\theta$ translated by $\boldsymbol{e}$ (then multiplied by an exponential factor): $\theta\left[\begin{array}{l}\boldsymbol{\delta} \\ \boldsymbol{\epsilon}\end{array}\right]\left(\boldsymbol{w}, \Pi_{\boldsymbol{a}, \boldsymbol{b}}\right)=$

$$
\begin{aligned}
& \exp \left({ }_{\frac{1}{2}} \boldsymbol{\delta} \Pi_{\boldsymbol{a}, \boldsymbol{b}}{ }^{\mathrm{t}} \boldsymbol{\delta}+(\boldsymbol{w}+2 \pi i \boldsymbol{\epsilon})^{\mathrm{t}} \boldsymbol{\delta}\right) \theta\left[\begin{array}{l}
\mathbf{0} \\
\mathbf{0}
\end{array}\right]\left(\boldsymbol{w}+\boldsymbol{e}, \Pi_{\boldsymbol{a}, \boldsymbol{b}}\right) \\
& =\sum_{\boldsymbol{m} \in \mathbb{Z}^{g}} \exp \left(\frac{1}{2}(\boldsymbol{m}+\boldsymbol{\delta}) \Pi_{\boldsymbol{a}, \boldsymbol{b}}{ }^{\mathrm{t}}(\boldsymbol{m}+\boldsymbol{\delta})+(\boldsymbol{w}+2 \pi i \boldsymbol{\epsilon})^{\mathrm{t}}(\boldsymbol{m}+\boldsymbol{\delta})\right) .
\end{aligned}
$$

There are $2^{2 g-1}+2^{g-1}$ choices of $(\boldsymbol{\epsilon}, \boldsymbol{\delta}) \in \frac{1}{2} \mathbb{Z} \bmod \mathbb{Z}$ giving even $\theta \mathrm{s}\left(4 \boldsymbol{\delta} \cdot{ }^{\mathrm{t}} \boldsymbol{\delta} \equiv 0\right.$ mod 2 - even characteristics) and $2^{2 g-1}-2^{g-1}$ choices of $(\boldsymbol{\epsilon}, \boldsymbol{\delta})$ giving odd $\theta \mathrm{s}$. Riemann's Theorem identifies the $\theta$ from Prop. B.1 with an even characteristic Fay73, Thm. 1.1].

B.4. Monodromy, characteristics and Pryms. Again consider $\boldsymbol{p} \in \mathcal{H}^{\mathrm{rd}}$, and let $P$ be a closed path in $\mathcal{H}^{\text {rd }}$ based at $\boldsymbol{p}$. Suppose $\boldsymbol{a}_{\boldsymbol{p}}, \boldsymbol{b}_{\boldsymbol{p}}$ is a choice of canonical homology basis for $H_{1}\left(X_{\boldsymbol{p}}, \mathbb{Z}\right)$. It is classical that analytic continuation around $P$ induces a linear transformation $\psi_{P}$ on $H_{1}\left(X_{\boldsymbol{p}}, \mathbb{Z}\right)$ : $\left(\boldsymbol{a}_{\boldsymbol{p}}, \boldsymbol{b}_{\boldsymbol{p}}\right) \mapsto \psi_{P}\left(\boldsymbol{a}_{\boldsymbol{p}}, \boldsymbol{b}_{\boldsymbol{p}}\right)=\left(\tilde{\boldsymbol{a}}_{\boldsymbol{p}}, \tilde{\boldsymbol{b}}_{\boldsymbol{p}}\right)$. Using Lem. B.3 this represents $\psi_{P}$ as an element $T_{P} \in \mathrm{Sp}_{2 g}(\mathbb{Z})$. For the modular curve case, the identification of $H_{1}\left(X_{\boldsymbol{p}}, \mathbb{Z}\right)$ with $\langle 2 \pi i, \tau\rangle$ (Fay's notation) makes this transparent because each path identifies with an element of $\mathrm{SL}_{2}(\mathbb{Z})$.

B.4.1. Monodromy acting on a $\theta$. [FaK01 Chap. 2], Fay73 p. 7] and Sh98, p. 179] use a transformation formula that explains how $\theta$ functions change with an application of $\psi_{P}$ a homology basis. Here are is a qualitative description.

(B.10a) Change $\boldsymbol{w}$ to $\tilde{\boldsymbol{w}}$ so analytic continuation around $P$ gives the new normalized period matrix $\tilde{\Pi}=\Pi_{\tilde{a}_{p}, \tilde{b}_{p}}$.

(B.10b) Express a translate of $\tilde{\boldsymbol{w}}$ that gives the new $\theta$, a function of $\left(\tilde{\boldsymbol{w}}, \Pi_{\tilde{\boldsymbol{a}}, \tilde{\boldsymbol{b}}}\right)$, as an explicit multiple of $\theta\left[\begin{array}{l}\boldsymbol{\delta} \\ \boldsymbol{\epsilon}\end{array}\right]\left(\boldsymbol{w}, \Pi_{\boldsymbol{a}, \boldsymbol{b}}\right)$.

Express $\Pi_{\tilde{\boldsymbol{a}} \tilde{\boldsymbol{b}}}$ as $\left(A_{P} \Pi_{\boldsymbol{a}, \boldsymbol{b}}+2 \pi i B_{P}\right)\left(C_{P} \Pi_{\boldsymbol{a}, \boldsymbol{b}}+2 \pi i D_{P}\right)^{-1}$. Denote $C_{P} \Pi_{\boldsymbol{a}, \boldsymbol{b}}+2 \pi i D_{P}$ by $M_{P}=M_{T_{P}}\left(\Pi_{\boldsymbol{a}, \boldsymbol{b}}\right)=M$. To simplify, refer to $T_{P} \in \mathrm{Sp}_{2 g}(\mathbb{Z})$ as $T$. Then, ${ }^{\mathrm{t}}\left(\tilde{\boldsymbol{a}}_{\boldsymbol{p}}, \tilde{\boldsymbol{b}}_{\boldsymbol{p}}\right)=\left(\begin{array}{cc}D & C \\ B & A\end{array}\right)^{\mathrm{t}}(\boldsymbol{a}, \boldsymbol{b})$ and $2 \pi i \boldsymbol{w}=\tilde{\boldsymbol{w}} M$. For a $g \times g$ matrix $U$, use bracket notation $\{U\}$ for the vector of diagonal elements (as in [Sh98]). The result:

$$
\begin{aligned}
\text { with } \quad\left[\begin{array}{c}
\tilde{\boldsymbol{\delta}} \\
\tilde{\boldsymbol{\epsilon}}
\end{array}\right]=\left(\begin{array}{cc}
D & -C \\
-B & A
\end{array}\right)\left[\begin{array}{l}
\boldsymbol{\delta} \\
\boldsymbol{\epsilon}
\end{array}\right]+\frac{1}{2}\left[\begin{array}{l}
\left\{C^{\mathrm{t}} D\right\} \\
\left\{A^{\mathrm{t}} B\right\}
\end{array}\right], \theta\left[\begin{array}{l}
\tilde{\boldsymbol{\delta}} \\
\tilde{\boldsymbol{\epsilon}}
\end{array}\right]\left(\tilde{\boldsymbol{w}}, \Pi_{\tilde{\boldsymbol{a}}, \tilde{\boldsymbol{b}}}\right)= \\
K_{\boldsymbol{\delta}, \boldsymbol{\epsilon}, T} \operatorname{det}(M)^{\frac{1}{2}} \exp \left(\frac{1}{2}\left(\boldsymbol{w} \nabla_{\Pi} \ln (\operatorname{det}(M))^{\mathrm{t}} \boldsymbol{w}\right) \theta\left[\begin{array}{l}
\boldsymbol{\delta} \\
\boldsymbol{\epsilon}
\end{array}\right]\left(\boldsymbol{w}, \Pi_{\boldsymbol{a}, \boldsymbol{b}}\right) .\right.
\end{aligned}
$$

Here $K_{\boldsymbol{\delta}, \boldsymbol{\epsilon}, T}$, having absolute value 1 , depends on which branch of $\operatorname{det}(M)^{\frac{1}{2}}$ we have chosen. When $g=1$, FaK01, p. 101] has $K_{\boldsymbol{\delta}, \boldsymbol{\epsilon}, T}$ explicitly, recognizing $K_{\mathbf{0}, \mathbf{0}, T}$ as 
an 8th root of 1 . View $\nabla_{\Pi}$ as producing the matrix whose $(i, j)$ entry is the partial with respect to the variable in the $(i, j)$ position of $\Pi$.

B.4.2. Monodromy on a Hurwitz fiber. Suppose $\mathcal{H}$ is a Hurwitz family (any $r$ ) for some absolute Nielsen class from $\left(G \leq S_{n}, \mathbf{C}\right)$ (or inner cases).

Definition B.4. Call $\left(G \leq S_{n}, \mathbf{C}\right) g$ (alois)c(losure)-ramified if for any cover $\varphi: X \rightarrow \mathbb{P}_{z}^{1}$ in the Nielsen class, there is no unramified cover $Y \rightarrow X$, of degree exceeding 1, fitting in a diagram $\hat{X} \rightarrow Y \rightarrow X$ with the Galois closure $\hat{X}$ of $\varphi$.

The notion gc-ramified (without the name) is from [Fri89 Lem. 3.3] which characterizes it using a branch cycle description $\boldsymbol{g}$ for $\varphi$. It is usual that $\varphi$ is gcramified (true in our cases), though nonpathological examples abound where it is not [Fri89 Ex. 3.4]. Let $H$ be any subgroup of $G(1)$. Consider the permutation representation $T_{H}: G \rightarrow S_{(G: H)}$ on $H$ cosets. There is a map from disjoint cycles of $T_{H}(g), g \in G$, to disjoint cycles of $g$ : Any $g$ orbit of $H$ cosets goes to the corresponding orbit of $G(1)$ cosets. Call $g$ unramified in $H$ if the lengths of the disjoint cycle of $T_{H}(g)$ and the corresponding disjoint cycle of $g$ are equal.

Lemma B.5. Let $H_{t}(\boldsymbol{g})$ be minimal among $H \leq G(1)$ with $g_{i}$ unramified in $H$, $i=1, \ldots, r$. Then, $(G, \mathbf{C})$ is gc-ramified if and only if $H_{t}(\boldsymbol{g})=G(1)$.

In the notation above, let $\boldsymbol{z}$ be the branch points of $\varphi, P_{1}, \ldots, P_{r}$ a set of classical generators of $\pi_{1}\left(U_{\boldsymbol{z}}, z_{0}\right)$ (1.2), and $g$ the genus of $X$. By a $\theta$-null we mean a function of the form $\theta\left[\begin{array}{l}\boldsymbol{\delta} \\ \boldsymbol{\epsilon}\end{array}\right]\left(\mathbf{0}, \Pi_{\boldsymbol{a}, \boldsymbol{b}}\right) \neq 0$, a $\theta$ with characteristic evaluated at $\mathbf{0}$. Let $S^{1}$ be the multiplicative group of complex numbers having absolute value 1 . The expression $f(\boldsymbol{p}) \bmod S^{1}$ refers to a function at $\boldsymbol{p} \in \mathcal{H}^{\text {rd }}$ up to multiplication by elements in $S^{1}$.

Proposition B.6. Assume $\left(G \leq S_{n}, \mathbf{C}\right)$ is gc-ramified. Then there is an effective procedure for computing $H_{r}$ on $\pi_{1}(X)$. Assume $r=4$. This action extends to $\bar{M}_{4}$ acting on the reduced Nielsen classes. For $\boldsymbol{g} \in \mathrm{Ni}(G, \mathbf{C})^{\mathrm{rd}} / N$, let $\bar{M}_{\boldsymbol{g}}$ be the stabilizer in $\bar{M}_{4}$ of the reduced class of $\boldsymbol{g}$. Then, there is an effective procedure for computing $\bar{M}_{\boldsymbol{g}}$ acting on $\pi_{1}(X)$ (extending the action of 8.2 and Prop. 4.4). This induces a map $\varphi_{*}: \bar{M}_{\boldsymbol{g}} \rightarrow \mathrm{Sp}_{2 g}(\mathbb{Z})$ through which $\bar{M}_{\boldsymbol{g}}$ acts as in (B.11).

Suppose elements in the classes $\mathbf{C}$ all have odd order and there is an even half-canonical class on $X_{\boldsymbol{p}}$ given by $d \varphi_{\boldsymbol{p}}$ according to the conclusion of Prop. B.1.

Let $\left[\begin{array}{l}\boldsymbol{\delta}_{\boldsymbol{p}} \\ \boldsymbol{\epsilon}_{\boldsymbol{p}}\end{array}\right]$ be the characteristic corresponding to it (relative to $\boldsymbol{a}_{\boldsymbol{p}}, \boldsymbol{b}_{\boldsymbol{p}}$ ) at the point $\boldsymbol{p} \in \mathcal{H}^{\mathrm{rd}}$. If $\theta\left[\begin{array}{l}\boldsymbol{\delta}_{\boldsymbol{p}} \\ \boldsymbol{\epsilon}_{\boldsymbol{p}}\end{array}\right]\left(\mathbf{0}, \Pi_{\boldsymbol{a}_{\boldsymbol{p}}, \boldsymbol{b}_{\boldsymbol{p}}}\right)$ is not 0 at all points $\boldsymbol{p}$, then the action of $\varphi_{*}$ gives a $\theta$-null $\bmod S^{1}$, an automorphic function on $\mathcal{H}^{\mathrm{rd}}$.

Comments. The effective procedure for computing the monodromy is Fri89 §3.2]. For $\gamma, \gamma^{\prime} \in \bar{M}_{4}$, consider $T_{\gamma}, T_{\gamma^{\prime}}$ the corresponding elements in $\operatorname{Sp}_{2 g}(\mathbb{Z})$, so and $M_{\gamma}\left(\Pi_{\boldsymbol{a}, \boldsymbol{b}}\right)$ and $M_{\gamma^{\prime}}\left(\Pi_{\boldsymbol{a}, \boldsymbol{b}}\right)$ are the corresponding $M$ factors. Then,

$$
M_{\gamma^{\prime} \gamma}\left(\Pi_{\boldsymbol{a}, \boldsymbol{b}}\right)=M_{\gamma^{\prime}}\left(\gamma\left(\Pi_{\boldsymbol{a}, \boldsymbol{b}}\right) M_{\gamma}\left(\Pi_{\boldsymbol{a}, \boldsymbol{b}}\right) \quad \bmod S^{1}\right. \text { Sh98, p. 177]. }
$$

So, $\bmod S^{1}$, the $M$ s are factors of automorphy.

From [Sh98 Thm. 27.2], however, finding factors of automorphy without modding out by $S^{1}$ improves if we know $T_{\gamma}$ is in the level 2 congruence subgroup of $\mathrm{Sp}_{2 g}(\mathbb{Z})$. Then, the 4 th power of the $\theta$-null is an automorphic function. 
B.4.3. Automorphic functions and Pryms along $\mathcal{H}^{\mathrm{rd}}$. Again, suppose elements in the classes $\mathbf{C}$ all have odd order and $d \varphi_{\boldsymbol{p}}$ gives an even half-canonical class on $X_{\boldsymbol{p}}$ as in the conclusion of Prop. B.1. Let $\hat{G} \rightarrow G$ be the spin cover. Let $\hat{\boldsymbol{w}}_{\boldsymbol{p}} \in \mathbb{C}^{\hat{g}}$ represent the unique $G$ invariant nontrivial 2-division point on $\operatorname{Pic}^{(0)}\left(\hat{X}_{\boldsymbol{p}}\right)$ corresponding to the unramified spin cover $\psi_{\boldsymbol{p}}: Y_{\boldsymbol{p}} \rightarrow \hat{X}_{\boldsymbol{p}}$ corresponding to spin separation. Denote the genus of $\hat{X}_{p}$ by $\hat{g}$, global holomorphic differentials on $Y_{\boldsymbol{p}}$ by $\Gamma\left(\Omega^{1}\left(Y_{\boldsymbol{p}}\right)\right)$. As in Mu76 p. 91], $\operatorname{Pic}\left(Y_{\boldsymbol{p}}\right)^{(0)}$ is isogenous to $\operatorname{Pic}\left(\hat{X}_{\boldsymbol{p}}\right)^{(0)} \times \mathcal{A}_{\boldsymbol{p}}$ with $\mathcal{A}_{\boldsymbol{p}}$ (the Prym) an abelian variety of dimension $\hat{g}-1$. Properties of this construction:

(B.12a) The tangent space at $0 \in \mathcal{A}_{p}$ identifies with the -1 eigenspace, of the involution of $Y_{\boldsymbol{p}}$ commuting with $\psi_{\boldsymbol{p}}$, on $\Gamma\left(\Omega^{1}\left(Y_{\boldsymbol{p}}\right)\right)$.

(B.12b) The isogenies between $\operatorname{Pic}\left(\hat{X}_{\boldsymbol{p}}\right)^{(0)} \times \mathcal{A}_{\boldsymbol{p}}$ and $\operatorname{Pic}\left(Y_{\boldsymbol{p}}\right)^{(0)}$ have degree $2^{2 g-1}$ in each direction, composing to multiplication by 2 , with $\hat{w}_{p}$ generating $\operatorname{ker}\left(\operatorname{Pic}\left(\hat{X}_{\boldsymbol{p}}\right)^{(0)} \rightarrow \operatorname{Pic}\left(Y_{\boldsymbol{p}}\right)^{(0)}\right) \stackrel{\text { def }}{=} \mathbb{Z} / 2(\boldsymbol{p})$.

More than just the involution of $Y_{\boldsymbol{p}}$, all of $\hat{G}$ acts on this construction. Analysis of this prym varying canonically with $\boldsymbol{p}$ will be in the final version of [Fri96] (see E.2.2 for the easiest appearance of a Prym).

Consider again $\boldsymbol{p} \in \mathcal{H}\left(A_{5}, \mathbf{C}_{3^{4}}\right)^{\text {in,rd }}$. Is there a nondegenerate $\theta$-null (nonzero and varying with $\boldsymbol{p}$ ) through $X_{\boldsymbol{p}}$ represented as a point on the moduli space of curves of genus 21? This is what Prop. B.6 has as an hypothesis. This requires at least that the image $\left[X_{\boldsymbol{p}}\right]$ of the curve for $\boldsymbol{p} \in \mathcal{H}_{0}^{\text {in,rd }}$ vary nontrivially in the moduli space $\mathcal{M}_{21}$ as $\boldsymbol{p}$ varies. FKK01 Main Thm] shows the moduli of all families of inner $\left(A_{n}, \mathbf{C}_{3^{r}}\right)$ covers, $r \geq n$ as in Ex. 3.17 varies nontrivially.

Our case, however, is inner covers with $r=n-1=4$, so this does not apply directly. It is more elementary. Let $\mathcal{H}(G, \mathbf{C})^{\text {in,rd }}$ be an inner reduced Hurwitz space with $r \geq 4$, where the genus $g$ of the covers exceeds 1 .

Lemma B.7. The moduli map $\boldsymbol{p} \in \mathcal{H}(G, \mathbf{C})^{\mathrm{in}, \mathrm{rd}} \mapsto\left[X_{\boldsymbol{p}}\right] \in \mathcal{M}_{g}$ is nonconstant.

ProOF. Restrict the argument to any geometrically connected component of $\mathcal{H}(G, \mathbf{C})^{\mathrm{in}, \mathrm{rd}}$. Assume the result is false and $X=X_{\boldsymbol{p}}$ is the constant image of the moduli map. So, with $\boldsymbol{p}$ running over $\mathcal{H}(G, \mathbf{C})^{\text {in,rd }}$, there are an infinite number of inequivalent Galois covers $\varphi_{\boldsymbol{p}}: X \rightarrow \mathbb{P}_{z}^{1}$ with group $G_{\boldsymbol{p}}$ (isomorphic to $G$, though not necessarily equal to it). Consider the group $G^{*}$ these $G_{p}$ s generate in the automorphism group of $X$. Since $g(X) \geq 2$, this is a finite group. So there are only finitely many covers up to equivalence in this class. The family is connected: There is only one cover up to equivalence, contradicting the above.

Fay73 Cor. 3.2] provides coordinates for period matrix degeneration as points on $\mathcal{M}_{g}$ approach its boundary. The simplest corollary considers being near that part of the boundary with period matrices close to products of period matrices of elliptic curves. All the even $\theta$-nulls are nonzero and all the odd $\theta$-nulls are nondegenerate on an elliptic curve. E.2.3 therefore shows $\mathcal{H}\left(A_{4}, \mathbf{C}_{3^{4}}\right)^{\mathrm{rd},+}$ has a nonzero even $\theta$ null.) So, these coordinates show the same holds for jacobians close to such points. For H-M components of Modular Tower levels, although the degeneration isn't quite so simple, this type of analysis also works. This is the Mumford half of the reason for calling them Harbater-Mumford representatives (continuing in [Fri96]). 


\section{Appendix C. Covers and lifting invariants}

Computations toward Main Conjecture Prob.1.1 - showing the genus of components of levels of a reduced Modular Tower go up - require recognizing the organization of $\gamma_{\infty}$ orbits within the $\bar{M}_{4}$ orbits on the Nielsen class. Every curve cover in a Nielsen class deforms to a cover associated with collections of cusps. Equivalencing covers according to which cusps are in their deformation range (call this $d$ (eformation)- equivalence) is equivalent to finding $\bar{M}_{4}$ orbits.

Acting with a braid generator on Nielsen classes is quite elementary. Yet, as the main examples of this paper show, finding $\bar{M}_{4}$ orbits is usually difficult. Further, even when we find from $\bar{M}_{4}$ action that there are several components, we need to know if these components are conjugate under $G_{\mathbb{Q}}$. In significant cases we expect intrinsic geometry (of covers in our case) to inform $G_{\mathbb{Q}}$ about d-equivalence. Our examples show that augmenting bare braid group computations with lifting invariants often reveals such intrinsic geometry. Two covers certainly have differences in their d-equivalence classes if one has unramified covers with a given group the other does not have. While there are other ways to say they have different geometries, this and its generalizations are d-equivalence invariants. The remainder of this section formulates this purely group theoretically.

C.1. The lifting invariant and Nielsen separation. Up to d-equivalence, assume two covers (in the same Nielsen class) whose $H_{r}$ orbits we wish to compare have the same branch points $\boldsymbol{z}$ so can compare them as covers by their branch cycles using the same classical generators of $\mathbb{P}_{z}^{1} \backslash\{z\}$. If the monodromy group of the cover is $G$, let these branch cycles be $\boldsymbol{g}=\left(g_{1}, \ldots, g_{r}\right) \in G^{r}$ and $\boldsymbol{g}^{\prime}=\left(g_{1}^{\prime}, \ldots, g_{r}^{\prime}\right) \in G^{r}$ (both in $\operatorname{Ni}(G, \mathbf{C})$; 3.1 .1 .

Consider $\psi_{H}: H \rightarrow G$, and a choice of $\mathbf{C}_{H}: r$ conjugacy classes lifting to $H$ those of $\mathbf{C}$.

For $\boldsymbol{g} \in \mathrm{Ni}(G, \mathbf{C})$, consider the $\left(H, \mathbf{C}_{H}\right)$-lifting invariant:

$$
s_{H, \mathbf{C}_{H}}(\boldsymbol{g})=\left\{\Pi\left(\boldsymbol{g}^{*}\right) \mid \boldsymbol{g}^{*}=\boldsymbol{g} \bmod \operatorname{ker}\left(\psi_{H}\right), \boldsymbol{g}^{*} \in \mathbf{C}_{H},\left\langle\boldsymbol{g}^{*}\right\rangle=H\right\} .
$$

Use $\mathcal{Y}_{\boldsymbol{g}}$ for the collection of $s_{H, \mathbf{C}_{H}}(\boldsymbol{g})$ running over all $\left(H, \mathbf{C}_{H}\right)$ to separate d-inequivalent elements in $\operatorname{Ni}(G, \mathbf{C})$. Two cases appear often in this paper.

(C.1a) $G \leq A_{n}$ for some integer $n$ and $H=\hat{G}$ is the pullback of $G$ to $\operatorname{Spin}_{n}$ in Prop. 5.9

(C.1b) $G=G_{k}$ is the $k$ characteristic quotient of the universal $p$-Frattini cover of the group $G_{0}$, and $H=R_{k}^{\prime}$ in Prop. 9.6

When $p=2$, and $G_{0}=A_{n}$, we may compare (C.17n with C.1b), and ask if the latter is a case of the former through some embedding of $G_{k}$ in an alternating group. That is exactly what happens in our main example at level 1 (Prop. 9.8). The following easy lemma slightly generalizes [Fri95a, Lem. 3.12]

Lemma C.1. $\mathcal{Y}_{\boldsymbol{g}}$ is an $H_{r}$ invariant:

$$
s_{H, \mathbf{C}_{H}}(\boldsymbol{g})=s_{H, \mathbf{C}_{H}}((\boldsymbol{g}) Q) \text { for } Q \in H_{r},
$$

any $\left(H, \mathbf{C}_{H}\right)$. It is a d-equivalence invariant.

Definition C.2. Call $\boldsymbol{g}$ and $\boldsymbol{g}^{\prime}$ in $\mathrm{Ni}(G, \mathbf{C})$ Nielsen separated if the collection $\mathcal{Y}_{g}$ differs from $\mathcal{Y}_{g^{\prime}}$ 
QuESTION C.3. If $\boldsymbol{g}$ and $\boldsymbol{g}^{\prime}$ are in separate d-equivalence classes, are $\mathcal{Y}_{\boldsymbol{g}}$ and $\mathcal{Y}_{\boldsymbol{g}}^{\prime}$ provably (computationally, significantly) different so as to detect this?

C.2. Frattini central extensions. The case of Nielsen separation from this paper is where $\psi: H \rightarrow G$ is a Frattini central extension with $\operatorname{ker}(\psi)$ of order prime to the orders of elements in $\mathbf{C}$. Construct $\mathbf{C}_{H}$ as the conjugacy classes $\mathbf{C}$ of $G$, each lifted (uniquely) to $H$ to have the same order. The next lemma follows easily from the technique of [FV91, App].

Lemma C.4. For $\boldsymbol{g} \in \mathrm{Ni}(G, \mathbf{C}), s_{H, \mathbf{C}_{H}}(\boldsymbol{g})=s(\boldsymbol{g})$ is a single element. Assume $\mathrm{Ni}(G, \mathbf{C}) \neq \emptyset$ and

(C.2) conjugacy classes in $\mathbf{C}$ appear many times.

Then $\{s(\boldsymbol{g}) \mid \boldsymbol{g} \in \operatorname{Ni}(G, \mathbf{C})\}=\operatorname{ker}(\psi)$. So, there are at least $|\operatorname{ker}(\psi)|$ orbits for $H_{r}$ on $\operatorname{Ni}(G, \mathbf{C})$.

General Nielsen separation uses complicated witnessing pairs $\left(H, \mathbf{C}_{H}\right)$. In the last example, the $\left(H, \mathbf{C}_{H}\right)$ witnessing separation is a small cover of $(G, \mathbf{C})$. Lem.C.4 shows that embeddings in alternating groups are only a tiny part of the story of general Nielsen separation of orbits of $H_{r}$.

For instance, consider the universal $p$-Frattini cover ${ }_{p} \tilde{G}$ of any simple group $G$ $(p|| G \mid$; Thm. 5.21) or $p$-split, $p$-perfect group with non-cyclic $p$-Sylow (Prop. 5.3). Then, ${ }_{p} \tilde{G}$ has infinitely many quotients $G^{\prime}$ with $H^{\prime} \rightarrow G^{\prime}$ a central (Frattini) extension with $\operatorname{ker}\left(H^{\prime} \rightarrow G^{\prime}\right)=\mathbb{Z} / p$. In any of these circumstances, consider such a group $G^{\prime}$ and corresponding $p^{\prime}$ conjugacy classes $\mathbf{C}^{\prime}$ satisfying the hypotheses of Lem. C.4. Then, the Hurwitz space $\mathcal{H}\left(G^{\prime}, \mathbf{C}^{\prime}\right)$ has at least $p$ components. For, however, a fixed $G, p$, and $r$ (say, $r=4$ ) consider for which $r$-tuples of $p^{\prime}$ conjugacy classes $\mathbf{C}$, does the collection of covers $H^{\prime} \rightarrow G^{\prime}$ Nielsen separate $H_{r}$ (equivalently $\left.\bar{M}_{4}\right)$ orbits on $\operatorname{Ni}\left(G^{\prime}, \mathbf{C}\right)$ (with $G^{\prime}$ running over the mentioned quotients of ${ }_{p} \tilde{G}$ ).

\section{Appendix D. The Open Image Theorem}

Use the notation of Prop.4.16 Figuring the image of $G_{K}$ (up to subgroups of finite index $)$ in $\operatorname{Hom}^{*}\left(\pi_{1}^{(p)}, \operatorname{ker}_{0}\right) / \operatorname{ker}_{0}$ or in $\left.\operatorname{Hom}^{*}\left(\mathbb{T}_{p}, \operatorname{ker}_{0}\right) /\left(\operatorname{ker}_{0}, \operatorname{ker}_{0}\right)\right)$ generalizes the goal of Serre's Theorem in Ser68. One goal would be to decide (find situations) when this action has an open orbit.

D.1. Interpreting and extending the Open Image Theorem. From the Hurwitz viewpoint, $\mathbf{S e r 6 8}$ is the case $G_{0}=D_{p}$, where $X_{\boldsymbol{p}_{0}}$ is an elliptic curve. It says, the image of $G_{K}$ is an open subgroup of the general linear group acting on the Tate module of $X_{\boldsymbol{p}_{0}}$, if $X_{\boldsymbol{p}_{0}}$ has no complex multiplications. Otherwise, its image is an open subgroup of the Tate module automorphisms commuting with the ring of endomorphisms.

Much of Ser68 describes the complex multiplication case. Still, the guiding theorem is Ser68, IV-20]: If $j(E)$ is not an algebraic integer (at $p$ ), then the image of $G_{K}$ in the Tate module of $E$ is the full general linear group. Further, in its closing pages, Ser68 outlines the case when $j(E)$ is integral and $E$ has no complex multiplication. Prop. 4.15 has a framework for considering these results applied to a Modular Tower where direct analogs would start with one prime $\ell$ (with $\ell$ not dividing $G$ ). We'll use $\boldsymbol{p}_{0} \in \mathcal{H}\left(A_{5}, \mathbf{C}_{3^{4}}\right)^{\text {in }}(\overline{\mathbb{Q}})$ for explicit comments.

Since $\mathcal{H}\left(A_{5}, \mathbf{C}_{3^{4}}\right)^{\text {in,rd }}$ covers the $j$-line, interpret $\boldsymbol{p}_{0} \in \mathcal{H}\left(A_{5}, \mathbf{C}_{3^{4}}\right)^{\text {in }}(\overline{\mathbb{Q}})$ as being $\ell$-adically close to $\infty$ if its $j$-invariant is sufficiently negative for the $\ell$-adic valuation. 
A first goal is to find the largest (up to equivalence according to the Lie algebra of the image) possible action of $G_{K}$ on projective systems of points lying over $\boldsymbol{p}_{0} \in \mathcal{H}\left(A_{5}, \mathbf{C}_{3^{4}}\right)^{\text {in }}(K)$. Finding how to estimate how $\ell$-adically close to $\infty$ would be part of this.

We make simplifying assumptions to propose a program. We haven't yet found a good analog on a general ( $p$-perfect) Modular Tower. There are two parts. SD.2 reminds of the place where a $p$-Frattini covering property enters in Serre's Open Image Theorem. $\mathrm{D} .3$ gives a precise test case for starting from Serre's situation using $j$ values $\ell$-adically close to $\infty$ for some prime $\ell$.

D.2. $p$-part of the outer monodromy group. Let $\operatorname{HMP}\left(A_{5}, \mathbf{C}_{3^{4}}\right)$ be the subset of projective systems $\left\{\boldsymbol{p}_{k}\right\}_{k=0}^{\infty}$ (points) on the $\left(A_{5}, \mathbf{C}_{3^{4}}, 2\right)$ Modular Tower lying over $\boldsymbol{p}_{0} \in \mathcal{H}\left(A_{5}, \mathbf{C}_{3^{4}}\right)^{\text {in,rd }}$ (so they correspond to elements of $\operatorname{Ni}\left({ }_{p} \tilde{G}, \mathbf{C}\right)^{\text {in }}$ (Prop. 4.15) with this additional property:

(D.1) Each $\boldsymbol{p}_{k}$ lies in a component at level $k$ containing H-M reps.

An absolute Galois group preserves the collection of H-M components Fri95a, proof Lem. 3.22]. So, it is a special case of the problem to describe the H-M components at every level (see Rem. D.3).

Let $K$ be a number field. Consider an arbitrary reduced Modular Tower (possibly inner, or absolute) $\left\{\mathcal{H}_{k}^{\text {rd }}\right\}_{k=0}^{\infty}$. Apply the notation of to a projective system of $K$ components $\left\{\mathcal{H}_{k}^{\prime}\right\}_{k=0}^{\infty}$. Specifically, let $H_{k, j}^{\prime}$ be the (geometric) monodromy group of the map $\mathcal{H}_{k}^{\prime} \rightarrow \mathcal{H}_{j}^{\prime}$ with $H_{k}^{\prime}$ the (geometric) monodromy group from $\mathcal{H}_{k}^{\prime} \rightarrow \mathbb{P}_{j}^{1} \backslash\{\infty\}$. The Ser68 case is $\cdots \rightarrow Y_{0}\left(p^{k+1}\right) \rightarrow Y_{0}\left(p^{k}\right) \rightarrow \cdots \rightarrow \mathbb{P}_{j}^{1} \backslash\{\infty\}$, the arithmetic monodromy groups are $\mathrm{GL}_{2}\left(p^{k+1}\right)$ while the geometric monodromy groups are $\mathrm{SL}_{2}\left(p^{k+1}\right)$ Ser92 p. 47]. Lem. 7.1 computes the geometric and arithmetic monodromy groups for level 0 of the $\left(A_{5}, \mathbf{C}_{3^{4}}, p=2\right)$ Modular Tower. The modular curve tower has $p$-groups for $H_{k, 0}$. notes that for the $\left(A_{5}, \mathbf{C}_{3^{4}}, p=2\right)$ Modular Tower, $H_{1,0}^{\prime}$ (for either component) is not a 2-group as its order is divisible by 1 power of 3 .

A basic lemma is that $\operatorname{PSL}_{2}\left(\mathbb{Z}_{p}\right) \rightarrow \operatorname{PSL}_{2}(\mathbb{Z} / p)$ is a Frattini cover if $p \geq 5$ Ser68 IV-23]. For $p=3$ : $\mathrm{PSL}_{2}(3)$ is $A_{4}$ - acting on the 4 points of the projective plane. So, it is not 3- perfect and its universal 3-Frattini cover is $K_{4} \times{ }^{s} \mathbb{Z}_{3}$.

For $p \geq 5$, the result is a very strong Hilbert's Irreducibility Theorem for this situation hidden in $\mathbf{S e r 6 8}$. It starts by assuming $\boldsymbol{p}_{0} \in Y_{0}(p)$, and the Galois closure of $K(\boldsymbol{p}) / K$ contains $\mathrm{SL}_{2}(\mathbb{Z} / p)$. The conclusion: For any $k \geq 0$ and $\boldsymbol{p}_{k} \in Y_{0}\left(p^{k+1}\right)$ over $\boldsymbol{p}_{0}$, the Galois closure of $K\left(\boldsymbol{p}_{k+1}\right) / K$ equals the full arithmetic monodromy group of $Y_{0}\left(p^{k+1}\right) \rightarrow \mathbb{P}_{j}^{1} \backslash\{\infty\}$. This is the strongest conclusion supporting the open image theorem. Considering Modular Towers requires addressing the next problem, especially in the $p$-split case (the topic of [Fr01]).

Problem D.1. Assume $G_{0}$ is $p$-perfect. Characterize when for some $j \geq 1$, $\lim _{\infty \leftarrow k} H_{k}^{\prime}$ is a $p$-Frattini cover of $H_{j}^{\prime}$ ?

To understand a general Modular Tower requires considering geometric components other than H-M types that could provide projective (unobstructed) systems of components. Here is an example. Suppose a collection of conjugacy classes $\mathbf{C}$ satisfies the following properties.

(D.2a) $\mathrm{Ni}\left(G_{0}, \mathbf{C}\right)$ contains no H-M reps (say, if $r$ is odd).

(D.2b) $\mathrm{Ni}\left(G_{k}, \mathbf{C}\right)$ is nonempty for all $k \geq 0$. 
Example D.2 (Geometry of other than H-M components). Example: Suppose $r=3 t$ with $t$ odd and with all conjugacy classes equal to $\mathrm{C}$, a rational $p^{\prime}$ conjugacy class of an element of order $t$. Assume also: $G_{0}=\left\langle g_{1}, g_{2}, g_{3}\right\rangle$ with $g_{i} \in \mathrm{C}, i=1,2,3$. If $\tilde{g}_{i}$ is a lift of $g_{i}$ to $\mathrm{C}$ in $G_{k}$, then

$$
(\underbrace{\tilde{g}_{1}, \ldots, \tilde{g}_{1}}_{t \text { times }}, \underbrace{\tilde{g}_{2}, \ldots, \tilde{g}_{2}}_{t \text { times }}, \underbrace{\tilde{g}_{3}, \ldots, \tilde{g}_{3}}_{t \text { times }}) \in \mathrm{Ni}\left(G_{k}, \mathbf{C}\right) .
$$

Remark D.3 (How many H-M components at each level?). It would help if there were just one H-M component at each level of a Modular Tower. For a general Modular Tower a simple assumption guarantees this [Fri95a Th. 3.21]. This assumption, however, holds in none of the cases when $r=4$ in this paper. The conclusion can even be false: Ex. 9.2 has two H-M components at level 1. Fri96. gives an explicit $r_{0}=r_{0}(n)$ for the Modular Towers associated to $\left(A_{n}, \mathbf{C}_{3^{r}}, 2\right)$ so that each level of the Modular Tower has exactly one H-M component (though possibly many components if the level $k$ is large) if $r \geq r_{0}$. If $r=n-1$ or $n$, however, the number of H-M components is still a mystery.

D.3. Finding the Frobenius. Consider $\left.\mathbb{P}^{4}(\overline{\mathbb{Q}((t))}) \backslash D\right) \times \mathbb{P}_{z}^{1}$ over the field $\mathbb{Q}((t))=K$ of Laurent series. Regard $\mathrm{PSL}_{2}(\bar{K})$ as acting as in $\$ 3.7$ (except extending to the $\mathbb{P}_{z}^{1}$ component). Let $d$ be a squarefree integer. Start with a tangential base point convenient for such an $\ell$-adic investigation. A convenient example is that from We01, §2]:

$$
\left.\operatorname{Spec}(\mathbb{Q}((t))) \rightarrow \mathbb{P}^{4}(\overline{\mathbb{Q}((t))}) \backslash D\right) \times \mathbb{P}_{z}^{1} / \mathrm{PSL}_{2}(\overline{\mathbb{Q}(t)})
$$

by using the unordered four points $S=\{ \pm \sqrt{d} \pm i \sqrt{t}\}=\left\{z_{1}, \ldots, z_{4}\right\}$ in the first position and $z_{0}=0$ (a fixed base point) in the last position. To extend the map to $\operatorname{Spec}(\mathbb{Q}[[t]])=\operatorname{Spec}(R)$ requires forming a special fiber in place of $\mathbb{P}_{z}^{1}$ over $j=\infty$. Extending the Stable Compactification Theorem appropriately to covers replaces $\mathbb{P}_{z}^{1}$ by three copies $\mathbb{P}_{z}^{1}, \mathbb{P}_{w_{5}}^{1}$ and $\mathbb{P}_{w_{6}}^{1}$ tied together as on the left side of Table 5.

TABle 5. The configuration of $\mathbb{P}^{1} \mathrm{~s}$ at a special fiber
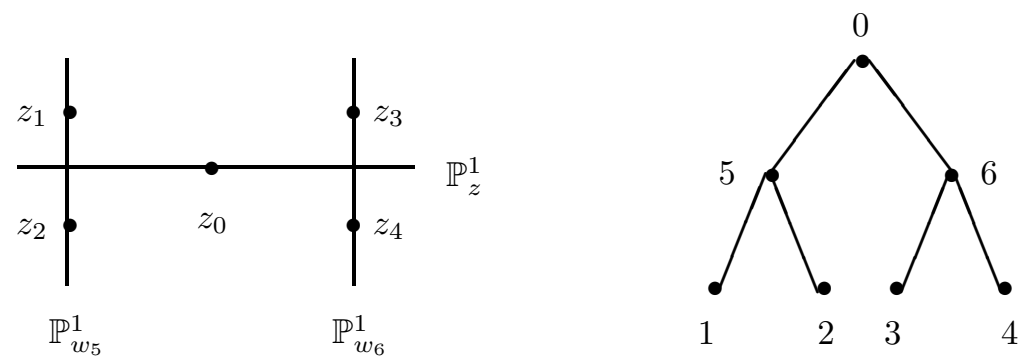

Each of the two pairs of points coming together (complex conjugate pairs) is wandering in $t$ space. Add a line of directions for the approach of each pair. Each pair degenerates to the common limit of the two points with the addition of the direction of their coming together in the copy of $\mathbb{P}_{z}^{1}$. The downward pointing tree on the right in Table 5 has root 0 labeled for the component $\mathbb{P}_{z}^{1}$, one edge each for $\mathbb{P}_{w_{5}}^{1}$ and $\mathbb{P}_{w_{6}}^{1}$ (with corresponding vertices 5 and 6 ) and four leaf edges terminating in vertices corresponding to the points $z_{1}, \ldots, z_{4}$. 
Computing with this amounts to finding explicit action of $G_{\mathbb{Q}((t))}$ on the elements of the Nielsen classes $\mathrm{Ni}\left(G_{k}, \mathbf{C}_{3^{4}}\right)^{\text {in,rd }}$ representing germs of covers approaching the special fiber. For example, in the complex conjugate pairs business above, identify each component with a choice of coordinate $w$. That identifies the function field of the copy of $\mathbb{P}_{K}^{1}$ (modulo the action of $\mathrm{PGL}_{2}(R)$ ). For the root component use $z$. For $\mathbb{P}_{w_{5}}^{1}$, the component from $z_{1}$ and $z_{2}$ coming together, choose $w_{5}=(z+\sqrt{d}) / \sqrt{t}$. For $\mathbb{P}_{w_{6}}^{1}$ choose $w_{6}=(z-\sqrt{d}) / \sqrt{t}$. Then,

$$
z_{1}=-\sqrt{d}+i \sqrt{t}, z_{2}=-\sqrt{d}-i \sqrt{t}, z_{3}=\sqrt{d}+i \sqrt{t}, z_{4}=\sqrt{d}-i \sqrt{t} .
$$

As $t \mapsto 0, z_{1}$ and $z_{2}$ have limit $-\sqrt{d}$ on $\mathbb{P}_{z}^{1}$. On, however, $\mathbb{P}_{w_{5}}^{1}$, the $w_{5}$ values representing the limit of $z_{1}$ and $z_{2}$ are $i$ and $-i$. Similarly for $z_{3}$ and $z_{4}$ coming together, given by $\sqrt{d}$ on $\mathbb{P}_{z}^{1}$ and $w_{6}$ values $i$ and $-i$.

Let $q_{0}$ be the generator of $G(\overline{\mathbb{Q}((t))} / \overline{\mathbb{Q}}((t)))$. The coordinates on each component give the $q_{0}$ action on the tree. Extend it to the fundamental group of the tree. The assumptions for this explicit action are in We01. First: Identify $t^{1 / n} \mapsto \zeta_{n} t^{1 / n}$ as a braid by recognizing its action on the tree. As in our example, this is from writing $z_{1}, \ldots, z_{r} \in \mathbb{C}\left(t^{1 / n}\right)$ explicitly as $z_{i}\left(t^{1 / n}\right)$. You get the image of the braid in $S_{r}$ just from its action on the power series for $z_{1}, \ldots, z_{r}$. Vertices $v$ of the tree consist of $S \cup\left\{z_{0}\right\}$ together with the components of the special fiber. Edges are from a point of $S$ lying on a component, or from the meeting of two components. Suppose an edge $e=\left(v_{1}, v_{2}\right)$ represents the component meeting at a singular point $x_{e} \in X^{\prime}$ (like $\mathbb{P}_{w_{5}}^{1}$ and $\mathbb{P}_{z}^{1}$ meeting). Then, the local ring around that point is $\mathcal{O}_{R, x_{e}}=R\left[\left[u_{1}, u_{2}\right]\right] /\left(u_{e, 1} u_{e, 2}=t^{n_{e}}\right)$ for some well-determined $n_{e}$ where $u_{e, i}=u_{i}=0$ defines the two components, $i=1,2$. Fix $t_{0}<\epsilon$, and form a neighborhood $V_{e}$ using the coordinates $\left(u_{e, 1}, u_{e, 2}, t\right)$ to include the arc

$$
c_{e}: s \in[0,1] \mapsto\left(t_{0}^{n_{e} / 2 n} e^{2 \pi i s}, t_{0}^{n_{e} / 2 n} e^{-2 \pi i s}, t_{0}^{1 / n}\right) .
$$

Let $U_{t_{0}}=\mathbb{P}_{z}^{1} \backslash\left\{z_{1}\left(t_{0}^{1 / n}\right), \ldots, z_{r}\left(t_{0}^{1 / n}\right)\right\}$.

For a leaf edge $e$ take the clockwise path around $z_{i}$. For $v \in V(T) \backslash\left\{v_{0}\right\}$, set $e=(\Re(v), v) \in E(T)$ and let $U_{v}$ be the connected component of $U_{t_{0}} \backslash\left(\cup_{e^{\prime}} c_{e^{\prime}}\right)$ containing the annulus

$$
\left\{\left(u_{e, 1}, u_{e, 2}, t\right) \in V_{e}|| u_{1, e}|>| u_{2, e} \mid\right\} .
$$

This is the topological realization of the ordered tree.

Reminder of those details: We01, §2.3] produces the presentation compatible with a given graph. For $v \in V(T) \backslash\left\{v_{0}\right\}$, let the unique path $p_{v}$ connecting $v_{0}$ to $v$ be denoted $\left(v_{0}, v_{1}, \ldots, v_{k-1}, v\right)$. Let $e=\left(v_{k-1}, v\right), z_{v}=c_{e}(0)$ and $z_{v_{0}}=z_{0}$. Choose a simple path $a_{v}:[0,1] \rightarrow \bar{U}_{v}$ leading from $z_{v_{k-1}}$ to $z_{v}$. Let $b_{v}=a_{v_{1}} \cdots a_{v}$, and $\gamma_{v}=b_{v} c_{e} b_{v}^{-1}$ (an element of $\pi_{1}\left(U, z_{0}\right)$ ).

As usual, use classical generators $\gamma_{1}, \ldots, \gamma_{4}$, clockwise around the $z_{1}, \ldots, z_{4}$ from $z_{0}$. Add these paths: $\gamma_{5}=\gamma_{1} \gamma_{2}, \gamma_{6}=\gamma_{3} \gamma_{4}$. We act on the opposite side of Wewers, following the notation of 3.2 .1 So, $q_{0}$ acts as

$$
\gamma_{1}^{q_{0}}=\gamma_{1}^{-1} \gamma_{2} \gamma_{1}, \gamma_{2}^{q_{0}}=\gamma_{1}, \gamma_{3}^{q_{0}}=\gamma_{3}^{-1} \gamma_{4} \gamma_{3}, \gamma_{4}=\gamma_{3}^{q_{0}} .
$$

Then, with $\Pi_{5}=\left\langle\gamma_{1}, \gamma_{2}\right\rangle$ and $\Pi_{6}=\left\langle\gamma_{3}, \gamma_{4}\right\rangle$, and $\sigma \in G_{\mathbb{Q}}$,

$$
\gamma_{i}^{\sigma}=\beta_{(i) \sigma}^{-1} \gamma_{(i) \sigma}^{\chi(\sigma)} \beta_{(i) \sigma} \text { with } \beta_{i} \in \Pi_{5}, i=1,2 \text {, and } \beta_{i} \in \Pi_{6}, i=3,4 \text {. }
$$


The action of $\sigma$ on the subscripts of the $\beta_{i}$ s is the obvious one from our the action of $\sigma$ on the Puiseux expression of the paths in $S$. Further, with $\epsilon: G_{\mathbb{Q}} \rightarrow\{ \pm 1\}$, $\gamma_{i}^{\sigma}=\gamma_{i}^{\epsilon(\sigma) \chi(\sigma)}, i=5,6$.

We01, Lem. 2.19] shows that if $\ell$ is prime to $|G|$ and the branch locus doesn't degenerate at $\ell$, we may take $t_{0}$ in the maximal ideal of the valuation ring for $\mathbb{Q}_{\ell}$ and specialize all the actions. There would be two steps in the program corresponding to two parts of Serre's result. Still, we restrict to the case of H-M components for the $\left(A_{5}, \mathbf{C}_{3^{4}}, p=2\right)$ Modular Tower.

(D.3a) For $a=t_{0} \in \overline{\mathbb{Q}}$, giving $j\left(t_{0}\right)$ suitably $\ell$-adically close to $\infty$ draw deductions about the action of $\mathbb{Q}_{\ell}$ on $\boldsymbol{p} \in \mathcal{H}\left(G_{k}, \mathbf{C}_{3^{4}}\right)^{\text {in,rd }}$ for $\boldsymbol{p}$ lying over $j(a)$ in an $\mathrm{H}-\mathrm{M}$ component.

(D.3b) Estimate from this the $\ell$-adic domain in the $j$-line to which the one may analytically continue from $j(a)$ the Lie algebra action of $G_{\mathbb{Q}_{\ell}}$ on the $\ell$-adic Grassmanian of 4.4 .3

Toward (D.3tan), an accomplishment would be to differentiate between the action of $G_{\mathbb{Q}_{\ell}}$ on H-M reps. and on near H-M reps., exactly as we did with $G_{\mathbb{R}}$ at all levels of the Modular Tower in Prop. 6.8 and Wewers did $\ell$-adically at level 0 of the same Modular Tower.

\section{Appendix E. Simple branching and $j$-awareness}

Two situations about a Modular Tower require extending considerations of this paper. The most classical situation doesn't satisfy $p$-perfectness. E.1 discusses a reasonable remedy. Further, given a reduced Modular Tower $\left\{\mathcal{H}_{k}^{\text {rd }}\right\}_{k=0}^{\infty}$, some significant part of the tower may come from a tower of modular curves. While monodromy groups of the levels over the $j$-line often preclude this, it is difficult to compute the monodromy groups even should that be sufficient to set the matter straight. $\mathrm{E} .2$ illustrates by example the problems.

E.1. More complicated Hurwitz spaces for non- $p$-perfect $G_{0}$. The pairing of dihedral groups and modular curves generalizes neatly into Modular Towers to include, for example, simple groups and any prime that divides the order of such a group. Still, the most classical case of moduli of covers does not: simple branching $\left(S_{n}, \mathbf{C}_{2^{r}}, p=2\right)$ with $\mathbf{C}_{2^{r}}$ being $r \geq 2(n-1)$ involutions in $S_{n}$ with $n \geq 4$. Since $\varphi: S_{n} \rightarrow \mathbb{Z} / 2$ has kernel $A_{n}, S_{n}$ is not 2-perfect, and 2 divides the orders of the conjugacy classes. Fr01 explains this situation in more generality (as in Ex. 3.27).

The remedy, however, is clear. Regard each level 0 curve in the reduced Hurwitz space as coming with a map to a hyperelliptic curve. Then view the (ramified) $A_{n}$ cover of hyperelliptic curves (of genus $g=\frac{r-2(n-1)}{2}$ ) as the starting point for unramified projective systems of covers coming from the universal 2-Frattini cover of $A_{n}$. Prop. 5.9 applies to this situation extending its application to Modular Towers starting with covers of $\mathbb{P}_{z}^{1}$. Fr01 illustrates this with $n=5$ using just the group theory from this paper.

E.2. Modular curve quotients from a Modular Tower. Continue with $r=4$ as at the beginning of this section. Ex. 3.27 gives situations automatically implying the jacobian of $\hat{X}_{\boldsymbol{p}}, \boldsymbol{p} \in \mathcal{H}^{\text {rd }}$ maps through the elliptic curve with $j$ invariant $j(\boldsymbol{p})$. (This is equivalent to $\hat{X}_{\boldsymbol{p}}$ itself mapping onto this elliptic curve.) This is the situation we call $j$-aware. Even if $\hat{X}_{\boldsymbol{p}}$ maps through an elliptic curve, it 
may not be the elliptic curve with $j$-invariant $j(\boldsymbol{p})$. For a general Modular Tower the jacobians of the corresponding curves at level $k$ go up in rank very quickly. Given the rank $\mathrm{rk}_{0}$ of $\mathrm{ker}_{0}$, inductively compute the rank of $\operatorname{ker}_{k+1}$ as $\mathrm{rk}_{k+1}=$ $1+\left(\mathrm{rk}_{k}-1\right) p^{\mathrm{rk}_{k}}$ from the Shreier formula. Given the genus $g_{0}$ of $\hat{X}_{0}$ (a curve at level $0)$, computed from the branch cycles as a cover of $\mathbb{P}_{z}^{1}$, then $g_{k+1}-1=p^{\mathrm{rk}_{k}}\left(g_{k}-1\right)$. For $\left(A_{5}, \mathbf{C}_{3^{4}}, p=2\right), g_{0}=21$ and $\mathrm{rk}_{0}=5$, so $g_{1}=1+5 \cdot 2^{7}, \mathrm{rk}_{1}=1+2^{7}$, $g_{2}=1+5 \cdot 2^{14}, \mathrm{rk}_{2}=1+2^{1+7+2^{7}}, \ldots$

E.2.1. Extending Ex. 9.2, $₫$ continues the Nielsen class $\mathrm{Ni}\left(A_{4}, \mathbf{C}_{3_{+}^{2} 3_{-}^{2}}\right)$ from Ex. 9.2 We show level 0 is not a modular curve.

The natural map from modding out by the Klein 4-group in $A_{4}$ gives $A_{4} \rightarrow A_{3}$. Regard $C_{4}=( \pm 1)^{4} /\langle(-1,-1,-1,-1)\rangle$ as a multiplicative group of order 8. Any element of $\mathrm{Ni}\left(A_{4}, \mathbf{C}_{3_{+}^{2} 3_{-}^{2}}\right)$ (the level 0 Nielsen class) gives a symbol $\left(\epsilon_{1}, \ldots, \epsilon_{4}\right) \in C_{4}$ where two entries are +1 and two entries are -1 . Let $\mathcal{H}_{0}^{\text {rd, }+}$ be the H-M component of this reduced Hurwitz space at level 0 (as in Ex. 3.17). The absolute and inner (nonreduced) spaces, $\mathcal{H}_{0}^{\text {abs, }+}$ and $\mathcal{H}_{0}^{\text {in, },+}$, at level 0 , have a natural degree 2 map $\psi^{\text {in,abs }}: \mathcal{H}_{0}^{\text {in, }+} \rightarrow \mathcal{H}_{0}^{\text {abs, }+}$. The element $\mathbf{s h}^{2} q_{1} q_{3}^{-1} \in \mathcal{Q}^{\prime \prime}$ takes the 4 -tuple ((123), (1 3 2), (134), (143)) to the 4-tuple ((143), (134), (132), (123)). The latter is conjugation of the former by $(24)$. This is exactly why $\psi^{\text {in,abs }}$ has degree 2 (illustrating Thm. 3.16). Then, $\mathcal{H}_{0}^{\text {abs, }+}$ is a family of genus 1 curves, while the curves of $\mathcal{H}_{0}^{\mathrm{in},+}$ have genus $g_{\mathrm{in}, 0}$ with $2\left(12+g_{\mathrm{in}, 0}-1\right)=4 \cdot 4 \cdot 2: g_{\mathrm{in}, 0}=5$. Since, however, we mod out by $\mathcal{Q}^{\prime \prime}$ to get reduced Nielsen classes, this induces an isomorphism of $\mathcal{H}_{0}^{\text {in,rd, }+}$ with $\mathcal{H}_{0}^{\text {abs,rd, }+}$. As in $\$ 2.8 .3$ and Prop. 6.8 the spaces still represent different moduli problems. Neither reduced space is a fine (or even b-fine) moduli space according to Prop.4.7. Still, to simplify, refer to both as $\mathcal{H}_{0}^{\text {rd, }+ \text {. }}$.

Choose a basepoint $j_{0} \in(1,+\infty)$ from $\mathbb{P}_{j}^{1}(\mathbb{R})$ (as in Lem. 6.5). Then, $\bar{M}_{4}$ acts on allowable symbols from $C_{4}$, giving a cover $\mathcal{H}\left(A_{3}\right) \rightarrow \mathbb{P}_{j}^{1} \backslash\{\infty\}$ with this branch cycle description: $\gamma_{0}^{\prime}=(123), \gamma_{1}^{\prime}=(13)$ and $\gamma_{\infty}^{\prime}=(12)$. One H-M rep. $\boldsymbol{g}^{+-+-} \in$ $\operatorname{Ni}\left(A_{4}, \mathbf{C}_{3_{+}^{2} 3_{-}^{2}}\right)$ lies over $(+1,-1,+1,-1) \in C_{4}$ and and one over $(+1,-1,-1,+1)$, $\boldsymbol{g}^{+--+} \in \operatorname{Ni}\left(A_{4}, \mathbf{C}_{3_{+}^{2} 3_{-}^{2}}\right): \mathbf{m p}\left(\boldsymbol{g}^{+-+-}\right)=2$ while $\mathbf{m p}\left(\boldsymbol{g}^{+--+}\right)=3$ (as in 2.9 .3$)$.

With $(+1,-1,+1,-1)$ over $j_{0}$ as a basepoint for the cover $\mathcal{H}_{0}^{\text {rd, },+} \rightarrow \mathcal{H}\left(A_{3}\right)$, the fiber over this point has representatives

$$
\begin{aligned}
& \boldsymbol{g}_{1}=((123),(132),(134),(143)), \boldsymbol{g}_{2}=((123),(124),(134),(234)), \\
& \boldsymbol{g}_{3}=((123),(124),(142),(132)) .
\end{aligned}
$$

It is easy from this to form a branch cycle description for $\overline{\mathcal{H}}_{0}^{\mathrm{rd},+} \rightarrow \mathbb{P}_{j}^{1}$ :

$$
\begin{aligned}
\gamma_{0}^{\prime \prime} & =(1,6,9)(2,5,8)(3,4,7), \\
\gamma_{1}^{\prime \prime} & =(1,8)(2,7)(3,9)(4,6), \\
\gamma_{\infty}^{\prime \prime} & =(1,5,2,4)(3,6)(7,8,9) .
\end{aligned}
$$

The group generated by these has size 648 and is centerless. Denote $\left\langle\gamma_{0}^{\prime \prime}, \gamma_{1}^{\prime \prime}, \gamma_{\infty}^{\prime \prime}\right\rangle$ by $H_{0}$. As the geometric monodromy group of a chain of two degree 3 extensions, it would naturally be a subgroup of the wreath product $S_{3} 2 S_{3}$, as in the argument of Lem. 7.1 The full wreath product has order 1296, while the kernel for the projection $H_{0} \rightarrow S_{3}$ is the subgroup of $(a, b, c) \in S_{3}^{3}$ for which $a b c \in A_{3}$.

E.2.2. $\mathcal{H}\left(A_{4}, \mathbf{C}_{3_{+}^{2} 3_{-}^{2}}\right)^{\text {abs,rd, }+}$ is not a modular curve. Our problem was to decide, for a general cover $\varphi_{\boldsymbol{p}}: X_{\boldsymbol{p}} \rightarrow \mathbb{P}_{z}^{1}$ in this family, lying over $j(\boldsymbol{p})$, if the Galois closure $\hat{X}_{\boldsymbol{p}}$ of the cover could factor through the elliptic curve with $j$ invariant $j(\boldsymbol{p})$. 
This is equivalent to $\mathrm{Pic}^{(0)}\left(\hat{X}_{\boldsymbol{p}}\right)$ having that elliptic curve as an isogeny factor. The generality of the next argument in $r$ will appear in later applications. Let $K_{4}$ be the copy of the Klein 4-group in $A_{4}$.

Lemma E.1. For $\boldsymbol{p}$ generic, $\mathrm{Pic}^{(0)}\left(\hat{X}_{\boldsymbol{p}}\right)$ is isogenous to

$$
\operatorname{Pic}^{(0)}\left(\hat{X}_{\boldsymbol{p}} / K_{4}\right) \times \operatorname{Pic}_{0}\left(X_{\boldsymbol{p}}\right) \times E_{\boldsymbol{p}, 1} \times E_{\boldsymbol{p}, 2}
$$

with $\operatorname{Pic}^{(0)}\left(\hat{X}_{\boldsymbol{p}} / K_{4}\right)$ a simple abelian variety of dimension 2, and $E_{\boldsymbol{p}, 1}$ and $E_{\boldsymbol{p}, 2}$ Prym (elliptic) varieties for the hyperelliptic curve $\hat{X}_{\boldsymbol{p}} / K_{4}$.

Terms And outline of Proof. Riemann-Hurwitz shows $\hat{X}_{p} / K_{4}$ has genus 2. Vö95a, p. 167] shows for any $r \geq 4$, the general cyclic degree 3 triple cover with $r$ branch points has a simple Jacobian. This result applies to available for $A_{4}$ covers no matter the number of branch points. The proof considers $K$, the function field $\mathbb{Q}\left(U_{r}\right)$ of the branch point locus, with the field of definition for the generic cover $C \rightarrow \mathbb{P}_{z}^{1}$ and its group of automorphisms adjoined. Then, for $\ell \geq 5$ a prime, $G_{K}$ acts on the $\ell$-division points $\operatorname{Pic}(C)_{\ell}^{(0)}=J_{\ell}$ according to this recipe:

(E.1a) If $\ell \equiv 1 \bmod 3$ then $J_{\ell}=V \oplus V^{\prime}$ with $V$ and $V^{\prime}$ a standard isotropic decomposition with respect to the Weil pairing, $G_{K}$ fixing $V$ and $V^{\prime}$, inducing a group between $\mathrm{SL}_{r-2}(\mathbb{Z} / \ell)$ and $\mathrm{GL}_{r-2}(\mathbb{Z} / \ell)$; and

(E.1b) if $\ell \equiv-1 \bmod 3$, then $\operatorname{Aut}\left(C / \mathbb{P}_{z}^{1}\right)$ makes $J_{\ell}$ an $\mathbb{F}_{\ell^{2}}$ vector space, with $G_{K}$ inducing a group between $\mathrm{SU}_{r-2}(\mathbb{Z} / \ell)$ and $\mathrm{GU}_{r-2}(\mathbb{Z} / \ell)$.

Suppose $J(C)=J$ is not simple. Let $K_{1}$ be a finite extension over which $J$ is isogenous to a product of abelian varieties, so $G_{K_{1}}$ acts through a reducible normal subgroup of one of the simple groups occurring in (E.1). Then, the finite group $G_{K} / G_{K_{1}}$ would contain infinitely many nonisomorphic simple groups, according to the composition factors appearing in the cases of E.1.

Finally, the cover $\hat{X}_{\boldsymbol{p}} \rightarrow \hat{X}_{\boldsymbol{p}} / K_{4}$ is unramified, and for each copy $A$ of a $\mathbb{Z} / 2$ in $K_{4}, \hat{X}_{\boldsymbol{p}} / A \rightarrow \hat{X}_{\boldsymbol{p}} / K_{4}$ is a Prym cover. So, $\mathrm{Pic}^{(0)}\left(\hat{X}_{\boldsymbol{p}} / A\right)$ has a degree 2 isogeny to $\operatorname{Pic}^{(0)}\left(\hat{X}_{\boldsymbol{p}} / K_{4}\right) \times E_{A}$ with $E_{A}$ an elliptic curve (B.4.3 and Mu76, Lecture IV, p. 90-94]). If $A_{1}$ and $A_{2}$ generate $K_{4}$, then their associated elliptic curves $E_{i}$, $i=1,2$, give the curves appearing in the statement of the lemma. These isogeny factors account for $\operatorname{Pic}^{(0)}\left(\hat{X}_{\boldsymbol{p}}\right)$ up to isogeny.

Many different maps from $\mathrm{PSL}_{2}(\mathbb{Z})$ to $\mathrm{PSL}_{2}(\mathbb{Z} / n)$ induce projective systems of covers of the $j$-line having the same geometric monodromy as the projective system of curve covers called modular curves. Even, however, if some of these covers did have significant moduli problems describing them, they would not be the same as those describing modular curves. For example, having monodromy group $S_{3}=\mathrm{PSL}_{2}(\mathbb{Z} / 2)$ does not imply an upper half plane quotient is the $\lambda$-line cover of the $j$-line. That is the case with our space $\mathcal{H}^{\text {rd, }+}$.

Lemma E.2. The cover $\bar{\psi}^{\mathrm{rd}}: \overline{\mathcal{H}}^{\mathrm{rd},+} \rightarrow \mathbb{P}_{j}^{1}$ does not factor through $\mathbb{P}_{\lambda}^{1}$. So, $\mathcal{H}^{\mathrm{rd},+}$ is not a modular curve.

Proof. Since the moduli space $\mathcal{H}^{\mathrm{rd},+}$ is a family of $r=4$ branch point covers, Ex. 3.30 shows it does not satisfy the moduli interpretation for factoring through $\mathbb{P}_{\lambda}^{1}$. As the monodromy group has $S_{3}$ as a quotient, this means $\mathcal{H}^{\mathrm{rd},+} \rightarrow \mathbb{P}^{1} \backslash\{\infty\}$ is not a quotient of a modular curve. 
E.2.3. Inspecting if $\mathcal{H}^{\mathrm{rd},+}$ is $j$-aware. Suppose $g \in A_{4}$ defines the degree 2 cover $\hat{X}_{\boldsymbol{p}} /\langle g\rangle \rightarrow \hat{X}_{\boldsymbol{p}} / K_{4}$. The other degree two covers (for example, $E_{\boldsymbol{p}, 2}$ ) in Lem. E.1 have the form $\hat{X}_{\boldsymbol{p}} /\left\langle h g h^{-1}\right\rangle \rightarrow \hat{X}_{\boldsymbol{p}} / K_{4}$ with $h$ having order 3 . So, if for a generic $j$ value $\hat{X}_{\boldsymbol{p}} /\langle g\rangle$ is isogenous to $E_{\psi(\boldsymbol{p})}$, then so will be $\hat{X}_{\boldsymbol{p}} /\left\langle h g h^{-1}\right\rangle$. According to Lem. E.1 to decide if $\mathcal{H}^{\mathrm{rd},+}$ is $j$-aware requires answering the following question.

Problem E.3. For general $\boldsymbol{p} \in \mathcal{H}^{\mathrm{rd},+}$, is either $X_{\boldsymbol{p}}$ or $E_{\boldsymbol{p}, 1}$ isogenous to the elliptic curve with $j$-invariant $\psi(\boldsymbol{p})$.

We leave this question after the following observations. Since the question is for general $\boldsymbol{p}$, and we know from [FKK01, Main Thm] we may assume $X_{\boldsymbol{p}}$ has no complex multiplication. The Riemann-Roch Theorem for genus 1 curves gives the dimension of linear systems of 2-division points as the dimension of $H^{0}\left(\kappa_{\boldsymbol{p}}-D_{\varphi_{\boldsymbol{p}}}\right)$ where $\kappa_{p}$ is the canonical class, which on a genus 1 curve is trivial. This dimension is 1 (odd) if $D_{\varphi_{p}}$ is linearly equivalent to 0 , and 0 (even) if not. So, according to Prop. B.1 the linear equivalence class of $D_{\varphi_{\boldsymbol{p}}}$ defines a non-trivial $\mathbb{Q}(\boldsymbol{p})$ 2-division point on $\operatorname{Pic}^{0}\left(X_{\boldsymbol{p}}\right)$.

\section{Appendix F. A representation difference between $\bar{M}_{4}$ on $\mathrm{Ni}_{1}^{+}$and $\mathrm{Ni}_{1}^{-}$}

The second author saw an S. Sternberg talk in Jerusalem based on GKS99. In that talk, three semi-simple lie group representations had appeared as associated with the existence of elementary particles, and the point was to see them as a natural multiplet. The shape of the final result was this.

They have $r \subset g$ two complex Lie algebras with $g$ semi-simple, $r$ reductive and both having the same Carton subalgebra $h$. So the following properties hold.

- $r$ and $g$ have the same rank.

- The Weyl group of $r$ is a subgroup of the Weyl group of $g$.

- With a choice of positive roots for each assume the positive Weyl chamber of $g$ is in the positive Weyl chamber of $r$.

- The killing form on $g$ gives an orthogonal splitting as $h \oplus$ po.

The last item allows embedding $r$ in the orthogonal Lie algebra acting on po. Further, this embedding has two representations $S^{+}$and $S^{-}$associated with the plus and minus representations of the orthogal Lie algebra on the even and odd part of the Clifford algebra for the orthogonal product (as in Prop. 5.10). Consider the elements $C_{g / r}$ of the Weyl group of $g$ that map the positive Weyl chamber of $g$ into the positive Weyl chamber of $r$. The highest weights $\lambda$ in the positive Weil chamber of $g$ correspond to irreducible representations $V_{\lambda}$ of $g$ [Fu92 p. 49].

In the Grothendieck group of $r$ representations, form $V_{\lambda} \otimes S^{+}-V_{\lambda} \otimes S^{-}$as a representation of $r$. Linear algebra magic occurs here: For each $c \in C$, GKS99 constructs a highest weight for $r$ from the pair $(c, \lambda)$. Denote the corresponding representation by $U_{c, \lambda}$. Their formula is

$$
V_{\lambda} \otimes S^{+}-V_{\lambda} \otimes S^{-}=\oplus_{c \in C} \pm U_{c, \lambda}
$$

with \pm indicating some choice of sign.

The analogy for us starts with a group $\bar{M}_{4}=\mathrm{SL}_{2}(\mathbb{Z})$, and $\boldsymbol{g}_{0} \in \mathrm{Ni}\left(A_{5}, \mathbf{C}_{3^{4}}\right)$ representing an H-M rep. at level 0 . Let $M_{\boldsymbol{g}_{0}}$ be the subgroup of $\bar{M}_{4}$ stabilizing the reduced class of $\boldsymbol{g}_{0}$. Then, Prop. 9.8 gives two representations $S^{+}$and $S^{-}$of $H_{\boldsymbol{g}_{0}}$, respectively corresponding to the actions on the sets of (9.5) and (9.6). The analogy would go further if there was a representation $V_{\lambda}$ of $\bar{M}_{4}$ that fit in the 
formula $V_{\lambda} \otimes S^{+}-V_{\lambda} \otimes S^{-}$to give the difference between the two representations on (9.5) and (9.6). The analogy misses a lot, though we can easily construct $p$-adic Lie algebras from the projective situation. Since there are infinitely many levels of a Modular Tower, our goal would be to understand if there are such paired representations at higher levels of the Modular Tower. This would give a direct translation of the Clifford (Spin) invariant of Prop. 9.8 and its relation to $\theta$-nulls for each component of $\mathcal{H}_{1}=\mathcal{H}\left({ }_{2}^{1} \tilde{A}_{5}, \mathbf{C}_{3^{4}}\right)^{\mathrm{in}, \mathrm{rd}}$ (as in $\sqrt{\mathrm{B}}$ ).

\section{Appendix G. Where are those regular realizations?}

Let $\left\{G_{k}\right\}_{k=0}^{\infty}$ the characteristic quotients of the universal $p$-Frattini cover ${ }_{p} \tilde{G}$ of a $p$-perfect group $G=G_{0}$. Our contributions to Main Conjecture (FK97, Main Conj. 0.1], Prob. 1.1 6.1.2 with $r \leq 4$ branch points suggest that no bounded set of branch points will allow regular realizations over a fixed number field of the complete set $\left\{G_{k}\right\}_{k=0}^{\infty}$.

Further, suppose for some $r_{0} \geq 1$, there are realizations of $\left\{G_{k}\right\}_{k=0}^{\infty}$, each requiring no more than $r_{0}$ branch points. Then, Thm. 6.1 says there must exist a Modular Tower for $\left(G_{0}, \mathbf{C}, p\right)$ with $\mathbf{C}$ a set of $p^{\prime}$ conjugacy classes having cardinality $r \leq r_{0}$, and rational points at every level. The tower levels are manifolds. So, having $\mathbb{Q}$ points at every level implies the Modular Tower levels have a projective system of $\mathbb{Q}$ components. [Fri95a Thm. 3.21] shows that if there is just one absolutely irreducible H-M component at every level of a $\mathbb{Q}$ Modular Tower, then this will be defined over $\mathbb{Q}$. There are variants on this, though this is the clearest case establishing this necessary condition. Further, assume $\mathrm{Ni}\left(G_{0}, \mathbf{C}\right)$ contains $\mathrm{H}-\mathrm{M}$ reps. For any integers $1 \leq i<j \leq r$, call $(i, j)$ an $\mathrm{H}-\mathrm{M}$ pair if $\mathrm{C}_{i}$ is inverse to the class $\mathrm{C}_{j}$. In this situation let $\mathbf{C}_{i, j}$ be $\mathbf{C}$ with $\mathrm{C}_{i}$ and $\mathrm{C}_{j}$ removed. The only successful hypotheses guaranteeing one $\mathrm{H}-\mathrm{M}$ component at every level are mildly variant on the other half of Fri95a Thm. 3.21]. There will be just one H-M component if these (H-Mgcomplete) hypotheses hold:

(G.1) For each H-M pair $(i, j)$, and $\boldsymbol{h} \in \mathbf{C}_{i, j},\left\langle h_{1}, \ldots, h_{r-2}\right\rangle=G_{0}$.

For example, this would hold if for each H-M pair $(i, j), \mathbf{C}_{i, j}$ contains every nontrivial conjugacy class of $G_{0}$.

There are results that encourage using H-Mgcomplete reps.: For any prime $\ell$, DDes01 Thm. 4.2] finds a $\mathbb{Q}_{\ell}$ regular realization of ${ }_{p} \tilde{G}$ using the H-Mgcomplete condition. The suggested generalization of the Open Image Theorem in $\$ \mathrm{D} .2$ would apply especially well to a sequence of H-Mgcomplete components. Reminder: Shafarevich's method with solvable groups does not give regular realizations. Only some version of the braid rigidity method has systematically produced regular realizations, and going beyond solvable groups the story has been regular realizations all the way. That even includes Shih's results using modular curves: These came from the first version of the method the second author told Shimura when he first worked it out at the Institute for Advanced Study at Princeton in 1967-69. We consider Modular Towers a general invention, extending through the analogy with modular curves, the potential for many applications. What, however, of someone who wants such regular realizations? Does this say anything of the next reasonable step in the search for them?

G.1. Reminder of the setup. For a large integer $k$, where would one find a regular realization of $G_{k}$. From Thm. 6.1 combined with the conclusion of the Main Conjecture, you will need many branch points, and so you need a model for 
where to get an indication of how many, and what kind of conjugacy classes (with elements of order divisible by $p$ ) you will use.

When $G_{0}$ is $p$-split, with cyclic $p$-Sylow, it is tempting to imitate realization of dihedral groups. No one knows how to get regular involution realizations of dihedral groups (when $r=4$, the moduli of these relates special Hurwitz spaces and modular curves 2.8 .2 . Yet, any elementary algebra book shows how to get regular realizations of dihedral groups. You must use $p$-cycles as branch cycles, and you require many branch points (see the implications of this elementary analysis in Fri94 or Fri95c). Yet, for a general finite group $G$ and prime $p$, no one has produced similar branch cycles. Results from 55.7 show divide the case where $M_{0}$ is cyclic from those where it is not. We refer to the former case as dihedral-like. It is the latter case that is serious. We consider finding such branch cycles when the situation is not dihedral-like, our basic assumption from here. So, we are seeking dihedral-like realizations in non-dihedral-like situations.

Recorded in FV91 is the following possibility: Given $\left(G_{0}, \mathbf{C}, p\right)$, there might be infinitely many $\mathbf{C}^{\prime}$ formed by suitably increasing the multiplicity of appearance of conjugacy classes in $\mathbf{C}$, so that $\mathcal{H}\left(G_{k}, \mathbf{C}\right)^{\text {in }}$ will be a uni- rational variety. (That result would make a mockery of the inverse Galois problem.) Our replacement would consider using special realizations at level 0, formed, say, from understanding simple groups. We acknowledge the Main Conjecture might be false; it has phenomenal implications for $r \geq 5$ (see the comments about [FKVo99 in 3.4.2).

G.2. From Chevalley realizations to the whole $p$-Frattini tower. An approach of Thompson-Völklein (TVo99 has an example, or the Thompson tuples of Ex. 3.14) uses intricate knowledge of Chevalley simple groups to locate conjugacy classes that show some Chevalley series over each finite field $\mathbb{F}_{q}$ has regular realizations excluding finitely many $q$. This has worked for many series, extending Völklein's production of high rank Chevalley group realizations. (Prior to his approach, a less abstract use of the main idea of [FV92, there had been essentially no higher rank realizations.) We consider as a starting point some successful realization cases where the Hurwitz space $\mathcal{H}\left(G_{0}, \mathbf{C}\right)$ in is uni-rational (it might even be an abelian cover of $\mathbb{P}^{r} \backslash D_{r}$ as in the Thompson-Völklein examples). Take a prime $p$ not dividing the orders of elements in $\mathbf{C}$.

Form, for each $k$, a sequence $\mathbf{C}_{k}$ of conjugacy classes, using the following principles. Reminder: Def. 3.5 explains rationalization of any conjugacy classes $\mathbf{C}^{\prime}$.

(G.2a) $\mathbf{C}_{k}=\left(\mathbf{C}, \mathbf{C}_{k}^{\prime}\right)$ is a rational union of conjugacy classes in $G_{k}$ with $\mathbf{C}_{k}^{\prime}$ (possibly empty) conjugacy classes whose elements have $p$ power order, $\mathbf{C}$ are $p^{\prime}$ classes, $\mathbf{C}_{k}^{\prime}$ are conjugacy classes of $p$-power order, and $\mathcal{H}\left(G_{0},\left(\mathbf{C}, \mathbf{C}_{0}^{\prime}\right)\right)^{\text {in }}$ is a rational (or uni-rational) variety over $\mathbb{Q}$.

(G.2b) $\mathbf{C}_{k}^{\prime}$ consists of $\left(\mathbf{C}_{k}^{*}, \mathbf{C}_{k}^{* *}\right)$ where $\mathbf{C}_{k}^{*}$ consists of the rationalization of $\mathbf{C}_{k-1}^{\dagger}$ whose terms consist of a lift to $G_{k}$ of the conjugacy class for $G_{k-1}$ for each member of $\mathbf{C}_{k}^{\prime}$, and $\mathbf{C}_{k}^{* *}$ is a rational union of conjugacy classes (for $G_{k}$ ) in $\operatorname{ker}\left(G_{k+1} \rightarrow G_{k}\right)=M_{k}$.

Since $G_{k} \rightarrow G_{0}$ is a Frattini cover, any lifts of generators of $G_{0}$ to $G_{k}$ generate $G_{k}$. So, as with the universal $p$-Frattini cover, it is only the product 1 condition that governs the possibility of braid orbits. There will be no obvious minimal choice. 
Given (nontrivial) $h \in \mathbf{C}_{k-1}^{\prime}$, any lift $\hat{h}$ to $G_{k}$ will have $p$ times the order of $h$ (Lem. 2.23). Further, since the situation is not dihedral-like, there will be many $G_{k-1}$ orbits on the collection of lifts of $h$. We use the notation established in the rest of the paper for Nielsen classes at levels of a Modular Tower.

Example G.1 (Start from $\left(A_{n}, \mathbf{C}_{3^{n-1}}, p=2\right)$ ). Consider $\mathbf{C}_{1}=\left(\mathbf{C}_{3^{n-1}}, \mathbf{C}_{1}^{\prime}\right)$ with $\mathrm{C}_{1}^{\prime}$ the conjugacy class of an element $c$ in $M_{0}=M_{0}\left(A_{n}\right)$. For $\operatorname{Ni}\left(G_{1}, \mathbf{C}_{1}\right)$ to be nonempty, the image of $c$ in $\operatorname{ker}\left(\operatorname{Spin}_{n} \rightarrow A_{n}\right)$ must be $(-1)^{n-1}$ (see Ex. 3.17). When $n=5$ we know this is sufficient to guarantee $\operatorname{Ni}\left(G_{1}, \mathbf{C}_{1}\right)$ is nonempty; the argument right after Princ. 5.13. (For $n=5$, there are two conjugacy class choices for such $c$, and several reasons (like Prop. 9.8) to see them as significantly different, though not for this particular obstruction.) For, however, $n \geq 6$, appearance of $\mathbf{1}_{A_{n}}$ beyond the first Loewy layer of $M_{0}$ might obstruct $\mathrm{Ni}\left(G_{1}, \mathbf{C}_{1}\right)$ from being nonempty for some choices of conjugacy class $\mathrm{C}_{1}$. That is, we don't know for certain if $\left\{\Pi(\boldsymbol{g}) \mid \boldsymbol{g} \in \mathrm{Ni}\left(G_{1}, \mathbf{C}_{1}\right)\right\}$ fills out all conjugacy classes lying over the conjugacy class of $(-1)^{n-1}$ in $\operatorname{Spin}_{n}$.

Assume the non-dihedral-like case, with the usual $p$-perfect assumption, and consider the list (G.2). The next problem asks if it is possible to remove choices after level 1 and still be certain higher level Nielsen classes are nonempty.

Problem G.2. Is it always possible to find some $\mathbf{C}_{1}$ so the following holds. For $k \geq 2$, with $\mathbf{C}_{k}^{* *}$ empty and any choice of a single lift of each class in $\mathbf{C}_{k-1}^{\prime}$ to $G_{k}$ to give $\mathbf{C}_{k-1}^{\dagger}$ and its rationalization $\mathbf{C}_{k}^{*}$, then $\mathrm{Ni}\left(G_{k}, \mathbf{C}_{k}\right)$ is nonempty?

\section{References}

[Ah79] L. Ahlfors, Introduction to the Theory of Analytic Functions of One, 3rd edition, Inter. Series in Pure and Applied Math., McGraw-Hill Complex Variable, 1979.

[AW67] M.F. Atiyah and C.T.C. Wall, Cohomology of groups, vol. Algebraic Number Theory, pp. 94-115, Thompson Book Co. and Academic Press, 1967, Editor J. W. S. Cassels.

[Ben91] D.J. Benson, I: Basic representation theory of finite groups and associative algebras, Cambridge Studies in advanced math., vol. 30, Cambridge U. Press, Cambridge, 1991.

[BF98] G. Berger and M. Fried, Rational cusps on noncongruence towers of the $j$-line, preprint (Sept. 1998), 1-28.

[BF82] R. Biggers and M. Fried, Moduli spaces of covers and the Hurwitz monodromy group, Crelles Journal 335 (1982), 87-121.

[Bi75] J. Birman, Braids, links and mapping class groups, Based on notes of J. Cannon: Princeton U. Press 82, 1975.

[Boh47] F. Bohnenblust, The algebraical braid group, Ann. of Math. (2) 48 (1947), 127-136.

[Ch44] F. Châtelet, Variations sur un thème de Poincaré, Annales ENX $3^{e}$ série 61 (1944), 249-300.

[CoH85] K. Coombes and D. Harbater, Hurwitz families and arithmetic Galois groups, Duke Math. J. 52 (1985), 821-839.

[CT88] J. Colliot-Thélene, Les Grands thèmes de François Châtelet, L'Ens. Math. 34 (1988), 387-405.

[DF90] P. Dèbes and M. Fried, Rigidity and real residue class fields, Acta Arith. 56 (1990), $13-45$.

[DF90b] P. Dèbes and M. Fried, Arithmetic variation of fibers in families: Hurwitz monodromy criteria for rational points ..., Crelles J. 409 (1990), 106-137.

[Deb95] P. Dèbes, Covers of $\mathbb{P}^{1}$ over the $\mathbb{Q}_{p}$-adics, Cont. Math. AMS, Recent Developments in the Inverse Galois Problem, Seattle 1995, Editors M. Fried, et. al., 186, 217-238.

[DF99] P. Dèbes and M. Fried, Integral specialization of families of rational functions, PJM 190 (1999) 45-85 (typos corrected at www.math.uci/ ${ }^{\sim}$ fried/\#math).

[Deb01] P. Dèbes, Descent Theory for Algebraic Covers, this volume. 
[DDes01] P. Dèbes et B. Deschamps, Corps $\psi$-libres et théorie inverse de Galois infinie, preprint as of October, 2001.

[DDE00] P. Dèbes, J.-C. Douai and M. Emsalem, Familles de Hurwitz et Cohomlogies non Abélienne, Annales de l'institut Fourier, 50 (2000), 113-149.

[Fal83] G. Faltings, Endlichkeitssätze über Zahlkörpern, Invent. Math. 73 (1983), 349-366.

[FaK01] H. Farkas and I. Kra, Theta Constants, Riemann Surfaces and the Modular Group, AMS graduate text series $\mathbf{3 7}, 2001$.

[Fay73] J. Fay, Theta Functions on Riemann Surfaces, Lecture notes in Mathematics 352, Springer Verlag, Heidelberg, 1973.

[FKVo99] G. Frey, E. Kani and H. Völklein, Curves with infinite K-rational geometric fundamental group, in: Aspects of Galois Theory, edts. H. Voelklein et. al., London Math. Soc. Lecture Notes 256, Cambridge Univ. Press (1999), 85-118.

[FJ86] M. Fried and M. Jarden, Field arithmetic, Ergebnisse der Mathematik III, vol. 11, Springer Verlag, Heidelberg, 1986.

[FK97] M. Fried and Y. Kopeliovic, Applying Modular Towers to the inverse Galois problem, Geometric Galois Actions II Dessins d'Enfants, Mapping Class Groups and Moduli, vol. 243, Cambridge U. Press, 1997, London Math. Soc. Lecture Notes, pp. 172-197.

[FKK01] M. Fried, E. Klassen, Y. Kopeliovic, Realizing alternating groups as monodromy groups of genus one covers, PAMS 129 (2000), 111-119.

[Fri70] M. Fried, On a conjecture of Schur, Mich. Math. J. 17 (1970), 41-55.

[Fri75] M. Fried, Brauer groups and Jacobians, Preprint from a talk at Oberwolfach Alg. No. Theory session, July 1975.

[Fri77] M. Fried, Fields of definition of function fields and Hurwitz families and groups as Galois groups, Communications in Algebra 5 (1977), 17-82.

[Fri78] M. Fried, Galois groups and Complex Multiplication, Trans.A.M.S. 235 (1978) 141-162.

[Fri89] M. Fried, Combinatorial computation of moduli dimension of Nielsen classes of covers, Cont. Math., vol. 89, pp. 61-79, AMS, Providence, RI, 1989.

[Fri90] M. Fried, Arithmetic of 3 and 4 branch point covers: A bridge provided by noncongruence subgroups of $\mathrm{SL}_{2}(\mathbb{Z})$, Progress in Math. Birkhauser 81 (1990), 77-117.

[Fri94] M. Fried, review-Topics in Galois Theory, J.-P. Serre, BAMS 30 \#1 (1994), $124-135$.

[Fri95a] M. Fried, Introduction to Modular Towers: Generalizing the relation between dihedral groups and modular curves, Proceedings AMS-NSF Summer Conference, vol. 186, 1995, Cont. Math series, Recent Developments in the Inverse Galois Problem, pp. 111-171.

[Fri95b] M. Fried, Extension of constants, rigidity, and the Chowla-Zassenhaus conjecture, Finite Fields and their applications; Carlitz Volume 1 (1995), 326-359.

[Fri95c] M. Fried, Enhanced review: Serre's Topics in Galois Theory, Proceedings of the Recent developments in the Inverse Galois Problem conferenc, AMS Cont. Math. 186 (1995), $15-32$.

[Fri96] M. Fried, Alternating groups and lifting invariants, Preprint 07/01/96 (1996), 1-34.

[Fri99] M. Fried, Separated variables polynomials and moduli spaces, Number Theory in Progress (Berlin-New York) (J. Urbanowicz K. Gyory, H. Iwaniec, ed.), Walter de Gruyter, BerlinNew York (Feb. 1999), Proceedings of the Schinzel Festschrift, Summer 1997, pp. 169228, http://www.math.uci.edu/ $\sim$ mfried/\#math.

[Fr01] M. Fried and D. Semmen, Relatively nilpotent algebraic extensions and work of Abel, Galois and Riemann, in preparation.

[Fr02] M. D. Fried, Moduli of relatively nilpotent extensions, Institute of Mathematical Science Analysis 1267, June 2002, Comm. in Arithmetic Fundamental Groups, 70-94.

[FV91] M. Fried and H. Völklein, The inverse Galois problem and rational points on moduli spaces , Math. Annalen 290 (1991), 771-800.

[FV92] M. Fried and H. Völklein, The embedding problem over an Hilbertian-PAC field, Annals of Math 135 (1992), 469-481.

[Fu92] J. Fuchs, Affine Lie algebras and quantum groups, Cambridge Univ. Press, Monographs on Mathematics physics, 1992.

[GB68] P. Gillette and van Buskirk, The word problem and its consequences for the braid groups and mapping class groups of the 2-sphere, TAMS 131 No. 2 (1968), 277-296.

[GS78] R. Griess and P. Schmid, The Frattini module, Archiv. Math. 30 (1978), 256266.

[GKS99] B. Gross, B. Kostant and S. Sternberg, Gainesville Lectures on Kostant's Dirac Operator, by Shlomo Sternberg, Feb. 23-26, 1999. 
[Gu67] R.C. Gunning, Special Coordinate Covering of Riemann Surfaces Math. Ann. 170 (1967), 67-86.

[Ha63] M. Hall, The theory of groups, Macmillan, 1963.

[Har77] R. Hartshorne, Algebraic Geometry, Graduate Texts in Math. 52, Springer-Verlag, 1977.

[Ha80] D. Harbater, Deformation theory and the tame fundamental group, TAMS, 262 (1980), 399-415.

[Ha84] D. Harbater, Mock covers and Galois extensions, J. Algebra 91 (1984), 281-293.

[Iha86] Y. Ihara, Profinite braid groups, Annals of Math. 123 (1986), 43-106.

[Iha91] Y. Ihara, Braids, Galois groups and some arithmetic functions, Proceedings of the International Congress, vol. Kyoto 1990, pp. 99-120, Springer-Verlag, Tokyo, 1991.

[Iha00] Y. Ihara, Shimura curves over finite fields and their rational points, Cont. Math., proceedings of AMS-IMS-SIAM Summer Conference 1997 on Applications of Curves over finite fields, Cont. Math. 245 (1999), 15-23.

[IM95] Y. Ihara and M. Matsumoto, On Galois actions on profinite completions of braid groups, Proceedings AMS-NSF Summer Conference, vol. 186, 1995, Cont. Math series, Recent Developments in the Inverse Galois Problem, 173-200.

[Ja99] M. Jarden, The projectivity of the fundamental group of an affine line, Turk. J. Math. 23 (1999), 531-547.

[Je41] S.A. Jennings, The structure of the group ring of a p-group over a modular field, TAMS 50 (1941), 175-185.

[KMS66] A. Karrass, W. Magnus and D. Solitar, Combinatorial group theory, Interscience Publishers, J. Wiley and Sons, 1966.

[Ma34] W. Magnus, Über Automorphismen von Fundamentalgruppen berandeter Flächen, Math. Ann. 109, 1934, 617-646.

[Mar45] A. Markoff, Foundations of the algebraic theory of tresses, Trav. Inst. Math. Steklov, vol. 16, Steklov, Moscow, 1945.

[MM99] G. Malle and B.H. Matzat, Inverse Galois Theory, ISBN 3-540-62890-8, Monographs in Mathematics, Springer,1999.

[Mes90] J.-F. Mestre, Extensions régulières de $\mathbb{Q}(t)$ de groupe de Galois $\tilde{A}_{n}$, J. of Alg. 131 (1990), 483-495.

[Mes94] J.-F. Mestre, Annulation, par changement de variable, d'éléments de $\mathrm{Br}_{2}(k(x))$ ayant quatre pǒles, CR. Acad. Sci., Paris 319 (1994), 781-782.

[Moc96] S. Mochizuki, A theory of ordinary p-adic curves, Publ. RIMS 32 \#6 (1996), 957-1151.

[Mu66] D. Mumford, Introduction to Algebraic Geometry; The Red Book, Harvard Lecture Notes, 1966.

[Mu72] D. Mumford, An analytic construction of degenerating curves over complete local rings, Comp. Math. 24 (1972), 129-174.

[Mu76] D. Mumford, Curves and their Jacobians, Progress in Mathematics, Univ. of Mich. Press, Ann Arbor, 1976.

[Nor62] D. G. Northcott, An introduction to homological algebra, Camb. U. press, 1962.

[Na98] H. Nakamura, Tangential base points and Eisenstein series, preprint, 1998, 1-16.

[Qu68] D.G. Quillen, On the associated graded ring of a group ring, J. Alg. 10 (1968), 411-418.

[Rig96] L.T. Rigatelli, Evariste Galois: 1811-1832, Vol. 11, translated from the Italian by John Denton, Vita Mathematica, Birkhäuser, 1996.

[Rob73] A. Robert, Elliptic curves, Lecture Notes, vol. 326, Springer Verlag, Heidelberg, 1973; second edition 1986.

[S+95] Martin Schönert et al. GAP - Groups, Algorithms, and Programming. Lehrstuhl D für Mathematik, Rheinisch Westfälische Technische Hochschule, Aachen, Germany, fifth edition, 1995.

[Se04b] D. Semmen, Jennings' theorem for p-split groups, J. Alg. 285 (2005), 730-742.

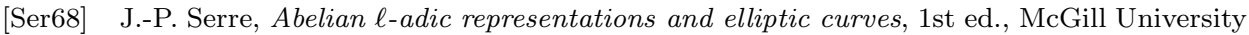
Lecture Notes, Benjamin, New York • Amsterdam, 1968, in collaboration with Willem Kuyk and John Labute.

[Ser72] J.-P. Serre, Propriétés galoisiennes des points d'ordre fini des courbes elliptiques, Inv. Math. 15 (1972), 259-331.

[Ser77] J.-P. Serre, Arbres, amalgames, SL 2 , vol. 46, Astérisque, New York, Amsterdam, 1977.

[Ser90a] J.-P. Serre, Relêvements dans $\tilde{A}_{n}$, C. R. Acad. Sci. Paris 311 (1990), 477-482. 
[Ser90b] J.-P. Serre, Revêtements a ramification impaire et thêta-caractéristiques, C. R. Acad. Sci. Paris 311 (1990), 547-552.

[Ser92] J.-P. Serre, Topics in Galois theory, no. ISBN \#0-86720-210-6, Bartlett and Jones Publishers, notes taken by H. Darmon, 1992.

[Ser96] J.-P. Serre, Galois Cohomology, 4th Edition, Springer: Translated from the French by Patrick Ion; original from Springer LN5 (1964), 1996.

[Sh01] A. Shalev, Asymptotic Group Theory, Notices of the AMS April 2001, 383-389.

[Sh94] G. Shimura, Introduction to the arithmetic of automorphic functions, Publications of Math. Soc. Japan, Princeton U. Press, 2nd Edition, First printing 1971, 1994.

[Sh98] G. Shimura, Abelian Varieties with Complex Multiplication and Modular Functions, Princeton U. Press, 1998.

[Sp57] G. Springer, Introduction to Riemann Surfaces, Addison-Wesley Mathematics Series 11, 1957.

[St94] M. Steinberger, Algebra, PWS publishing, 1994.

[Ta58] J. Tate, WC-groups over p-adic fields, Sém. Bourbaki, Exposé 156, 1957-1958.

[TVo99] J.G. Thompson, and H. Völklein, Braid abelian tuples in $\operatorname{Sp}_{n}(K)$, in: Aspects of Galois Theory, edts. H. Voelklein et. al., London Math. Soc. Lecture Notes 256, Cambridge Univ. Press (1999), 85-118.

[Vö95a] H. Völklein, Cyclic covers of $\mathbb{P}^{1}$ and Galois action on their Division Points, Proceedings AMS-NSF Summer Conference, vol. 186, 1995, Cont. Math series, Recent Developments in the Inverse Galois Problem, 91-107.

[Vö96] H. Völklein, Groups as Galois Groups 53, Cambridge Studies in Advanced Mathematics, Camb. U. Press, Camb. England, 1996.

[Vö01] H. Völklein, Galois realizations of profinite projective linear groups, this volume.

[We56] A. Weil, The field of definition of a variety, Amer. J. Math. 78 (1956), 509-524.

[We98] S. Wewers, Construction of Hurwitz spaces, Thesis, Institut für Experimentelle Mathematik 21 (1998), 1-79.

[We01] S. Wewers, Field of moduli and field of definition of Galois covers, this volume.

UC Irvine, Irvine, CA 92697, USA

E-mail address: pbailey@math.uci.edu

UC Irvine, Irvine, CA 92697, USA

E-mail address: mfried@math.uci.edu 